\title{
FLOOR TILE AND MASTIC REMOVAL PROJECT REPORT
}

NOVEMBER 1992

Through The

TULSA DISTRICT, CORPS OF ENGINEERS 


\section{DISCLAIMER}

This report was prepared as an account of work sponsored by an agency of the United States Government. Neither the United States Government nor any agency thereof, nor any of their employees, make any warranty, express or implied, or assumes any legal liability or responsibility for the accuracy, completeness, or usefulness of any information, apparatus, product, or process disclosed, or represents that its use would not infringe privately owned rights. Reference herein to any specific commercial product, process, or service by trade name, trademark, manufacturer, or otherwise does not necessarily constitute or imply its endorsement, recommendation, or favoring by the United States Government or any agency thereof. The views and opinions of authors expressed herein do not necessarily state or reflect those of the United States Government or any agency thereof. 


\section{DISCLAIMER}

Portions of this document may be illegible in electronic image products. Images are produced from the best available original document. 


\section{INTER AGENCY FUNDED NON-FRIABLE ASBESTOS DEMONSTRATION PROGRAM SUPPORTED BY THE ASBESTOS DEVELOPMENT AND DEMONSTRATION INITIATIVES GROUP (ADDIG) THROUGH THE TULSA DISTRICT CORPS OF ENGINEERS}

\section{FIOOR TIIB IND WASTIC RELOVAL PROJBCT GUYIARY RBPORT BEPTEIMBR 1992}

\section{I - BUMMARY OF RESULTS}

A test program was developed and coordinated with state and Federal Regulators and carried out at Fort Sill, oklahoma. This program was carefully designed to create the worst conditions in order to evaluate whether asbestos fibers are released when asbestos containing floor tile and mastic are removed. There were over 1,000 samples taken and analyzed during the execution of the program. The conclusions reached were based upon analysis of the critical samples using the Transmission Electron Microscope (TEM) technology. Additionally, the TEM procedures were used to evaluate personnel samples to determine whether those fibers found were asbestos or other materials. Most of the (TEM) samples were analyzed by the U.S. Environmental Protection Agency (EPA) Risk Reduction Engineering Laboratory in cincinnati, Ohio. Based upon all data, the following are evident:

1. Based upon the current occupational Safety and Health Administration (OSHA) regulations, the action level of 0.1 regulated fibers or structures per cubic centimeter ( $f / c c$ or s/cc) was never approached. To be regulated, current OSHA regulations require that a fiber must have both 1) a length to width ratio of at least 3 to 1 and 2) a length greater than 5 
microns. The substantial increase in unregulated fiber concentrations is noted as scientific information.

2. Soaking of floor tile with amended water reduces nuisance dust and loosens the tile which makes removal of floor tile easier.

clarification of Floor Tile in Good condition.

In association with this study, a clarification of the requirements of the Asbestos National Emission Standards for Hazardous Air Pollutants (NESHAP) was discussed with representatives of the Federal EPA. Floor tile and mastic that are in good condition and are not friable, and contain more than 1 percent asbestos are classified as Category I Asbestos Containing Materials (ACM) under the Asbestos NESHAP.

\section{Demolition of Buildings Involving Floor Tile and Mastic}

Floor tile and mastic that are Category I ACM do not have to be removed before demolition unless it is in poor condition and is friable. "In poor condition" means that the binding of the material is loosing its integrity as indicated by peeling, cracking, or crumbling of the material. "Friable" means that the material can be crumbled, pulverized, or reduced to powder by hand pressure. The waste that results from the demolition of a building which contains floor tile and mastic that is not friable is not regulated by the Asbestos NESHAP when using normal demolition methods. Normal demolition methods of waste collection would include picking the waste material up with a front end loader or other similar device to load into trucks, 
pushing the material into piles for more efficient loading, and reducing the size of the material so it can be loaded. It does not include the intentional compacting of the material by driving on top of the waste with a bulldozer or other similar device. Methods of waste reduction such as grinding the waste into small pieces so that it can be used for general fill or recycled is regulated by the Asbestos NESHAP.

\section{Renovation of Buildings Involving Floor Tile and Mastic}

In this situation the building owner decides to remove the asbestos-containing floor tile and mastic. Floor tile and mastic that are in poor condition and friable before removal are regulated by the NESHAP. Floor tile and mastic that will be subject to sanding, grinding, cutting, or abrading is regulated by the Asbestos NESHAP. "Grinding" means to reduce to powder or small fragments and includes mechanical chipping and drilling. Most nonfriable materials can be broken without releasing significant quantities of airborne fibers. It is only when the material is is extensively damaged that the potential for significant fiber release is greatly increased. The extent of breakage that will render the ACM friable depends to some degree on the condition of the ACM prior to the abatement operation. Therefore, it is difficult to make a general statement on the level of breakage which causes ACM to become regulated. Generally, if floor tile is in good condition, is not friable, and each tile is only broken into a few pieces during removal, the asbestos removal operation would not be subject to the 
regulation. A case-by-case determination must be made considering the condition of the material and the forces which have or will act upon it. 
FLOOR TILE AND MASTIC REMOVAL REPORT

AN INTER AGENCY FUNDED PROGRAM SUPPORTED BY THE ASBESTOS DEMONSTRATION AND DEVEIOPMENT INITIATIVES GROUP THROUGH THE TULSA DISTRICT CORPS OF ENGINEERS NOVEMBER 1991

TABLE OF CONTENTS

I. SUMMARY OF RESULTS $\ldots \ldots \ldots \ldots \ldots \ldots \ldots \ldots \ldots \ldots \ldots \ldots$

II. REFERENCES $\ldots \ldots \ldots \ldots \ldots \ldots \ldots \ldots \ldots \ldots \ldots \ldots \ldots \ldots$

III. DISCUSSION............................
A. Background
B. General
C. Industrial Hygiene and Safety Program
D. Testing Program.
E. Discussion

IV. FINDINGS DURING TILE REMOVAL................ 10
A. Dry Removal
B. Wet Removal.
c. Machine Removal.

V. EPA'S INTERPRETATION OF THE ASBESTOS NESHAP........12

VI. ADDITIONAL INFORMATION...................... 13 
FIOOR TILE AND MASTIC REMOVAL

\section{II - REFERENCES}

1. Report titled, "FLOOR TILE AND MASTIC REMOVAL", prepared by stanley Engineering, Inc. Oklahoma City, OK. dated July/August 1990.

2. Draft report titled; "EVALUATION OF TILE AND MASTIC REMOVAL AT FORT SILL", prepared by David C. Cox \& Associates, Washington, D.C. dated 22 Aug 1991. 


\section{III - DI8CUSSION}

\section{A. Background}

This program was developed to evaluate a safe, economical method of removing non-friable asbestos. The phase under study in this report is the asbestos containing floor tile and mastic which was supported by the ADDIG and funded by various federal agencies including the Army, Navy, Air Force, Department of Defense Dependent Schools (DODDS), Department of Energy (DOE), and the Environmental Protection Agency (EPA). The Tulsa District Corps of Engineers was the agent for coordinating and executing the program. A video that provides a visual record of this program is available from the Tulsa District.

\section{B. General}

This segment of the program was designed to investigate the removal of asbestos containing Floor Tile and Mastic under conditions that would provide the greatest opportunity to have a release of fibers and to measure those releases, if any. Stanley Engineering Inc. Oklahoma City, OK., was the ArchitecturalEngineering ( $\mathrm{AE}$ ) consultant selected to design the program, develop the protocol, provide technical assistance during the execution of the test, take the samples, and analyze most of the samples. The EPA Risk Reduction Engineering Laboratory in Cincinnati, Ohio analyzed over 130 TEM samples, provided technical assistance and guidance, and through their consultant Mr. Cox, provided the reference araft report. The program was carried out in three buildings at Fort sill, OK. The special characteristics of each building are provided later. 


\section{c. Industrial Hygiene and safety Program.}

Protecting the worker and the environment was always the number 1 priority during this program. The project was designed to protect the environment through the use of critical barriers and enclosures. The technicians who hand removed the tile were carefully monitored to assure they were not exposed to fibers, chemicals or extreme physical exertion. Daily meetings were held to discuss the program and to review procedures to assure that the project would remain safe.

\section{Testing Program.}

The testing program had been designed, coordinated and approved through the reference protocol before the program began. Testing included: Polarized Light Microscopy (PLM) for bulk samples, Phase Contrast Microscopy (PCM) and TEM procedures. This was done in order to identify any existing ACM, comply with all regulatory requirements, and measure asbestos fibers released during the removal process. The referenced Stanley Engineering report (ref.1) with the Protocol (Tab AA) provides detail on how each test procedure was used.

\section{Buildings Selected for Testing. Building 1436 was} selected as the test facility for removal of tile and mastic on a concrete floor. This tile and mastic was placed in the 1960's and contained over $20 \%$ (tile) and $5 \%$ (mastic) asbestos. The building had been a youth center and had received heavy traffic and typically was stripped and buffed every three months. A barrack (building 4387) was selected to test tile removal on wood 
flooring. A storage facility (building 4375) was selected to test removal of multiple layers of floor tile and mastic. Each of these facilities and the testing performed is briefly outlined below and provided in detail in the referenced documents.

\section{E. Discussion}

Building 1436. Inside this concrete floor structure, 7 cells were constructed in order to provide ideal testing areas. This allowed the selected procedures to be repeated under controlled conditions. The cells were unventilated. They were cleaned and recleaned and a negative air machine was used to circulate air inside each cell to remove and free fiber before the initiation of removal activities. It was important to have very low fiber counts before the actual removal began in order to determine if any fibers were released during the removal process. Tile was removed from each cell, then the mastic was removed. The cells were completely recleaned and tested between the tile and the mastic removal test. The results of those tests are summarized in references 1 and 2 .

Building 4387. This WWII two story, wood floor barracks structure had 12" X 12" asbestos containing floor tile (7\%) and mastic (5\%). The first floor of this structure was divided into two cells. One cell was used to test hand removal procedures and the second cell was used for testing during machine removal. The tile was removed along the underlying particle board which had the mastic on its surface. Results of the tests are contained in reference \#I. 
Building 4375. This concrete floor structure had two layers of tile and mastic in three adjoining rooms. These rooms were combined into one testing area in order to test machine removal of more than one layer of tile. The results of these tests are contained in the referenced reports.

\section{IV - Findings During Tile Removal.}

\section{A. Dry Removal}

Floor tile in cells in Building 1436, 4387, and 4375 were removed by primary inexperienced workers using hand tools. This was the most aggressive method of removing tile. Since the tile was securely glued and had very heavy traffic, the tile was broken into very small fragments. Six air samples were taken inside the cell during each phase of the program. Three of these were analyzed using the National Institute for occupational Safety and Health (NIOSH) 7402 procedures by Stanley Engineering and three were analyzed using Asbestos Hazard Emergency Response Act of 1986 (AHERA) protocol by the EPA Risk Reduction Engineering Laboratory. The AHERA clearance procedures were used to test the area before beginning the removal and again during the execution of the program, and at the end of the program. This assured that the areas were free of asbestos fibers, or at least equal to background concentrations, prior to initiating the floor tile removal test, prior to initiating the mastic removal test after completion of the floor tile removal, and after completion of all asbestos removal for an 
area. Workèrs were also monitored (PCM samples) as well as adjacent areas, plus, upwind and downwind samples. The results of all samples are contained in references 1 and 2 . Results of the PCM indicate that very few fibers were present. The samples taken and analyzed during the removal process using the NIOSH 7402 procedures indicated a low level of asbestos fibers. The samples taken and analyzed during the AHERA procedures indicated an increase in the very small fibers. These very small fibers were found during the removal of both the tile and mastic. However, the time waited average of $\mathrm{f} / \mathrm{cc}$ was well below the action level.

\section{B. Tet Removal.}

Floor tile in two cells in building 1436 were removed while wet. One cell employed a mist which contained a wetting agent and one floor was soaked for two days before the tile was removed with hand tools. Water was also used with the machine removal tests which are described below. It is significant that the fiber levels (both regulated and unregulated) in the wet tile removal test was lower than when removed dry.

\section{Machine Removal.}

The seven cells constructed inside building 1436 were removed once the testing was completed and the cells were clean. The tile from the remaining accessible areas inside this building was removed with a machine designed to remove tile from large areas. The machine had three rotating disc with cutting heads that cut the tile from the floor. Since the tile was so securely 
attached, the tile broke into small pieces making clean up easy. A second machine that used hydraulic power to push a scraper blade was used in the second cell in building 4387 and in the cell in building 4375. Fiber levels were very low for each of these tests.

\section{V - EPA'S INTERPRETATION OF THE ASBESTOS NESHAP.}

To assist the user of this report with the EPA's current interpretations of the Asbestos NESHAP, a letter from the author of the the Asbestos NESHAP is provided. Attached to this letter are 4 letters of response to various scenarios by EPA's Compliance Division.

1) Letter dated March 29, 1991 concerns a wet method of shot blasting removal of mastic.

2) Letter dated April 9, 1991 concerns categorizing the condition of mastic both prior to and during demolition. 3) Letter dated January 8, 1992 provides Asbestos NESHAP clarification on what constitutes crumbled, pulverized, or reduced to powder.

4) Letter dated March 18, 1992 concerns demolition wetting and debris disposal of nonfriable ACM.

It is anticipated that these responses will provide general compliance guidance for asbestos projects. Specific questions on compliance requirements may be addressed to the Washington office. 


\section{VI - ADDITIONAL INFORMATION.}

Additional information for applications of this project data to specific projects can be obtained by contacting Mr. Richard Smith or Mr. Burl Ragland at the Tulsa District, Corps of Engineers; P.O. Box 61; Tulsa, Oklahoma 74121; Phone (918) $581-6641$. 
ENCLOSURE 2

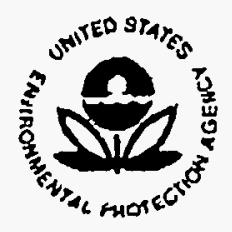

\section{UNITED STATES ENVIRONMENTAL PROTECTION AGENCV WASHINGTON, D.C. 20460}

MAR 291991

OFFKCE OF AIR AND RADLATION

Mr. Jim Sander

Manager

OCRIC, Inc.

R.O. Box 99381

San Diego, California 92169

Dear Mr. Sander:

Th1s letcer is in response to your lanuary 24 , 1991 letter in which you discussed a wet method of the shot blasting removal technique for asbestos containing masti:. Included with your letter was a video tape of the process which members of my staff and I viewed.

In a September 21, 1990 applicability determination, we stated that nonfriable material (includirg floor tile mastic) which is removed using abrasive torces "jich as grinding, sanding or other abrasive methods, is subject to the Asbestos NESHAP regulation. The shot blasting operation would cause nonfriable asbestos materlal (mastic) to be crumblad, pulverized, or reduced to powder, and therefore, Asbestos NeSHnl work practices must be followed. This meang adequately wettin.l liw nonfriable materlal (mastic) before and during the shot bla:.l im! operation, kefping the shot blasted material adequately wet hefore collection, and disposing of the waste according to the reillirements of the Asbestos NESHAP.

You have incorporated a wetting process in the shot blasting and waste collection stages of your syst im which appears to meet the above requirements. To the extenl 111.4 L Llusie requirements are being met, this wet shot blasting systen would be ar acceptable method of mastic removal. Please be awirt lhat ERA does not tormaliy approve or endorse specific relmivil techniques. This letter should not be interpreted as an ulpisval from EP $\lambda$ regarding OCRIC's process. 


$$
-2-
$$

This response has been coordinated with EPA's office of Enforcement and the Emission Standards bivision of the Office of Air Quality Planning and Standards. If you have any further questions, please contact scot.t Throwe if my staff at (703) $308-8699$.

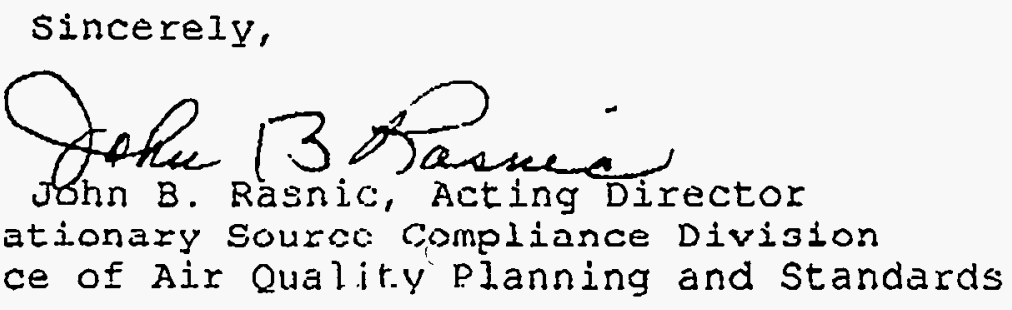

CC: Sims ROY, ESD (MD-13)

EIIse Hoerath, OE (LE-134A)

Omayra Salgado, SSCD (EN-34JW)

TOM RIpp, SSCD, (EN-34IW) 


\section{UNITED STATES ENVIRONMENTAL PROTECTION AGENCY \\ WASHINGTON, D.C. 20460}

\section{ENCLOSURE 3 \\ APR $9199 \mid$}

OFFICE OF

AIR AND RADIATION

Mr. William Loch

Project Manager

Warren and Panzer Engineers P.C.

10 East 38th Street

New York, New York 10016

Dear Mr. Loch:

This letter is in response to your December 21, 1990 letter in which you requested clarification regarding the categorization of mastic in the Asbestos National Emission Standard for Hazardous Air Pollutants (NESHAP).

In the recently revised Asbestos NESHAP, promulgated on November 20, 1990, EPA established two categories of nonfriable asbestos containing material ( $A C M$ ): Category I nonfriable $A C M$ and Category II nonfriable ACM. As defined in 40 CFR $\$ 61.141$, "Category I nonfriable ACM means asbestos containing packings, gaskets, resilient floor covering and asphalt roofing products containing more than 1 percent asbestos determined using the methods specified in Appendix A, Subpart F, 40 CFR Part 763, Section 1, Polarized Light Microscopy. Category II nonfriable. ACM means any material, excluding Category I nonfriable ACM, containing more than 1 percent asbestos as determined using the methods [cited above] that when dry cannot be crumbled, pulverized or reduced to powder by hand pressure."

Mastic which is in good condition, that is, it is not peeling, cracking, or crumbling, would be considered category I nonfriable ACM. Therefore, mastic which is in good condition will not be subject to the Asbestos NESHAP unless it is subjected to sanding, grinding, cutting or abrading. In cases where floor tile is not friable and is not being removed from a building prior to demolition, we believe the mastic underneath the floor tile will be in good condition and not friable and therefore, can remain in the building during the demolition process.

However, if the demolition process involves the intentional burning of a building, all of the asbestos containing material in the building, including the floor tile mastic, must be removed in accordance with the NESHAP before the burning. In addition, it 


$$
\text { - } \quad-2-
$$

should be noted that the condition of the mastic may be affected by the process used to remove the floor tile. Mastic should be closely inspected after floor tile removal to determine its condition. In reference to your question on the use of solvents to remove mastic, we believe the use of solvents alone would not cause the mastic to be crumbled, pulverized or reduced to powder and therefore would not be subject to the Asbestos NESHAP.

This determination has been coordinated with EPA's Office of Enforcement and with the Emission Standards Division of the Office of Air Quality Planning and Standards. If you have any questions, please contact Scott Throwe of my staff at (703) 308-8699.

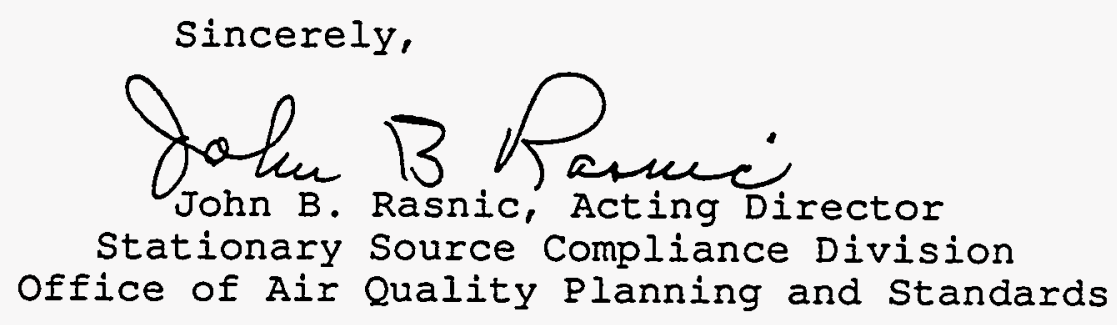

CC: Sims Roy, ESD (MD-13)

Elise Hoerath, OE, (LE-134A)

- Omayra Salgado, SSCD, (EN-341W)

Tom Ripp, SSCD (EN-34IW) 
ENCLOSURE 4

\section{UNITED STATES ENVIRONMENTAL PROTECTION AGENCY \\ WASHINGTON, D.C. 20460}

\section{JAN 81992}

OFFICE OF

AIR AND RADIATION

\section{MEMORANDUM}

SUBJECT: Asbestos NESHAP Clarification on what Constitutes Crumbled, Pulverized, or Reduced to Powder

FROM: John B. Rasnic, Director Stationary Source Compliance Division

TO: James J. Burke, Chief

Toxics and Pesticides Branch

Region III (3AT30)

This memorandum is in response to your October 17, 1991 memorandum in which you requested a policy determination on when asbestos containing materials ( $A C M)$, which are damaged during the course of demolition and renovation, become friable ACM. Specifically, you inquired at what point does damaged nonfriable ACM, such as transite siding, become regulated. As you stated in your memorandum, the word "broken" has been deleted from the definition section because it could be mistakenly interpreted as substantially increasing the scope of the standard. Most nonfriable materials can be broken without releasing significant quantities of airborne fibers. It is only when the material is extensively damaged that the potential for significant fiber release is greatly increased.

Transite siding, which is a Category II nonfriable ACM, becomes regulated ACM if it has a high probability of becoming or has become crumbled, pulverized or reduced to powder by the forces expected to act on the material in the course of the demolition and renovation operations. There is a difference between merely breaking a transite panel, and crumbling, pulverizing or reducing it to powder. If a Category II material, such as transite, is in good condition it can be broken without causing the material to become regulated. Transite panels are typically bolted or nailed to buildings on which they are attached. The extent of breakage which would normally result from carefully removing a transite panel from a building and lowering it to the ground prior to demolition would not result in crumbling, pulverizing or reducing the panel to powder. 
The extent of breakage which will render the ACM friable depends to some degree on the condition of the ACM prior to the abatement operation. This is true for both Category I and II ACM. Therefore, it is difficult to make a general statement on the level of breakage which causes $A C M$ to be regulated. A case by case determination must be made considering the condition of the material and the forces which have or will act upon it.

Under the definition of "regulated asbestos containing material" (RACM) in the asbestos NESHAP, EPA listed sanding, grinding, cutting or abrading as work practices which when subjected to Category I ACM, would cause that material to be regulated. Similarly, we can give examples of work practices which will cause Category II ACM, such as transite, in any condition to become RACM. Any demolition operation (i.e., use of a wrecking ball; implosion; use of a bulldozer, backhoe or other heavy machinery to knock the building over) will extensively damage Category II ACM such that it is crumbled, pulverized or reduced to powder. Dropping Category II material, such as transite panels, from a building as a means of transporting it to the ground, will also cause the ACM to be regulated in most conditions. The burning of any $A C M$, including category $I$ and II, will also cause that material to become regulated. This is not meant to be an exhaustive list of work practices which will cause Category II ACM to be crumbled, pulverized or reduced to powder. It does, however, provide guidance on the type of work practices which we believe will cause Category II ACM to be subject to the asbestos NESHAP.

This determination has been coordinated with EPA's office of Enforcement and the Emission Standards Division of the Office of Air Quality Planning and Standards. If you have any questions, please contact Scott Throwe of my staff at FTS 678-8699.

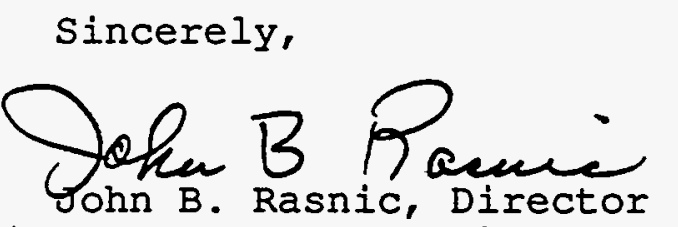

Stationary Source Compliance Division office of Air Quality Planning and Standards

CC: Sims ROY, ESD (MD-13)

Charles Garlow, OE (IE-134A)

Omayra Salgado, SSCD (EN-341W)

Tom Ripp, SSCD (EN-34IW)

Regional Asbestos NESHAP Coordinators 


\title{
Enclosure 5
}

\author{
UNITED STATES ENVIRONMENTAL PROTECTION AGENCY \\ WASHINGTON, D.C. 20460
}

\section{MAR 181992}

Ms. Ann Bieller, Area Manager

Environmental Management

Southwestern Bell Telephone

Procurement Organization

500 North Broadway, Room 1400

St. Louis, Missouri 63102

Dear Ms. Bieller:

This letter is in response to your January 28, 1992 letter requesting a clarification of $40 \mathrm{CFR} \$ 61.150(\mathrm{a})(3)$ as it relates to $\$ 61.145$ (c) ( 1 ) (i) and (iv), of the Asbestos NESHAP Revision; Final Rule, dated November 20, 1990.

In your letter, you present your understanding that there are no special requirements for adequately wetting Category I or category II nonfriable asbestos-containing material (ACM) during the course of a demolition or renovation if it does not meet the definition of regulated asbestos-containing material (RACM). You also state that this conclusion is further supported by $\$ 61.150(a)(5)$, until $\$ 61.150(a)(3)$ is taken into consideration. Section $61.150(a)(3)$ requires, "for facilities demolished where RACM is not removed prior to demolition according to

$\S \S 61.145$ (c)(1)(i), (ii), (iii) and (iv) or for facilities demolished according to $\$ 61.145(c)(9)$, adequately wet asbestos-containing waste material at all times after demolition and keep wet during handling and loading for transport to a disposal site." You question the correctness of the $\$ 61.150$ (a) (3) reference to $\$ 61.145(c)(1)(i)$ and (iv).

section $61.150(a)(3)$ correctly cites $\$ 61.145(\mathrm{c})(1)(i),(i i),(i i i)$ and (iv). It is required under $\$ 61.150$ (a) (3) that asbestos-containing waste material be kept adequately wet. Asbestos-containing waste material as applied to demolitions and renovations includes RACM waste and materials contaminated with asbestos including disposable equipment and clothing. Category I or Category II nonfriable ACM that has been contaminated by RACM, and cannot be decontaminated (e.g., building debris in a pile contaminated with RACM) must be treated as asbestos-containing waste material. Category I or Category II ACM that does not meet the definition of RACM after a demolition or renovation, and is not contaminated with RACM, is not asbestoscontaining waste material and is not subject to the wetting requirement of $\$ 61.150(a)(3)$. 
If, after a demolition, material left in the facility according to $\$ 61.145$ (c) (1) (i) or (iv) is intentionally ground-up (such as by repeatedly running over the debris with a bulldozer to compact the material), then $\$ 61.150$ (a) (3) applies. The material must be adequately wetted and kept adequately wet during collection and transport to a site or facility operated in accordance with $\$ 61.154$ or $\$ 61.155$. However, if the bulldozer is moving the debris or picking it up to be put in a vehicle and inadvertently runs over Category I material, then it is not subject to the NESHAP standard.

Category I or II nonfriable ACM that is not subject to $\$ 61.150(a)(3)$ would still have to be disposed of in a landfill that accepts building debris, in a landfill that operates in accordance with $\$ 61.154$, or at a facility that operates in accordance with $\$ 61.155$. This waste material would not be allowed to go to any facility that would sand, grind, cut or abrade the non-RACM waste or otherwise turn it into RACM waste (such as a cement recycling facility). In addition, if category I or II nonfriable ACM is sanded, ground, cut or abraded during disposal at a landfill, before it is buried, it is subject to the NESHAP.

This response has been coordinated with EPA's office of Enforcement and with the Emission Standards Division of the office of Air Quality Planning and standards. If you have any questions, please contact Tom Ripp of my staff at (703)308-8727.

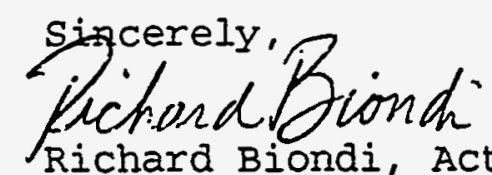

Richard Biondi, Acting Director

Stationary Source Compliance Division Office of Air Quality Planning and Standards

CC: Sims ROY, ESD (MD-13)

Omayra Salgado, SSCD (EN-341W)

Scott Throwe, SSCD (EN-341W)

Charlie Garlow, OE (LE-134A)

Regional Asbestos NESHAP Coordinators 
DEPARTMENT OF THE ARMY CORPS OF ENGINEERS, TULSA DISTRICT DEMONSTRATION PROJECT REMOVAL OF ASBESTOS CONTAINING FLOOR TILE

CONTRACT NO. DACA56-88-D-0054 DELIVERY ORDER NO. "3"

PROJECT DATE: JULY/AUGUST 1990

CERTIFIED INDUSTRLAL HYGIENE SERVICES BY:

STANLEY ENGINEERING, INC.

2700 N.W. 39TH STREET

OKLAHOMA CITY, OKLAHOMA 73112 


\section{DEPARTMENT OF THE ARMY} CORPS OF ENGINEERS, TULSA DISTRICT DEMONSTRATION PROJECT REMOVAL OF ASBESTOS CONTAINING FLOOR TILE
CONTRACT NO. DACA56-88-D-0054 DELIVERY ORDER NO. "3"

PROJECT DATE: JULY/AUGUST 1990

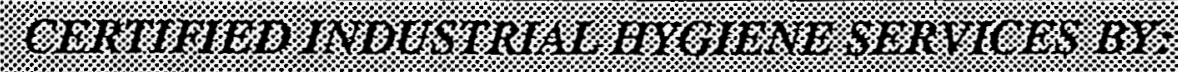

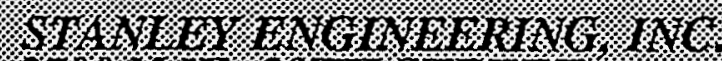

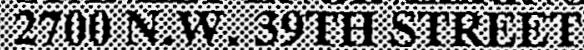

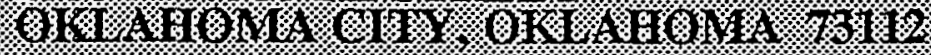




\section{TABLE OF CONTENTS}

Acknowledgment .................................................................... A

Certification.......................................................................... B

Executive Summary................................................................. C

Introduction ....................................................................... D

Air Monitoring .................................................................... E

Conclusions........................................................................ F

Abatement Orders ....................................................................G

Figure - TEM Sample Locations .............................................. H

Summary of Analytical Results................................................. I

Transmission Electron Microscopy

Cell 6 Floor Tile Removal ............................................................................ J

Cell 4 Floor Tile Removal ............................................................................

Cell 5 Floor Tile Removal ............................................................................

Cell 3 Floor Tile Removal .........................................................................M

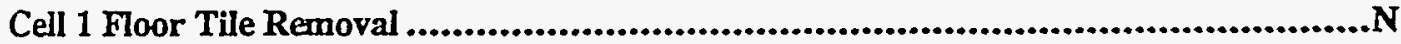

Summary of Results Mastic................................................................................ 0

Cell 6 Mastic Removal .....................................................................................P

Cell 5 Mastic Removal ..............................................................................Q.Q.

Cell 2 Mastic Removal ..................................................................................

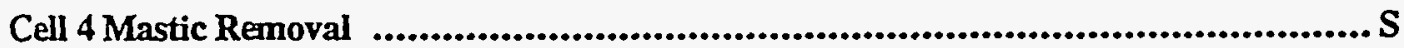

Cell 2 Mist (High Humidity) Floor Tile Removal .....................................................T

Cell 7 Wet (Soaked Floor) Floor Tile Removal .....................................................U

Cell 3 Mastic Removal ...............................................................................V

Cell 1 Mastic Removal ...............................................................................W

Cell 7 Mastic Removal

Final Clearance Samples.......................................................................Y

Phase Contrast Microscopy Results ............................................. Z

Removal Protocol ................................................................ AA

Peripheral Phase Contrast Microscopy ..................................... BB

Building 1436 Final Clearance Figures ...................................... CC 


\section{ACKNOWLEDGMENT}

The principals of Stanley Engineering, Inc., would like to thank the Tulsa District Corps of engineers for their selection, utilization, and support of Stanley Engineering, throughout the duration of this floor tile and mastic Removal Demonstration Project. Special appreciation is extended to Mr. Burl Ragland of the Tulsa District Corps of Engineers, whose efforts guided the project to completion in a timely fashion. We would also like to extend a special thank you to Mr. Bob Vandegriff of the Tulsa District Corps of Engineers, and Mr. Bob Stout of the Headquarters, U.S. Army Corps of Engineers, for their input and direction with specific regard to Industrial Hygiene aspects of the project.

Special appreciation is also extended to the staff of the United States Environmental Protection Agency (USEPA) and in particular, Mr. Sims Roy, Mr. Darl Mount, and Mr. Bruce Moore. Special appreciation is also extended to Mr. Tom Powers of the USEPA Research and Development Risk Reduction Engineering Laboratory. Mr. Powers was present for a significant portion of the project, and contributed to establishing the sample identification and nomenclatural system utilized for the project. Also, we would like to thank Mr. Roger Wilmouth and Mr. Pat Clark, whose assistance was greatly appreciated in the analysis of samples by Transmission Electron Microscopy (TEM) as well as significant participation in the data presentation programs (pre and post project). A special thank you is also extended to Ms. Jean Chesson and Dave Cox who provided statistical support for this project.

Beyond this, we would also like to take the opportunity to thank Mr. Steve Allen, Director of the Oklahoma State Department of Labor Asbestos Division and Mr. Jim Millsap of the Oklahoma State Department of Health Air Quality Service. Both these individual contributed significantly to the definition of friable/non-friable asbestos containing materials in the interpretation of its' applicability to the removal of asbestos containing floor tiles/mastic.

A very special appreciation is extended to the Fort Sill Environmental Division, who participated in the successful guidance of the project leading to a timely conclusion, the Fort Sill Asbestos Remediation Workers who established the controlled areas and participated in the removal of floor tile and mastic, and the Fort Sill Department of Master Planning, Mr. Serge Saltiel who aided in the selection of the structures. Finally, the entire staff of Stanley Engineering would like to thank Mr. Wayne Kaiser, as well as the many other individuals who provided assistance in the administration of this project, and without whose support the project would not have been possible.

Our thanks also to Mr. Rex Rudy, who provided protective garments and laundry service throughout the project as well as Mr. Jerry McKinley and Mr. Jerry Cooper of Sublett \& Associates, Inc., who subsequent to the dry hand removal, demonstrated mechanized removal techniques utilizing equipment developed specifically for floor tile removal. 
Our apologies to the many other persons who participated and provided valuable assistance to this project, and whom we have not named individually. Your assistance was greatly appreciated. 
 \\ CERTIFIED INDUSTRIAL HYGIENIST \\ REGISTERED PROFESSIONAL ENGINEER \\ INDEPENDENT CERTIFICATION \\ DEMONSTRATION PROJECT \\ FT. SULL ARMY INSTALLATION \\ BUILDING 1436 \\ FLOOR TILE REMOVAL
}

Stanley Engineering, Inc., a professional engineering firm licensed to practice in the State of Oklahoma, under the provisions of Article I, Chapter 245, Section 21, of the Oklahoma Session Laws, 1968, Certificate No. Ca 693, and I, Keith L. Stanley, Ph.D., P.E., C.I.H., a Registered Professional Engineer in the State of Oklahoma Session Laws, 1968, P.E., Certificate Number 12686, and C.I.H. Certificate Number 1203, acting in the capacity of an independent consultant to the Tulsa District Corps of Engineers, do hereby certify, attest, acclaim or otherwise make known to all persons with particular regard to the floor tile removal project, Building 1436, Ft. Sill Army Base, that the requirements and formalities of all applicable regulations, were followed for the duration of the project and compliance with the same achieved. All relative documentation, with regard to this project, which is identified as the Ft. Sill Building 1436 Floor Tile Removal Demonstration Project, has been closely monitored in order to verify that all regulatory agency requirements were achieved.

Dr. Keith L. Stanley, P.E., C.I.H. Date

C.I.H. Number 1203

P.E. No. 12686 


\section{EXECUTIVE SUMMARY}

Duplicate efforts to demonstrate increase airborne structure concentration from the dry hand removal of asbestos containing floor tile, has been strongly documented within this report. This documentation is reflected only through the utilization of Transmission Electron Microscopy and the AHERA Analytical Protocol. Other measurement techniques utilized to measure the presence of regulated airborne fiber concentrations, i.e., Phase Contrast Microscopy, do not document or reflect any significant increase in airborne concentrations during floor tile and mastic removal. Likewise, NIOSH 7402, analytical protocols, are superfluous to the issue since Phase Contrast Microscopy does not document elevated fiber concentrations.

Of particular importance to the documented increase in airborne asbestos structures as identified by AHERA Analytical Protocol, is the absence of significant concentrations when compared to Permissible Exposure Limits established by the Occupational Safety and Health Administration. Throughout the duration of this project, levels equal to or exceed 0.2 fiber (structures)/cc were noticeably absent.

Findings reflected within this report suggest a recommended utilization of personal protective equipment (dual cartridge High Efficiency Particle Absolute filtration, in conjunction with full face respirators) and critical barriers, in order to assure minimizing of dust migration to adjacent areas. Wet removal, when feasible, is recommended as a dust control technique, however, misting (high humidity) should be avoided due to increased respiratory demand placed on workers.

Analytical data review from all Cells which were duplicated both during floor tile and mastic removal, reflect small structure generation when analyzed by TEM. Phase Contrast Microscopy, however, which reflects the regulated fibers, does not indicate that utilization of this technique will contribute to an evaluation of floor tile removal. The following schematic reflects structure sizes as counted by the techniques utilized. 


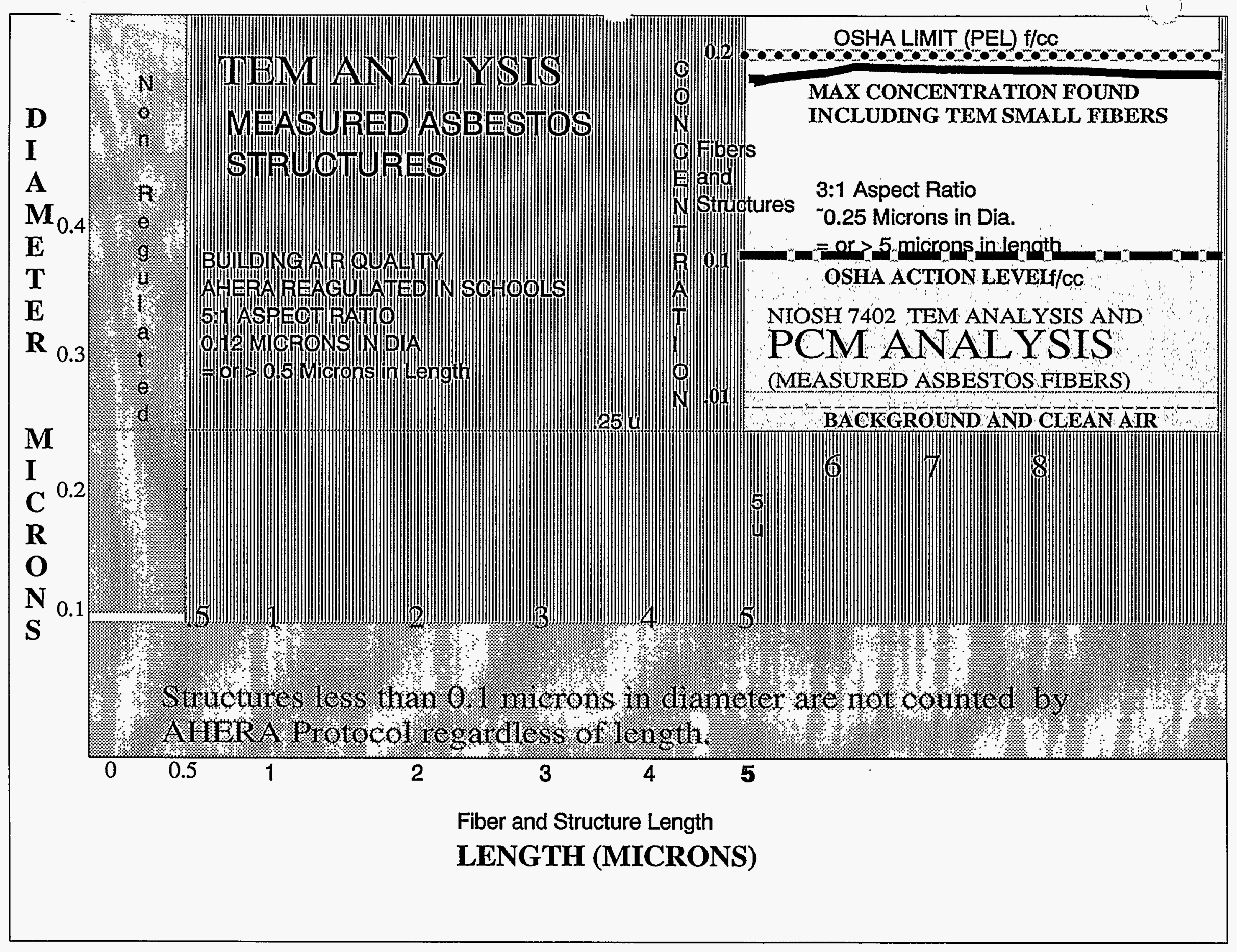




\section{INTRODUCTION}

Considerable scientific effort, over the past two years, has gone toward gaining further insight into the potential hazards of asbestos containing floor tile and mastic abatement. With specific regard to the liberation of asbestos fibers from asbestos containing floor tile and mastic during removal, debate within the scientific community has ranged from projections of no potential for exposure above acceptable Permissible Exposure Limits to projections of a necessity to address floor tile remediation by utilization of maximum respiratory protection. Other significant observations in association with floor tile and mastic, are their potential to produce elevated concentrations of airborne asbestos related to the type of "floor tile care" and debates with regard to whether or not airborne concentrations of asbestos fibers (structures) are related to breakage of floor tile during remediation or liberation of trapped "short fibers" deposited during floor tile care activities.

This project was specifically intended to address whether or not significant liberation of airborne asbestos fibers will occur in association with the removal of asbestos containing floor tile and mastic when this activity is accomplished by dry hand removal techniques within a contained area and in the absence of dilution air. In an effort to affect this scenario, seven identical enclosed areas were constructed within Building 1436, Fort Sill Lawton, Oklahoma.

The selection process and search for an appropriate structure within which this demonstration project could be accomplished included the collection and analysis of numerous floor tile samples and an on-going search which persisted for several months prior to the final selection of Building 1436. This facility contained floor tile with an asbestos content of $30 \%$ as determined by Polarized Light/Dispersion Staining Microscopy (NVLAP Certified Laboratory) and 25\% as reflected by Scanning Electron Microcopy analysis and special preparation techniques. Mastic was also found to contain in excess of $20 \%$ asbestos fiber. Beyond this, the area within Building 1436 was large enough to accommodate multiple containments $(7)$ of sufficient area to require several man hours of dry hand removal activity and multiple hours of mastic removal.

Materials of construction for each Cell were those typically utilized in asbestos remediation projects, i.e., $2^{n} \times 4^{n}$ frame with polyethylene sheeting (two layers of six mil). This material was utilized for walls as well as ceiling with only the floor remaining uncovered. One doorway (double flapped) exited each Cell and opened into an enclosed hallway which lead to the decontamination facility. Individual drawings depicting each Cell as well as sample locations are included within the body of this report and representative of each Cell activity.

Finally, a testing protocol was developed and was intended to allow for the measurement of any increase in airborne concentrations of asbestos which may occur between measurements identified as "Passive Background" samples and "Dry Hand Removal" samples. The entire process was then repeated for mastic removal. Analytical techniques included Phase Contrast Microscopy which was accomplished both at the site (screening) and in the Laboratory, Transmission Electron Microscopy (AHERA Analytical Protocol), and Transmission Electron Microscopy (NIOSH 7402 Analytical Protocol). 
The primary phase of air monitoring included sample collection in association with all workers and adjacent building occupants in order to demonstrate the absence of any impact due to the floor tile remediation project. These samples were analyzed on a daily basis at the site, by Phase Contrast Microscopy. Results of this technique, which counts all fibers equal to or greater than five microns in length, with at least a 3:1 aspect ratio, at $400 \mathrm{X}$ to $450 \mathrm{X}$ and a limit of resolution in the range of 0.25 microns in diameter, was noticeably absent of significant airborne concentrations. Routine analysis did reflect a significant presence of dust and other non fibrous materials.

Secondarily to the monitoring that was conducted to demonstrate personnel and occupant safety, as reflected above, monitoring was also conducted to reflect any impact to adjacent unoccupied spaces and the environment. On site analysis of these samples was also accomplished on a daily basis by Phase Contrast Microscopy and results were typically those of background and/or at the limit of detection for the methodology utilized. All samples were retained for confirmation in the laboratory, subsequent to their initial analysis in the field.

Monitoring specific to this demonstration project, and which was intended to reflect any significant increase in airborne fiber concentrations as demonstrated by Transmission Electron Microscopy analysis, AHERA Analytical Protocol, was directed at passive background sampling and during dry hand removal sampling. This process was repeated for mastic removal. Additional samples were collected and analyzed by TEM in order to reflect the completeness of the project, i.e., periodic aggressive sampling and clean air sampling. Incidental to the project were other building floor tile remediation peripheral projects, i.e., Building 4387 and Building 4375. Additionally, automatic removal of floor tile was also accomplished. Complete data for each of the peripheral activities is included in this report, as accomplished by Phase Contrast Microscopy, however, all results reflect a minimal presence of airborne fiber concentrations due to floor tile removal.

Samples collected specifically to reflect elevations between passive background sampling and dry hand removal sampling, were located at breathing zone height and eighteen inches apart. A total of six samples and two blanks were collected during each activities, i.e., passive background and removal. Specific locations of the samples, by cells, are reflective within the associated drawings and summaries. Samples designed with the trailing Alpha Character "E", (inclusive of blanks), were analyzed by the US Environmental Protection Agency, at their laboratory located in Cincinnati, Ohio utilizing the AHERA Analytical Protocol. Samples designated with the trailing alpha character "N" (inclusive of blanks) were analyzed by Stanley Engineering, Inc./Alpha Analytical Laboratories, utilizing the AHERA Analytical Protocol for passive background samples, and, the NIOSH 7402 
methodology for the removal samples. Samples analyzed by USEPA, utilizing

AHERA analytical protocol, were subjected to statistical tests, designed to reflect any significant increases in measured concentrations of airborne asbestos structures as defined within the AHERA Analytical Protocol.

NIOSH 7402 collection, preparation and analytical methodology, was applied to samples collected during dry hand removal in order to demonstrate the percent of asbestos structures compared to non-asbestos structures. Determined percentages are then applied to Phase Contrast Microscopy (NIOSH Method 7400) results, in order to differentiate asbestos/non-asbestos fibers.

Stanley Engineering provided air monitoring and industrial hygiene oversite during the length of the project. All air monitoring was conducted in compliance with sampling requirements made by Certified Industrial Hygienist, Dr. Keith L. Stanley.

Test results are provided for review and permanent records, within the content of this report. 


\section{CONCLUSIONS}

A systematic review of all data reflects the following findings:

\section{Phase Contrast Microscopy (PCM)}

Samples were collected for Phase Contrast Microscopy throughout the duration of the project and within all areas of activity and occupancy. This methodology has been developed and utilized extensively for the purpose of identifying airborne asbestos and other fibers which may be associated with asbestos remediation projects. It is recognized that Phase Contrast Microscopy generally has, as a limited resolution, 0.25 microns. Therefore, the regulated fiber, identified in association with remediation projects, where PCM is utilized as a tool of analysis, is a fiber with an aspect ratio of at least 3:1, but having as a length, 5 microns or greater.

Analytical results from all PCM do not indicate the presence of airborne asbestos fibers. Stated alternatively, the regulated fiber as related to asbestos remediation, was noticeably absent from this floor tile remediation project, wherein dry hand removal was accomplished without the support of dilution air. A worst case was created and there was no indication of elevated concentrations of total fibers (asbestos or otherwise) as identified by Phase Contrast Microscopy. It should be pointed out that there was a significant presence of dust in association with all work related samples.

\section{Transmission Electron Microscopy (NIOSH 7402 Analytical Protocol)}

The NIOSH 7402 Analytical Protocol was established as a desirable method of analysis prior to the start of the project and for the purpose of identifying and quantifying asbestos related fibers as may be present during dry hand removal of floor tile. This technique presumes the presence of significant concentrations of airborne fibers as determined by Phase Contrast Microscopy (Method 7400).

This project utilized the NIOSH 7402 Analytical Protocol, on samples collected in paralleled with samples identified for analysis by the AHERA Analytical Protocol. Samples collected for analysis by this technique, were collected only during removal activities. Each sample was first counted by Phase Contrast Microscopy and subsequently by Transmission Electron Microscopy NIOSH 7402 Analytical Protocol.

This Transmission Electron Microscopy technique, has been established to confirm only those fibers identified by Phase Contrast Microscopy, therefore, even though the 
confirmation is carried out, at magnifications of $10,000 \mathrm{X}$, only those fibers meeting "regulated fiber" criteria, i.e., limited resolution of 0.25 microns in diameter, at least a 3:1 aspect ratio, and equal to or greater than 5 microns in length. Since all Phase Contrast analytical results carried out by the NIOSH 7400 Analytical technique, were low in concentrations (fibers/cc), then also, confirming results, which establish a percentage of asbestos vs non-asbestos fibers, was also low.

Finally, analytical results as accomplished by TEM NIOSH 7402 Analytical Protocol, establish the presence of regulated fibers in association with floor tile removal, to be noticeably absent from all analytical results.

\section{Transmission Electron Microscopy AHERA Analytical Protocol}

The AHERA analytical Protocol was utilized extensively in association with this floor tile remediation project. Samples were collected and subjected to this analytical technique as follows:

Aggressive Pre-start Samples:

Passive Background Samples

During Hand Removal/Floor tile Samples

Clearance/Mastic Passive Background Samples

During Mastic Removal Samples

Building Clearance Samples

This analytical technique which is carried out at $20,000 \mathrm{X}$, and is designed to reflect clean air within schools following asbestos remediation projects, was applied to this project in order to achieve greater sensitivity and recognition of structures (inclusive of fibers) below those identified as the "regulated structure". A structure is defined as a clump, matrix, cluster, fiber, bundle, etc., having a 5:1 aspect ratio and a length equal to or greater than 0.5 microns. The limit of diameter resolution is generally recognized to be 0.01 microns. This technique also affords the opportunity to apply a cut size in recognition of fibers (structures) meeting the definition of the regulated fiber, i.e., 5 microns in length and with a diameter of 0.25 microns (limit of resolution PCM). ' 
One additional difference in preparation of Transmission Electron Microscopy samplestish recognized and may impact analytical results. This difference is reflected in the absence of sample ashing within the NIOSH 7402 preparation technique, whereas the AHERA analytical protocol requires ashing. The significance of this difference is that "ashing" while it is utilized to uncover or reveal small fibers trapped with the filter pores, nonasbestos fibers would be removed in the process. Since the NIOSH 7402 analytical protocol, is a discriminating technique, designed to reveal the percent of asbestos and non asbestos fibers (structures), ashing is not accomplished within the preparation technique. As a result of this difference, minor differences in results as determined by the 7402 and AHERA analytical protocol, may exist.

Results as achieved by the AHERA analytical protocol reflect a significant difference between passive background levels and during work samples, both in floor tile and mastic removal. The significance of the presence of small unregulated asbestos structures at low concentrations (refer to individual data sheets) in association with this project, cannot be determined since they are below the identified "regulated fiber" in size. Beyond this, one other conclusion may be drawn, the presence of the small "unregulated structure (fiber)" would likely result in the failure of any given area to achieve compliance with the AHERA requirements as established for schools. Stated alternatively, the criteria for clean air as determined by the AHERA Analytical Protocol, would likely not be achieved, due to the presence of small asbestos structures.

This finding emphasizes the need for additional work to establish the significance of small asbestos type fibers as they may impact the public health. At present, there are no regulations which identify limitations in concentration of small asbestos fibers as may be identified by the AHERA analytical Protocol. 


\section{ABATEMENT ORDERS:}

The project was executed through a series of Abatement Orders which dictated the procedures to be followed during the Tile or Mastic Removal and the Building and Cell in which the Abatement was to take place.

Abatement Orders for the project were as follows:

$\begin{array}{cllcll}\text { ABATEMENT } & \text { DATE } & \text { BLDG } & \text { CELL } & \text { ACTIVITY } & \text { PROCEDURE } \\ 1 & & & & & \\ 2 & 7 / 25 / 90 & 1436 & 6 & \text { Tile } & \text { Dry hand removal } \\ 3 & 7 / 26 / 90 & 1436 & 4 & \text { Tile } & \text { Dry hand removal } \\ 4 & 7 / 27 / 90 & 1436 & 2 & \text { Tile } & \text { Wet hand removal } \\ 5 & 7 / 28 / 90 & 1436 & 5 & \text { Tile } & \text { Dry hand removal } \\ 6 & 7 / 29 / 90 & 1436 & 3 & \text { Tile } & \text { Dry hand removal } \\ 7 & 7 / 30 / 90 & 1436 & 1 & \text { Tile } & \text { Dry hand removal } \\ 8 & 7 / 31 / 90 & 1436 & 6 & \text { Mastic } & \text { Sand Machine } \\ 9 & 7 / 31 / 90 & 1436 & 3 & \text { Mastic } & \text { Citric Acid } \\ 10 & 8 / 1 / 90 & 1436 & 2 & \text { Mastic } & \text { Sand Machine } \\ 11 & 8 / 1 / 90 & 1436 & 5 & \text { Mastic } & \text { Sand Machine } \\ 12 & 8 / 2 / 90 & 1436 & 7 & \text { Tile } & \text { Wet Removal } \\ 13 & 8 / 2 / 90 & 1436 & 1 & \text { Mastic } & \text { Citric Acid/Towels } \\ 13 & 8 / 3 / 90 & 1436 & 4 & \text { Mastic } & \text { Sand Machine } \\ 14 & 8 / 3 / 90 & 1436 & 7 & \text { Mastic } & \text { Citric Acid/Towels } \\ 15 & 8 / 4 / 90 & 1436 & \text { All } & & \text { Final Clearances } \\ 16 & 8 / 7 / 90 & 4387 & 1 & \text { Tile } & \text { Dry hand removal } \\ 17 & 8 / 7 / 90 & 4387 & 1 & \text { Sub Fir/Mastic } & \text { Dry hand removal } \\ 18 & 8 / 9 / 90 & 4387 & 2 & \text { Tile/Sub Flr/Mastic } & \text { Machine Dry } \\ 19 & 8 / 9 / 90 & 1436 & \text { All } & \text { Tile/Mastic } & \text { Machine Dry } \\ 20 & 8 / 9 / 90 & 4375 & & \text { 2 Layers Tile } & \text { Machine Dry } \\ 21 & 8 / 23 / 90 & 4387 & & \text { Pick Up Tile } & \\ 22 & 8 / 23 / 90 & 4375 & & \text { Pick Up Tile } & \\ & 8 / 24 / 90 & 1436 & & \text { Pick Up Tile } & \end{array}$




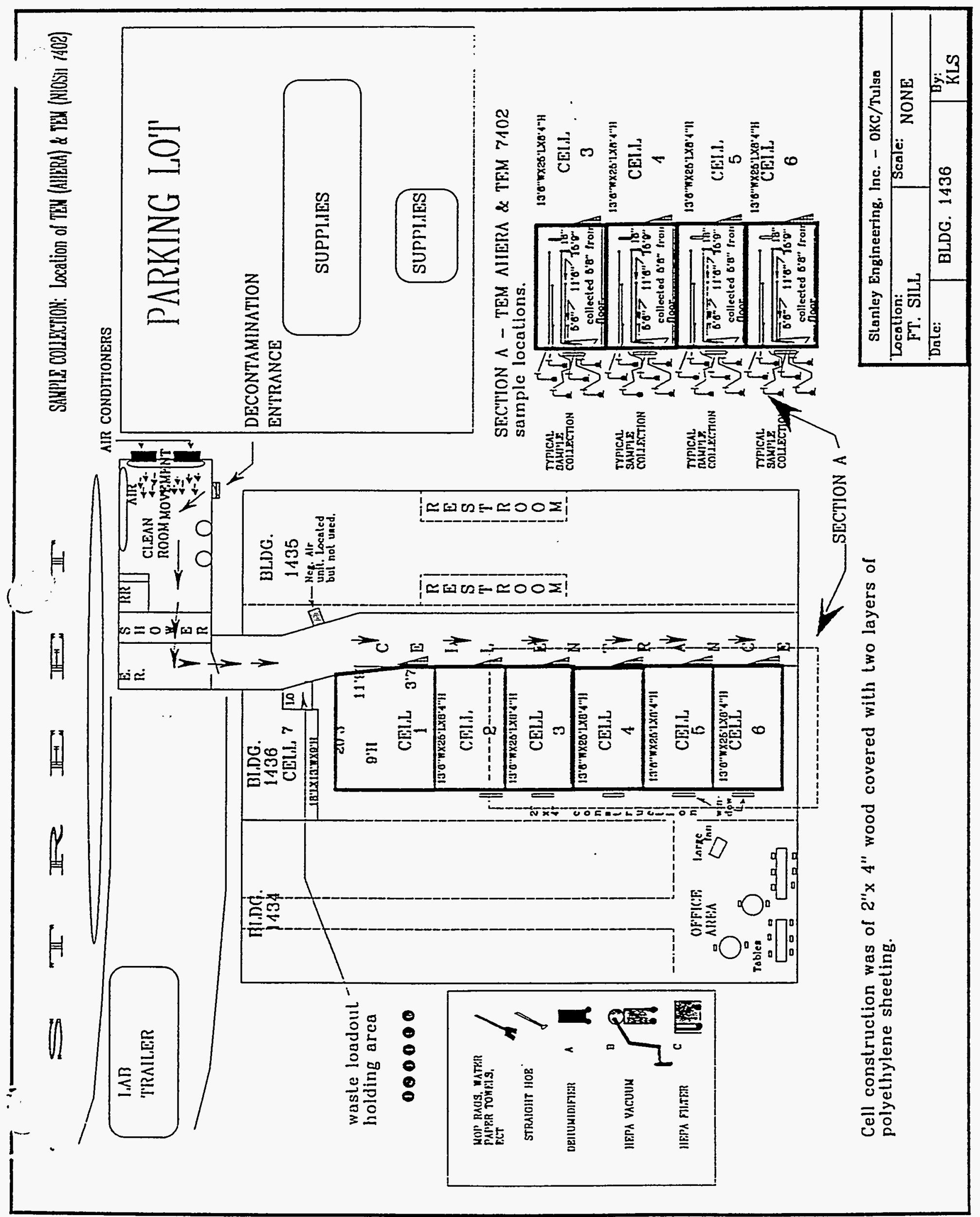


NOTE: EPA LEVELS OBTAINED DURING THE REMOVAL INCLUDE FIBERS/STRUCTURES THAT ARE NOT REGULATED BY OSHA FT.SILL DEMONSTRATION PROJECT- CELL 3 SUMMARY

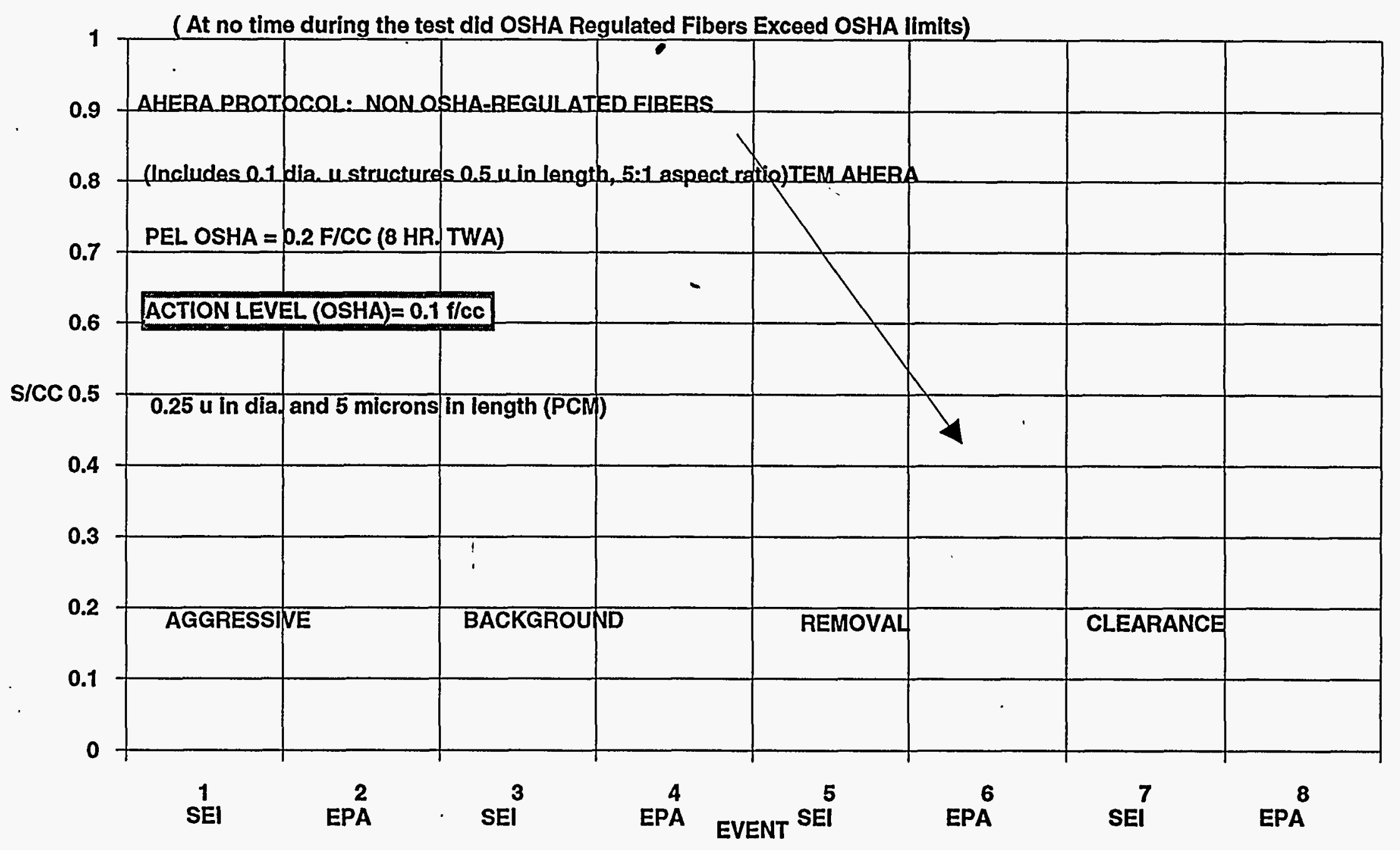

FT. SILL FLOOR TILE 
T.E.M. ANALYSIS

PEL OSHA $=0.2$ F/CC $(8 \mathrm{HR}$. TWA)

FT.SILL DEMONSTRATION PROJECT-CELL 1 SUMMARY

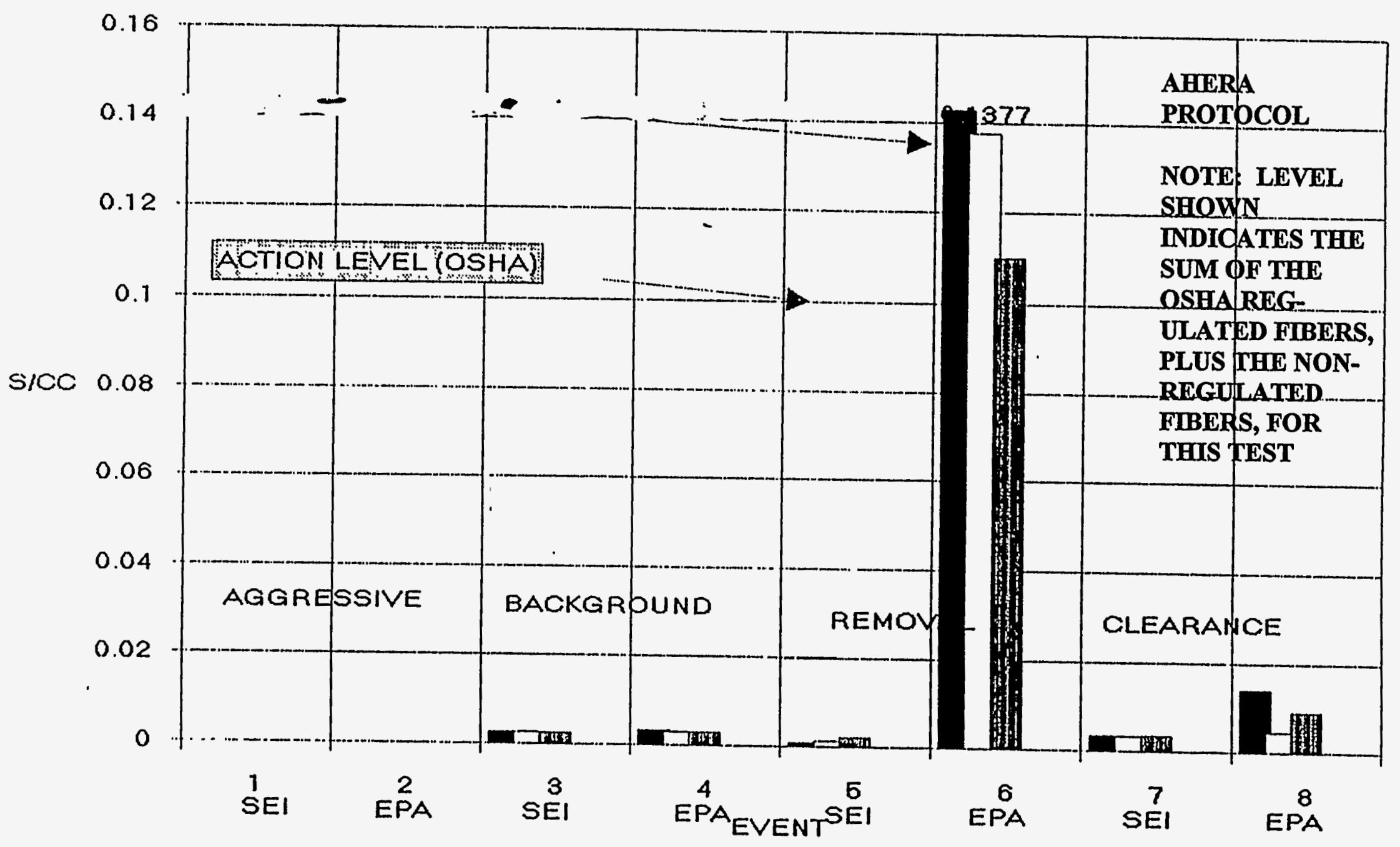

FT. SILL FLOOR TILE 


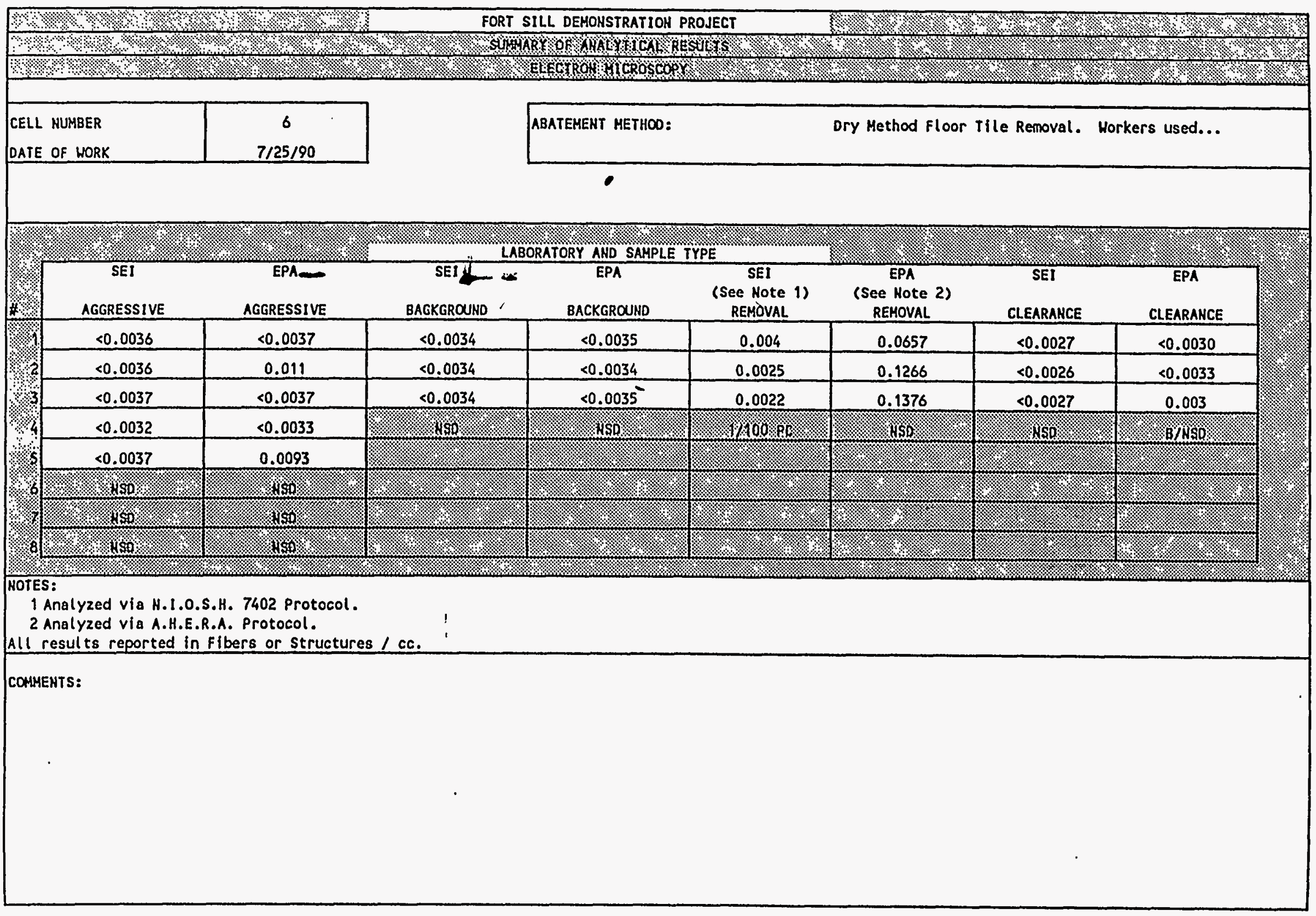




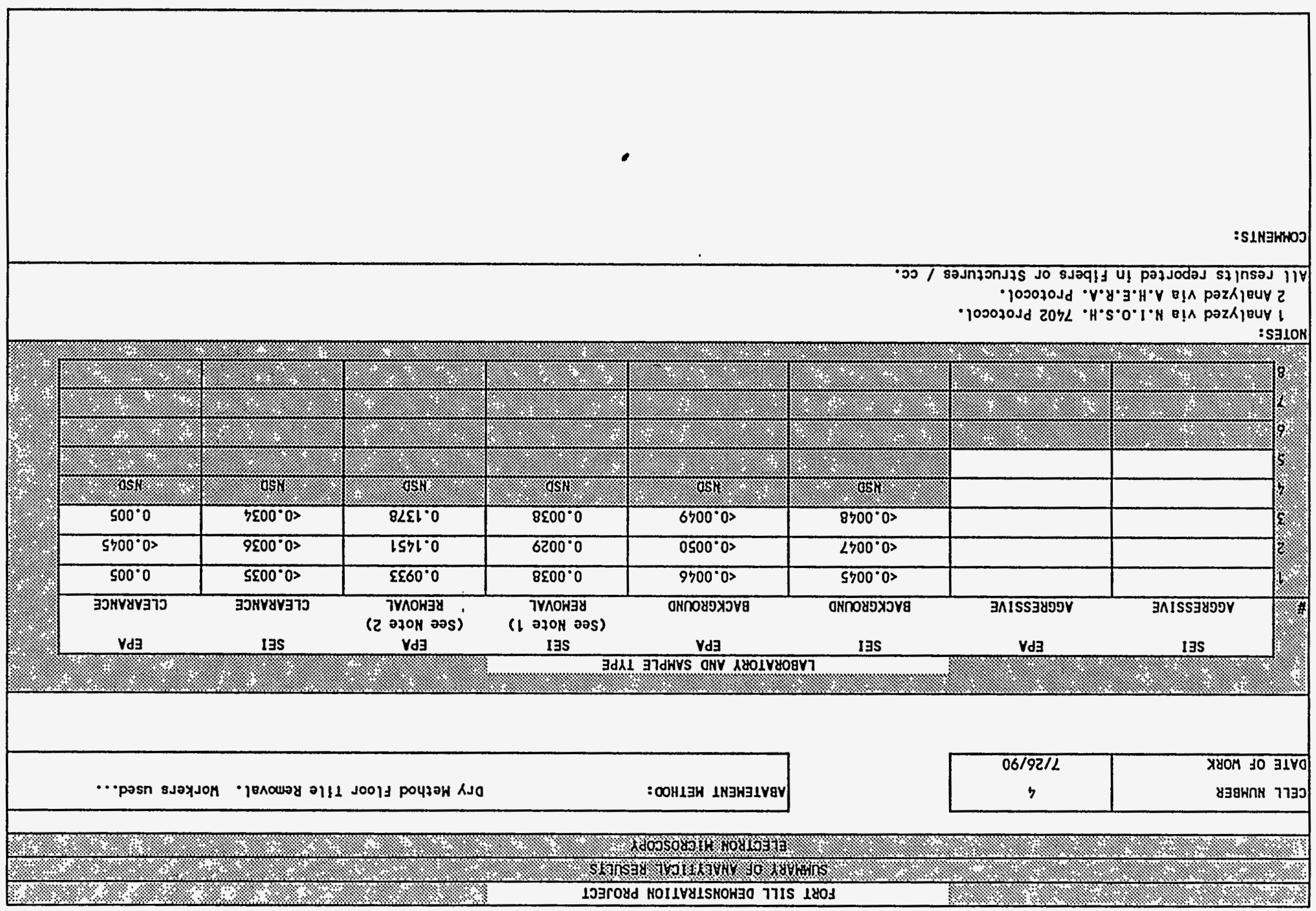


PEL OSHA $=0.2 \mathrm{~F} / \mathrm{CC}(8 \mathrm{HR}$. TWA $)$

FT.SILL DEMONSTRATION PROJECT-CELL 6 SUMMAR AHERA

PROTOCOL

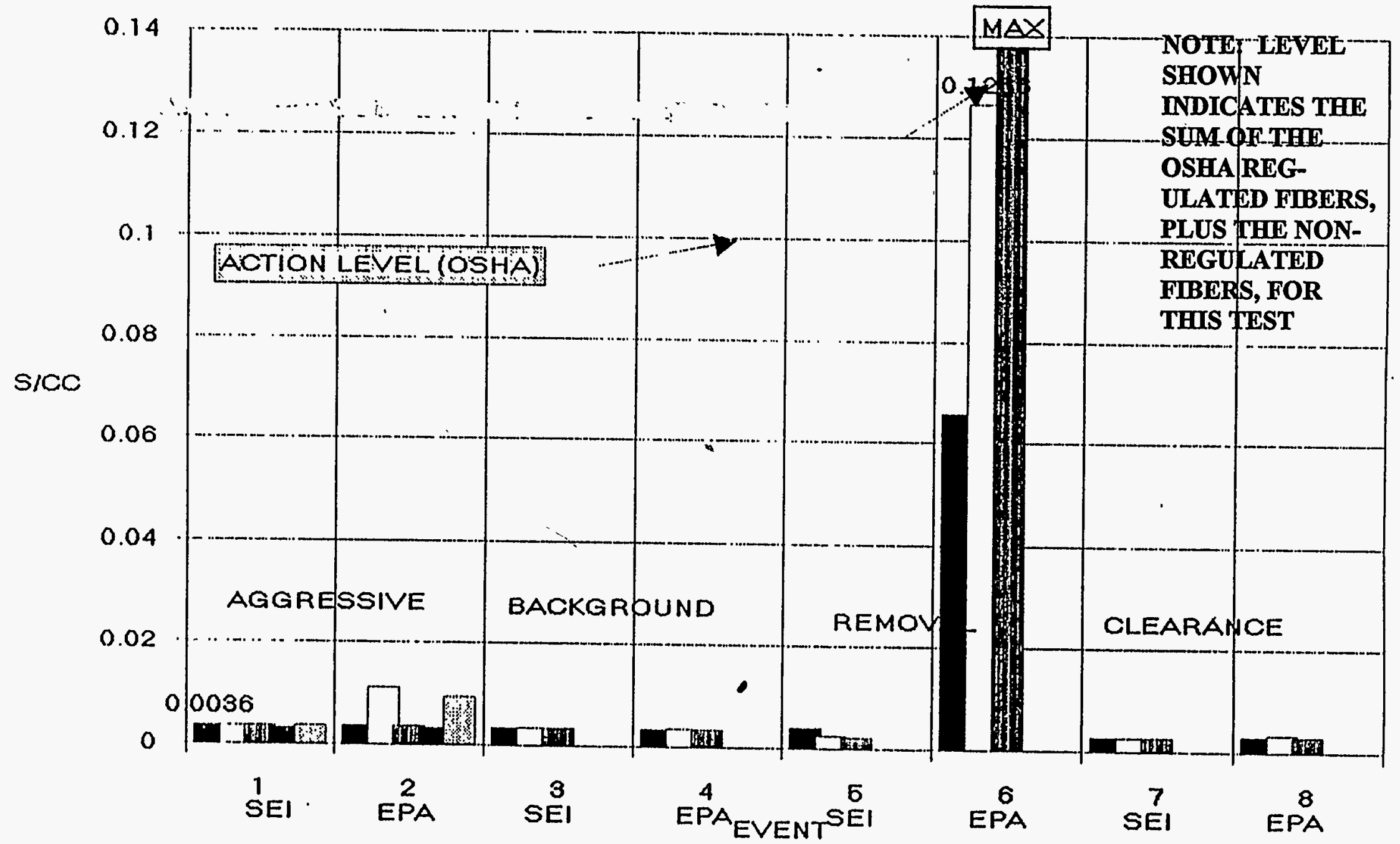




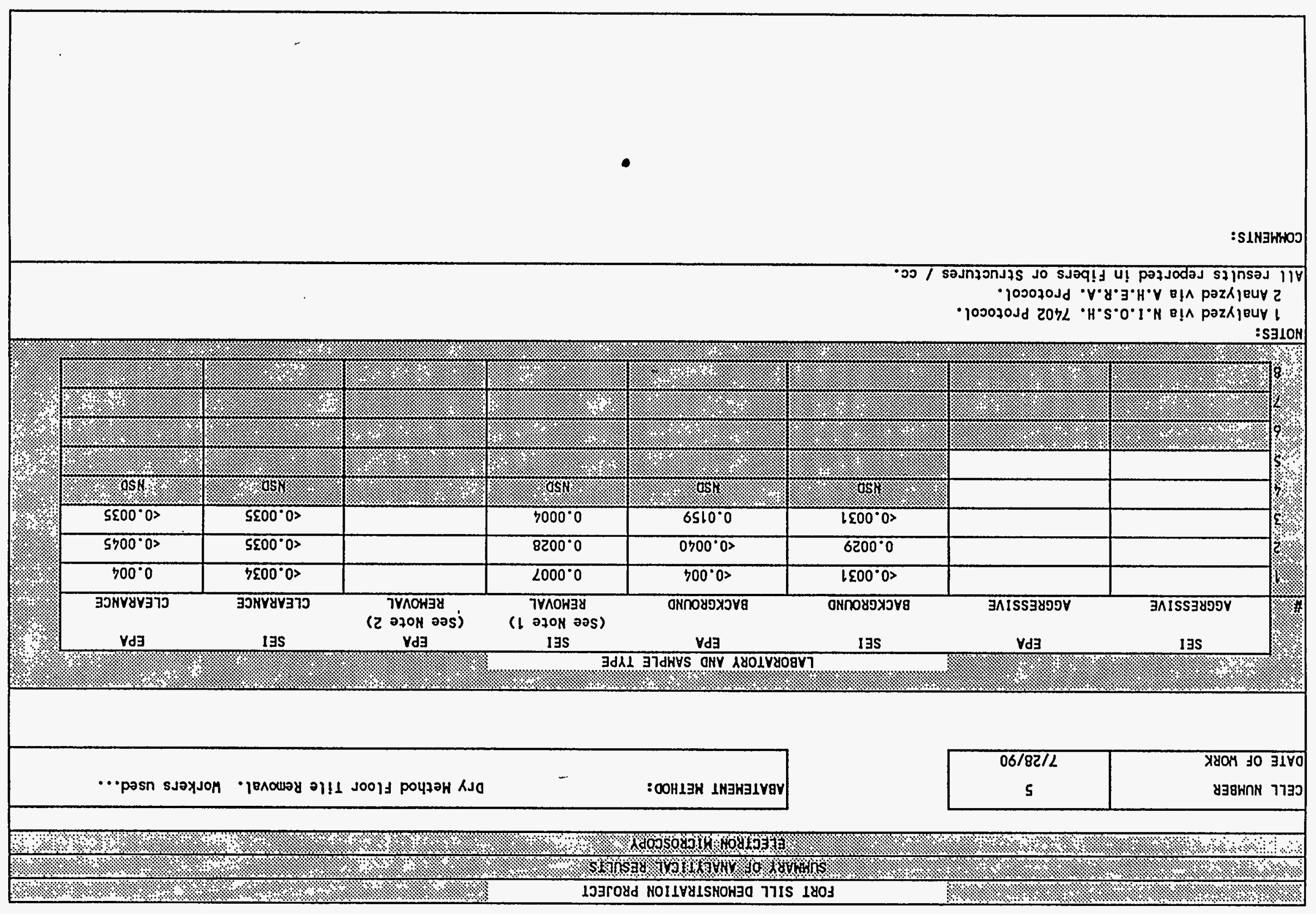




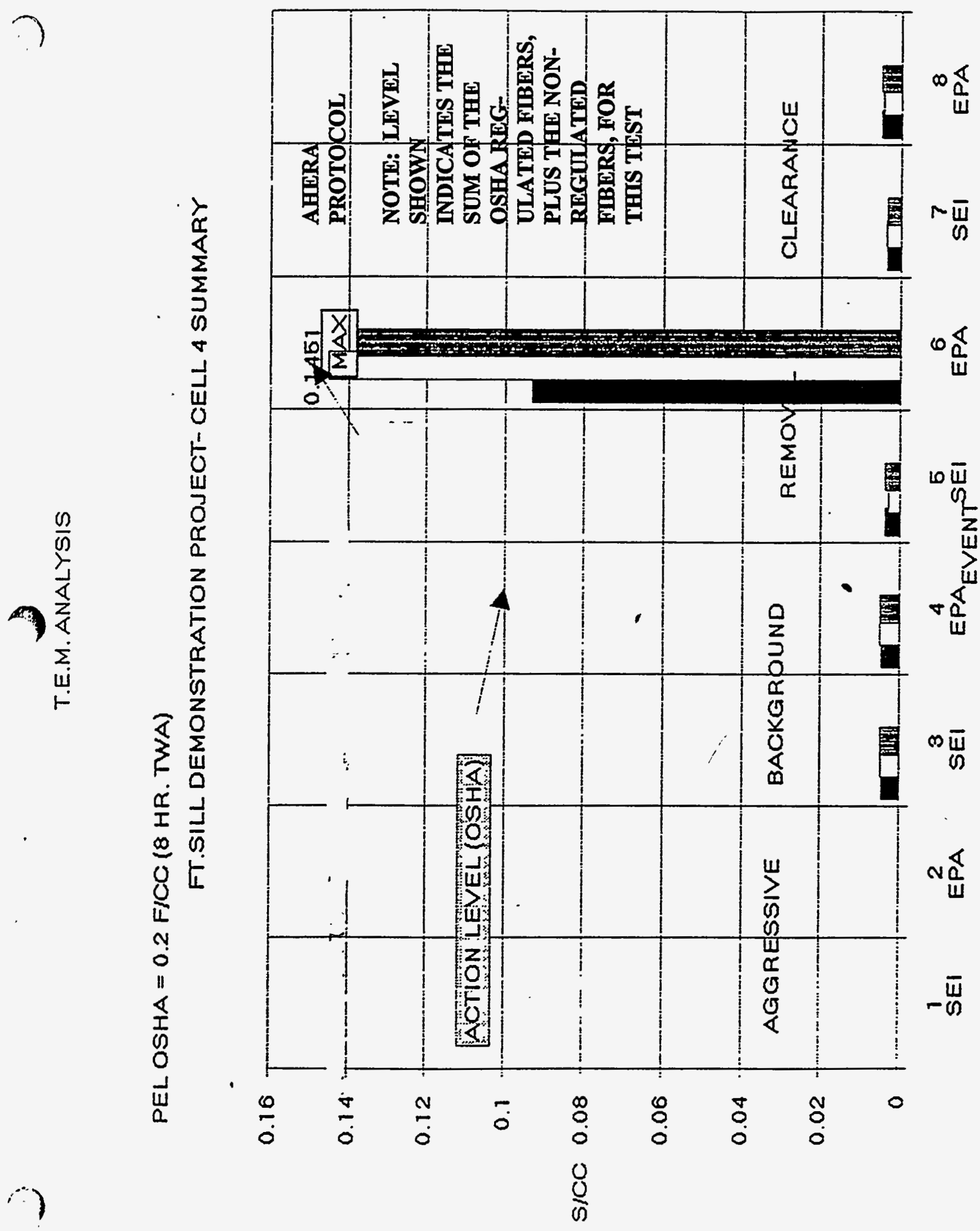

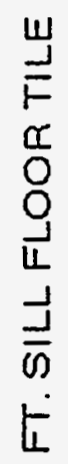




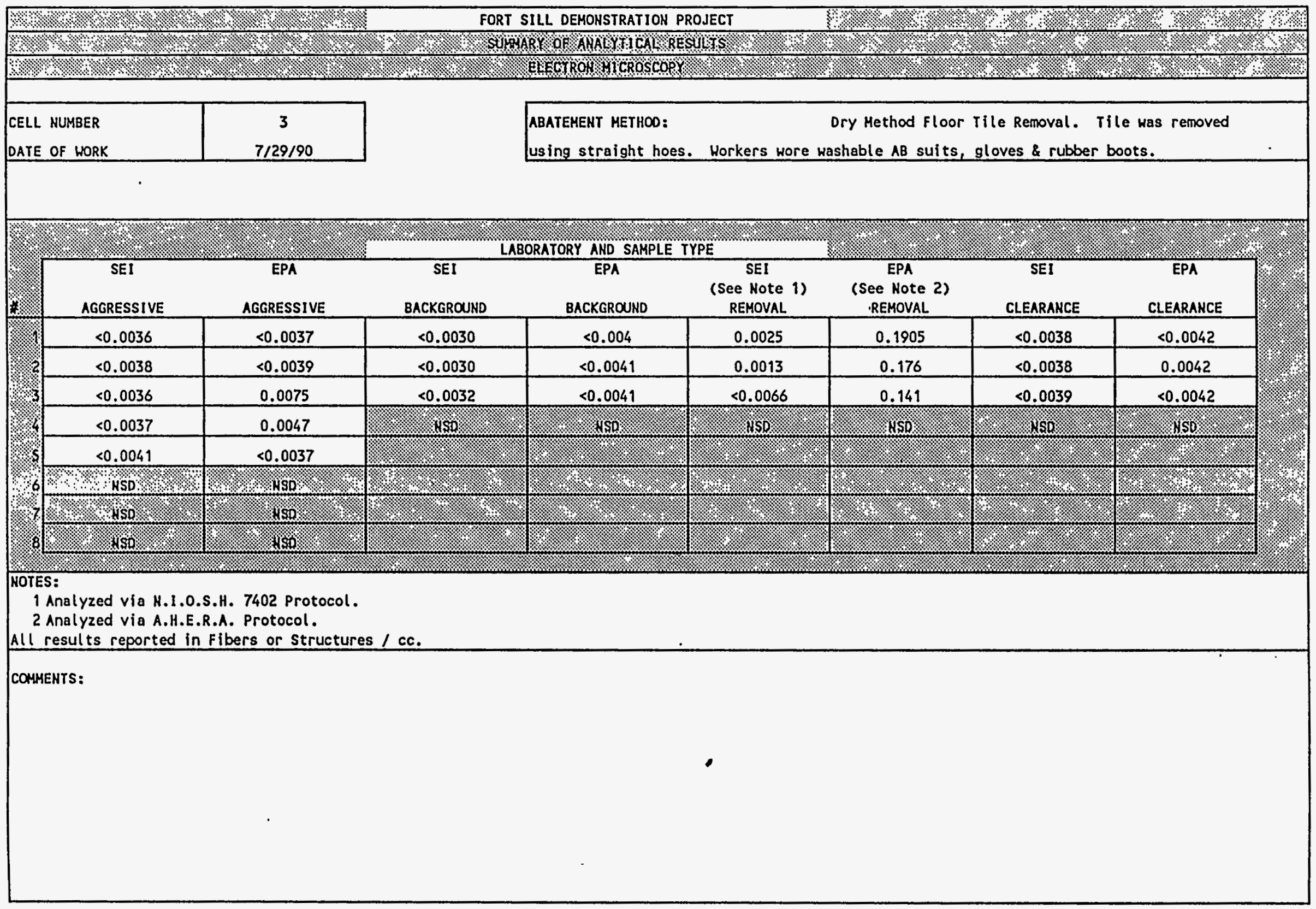


PEL OSHA $=0.2$ FICC $(8$ HR. TWA $)$

FT.SILL DEMONSTRATION PROJECT-CELL 5 SUMMARY

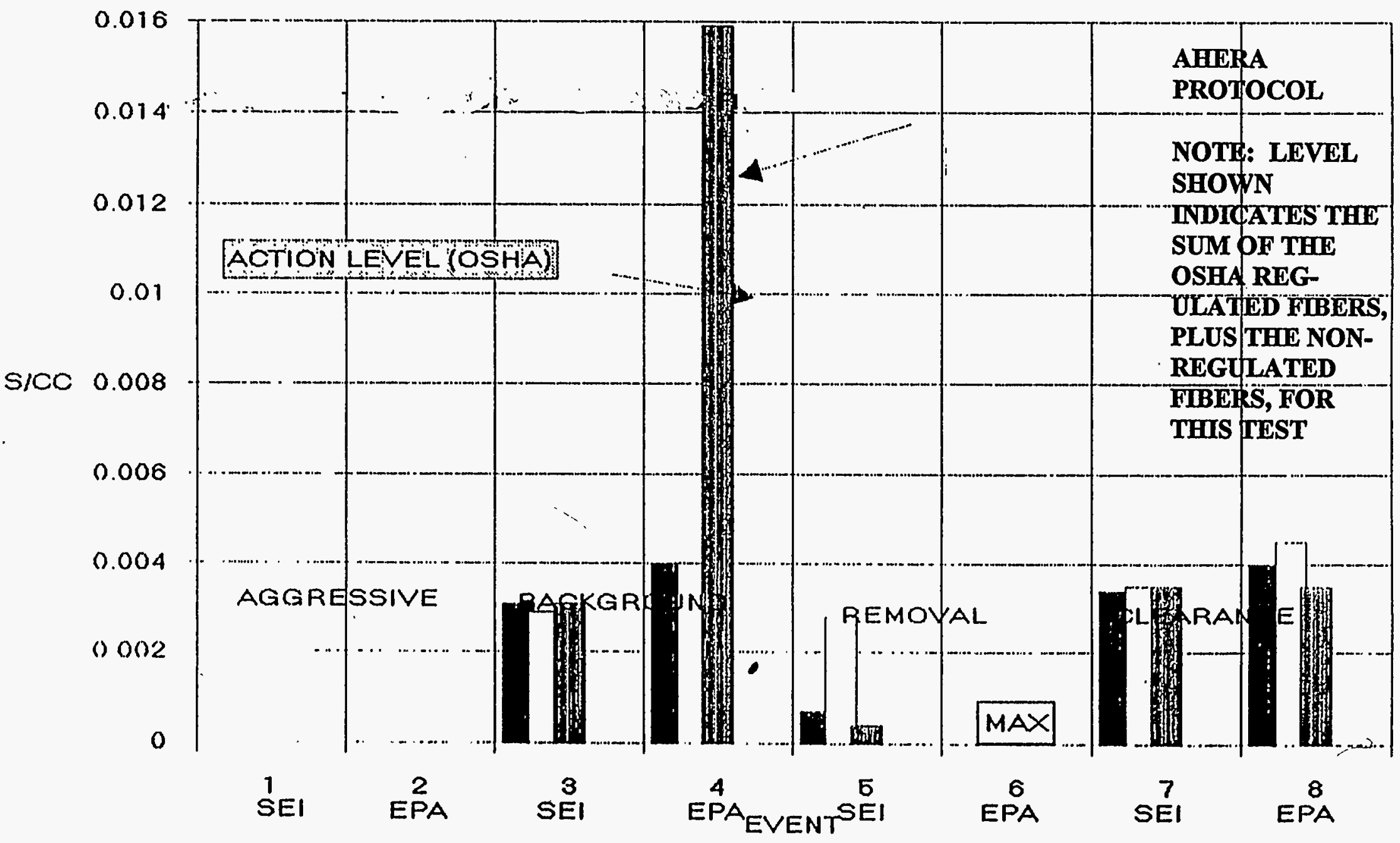




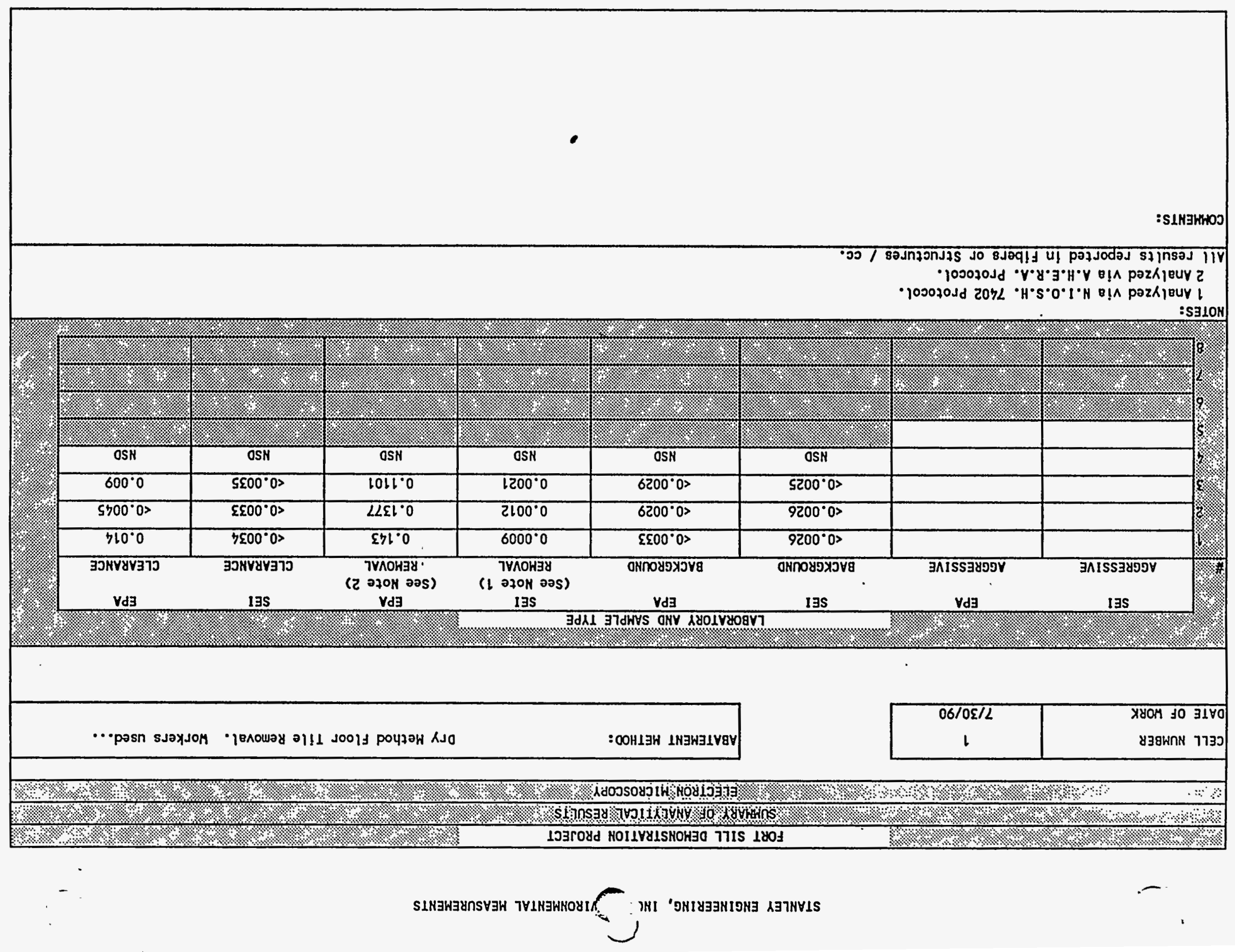



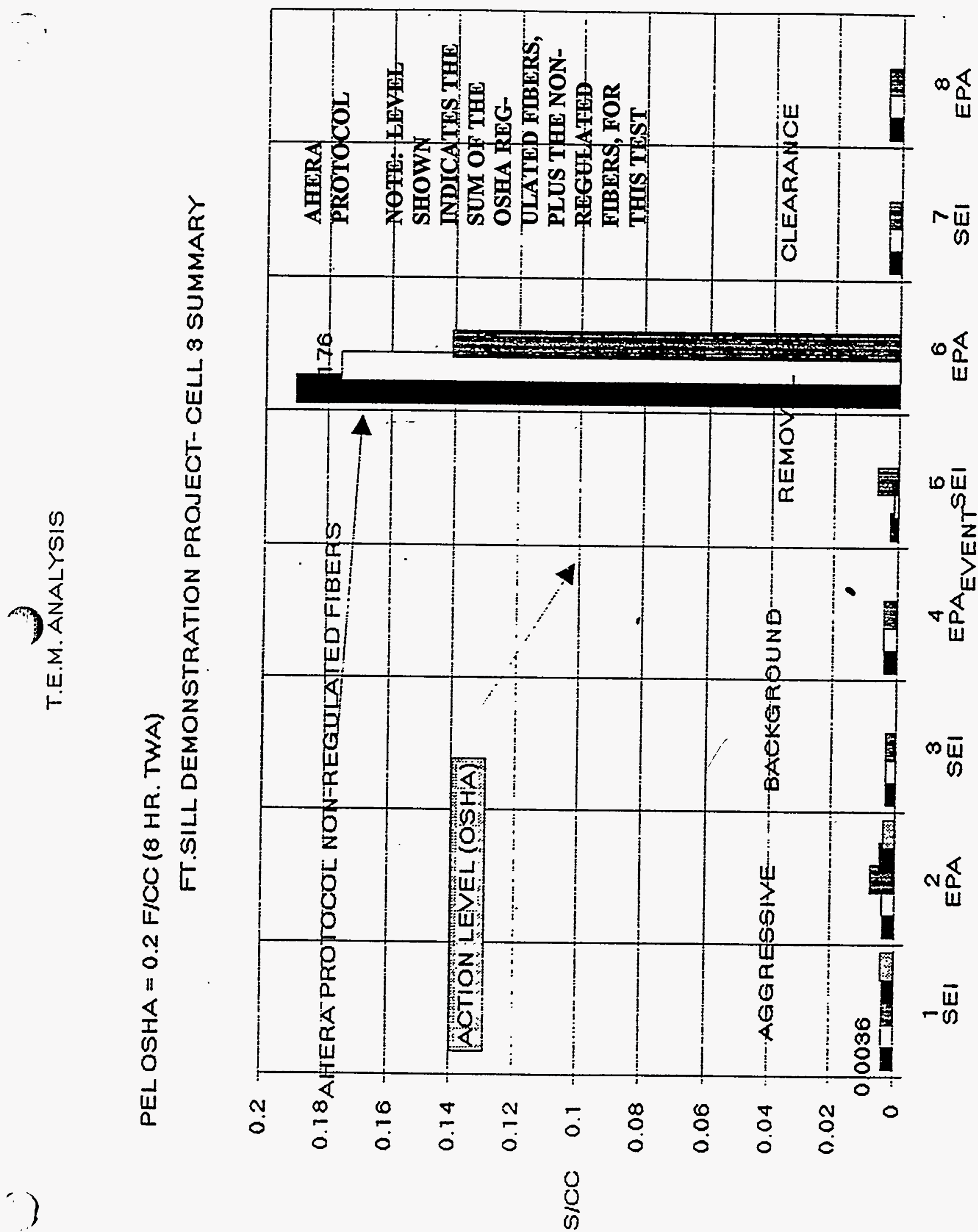

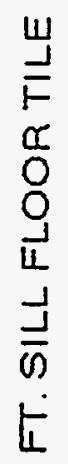




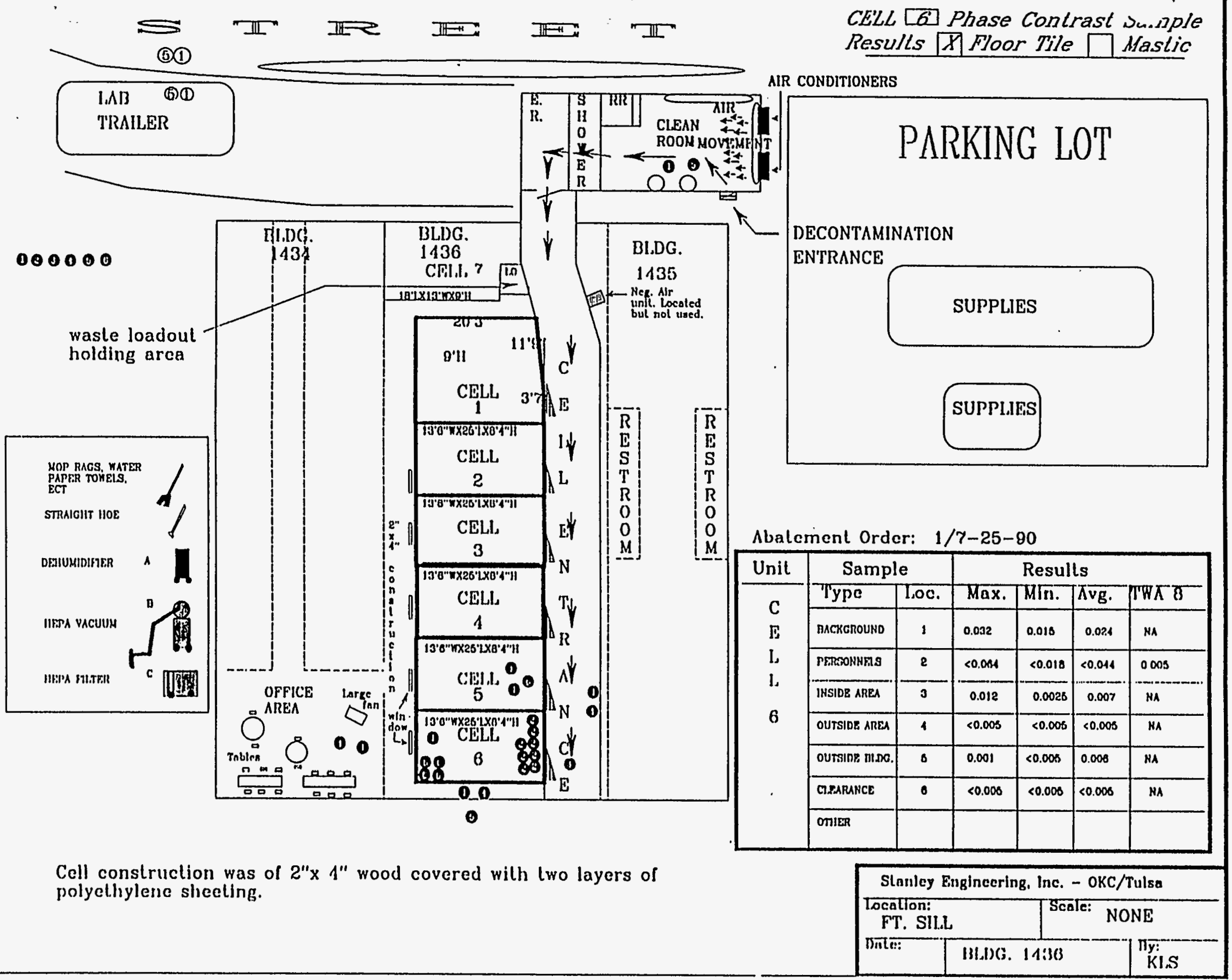




\begin{tabular}{|c|c|c|c|c|c|c|c|c|c|c|c|}
\hline & & & & & TRAHSHISSIC & IOH ELECTRON & MICROSCOPI & Y (T.E.M.) & & & \\
\hline & & & & A.H.E.R.A. & Analytical & 1 Protocol & CIniti & ial Screenin & g Test) & & \\
\hline Client: & & TULSA CORPS & $S$ OF ENGINEE & ERS & & NVLAP & NLWIBER - & -1568 & & & \\
\hline Project: & & FT. SILL AB & BATEMENT PRO & OOJECT & & 2700 & M.H. 39th S & Street & & Grid Lot I.D. & \\
\hline PPE: & & DUAL CARTRI & IDGE - FULL & FACE MASK & & Oklahom & ma city, OK & K 73112 & & Number of Samples Counted: & \\
\hline Date: & & $7 / 25 / 90$ & Analyzed & $7 / 30 / 90$ & Reported & & 405) 948-65 & 505 & & Grid Open Area: $\left(\mathrm{mm}^{\wedge} \mathrm{2}\right)$ & 0.0081 \\
\hline FAX Numbe & & & & & & Analyst: & KEITH L.S & STANLEY, Ph. & & Filter Area: $\left(m m^{\wedge} 2\right)$ & 385 \\
\hline $\begin{array}{l}\text { SALPrib\% } \\
\text { Prior to } C\end{array}$ & $\begin{array}{l}\text { oupinoNs } \\
6 \text { Abatement }\end{array}$ & 1. & Aggressive & Background & Samples $\mathrm{Ta}$ & aken & $\begin{array}{l}\text { DES } \\
\text { C6 and Lea }\end{array}$ & $\begin{array}{l}\text { Oof ERSS } \\
\text { af Blower. }\end{array}$ & \%ে & Aggressive $=$ HEPA Unit Operatin & Inside \\
\hline 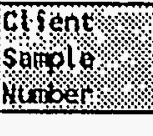 & hipho & Pobestos & 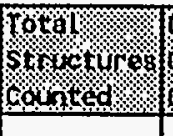 & connos & (1) & 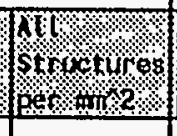 & surocturos & 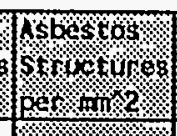 & 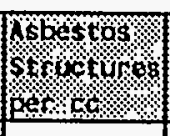 & $31.1 \%$ \% & \\
\hline C601N & 901204 & 0 & 9 & 10 & 1320 & 111.111111 & 0.03240741 & & 0 & BLDG 1436 - Cell 6 East & \\
\hline $\mathrm{C} 602 \mathrm{~N}$ & 901205 & of & 4 & 10 & 1303.05 & 49.382716 & 0.01459065 & & 0 & BLOG 1436 - Cell 6 East Center & \\
\hline $\mathrm{C} 603 \mathrm{~N}$ & 901206 & of & 5 & 10 & 1280.3 & 61.7283951 & 0.01856239 & 18 & 0 & BLDG 1436 - Cell 6 Center & \\
\hline $\mathrm{C} 604 \mathrm{~N}$ & 901207 & 0 & 12 & 10 & 1475.6 & 148.148148 & 0.03865345 & 1. & 0 & BLDG 1436 - Cell 6 Hest Center & \\
\hline C605N & 901208 & of & 8 & 10 & 1268.5 & 98.7654321 & 0.02997611 & ig & 0 & BLDG 1436 - Cell 6 Hest & \\
\hline & & & & & & 0 & 0 & 0. & 0 & HOTE: EPA also analyzed under & \\
\hline & & & & & & 0 & 0 & 08. & 0 & Sample numbers C6001E-C6005E & \\
\hline & & & & & & 0 & 0 & 0 & 0 & & \\
\hline & & & & & & o & 0 & 0 & 0 & & \\
\hline & & & & & & 0 & 0 & 10. & 0 & & \\
\hline SUMMARY: & & & & & $\begin{array}{l}\text { TOTAL = } \\
\text { MEAN = }\end{array}$ & $\begin{array}{l}469.135802 \\
93.8271605 \\
\end{array}$ & 0.026838 & భै\% & 이 & & \\
\hline & ASBESTOSOSY & TRGGKORES R & ER $12 \%$ & \% & & & Hen 140 & 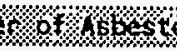 & 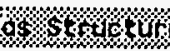 & 6) & 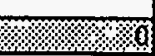 \\
\hline & $\begin{array}{l}\text { Mean }<=70 \\
\text { PASS }\end{array}$ & 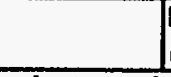 & $\begin{array}{l}\text { Mean }>70 \\
\text { FAIL }\end{array}$ & & & & Wenowno & \% & 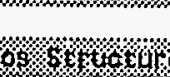 & - & \\
\hline
\end{tabular}


TRANSMISSION ELECTRON MICROSCOPY (T.E.M.)

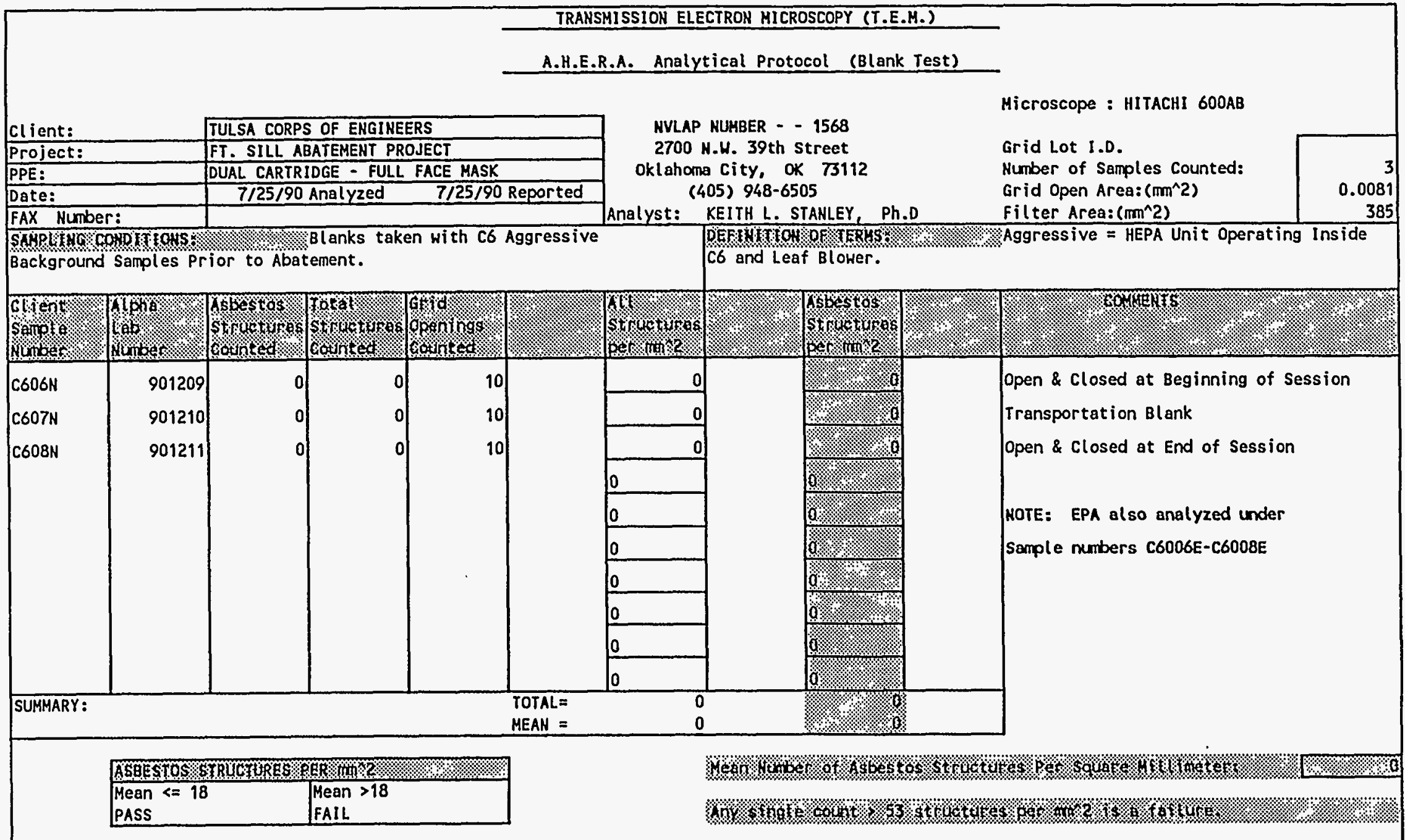




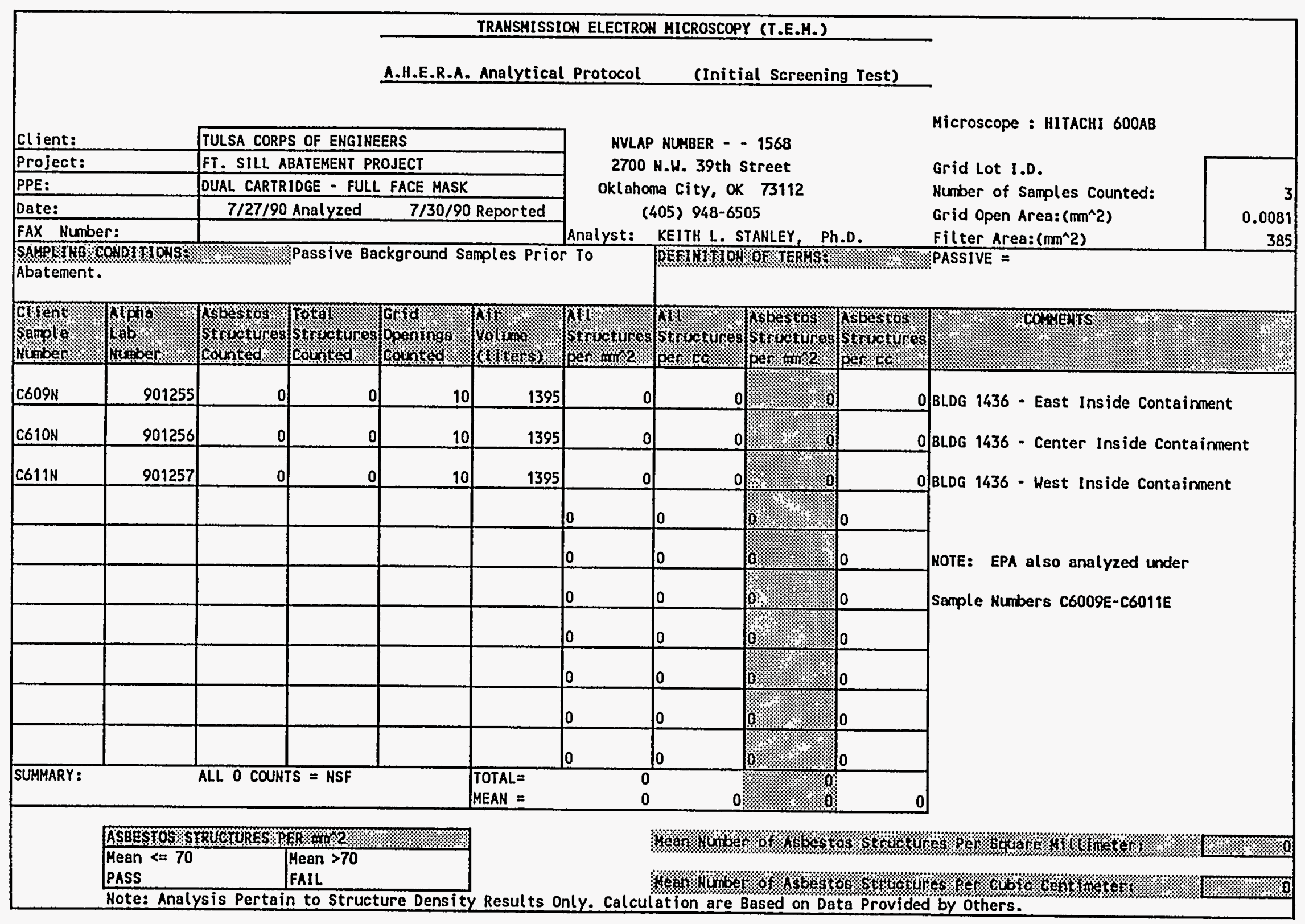




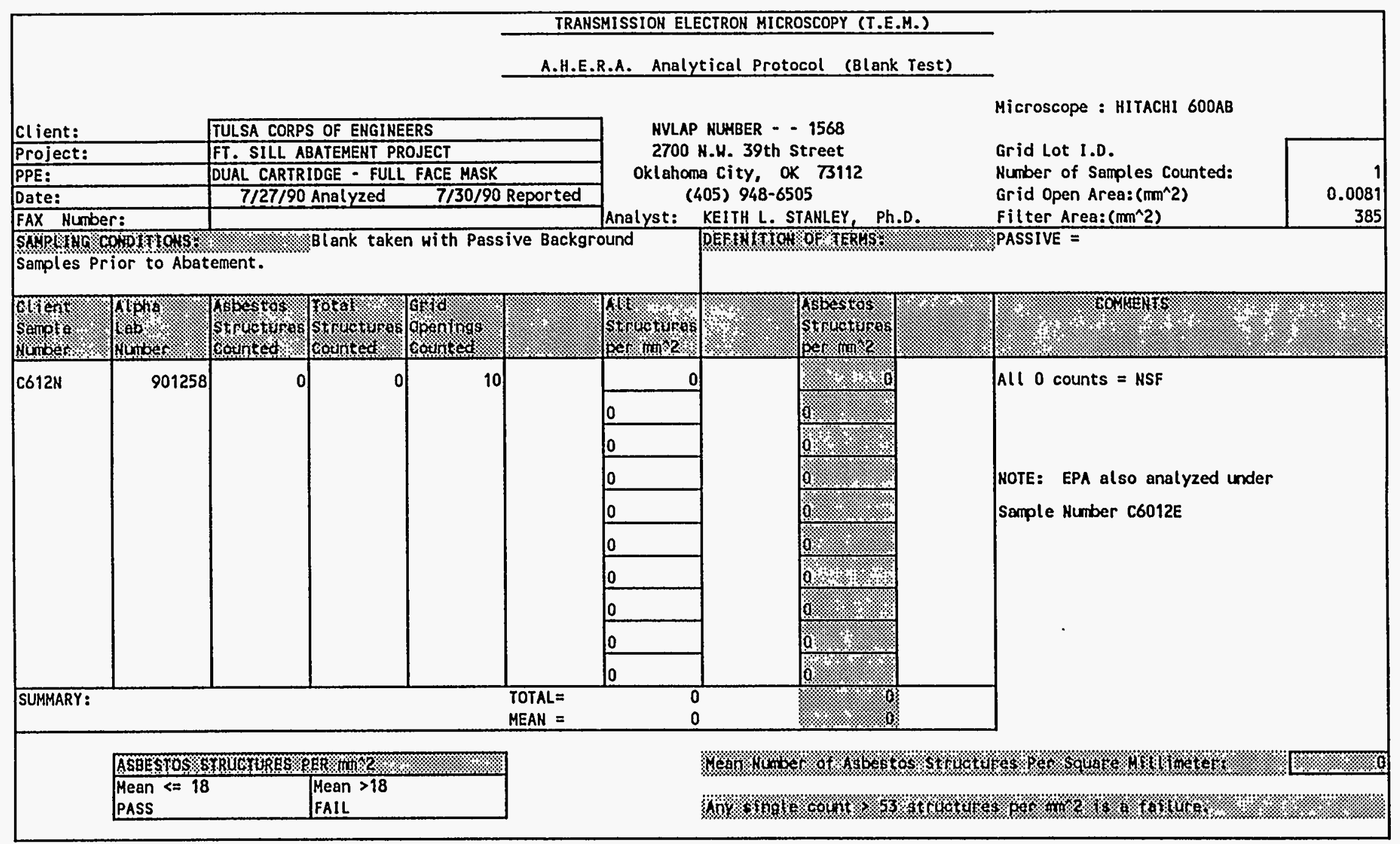




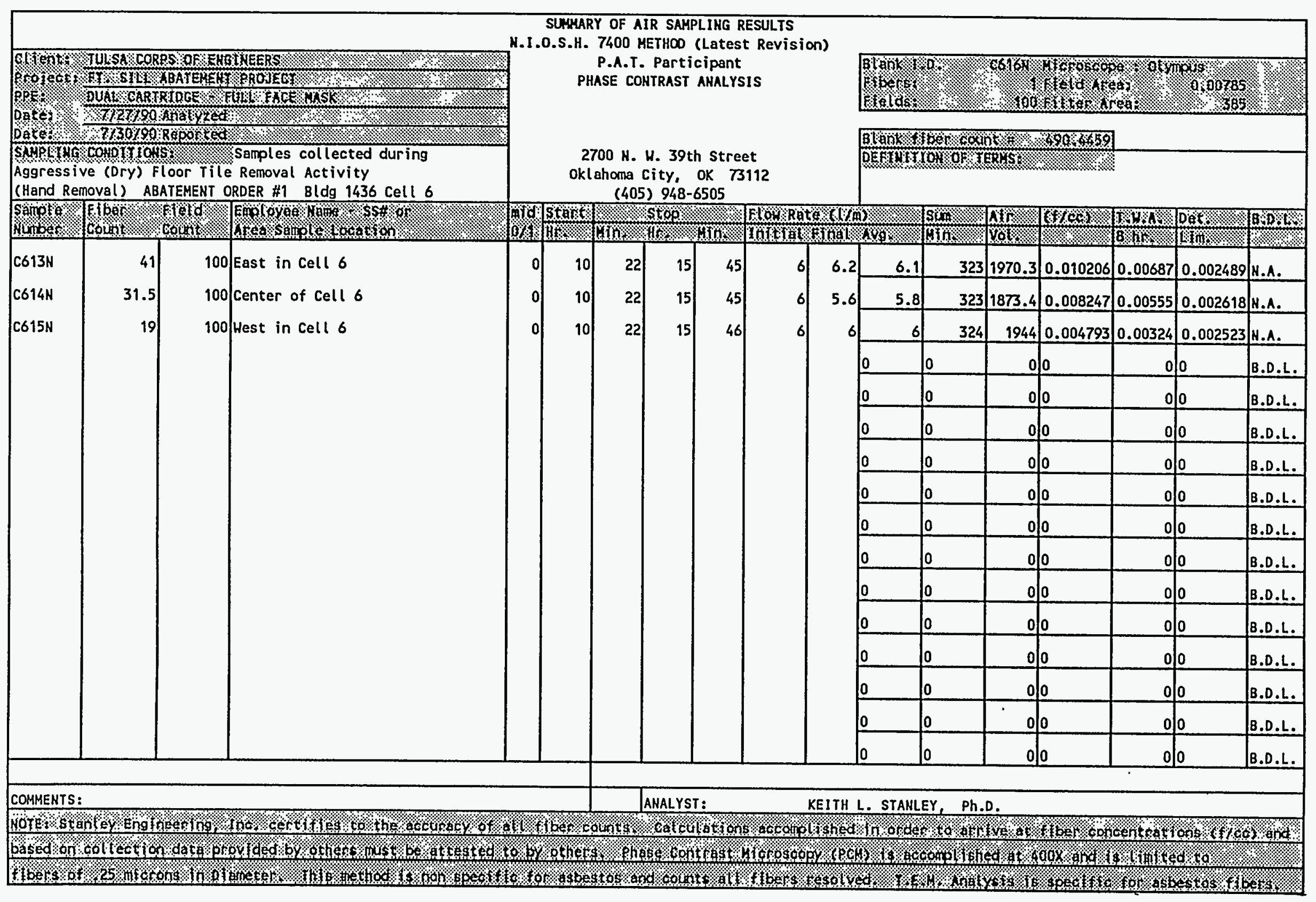




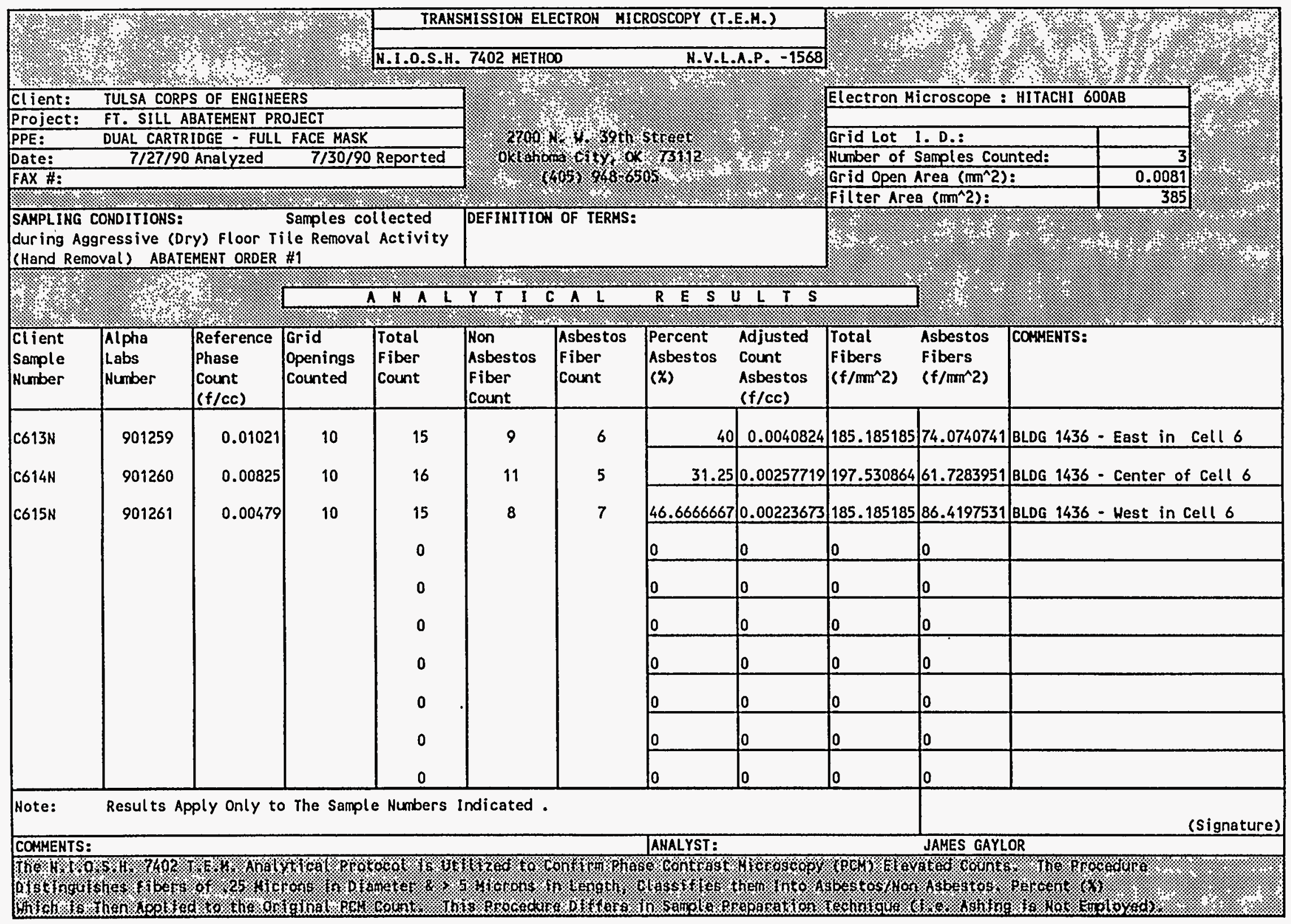




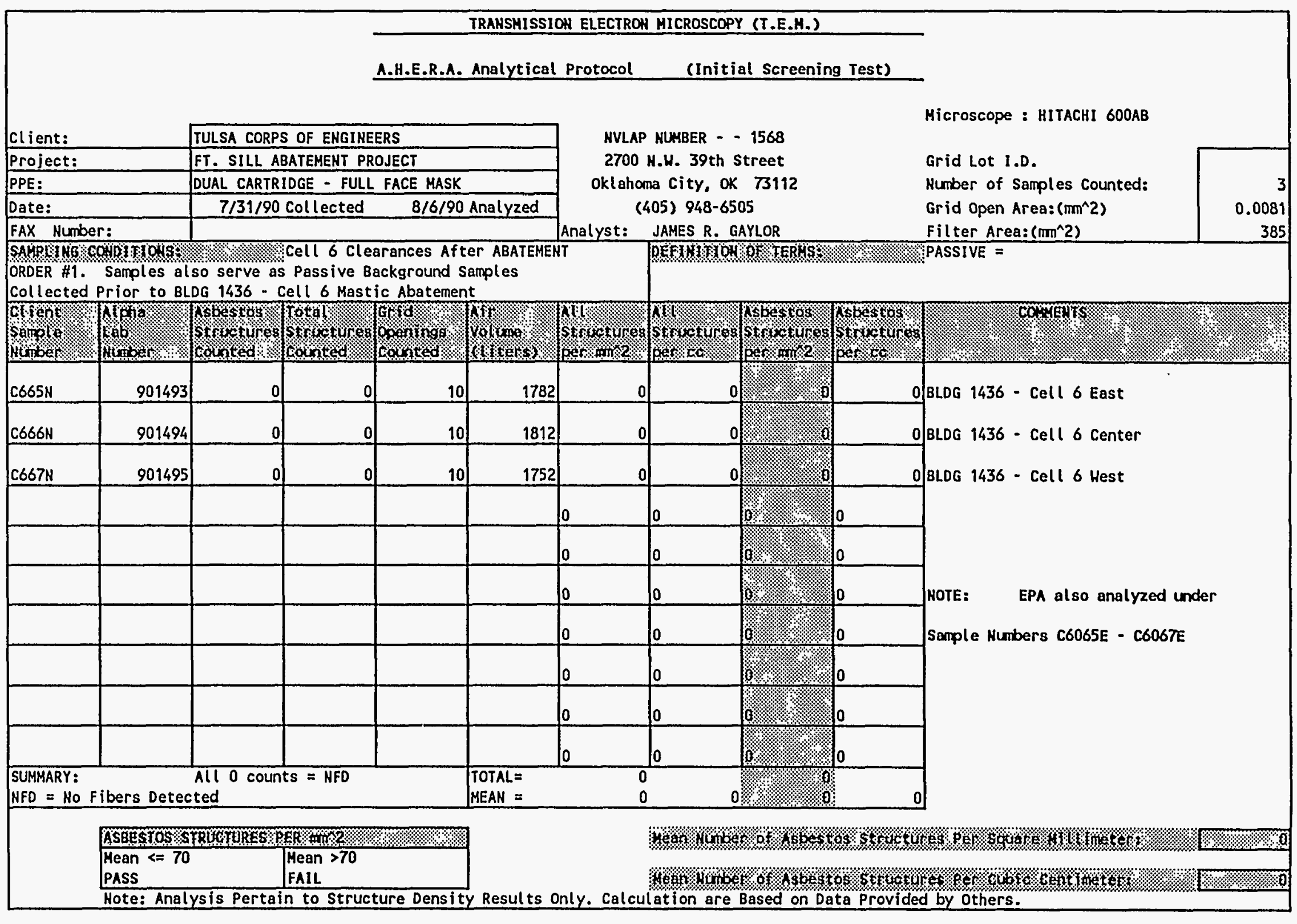




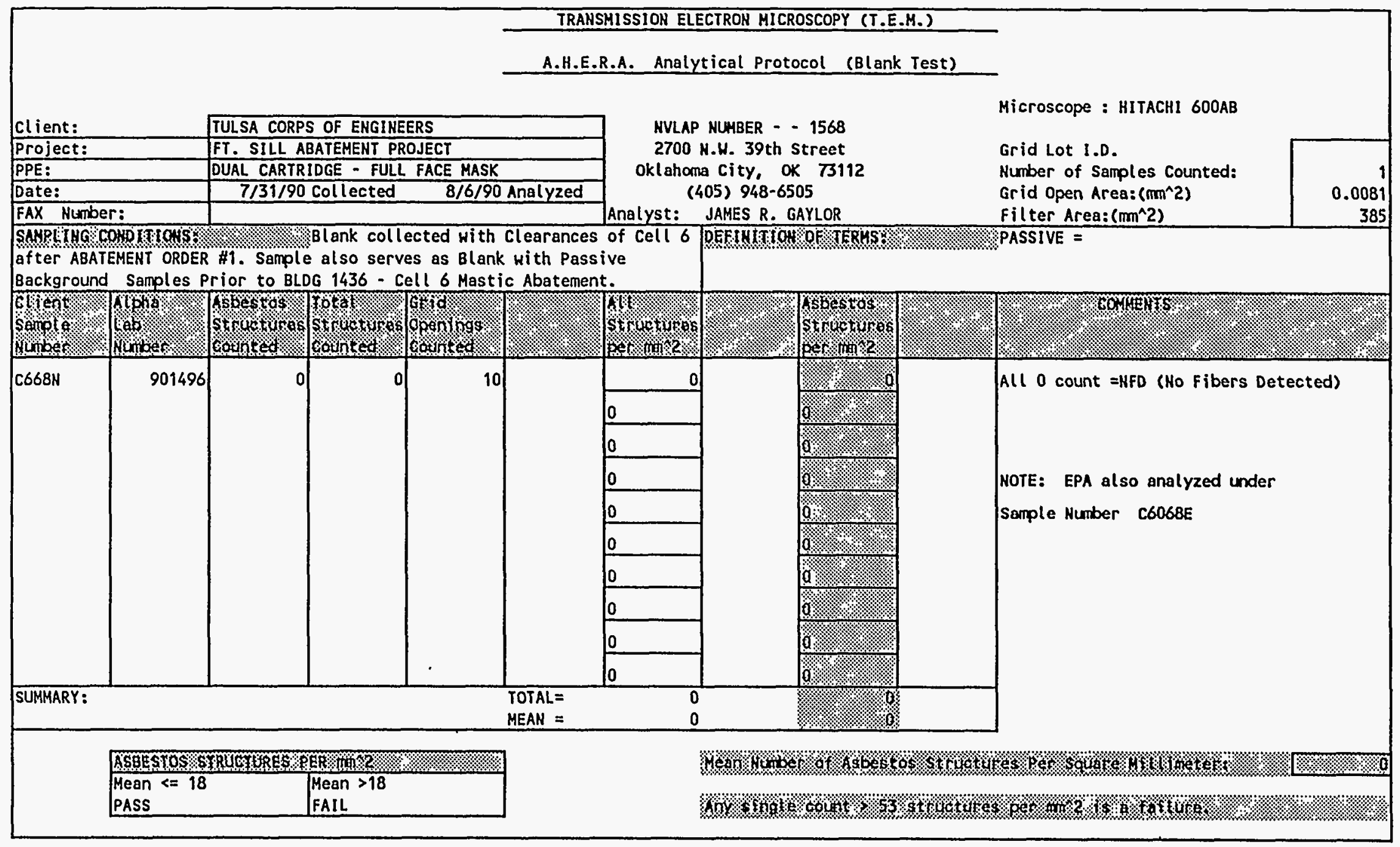




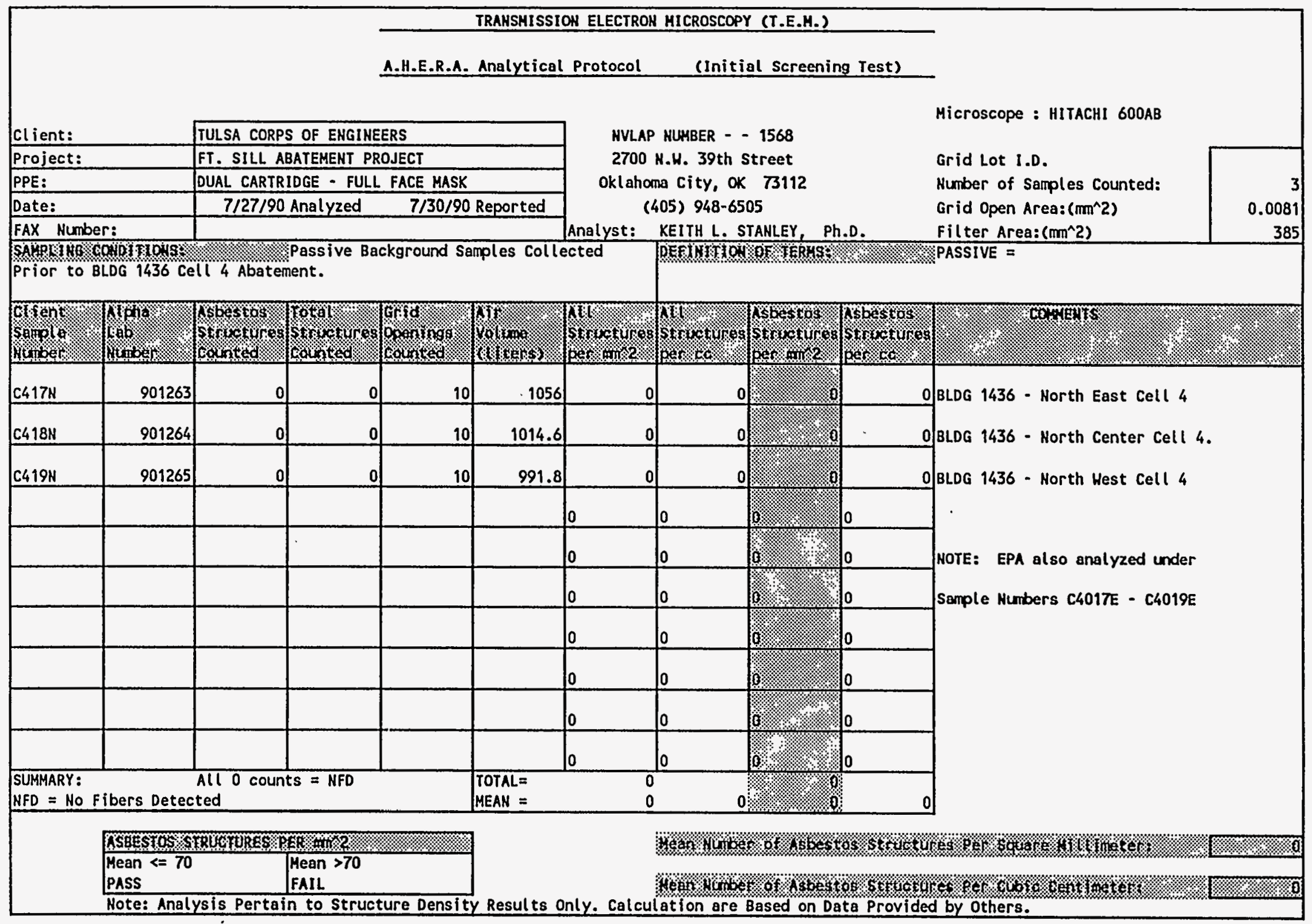




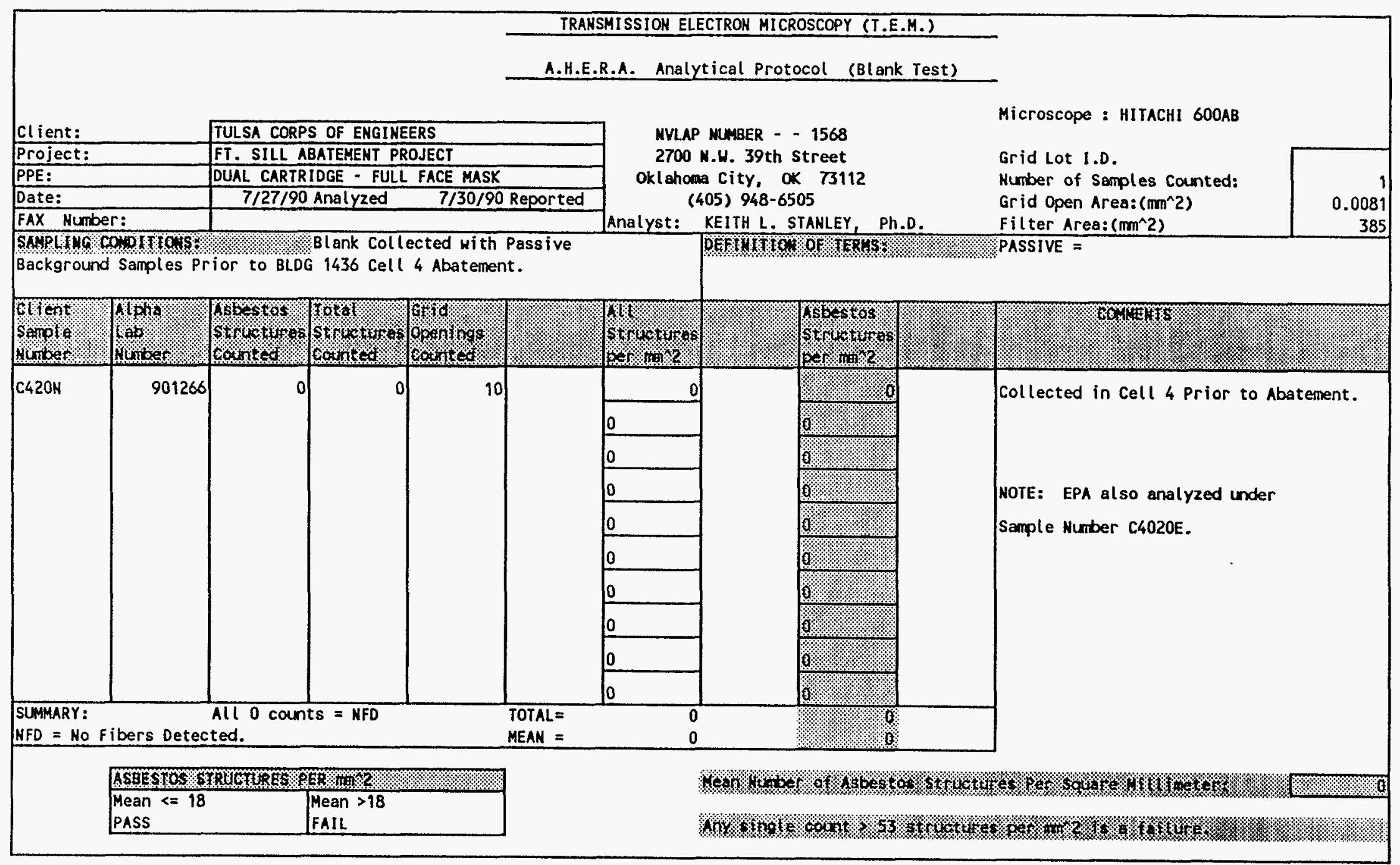




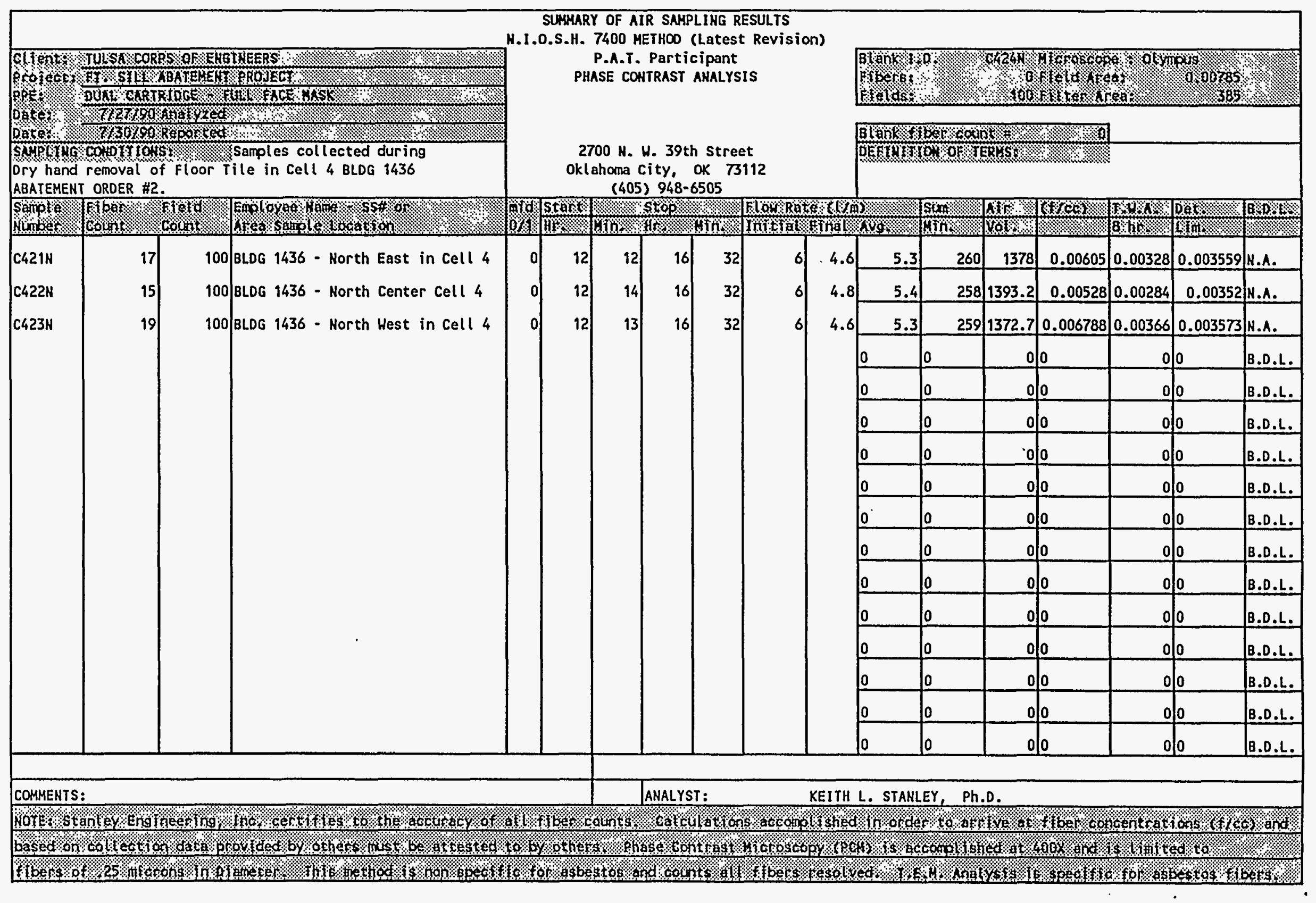




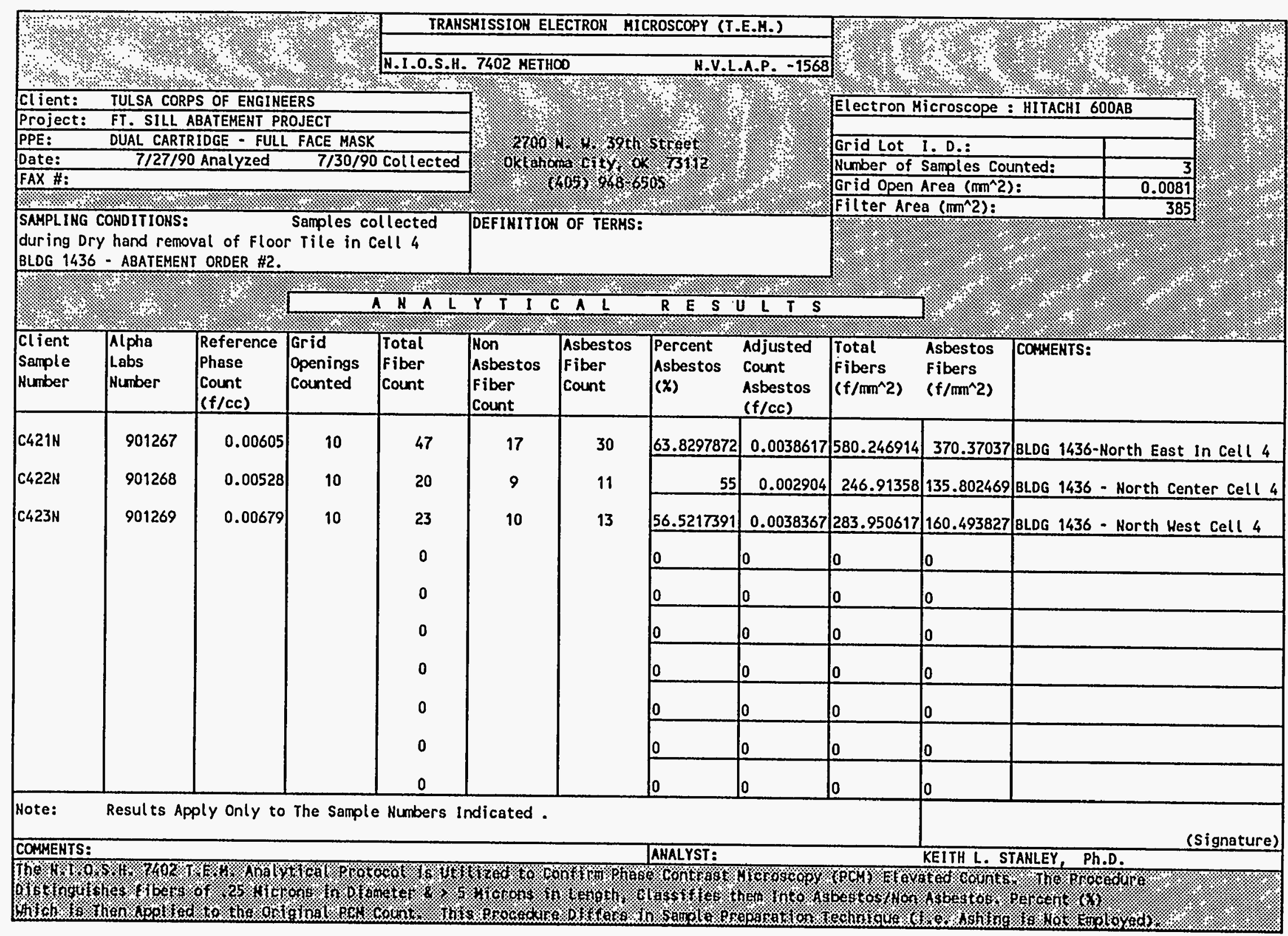




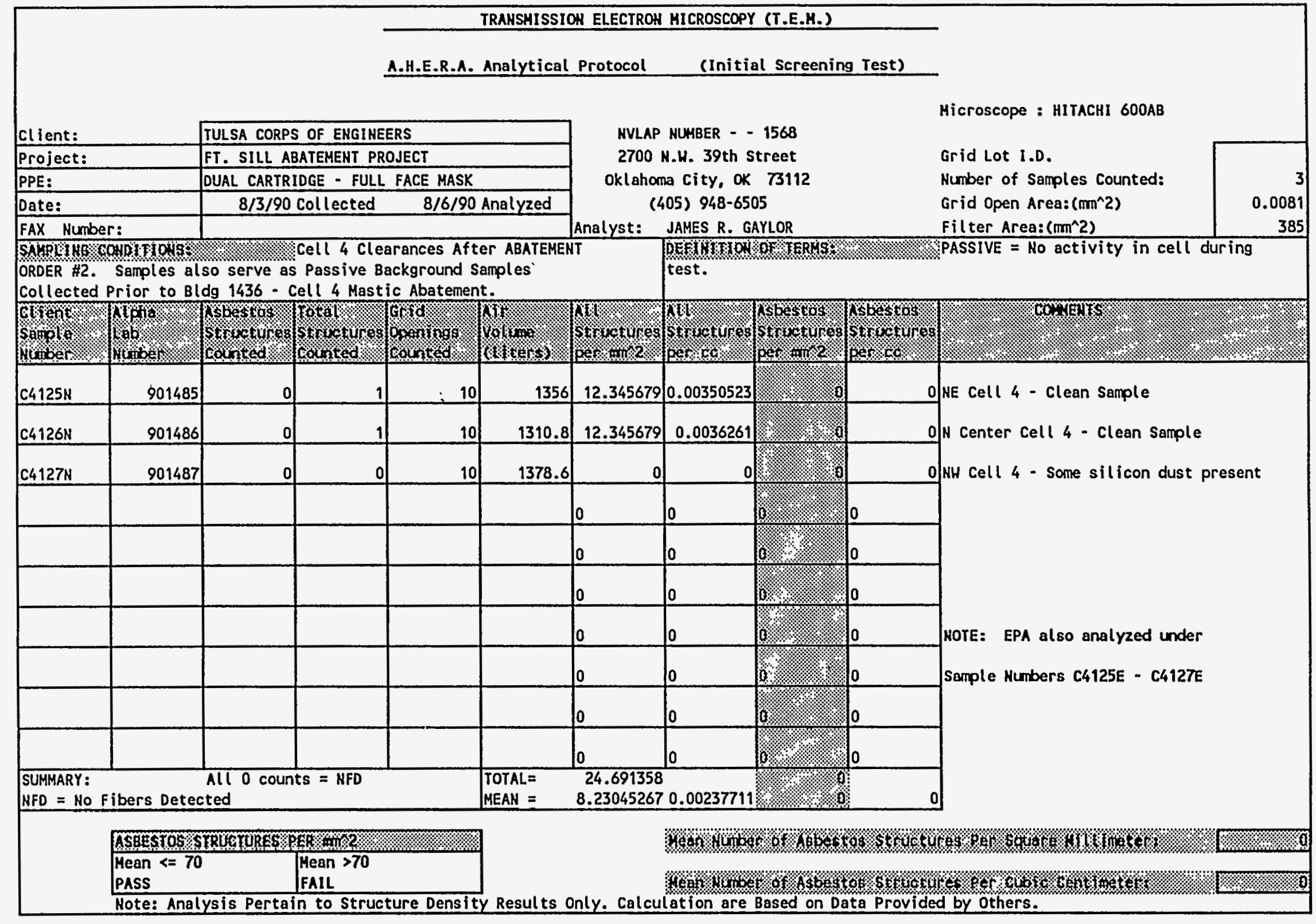


TRANSMISSION ELECTROH MICROSCOPY (T.E.M.)

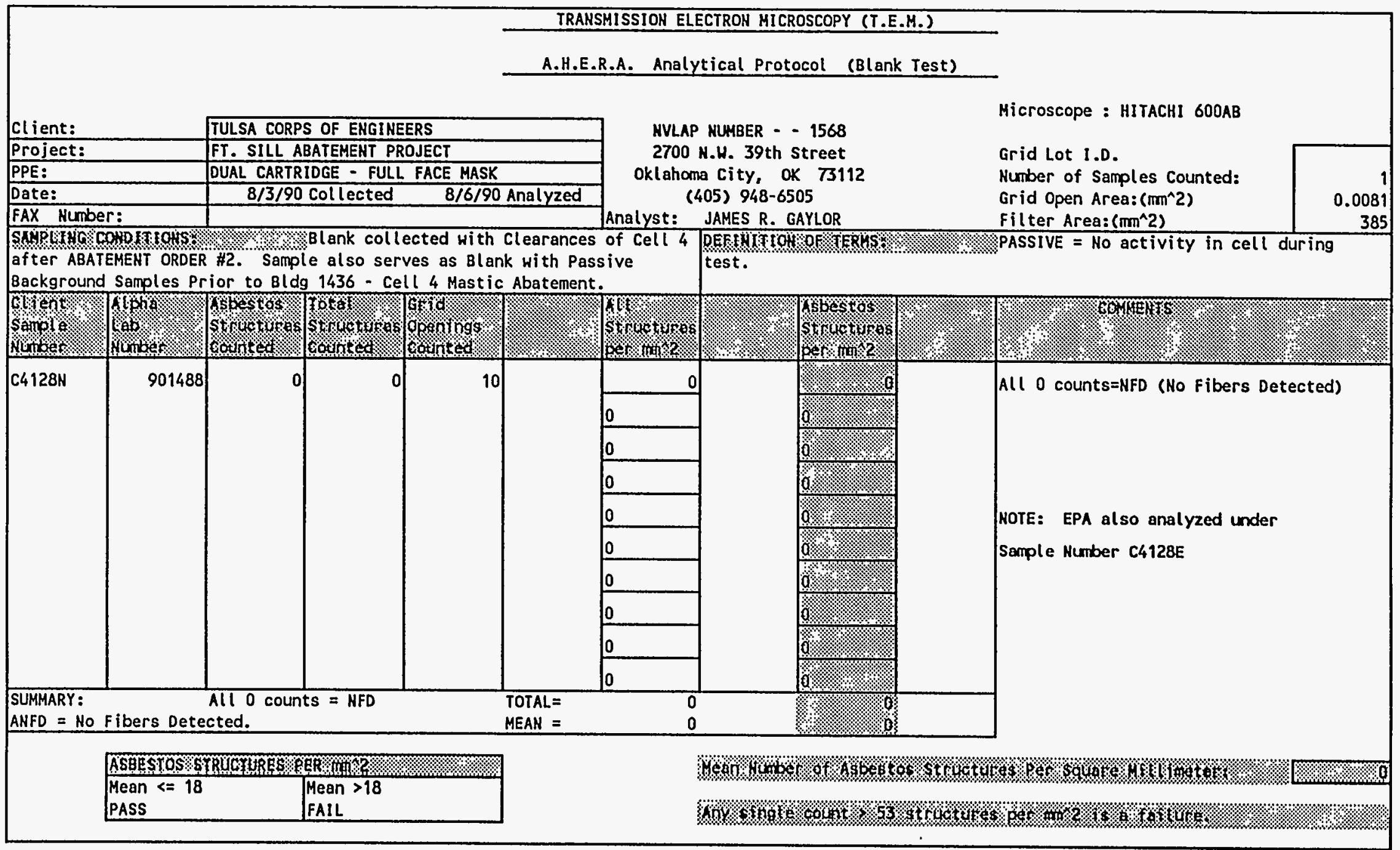




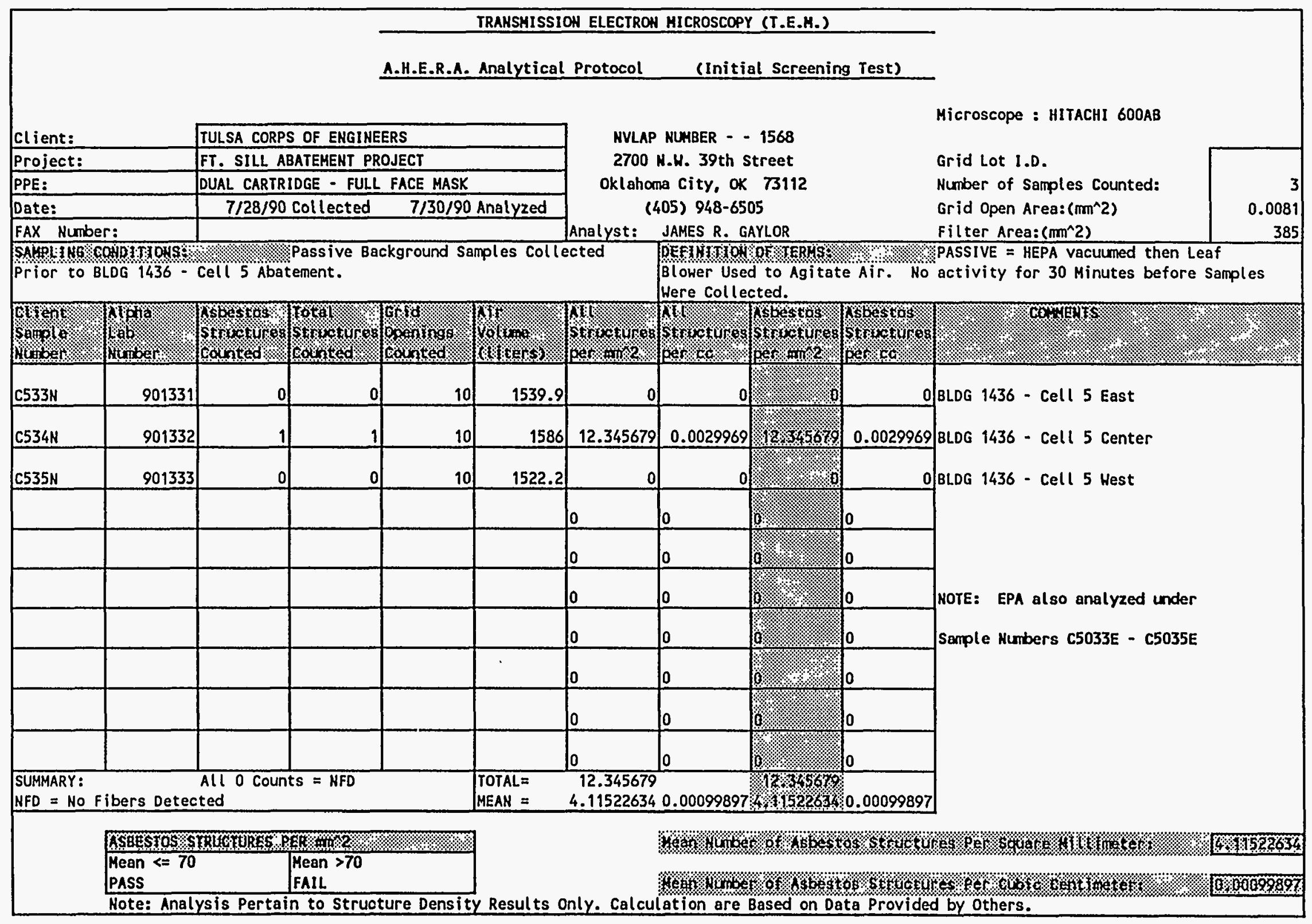


STAHLEY ENGINEERING, INC. - UIRONMENTAL MEASUREMENTS

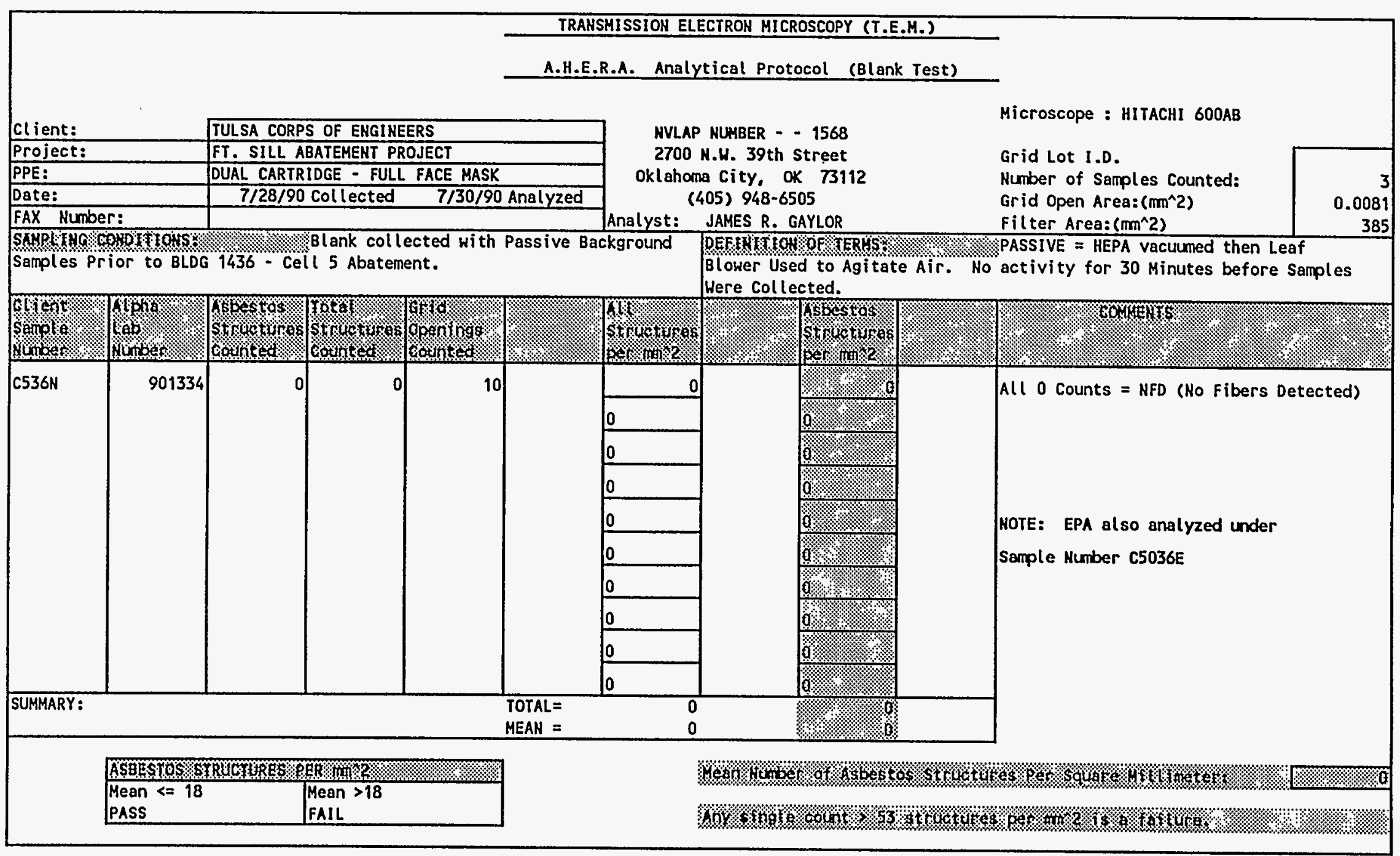




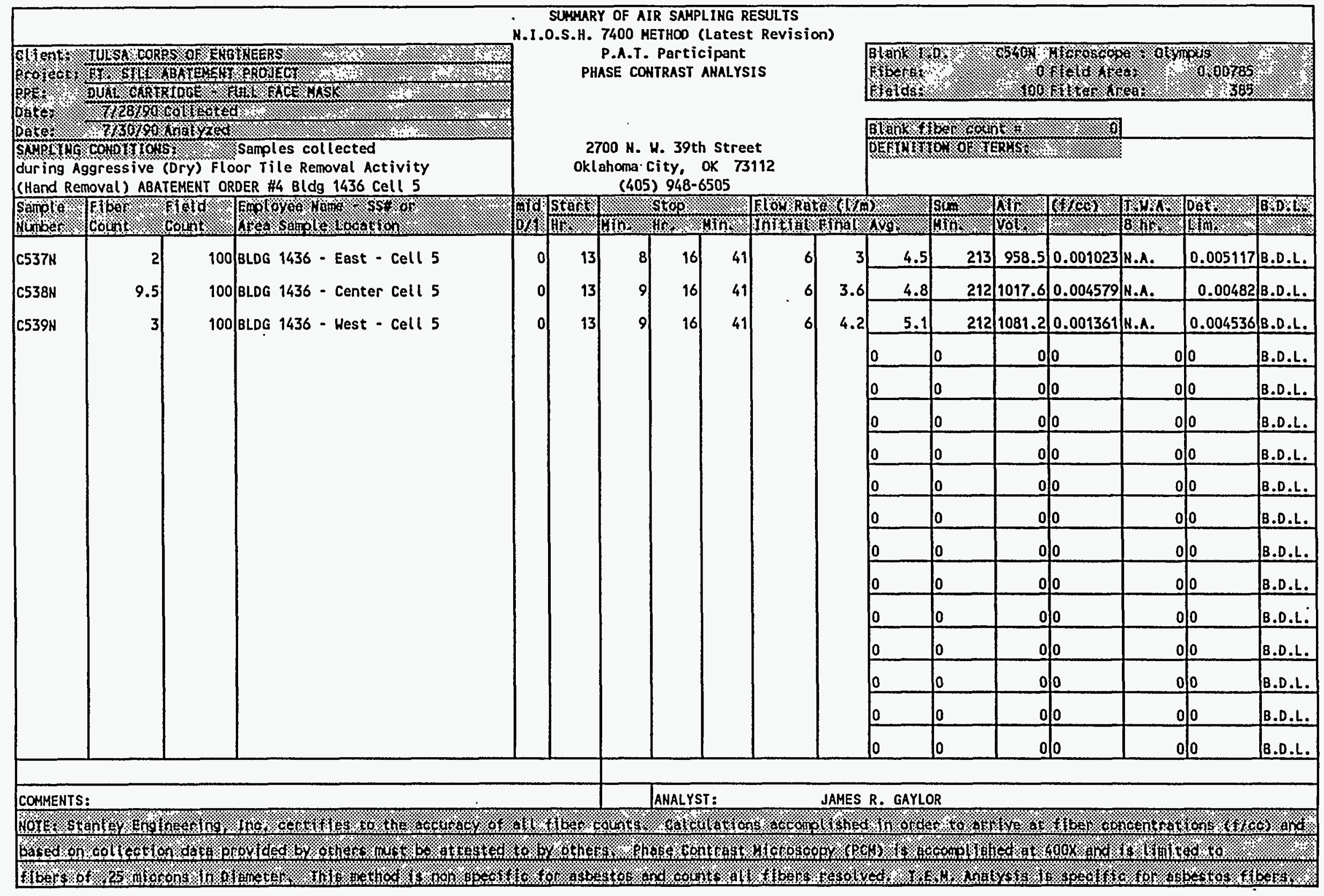




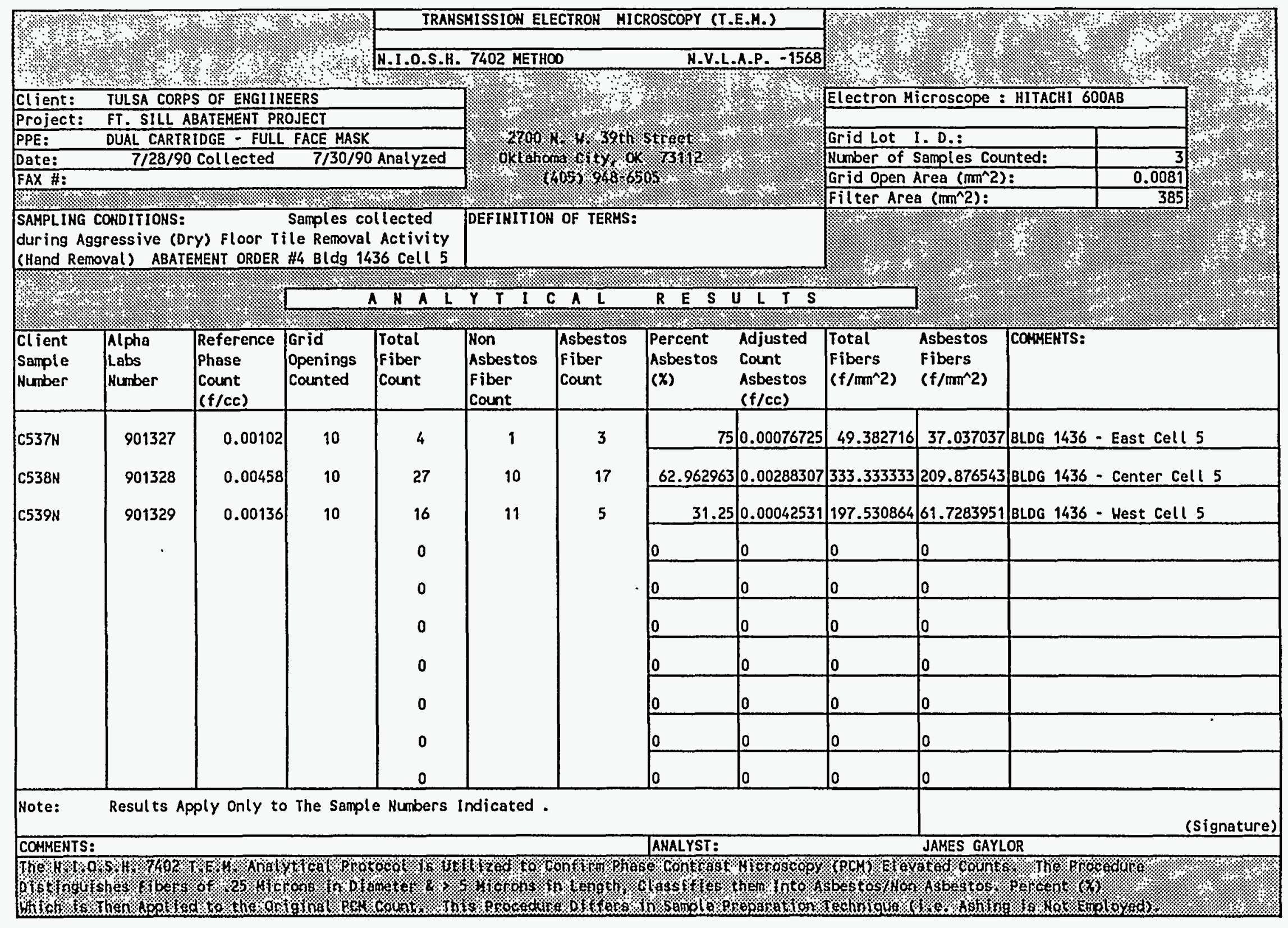




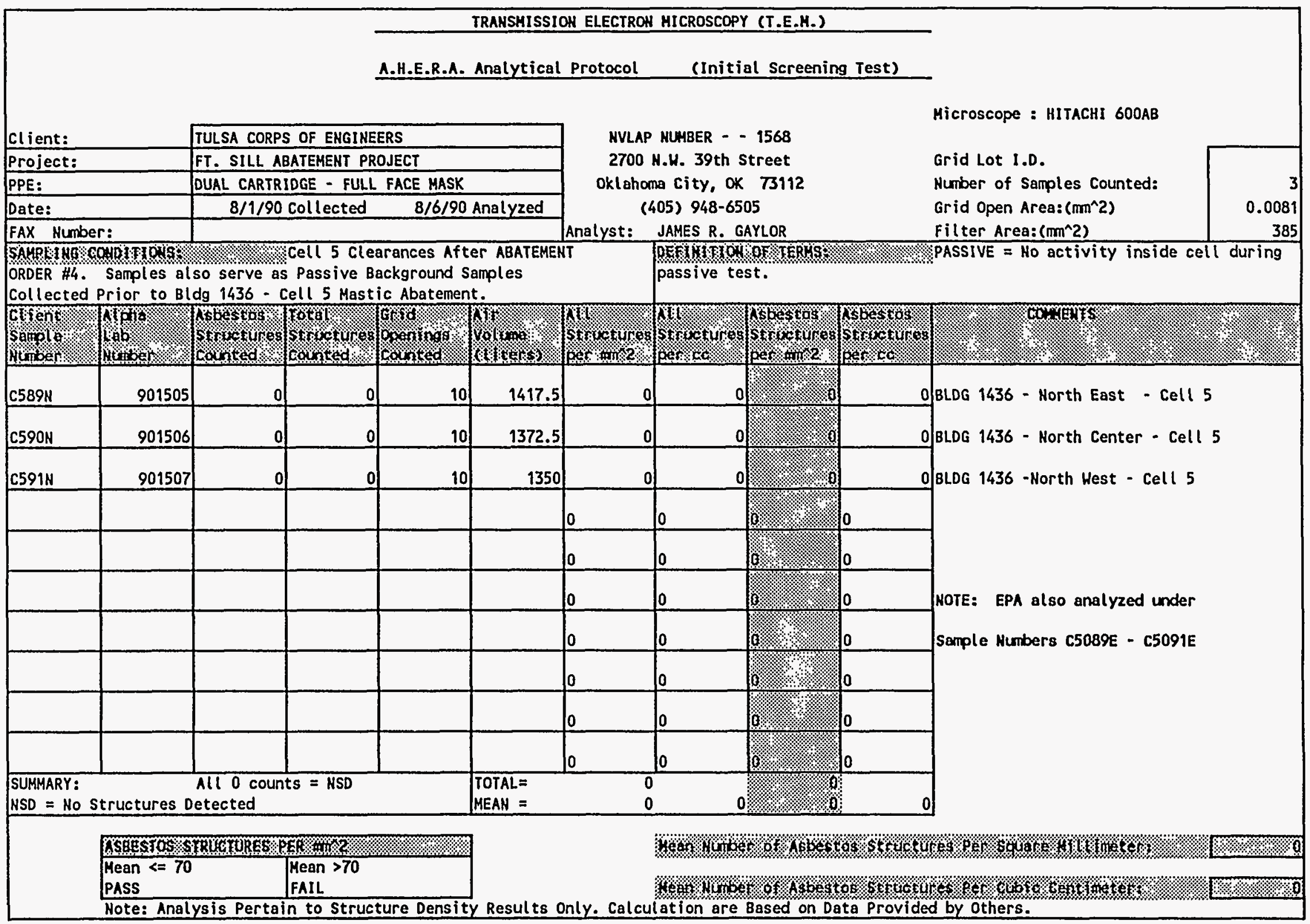




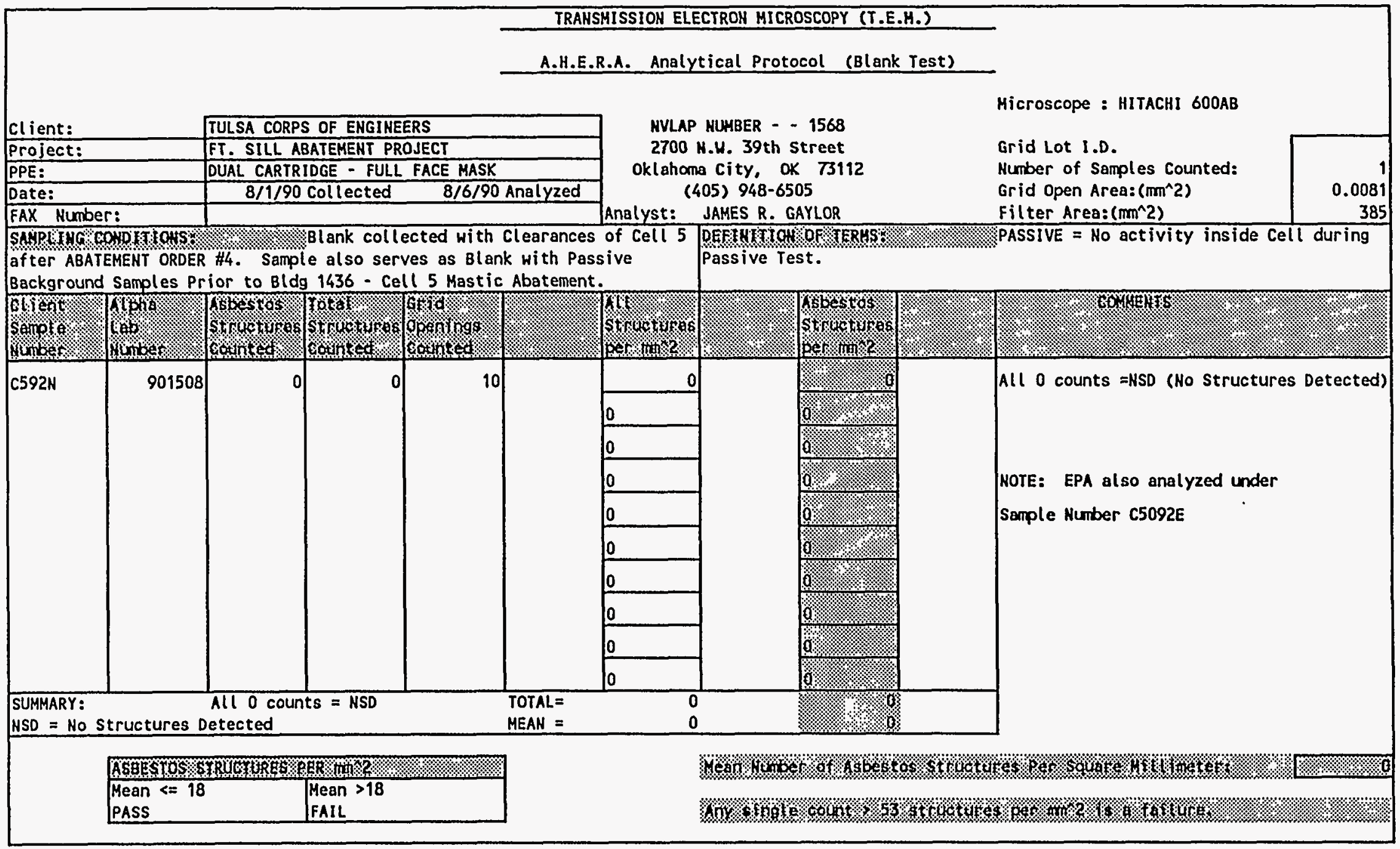




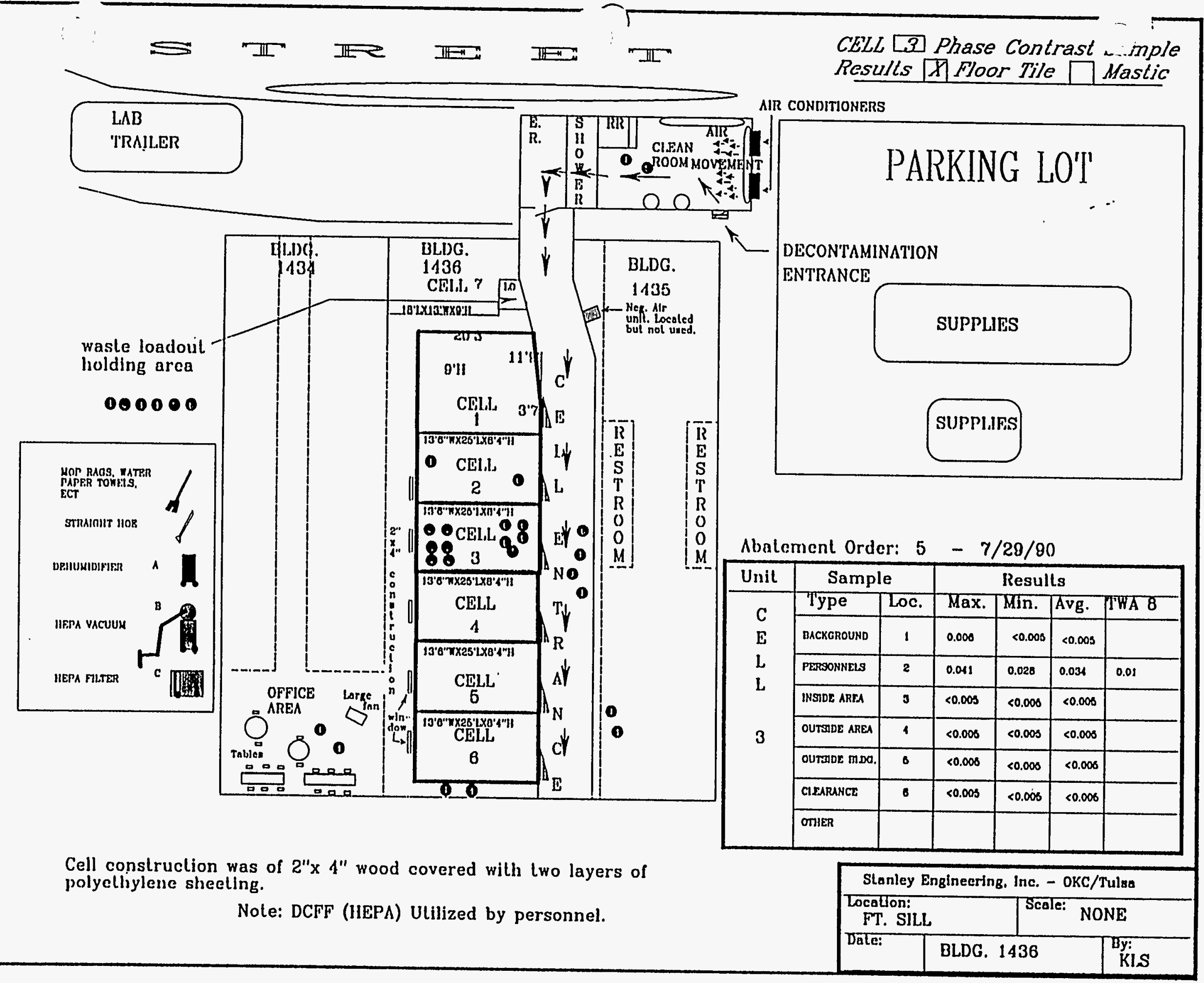




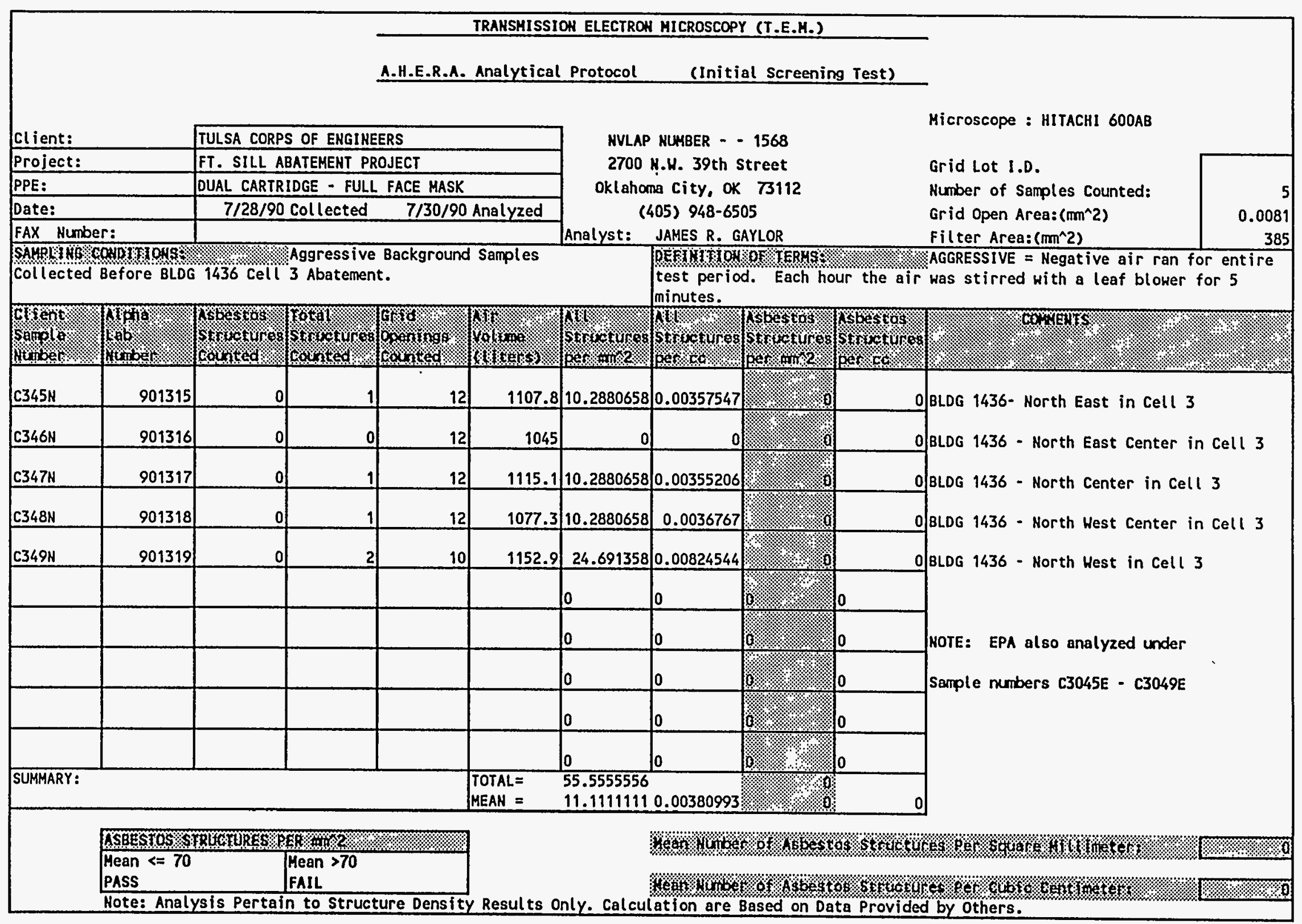




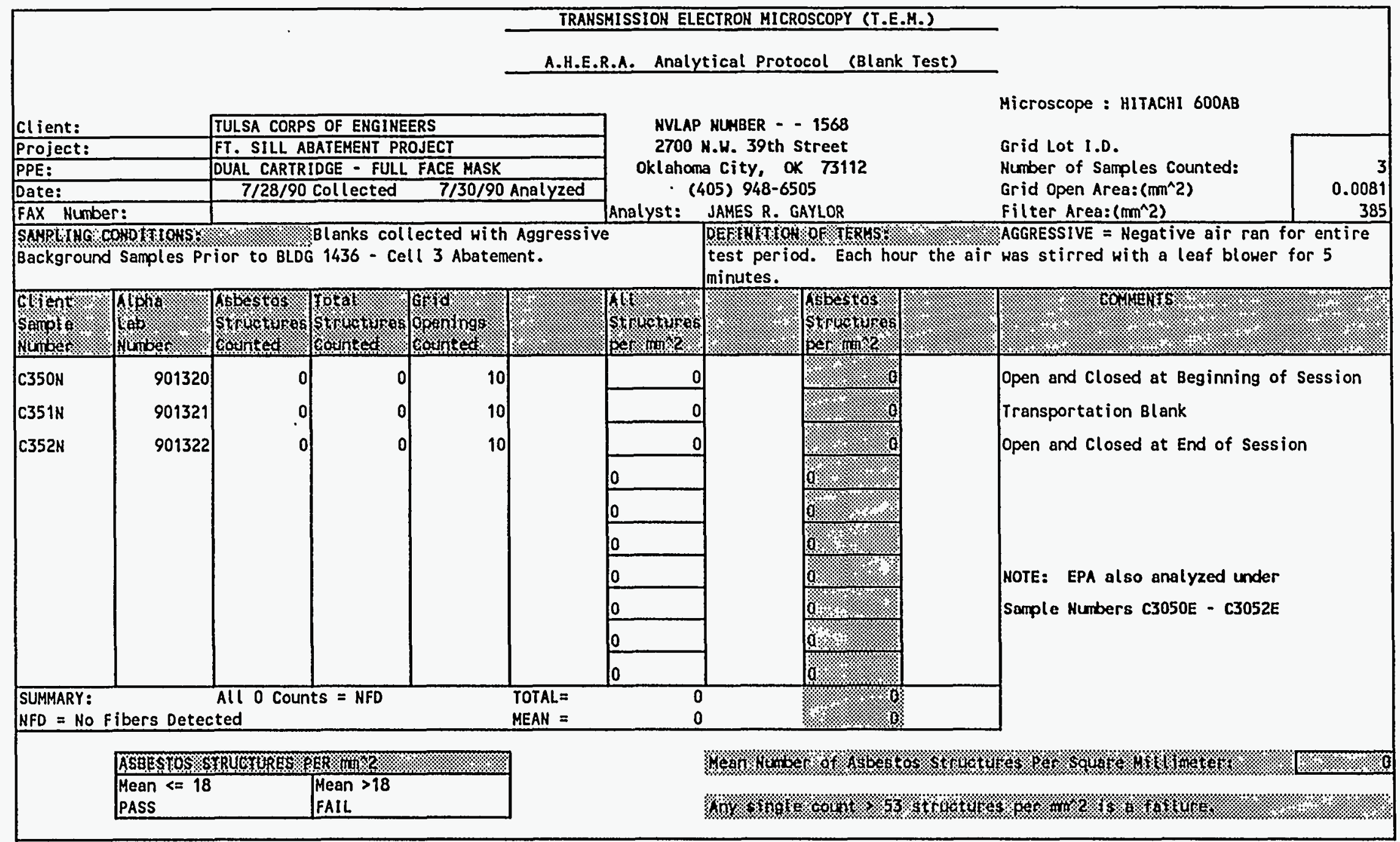




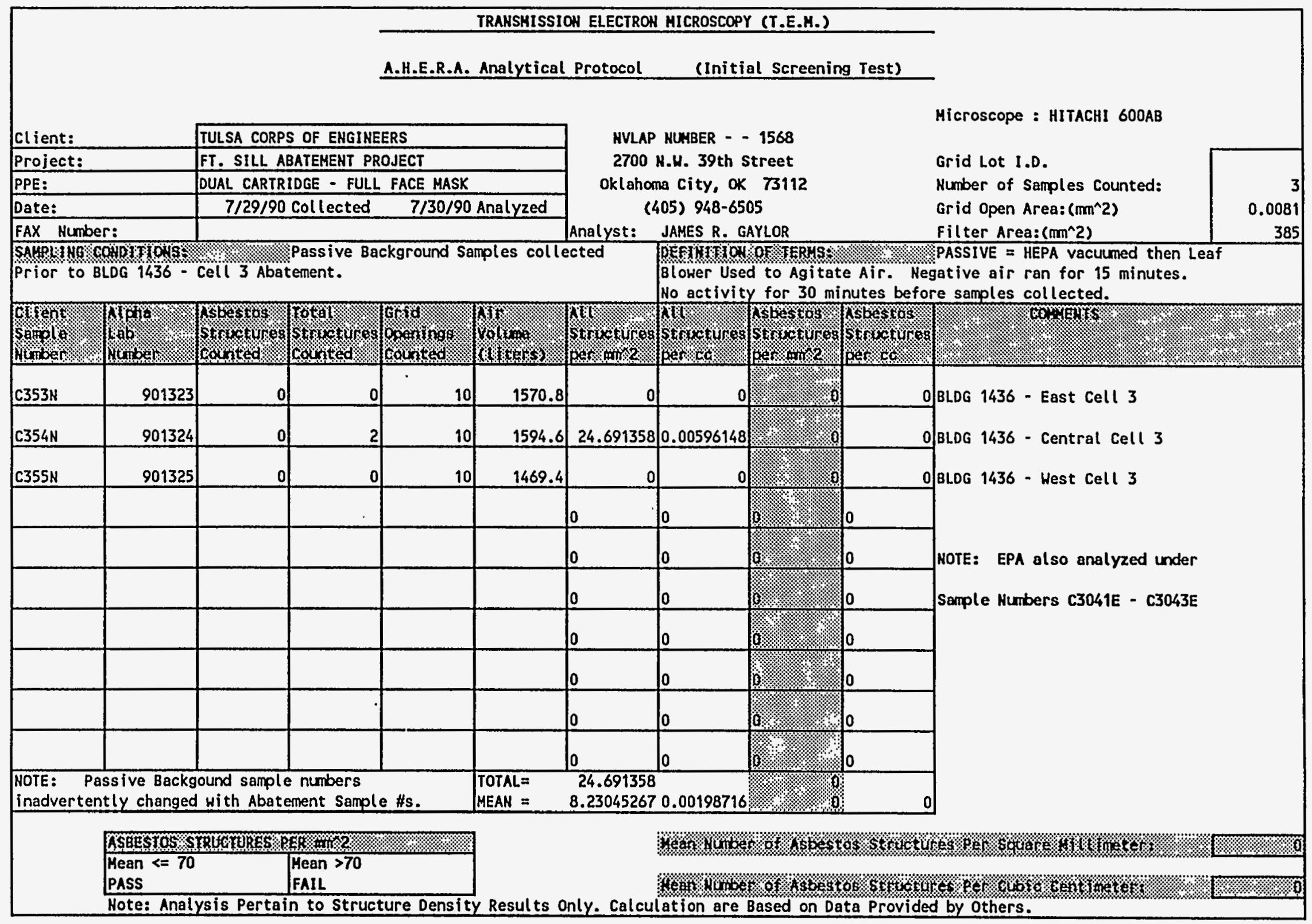




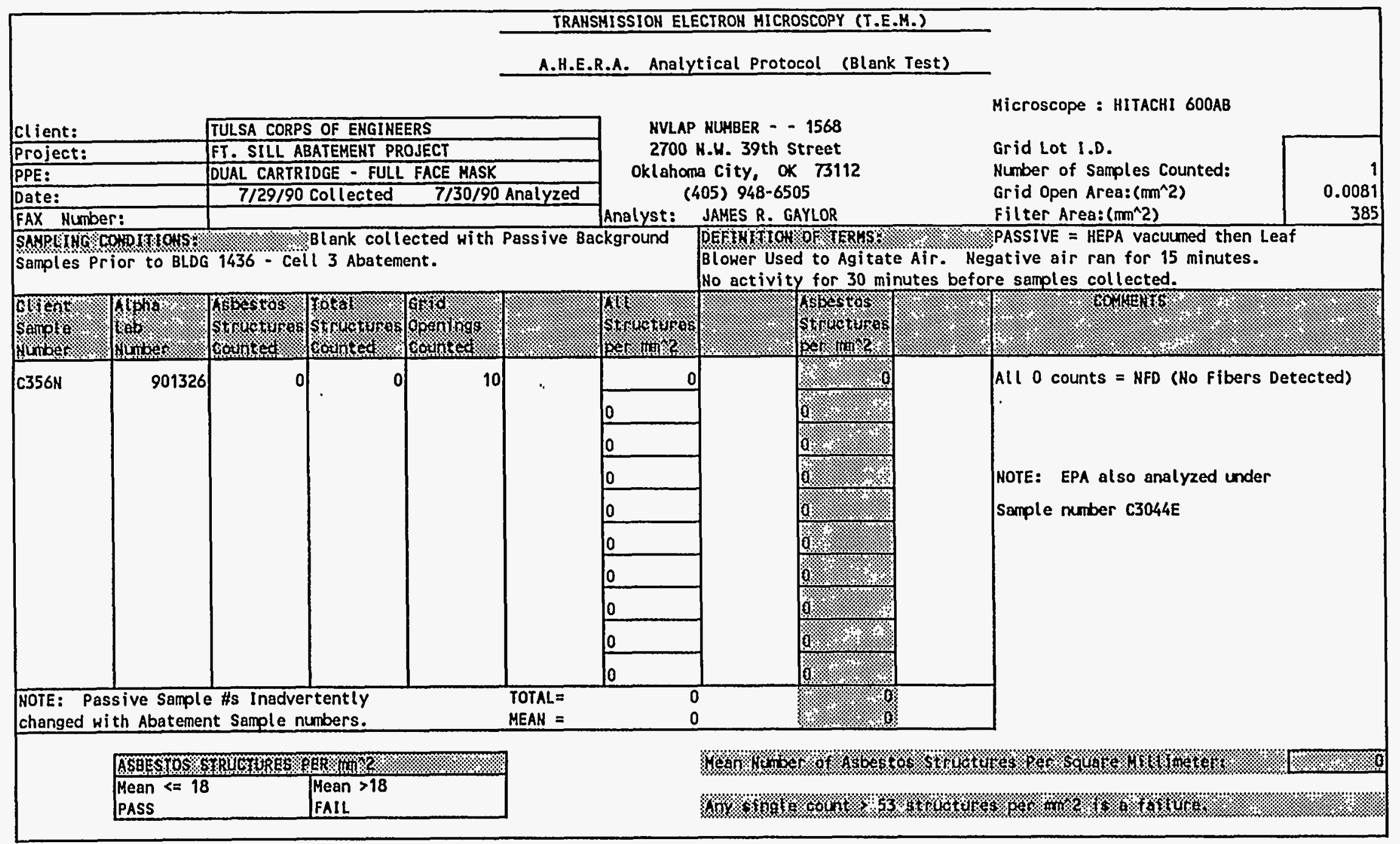




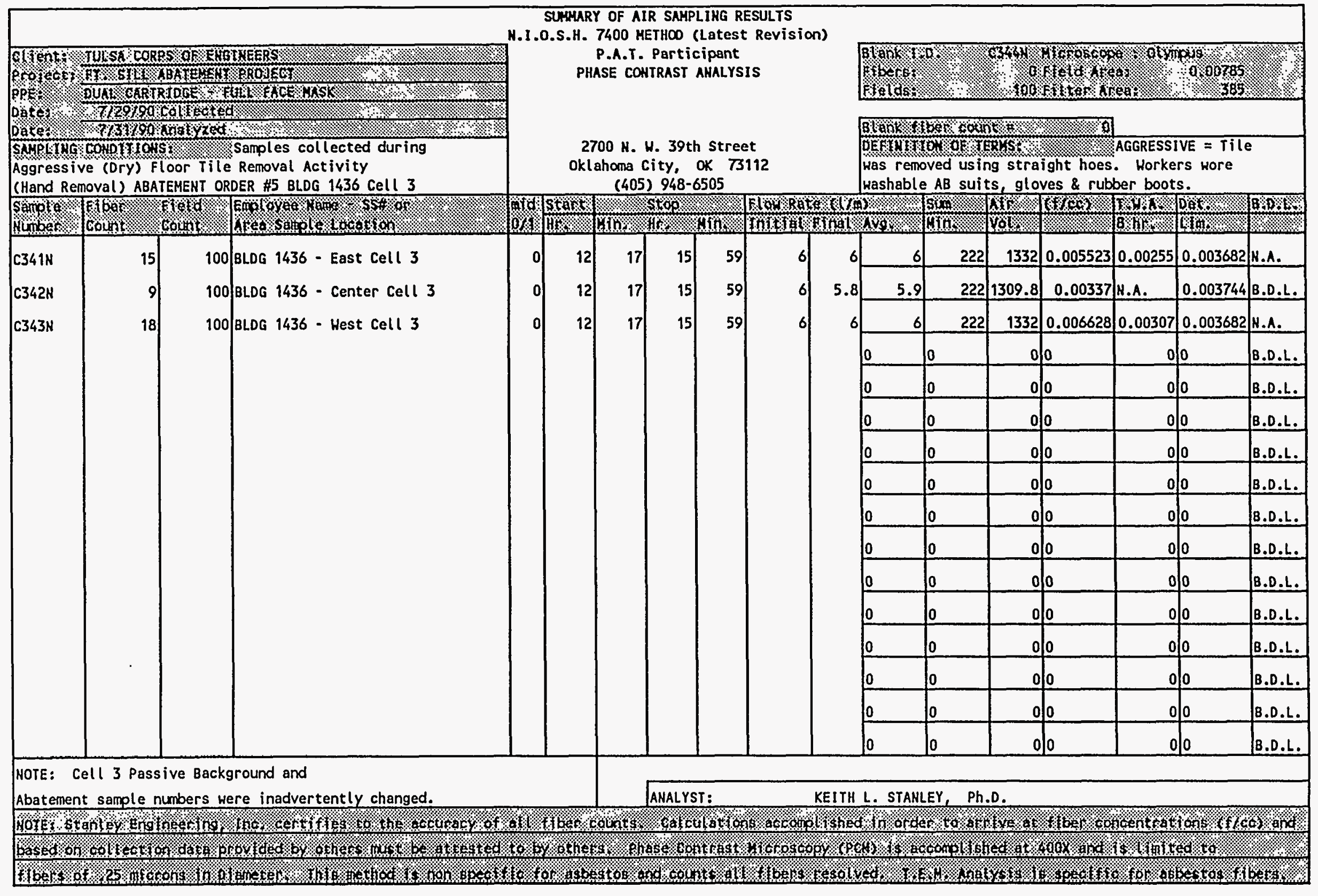




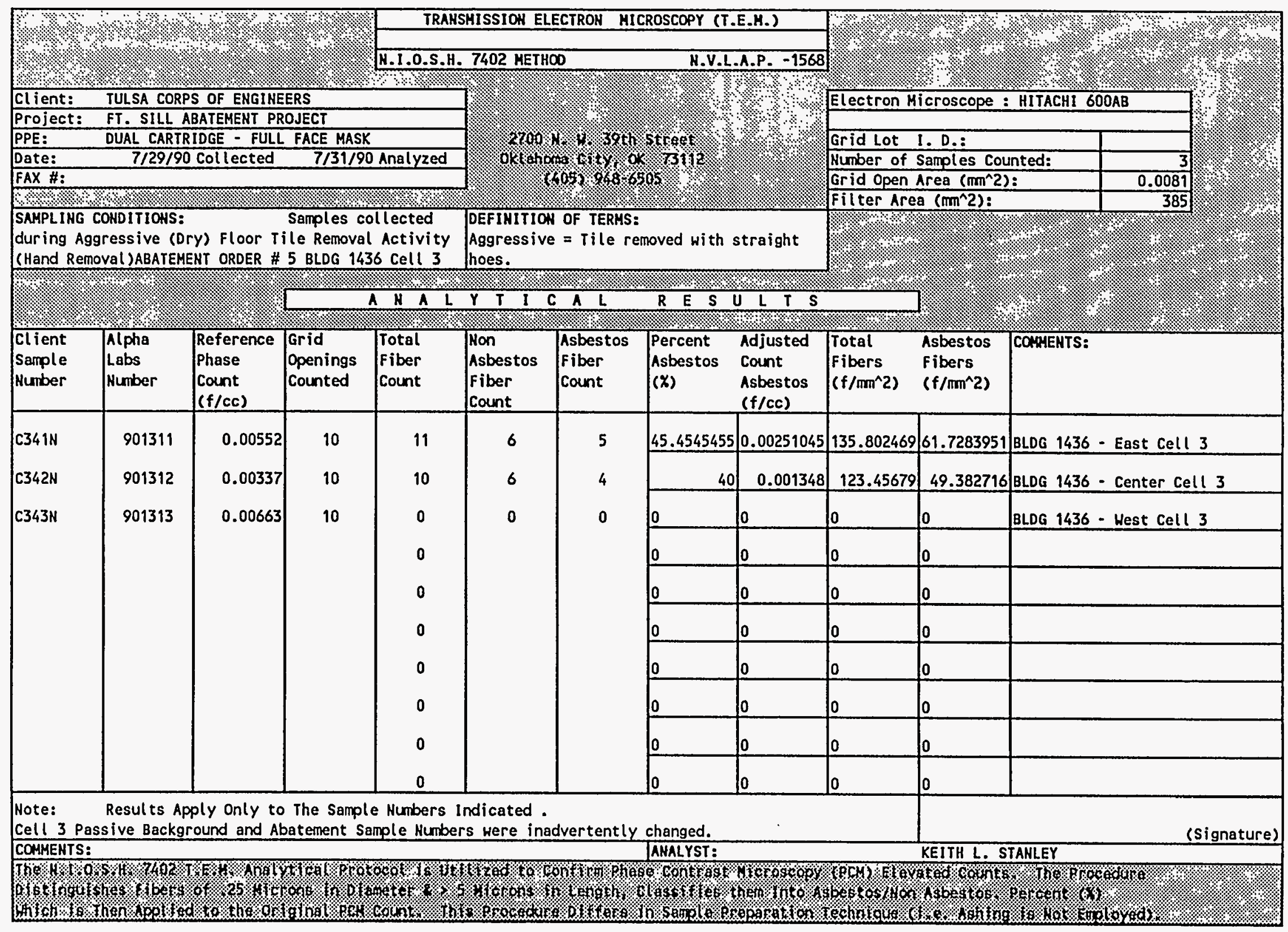




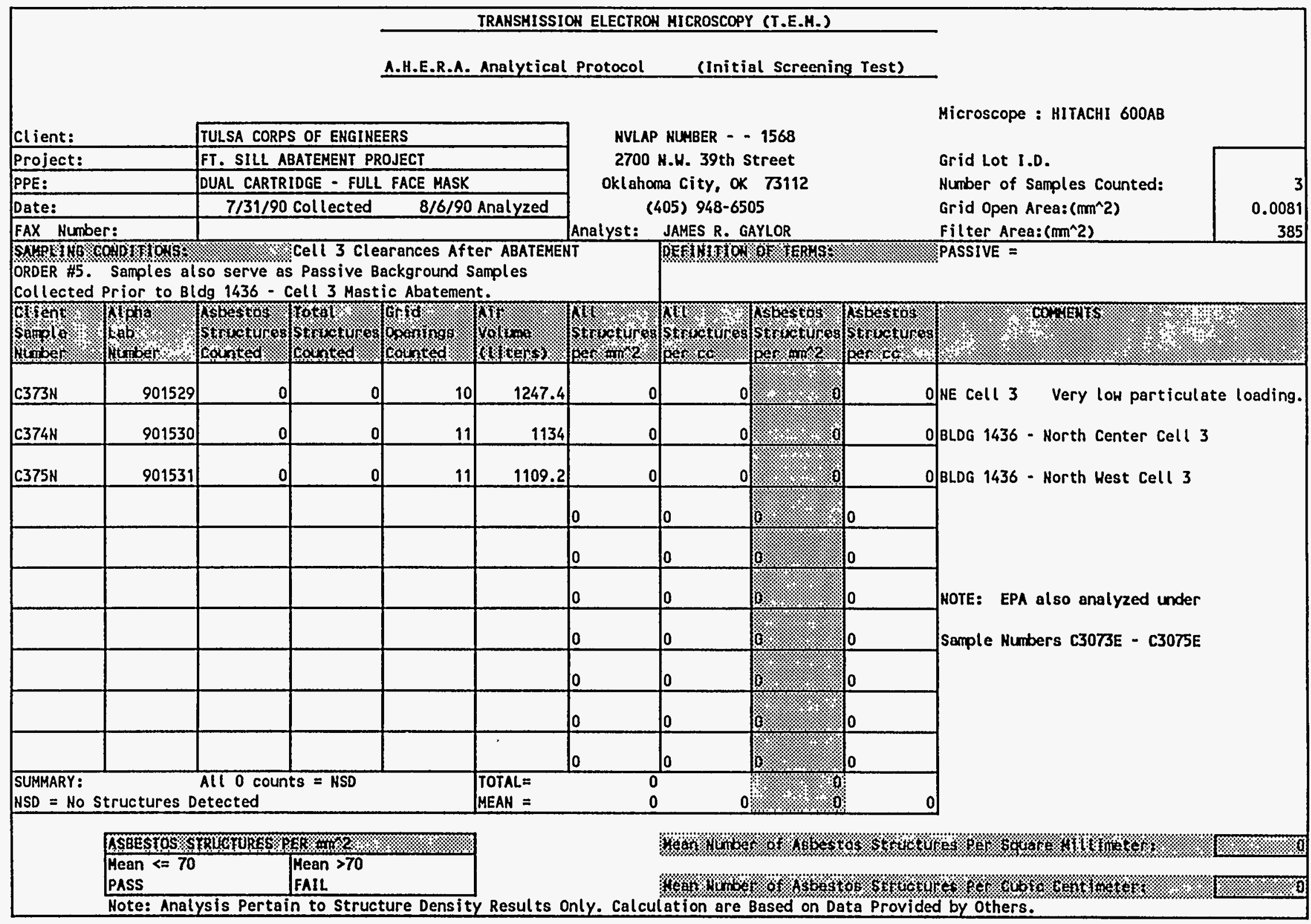


TRAHSHISSION ELECTRON MICROSCOPY (T.E.M.)

A.H.E.R.A. Analytical Protocol (Blank Test)

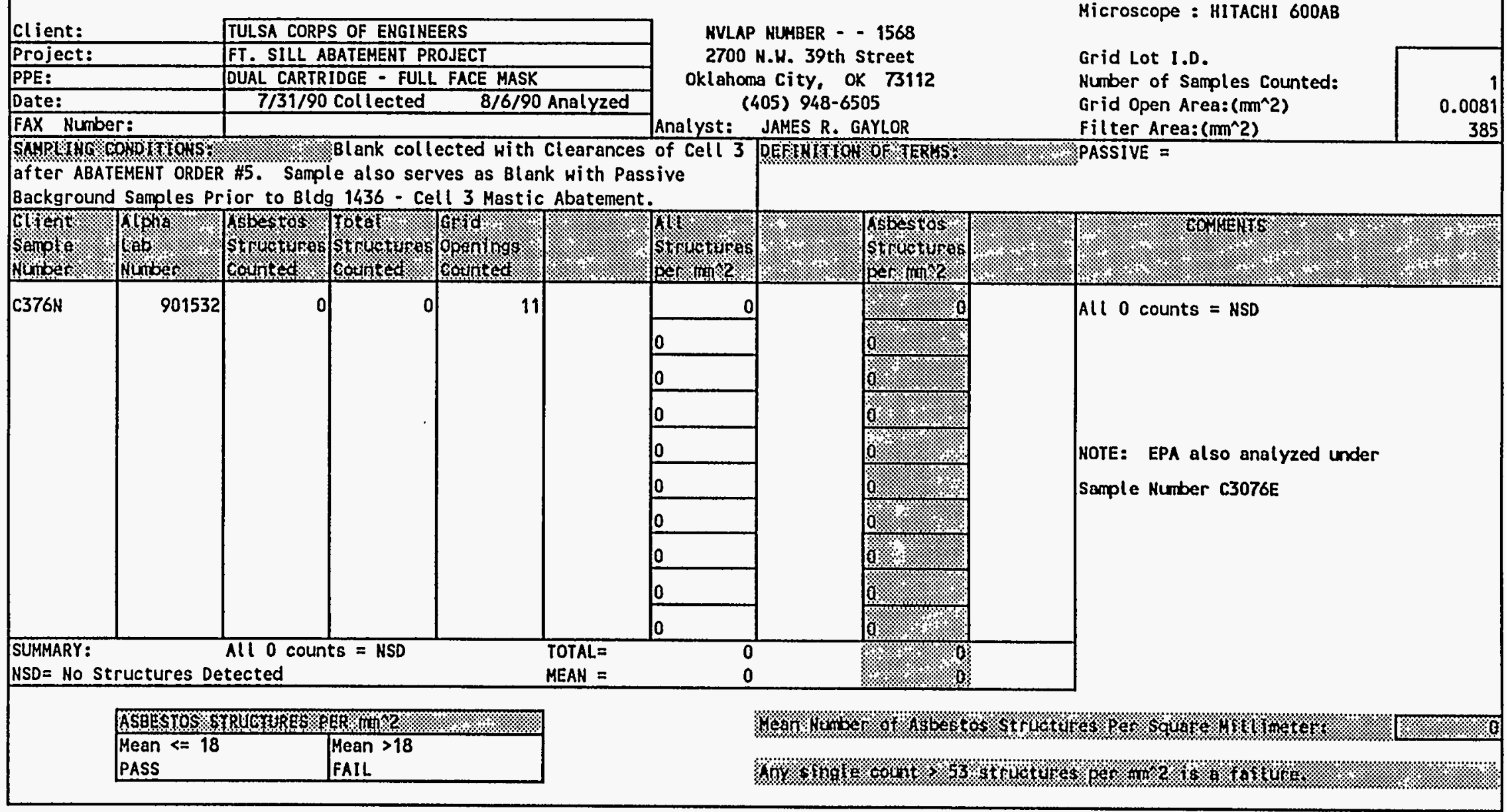




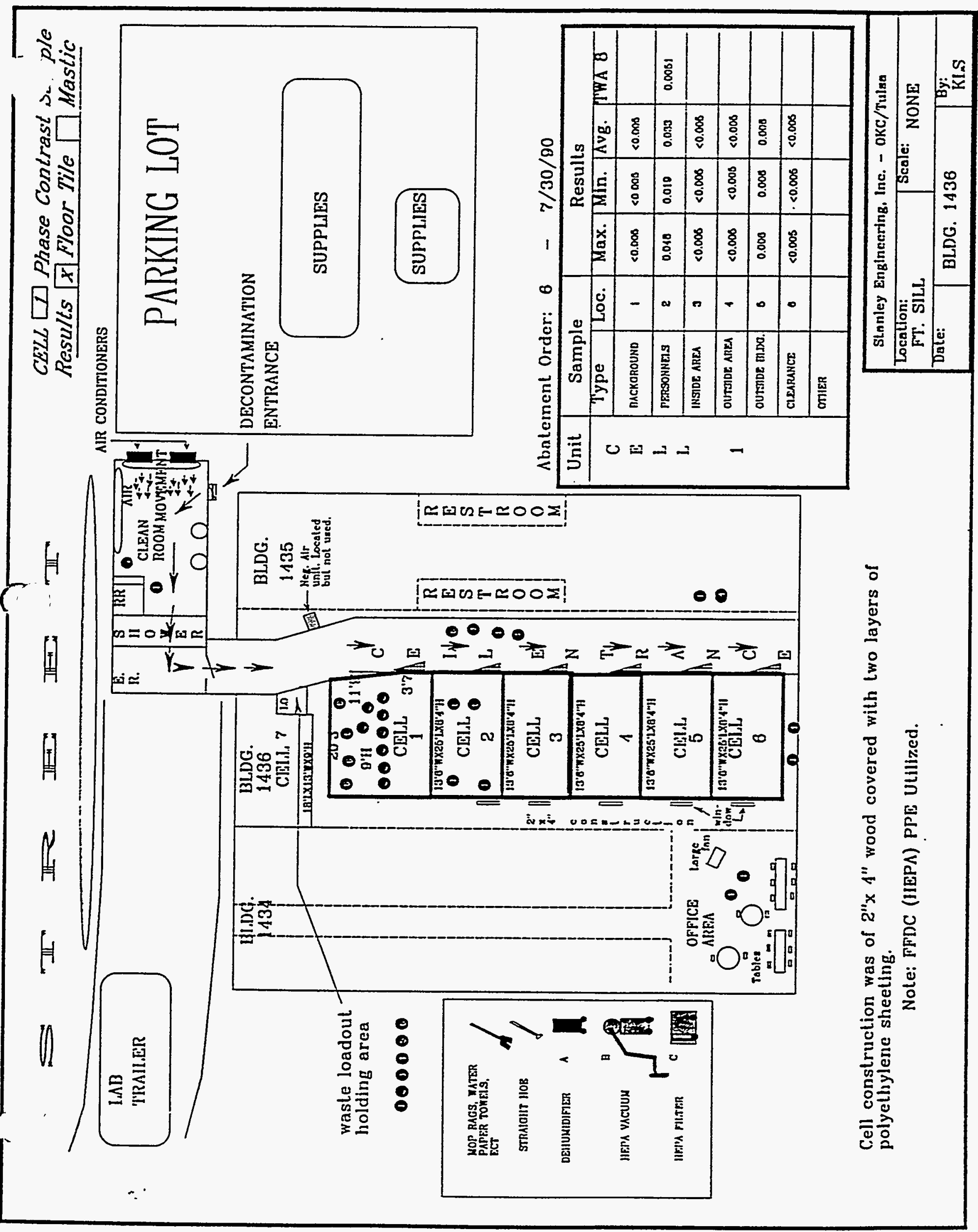


StANLEY ENGINEERING, INC:-E WMMENTAL MEASUREMENTS

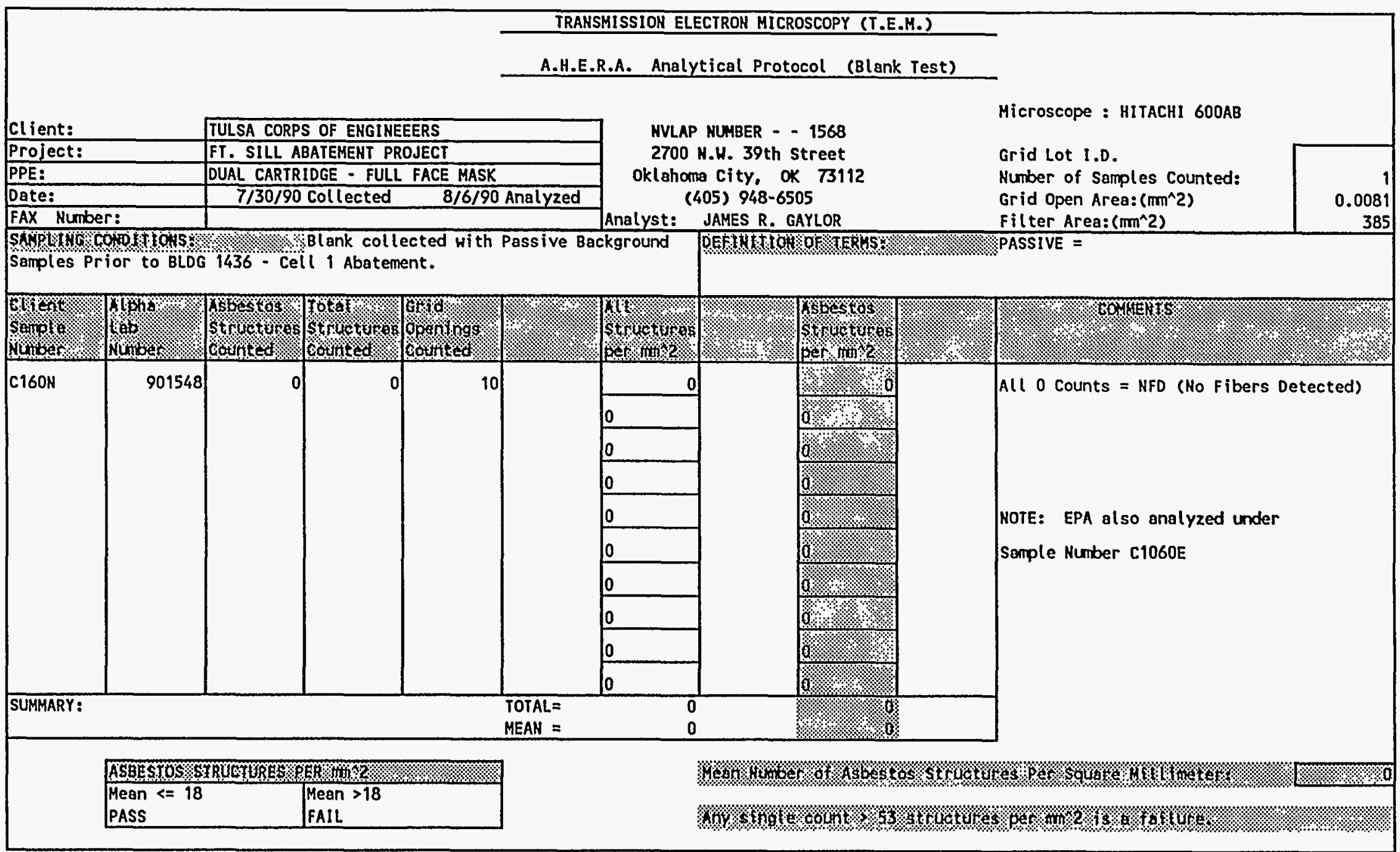




\begin{tabular}{|c|c|c|c|c|c|c|c|c|c|c|}
\hline & & & & & TRAHSHISSIC & IOH ELECTROH & N MICROSCOPY (T.E.H.) & & & \\
\hline & & & & A.H.E.R.A. & Analytical & 1 Protocol & (Initial Screenin & ng Test) & & \\
\hline Cl ient: & & TULSA CORPS & $S$ OF ENGINE & EERS & & NVLAP & P NUABER - - 1568 & & Microscope : HITACHI 600AB & \\
\hline Project: & & FT. SILL AB & BATEMENT PR & ROJECT & & 2700 & H.H. 39th Street & & Grid Lot I.D. & \\
\hline PPE: & & DUAL CARTRI & IDGE - FULL & $\angle$ FACE MASK & & Oklahom & ma City, ok 73112 & & Number of Samples Counted: & \\
\hline Date: & & $7 / 30 / 90$ & Collected & $8 / 6 / 90$ & Analyzed & & 405) $948-6505$ & & Grid Open Area: $\left(\mathrm{m}^{\wedge} \mathrm{2}\right)$ & 0.0081 \\
\hline FAX Numbe & & & & & & Analyst: & JAMES R. GAYLOR & & Filter Area: $\left(\mathrm{m}^{\wedge} \mathbf{2}\right)$ & 385 \\
\hline $\begin{array}{l}\text { Soging } \\
\text { Prior to }\end{array}$ & WLG 1436 - & Cell 1 Abat & $\begin{array}{l}\text { Passive Bac } \\
\text { tement. }\end{array}$ & ackground San & imples colle & ected & 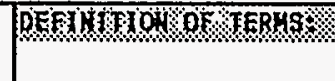 & & PASSIVE $=$ & \\
\hline Sonolvit & Who & 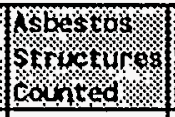 & Sorotion & Coninged & Wor & 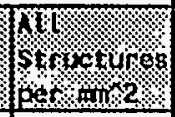 & S & Lsbestor & (4) & ?... \\
\hline C157N & 901545 & of & $\underline{0}$ & 10 & 1812 & 0 & 0 & & BLDG 1436 - North East Cell 1 & \\
\hline C158N & 901546 & 0 & 0 & 10 & 1812 & $\underline{0}$ & 28 & & BLDG 1436 - North Center Cell 1 & \\
\hline C159N & 901547 & 0 & $\underline{0}$ & 10 & 1878.6 & $\underline{0}$ & 8 & & BLDG 1436 - North Hest Cell 1 & \\
\hline & & & & & & 10 & 0 & 0 & & \\
\hline & & & & & & 0 & 6. & 0 & & \\
\hline & & & & & & 0 & (1) & 0 & |HOTE: EPA also analyzed under & \\
\hline & & & & & & 0 & 6. & 0 & Sample Numbers C1057E - C1059E & \\
\hline & & & & & & lo & 18. & 0 & & \\
\hline & & & & & & 10 & $10 \%$ & 0 & & \\
\hline & & & & & & 10 & (6.1. & & & \\
\hline $\begin{array}{l}\text { SUMMARY: } \\
\text { NFD = NO }\end{array}$ & ibers Detec & $\begin{array}{l}\text { All } 0 \text { count } \\
\text { sted }\end{array}$ & ts $=$ NFD & & $\begin{array}{l}\text { TOTAL }= \\
\text { MEAN }=\end{array}$ & $\begin{array}{l}0 \\
0\end{array}$ & (2) & 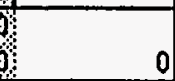 & & \\
\hline & $\begin{array}{l}\text { ASBESTOSPS } \\
\text { Mean }<=70 \\
\text { PASS }\end{array}$ & 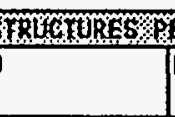 & $\begin{array}{l}\text { Mean }>70 \\
\text { FAIL }\end{array}$ & 1. & & & 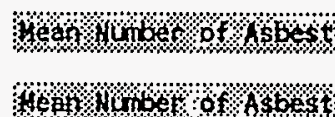 & 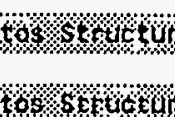 & 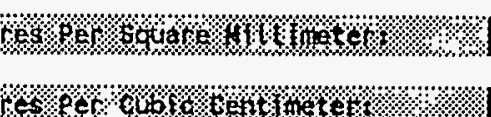 & \\
\hline
\end{tabular}




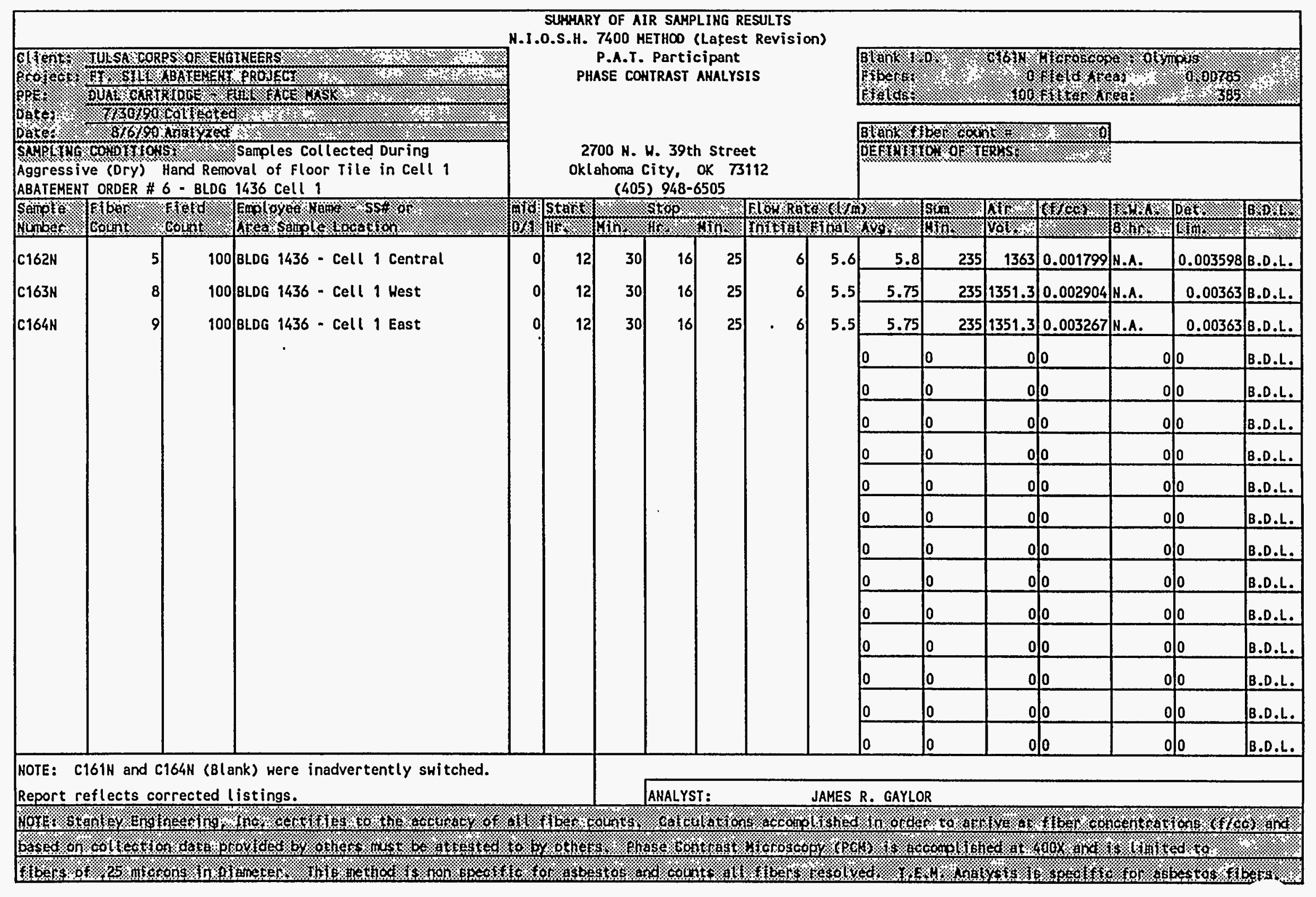




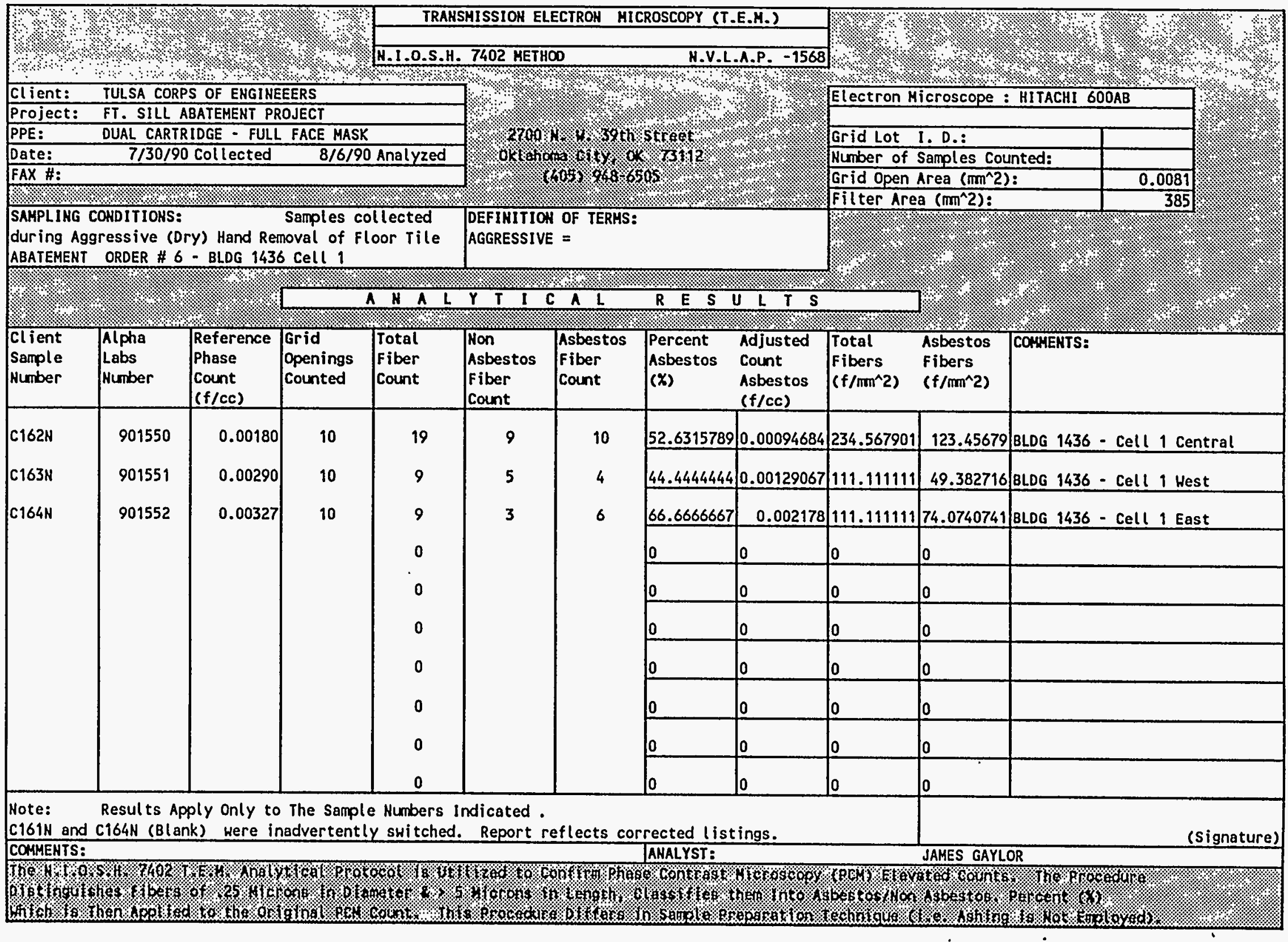


TRANSHISSION ELECTROH MICROSCOPY (T.E.H.)

A.H.E.R.A. Analytical Protocol (Initial Screening Test)

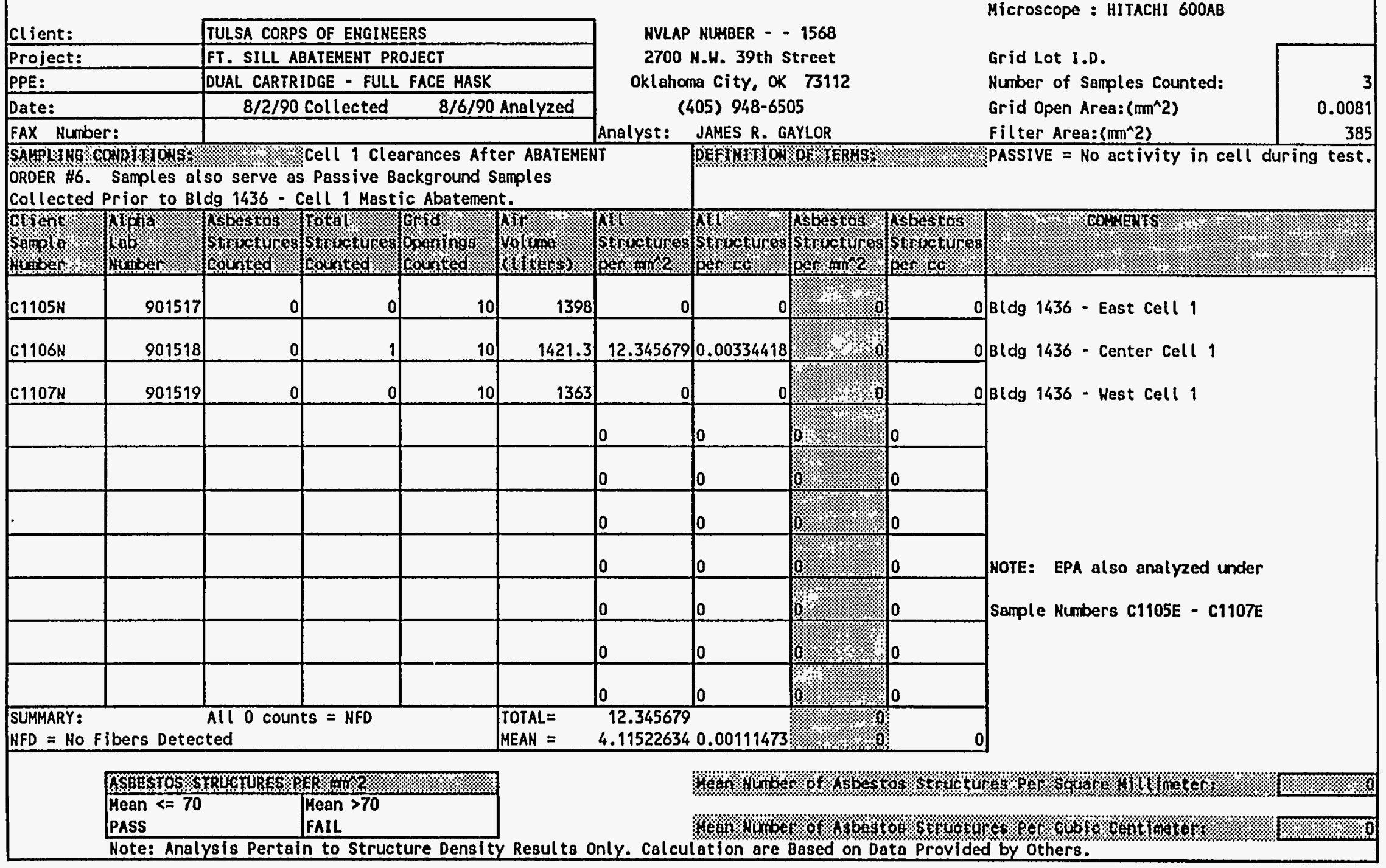




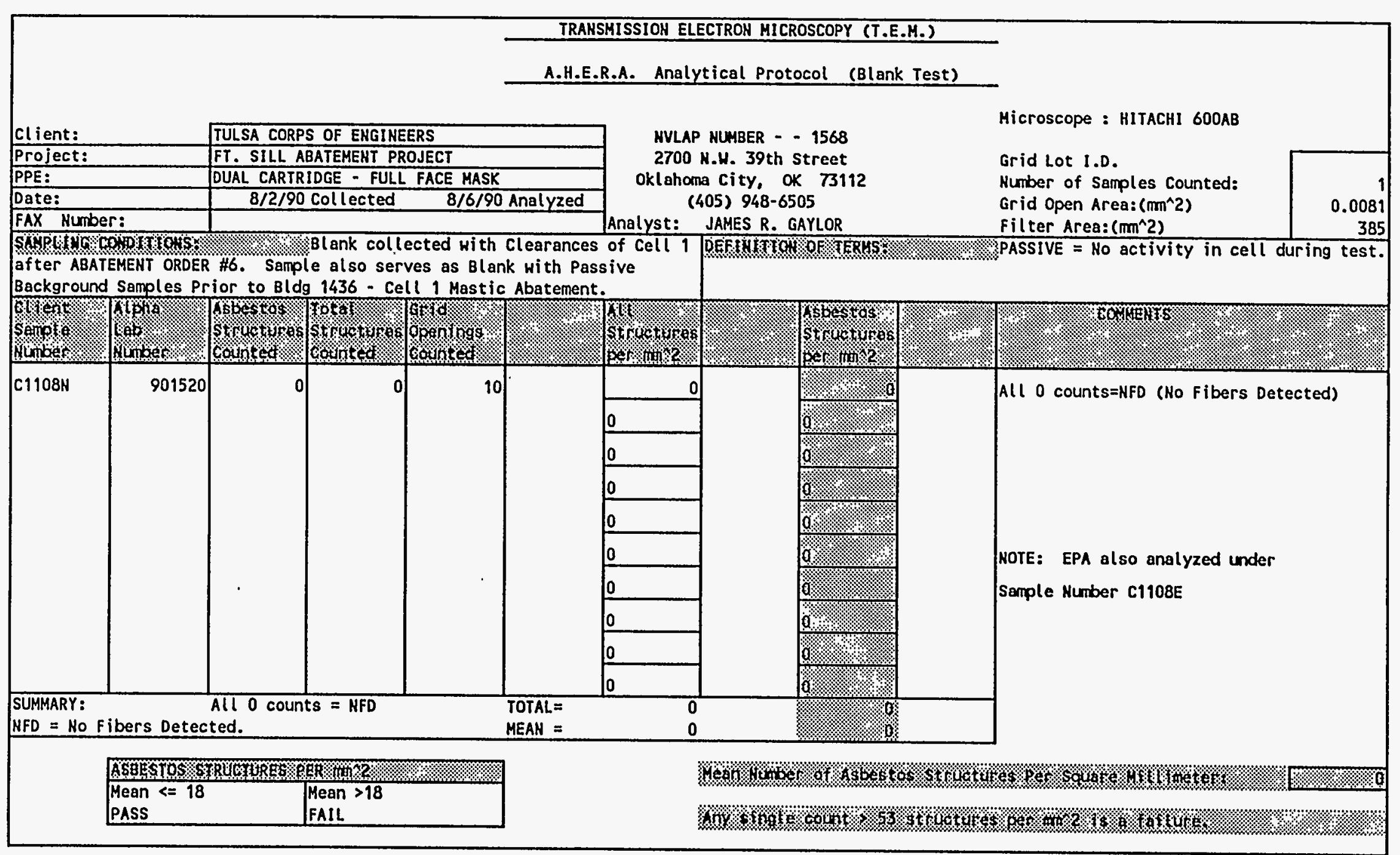




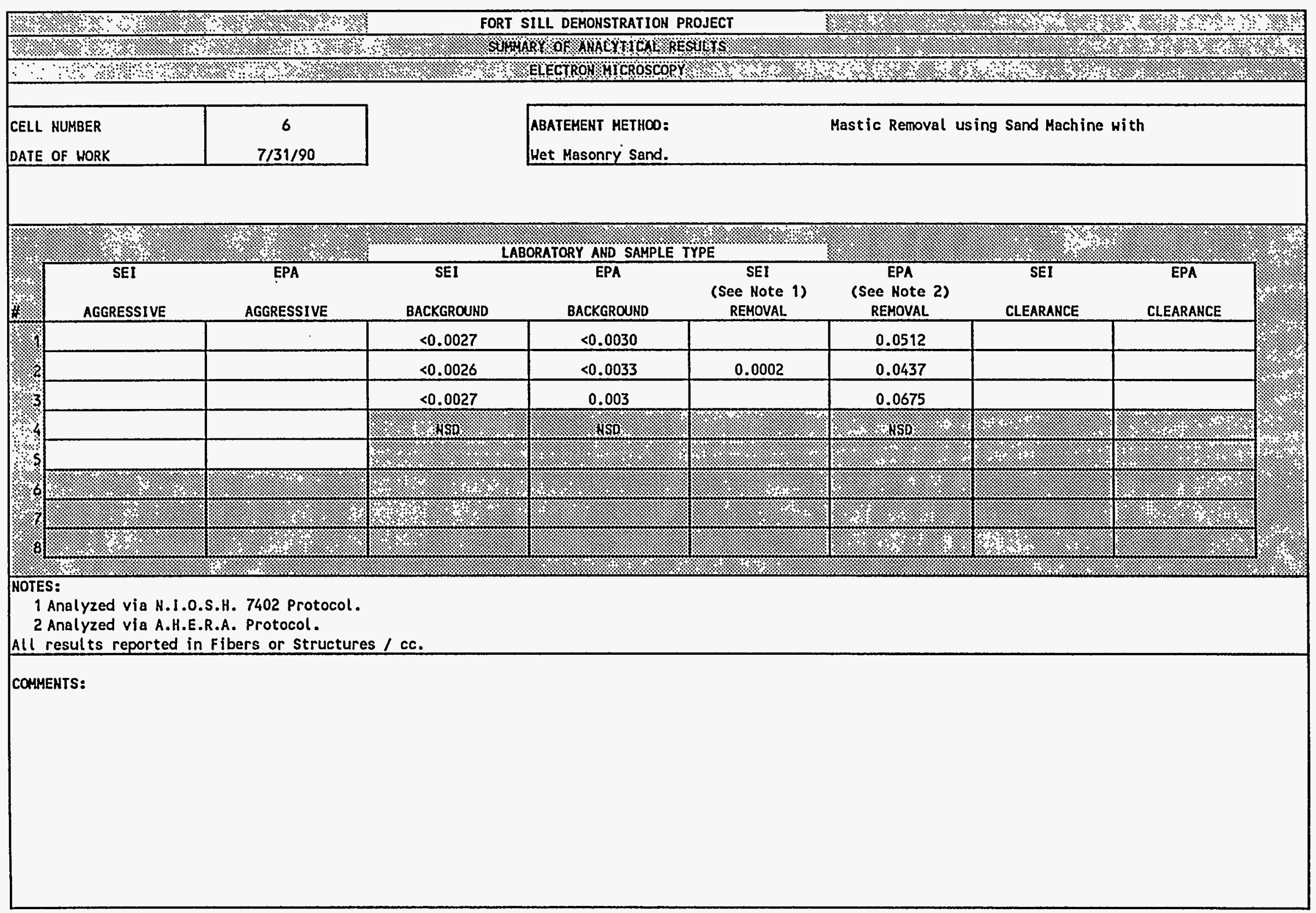




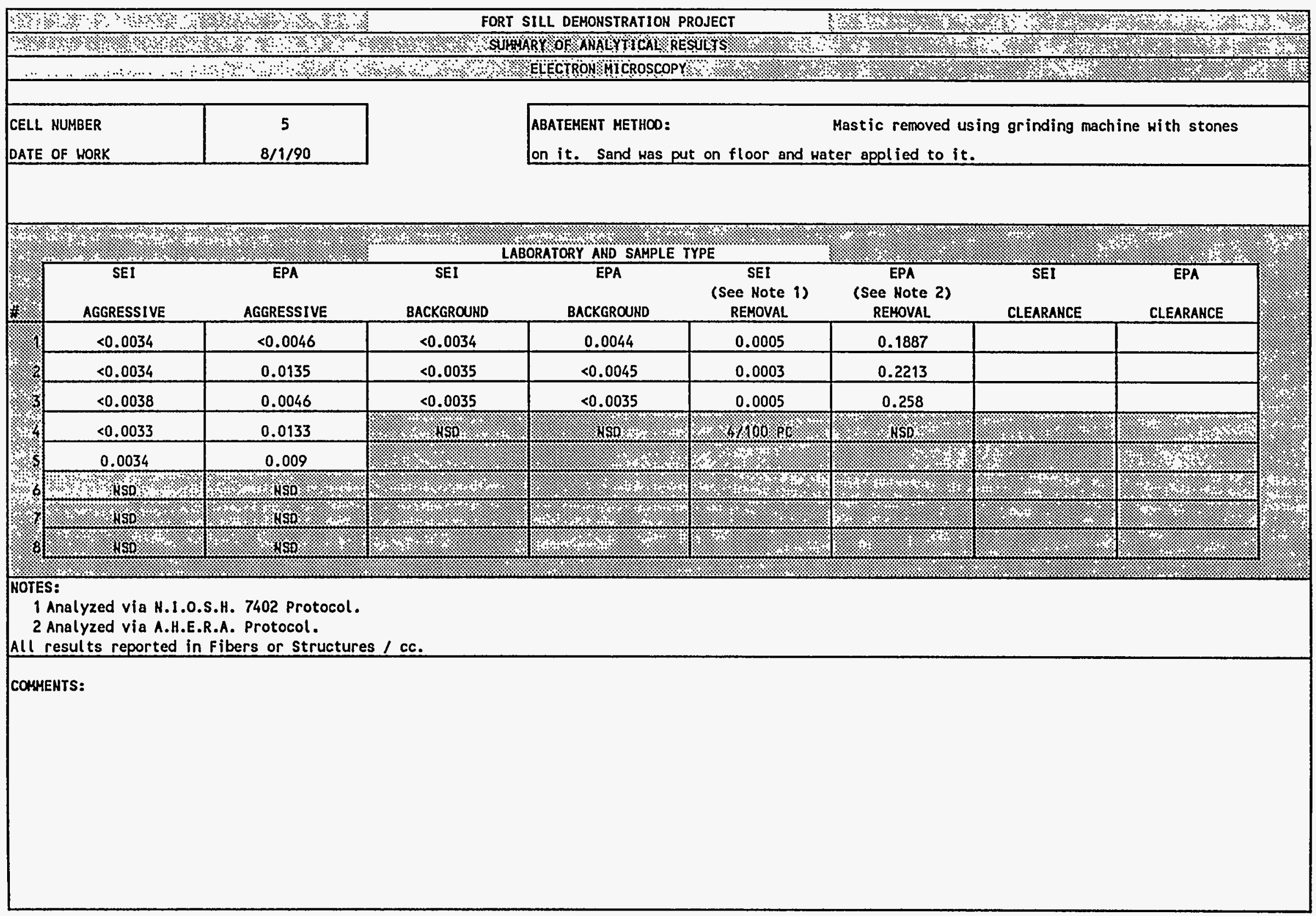




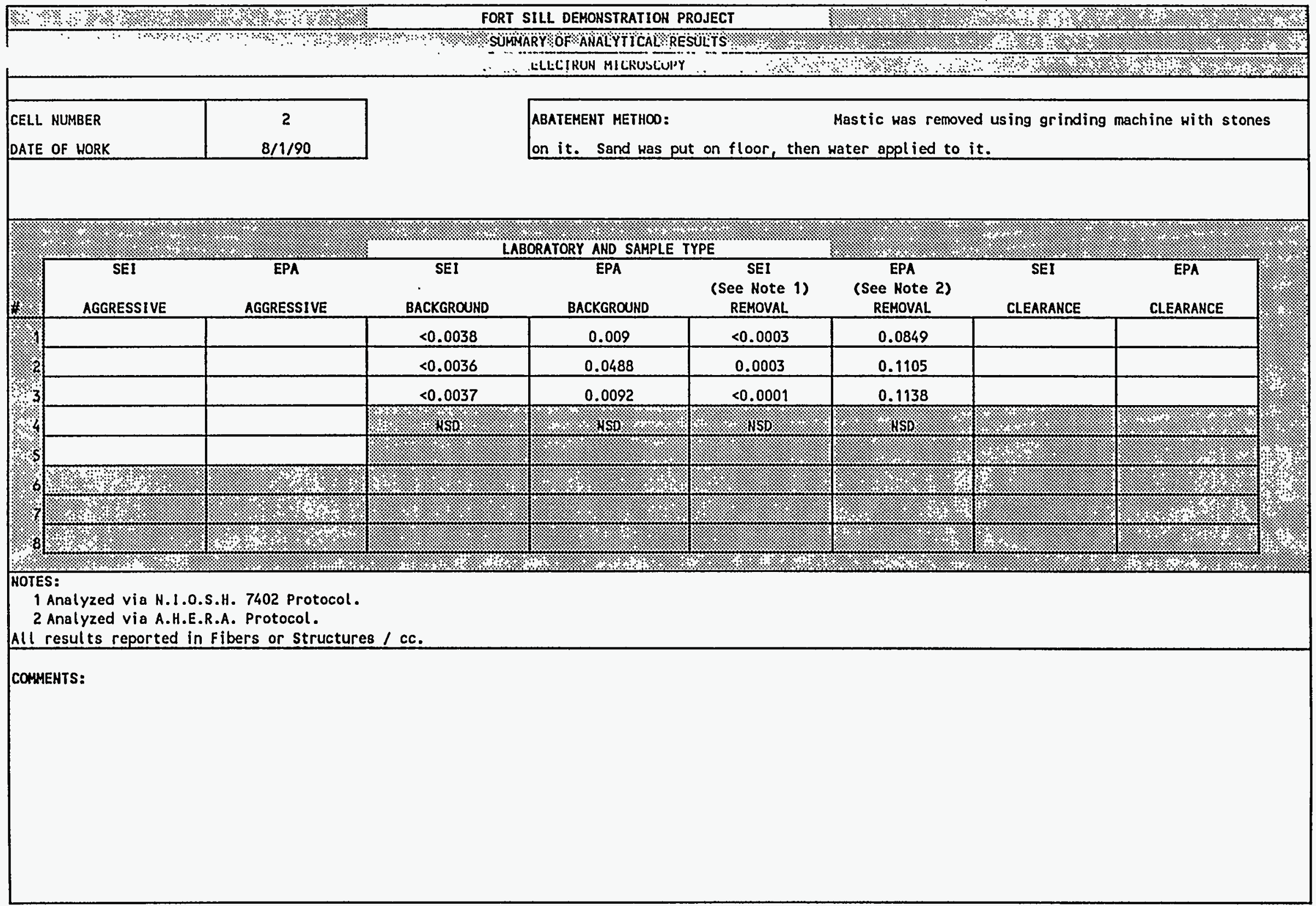




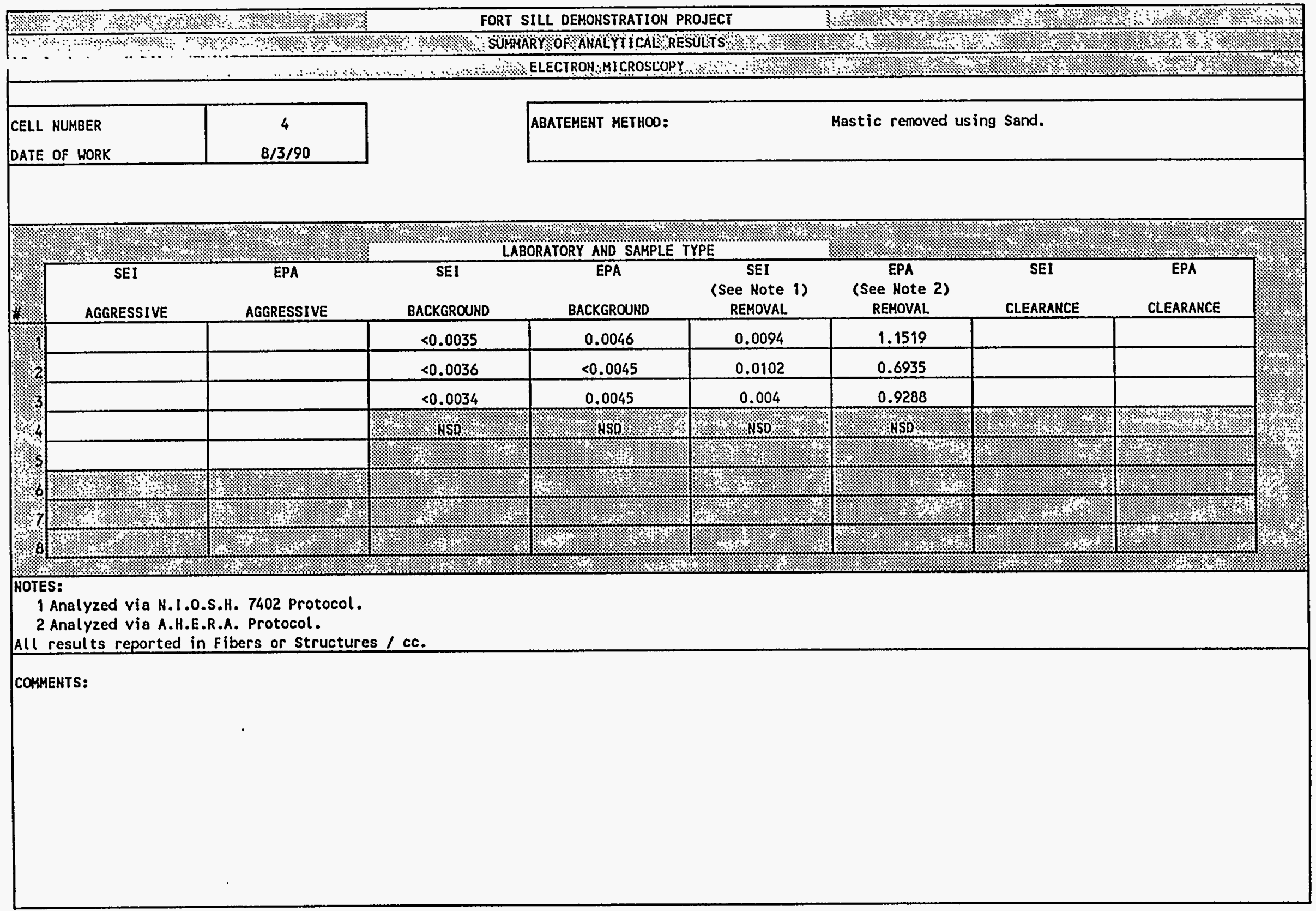




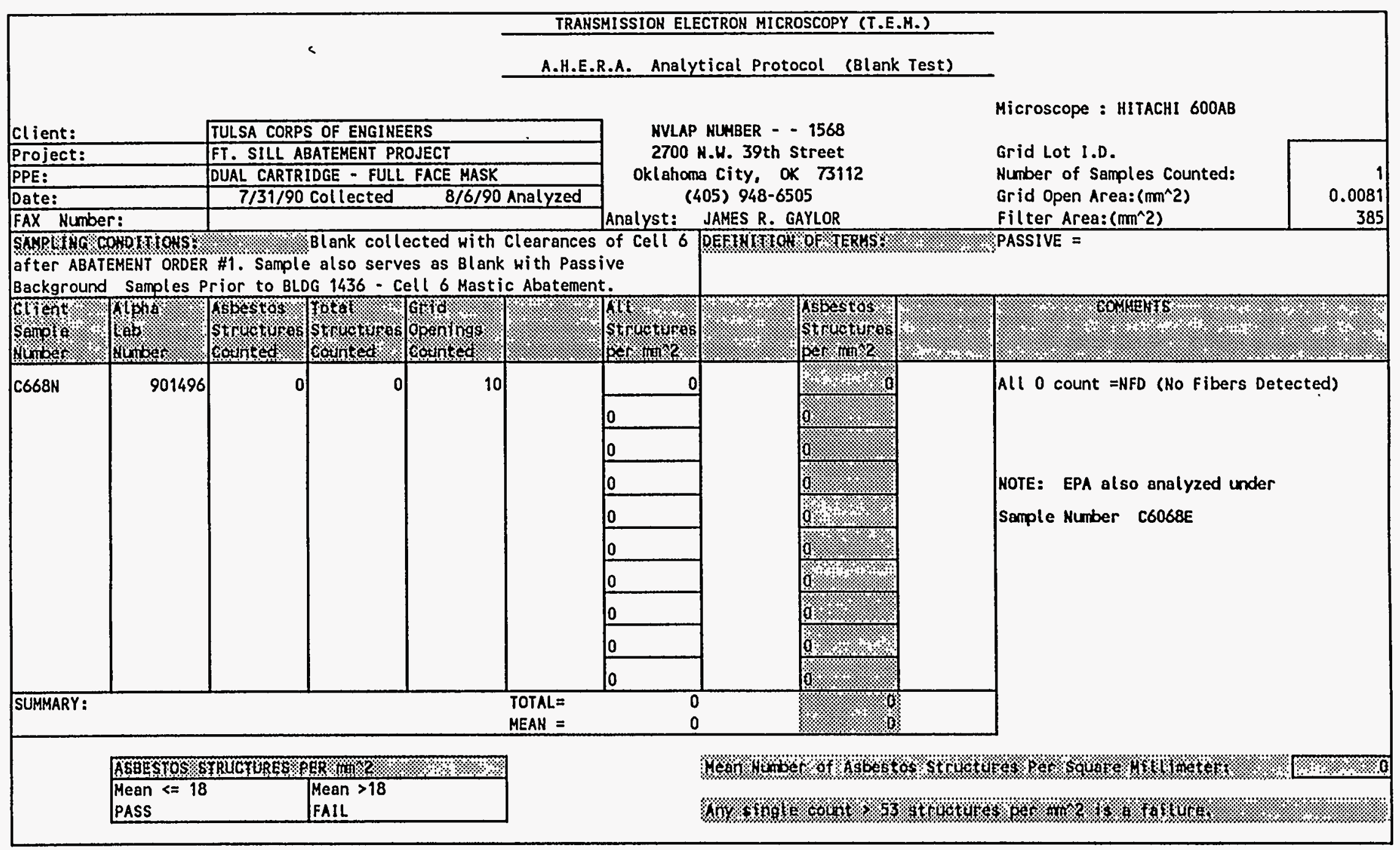




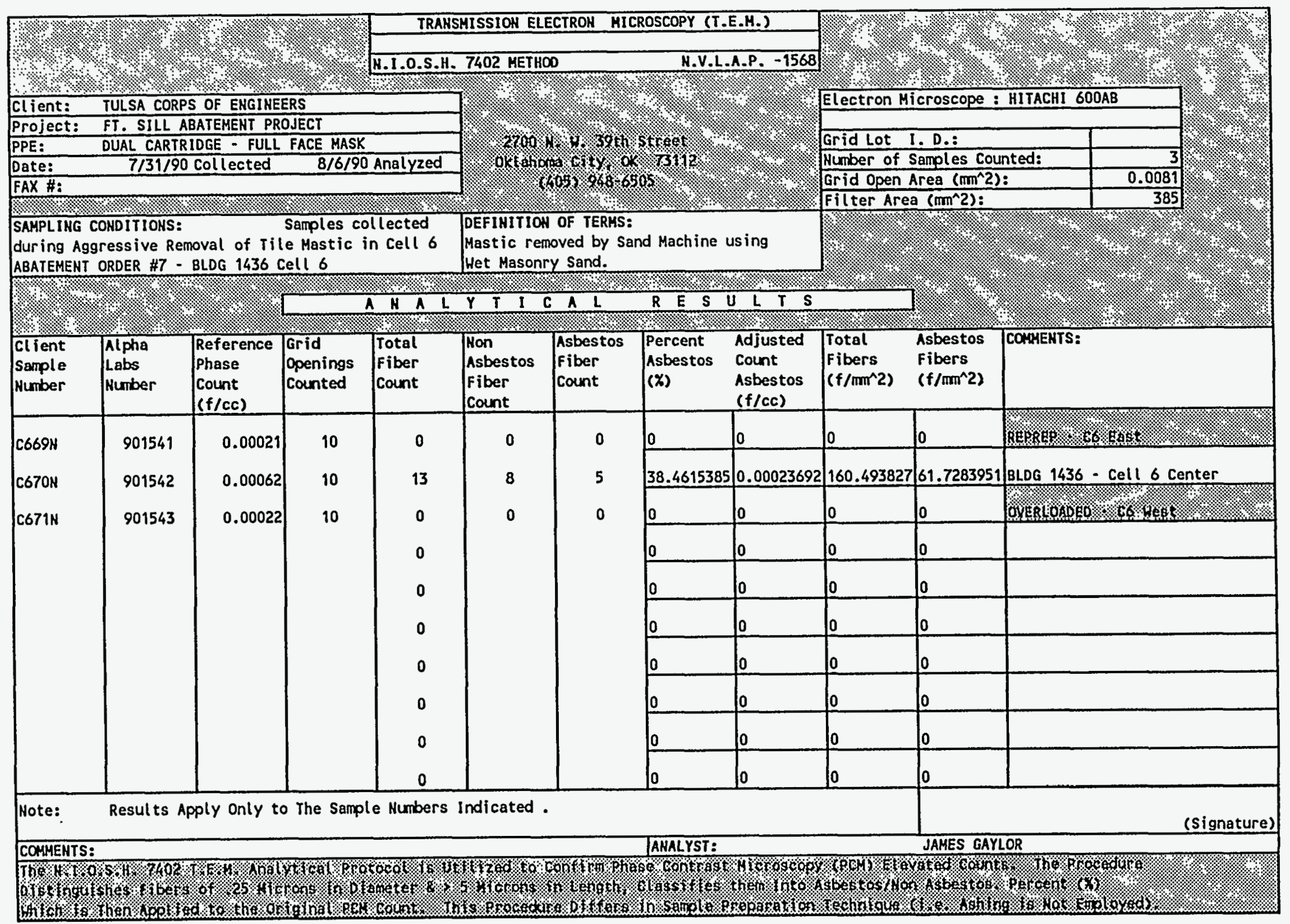


TRANSHISSIOH ELECTRON MICROSCOPY (T.E.M.)

A.H.E.R.A. Analytical Protocol (Initial Screening Test)

\begin{tabular}{|l|l|}
\cline { 2 - 2 } Client: & TULSA CORPS OF ENGIMEERS \\
\hline Project: & FT. SILL ABATEMENT PROJECT \\
\hline PPE: & DUAL CARTRIDGE - FULL FACE MASK \\
\hline Date: & $7 / 31 / 90$ Collected 8/6/90 Analyzed \\
\hline FAX Number: &
\end{tabular}

NVLAP MUMBER - - 1568

2700 N.W. 39th Street

oklahoma city, OK 73112

(405) $948-6505$

\section{Analyst: JAMES R. GAYLOR}

FAX Number:

DESTH IFION OD TERHS $\$$ Filter Area: $\left(\mathrm{mm}^{\wedge} 2\right)$

Microscope : HITACHI 600AB

ORDER \#1. Samples also serve as Passive Background Samples

Collected Prior to BLDG 1436 - Cell 6 Mastic Abatement

CGESF

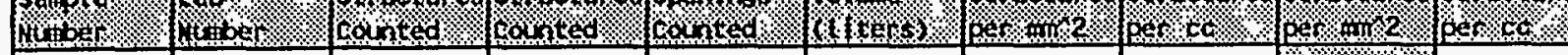

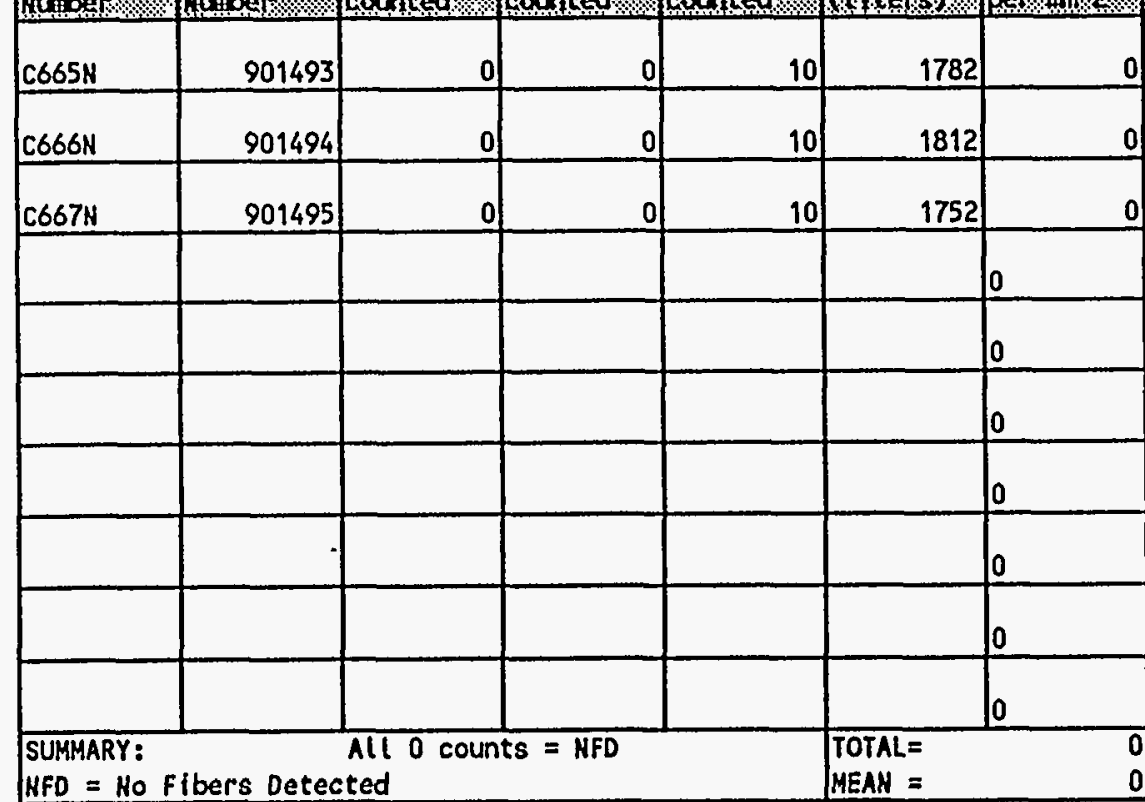

MFD $=$ Ho Fiber's Detected

MEAH $=$

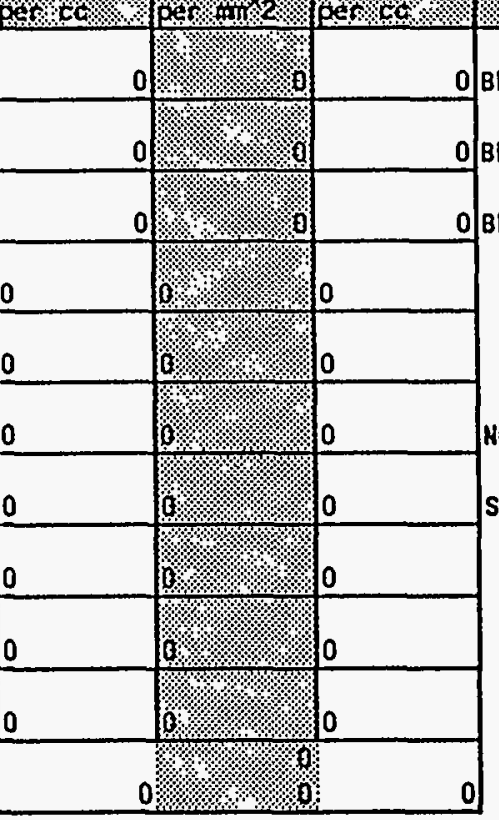

0 BLDG 1436 - Cell 6 East

BLDG 1436 - Cell 6 Center

O BLDG 1436 - Cell 6 West

HOTE: EPA also analyzed under Sample Numbers C6065E - C6067E

\begin{tabular}{|c|c|}
\hline KSBESTOS SY & $\mathrm{C}_{\mathrm{K}} \mathrm{x}$ \\
\hline $\begin{array}{l}\text { Mean }<=70 \\
\text { PASS }\end{array}$ & $\begin{array}{l}\text { Mean }>70 \\
\text { FAIL }\end{array}$ \\
\hline
\end{tabular}

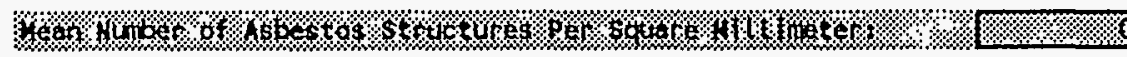

PASS

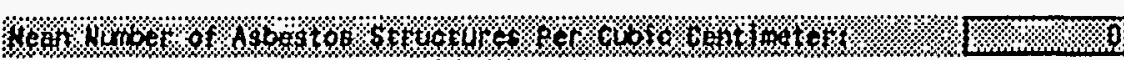
Hote: Analysis Pertain to Structure Density Results only. Calculation are Based on Data Provided by Others. 


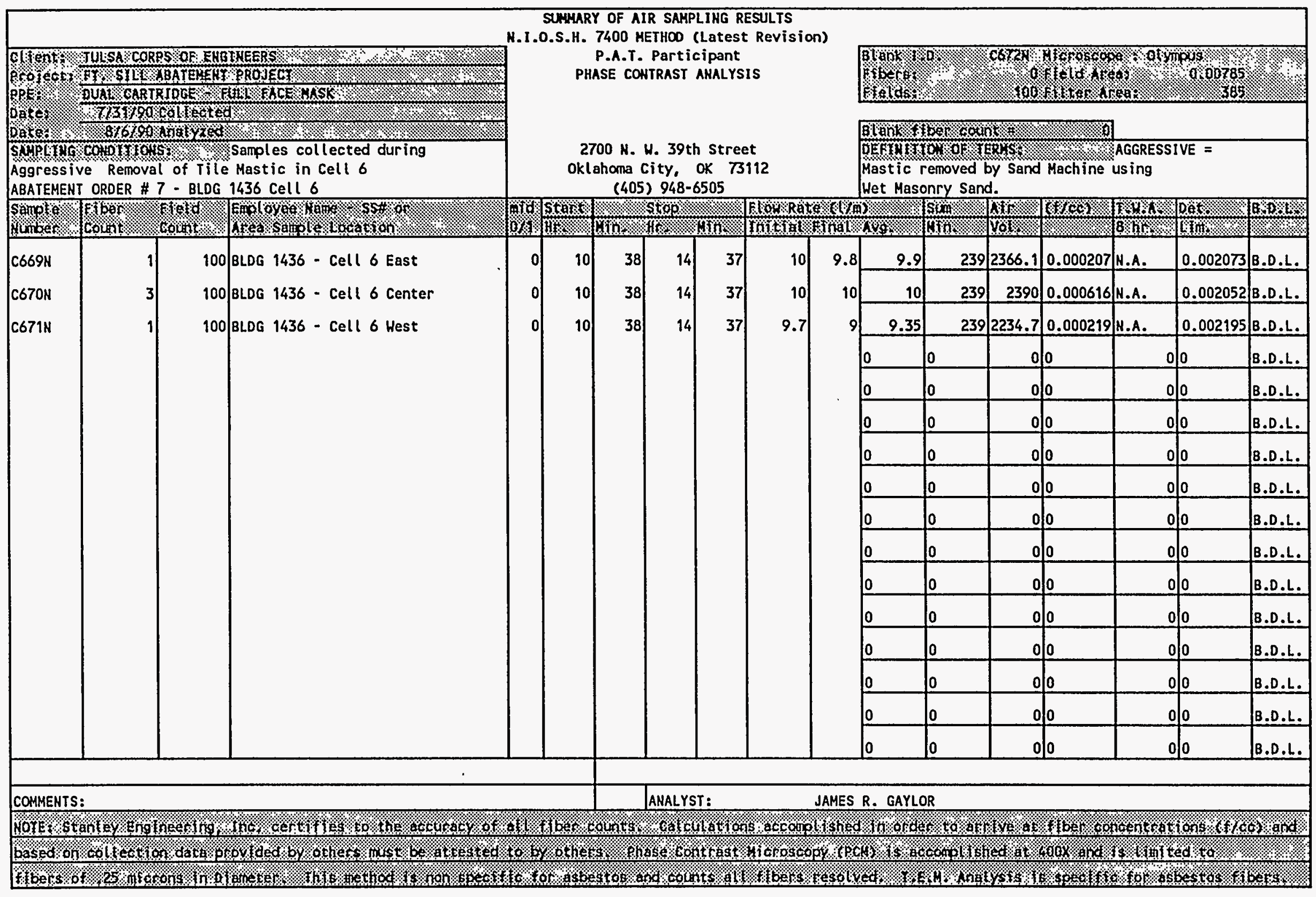




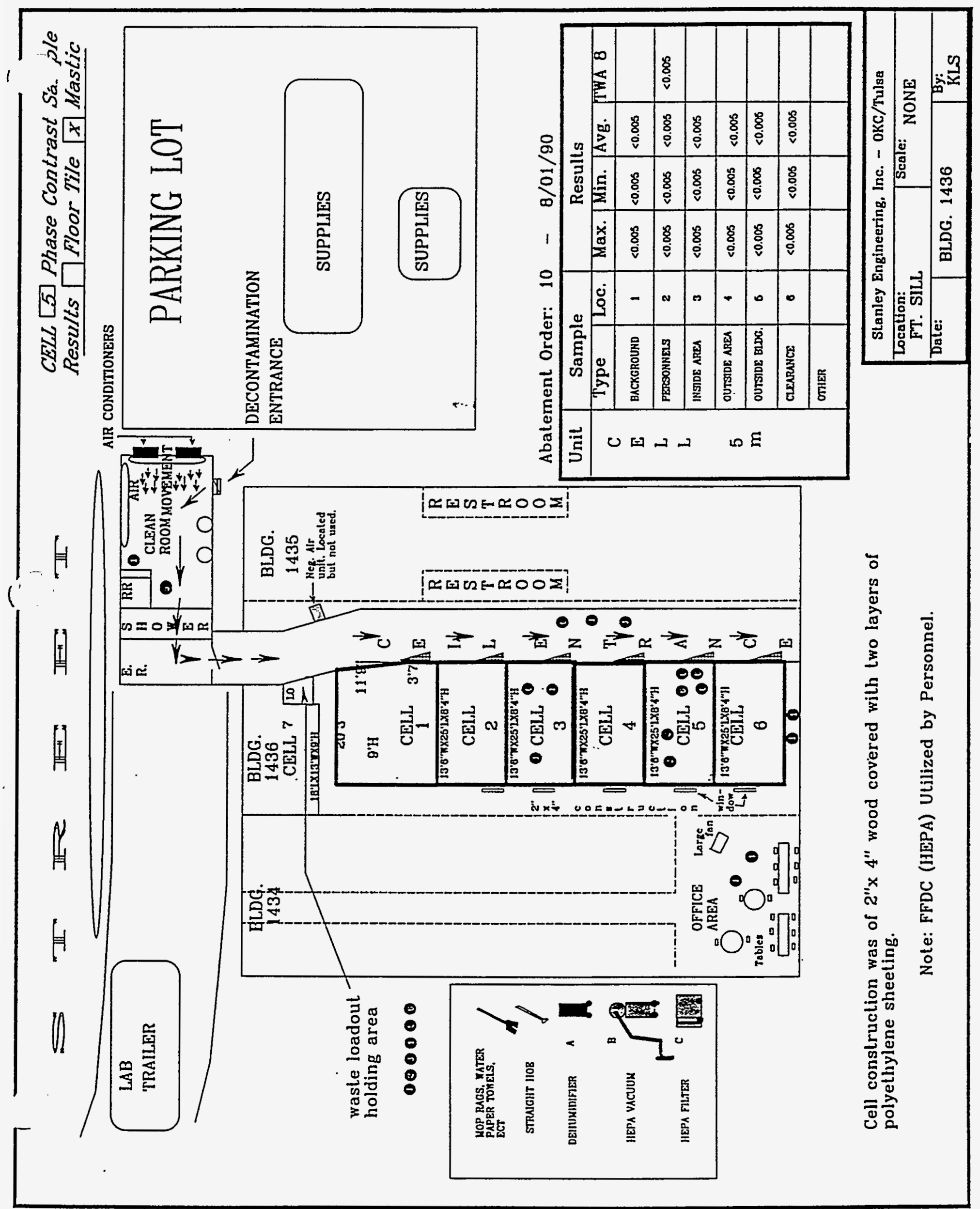


TRANSMISSIOH ELECTRON MICROSCOPY (T.E.M.)

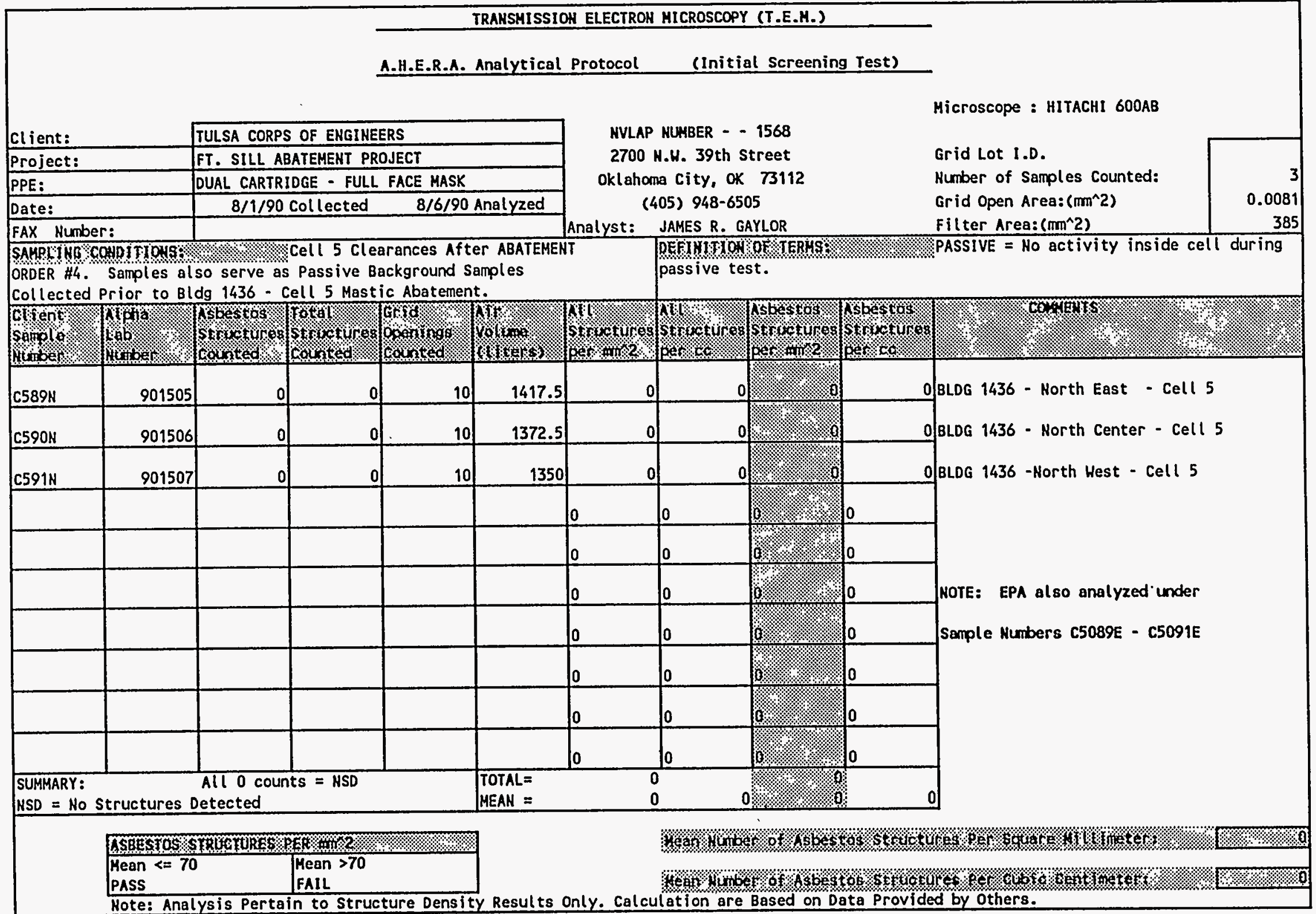




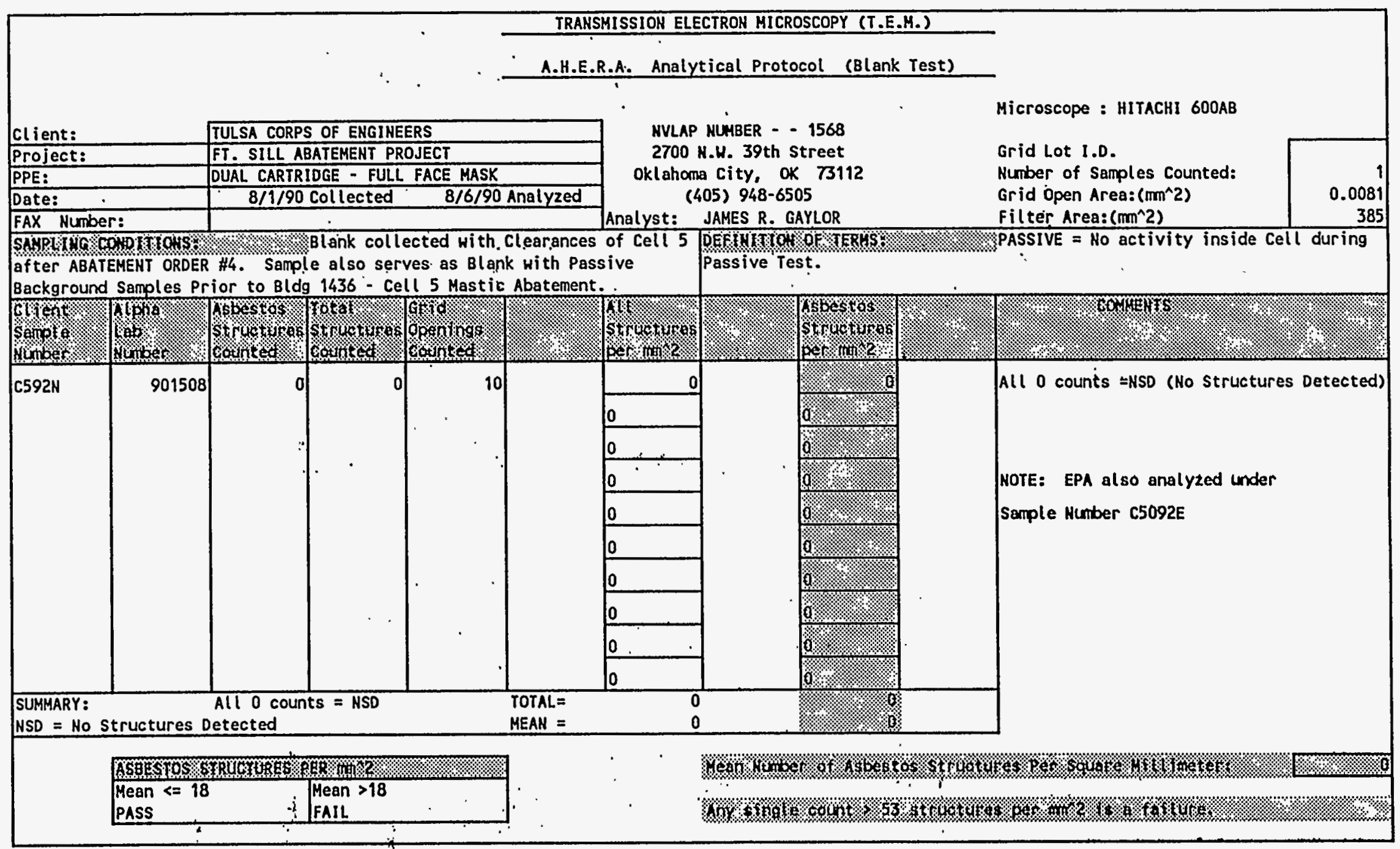




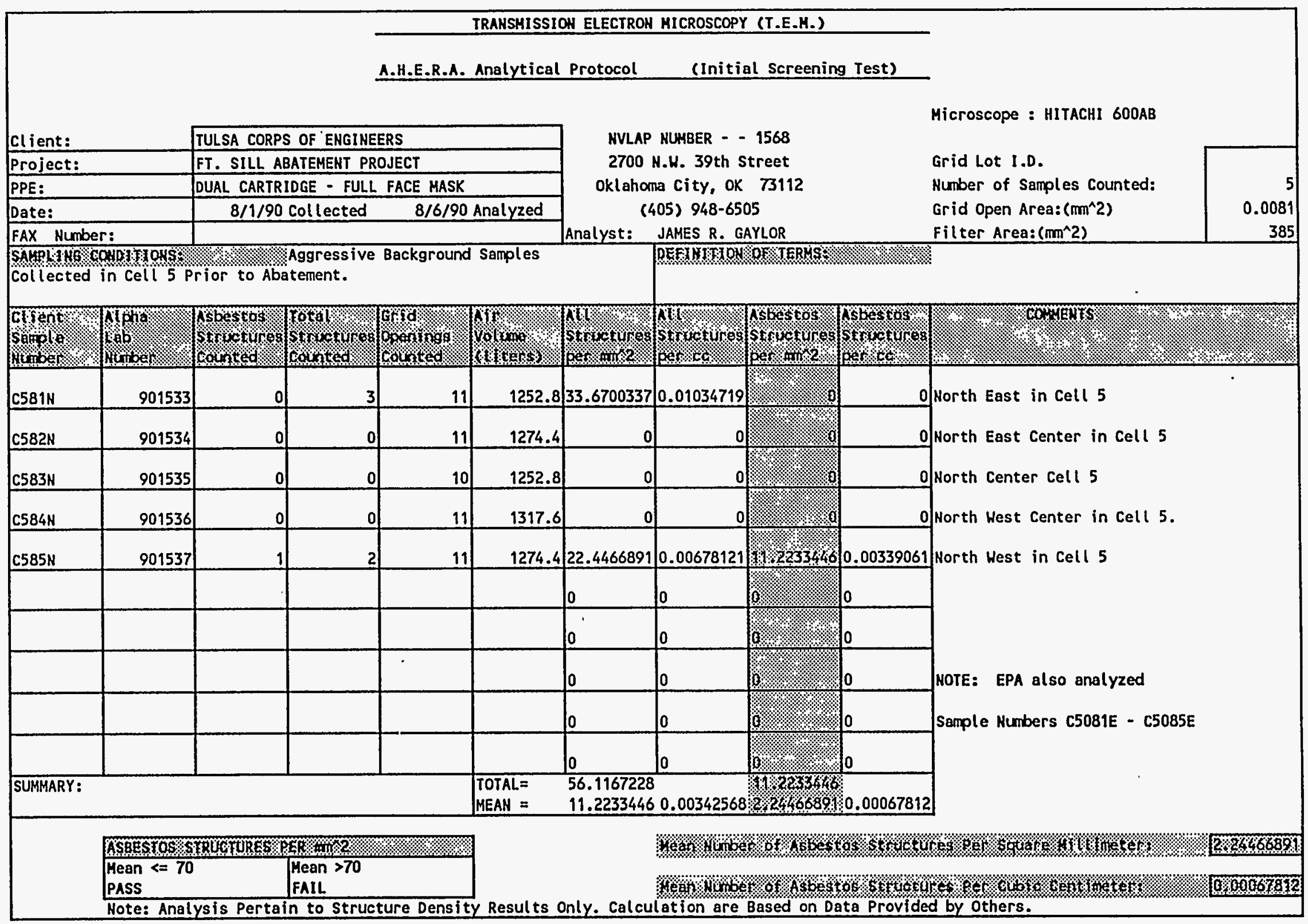


STANLEY ENGINEERING, INC. . IVIRONMENTAL MEASUREMENTS

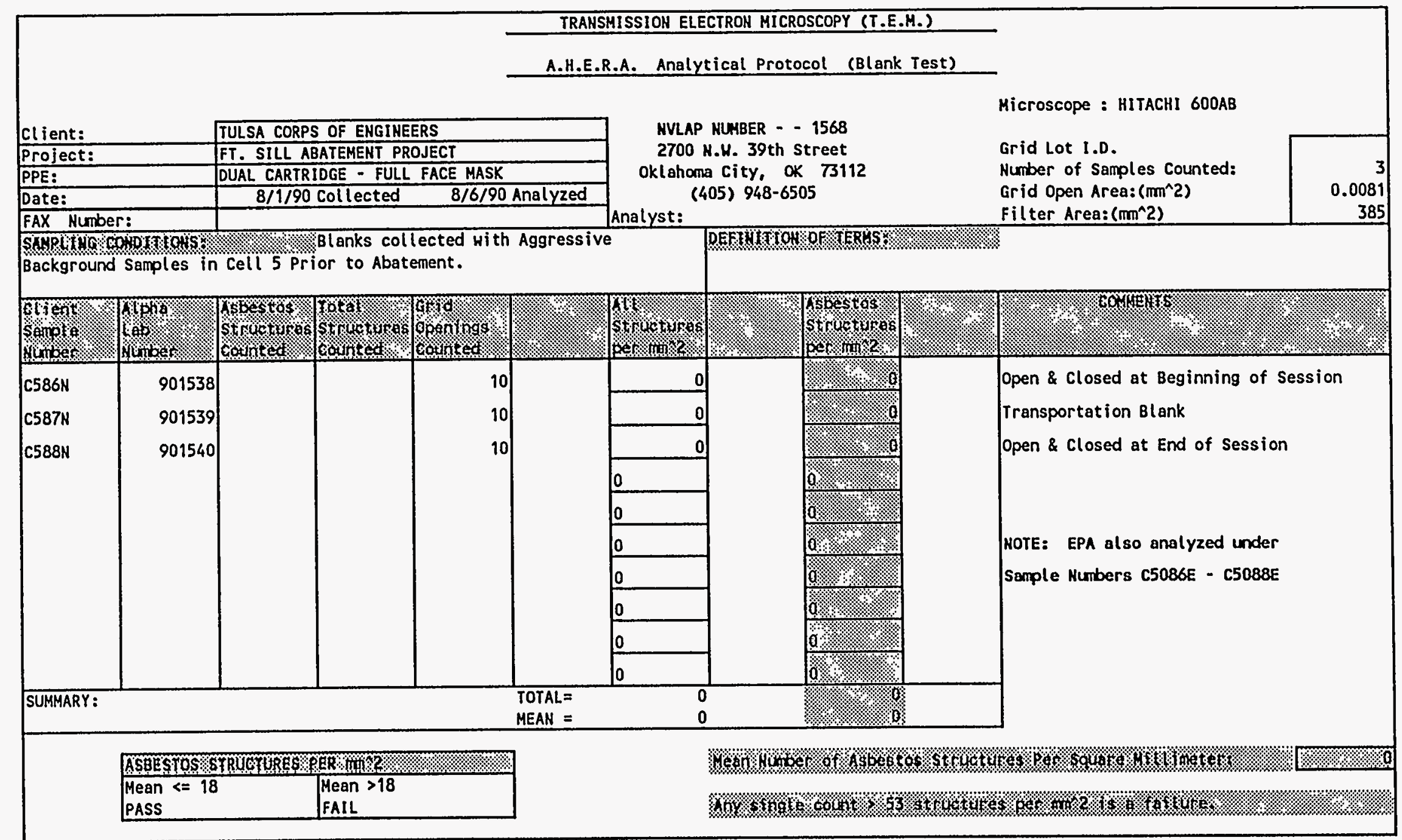




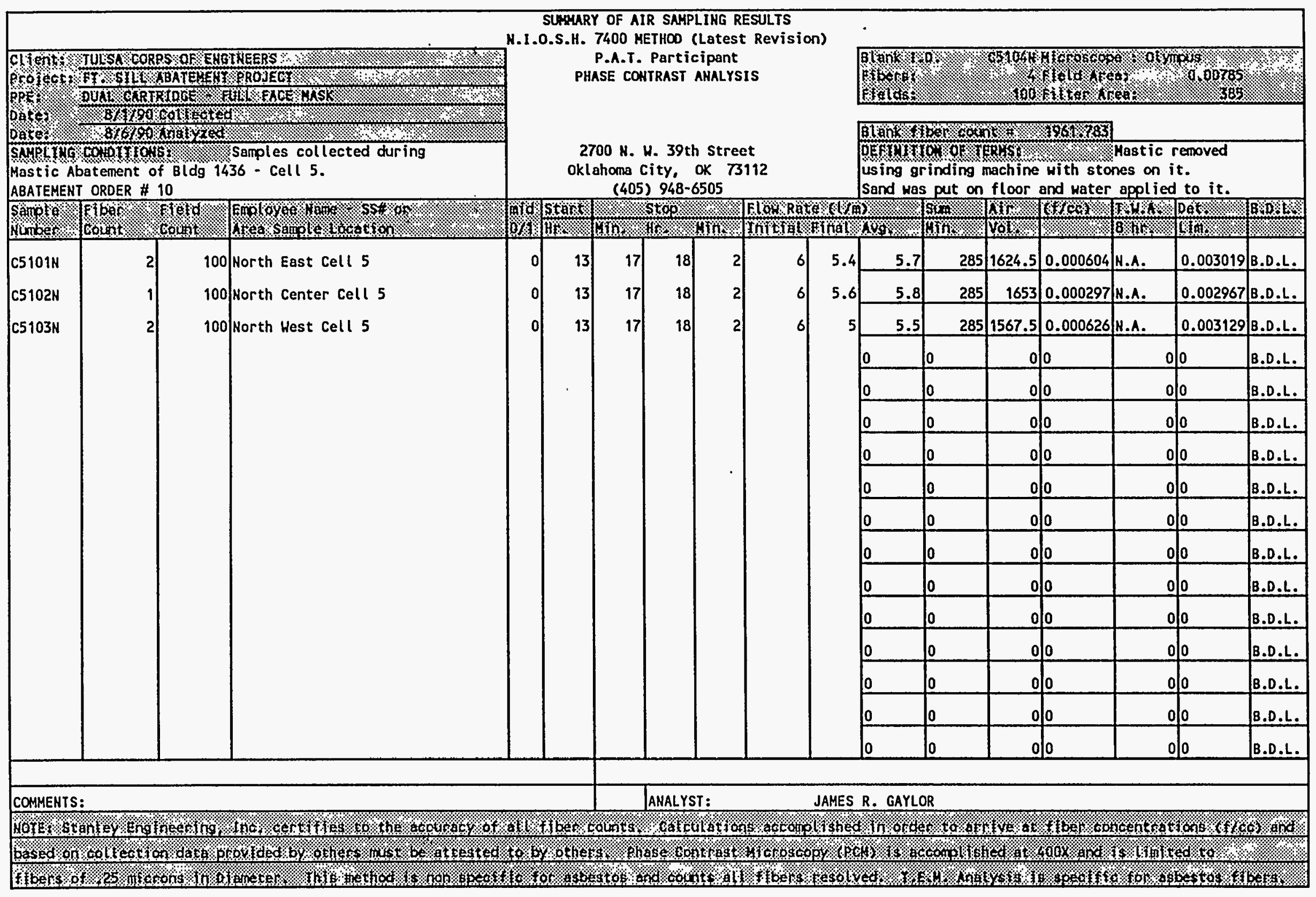




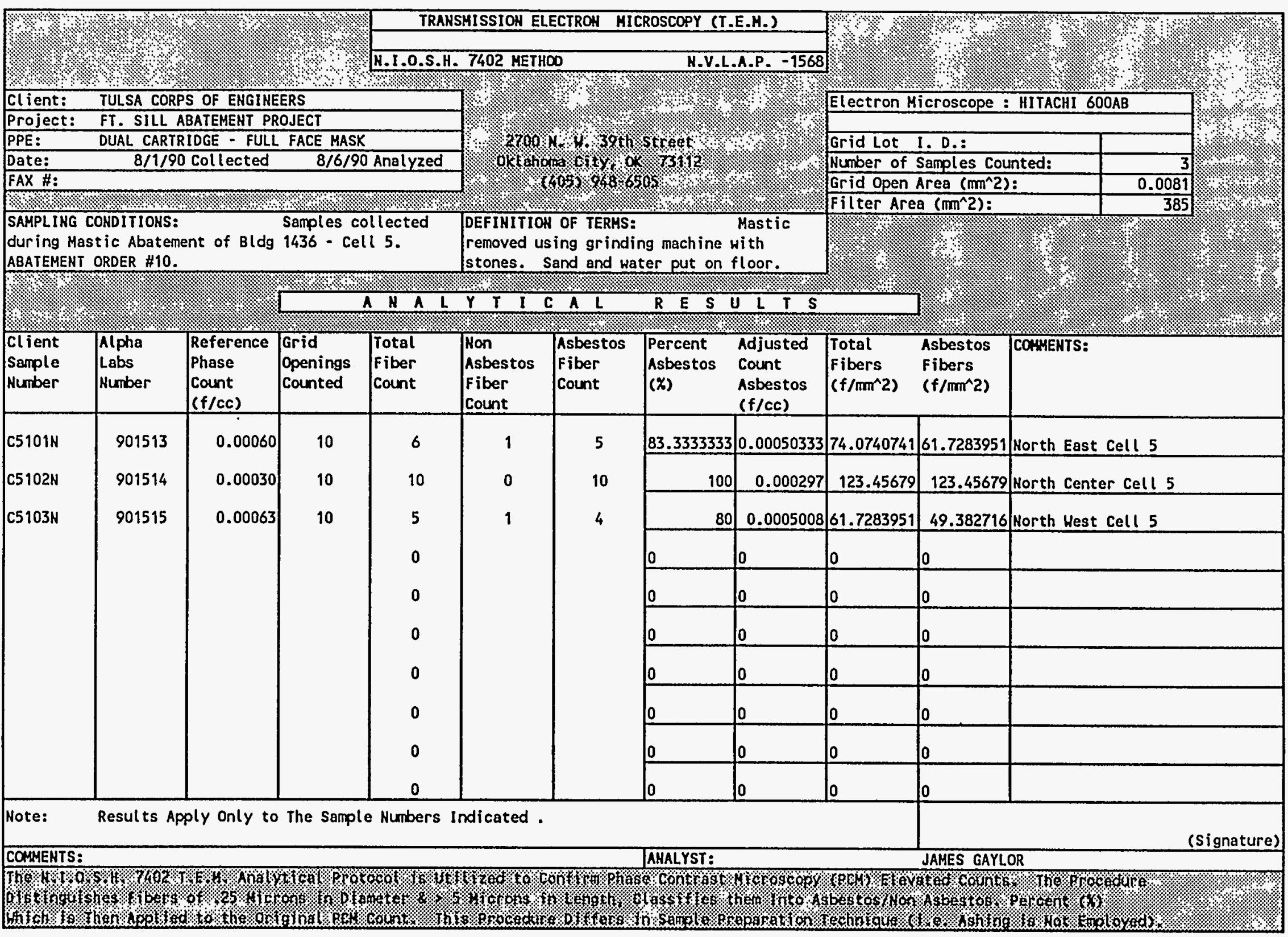




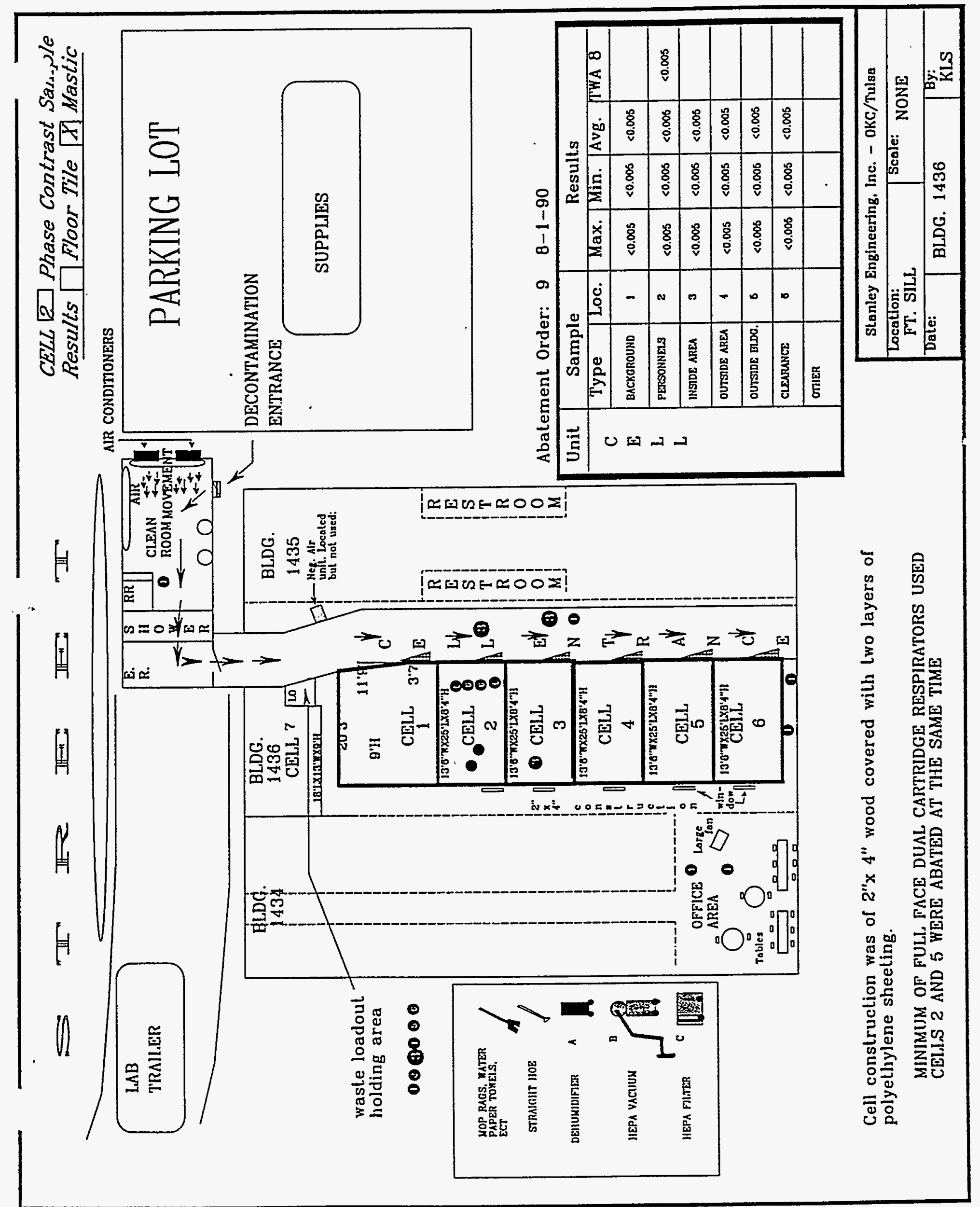




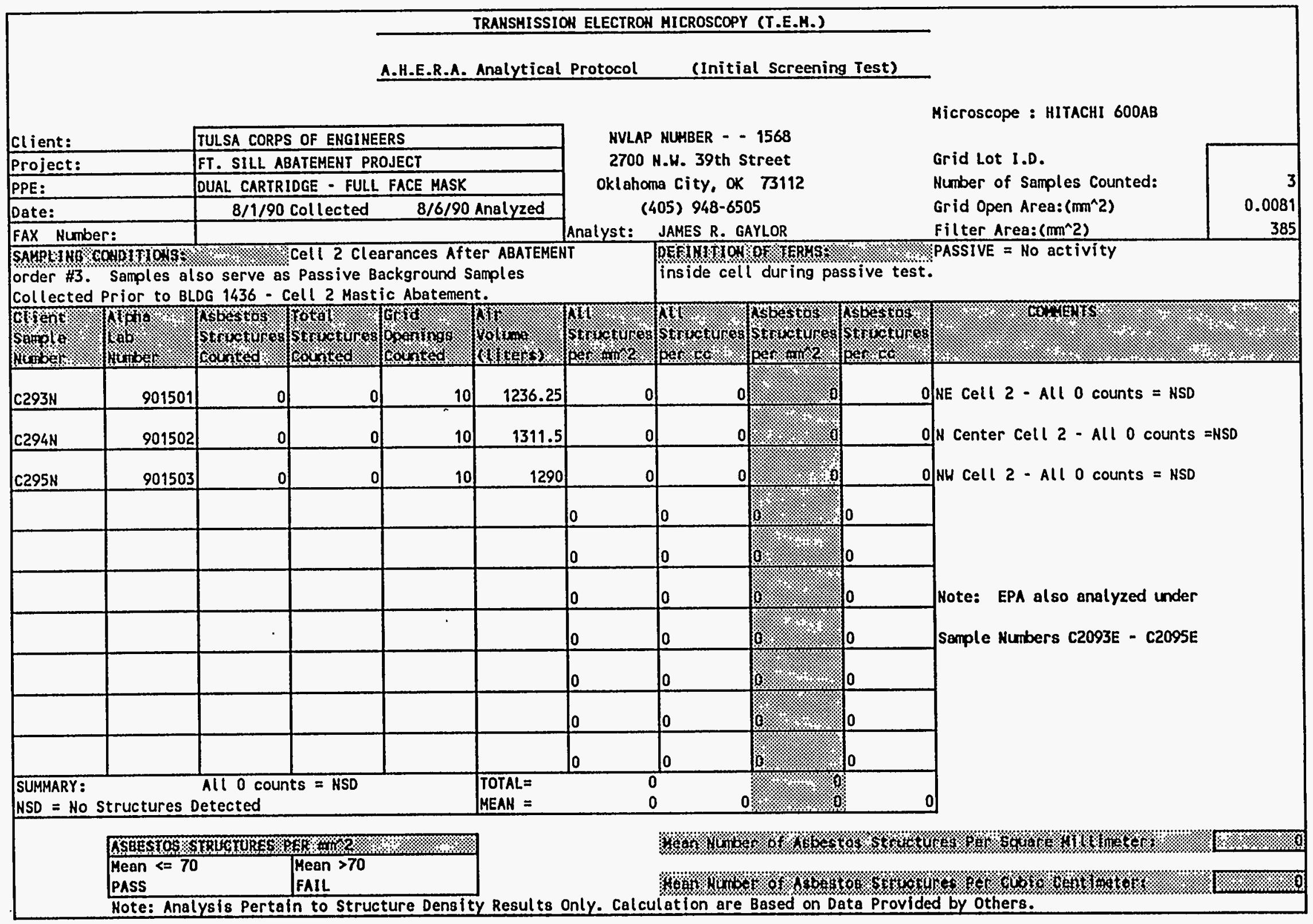




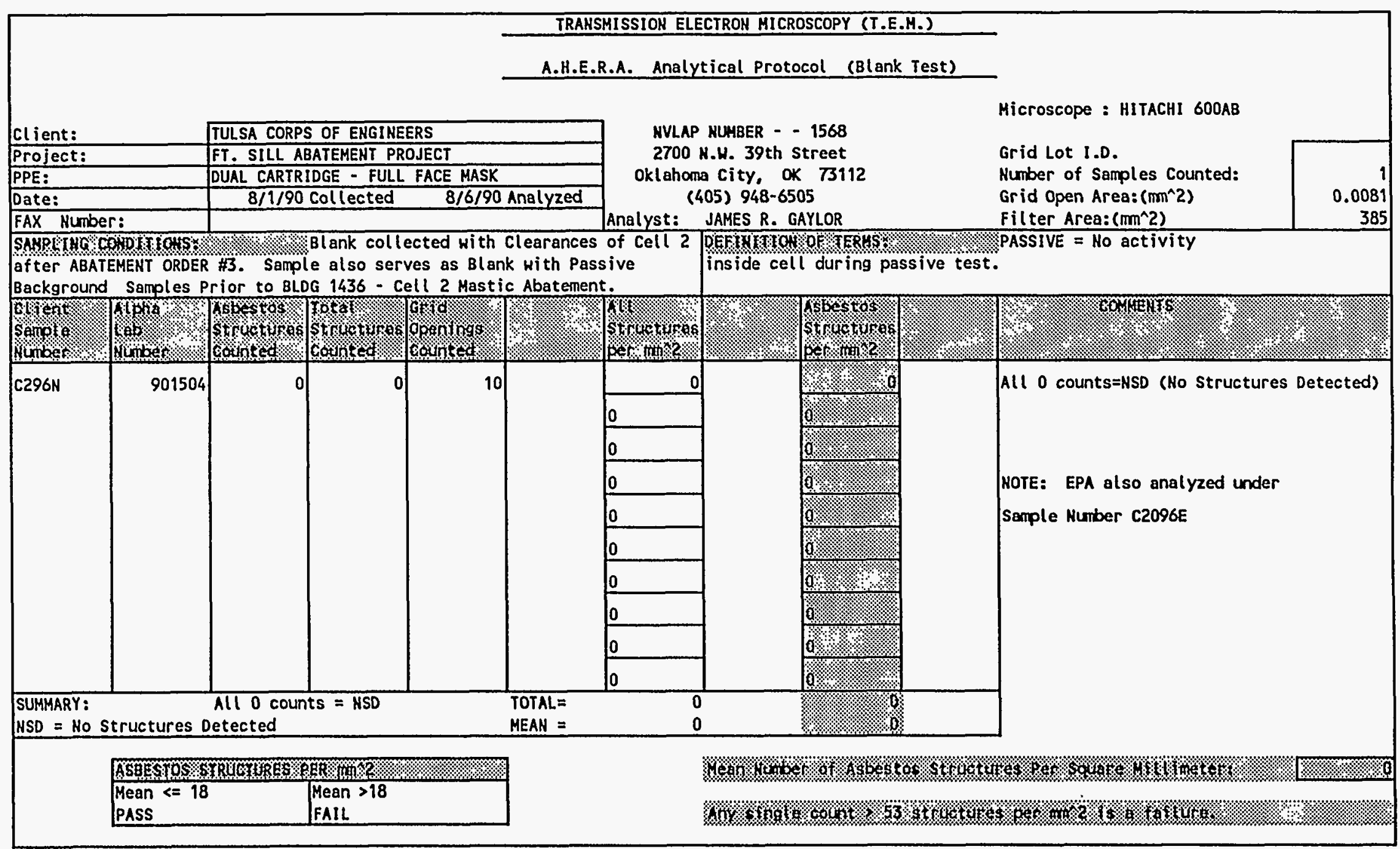




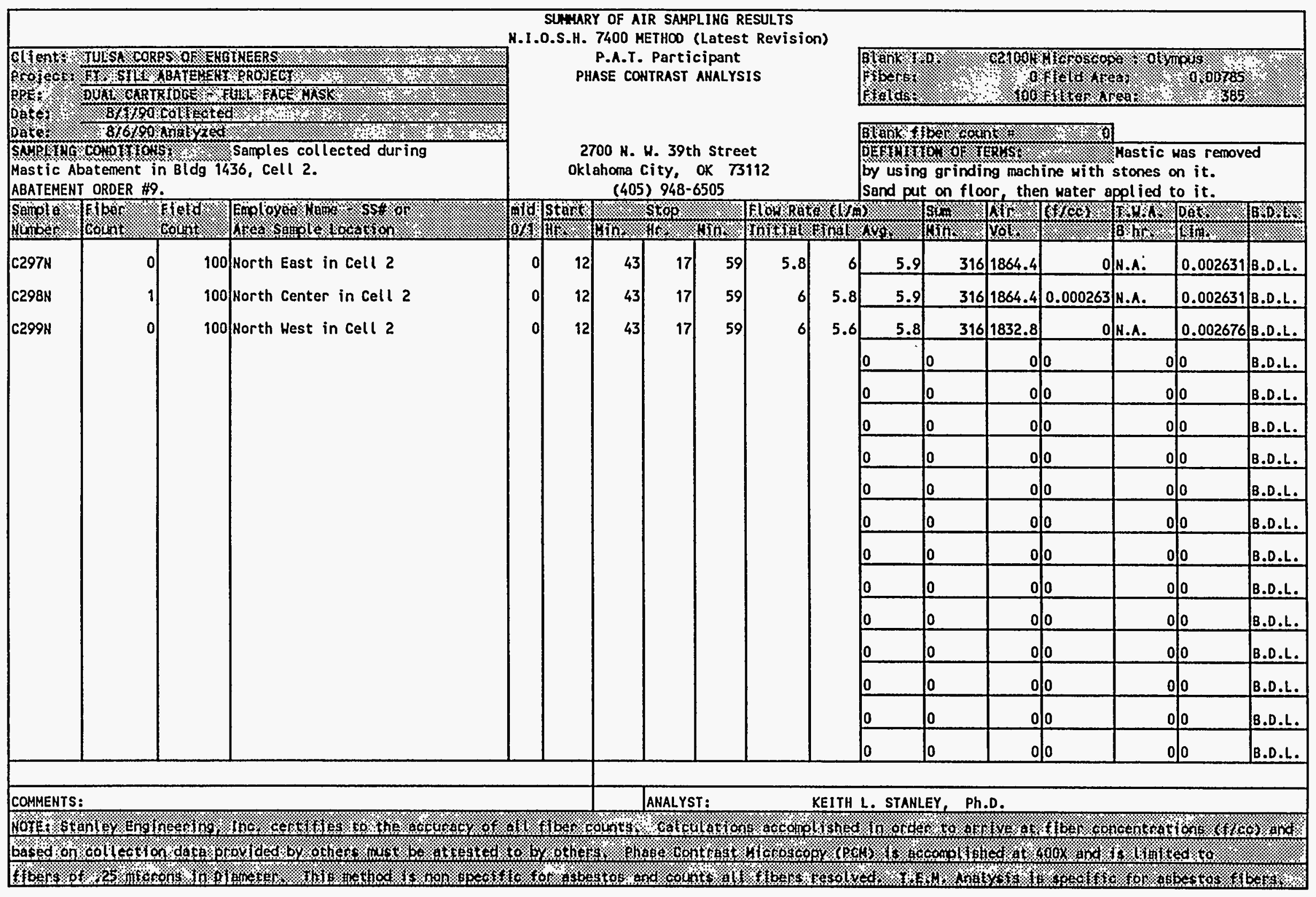




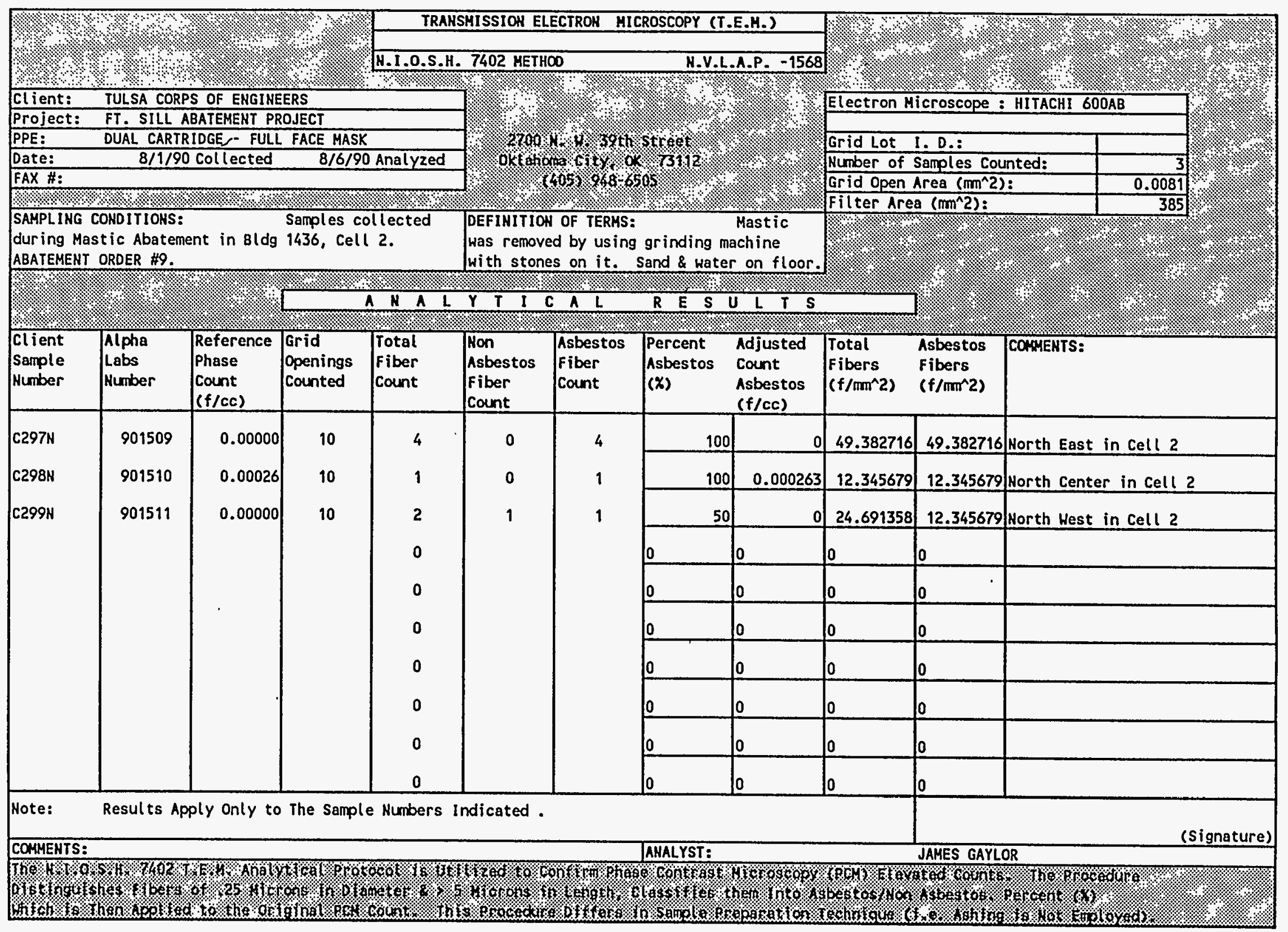




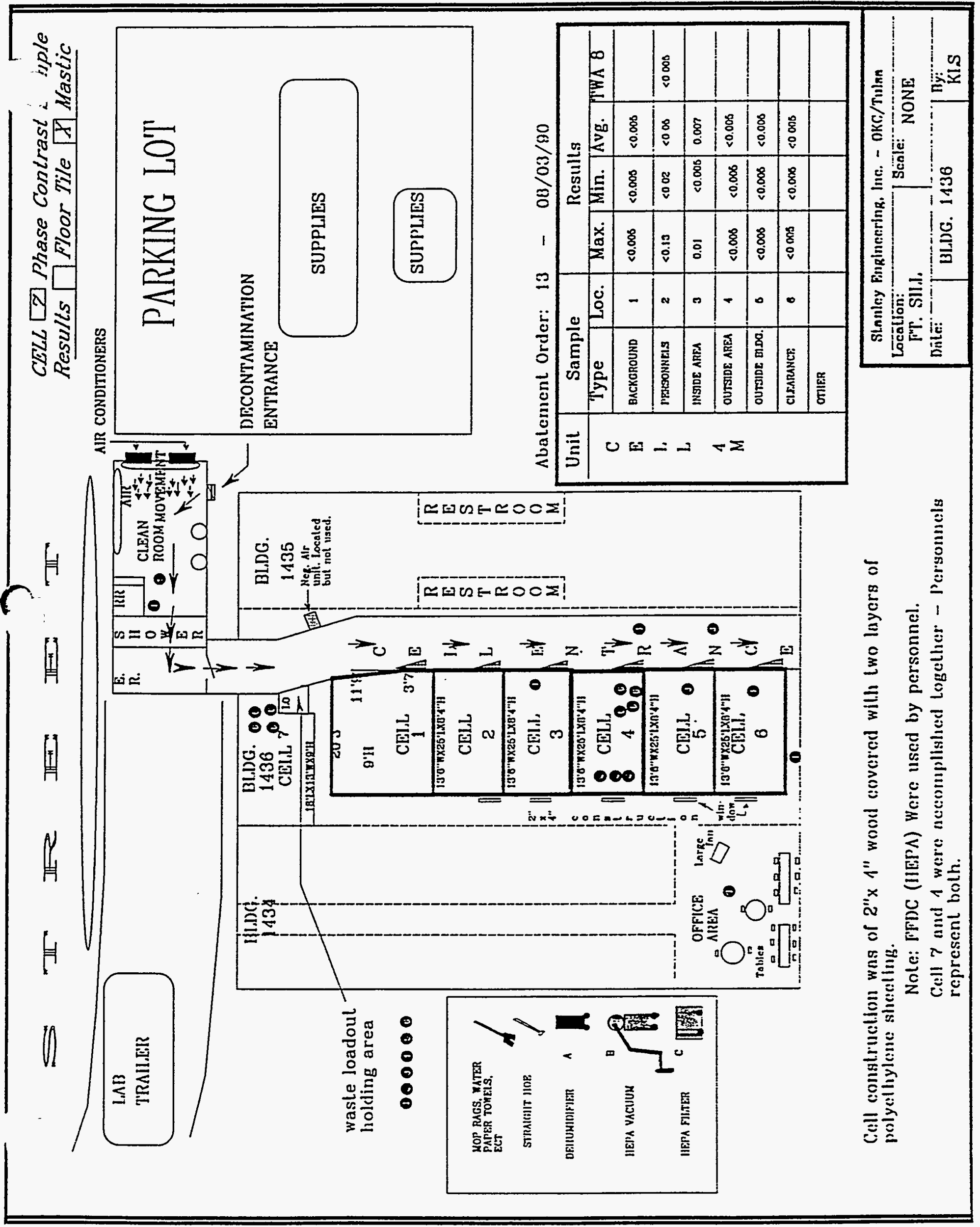




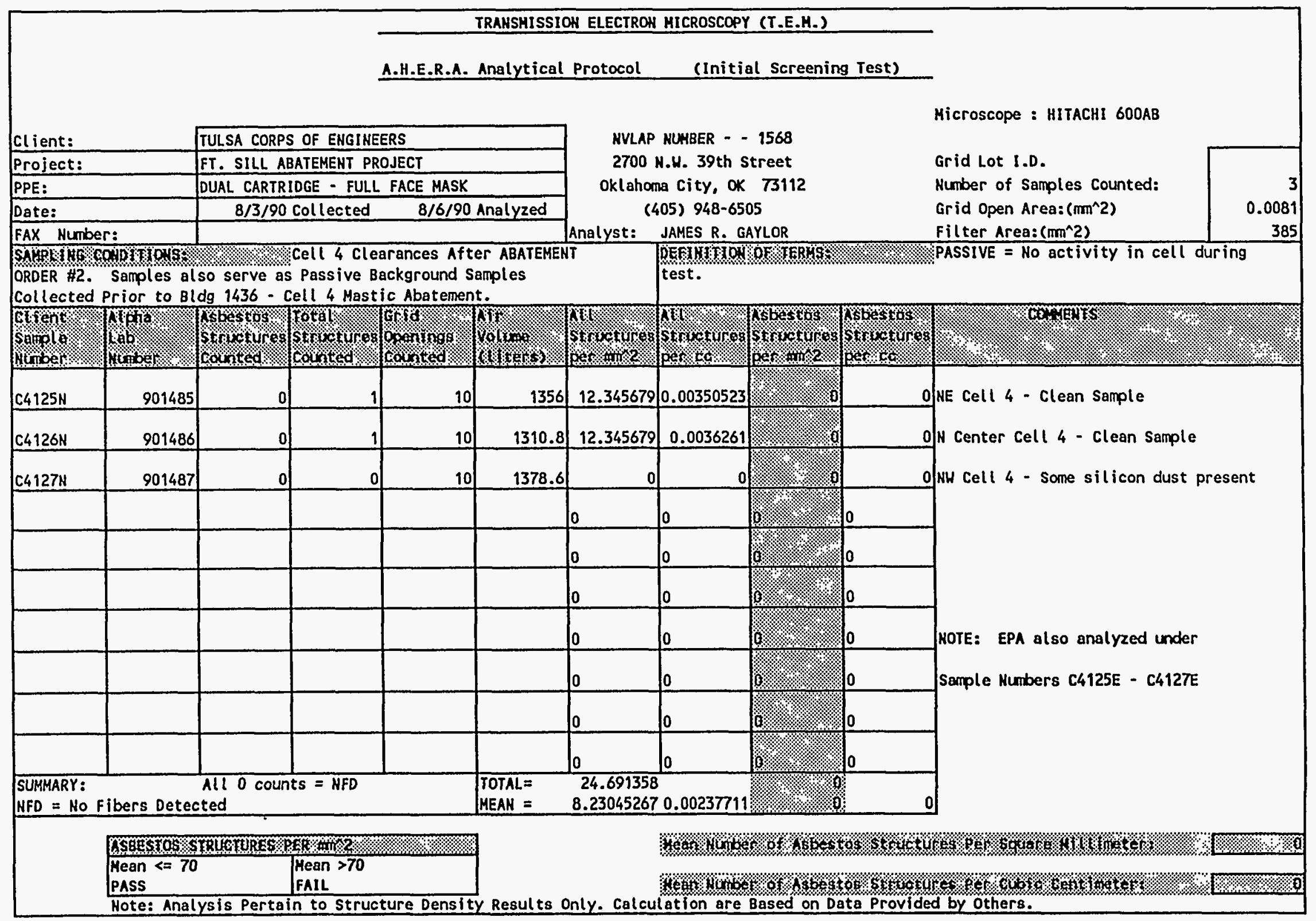


TRAHSMISSIOH ELECTROH MICROSCOPY (T.E.H.)

\section{A.H.E.R.A. Analytical Protocol (Initial Screening Test)}

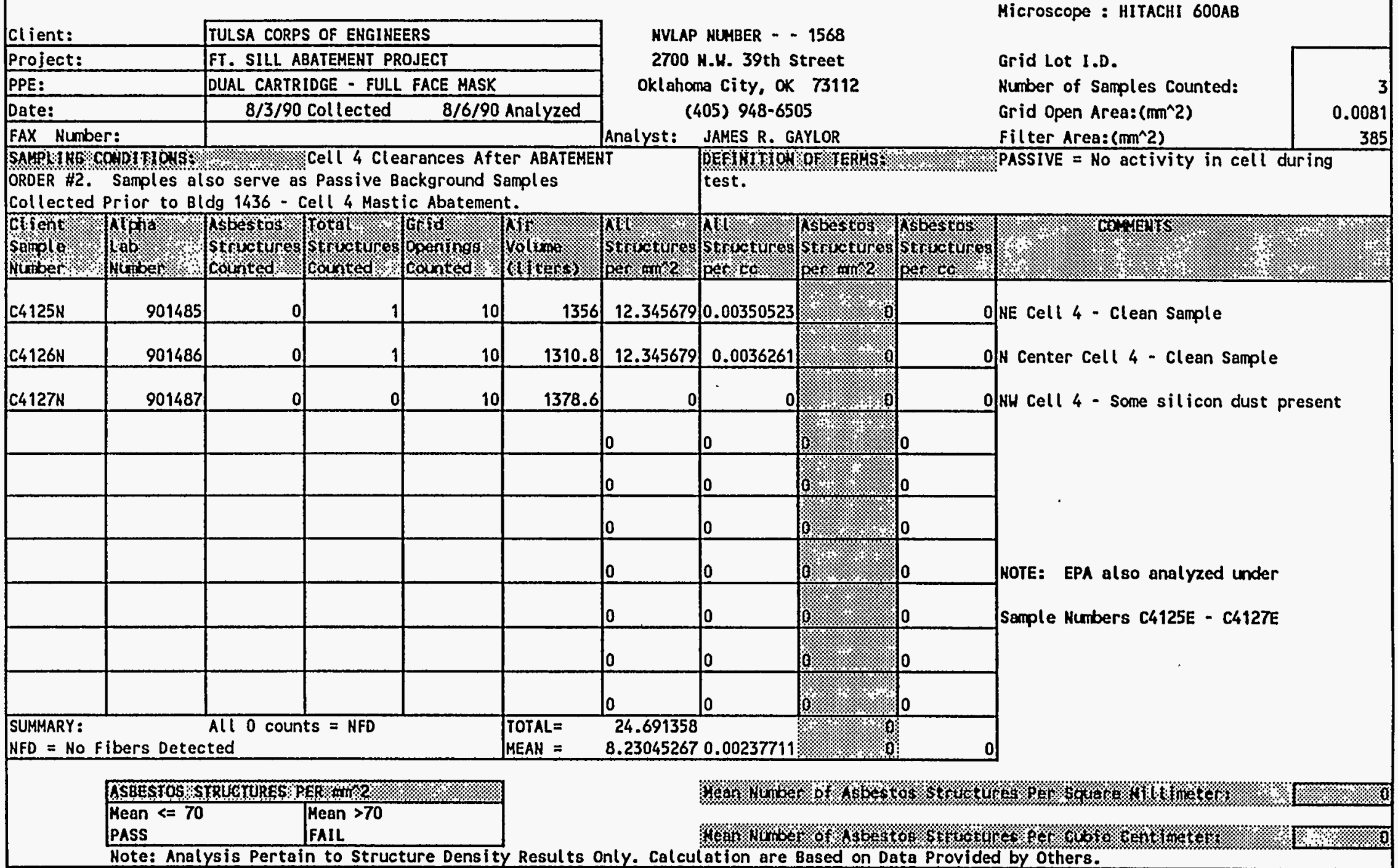


STANLEY ENGINEERING, INC. - .RONMEHTAL MEASUREMENTS

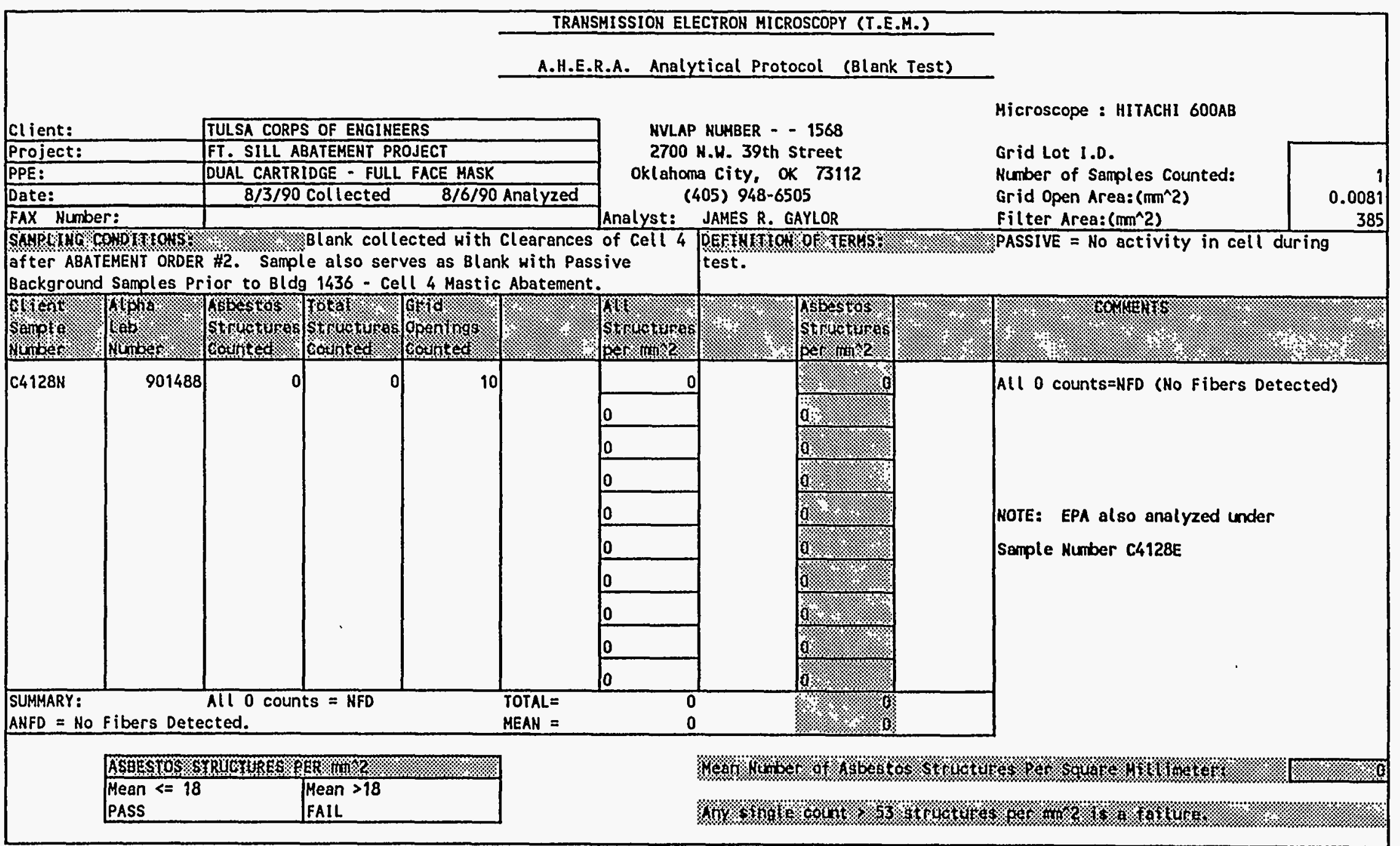




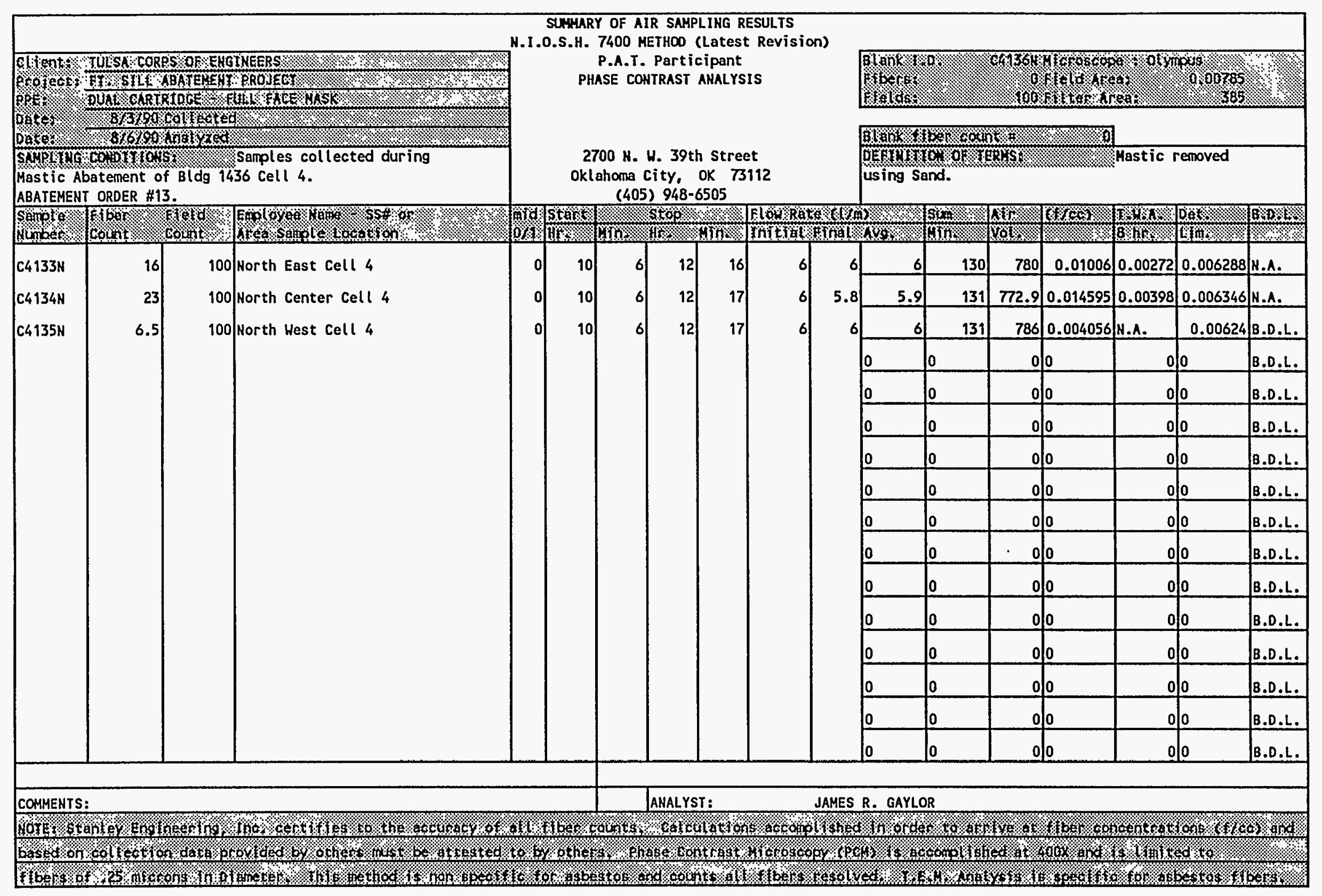




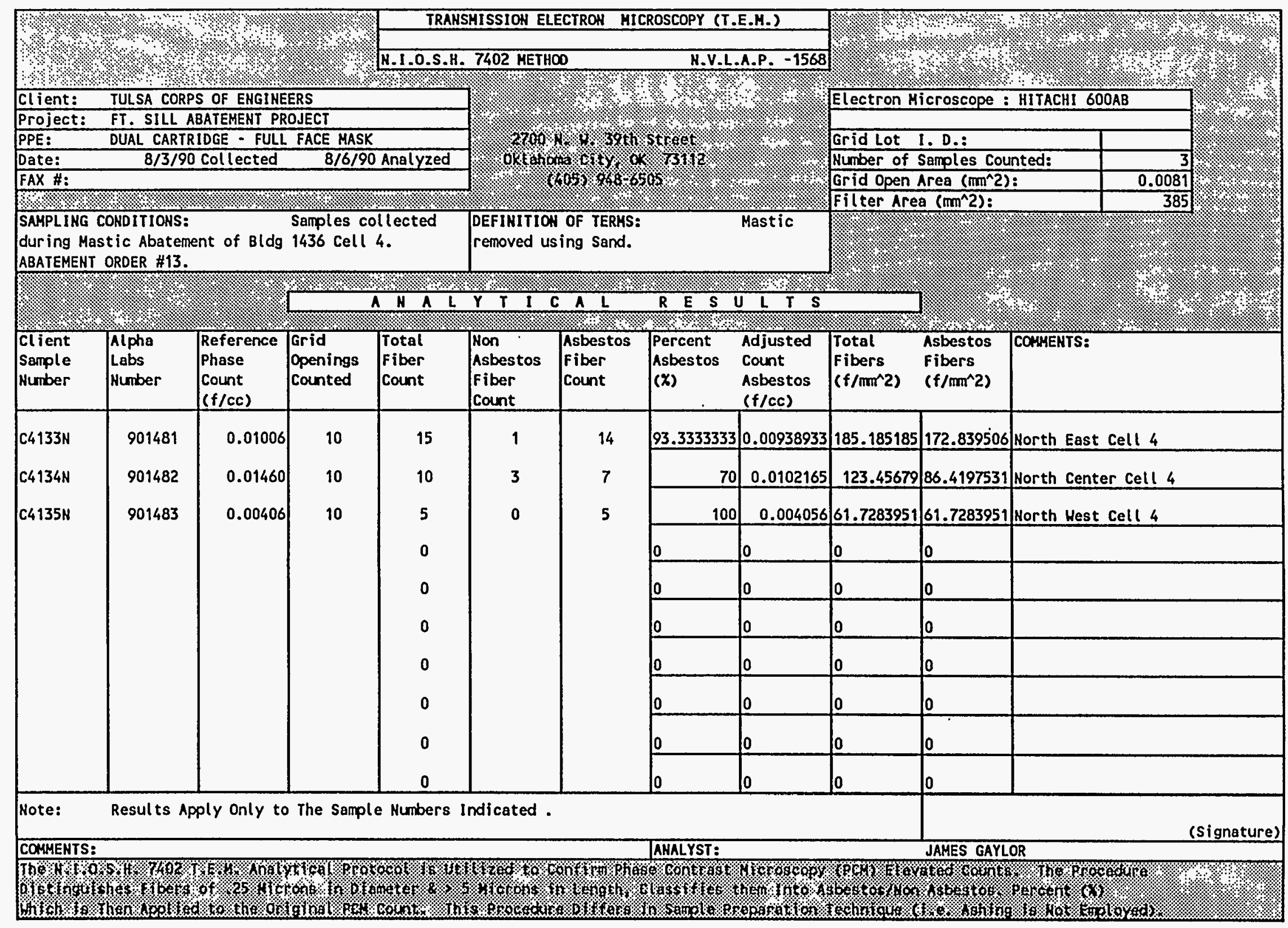




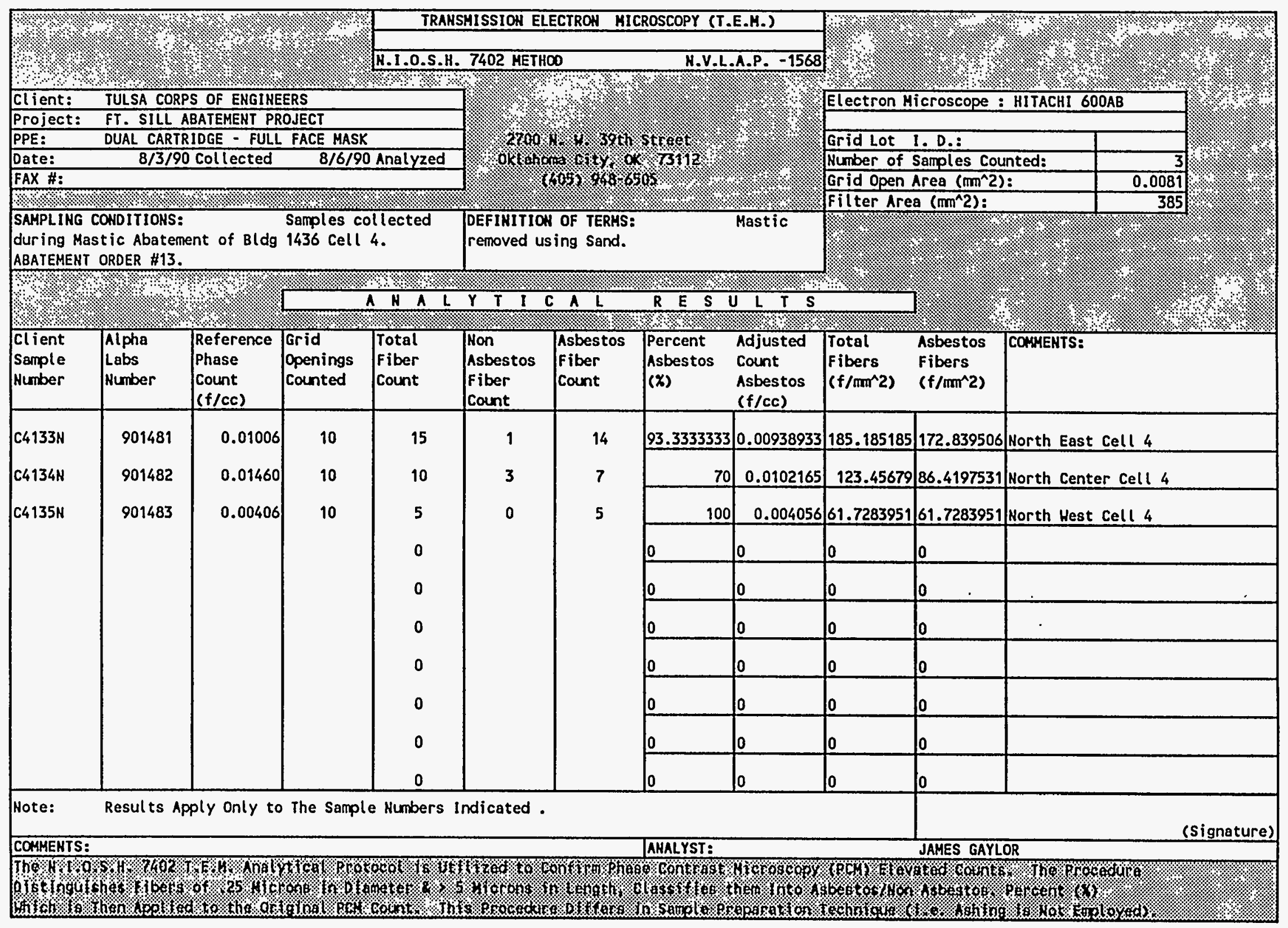




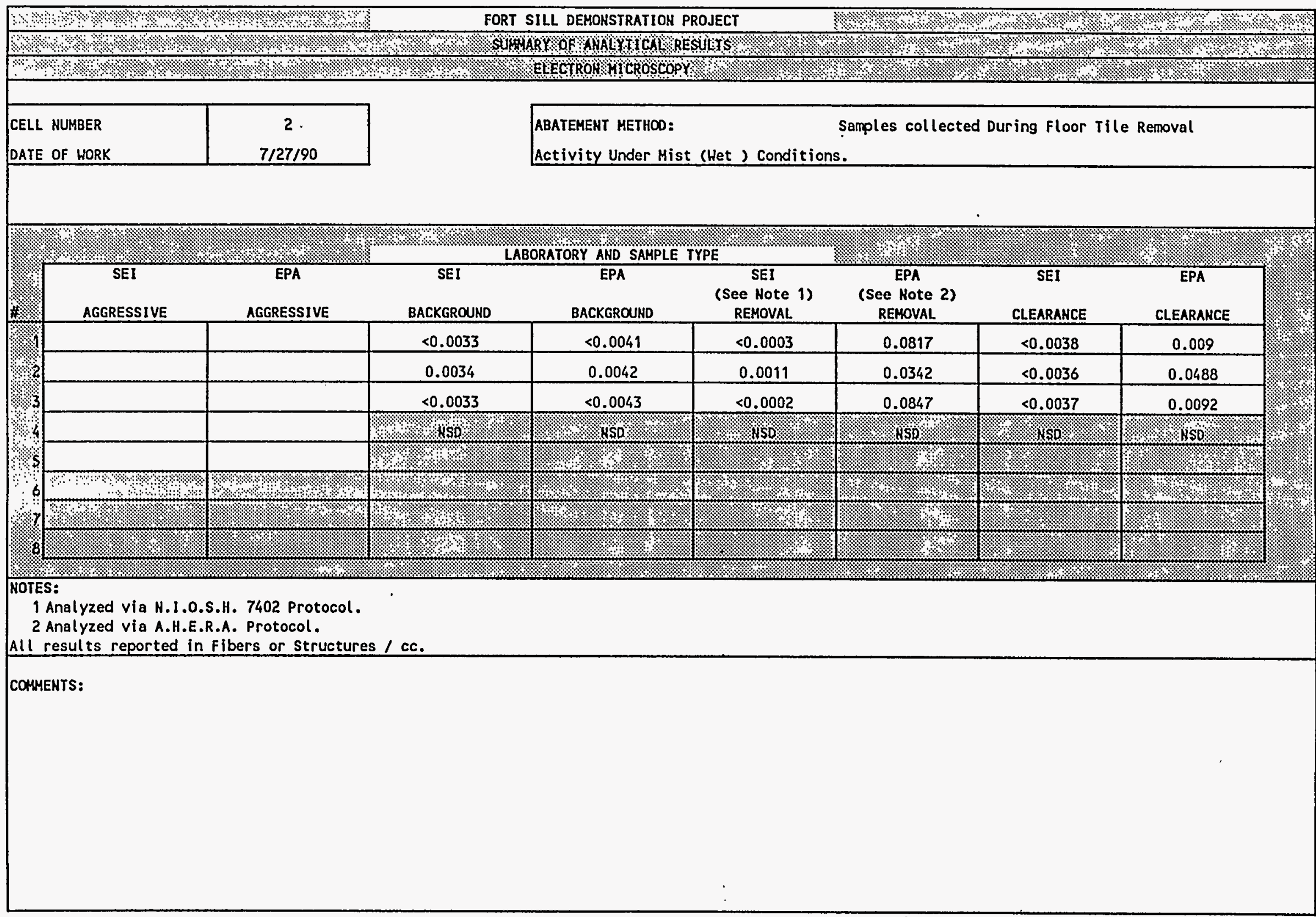




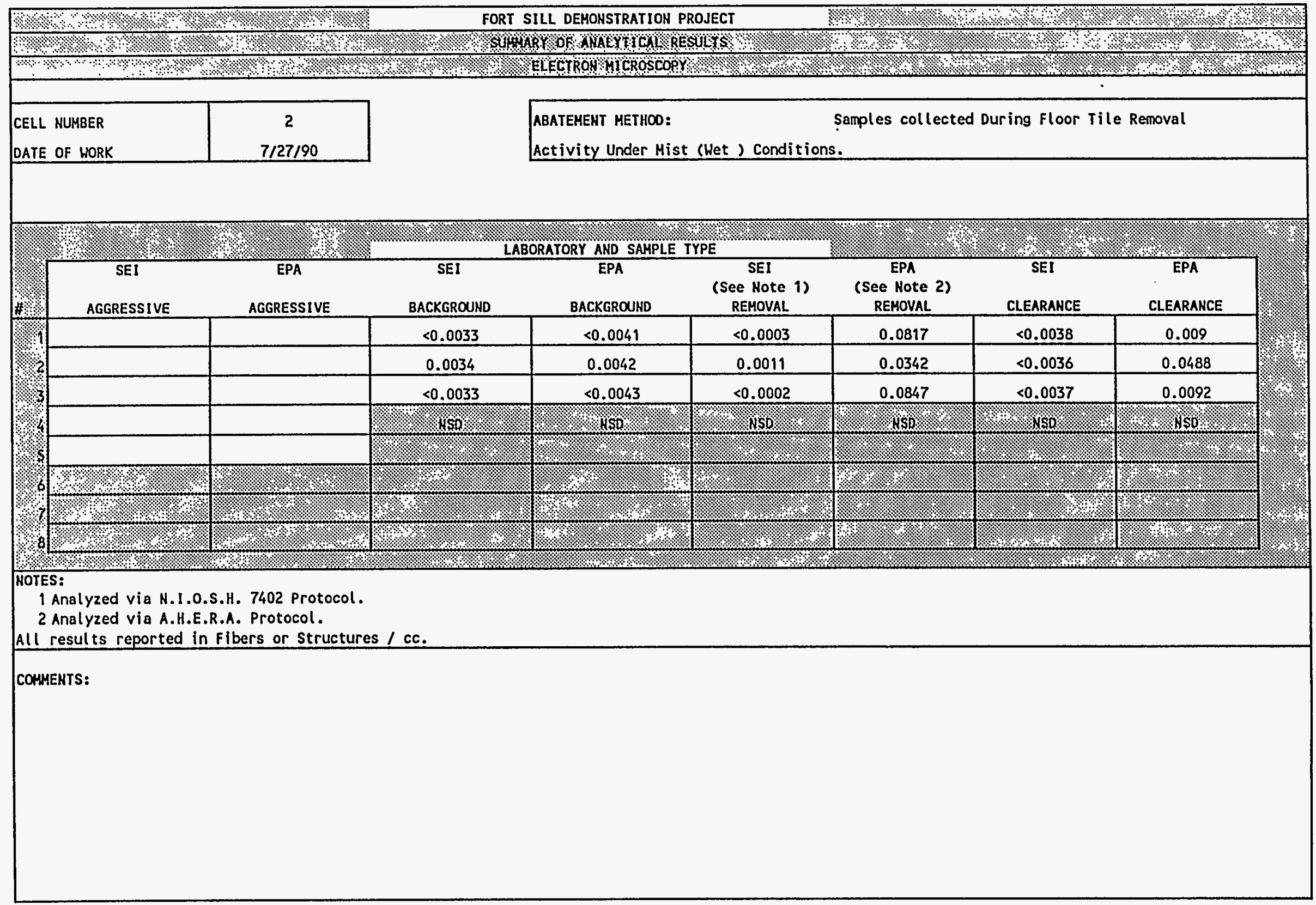




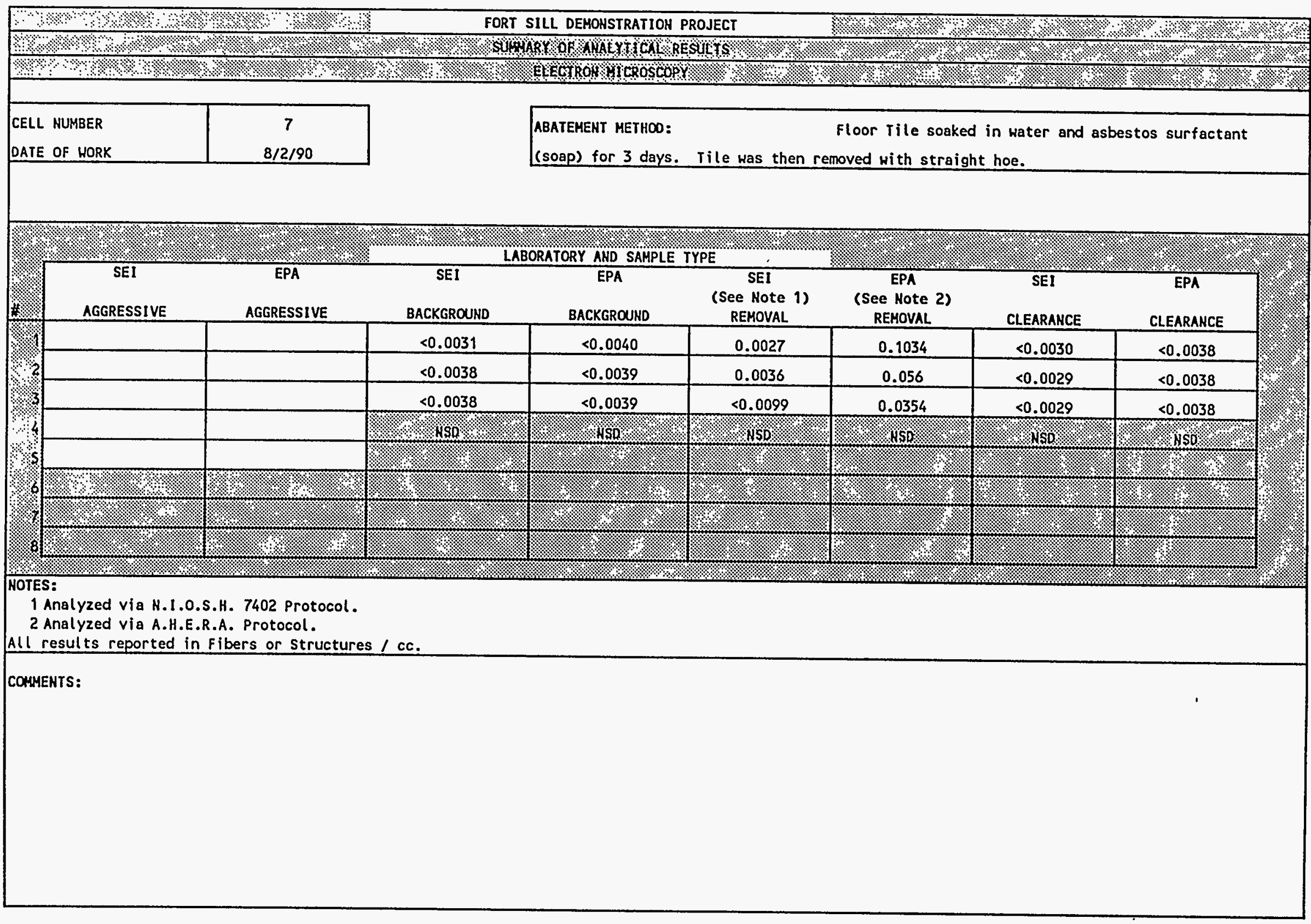




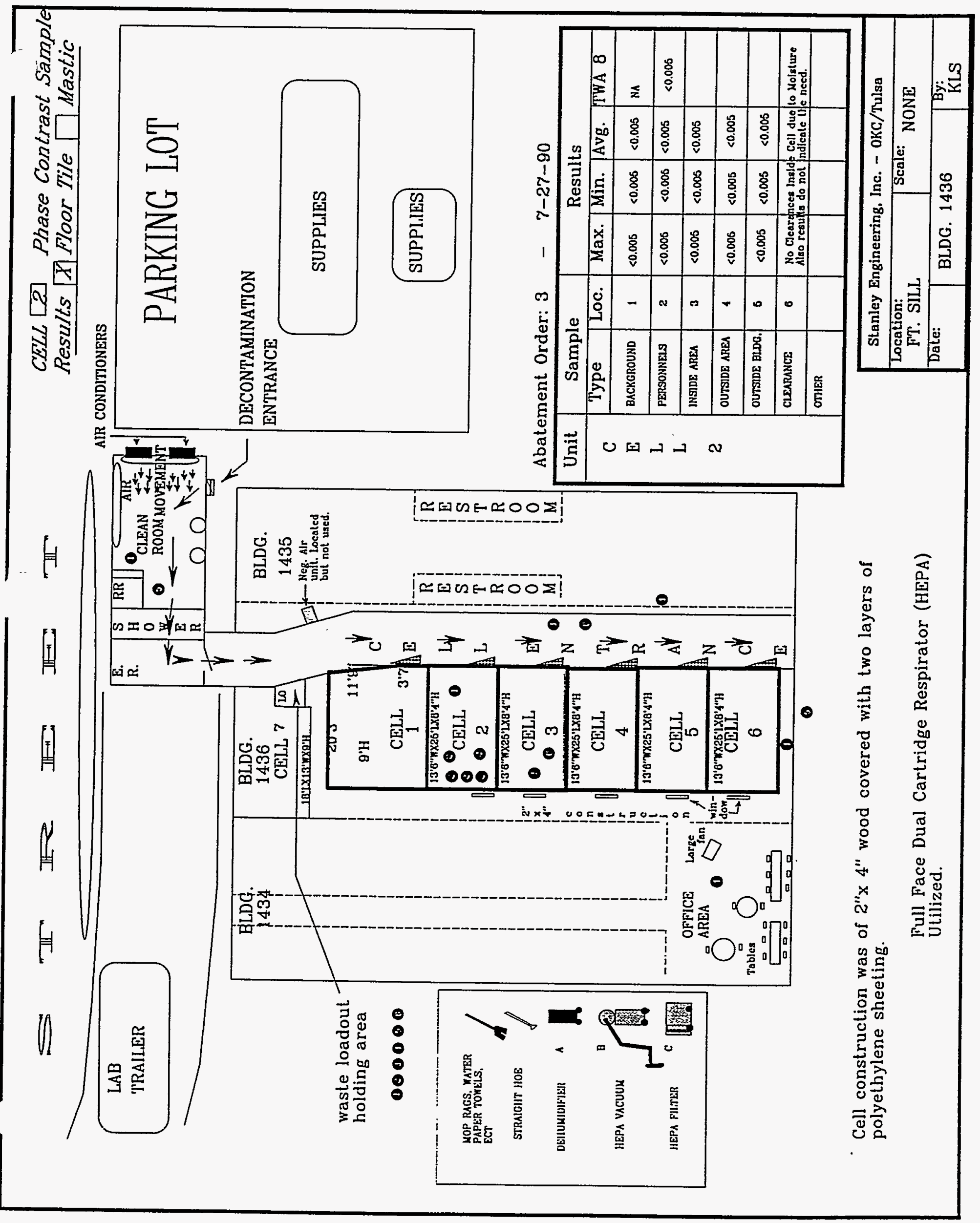




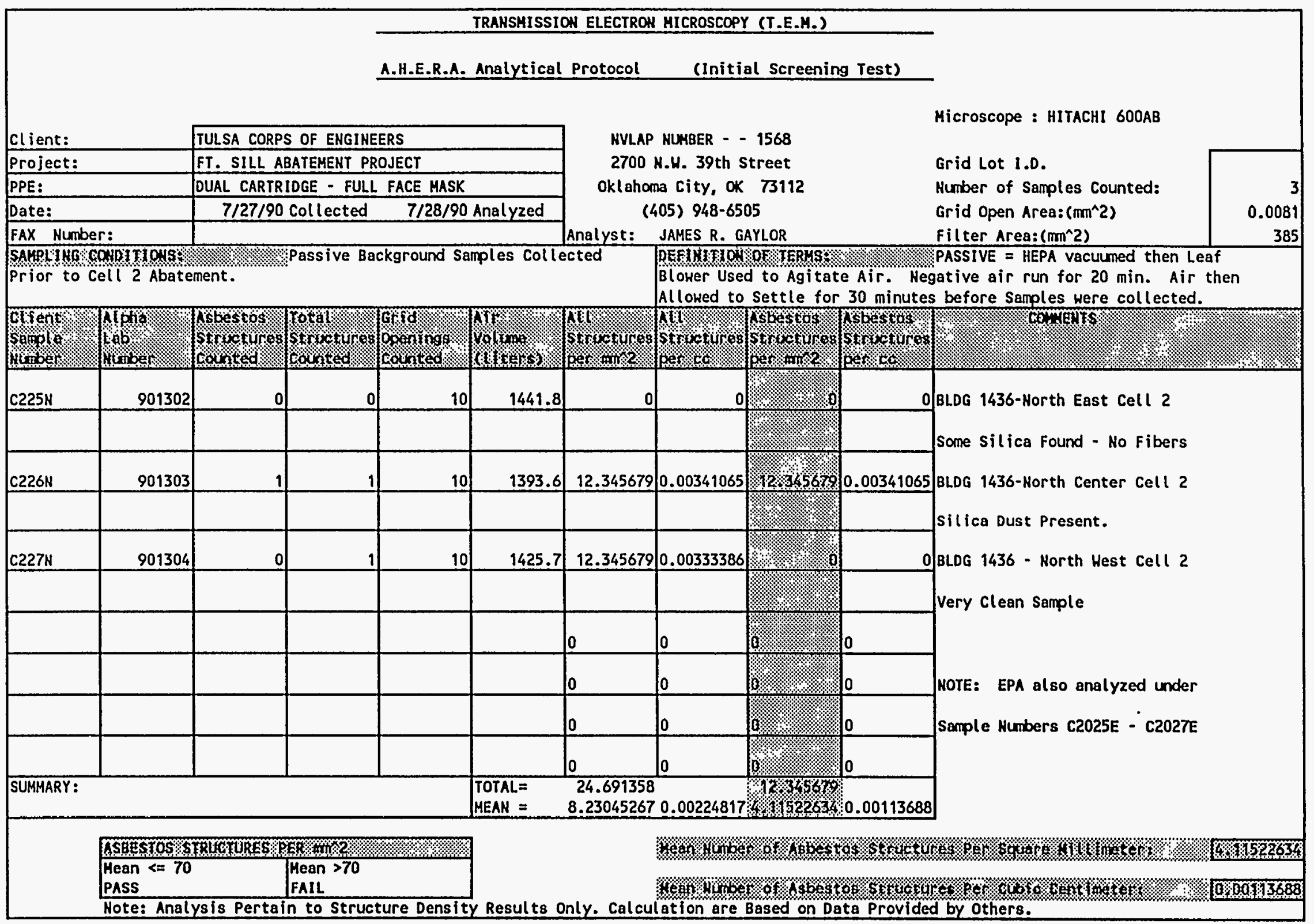




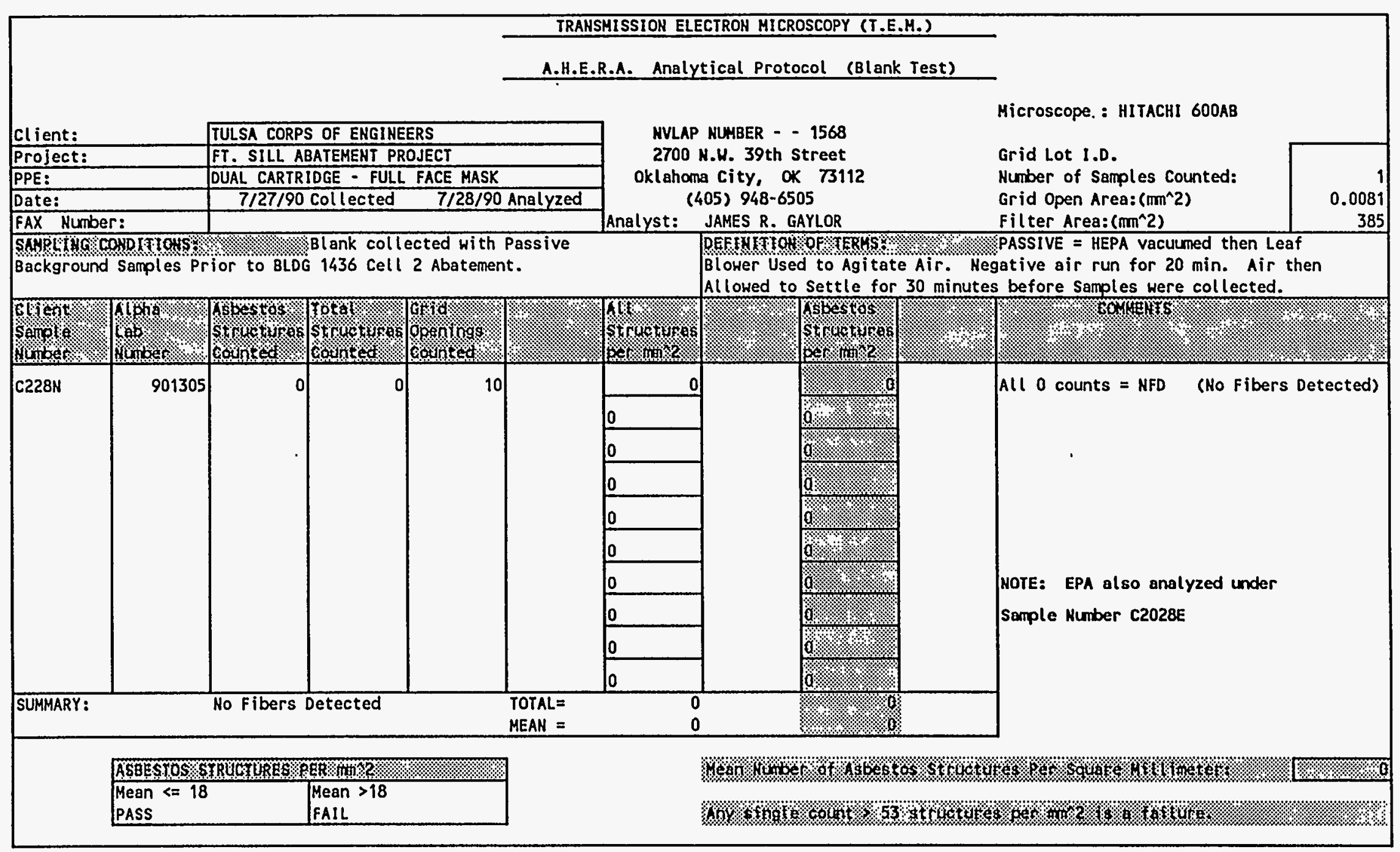




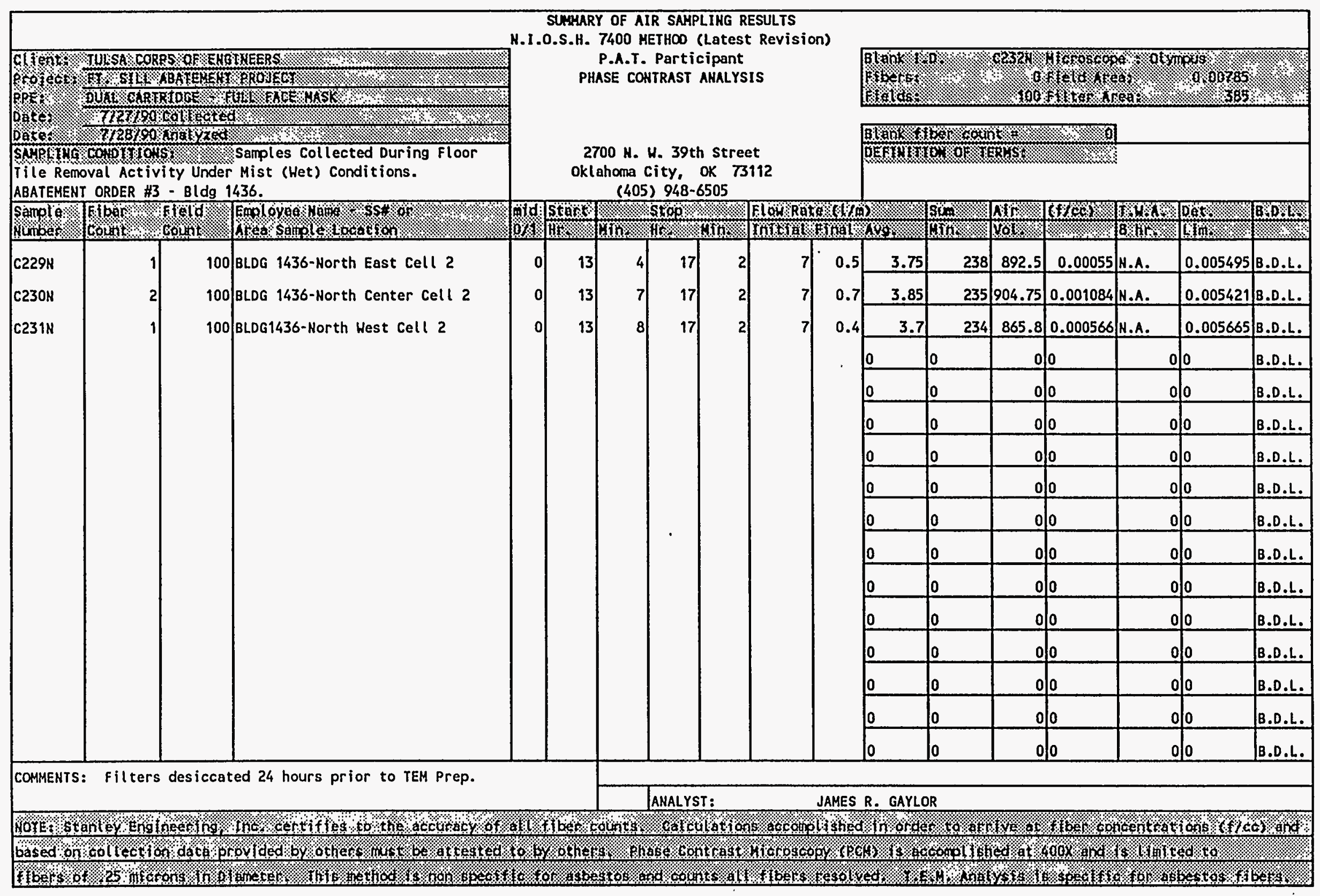




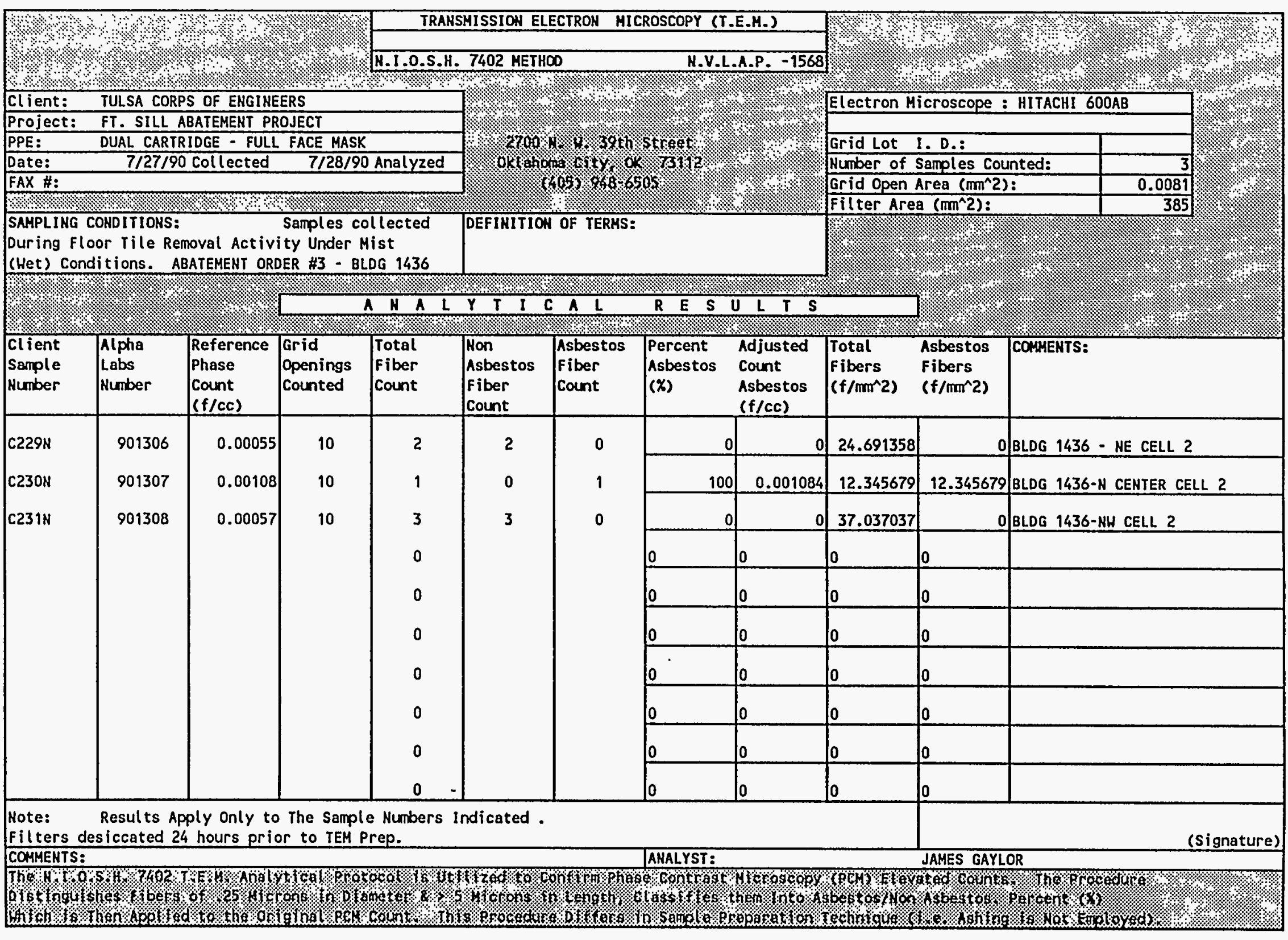




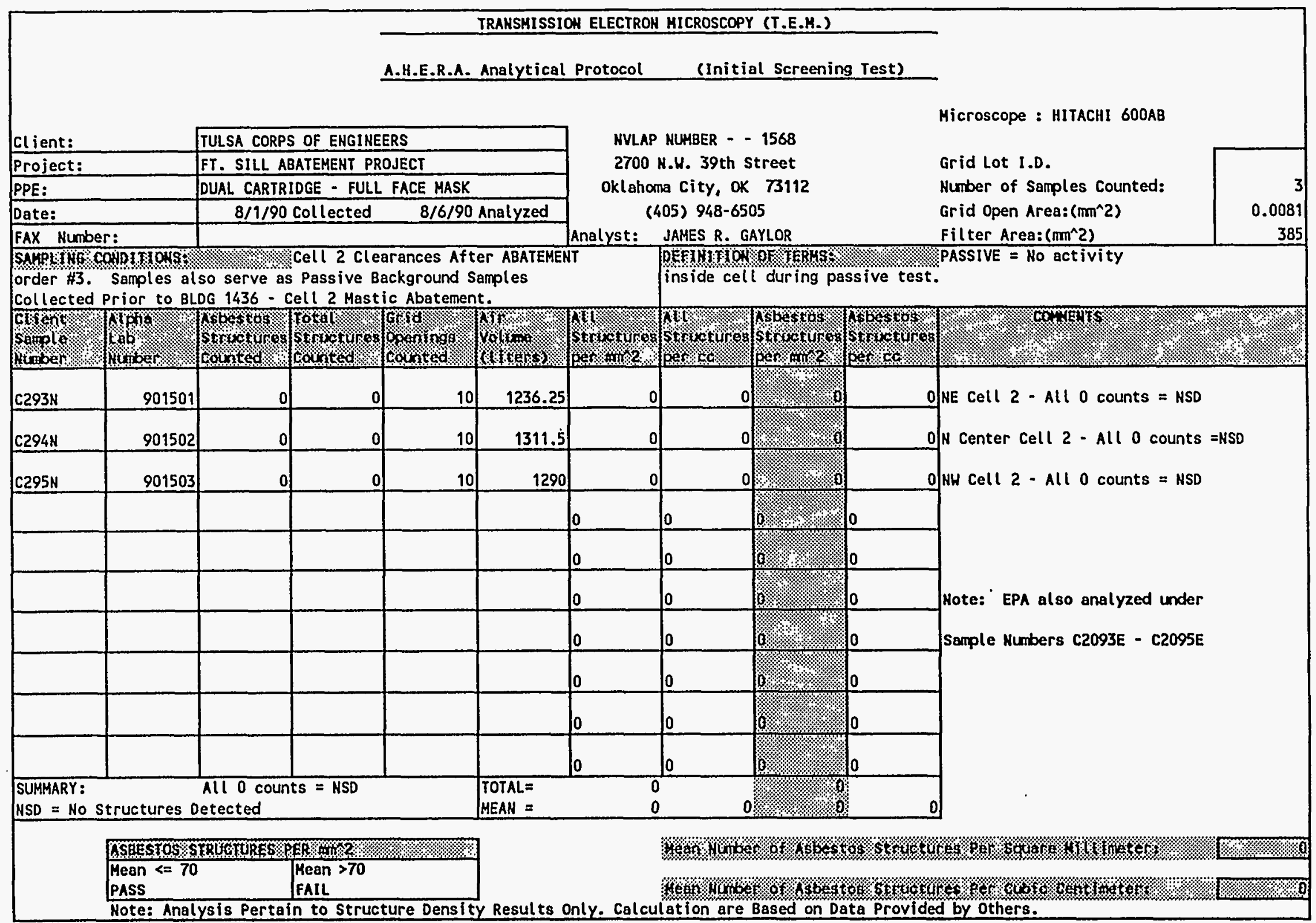




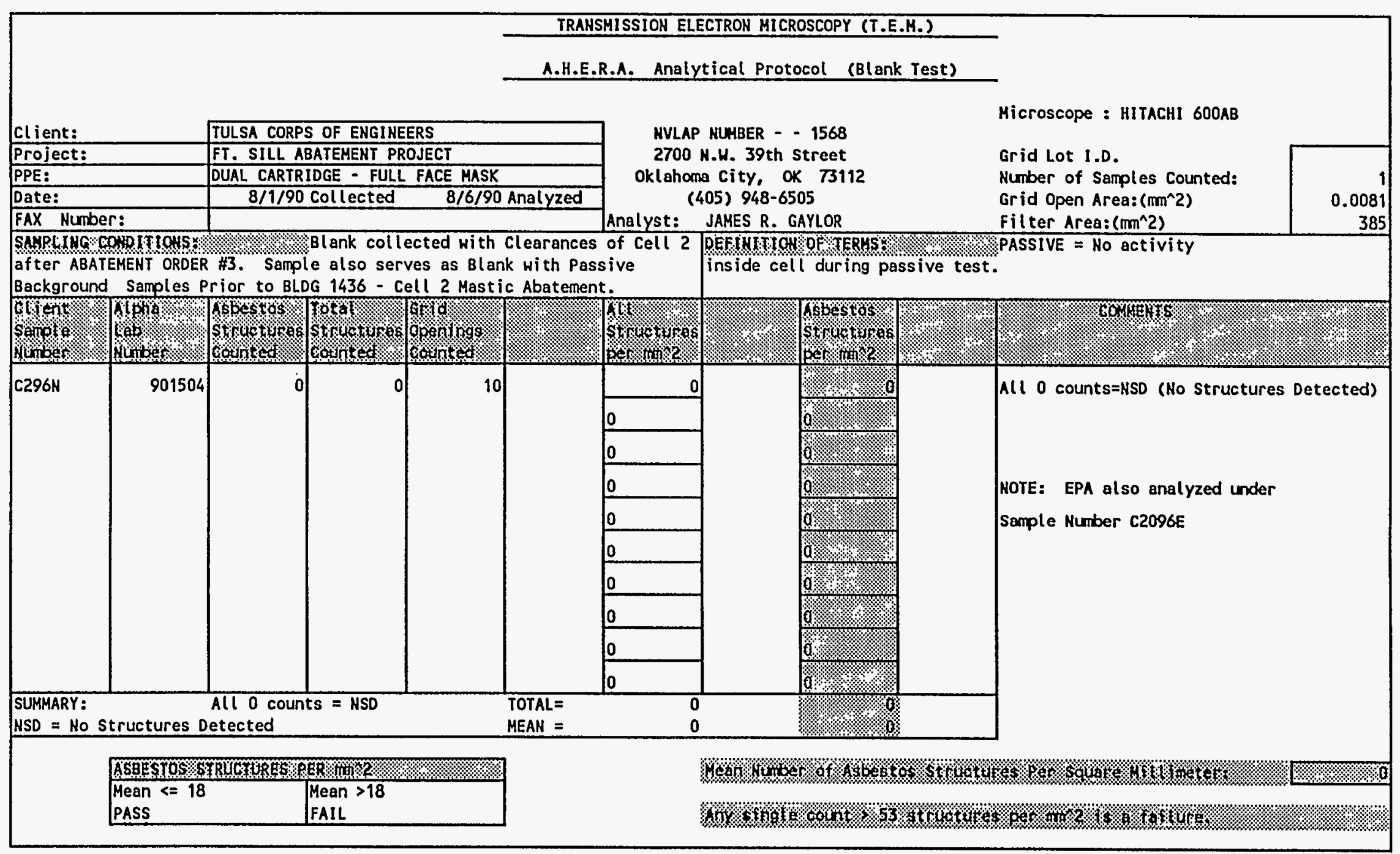




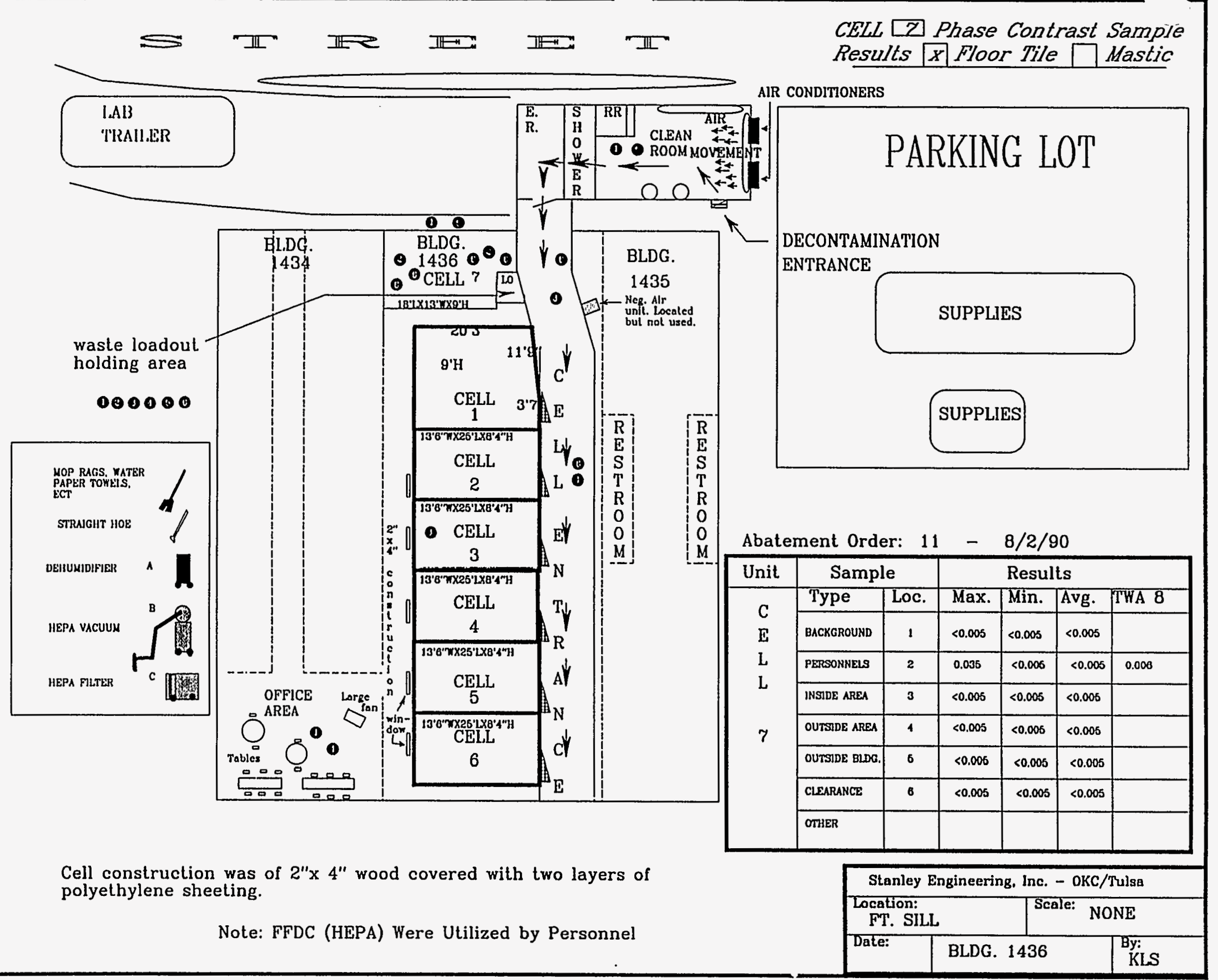




\begin{tabular}{|c|c|c|c|c|c|c|c|c|c|c|}
\hline \multirow[b]{4}{*}{ Client: } & & \multicolumn{7}{|c|}{ TRANSHISSIOH ELECTROH MICROSCOPY (T.E.H.) } & \multirow{3}{*}{\multicolumn{2}{|c|}{ Microscope : HITACHI 600AB }} \\
\hline & & & & \multicolumn{3}{|c|}{ A.H.E.R.A. Analytical Protocol } & \multicolumn{2}{|c|}{ (Initial Screening Test) } & & \\
\hline & & & & & & \multirow{2}{*}{\multicolumn{3}{|c|}{ NVLAP NLMBER - - 1568}} & & \\
\hline & & \multicolumn{4}{|c|}{ TULSA CORPS OF ENGINEERS } & & & & & \\
\hline \multicolumn{2}{|l|}{ Project: } & \multicolumn{4}{|c|}{ FT. SILL ABATEMENT PROJECT } & \multirow{3}{*}{\multicolumn{2}{|c|}{$\begin{array}{l}2700 \text { N.H. 39th Street } \\
\text { Oklahoma City, OK } 73112 \\
\text { (405) } 948-6505\end{array}$}} & & \multirow{4}{*}{$\begin{array}{l}\text { Grid Lot } 1 . D \text {. } \\
\text { Number of Samples Counted: } \\
\text { Grid Open Area: }\left(m^{\wedge} 2\right) \\
\text { Filter Area: }\left(m^{\wedge} 2\right)\end{array}$} & \\
\hline & \multicolumn{4}{|c|}{ DUAL CARTRIDGE - FULL FACE MASK } & & & & & \\
\hline & \multirow{2}{*}{\multicolumn{2}{|c|}{$8 / 2 / 90$ Collected }} & \multicolumn{2}{|c|}{$8 / 6 / 90$ Analyzed } & \multirow{2}{*}{\multicolumn{3}{|c|}{$\begin{array}{ll} & (405) 948-6505 \\
\text { Analyst: } & \text { JAMES R. GAYLOR }\end{array}$}} & & 0.0081 \\
\hline \multicolumn{2}{|c|}{ FAX Number: } & & & & & & & & & 385 \\
\hline \multicolumn{2}{|c|}{ 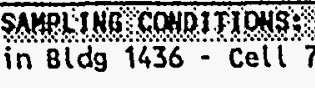 } & 7 prior to a & $\begin{array}{l}\text { Passive ba } \\
\text { batement. }\end{array}$ & ckground sar & imples colle & ected & 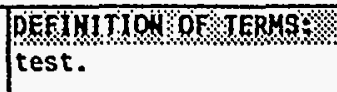 & $\sqrt{1} \times$ & PASSIVE = No activity inside cel & during \\
\hline Stfert & Kiturbert & 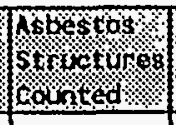 & Sotal & Foring & Wro, & 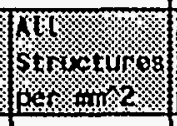 & 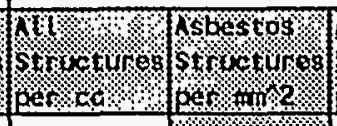 & S & 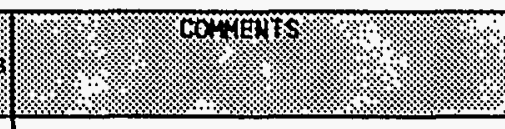 & ?. \\
\hline C7109N & 901525 & 0 & $\underline{0}$ & 10 & 1557.6 & 요 & 0 & & 8 ldg 1436 - South East Cell 7 & \\
\hline C7110N & 901526 & 0 & $\underline{0}$ & 8 & 1557.6 & 0 & 0,8 & & Bldg 1436 - South Center Cell 7 & \\
\hline$C 7111 \mathrm{~N}$ & 901527 & 0 & $\underline{0}$ & 8 & 1584 & 0 & of: & & Bldg 1436 - North East Cell 7 & \\
\hline & & & & & & 0 & 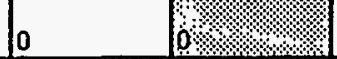 & 0 & & \\
\hline & & & & & & 0 & $\sqrt[6]{6}, 1.2 .8$. & 0 & & \\
\hline & & & & & & o & $\sqrt{6.1 \%}$ & 0 & & \\
\hline & & & & & & 0 & 6. & 0 & HOTE: EPA also analyzed under & \\
\hline & & & & & & 0 & (1. & 0 & Sample Number C7109E - C7111E & \\
\hline & & & & & & 0 & (6) & 0 & & \\
\hline & & & & & & 0 & 1.1.:. & 0 & & \\
\hline $\begin{array}{l}\text { SUMMARY: } \\
\text { NSD = NO S } \\
\end{array}$ & Structures D & $\begin{array}{l}\text { All } 0 \text { count } \\
\text { Detected }\end{array}$ & ts = HSD & & $\begin{array}{l}\text { TOTAL }= \\
\text { MEAN }=\end{array}$ & $\begin{array}{l}0 \\
0\end{array}$ & 0 & & & \\
\hline & ASGESTOSS & SYRUGYORES & EP & $\sqrt[1]{1}$ & & & 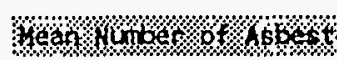 & $40 \% 5 \% 100$ & 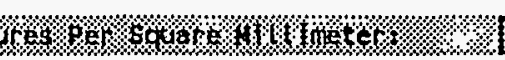 & \\
\hline & $\begin{array}{l}\text { Mean }<=70 \\
\text { PASS }\end{array}$ & 0 & $\begin{array}{l}\text { Hean }>70 \\
\text { FAIL }\end{array}$ & & & & 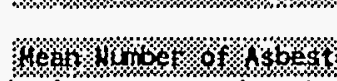 & $15.6 \%$ & U⿰冫欠 & \\
\hline
\end{tabular}




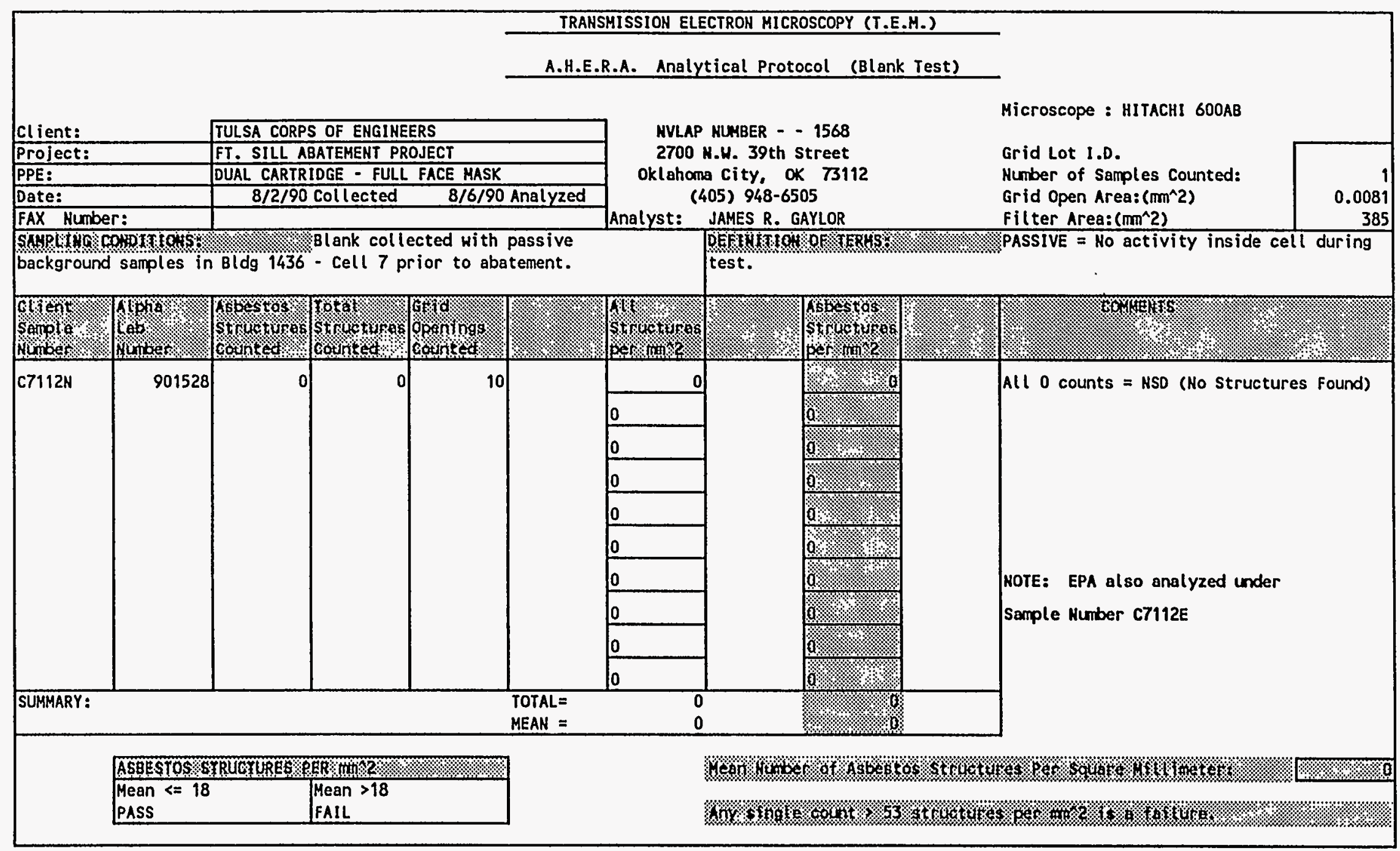




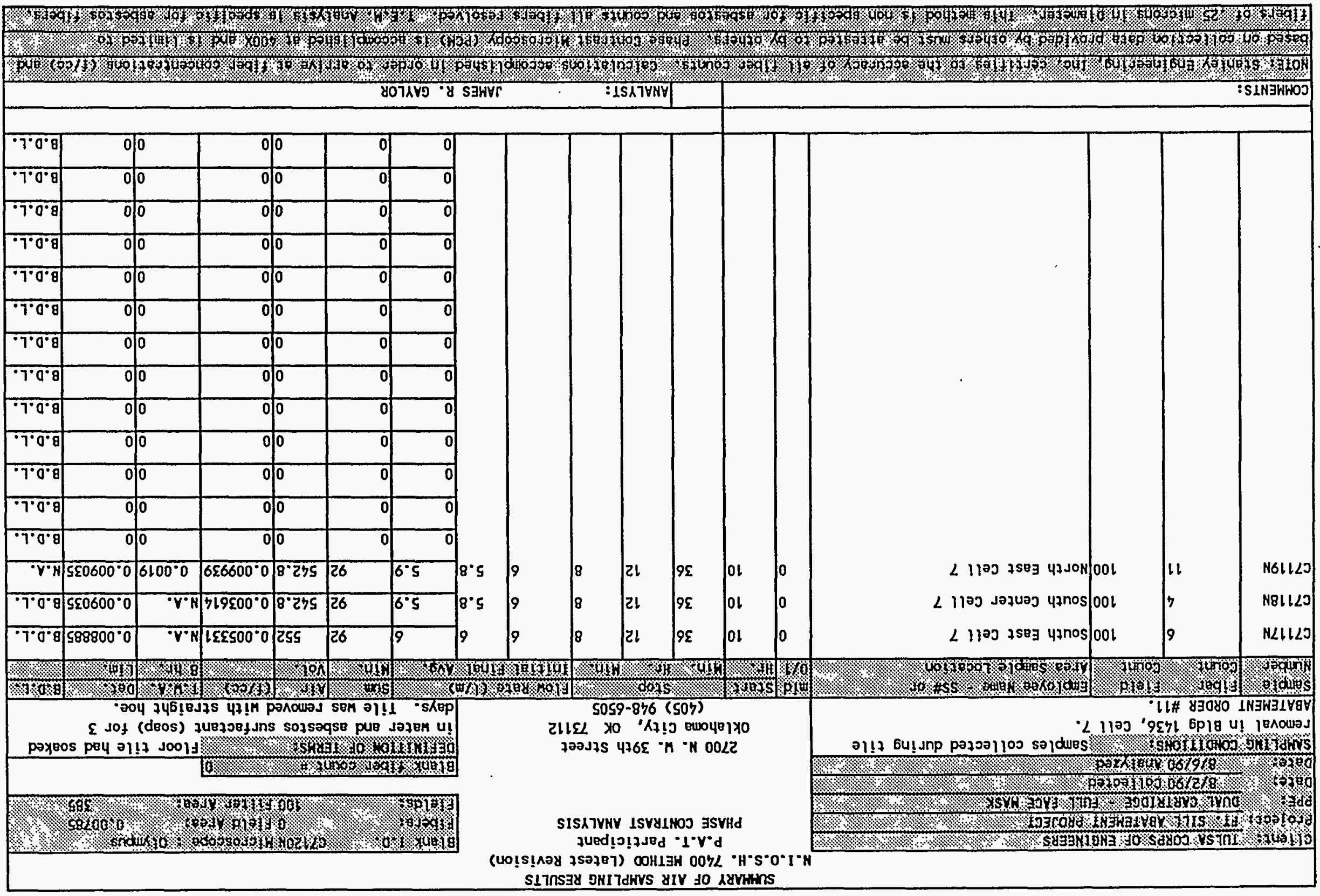

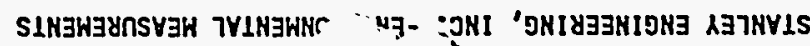




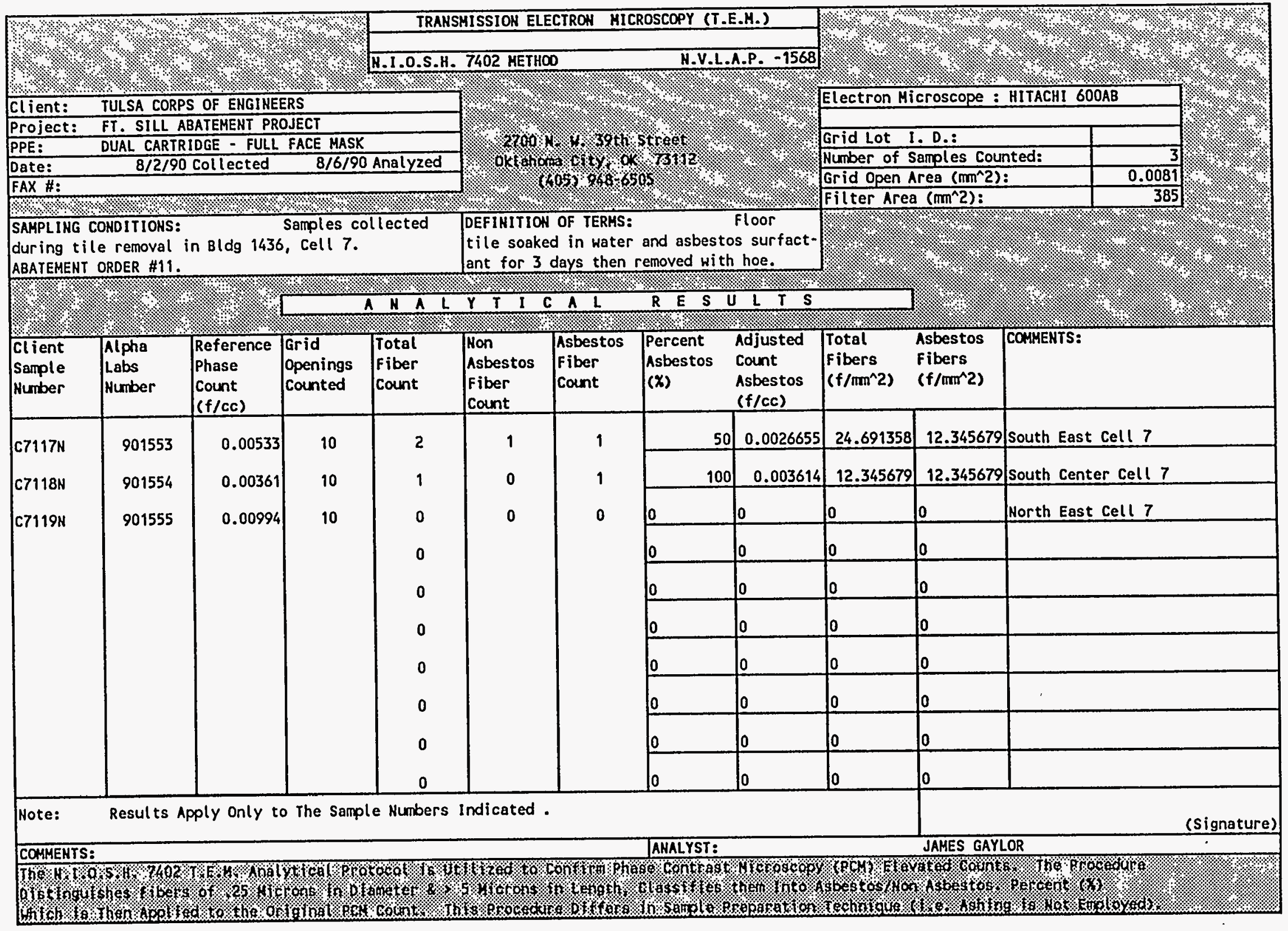




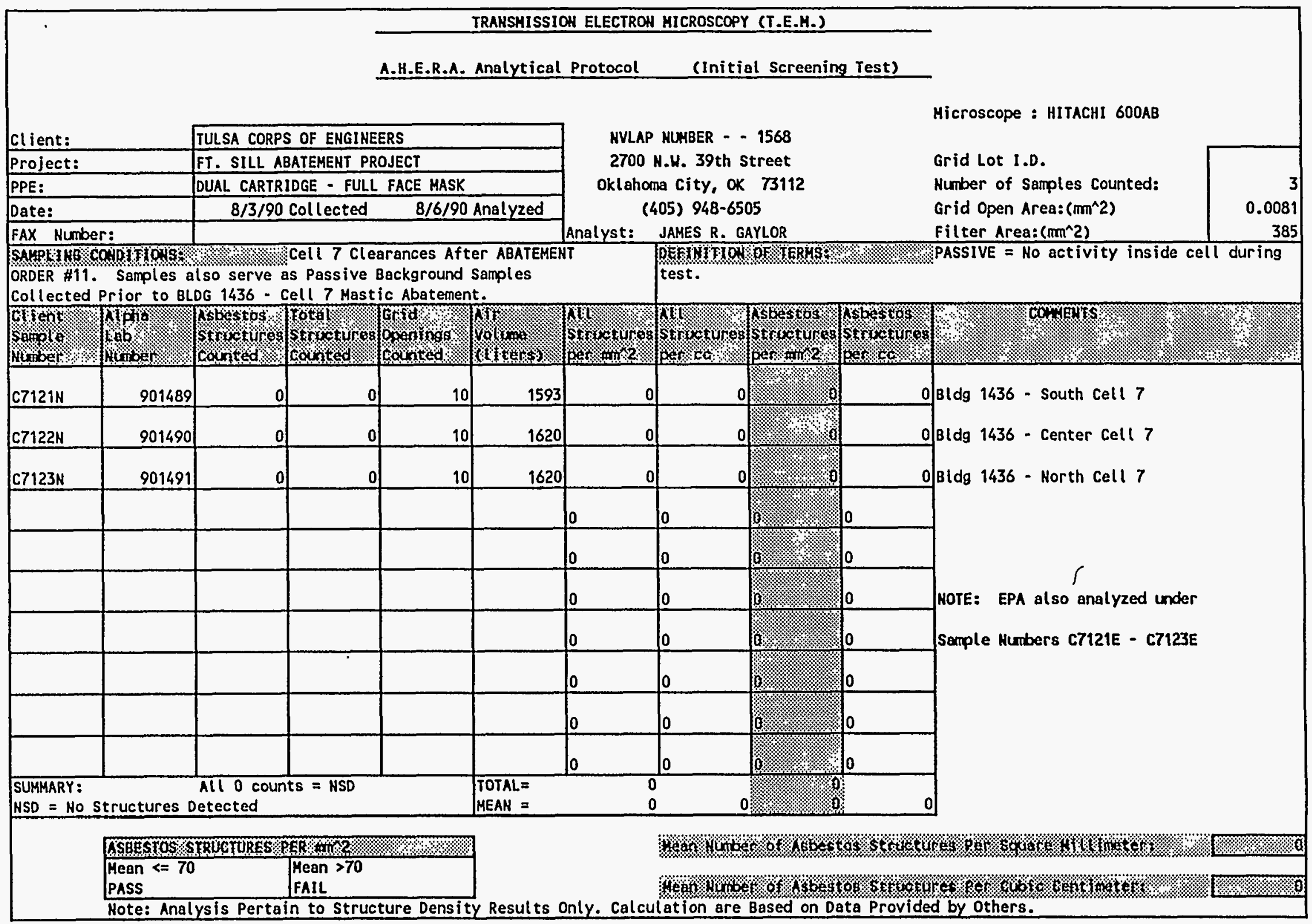




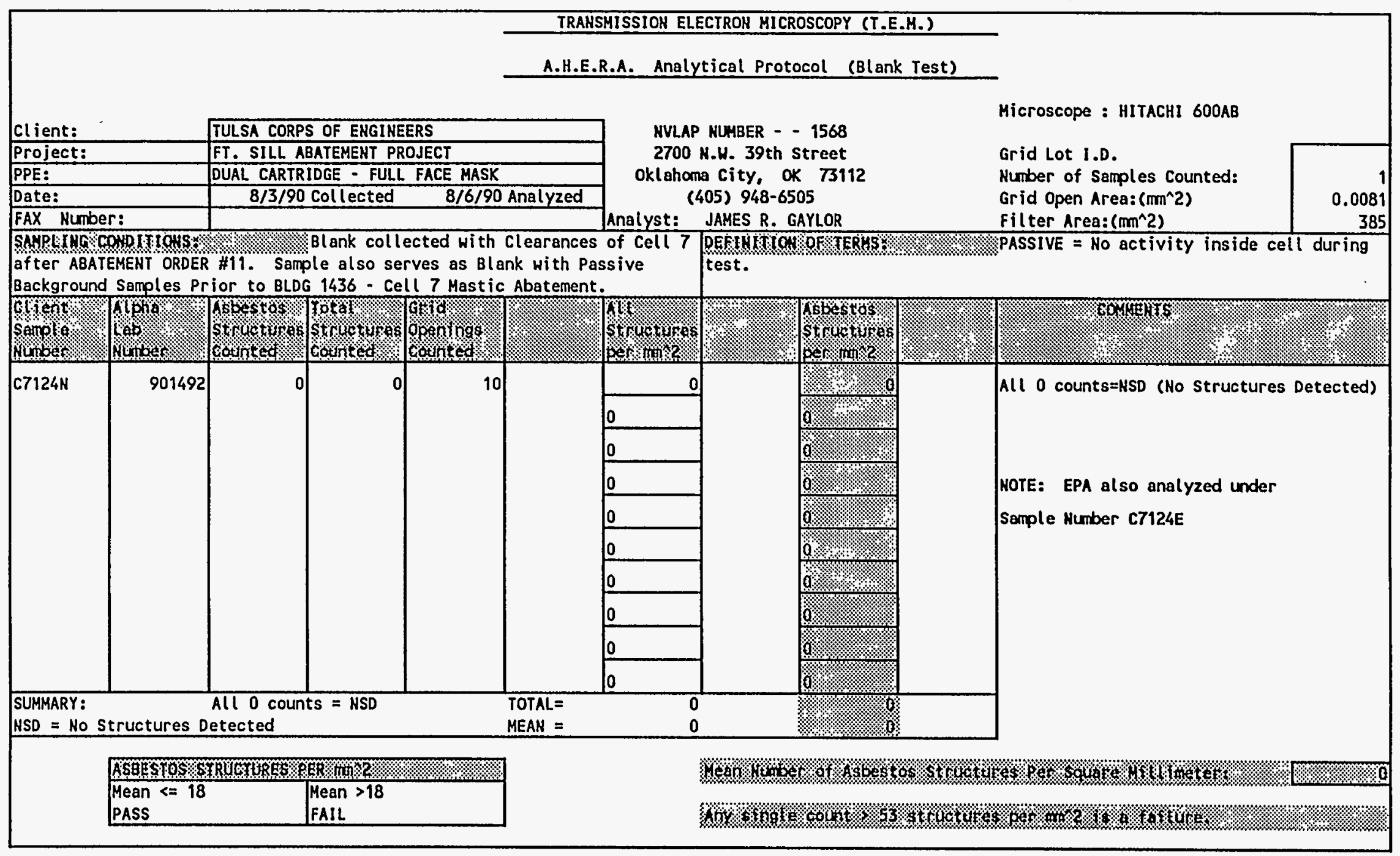




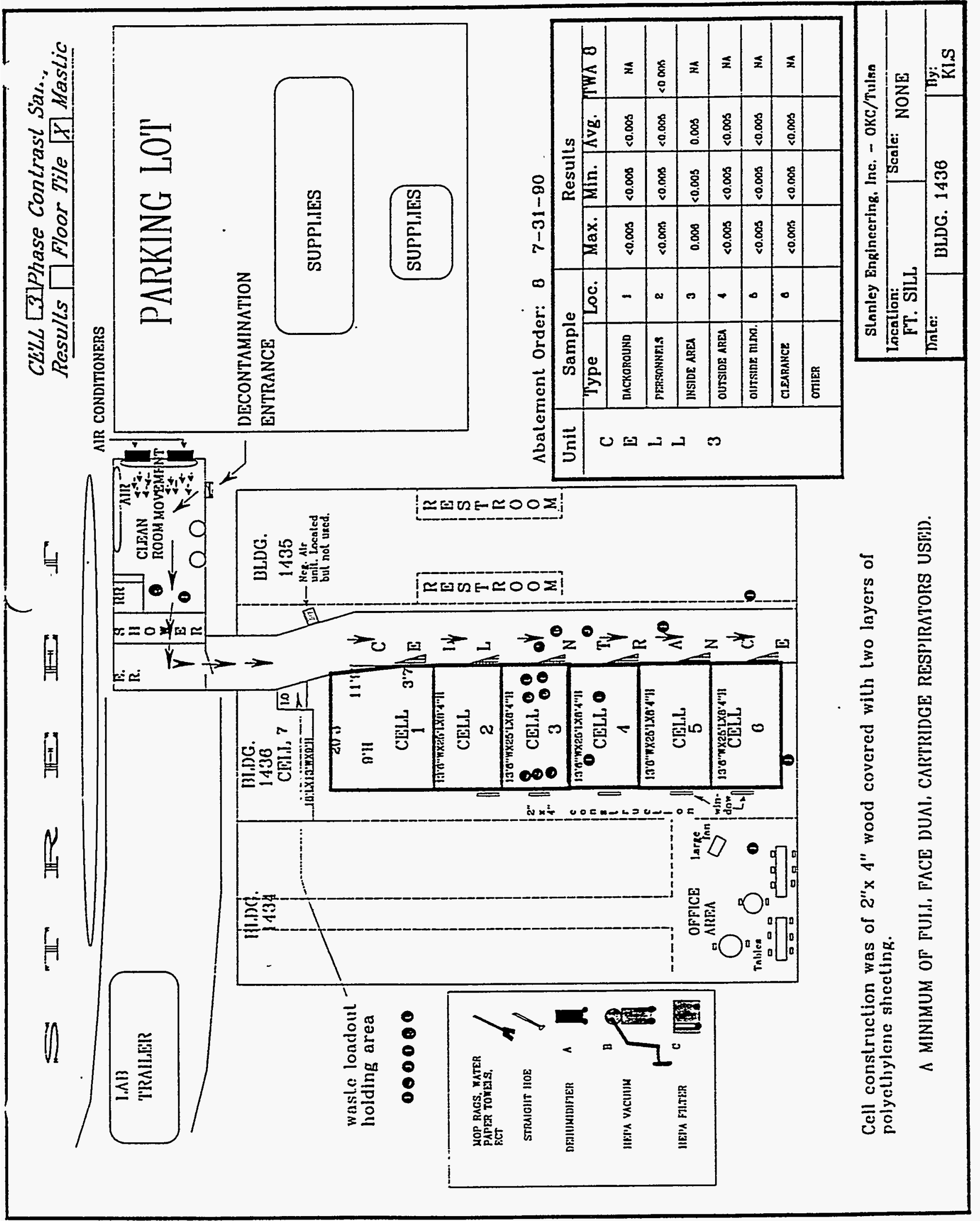


TRAHSHISSIOH ELECTRON MICROSCOPY (T.E.H.)

A.H.E.R.A. Analytical Protocol (Initial Screening Test)

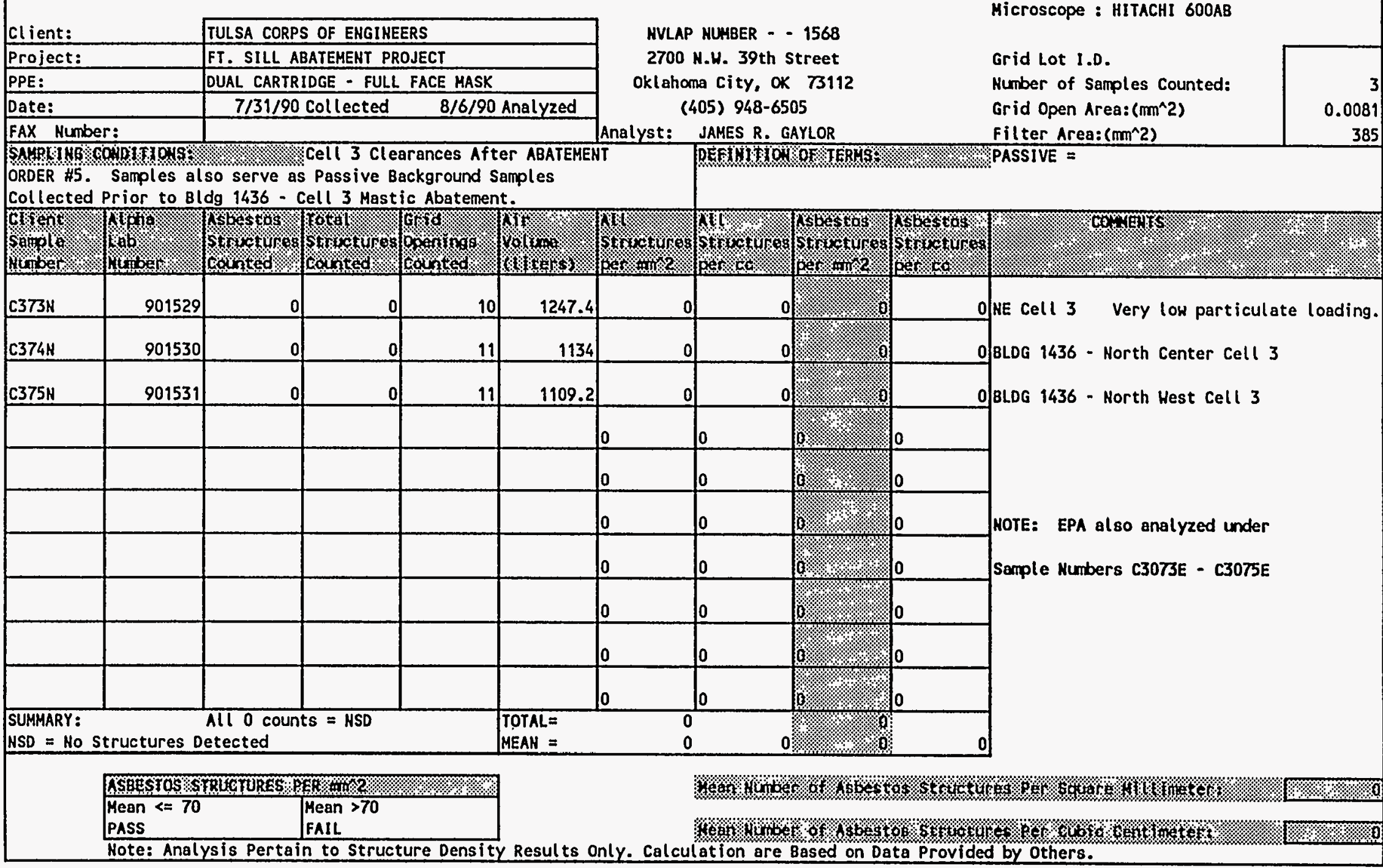




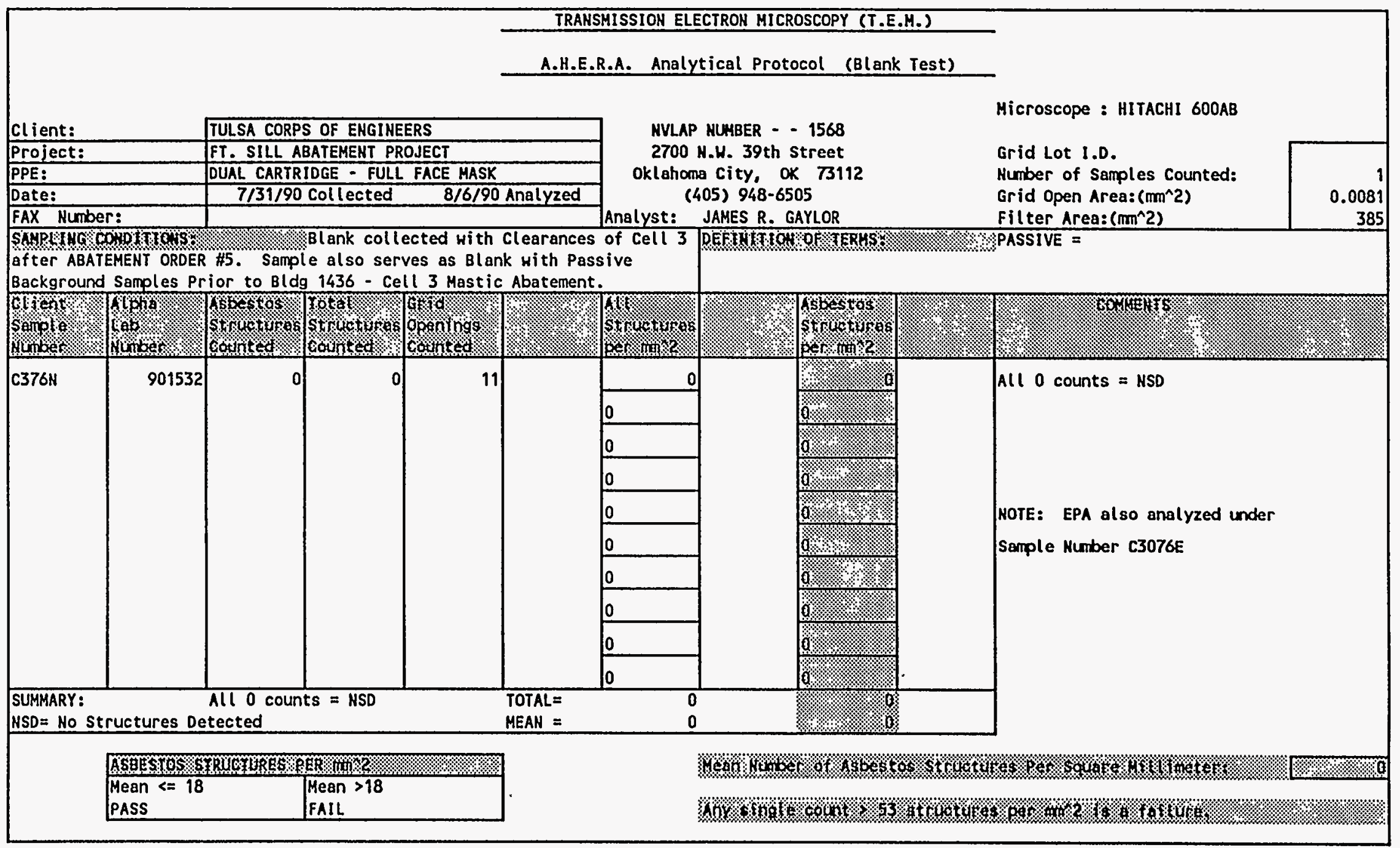




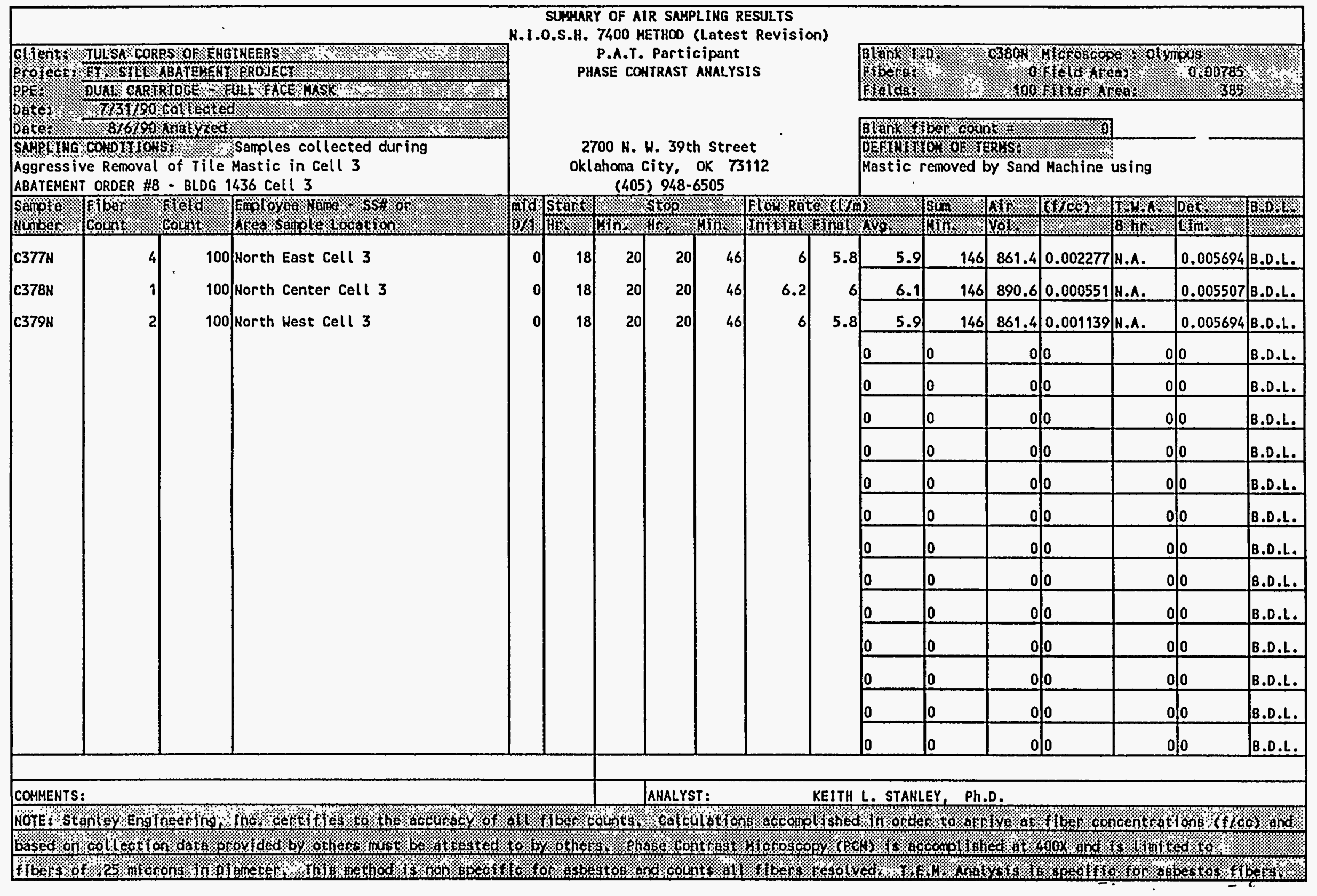




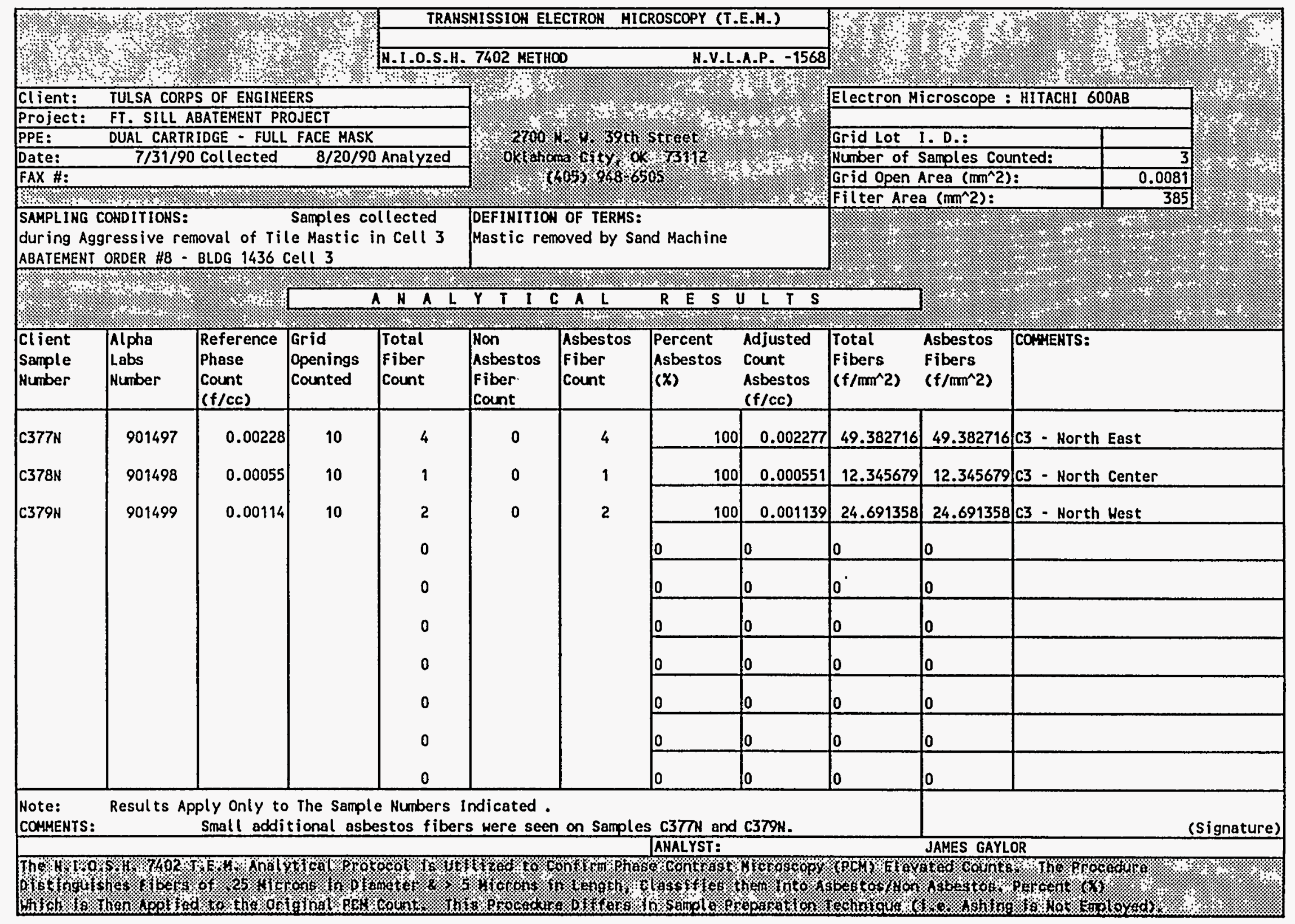




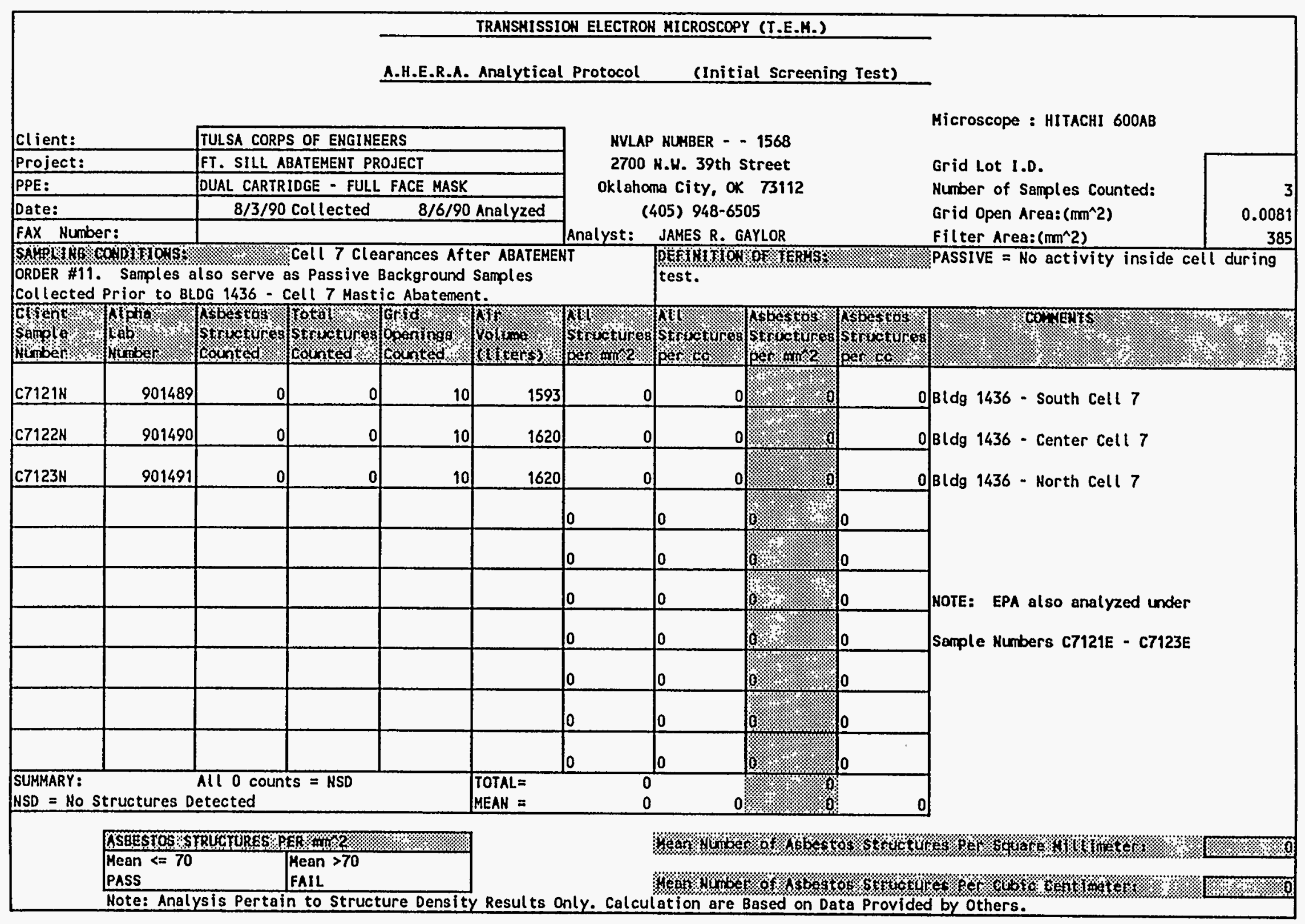




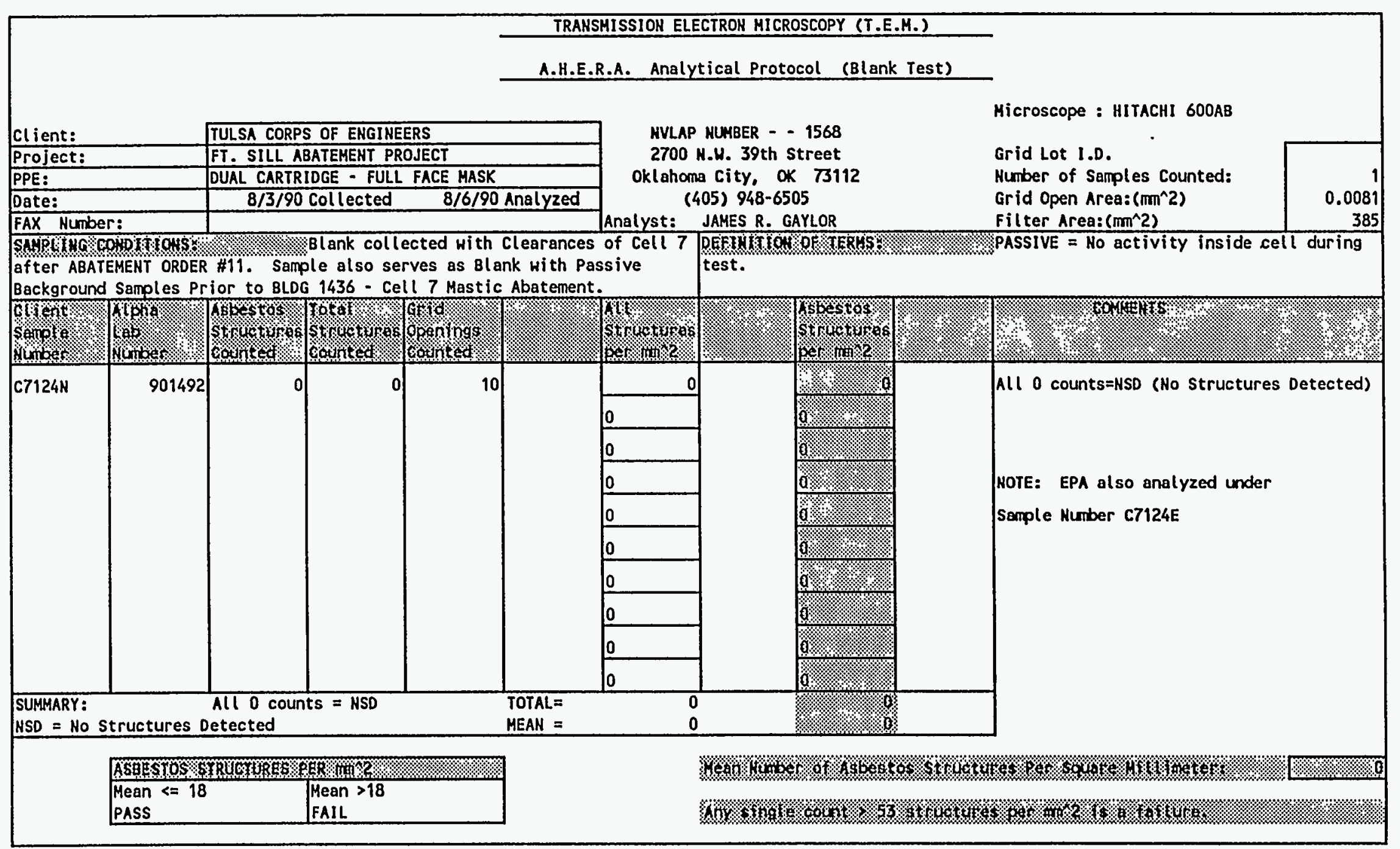




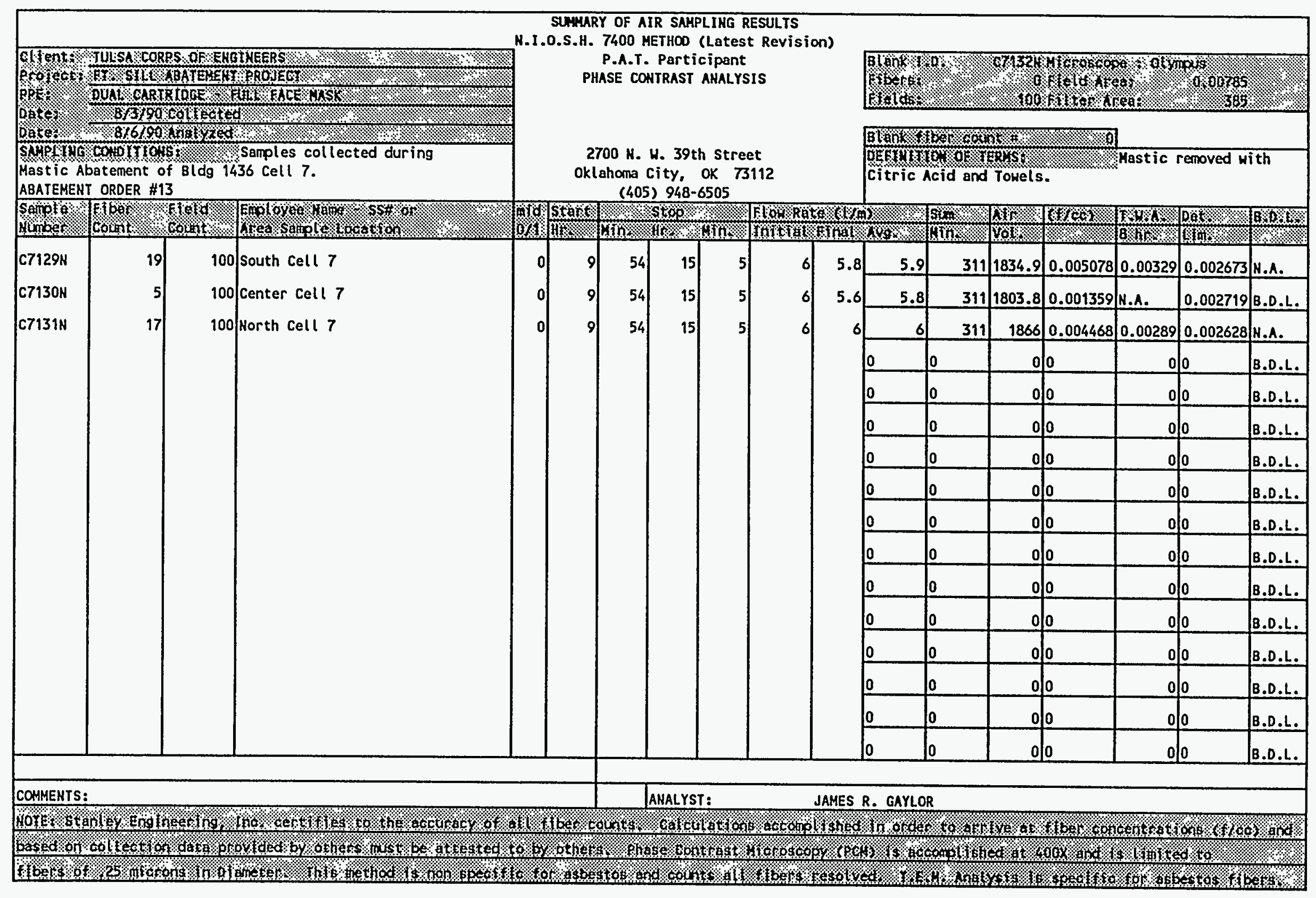


11

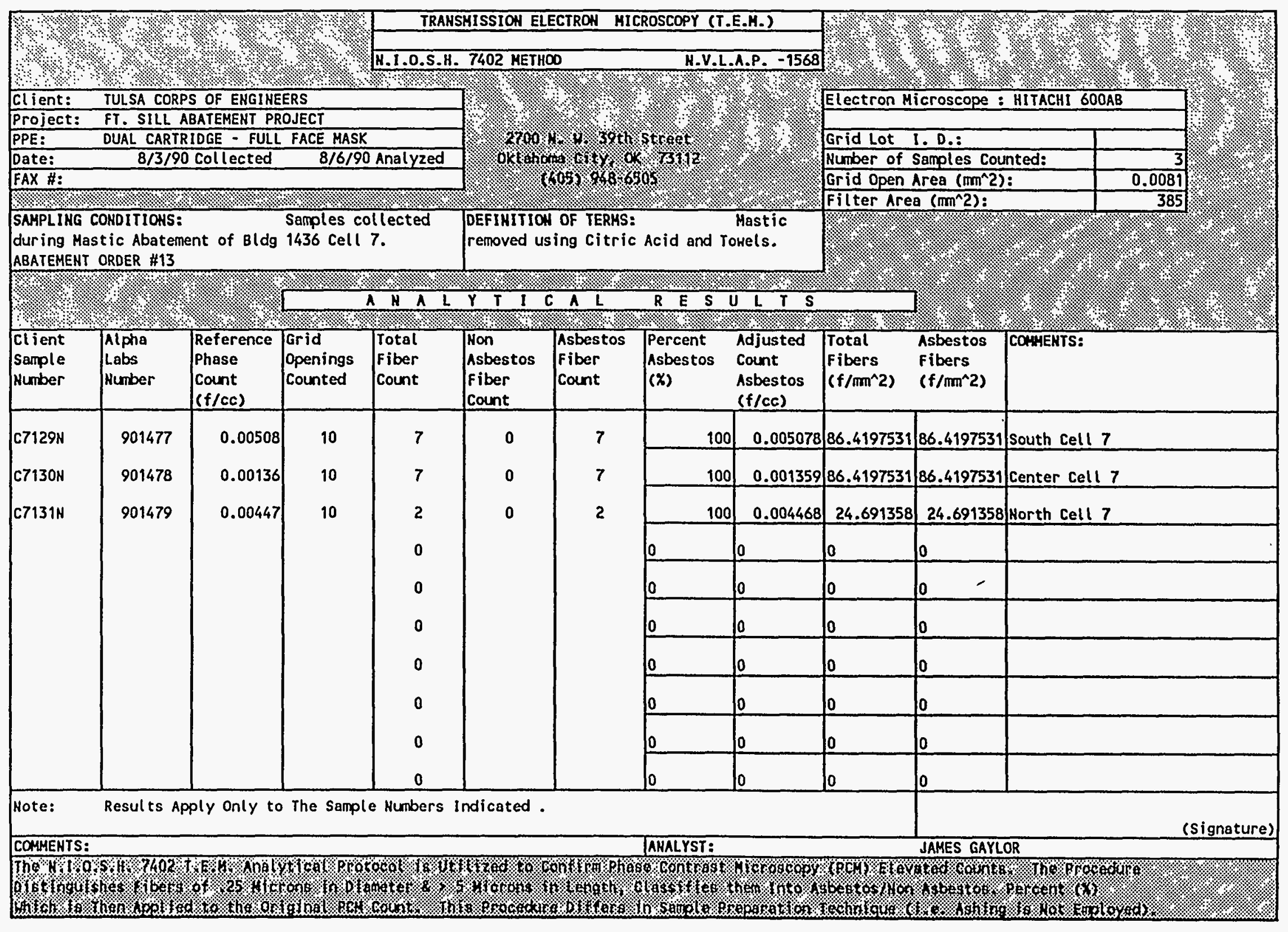




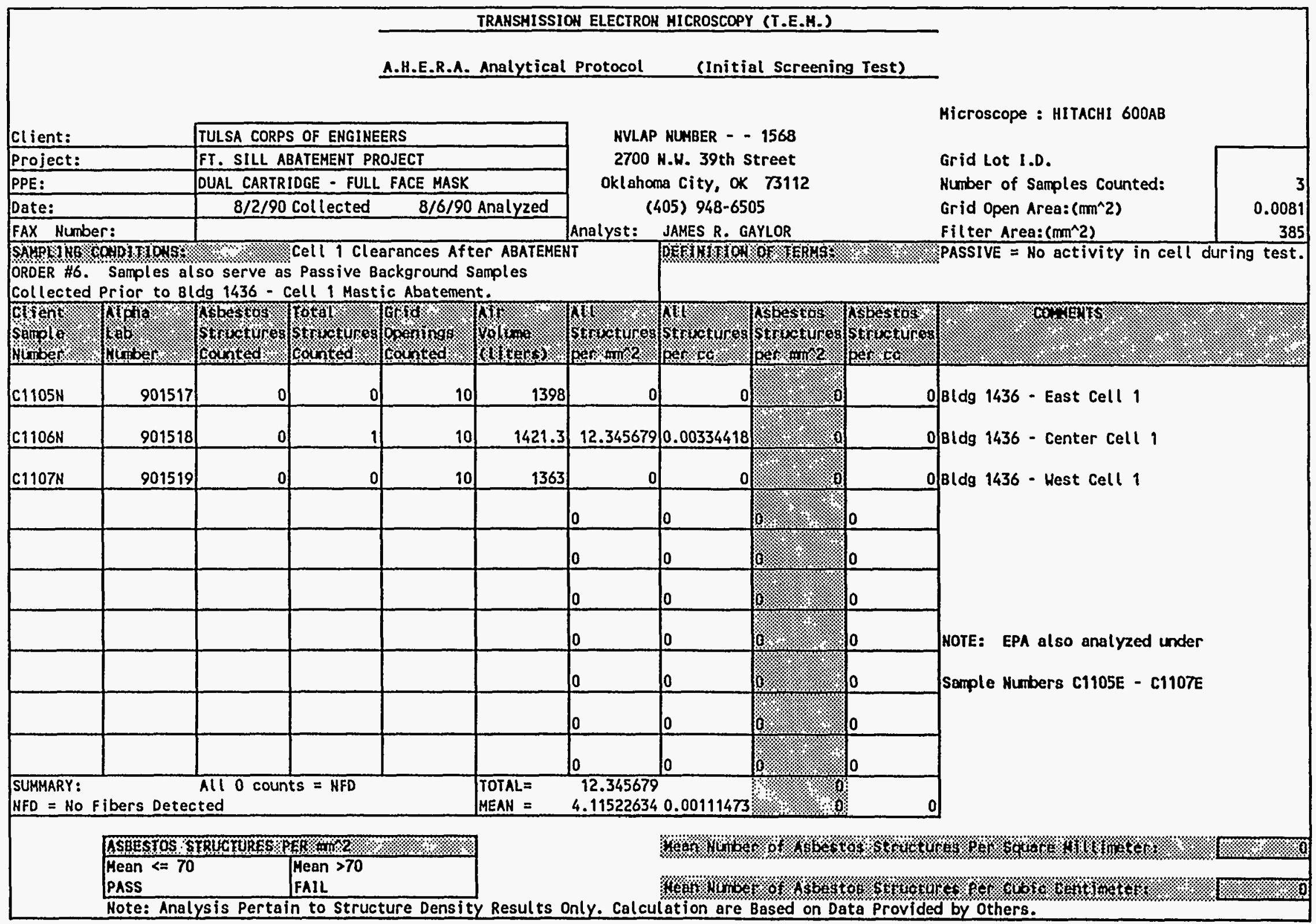




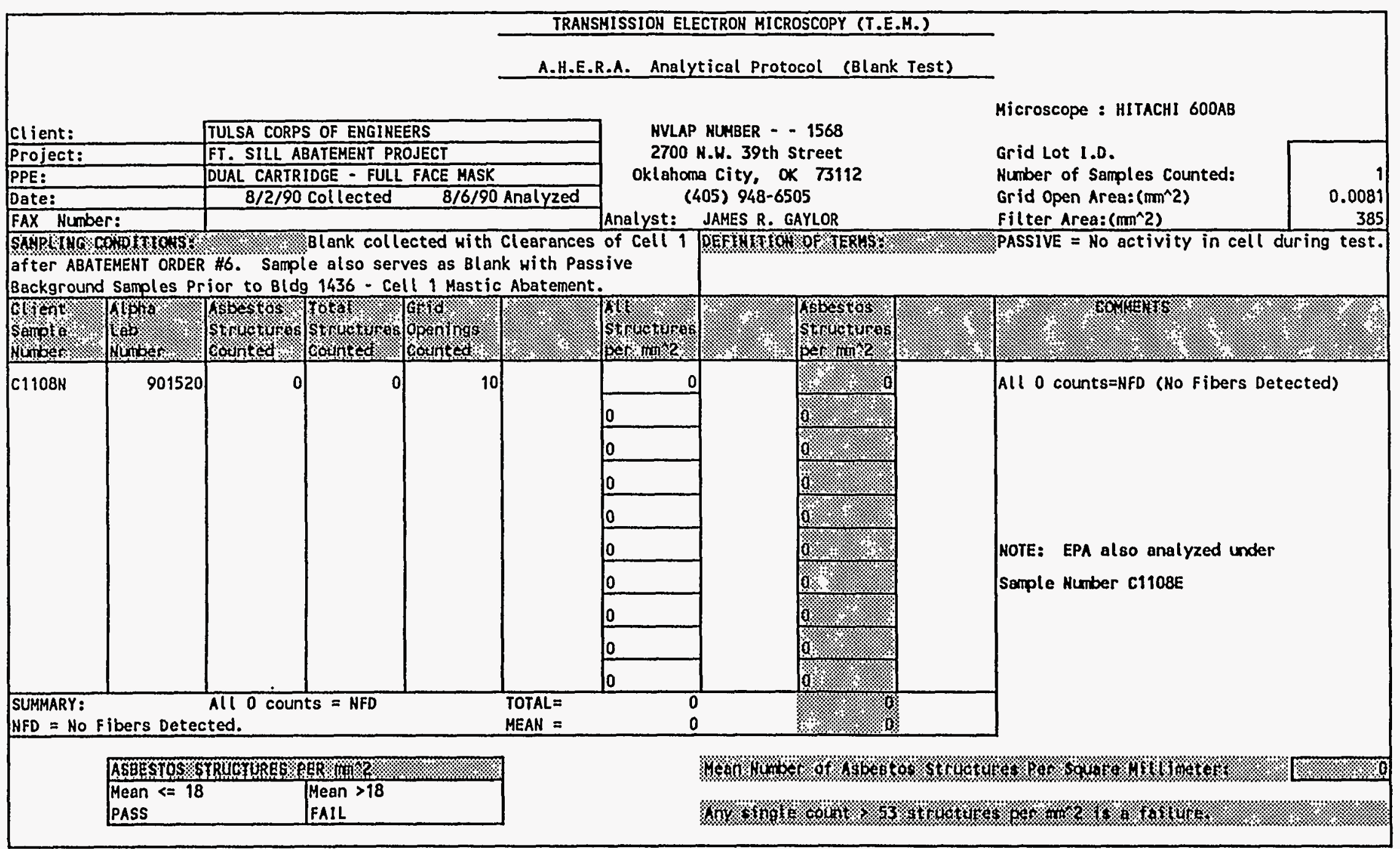




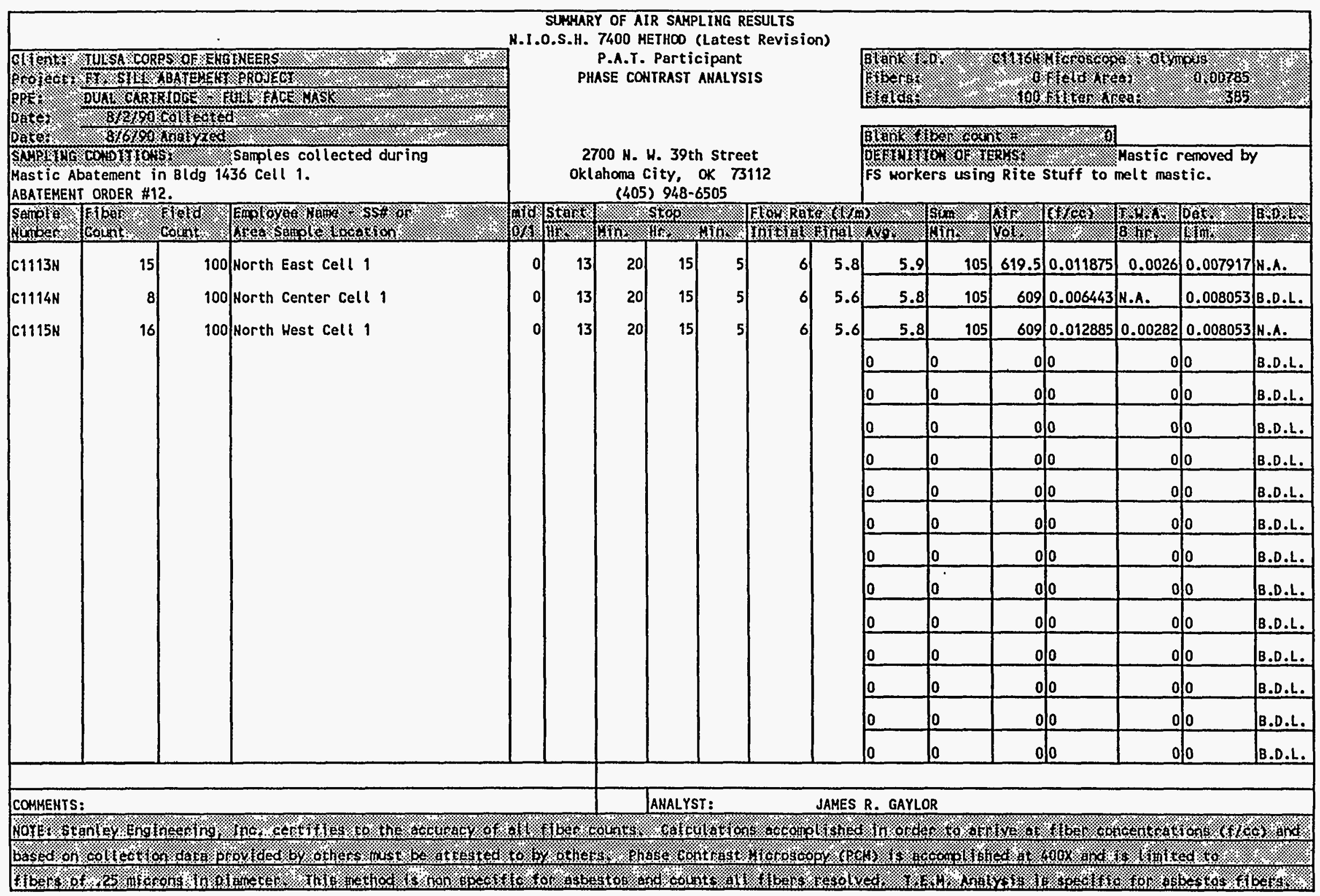




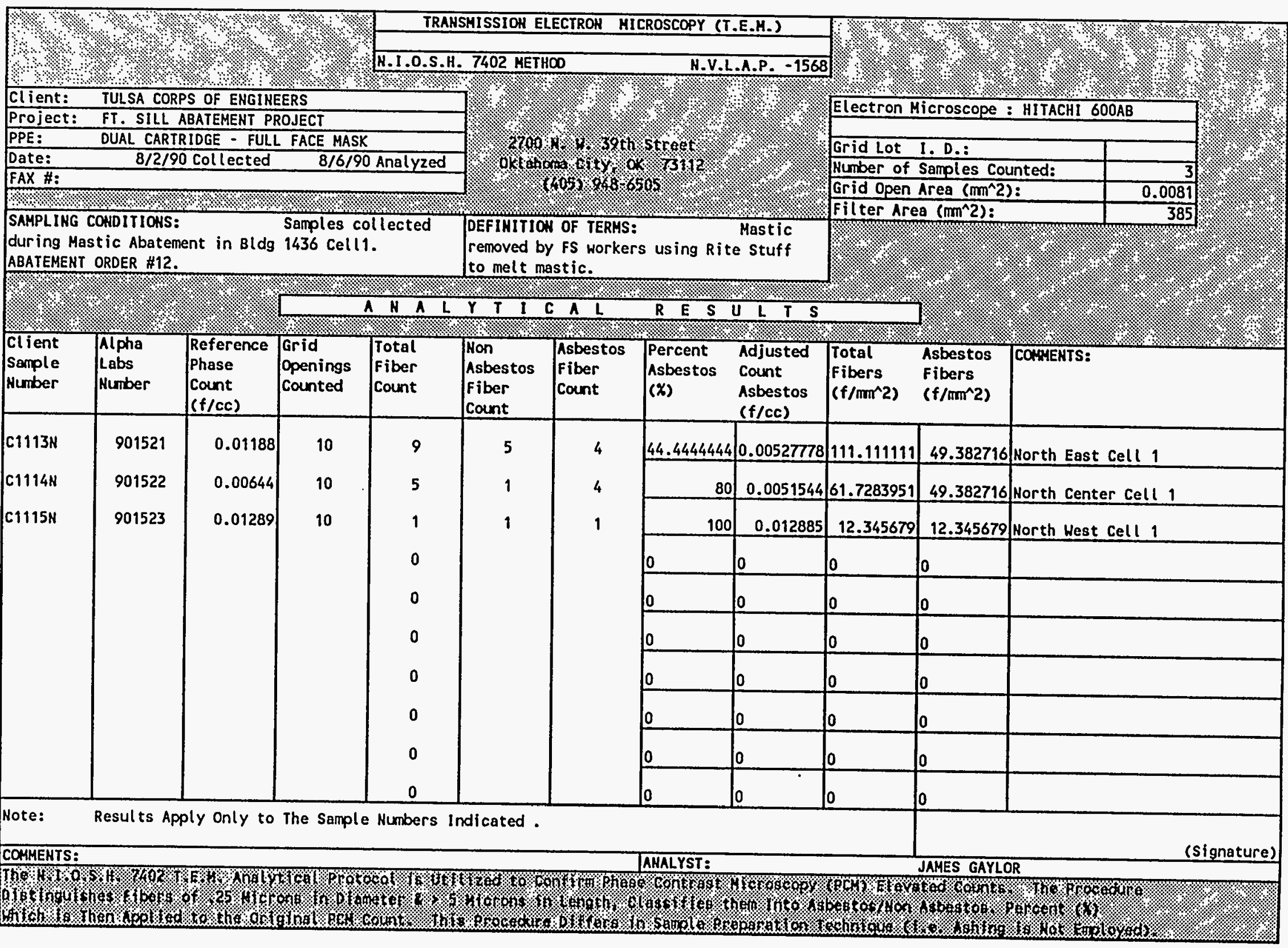


TRANSHISSIOH ELECTROH HICROSCOPY (T.E.M.)

\section{A.H.E.R.A. Analytical Protocol (Initial Screening Test)}

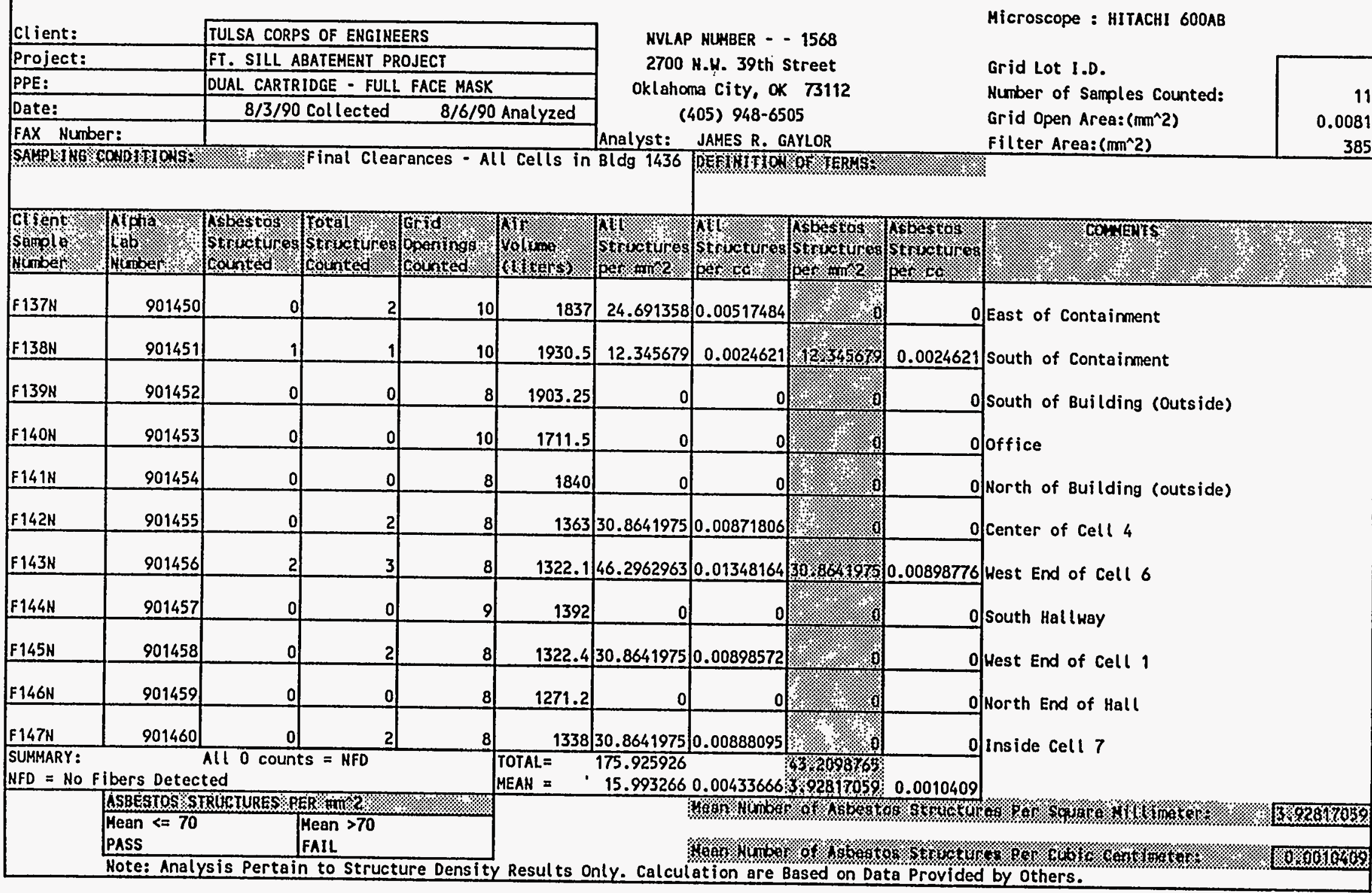




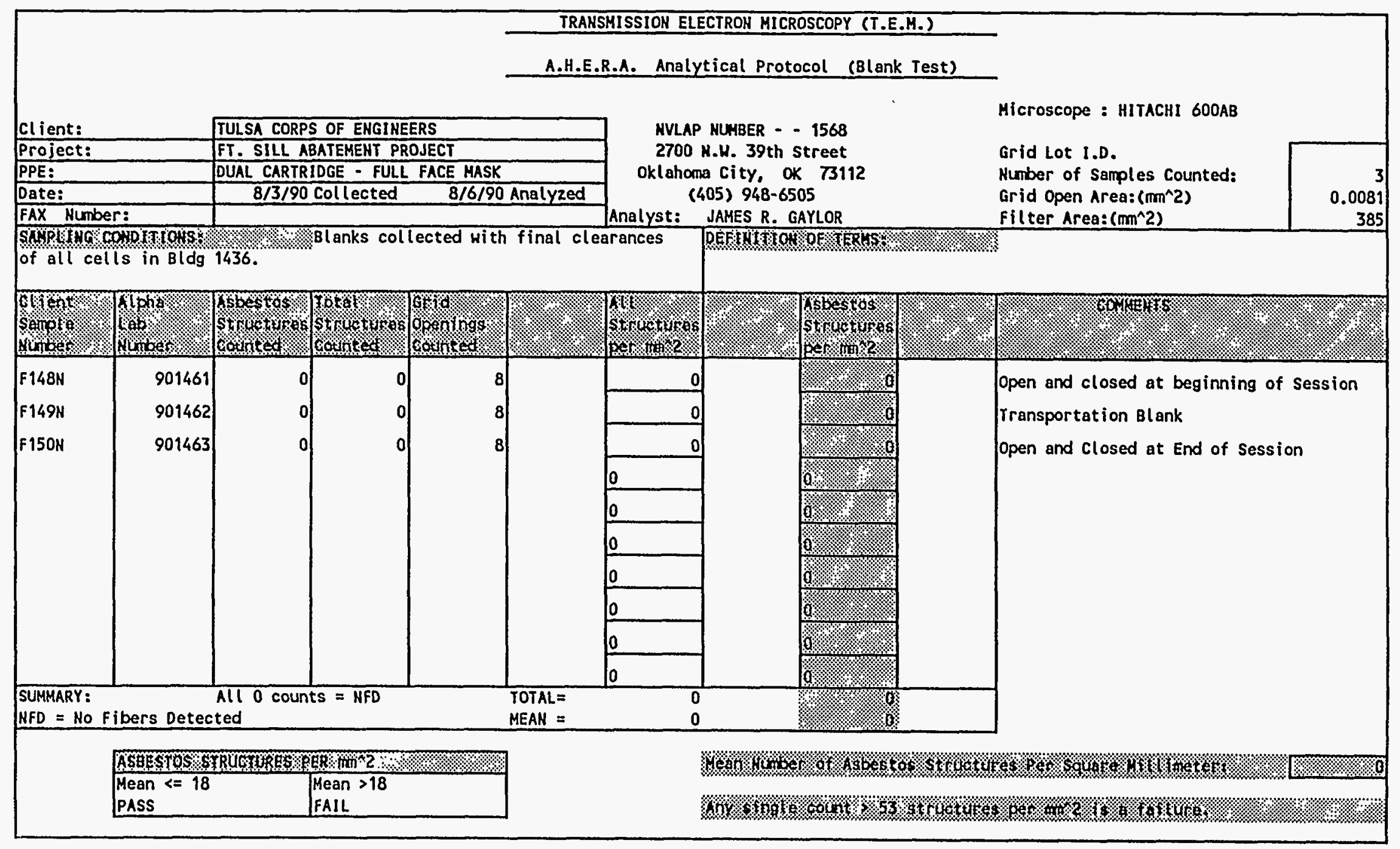




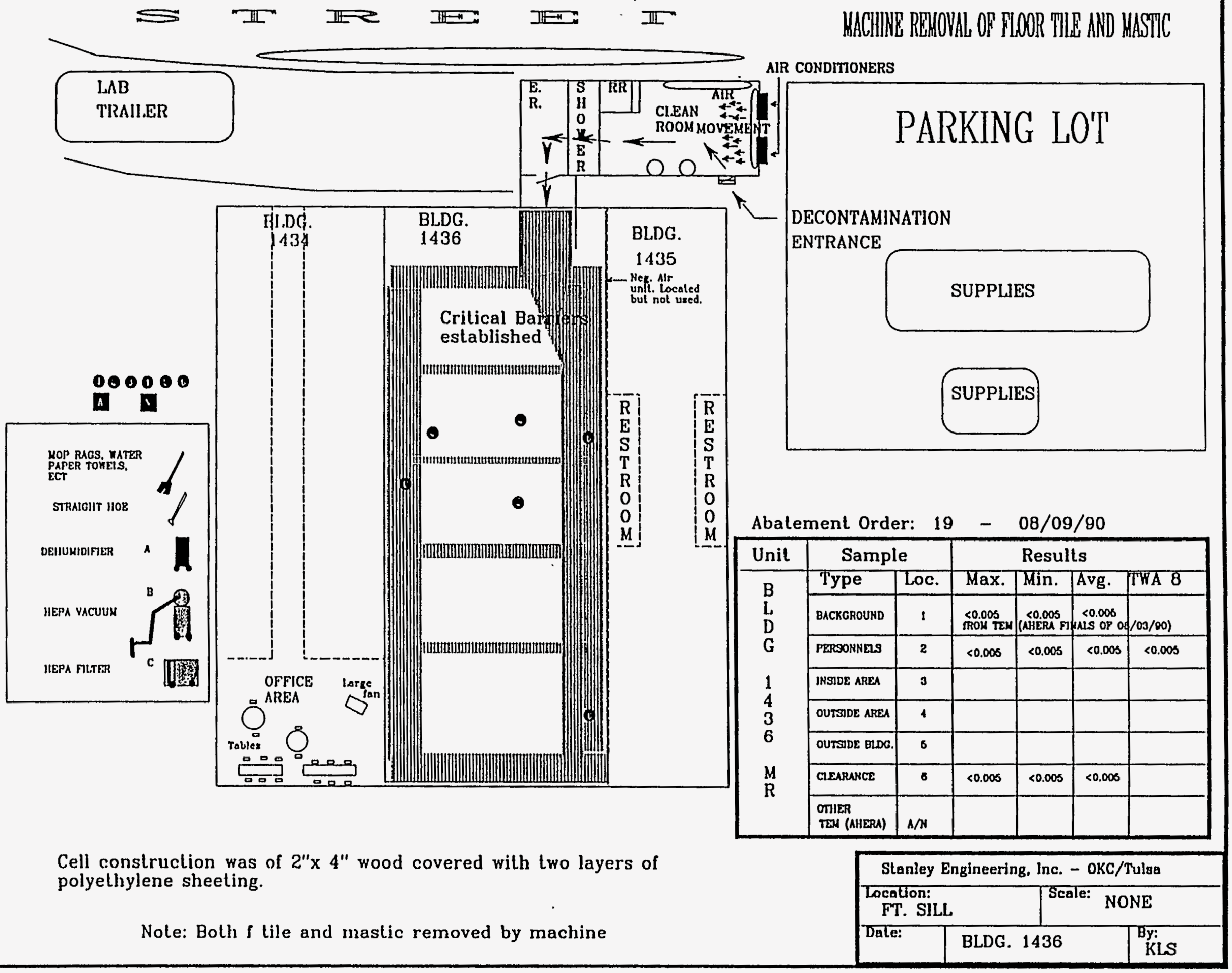




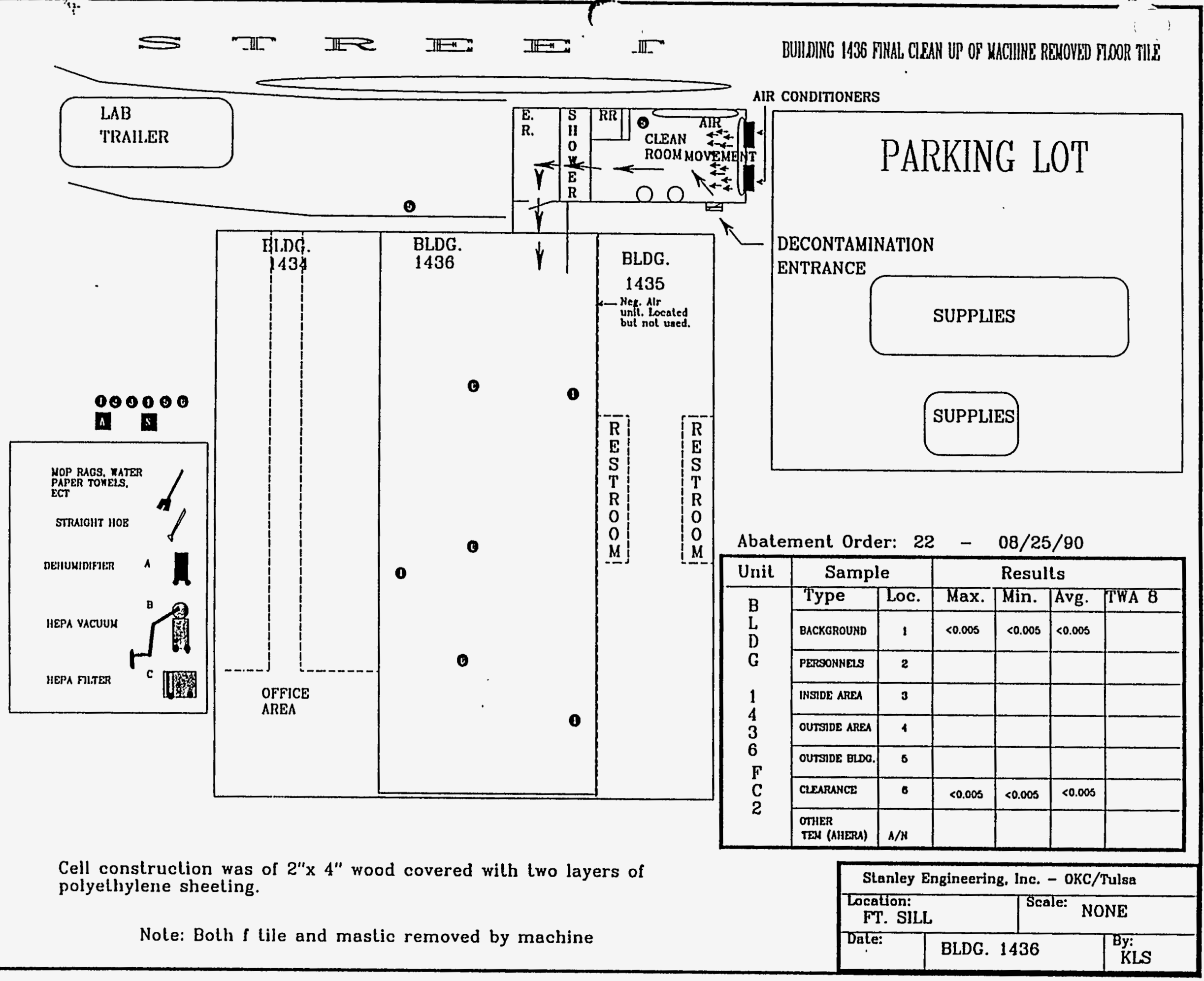




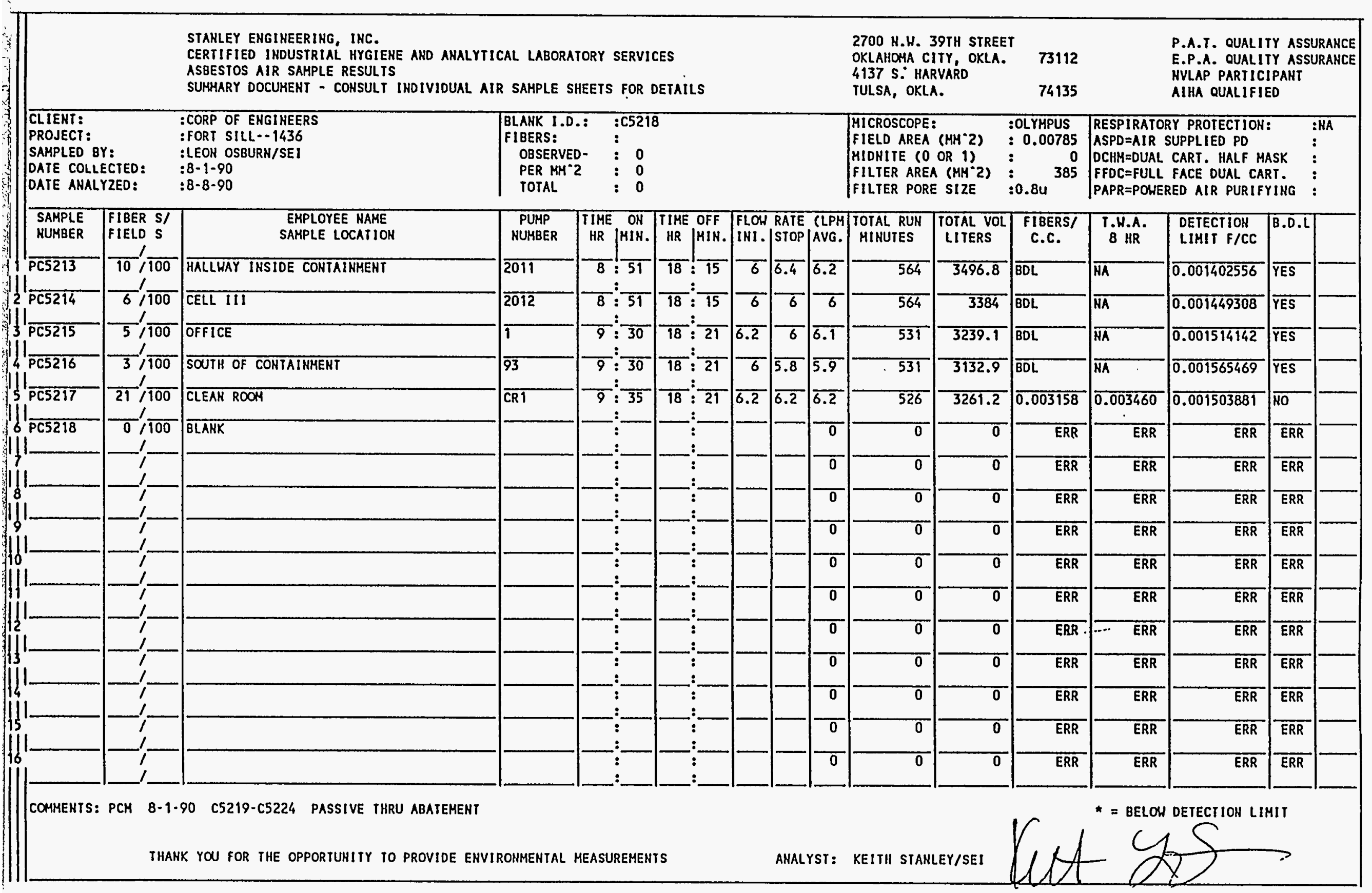




\begin{tabular}{|c|c|c|c|c|c|c|c|c|c|c|c|c|c|c|c|}
\hline & & \multicolumn{7}{|c|}{$\begin{array}{l}\text { STANLEY ENGINEERING, INC. } \\
\text { CERTIFIED INDUSTRIAL HYGIENE AND ANALYTICAL LABORATORY SERVICES } \\
\text { ASESTOS AIR SAMPLE RESUTS } \\
\text { SUMMARY DOCUMENT - CONSULT INDIVIDUAL AIR SAMPLE SHEETS FOR DETAILS }\end{array}$} & \multicolumn{2}{|c|}{$\begin{array}{l}\text { 2700 H.H. 39TH STREET } \\
\text { OKLAHOMA CITY, OKLA. } \\
4137 \text { S. HARVARD } \\
\text { TULSA, OKLA. }\end{array}$} & 73112 & & \multicolumn{3}{|c|}{$\begin{array}{l}\text { P.A.T. QUALITY ASSURANCE } \\
\text { E.P.A. QUALITY ASSURANCE } \\
\text { HVLAP PARTICIPANT } \\
\text { AIHA QUALIFIED }\end{array}$} \\
\hline \multicolumn{2}{|c|}{$\begin{array}{l}\text { CLIENT: } \\
\text { PROJECT: } \\
\text { SAMPLED BY: } \\
\text { DATE COLLECTED: } \\
\text { DATE ANALYZED: }\end{array}$} & \multirow{2}{*}{ 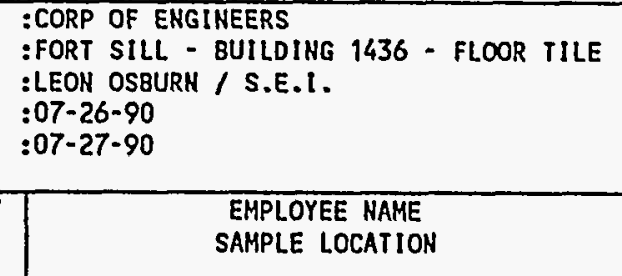 } & \multicolumn{3}{|c|}{\begin{tabular}{|ll} 
BLANK I.D.: & $:$ C4075 \\
FIBERS: & $\vdots$ \\
OBSERVED- & $\vdots 0$ \\
PER MM-2 & $\vdots 0$ \\
TOTAL & $: 0$
\end{tabular}} & & & & \multicolumn{2}{|c|}{$\begin{array}{l}\text { MICROSCOPE: } \\
\text { FIELD AREA (MM-2) } \\
\text { MIDNITE }(0 \text { OR } 1) \\
\text { FILTER AREA (MM־2) } \\
\text { FILTER PORE SIZE }\end{array}$} & $\begin{array}{l}\text { :OLYMPUS } \\
: 0.00785 \\
\vdots \\
: 0.8 u^{\quad 385}\end{array}$ & \multicolumn{3}{|c|}{$\begin{array}{l}\text { RESPIRATORY PROTECTION: } \\
\text { ASPD =AIR SUPPLIED PD } \\
\text { DCHM=DUAL CART. HALF MASK } \\
\text { FFOC=FULL FACE DUAL CART. } \\
\text { PAPR }=\text { POHERED AIR PURIFYING }\end{array}$} & $\begin{array}{l}: N / A \\
\vdots \\
\vdots \\
:\end{array}$ \\
\hline $\begin{array}{l}\text { SAMPLE } \\
\text { NUMBER }\end{array}$ & $\begin{array}{c}\text { FIBER SI } \\
\text { FIELD S }\end{array}$ & & $\begin{array}{c}\text { PUMP } \\
\text { NUMBER }\end{array}$ & \begin{tabular}{|c|c|} 
TIME ON \\
HR PMIN.
\end{tabular} & $\begin{array}{l}\text { TIME OFF } \\
\text { HR PIN. }\end{array}$ & $\begin{array}{l}\text { FLON } \\
\text { IIII. }\end{array}$ & $\begin{array}{l}\text { RATE } \\
\text { STOP }\end{array}$ & $\begin{array}{l}\text { (LPM } \\
\text { AVG. }\end{array}$ & $\begin{array}{l}\text { TOTAL RUN } \\
\text { MINUTES }\end{array}$ & \begin{tabular}{|c|} 
TOTAL VOL \\
LITERS
\end{tabular} & $\begin{array}{c}\text { FIBERS/ } \\
\text { C.C. }\end{array}$ & $\begin{array}{c}\text { T.H.A. } \\
\text { B HR }\end{array}$ & $\begin{array}{l}\text { DEIECTION } \\
\text { LIMIT F/CC }\end{array}$ & $\overline{B . D . L}$ & \\
\hline 64069 * & $6 / 100$ & NORTHEAST INSIDE CELL 4 & $\overline{2019}$ & $16: \overline{45}$ & $6: 15$ & $\overline{6.2}$ & $\overline{4.6}$ & 5.4 & $\overline{810}$ & $\overline{4374}$ & $\overline{B D L}$ & $\overline{N A}$ & 0.001121275 & $\overline{\text { YES }}$ & \\
\hline 4070 & $8 / 100$ & NORHT CENTER INSIDE CELL 4 & $\overline{2021}$ & $16: \overline{45}$ & $6: \overline{15}$ & $\overline{6.2}$ & $\overline{5.2}$ & $\overline{5.7}$ & $\overline{810}$ & $\overline{4617}$ & $\overline{B D L}$ & NA & 0.001062260 & $\overline{\text { YES }}$ & \\
\hline 4071 & $11 / 100$ & NORTHHEST INSIDE CELLL & 2013 & $16: \overline{45}$ & $6: \overline{16}$ & 6 & $\overline{4.8}$ & 5.4 & 811 & 4379.4 & $\overline{0.001231}$ & $\overline{0.002081}$ & $\overline{0.001119892}$ & NO & \\
\hline$\overline{c 4072}$ & $7.5,100$ & SOUTHWEST INSIDE CELL 4 & 2012 & $76: \overline{45}$ & $6: \overline{16}$ & 6 & 6 & 6 & 811 & 4866 & $\overline{B O L}$ & $\overline{N A}$ & $\overline{0.001007903}$ & YES & \\
\hline C4073 & $8 / \overline{100}$ & HALLHAY BY CELLL 4 & 6 & $16: \overline{46}$ & $6: \overline{18}$ & 6 & $\overline{5.6}$ & $\overline{5.8}$ & $\overline{812}$ & 4709.6 & $\overline{B D L}$ & $\overline{\mathrm{NA}}$ & $\overline{0.001041374}$ & $\overline{Y E S}$ & \\
\hline$\overline{C 4074}$ & 5,100 & CELL 5 & $\overline{2011}$ & $16: \overline{47}$ & $6: \overline{19}$ & 6 & 5.6 & $\overline{5.8}$ & $\overline{812}$ & 4709.6 & $\overline{B D L}$ & $\overline{N A}$ & $\overline{0.001041374}$ & $\overline{\text { YES }}$ & \\
\hline . & $0, \overline{100}$ & BLANK & & & & & & T & $\overline{1440}$ & $\overline{0}$ & $\overline{E R R}$ & $\overline{E R R}$ & $\overline{\text { ERR }}$ & $\overline{\text { ERR }}$ & \\
\hline & & & & $:$ & $\because$ & & & $\overline{0}$ & $\overline{1440}$ & 0 & $\overline{E R R}$ & $\overline{\text { ERR }}$ & ERR & $\overline{\text { ERR }}$ & \\
\hline & & & & & & & & 0 & 1440 & $\overline{0}$ & $\overline{\text { ERR }}$ & $\overline{E R R}$ & ERR & $\overline{\text { ERR }}$ & \\
\hline & & & & & $:$ & & & 0 & 1440 & $\overline{0}$ & $\overline{E R R}$ & $\overline{\text { ERR }}$ & ERR & $\overline{\text { ERR }}$ & \\
\hline & & & & $\because$ & $\because$ & & & 0 & $\overline{1440}$ & $\overline{0}$ & $\overline{\text { ERR }}$ & $\overline{E R R}$ & ERR & $\overline{\text { ERR }}$ & \\
\hline & & & & & & & & 0 & 1440 & $\overline{0}$ & $\overline{E R R}$ & $\overline{\text { ERR }}$ & ERR & $\overline{\text { ERR }}$ & \\
\hline & & & & & $\because$ & & & 0 & 1440 & 0 & $\overline{\text { ERR }}$ & $\overline{E R R}$ & ERR & $\overline{\text { ERR }}$ & \\
\hline & 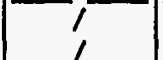 & & & $\because$ & & & & 0 & 1440 & $\overline{0}$ & $\overline{\text { ERR }}$ & $\overline{E R R}$ & $\overline{E R R}$ & $\overline{E R R}$ & \\
\hline & 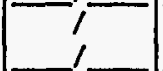 & & & $\because$ & & & & $\overline{0}$ & $\overline{1440}$ & $\overline{0}$ & $\overline{\text { ERR }}$ & $\overline{\text { ERR }}$ & ERR & $\overline{E R R}$ & \\
\hline & & & & $:$ & $\because$ & & & 0 & 1440 & $\overline{0}$ & ERR & ERR & ERR & $\overline{\text { ERR }}$ & \\
\hline COMMENTS: & $\begin{array}{l}\text { THESE WERE } \\
\text { * SAMPLE \# } \\
\text { CLEARANCES } \\
\text { THANK }\end{array}$ & $\begin{array}{l}\text { E PUT ON AFTER ABATEMENT IN CELL } 4 . \\
\text { HC4069 HAS VERY DUSTY. } \\
\text { I } \\
\text { K YOU FOR THE OPPORTUNITY TO PROVIDE EN }\end{array}$ & & MEASUREMENT & & & ANALY & YST: & KEITH L. ST & TANLEY & & $*=$ BELOH & DETECTION LIM & & \\
\hline
\end{tabular}




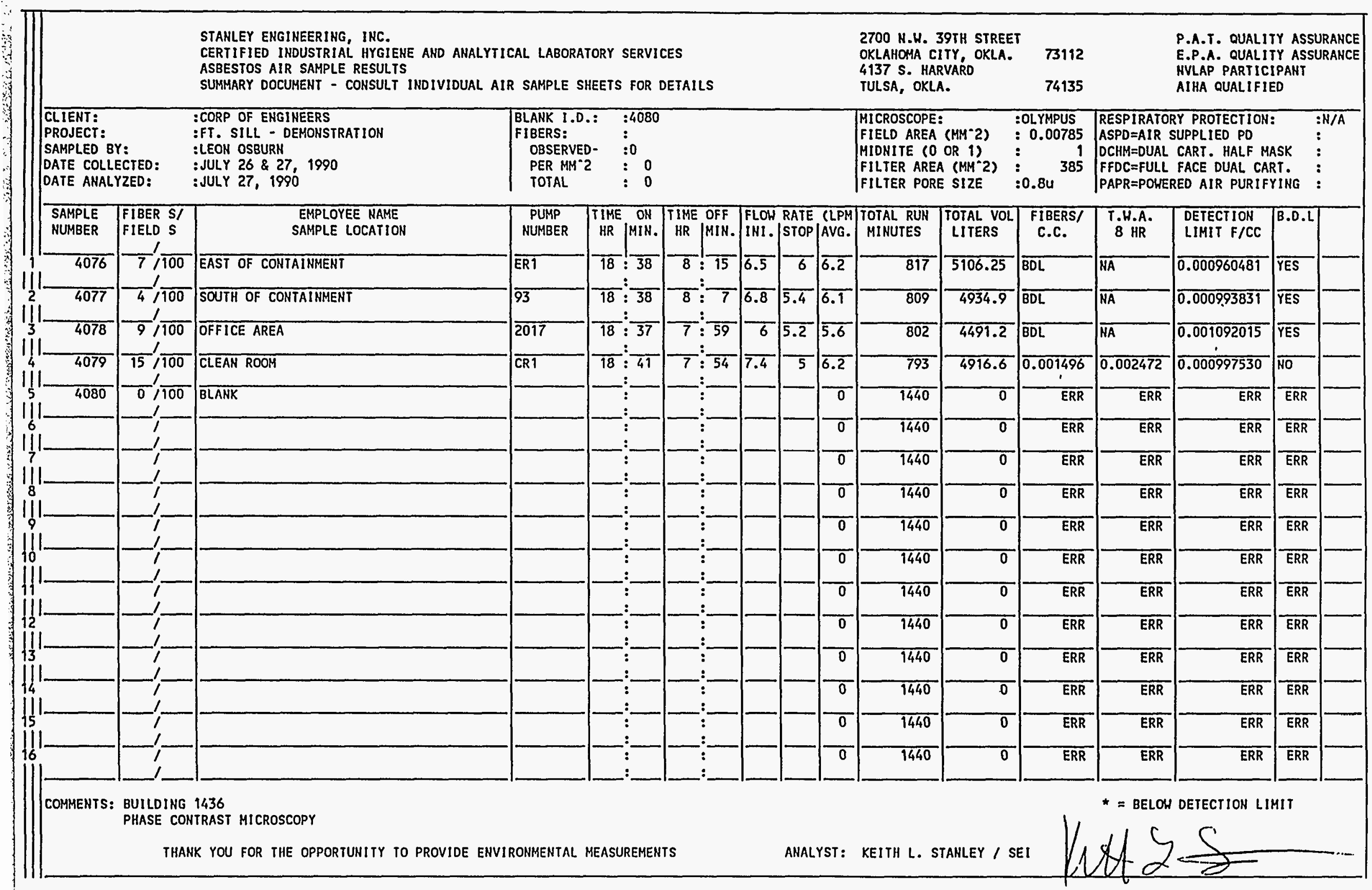


STANLEY ENGINEERING, INC.

CERTIFIED INDUSTRIAL HYGIENE AND ANALYTICAL LABORATORY SERVICES

ASBESTOS AIR SAMPLE RESULTS

SUMMARY DOCUMENT - CONSULT INDIVIDUAL AIR SAMPLE SHEETS FOR DETAILS

\begin{tabular}{ll|ll}
\hline CLIENT: & :CORP OF ENGIMEERS & BLANK I.D.: & $: 0$ \\
PROJECT: & $:$ FORT SILL-DEMONSTRATION & FIBERS: & $:$ \\
SAMPLED BY: & $:$ SEI & OBSERVED- & 0 \\
DATE COLLECTED: & $: 7-26-90$ & PER MM-2 & $: 0$ \\
DATE ANALYZED: & $: 7-30-90$ & TOTAL & $: 0$
\end{tabular}
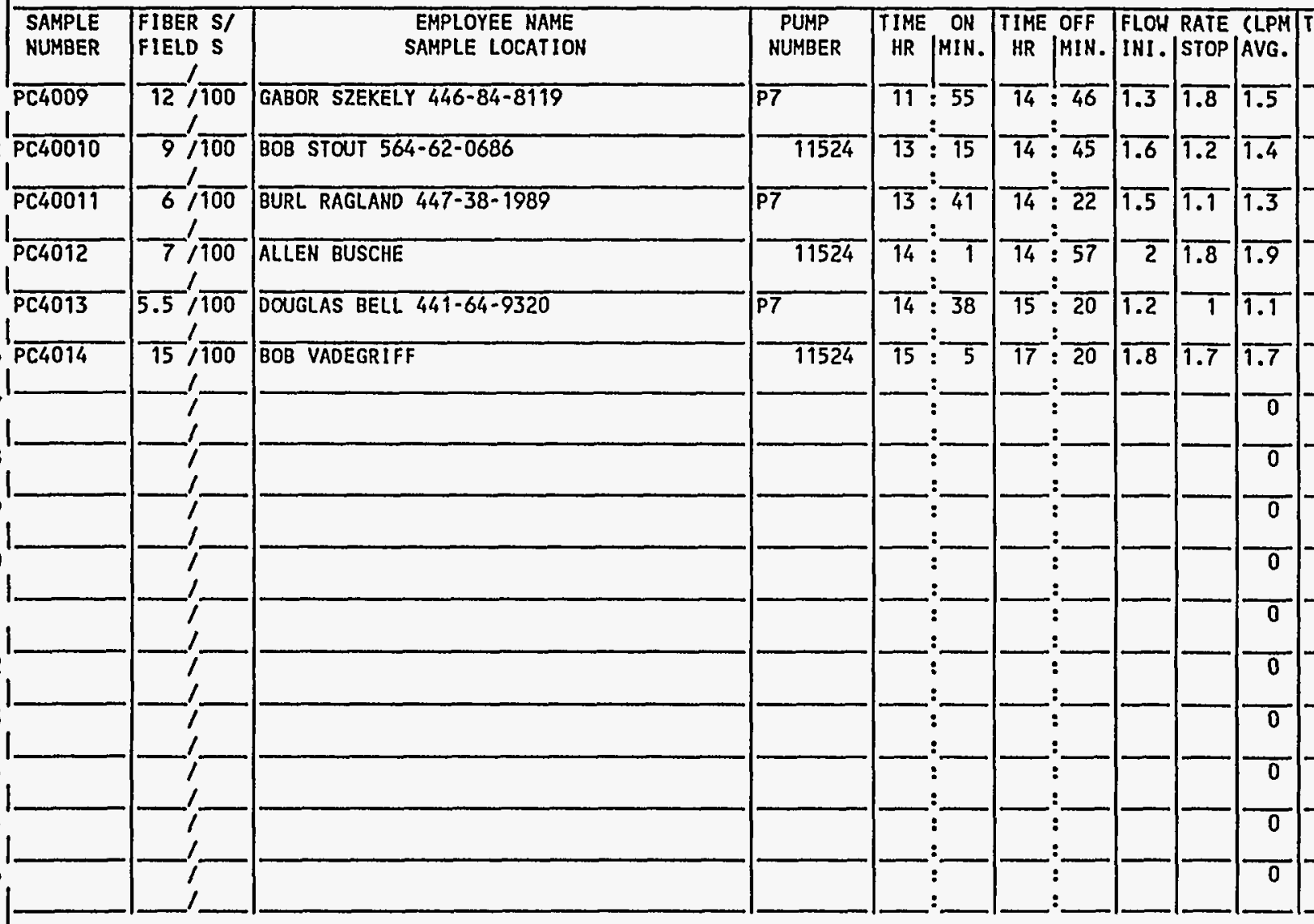

2700 N.H. 39TH STREET

OKLAHOHA CITY, OKLA.

4137 S. HARVARD

TULSA, OKLA.

MICROSCOPE:

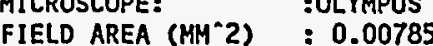

MIDNITE (O OR 1):

FILTER AREA (MM-2)

FILTER PORE SIZE$$
\text { TTOTAL VOL FIBERST T.H.A. TO DETECTION }
$$

TIAL RUH TOTAL YOL FIBERSI TT THA

\begin{tabular}{|c|c|}
\hline $\begin{array}{l}\text { TOTAL RUN } \\
\text { MINUTES }\end{array}$ & $\begin{array}{l}\text { TOTAL VOL } \\
\text { LITERS }\end{array}$ \\
\hline 171 & 265.05 \\
\hline 90 & 126 \\
\hline 49 & $53 . \overline{3}$ \\
\hline 56 & 106.4 \\
\hline 42 & 46.2 \\
\hline 135 & 236.25 \\
\hline 0 & 0 \\
\hline 0 & 0 \\
\hline 0 & $\overline{0}$ \\
\hline 0 & 0 \\
\hline 0 & $\overline{0}$ \\
\hline 0 & $\overline{0}$ \\
\hline 0 & 0 \\
\hline 0 & 0 \\
\hline 0 & 0 \\
\hline 0 & 0 \\
\hline
\end{tabular}

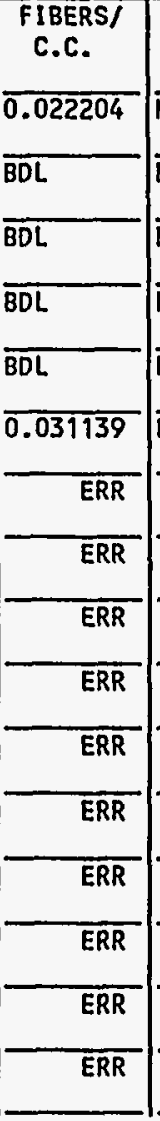

COMMENTS: LIST OF PERSONS HHO REMOVED THE TILE FROM THE FLOOR IN CELL IV.

THANK YOU FOR THE OPPORTUNITY TO PROVIDE ENVIRONMENTAL MEASUREMENTS

ANALYST: KEITH STANLEY/SEI
73112

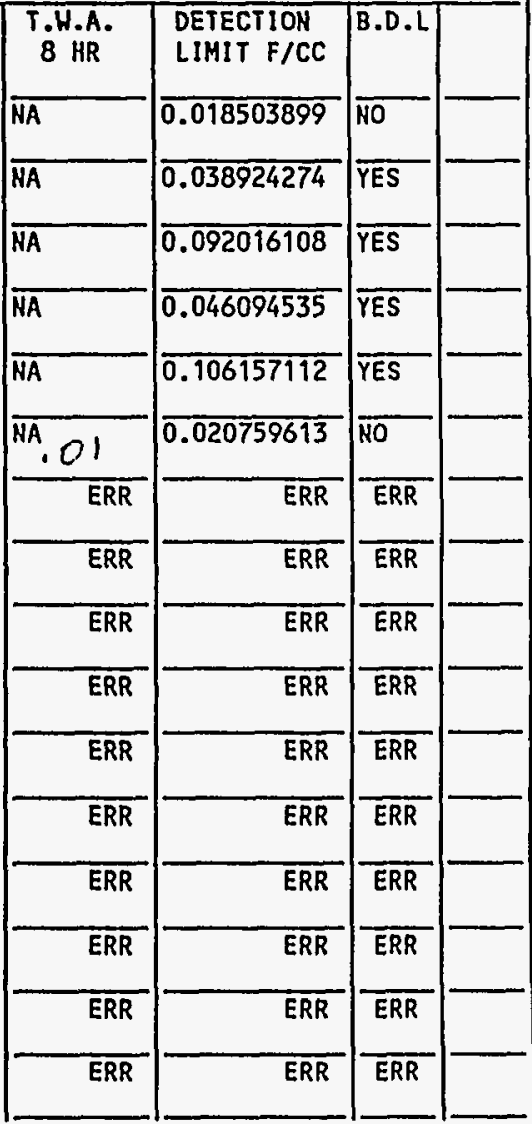

* = BELOW DETECTION UIMH -
P.A.T. QUALITY ASSURANCE

E.PA. QUALITY ASSURANCE

HVLAP PARTICIPANT

AIHA QUALIFIED

RESPIRATORY PROTECTION: :NA

ASPD $=A I R$ SUPPLIED PD
DCHM=DUAL CART. HALF MASK :

FFDC=FULL FACE DUAL CART
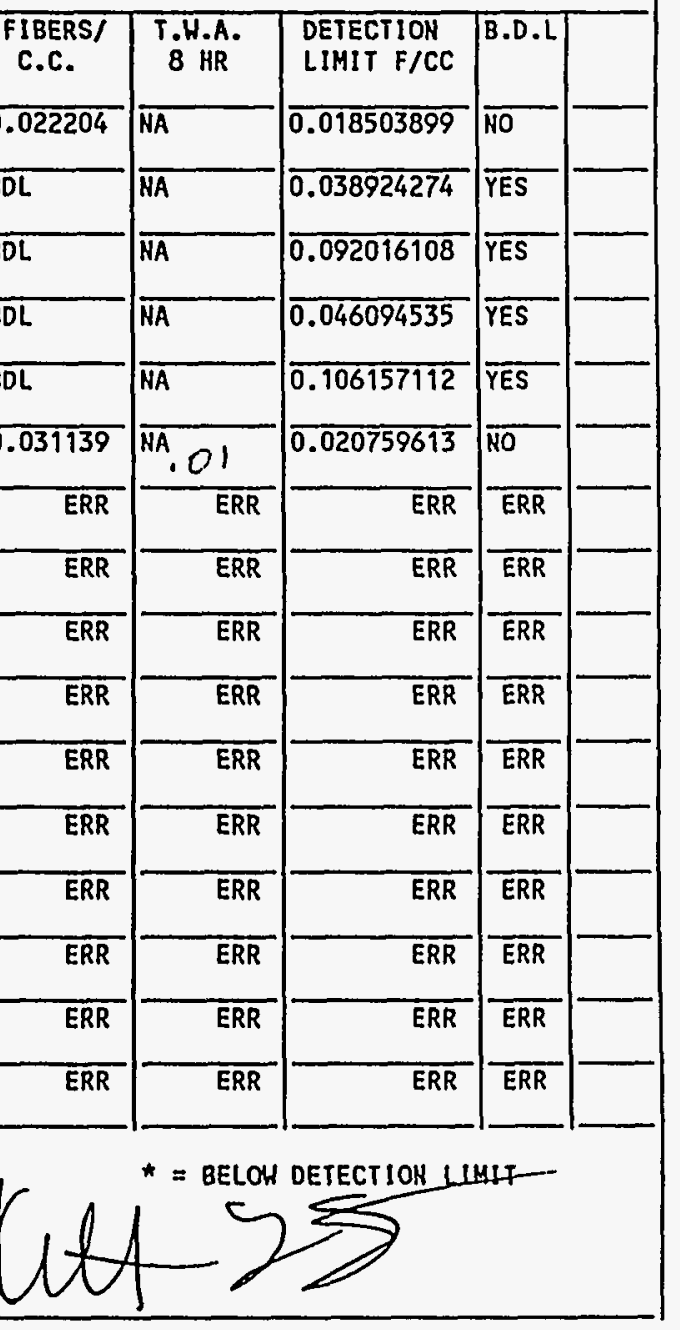


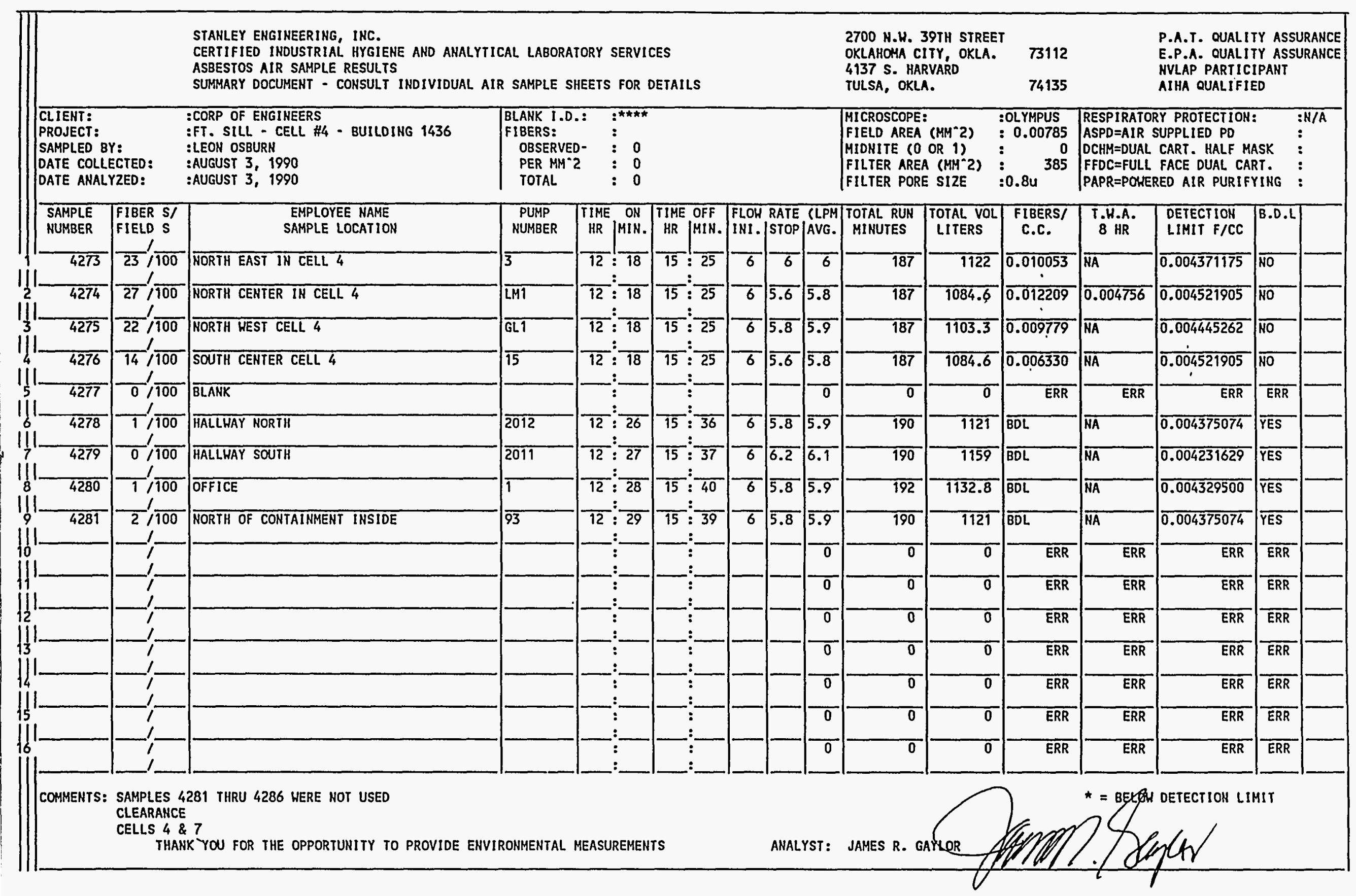




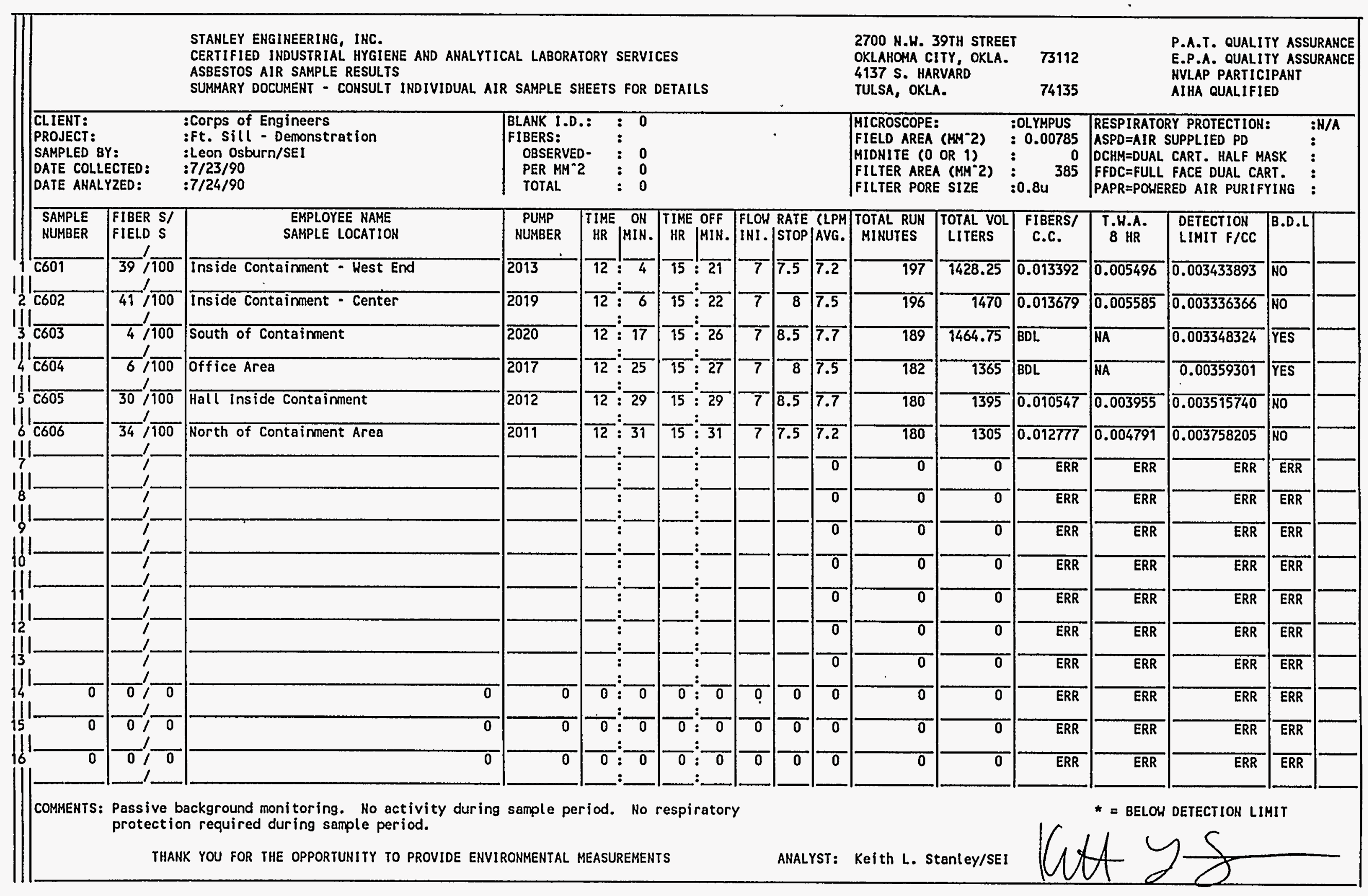




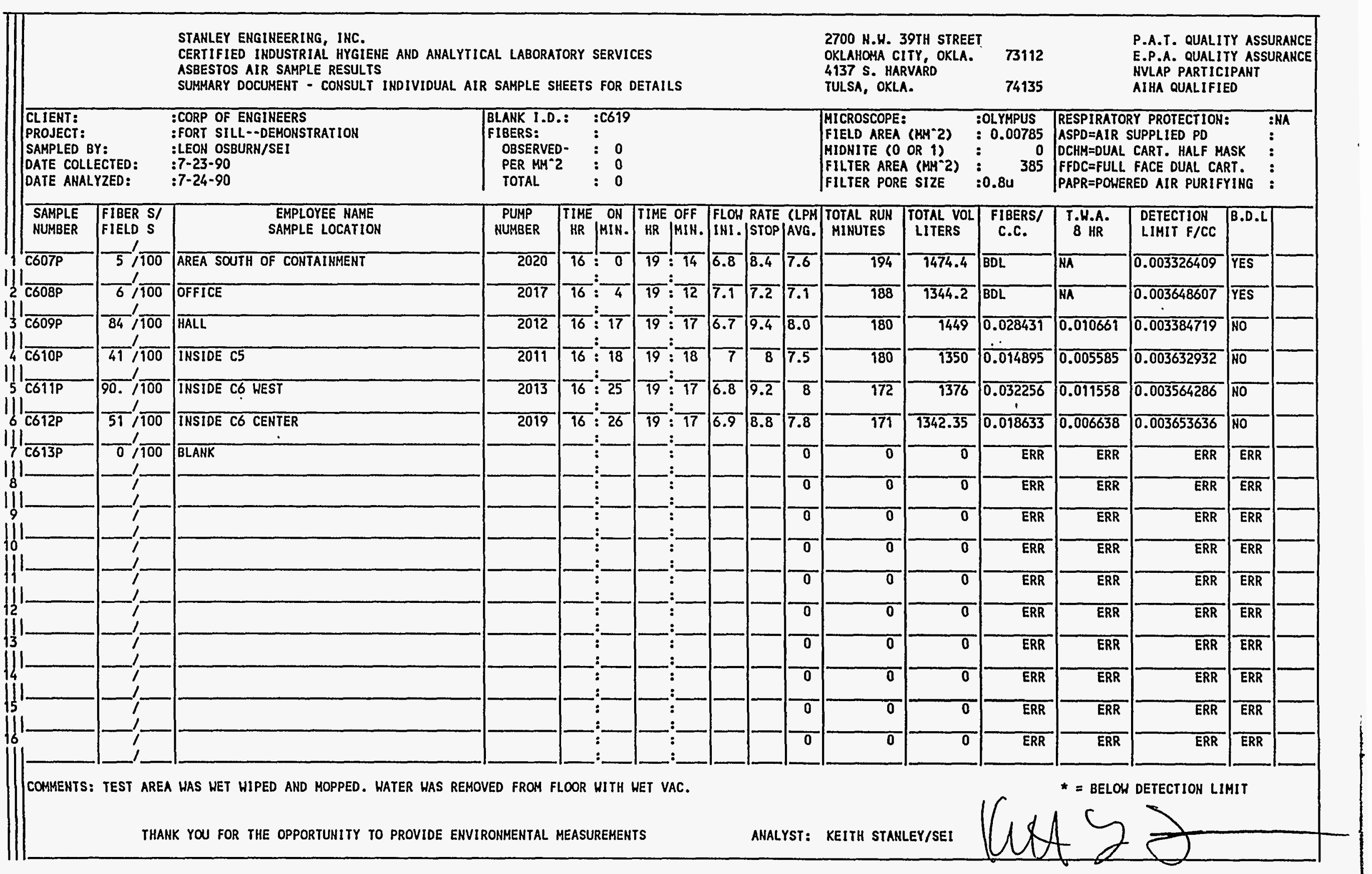




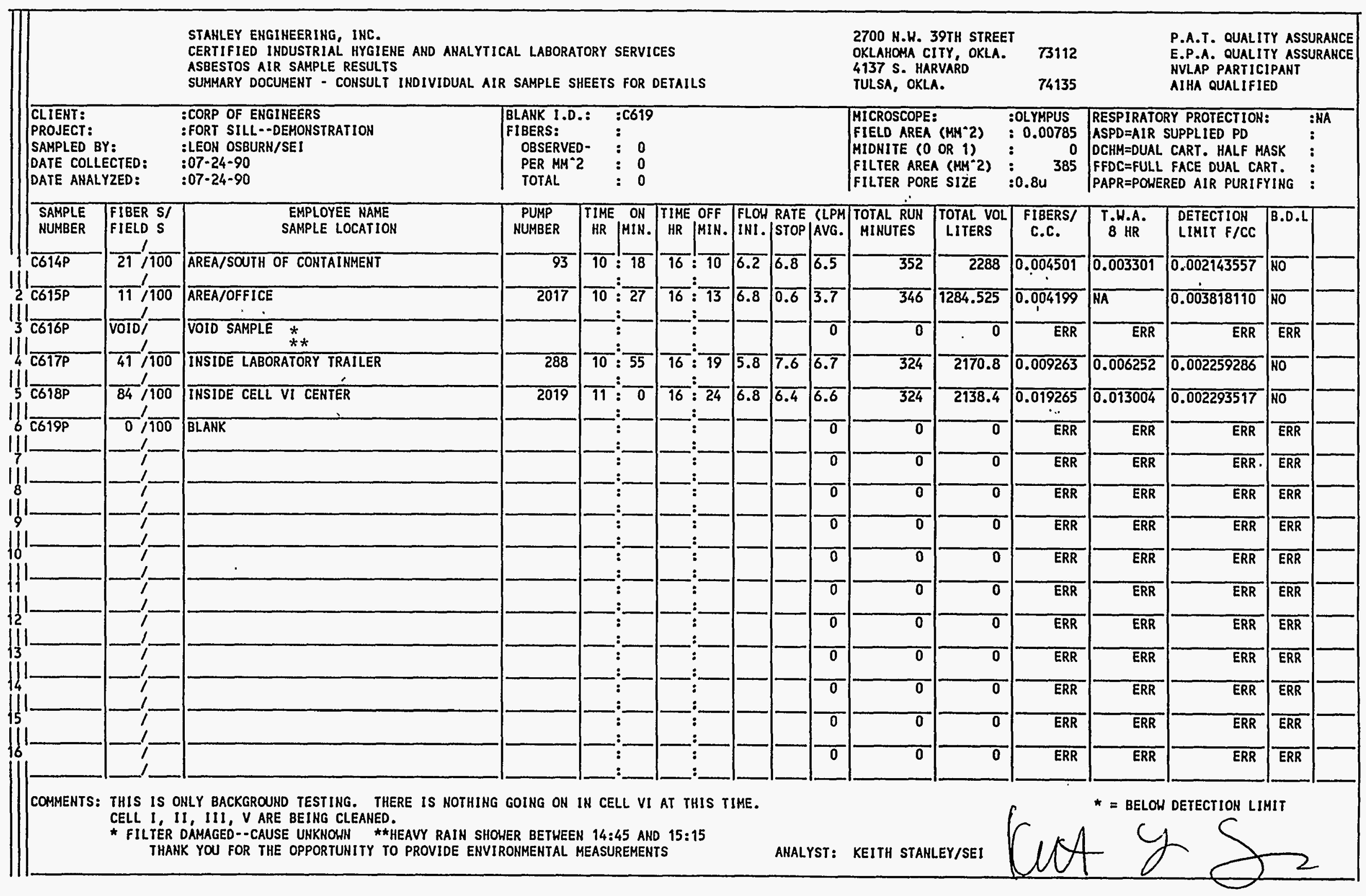




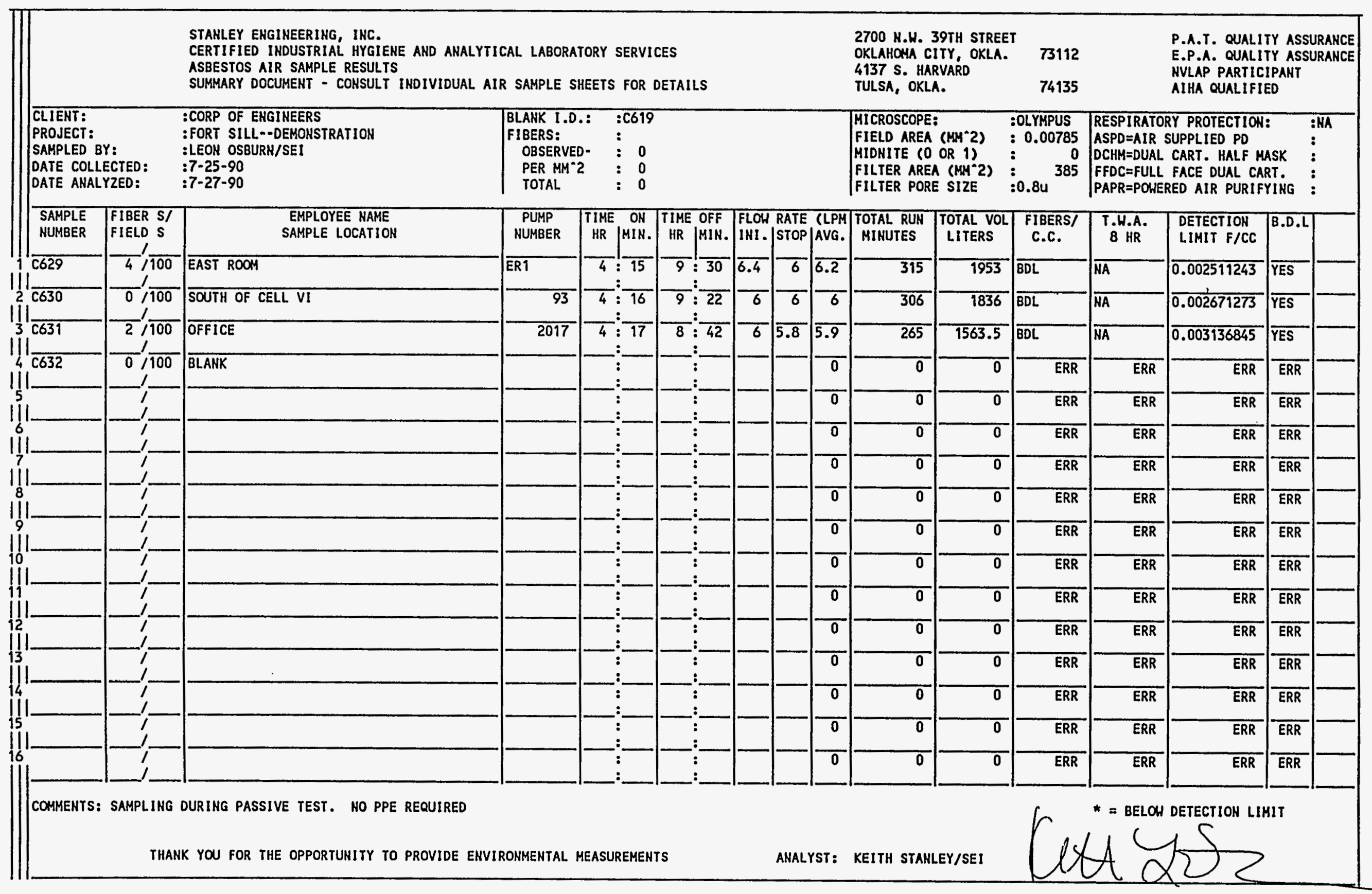




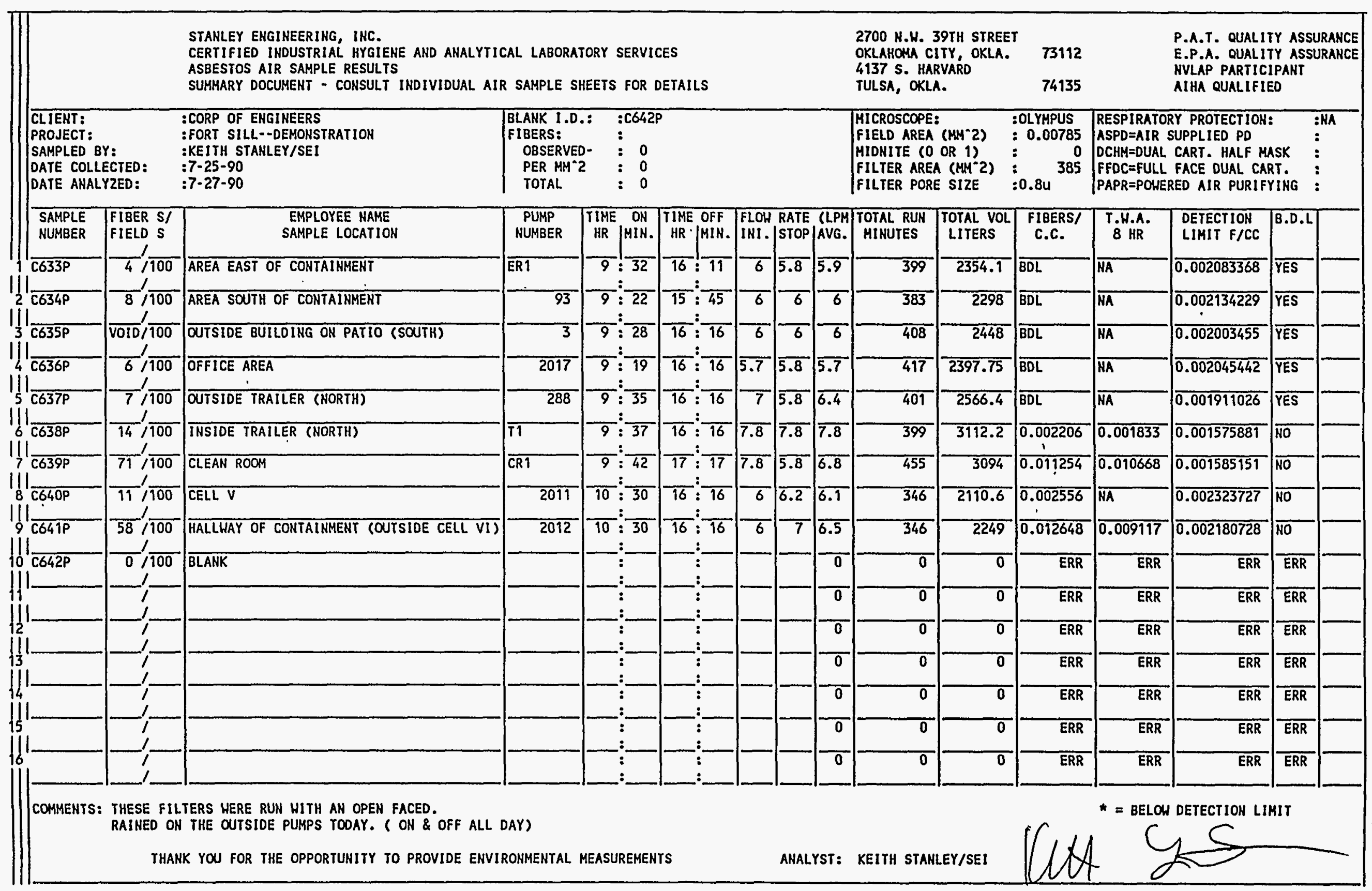




\begin{tabular}{|c|c|c|c|c|c|c|c|c|c|c|c|c|c|c|c|}
\hline \multicolumn{2}{|c|}{$\mid$\begin{tabular}{l||} 
\\
CLIENT: \\
PROJECT: \\
SAMPLED BY: \\
DATE COLLECTED: \\
DATE ANALYZED:
\end{tabular}} & \multicolumn{7}{|c|}{$\begin{array}{l}\text { STANLEY ENGINEERING, INC. } \\
\text { CERTIFIED INDUSTRIAL HYGIENE AND ANALYTICAL LABORATORY SERVICES } \\
\text { ASBESTOS AIR SAMPLE RESULTS } \\
\text { SUMMARY DOCUMENT - CONSULT INDIVIDUAL AIR SAMPLE SHEETS FOR DETAILS }\end{array}$} & \multicolumn{2}{|c|}{$\begin{array}{l}\text { 2700 N.H. 39TH STREET } \\
\text { OKLAHOMA CITY, OKLA. } \\
4137 \text { S. HARVARD } \\
\text { TULSA, OKLA. }\end{array}$} & $\begin{array}{l}73112 \\
74135\end{array}$ & & \multicolumn{3}{|c|}{$\begin{array}{l}\text { P.A.T. QUALITY ASSURAHCE } \\
\text { E.P.A. QUALITY ASSURANCE } \\
\text { NVLAP PARTICIPANT } \\
\text { AIHA QUALIFIED }\end{array}$} \\
\hline \multicolumn{2}{|c|}{$\begin{array}{l}\text { CLIENT: } \\
\text { PROJECT: } \\
\text { SAMPLED BY: } \\
\text { DATE COLLECTED: } \\
\text { DATE ANALYZED: }\end{array}$} & $\begin{array}{l}\text { :CORP OF ENGINEERS } \\
\text { :FT. SILL - AFTER ABATEMENT } \\
: \text { :LON OSBURN } \\
: 7-25-90 \\
: 7-27-90\end{array}$ & $\begin{array}{l}\text { BLANK I.D } \\
\text { FIBERS: } \\
\text { OBSERVE } \\
\text { PER MM } \\
\text { TOTAL }\end{array}$ & $\begin{array}{ll}: & : 656 \\
0- & \vdots 0 \\
2- & \vdots \\
2 & 0\end{array}$ & & & & & $\begin{array}{l}\text { MICROSCOPE } \\
\text { FIELD AREA } \\
\text { MIDHITE CO } \\
\text { FILTER ARE } \\
\text { FILTER POR }\end{array}$ & $\begin{array}{l}\left(M H H^{-2}\right) \\
\text { OR } 11) \\
\left(M H^{-2} 2\right) \\
\text { SIZE }\end{array}$ & $\begin{array}{l}: \text { OLYMPUS } \\
: 0.00785 \\
\vdots \\
: 0.84^{\quad 385}\end{array}$ & $\begin{array}{l}\text { RESPIRATOR } \\
\text { ASPD=AIR S } \\
\text { OCHM=DUAL } \\
\text { FFDC=FULL } \\
\text { PAPR=POHER }\end{array}$ & $\begin{array}{l}\text { RY PROTECTION } \\
\text { SUPPLIED PD } \\
\text { CART. HALF M } \\
\text { FACE DUAL CA } \\
\text { RED AIR PURIF }\end{array}$ & $\begin{array}{l}\text { IASK } \\
\text { RT. } \\
\text { YSING }\end{array}$ & $\begin{array}{l}: \mathrm{N} / \mathrm{A} \\
\vdots \\
\vdots \\
\vdots\end{array}$ \\
\hline $\begin{array}{l}\text { SAMPLE } \\
\text { NUMBER }\end{array}$ & $\begin{array}{l}\text { FIBER SI } \\
\text { FIELO S }\end{array}$ & $\begin{array}{l}\text { EMPLOYEE NAME } \\
\text { SAMPLE LOCATION }\end{array}$ & $\begin{array}{l}\text { PUMP } \\
\text { NUMBER }\end{array}$ & \begin{tabular}{|c|c|} 
TIME & ON \\
HR & MIN. \\
\end{tabular} & \begin{tabular}{|l|} 
TIME OFF \\
HR MIN.
\end{tabular} & $\begin{array}{l}\text { FLOW } \\
\text { INI. }\end{array}$ & $\begin{array}{l}\text { RATE } \\
\text { STOP }\end{array}$ & $\begin{array}{l}\text { CLPM } \\
\text { AVGG. }\end{array}$ & $\begin{array}{l}\text { TOTAL RUN } \\
\text { MINUTES }\end{array}$ & $\begin{array}{l}\text { TOTAL VOL } \\
\text { LITERS }\end{array}$ & $\begin{array}{l}\text { FIBERS/ } \\
\text { C.C. }\end{array}$ & $\begin{array}{c}\text { T.H.A. } \\
B \text { HR }\end{array}$ & $\begin{array}{l}\text { DETECTION } \\
\text { LIMIT F/CC }\end{array}$ & 8.0 .1 & \\
\hline 643 & 12. $/ \overline{100}$ & EAST OF CONTAINMENT - CELL 6 & $\overline{\text { ER1 }}$ & $16: 7$ & $\overline{36}$ & $\overline{6}$ & $\overline{5.4}$ & $\overline{5.7}$ & $\overline{982}$ & 5597.4 & 0.001095 & 0.002240 & $\overline{0.000876203}$ & $\overline{\text { SO }}$ & \\
\hline$\overline{644}$ & $\overline{14} / \overline{100}$ & SOUTH OF CONTAINMENT - C6 & 93 & $16: \overline{4}$ & & 6 & $\overline{6.2}$ & $\overline{6.1}$ & 990 & 6039 & 0.001136 & $\overline{0.002345}$ & $\overline{0.000812130}$ & $\overline{\text { NO }}$ & \\
\hline 645 & $9 / \overline{100}$ & OUTSIDE BLDG. ON PATIO (SOUTH) & $\overline{3}$ & $76:-$ & & 6 & $\overline{4.8}$ & $\overline{5.4}$ & 996 & 5378.4 & $\overline{B D L}$ & $\overline{\text { NA }}$ & $\overline{0.000911880}$ & $\overline{\text { YES }}$ & \\
\hline 646 & $10, \overline{100}$ & OFFICE AREA & 2017 & $16: 1$ & $\overline{49}$ & 6 & $\overline{5.6}$ & $\overline{5.8}$ & 1008 & 5846.4 & $\overline{B D L}$ & $\overline{\mathrm{NA}}$ & $\overline{0.000838885}$ & $\overline{\text { YES }}$ & \\
\hline 647 & $17 / \overline{100}$ & QUTSIDE TRAILER (NORTH) & 288 & $16: \overline{56}$ & & $\overline{5.8}$ & $\overline{6}$ & $\overline{5.9}$ & 958 & 5652.2 & 0.001475 & 0.002944 & $\overline{0.000867707}$ & $\overline{\text { HO }}$ & \\
\hline 648 & 12. & INSIDE TRAILER (NORTH) & T1 & $\overline{16}: \overline{51}$ & & $\overline{7.8}$ & $\overline{7.2}$ & $\overline{7.5}$ & 964 & 7230 & 0.000847 & $\overline{0.001702}$ & $\overline{0.000678348}$ & MO & \\
\hline 649 & $7 / \overline{100}$ & $\overline{C E L L ~} 6$ & $\overline{\mathrm{NP}}$ & $16: 39$ & & $\overline{4.2}$ & $\overline{5.8}$ & 5 & 838 & 4190 & $\overline{B D L}$ & $\overline{\mathrm{NA}}$ & $\overline{0.001170515}$ & $\overline{Y E S}$ & \\
\hline 650 & $\overline{14 .}, \overline{100}$ & HALLHAY BY CELL 6 & $\overline{2012}$ & $16: 39$ & $\overline{37}$ & 7 & $\overline{7.8}$ & $\overline{7.4}$ & 838 & 6201.2 & 0.001146 & $\overline{0.002002}$ & $\overline{0.000790888}$ & NO & \\
\hline 651 & $9, \overline{100}$ & HALLWAY OF CONTAINMENT (OUTSIDE CELLL 6) & $\overline{N P}$ & $16: \overline{40}$ & 6 & 5 & 7 & $\overline{5.5}$ & $\overline{837}$ & 4603.5 & $\overline{B D L}$ & MA & $\overline{0.001065376}$ & $\overline{\text { YES }}$ & \\
\hline 652 & $8 / \overline{100}$ & CELL 6 & $\overline{\mathrm{MP}}$ & $16: 39$ & $6: \overline{37}$ & $\overline{4.2}$ & 5 & $\overline{4.6}$ & 838 & 3854.8 & $B D$ & $\overline{N A}$ & $\overline{0.001272299}$ & $\overline{\text { YES }}$ & \\
\hline 653 & $11 / \overline{100}$ & CELL 6 & NP & $\overline{16}: \overline{43}$ & $6: 37$ & 5 & $\overline{5.8}$ & $\overline{5.4}$ & $\overline{834}$ & 4503.6 & 0.001197 & 0.002081 & $\overline{0.001089008}$ & $\overline{N O}$ & \\
\hline 654 & $7 \longdiv { 1 0 0 }$ & $\overline{C E L L ~} 5$ & 2011 & $16: \overline{45}$ & $6: \overline{37}$ & 6 & $\overline{6.4}$ & $\overline{6.2}$ & 832 & 5158.4 & $\overline{B D L}$ & $\overline{\mathrm{HA}}$ & $\overline{0.000950771}$ & $\overline{\text { YES }}$ & \\
\hline 6008 & $0 / \overline{100}$ & BURL RAGLAND: $447-38-1989$ & $\overline{\mathrm{P} 7}$ & $16: 50$ & $17: \overline{35}$ & 2 & $\overline{1.7}$ & $\overline{1.8}$ & 1485 & 2747.25 & $\overline{B D L}$ & MA & $\overline{0.001785224}$ & $\overline{\text { YES }}$ & \\
\hline 655 & $11 / \overline{100}$ & CLEAN ROOM & $\overline{C R 1}$ & $17: 10$ & $8: \overline{59}$ & $\overline{5.8}$ & $\overline{6.2}$ & 6 & 949 & 5694 & 0.000947 & $\overline{0.001873}$ & 0.000861338 & NO & \\
\hline 656 & $0 / \overline{100}$ & BLANK & & & & & & 0 & 1440 & $\overline{0}$ & ERR & ERR & ERR & $\overline{\text { ERR }}$ & \\
\hline & & & & & & & & 0 & 1440 & $\overline{0}$ & ERR & $\overline{\text { ERR }}$ & ERR & ERR & \\
\hline COHMENTS: & AFTER ABA & ITEMENT IN CELL 6 & & & & & & & & & & BELO & W LI & & \\
\hline & & IK YOU FOR THE OPPORTUNITY TO PROVIDE & ONM & SUR & & & & YST: & EITH & NIEY 18 & & & & & \\
\hline
\end{tabular}




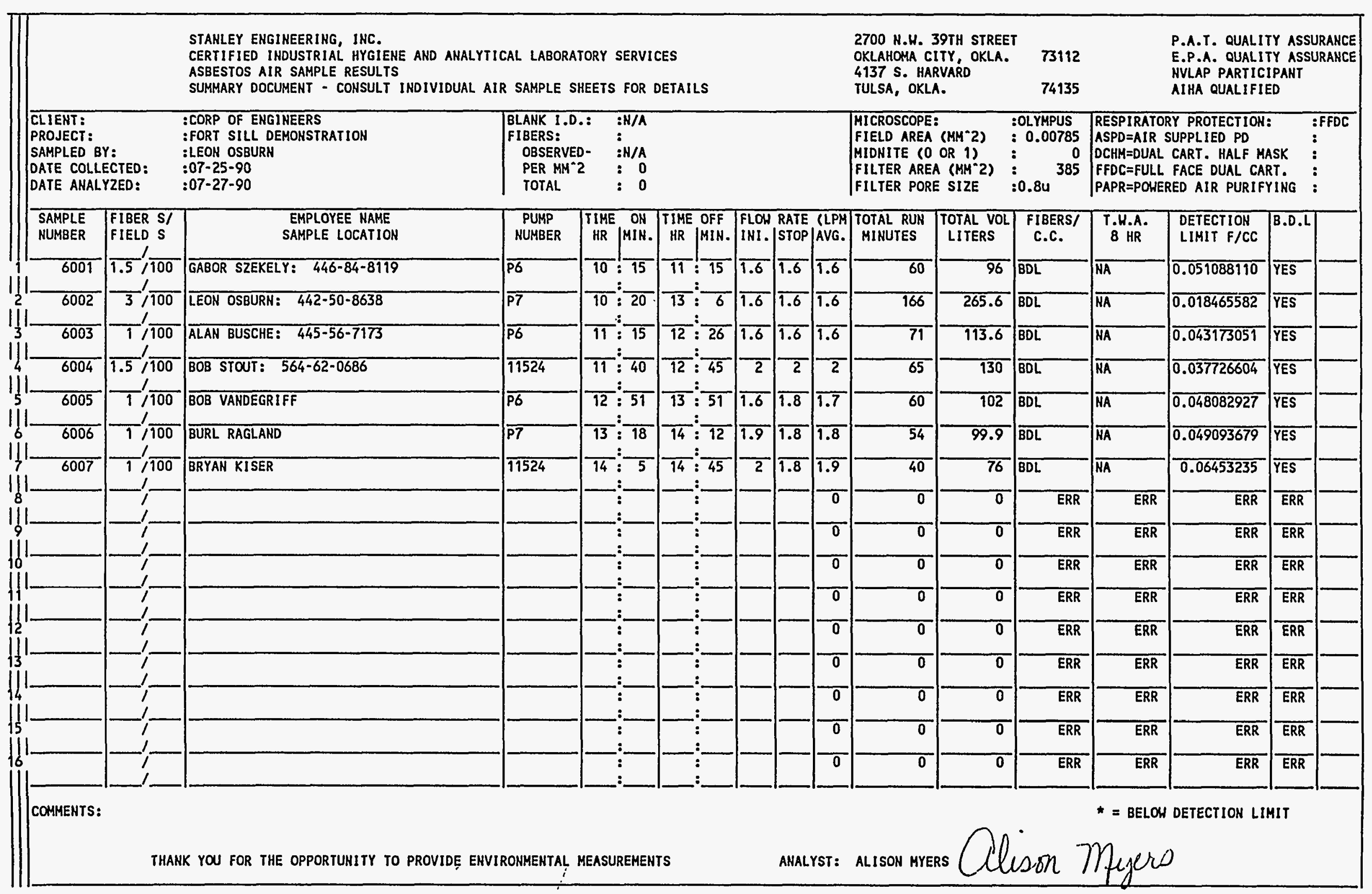




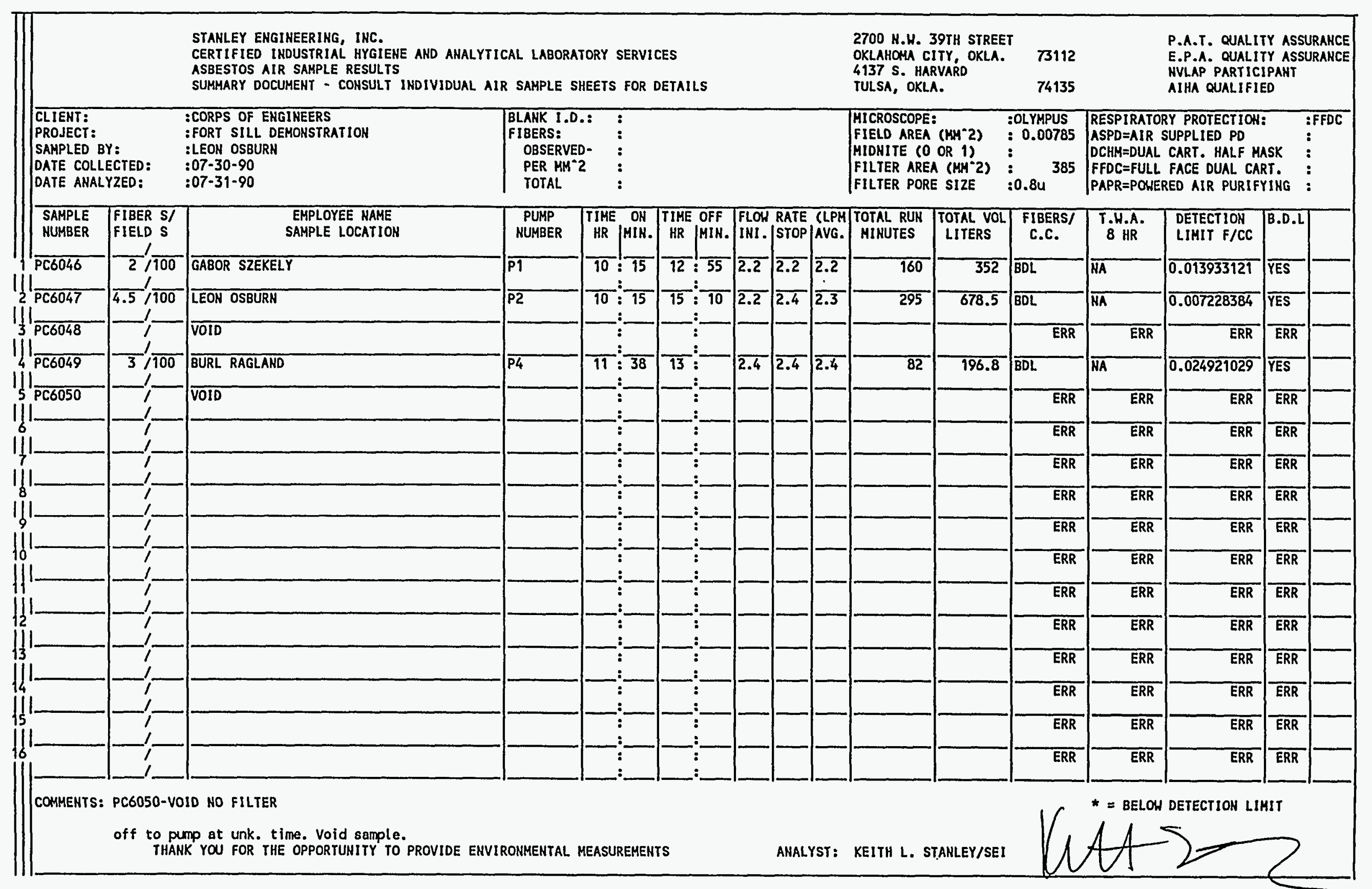




\begin{tabular}{|c|c|c|c|c|c|c|c|c|c|c|c|c|c|c|c|}
\hline & & \multicolumn{7}{|c|}{$\begin{array}{l}\text { STANLEY ENGINEERING, INC. } \\
\text { CERTIFIED INDUSTRIAL HYGIENE AND ANALYTICAL LABORATORY SERVICES } \\
\text { ASBESTOS AIR SAMPLE RESULTS } \\
\text { SUMMARY DOCUMENT - CONSULT INDIVIDUAL AIR SAMPLE SHEETS FOR DETAILS }\end{array}$} & \multicolumn{2}{|c|}{$\begin{array}{l}2700 \text { H.H. 39TH STREET } \\
\text { OKLAHOMA CITY, OKLA. } \\
4137 \text { S. HARVARD } \\
\text { TULSA, OKLA. }\end{array}$} & $\begin{array}{ll}73112 \\
74135\end{array}$ & & \multicolumn{3}{|c|}{$\begin{array}{l}\text { P.A.T. QUALITY ASSURANCE } \\
\text { E.P.A. QUALITY ASSURANCE } \\
\text { NVLAP PARTICIPANT } \\
\text { AIHA QUALIFIED }\end{array}$} \\
\hline \multicolumn{2}{|c|}{$\begin{array}{l}\text { CLIENT: } \\
\text { PROJECT: } \\
\text { SAMPLED BY: } \\
\text { DATE COLLECTED: } \\
\text { DATE ANALYZED: }\end{array}$} & \multirow{2}{*}{ 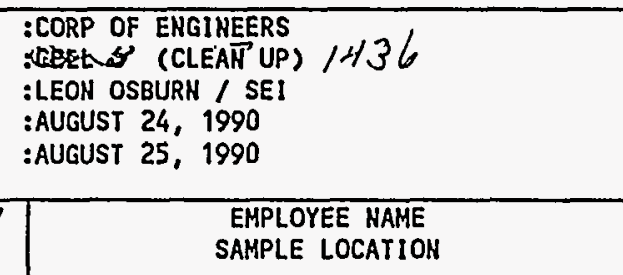 } & \multicolumn{3}{|c|}{ 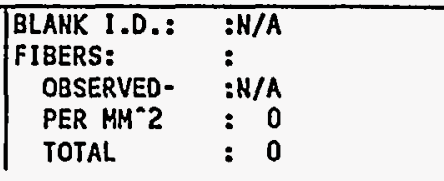 } & & & & \multicolumn{2}{|c|}{$\begin{array}{l}\text { MICROSCOPE: } \\
\text { FIELD AREA (MH } 2) \\
\text { MIDNITE (O OR } 1) \\
\text { FILTER AREA (MH'2) } \\
\text { FILTER PORE SIZE }\end{array}$} & $\begin{array}{l}: \text { OLYMPUS } \\
: 0.00785 \\
: \quad 0 \\
\vdots \\
: 0.8 u^{r}\end{array}$ & \multicolumn{3}{|c|}{$\begin{array}{l}\text { RESPIRATORY PROTECTION: } \\
\text { ASPD=AIR SUPPLIED PD } \\
\text { DCHM=DUAL CART. HALF MASK } \\
\text { FFDCFULL FACE DUAL CART. } \\
\text { PAPR=POWERED AIR PURIFYING }\end{array}$} & $\begin{array}{l}\text { :DCHM } \\
\vdots \\
\vdots \\
\vdots\end{array}$ \\
\hline $\begin{array}{l}\text { SAMPLE } \\
\text { NUMBER }\end{array}$ & $\begin{array}{l}\text { FIBER S/ } \\
\text { FIELD S }\end{array}$ & & $\begin{array}{c}\text { PUMPP } \\
\text { NUMBER }\end{array}$ & $\begin{array}{l}\text { TIME ON } \\
\text { HR JMIN. }\end{array}$ & $\begin{array}{l}\text { TIME OFF } \\
\text { HR J MIN. }\end{array}$ & $\begin{array}{l}\text { FL.W } \\
\text { INI. }\end{array}$ & $\begin{array}{l}\text { RATE } \\
\text { STOP }\end{array}$ & $\begin{array}{l}\text { (LPH } \\
\text { AVG. }\end{array}$ & $\begin{array}{c}\text { TOTAL RUN } \\
\text { MINUTES }\end{array}$ & $\begin{array}{l}\text { TOTAL VOL } \\
\text { LITERS }\end{array}$ & $\begin{array}{l}\text { FIBERS/ } \\
\text { C.C. }\end{array}$ & $\begin{array}{c}\text { T.H.A. } \\
8 \mathrm{HR}\end{array}$ & $\begin{array}{l}\text { DETECTION } \\
\text { LIMIT F/CC }\end{array}$ & B.D.1 & \\
\hline 6015 & $6 / \overline{100}$ & SOUTH & 2017 & 4 & $12 ! \overline{45}$ & 6 & 6 & 6 & $\overline{475}$ & 2850 & $\overline{B D L}$ & $\overline{M A}$ & 0.001720862 & YES & \\
\hline 6016 & $\overline{4}, \overline{100}$ & CENTER & 8 & $4: \overline{50}$ & $\overline{12}: \overline{45}$ & $\frac{6}{6}$ & $\overline{5.7}$ & $\overline{5.8}$ & 475 & $27 \overline{778.75}$ & $\overline{\mathrm{BDL}}$ & $\overline{M A}$ & 0.001764987 & $\overline{Y E S}$ & \\
\hline$\overline{6017}$ & $\sqrt{2}, \overline{100}$ & NORTH & 2021 & $4: \overline{50}$ & $\overline{12}: \overline{45}$ & 6 & 5.9 & 5.9 & 475 & 2826.25 & $\overline{B D L}$ & $\overline{N A}$ & 0.001735323 & $\overline{\text { YES }}$ & \\
\hline 6018 & $5 / 100$ & CLEAN ROOM & 2019 & $4: \overline{45}$ & $\overline{12}: \overline{55}$ & 6 & 6 & 6 & 490 & $\overline{2940}$ & $\overline{B D L}$ & $\overline{\text { NA }}$ & 0.001668183 & $\overline{Y E S}$ & \\
\hline 6019 & $\overline{3} / 100$ & NORTH OF BUILDING & 3 & $4: \overline{40}$ & $73: \overline{15}$ & $\overline{6}$ & $\overline{5.8}$ & $\overline{5.9}$ & 515 & 3038.5 & $\overline{B D L}$ & $\overline{\mathrm{NA}}$ & 0.001614105 & $\overline{\text { YES }}$ & \\
\hline 6020 & & VOID (NOT USED) & & & & & & 0 & $\overline{0}$ & 0 & ERR & ERR & ERR & $\overline{E R R}$ & \\
\hline $6021 \mathrm{P}$ & $2 / 100$ & GABOR SZEKELY & $\overline{p-1}$ & $4: \overline{47}$ & $\overline{12}: \overline{45}$ & $\overline{2}$ & $\overline{2}$ & 2 & 478 & 956 & $\overline{B D L}$ & $\overline{N A}$ & 0.005130186 & YES & \\
\hline $6022 \mathrm{P}$ & $\overline{4.5}, \overline{100}$ & BURL RAGLAND & $\overline{P-2}$ & $\overline{4}: \overline{47}$ & $\overline{12}: \overline{45}$ & 2 & 2 & 2 & $\overline{478}$ & $\overline{956}$ & $\overline{B D L}$ & $\overline{N A}$ & 0.005130186 & $\overline{Y E S}$ & \\
\hline$\overline{6023 \mathrm{P}}$ & $\overline{\mathrm{TD}} / \overline{\mathrm{TC}}$ & LEON OSBURN & $\overline{p-3}$ & $4:$ & $\overline{12}: \overline{45}$ & $\overline{2}$ & 2 & 2 & 478 & 956 & ERR & $\overline{E R R}$ & 0.005130186 & $\overline{\text { ERR }}$ & \\
\hline & & & & & & & & 0 & 0 & $\overline{0}$ & ERR & ERR & $\overline{\text { ERR }}$ & $\overline{\text { ERR }}$ & \\
\hline & & & & : & : & & & 0 & 0 & 0 & ERR & $\overline{\text { ERR }}$ & $\overline{\text { ERR }}$ & $\overline{\text { ERR }}$ & \\
\hline & & & & : & : & & & 0 & $\overline{0}$ & $\overline{0}$ & ERR & ERR & ERR & $\overline{\text { ERR }}$ & \\
\hline & $\gamma$ & & & : & & & & 0 & $\overline{0}$ & $\overline{0}$ & $\overline{E R R}$ & ERR & ERR & $\overline{\text { ERR }}$ & \\
\hline & & & & & & & & 0 & $\overline{0}$ & $\overline{0}$ & $\overline{\text { ERR }}$ & ERR & ERR & ERR & \\
\hline & & & & : & & & & 0 & 0 & $\overline{0}$ & ERR & $\overline{E R R}$ & ERR & ERR & \\
\hline & 1 & & & $:$ & $:$ & & & 0 & 0 & 0 & $\overline{E R R}$ & ERR & $\overline{E R R}$ & $\overline{\text { ERR }}$ & \\
\hline COMMENTS: & $\begin{array}{l}\text { LOG \#9120 } \\
\text { INSIDE } 6 \\
\text { SEALED W } \\
\text { THAI }\end{array}$ & $\begin{array}{l}002 \text { - PHASE CONTRAST MICROSCOPY - BUILDINC } \\
\text { MIL AB BAGS SEALD WITH DUCT TAPE THEN PL } \\
\text { ITH DUCT TAPE. WORKERS HORE DCHM RESP. D } \\
\text { NK YOU FOR THE OPPORTUNITY TO PROVIDE ENVI }\end{array}$ & $\begin{array}{l}\text { 1436: } \\
\text { ACED INSI } \\
\text { SPOSABLE } \\
\text { ROSMENTAL }\end{array}$ & $\begin{array}{l}\text { OOR TILE } \\
\text { E A SECON } \\
\text { B SUITS, } \\
\text { MEASUREME }\end{array}$ & $\begin{array}{l}\text { AS PLACED } \\
6 \text { MIL AB } \\
\text { UBBER BOOT } \\
\text { TS }\end{array}$ & $\begin{array}{l}\text { BAG } \\
S \text { AND }\end{array}$ & $\begin{array}{l}\text { GLOVE } \\
\text { ANALY }\end{array}$ & & ALISON A & RS / SEI & & $*=B E L O$ & & & \\
\hline
\end{tabular}




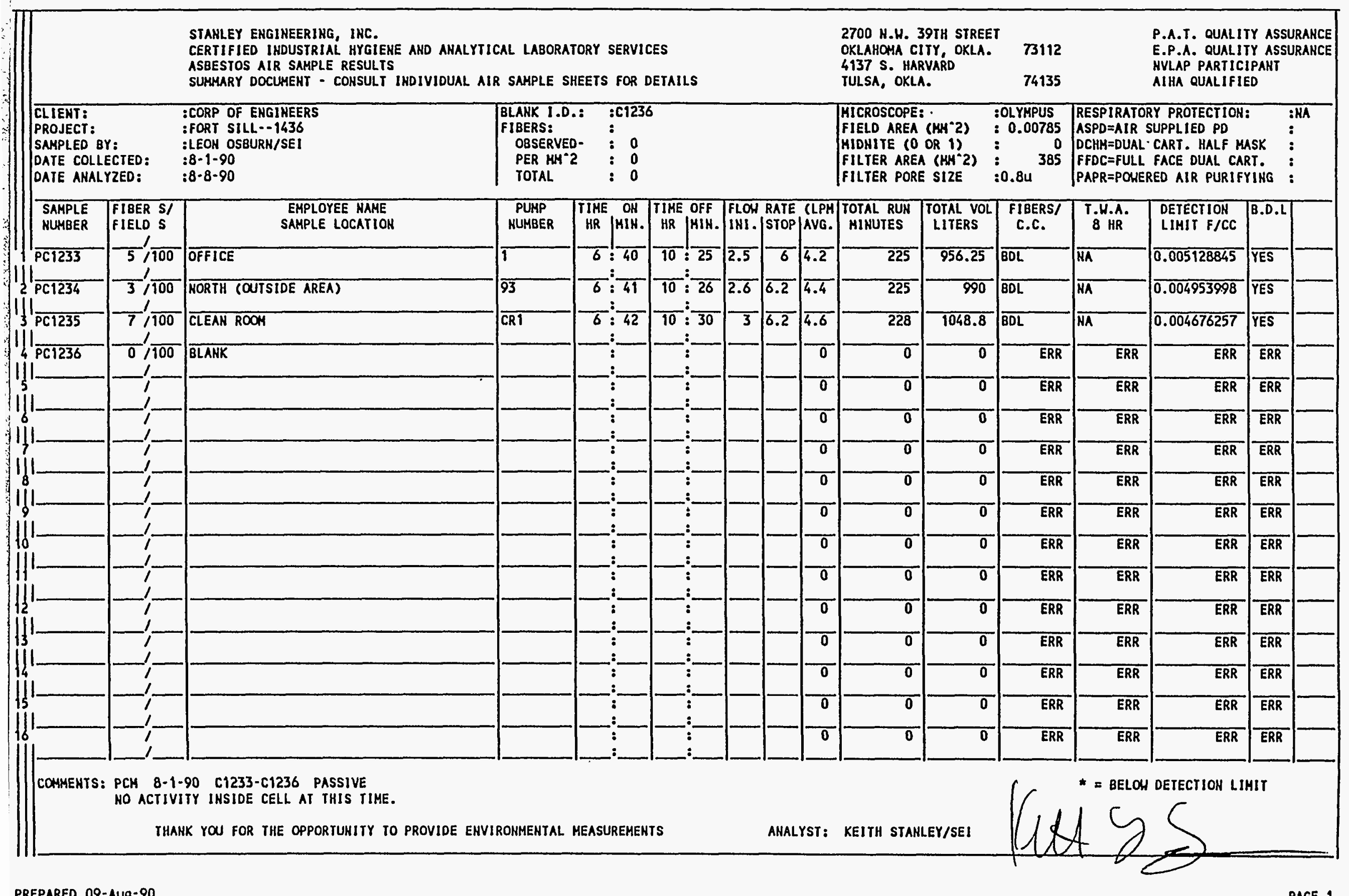




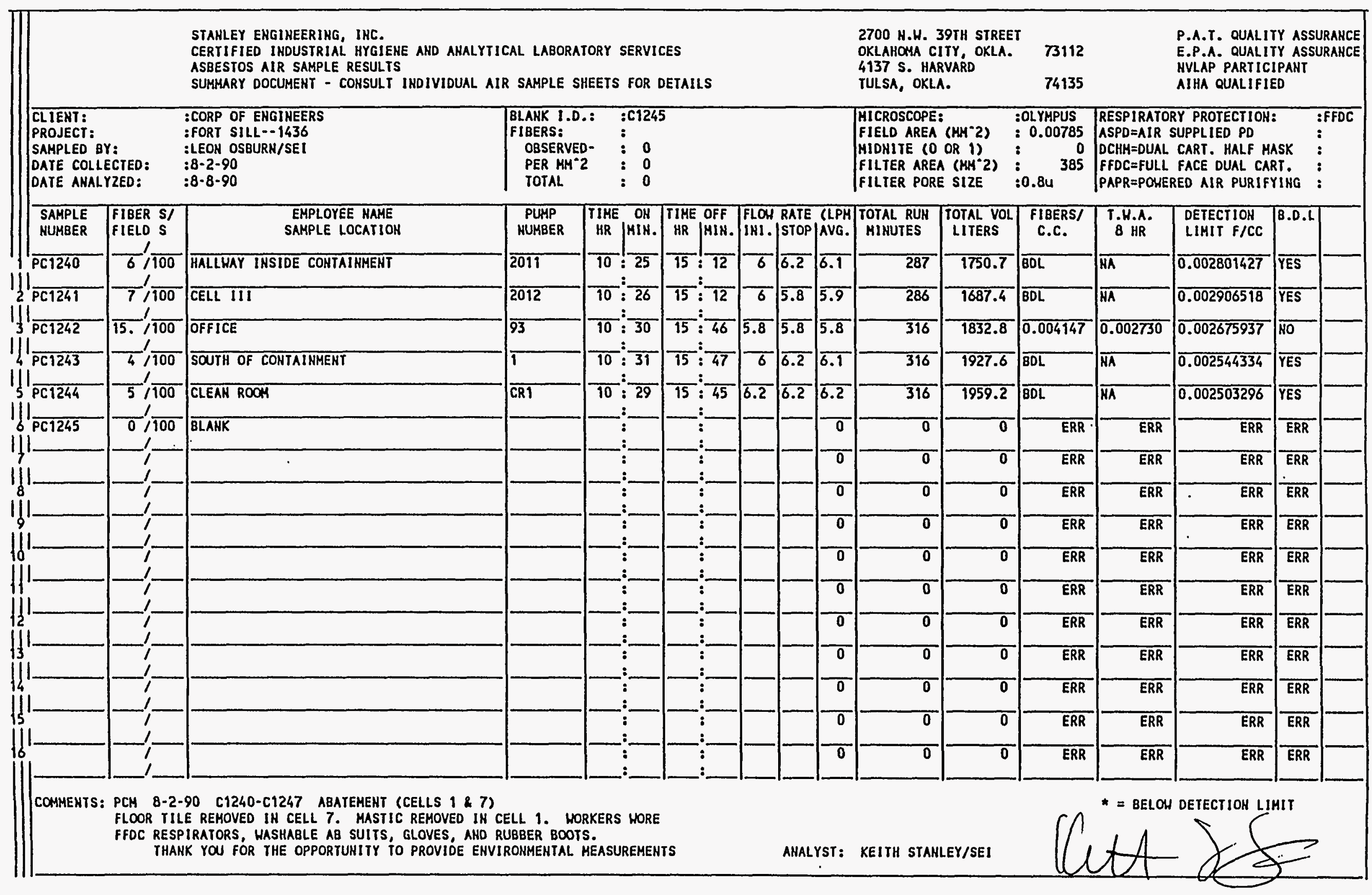




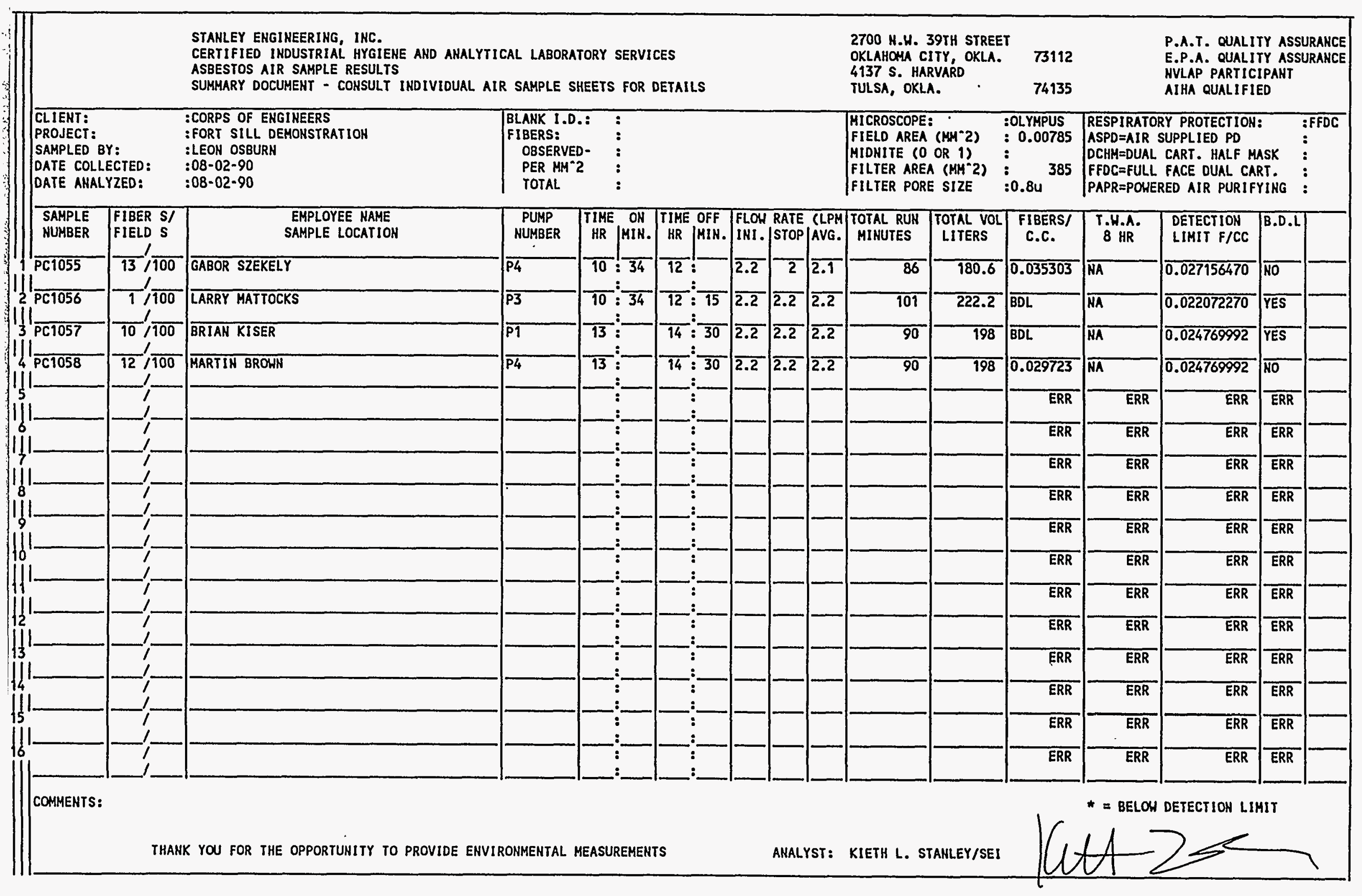




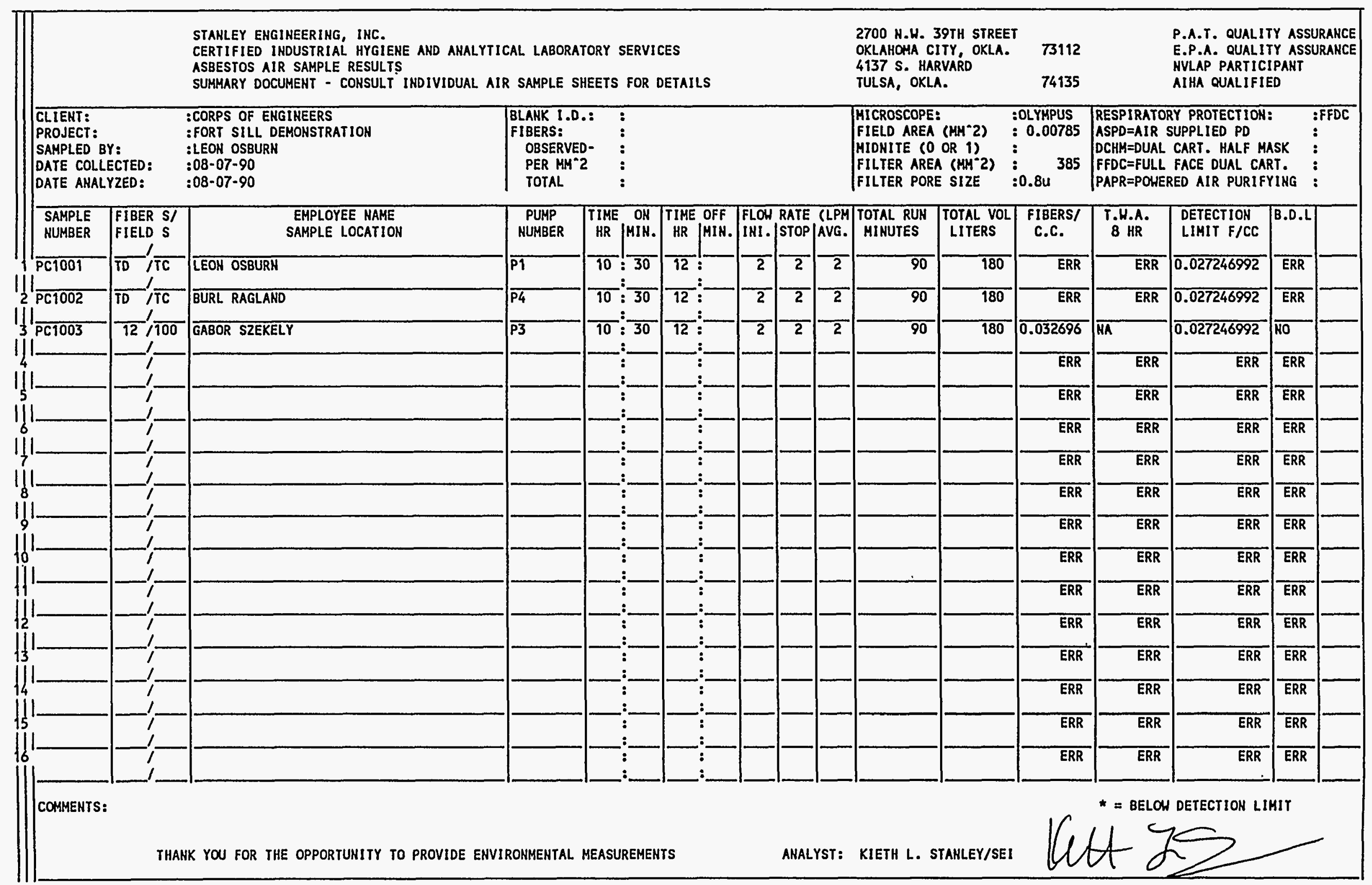




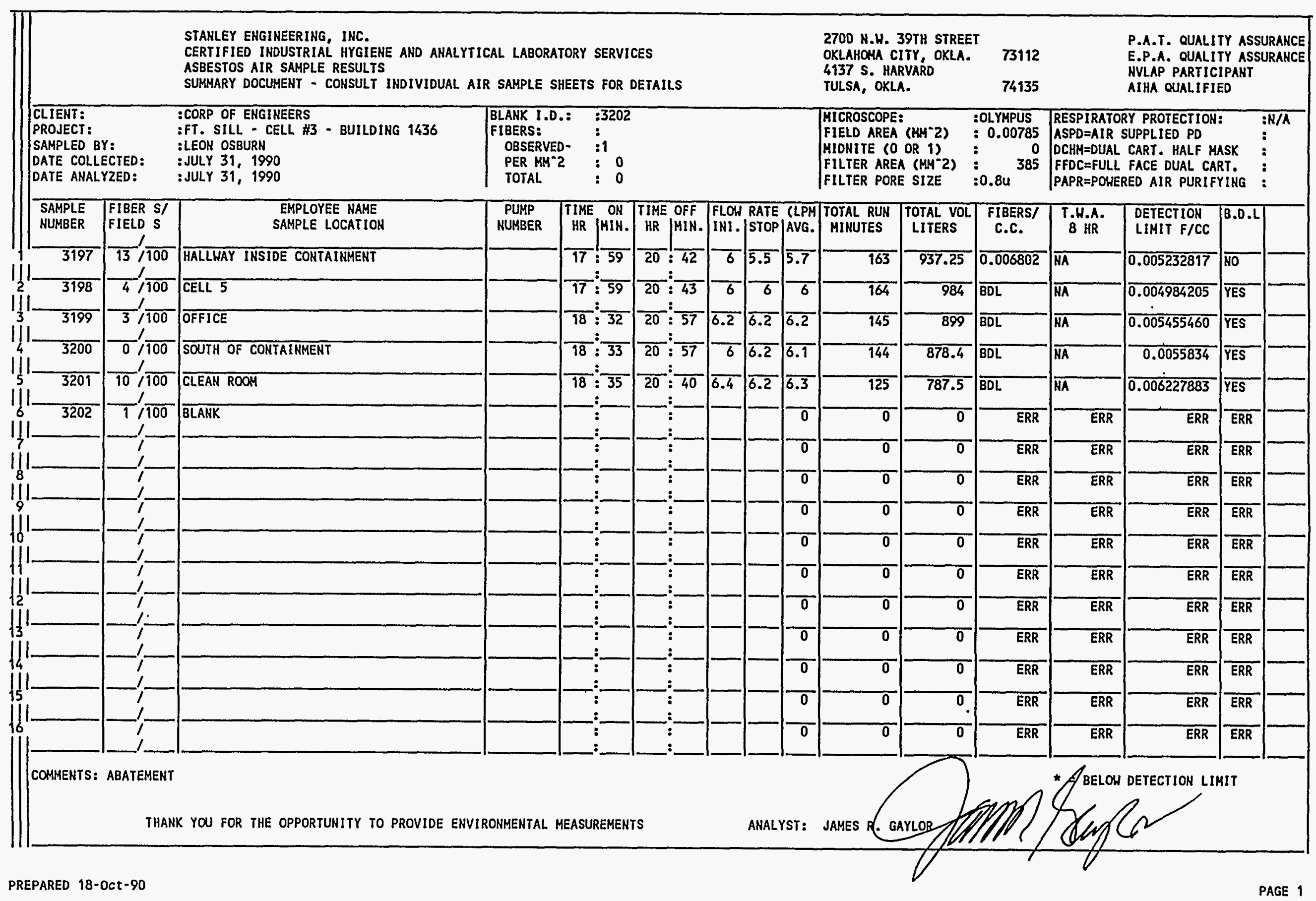




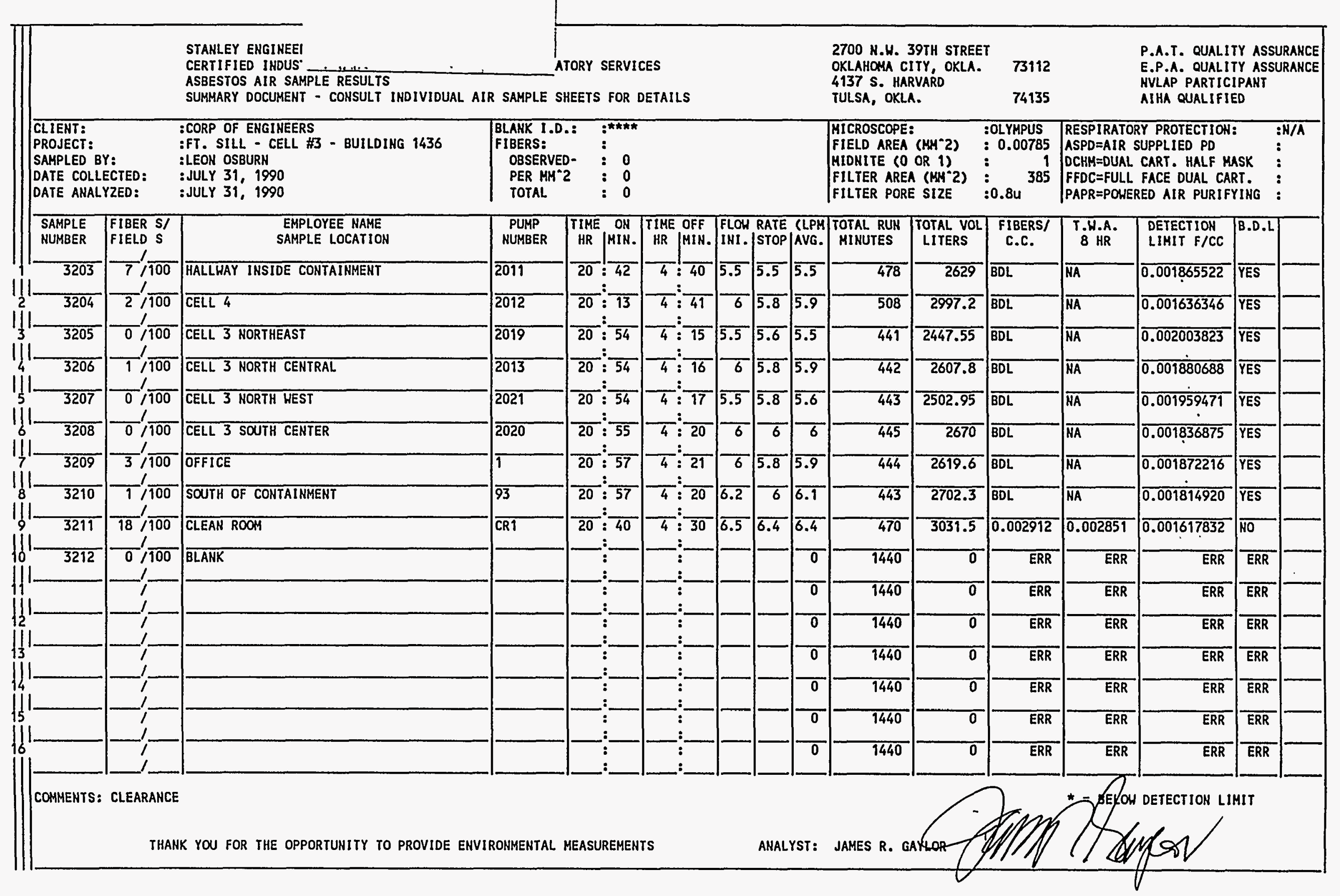




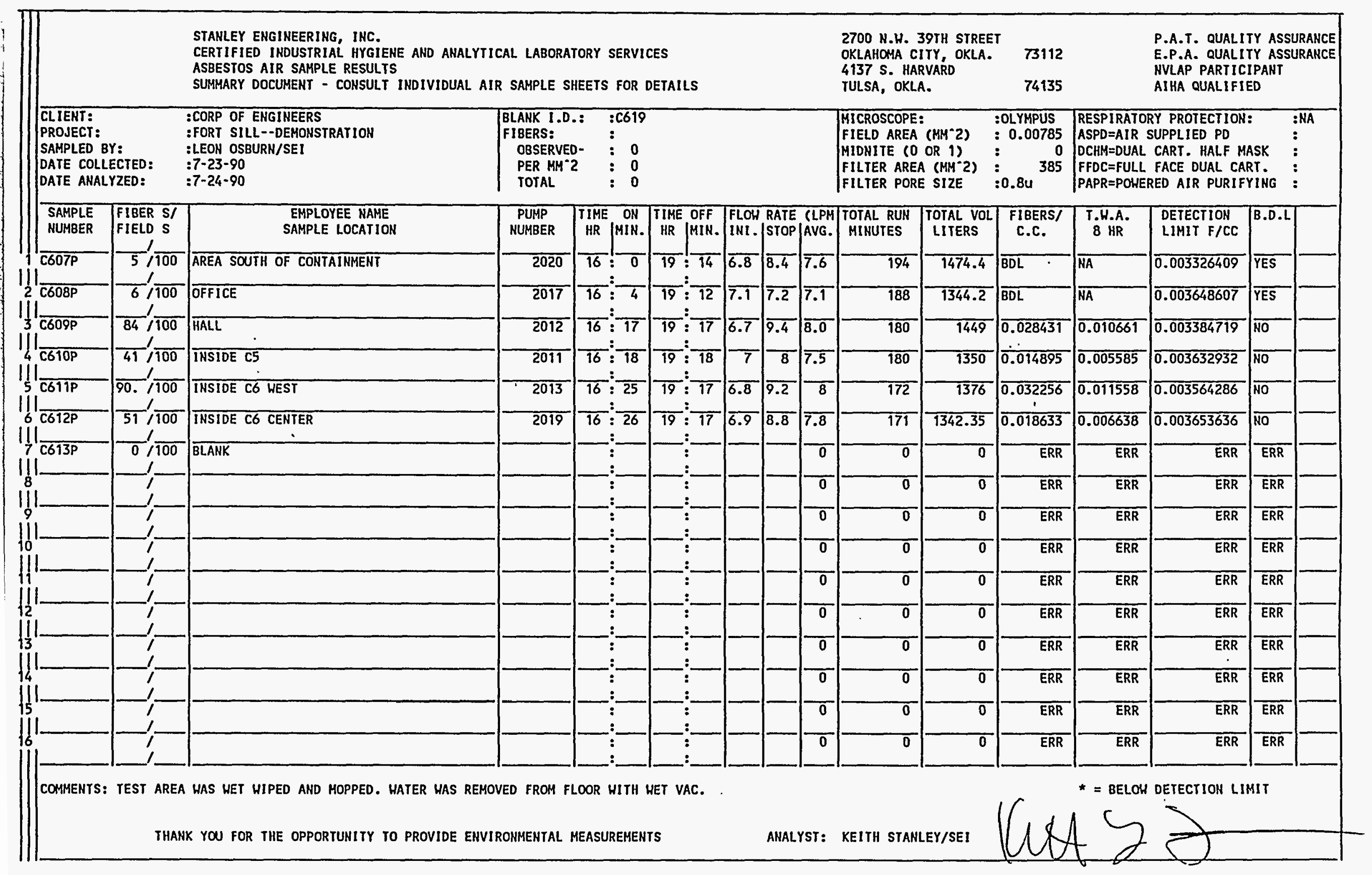




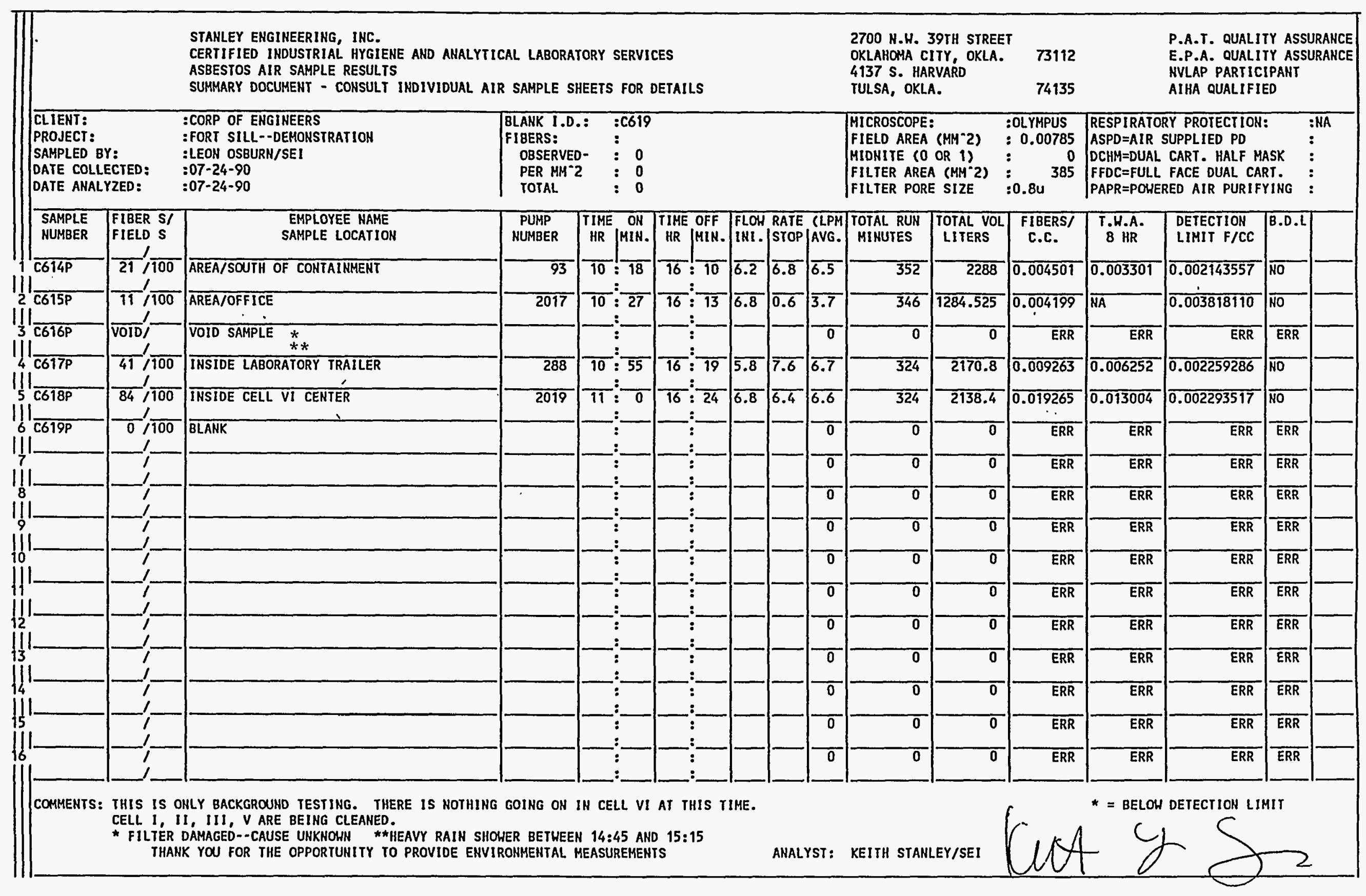




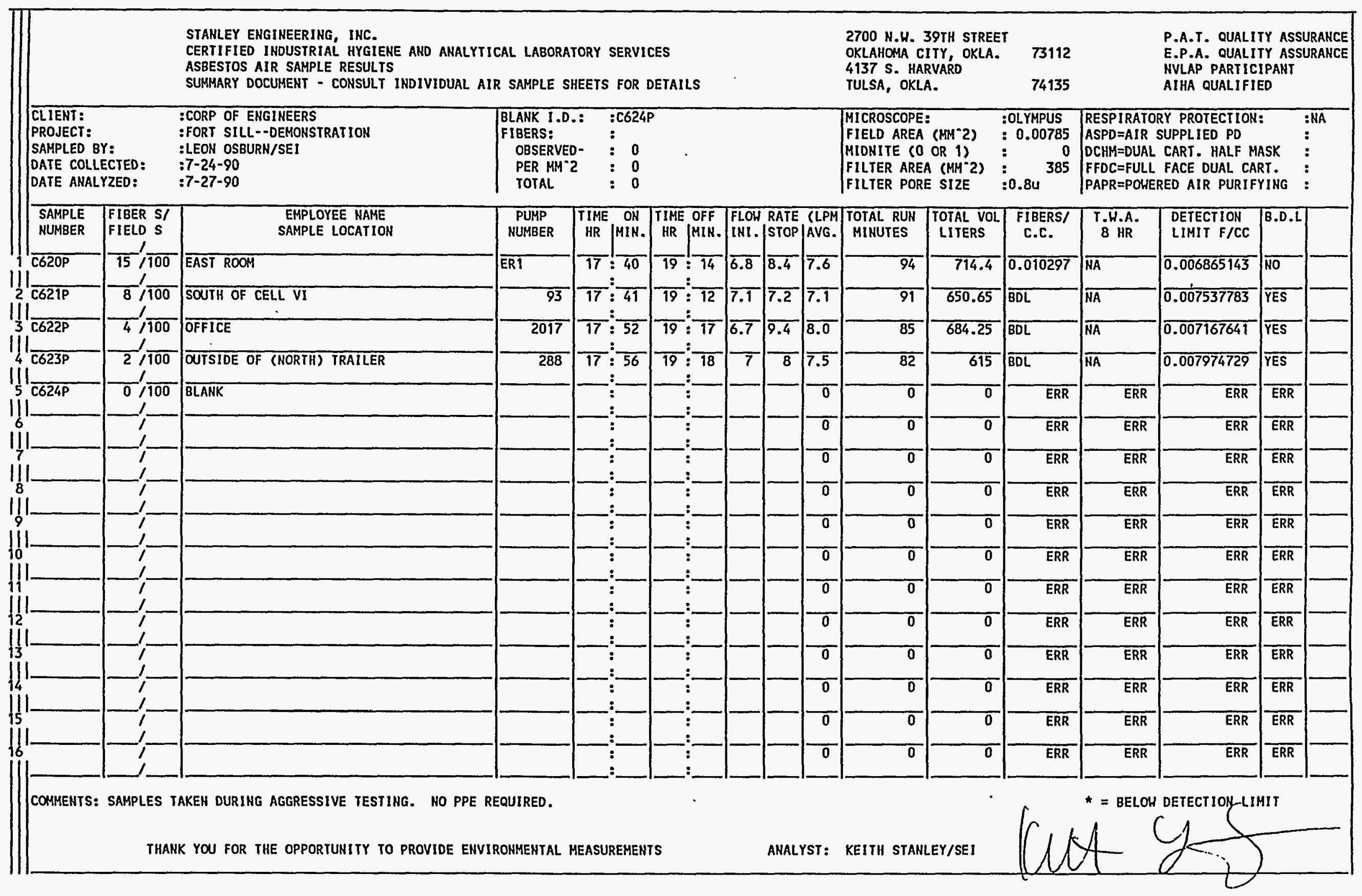




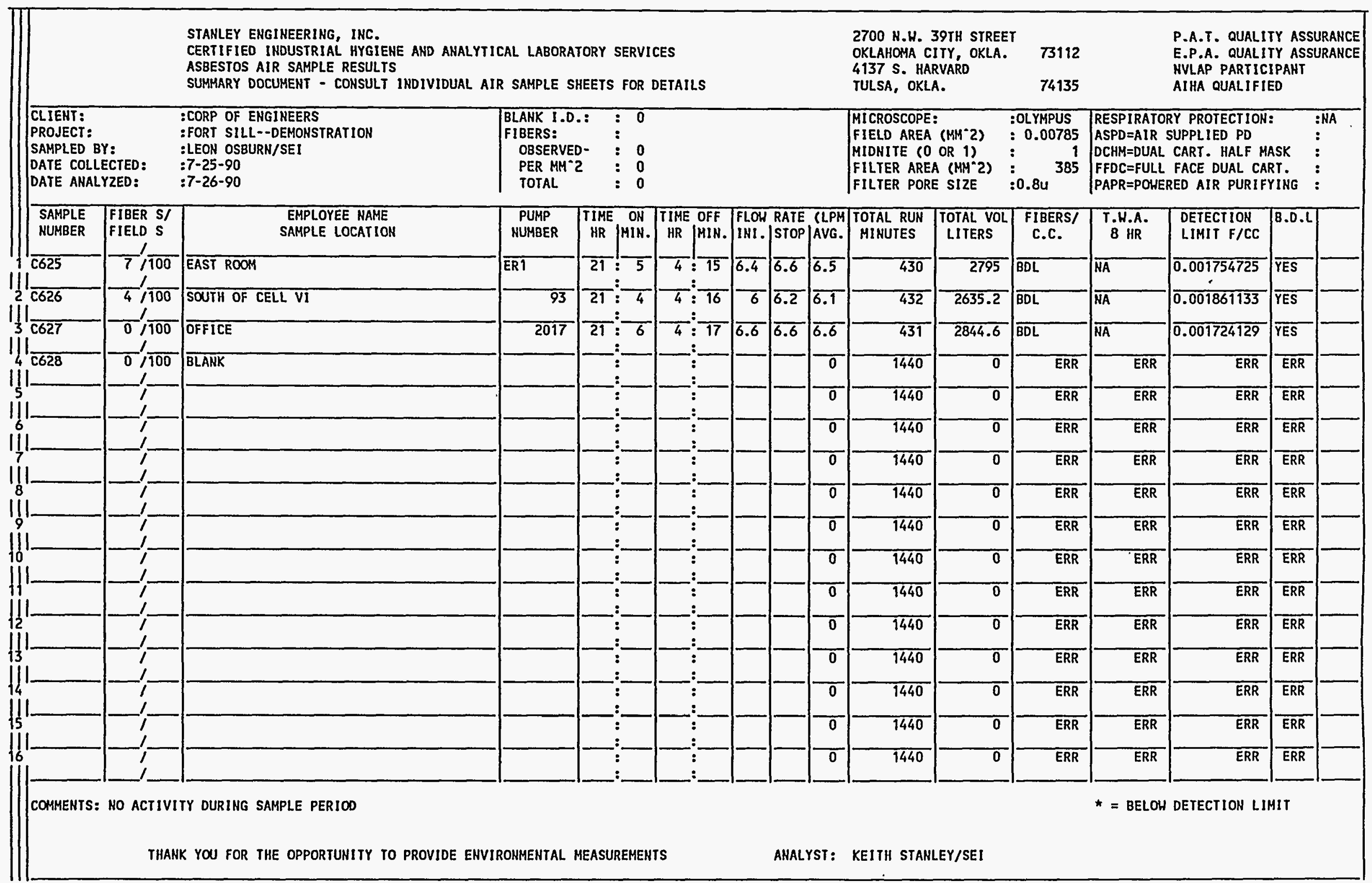




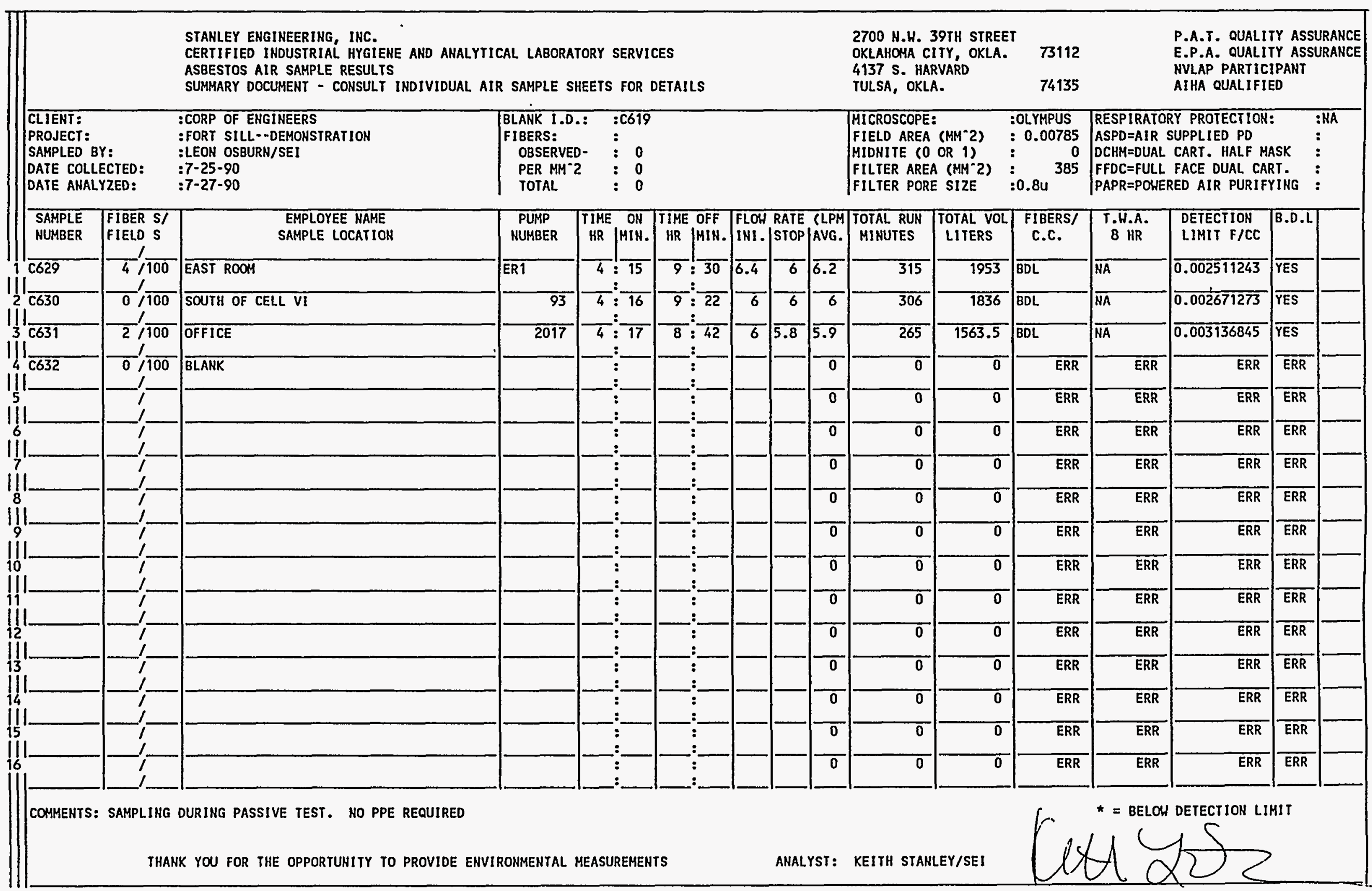




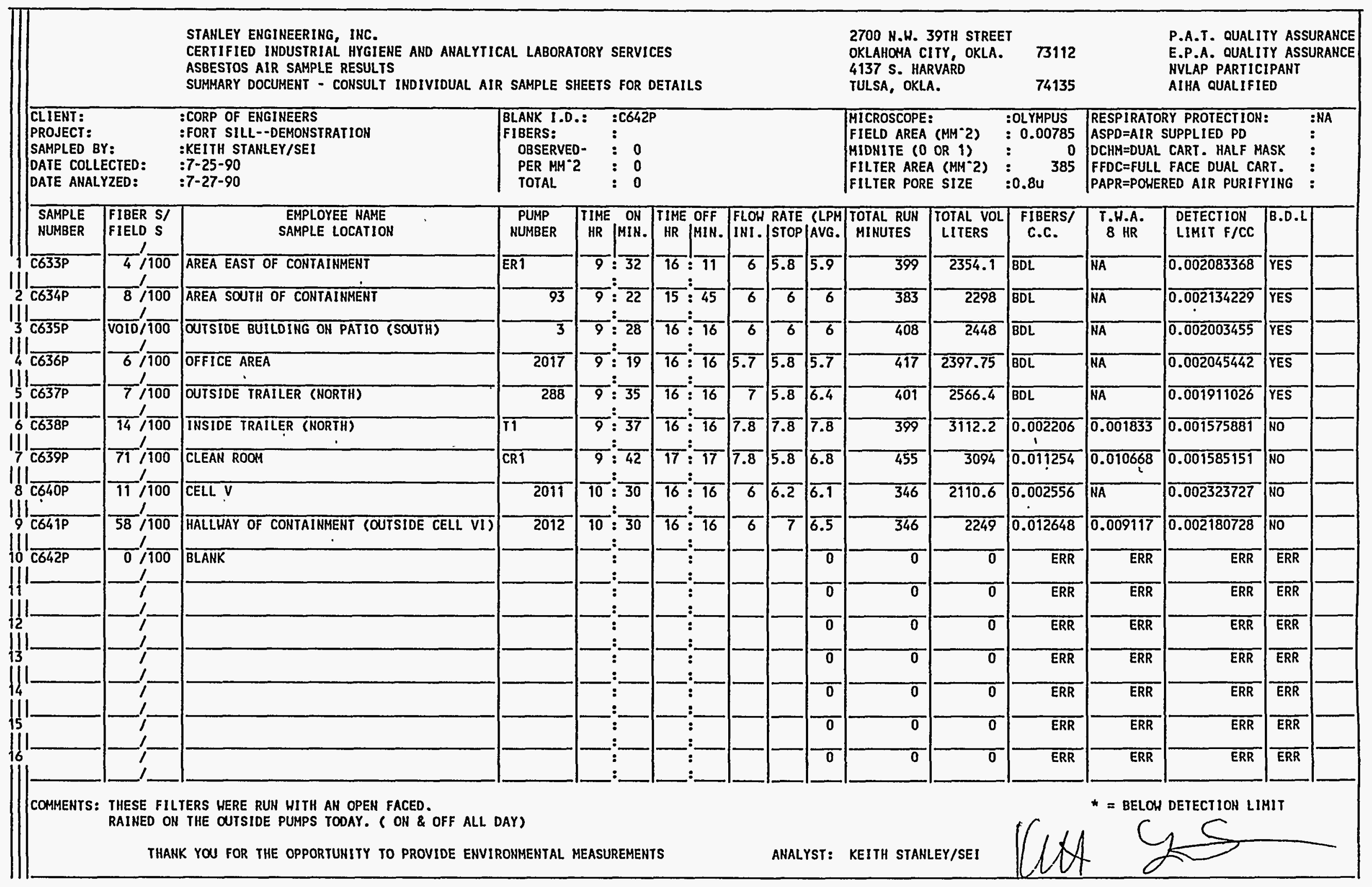




\begin{tabular}{|c|c|c|c|c|c|c|c|c|c|c|c|c|c|c|c|}
\hline & & \multicolumn{7}{|c|}{$\begin{array}{l}\text { STANLEY ENGINEERING, INC. } \\
\text { CERTIFIED INDUSTRIAL HYGIENE AND ANALYTICAL LABORATORY SERVICES } \\
\text { ASBESTOS AIR SAMPLE RESULTS } \\
\text { SUMMARY DOCUMENT - CONSULT INDIVIDUAL AIR SAMPLE SHEETS FOR DETAILS }\end{array}$} & \multicolumn{2}{|c|}{$\begin{array}{l}2700 \text { N.H. 39TH STREET } \\
\text { OKLAHOHA CITY, OKLA. } \\
4137 \text { S. HARVARD } \\
\text { TULSA, OKLA. }\end{array}$} & $\begin{array}{l}73112 \\
74135\end{array}$ & & \multicolumn{3}{|c|}{$\begin{array}{l}\text { P.A.T. QUALITY ASSURANCE } \\
\text { E.P.A. QUALIIY ASSURANCE } \\
\text { NVLAP PARTICIPANT } \\
\text { AIHA QUALIFIED }\end{array}$} \\
\hline \multicolumn{2}{|c|}{$\begin{array}{l}\text { CLIENT: } \\
\text { PROJECI: } \\
\text { SAMPLED BY: } \\
\text { DATE COLLECTED: } \\
\text { DATE ANALYZED: }\end{array}$} & \multirow{2}{*}{$\begin{array}{l}\text { :CORP OF ENGINEERS } \\
\text { :FT. SILL - AFTER ABATEMENT } \\
\text { :LEON OSBURM } \\
: 7-25-90 \\
: 7-27-90 \\
\mid \begin{array}{l}\text { EMPLOYEE NAME } \\
\text { SAMPLE LOCATION }\end{array}\end{array}$} & \multicolumn{3}{|c|}{\begin{tabular}{|ll} 
BLAHK I.D.: & $: 656$ \\
FIBERS: & $:$ \\
OBSERVED- & $: 0$ \\
PER MM & $: 0$ \\
TOIAL & $: 0$
\end{tabular}} & & & & \multicolumn{2}{|c|}{$\begin{array}{l}\text { MICROSCOPE: } \\
\text { FIELD AREA (MM־2) } \\
\text { MIDNITE }(0 \text { OR } 1) \\
\text { FILTER AREA (MM־2) } \\
\text { FILTER PORE SIZE }\end{array}$} & $\begin{array}{l}\text { :OLYMPUS } \\
: 0.00785 \\
\vdots \\
\vdots \\
: 0.8 u^{\quad} \quad 385\end{array}$ & \multicolumn{3}{|c|}{$\begin{array}{l}\text { RESPIRATORY PROTECTION: } \\
\text { ASPD=AIR SUPPLIED PD } \\
\text { DCHM=DUAL CART. HALF MASK } \\
\text { FFDC=FULL FACE DUAL CART. } \\
\text { PAPR=POWERED AIR PURIFYING }\end{array}$} & $\begin{array}{l}: N / A \\
\vdots \\
\vdots \\
\vdots\end{array}$ \\
\hline $\begin{array}{l}\text { SAMPLE } \\
\text { NUMBER }\end{array}$ & $\begin{array}{l}\text { FIBER S/ } \\
\text { FIELD S }\end{array}$ & & $\begin{array}{c}\text { PUMP } \\
\text { NUMBER }\end{array}$ & $\begin{array}{l}\text { TIME ON } \\
\text { HR J } \text { MIN. }\end{array}$ & \begin{tabular}{|c|c|c|c|c|c|} 
TIME OFF \\
HR & MIN.
\end{tabular} & $\begin{array}{l}\text { FLOW } \\
\text { INI. }\end{array}$ & $\begin{array}{l}\text { RATE } \\
\text { STOP }\end{array}$ & $\begin{array}{l}\text { CLPM } \\
\text { |AVG. }\end{array}$ & {$\left[\begin{array}{c}\text { TOTAL RUN } \\
\text { MINUTES }\end{array}\right.$} & $\begin{array}{l}\text { TOTAL VOL } \\
\text { LITERS }\end{array}$ & $\begin{array}{l}\text { FIBERSI } \\
\text { C.C. }\end{array}$ & $\begin{array}{c}\text { T.H.A. } \\
8 \text { HR }\end{array}$ & $\begin{array}{l}\text { DETECTION } \\
\text { LIMIT F/CC }\end{array}$ & $\overline{B . D . L}$ & \\
\hline 643 & $\overline{12 .} / \overline{100}$ & EAST OF CONTAINMENT - CELL 6 & $\overline{\mathrm{ER} 1}$ & $\overline{16}: \overline{14}$ & $8 ! 36$ & 6 & $\overline{5.4}$ & $\overline{5.7}$ & $\overline{982}$ & 5597.4 & $\overline{0.001095}$ & 0.002240 & $\overline{0.000876203}$ & WO & \\
\hline$\overline{644}$ & $14 / 100$ & SOUTH OF CONTAINMENT - C6 & $\overline{93}$ & $16:$ & $8: \overline{34}$ & 6 & $\overline{6.2}$ & $\overline{6.1}$ & $\overline{990}$ & $\overline{6039}$ & $\overline{0.001136}$ & $\overline{0.002345}$ & $\overline{0.000812130}$ & NO & \\
\hline$\overline{645}$ & $9 / \overline{100}$ & OUTSIDE BLDG. ON PATIO (SOUTH) & 3 & $16:$ & 8 & 6 & $\overline{4.8}$ & $\overline{5.4}$ & 996 & 5378.4 & BDL & $\overline{N A}$ & $\overline{0.000911880}$ & $\overline{\text { YES }}$ & \\
\hline 646 & $10, \overline{100}$ & OFFICE AREA & 2017 & $16: \overline{1}$ & $8: \overline{49}$ & 6 & $\overline{5.6}$ & $\overline{5.8}$ & 1008 & 5846.4 & $\overline{B D L}$ & $\overline{\mathrm{MA}}$ & $\overline{0.000838885}$ & $\overline{Y E S}$ & \\
\hline 647 & $17 / \overline{100}$ & OUTSIDE TRAILER (NORTH) & 288 & $16:-$ & & 5.8 & 5 & $\overline{5.8}$ & 958 & 5652.2 & 0.001475 & 0.002944 & $\overline{0.000867707}$ & NO & \\
\hline 648 & $\overline{12 .} / \overline{100}$ & INSIDE TRAILER (NORTH) & $\overline{T 1}$ & $16: \overline{51}$ & 8 & $\overline{7.8}$ & $\overline{7.2}$ & $\overline{7.5}$ & 964 & 7230 & $\overline{0.000847}$ & $\overline{0.001702}$ & $\overline{0.000678348}$ & $\overline{\text { NOO }}$ & \\
\hline 649 & $7 / \overline{100}$ & $\overline{C E L L L} 6$ & $\overline{\mathrm{NP}}$ & $16: \overline{39}$ & $6: \overline{37}$ & 4.2 & $\overline{5.8}$ & 5 & $\overline{838}$ & 4190 & $\overline{B D L}$ & $\overline{N A}$ & $\overline{0.001170515}$ & YES & \\
\hline 650 & 14. & HALLWAY BY CELL 6 & $\overline{2012}$ & $16: \overline{39}$ & $6: \overline{37}$ & 7 & $\overline{7.8}$ & $\overline{7.4}$ & 838 & 6201.2 & $\overline{0.001146}$ & $\overline{0.002002}$ & $\overline{0.000790888}$ & NO & \\
\hline 651 & $9 / \overline{100}$ & HALLHAY OF CONTAINMENT (OUTSIDE CELL 6) & $\overline{N P}$ & $16: \overline{40}$ & 6 & 5 & 6 & $\overline{5.5}$ & $\overline{837}$ & 4603.5 & $\overline{B O L}$ & $\overline{N A}$ & $\overline{0.001065376}$ & $\overline{Y E S}$ & \\
\hline 652 & $8 / \overline{100}$ & $\overline{\text { CELLL } 6}$ & $\overline{\mathrm{NP}}$ & $16: \overline{39}$ & $6: \overline{37}$ & 4.2 & 5 & $\overline{4.6}$ & $\overline{838}$ & $\overline{3854.8}$ & $\overline{B O L}$ & $\overline{N A}$ & $\overline{0.001272299}$ & $\overline{Y E S}$ & \\
\hline 653 & $11 / \overline{100}$ & $\overline{\text { CELL } 6}$ & $\overline{\mathrm{NP}}$ & $16: \overline{43}$ & $6: \overline{37}$ & 5 & $\overline{5.8}$ & 5.4 & $\overline{834}$ & 4503.6 & $\overline{0.001197}$ & $\overline{0.002081}$ & $\overline{0.001089008}$ & $\overline{\text { NO }}$ & \\
\hline 654 & $7 / 100$ & CELL 5 & 2011 & $\overline{16}: \overline{45}$ & $6: \overline{37}$ & 6 & $\overline{6.4}$ & $\overline{6.2}$ & 832 & 5158.4 & $\overline{B D L}$ & $\overline{\mathrm{NA}}$ & $\overline{0.000950771}$ & $\overline{Y E S}$ & \\
\hline 6008 & $0 / \overline{100}$ & BURL RAGLAND: $447-38-1989$ & $\overline{p 7}$ & $16: \overline{50}$ & $17: 35$ & 2 & 1.7 & $\overline{1.8}$ & 1485 & 2747.25 & $\overline{B D L}$ & $\overline{N A}$ & $\overline{0.001785224}$ & $\overline{\text { YES }}$ & \\
\hline$\overline{655}$ & $11 / \overline{100}$ & CLEAN ROOM & $\overline{\mathrm{CR} 1}$ & $17: \overline{10}$ & $8: 59$ & 5.8 & $\overline{6.2}$ & $\overline{6}$ & 949 & $\overline{5694}$ & $\overline{0.000947}$ & $\overline{0.001873}$ & $\overline{0.000861338}$ & $\overline{\text { NO }}$ & \\
\hline 656 & $0 / \overline{100}$ & $\overline{\text { BLANK }}$ & & $:$ & & & & $\overline{0}$ & $\overline{1440}$ & $\overline{0}$ & ERR & ERR & $\overline{E R R}$ & $\overline{\text { ERR }}$ & \\
\hline & & & & & & & & $\overline{0}$ & 1440 & 0 & $\overline{\text { ERR }}$ & $\overline{\text { ERR }}$ & $\overline{\text { ERR }}$ & $\overline{E R R}$ & \\
\hline COMMENTS: & AFTER ABA & ITEMENT IN CELL 6 & & & & & & & & & & BELO & Crion & & \\
\hline
\end{tabular}




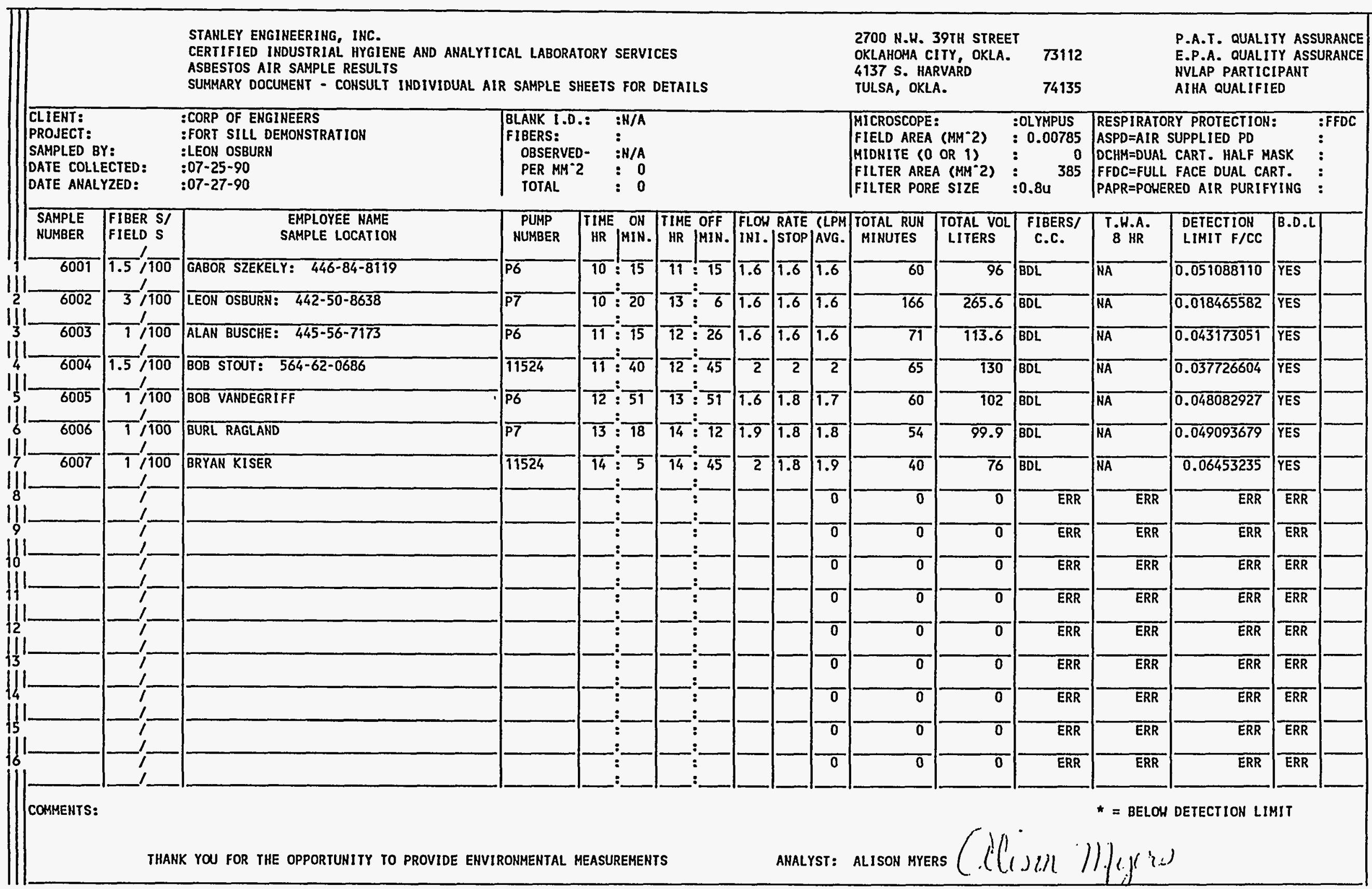




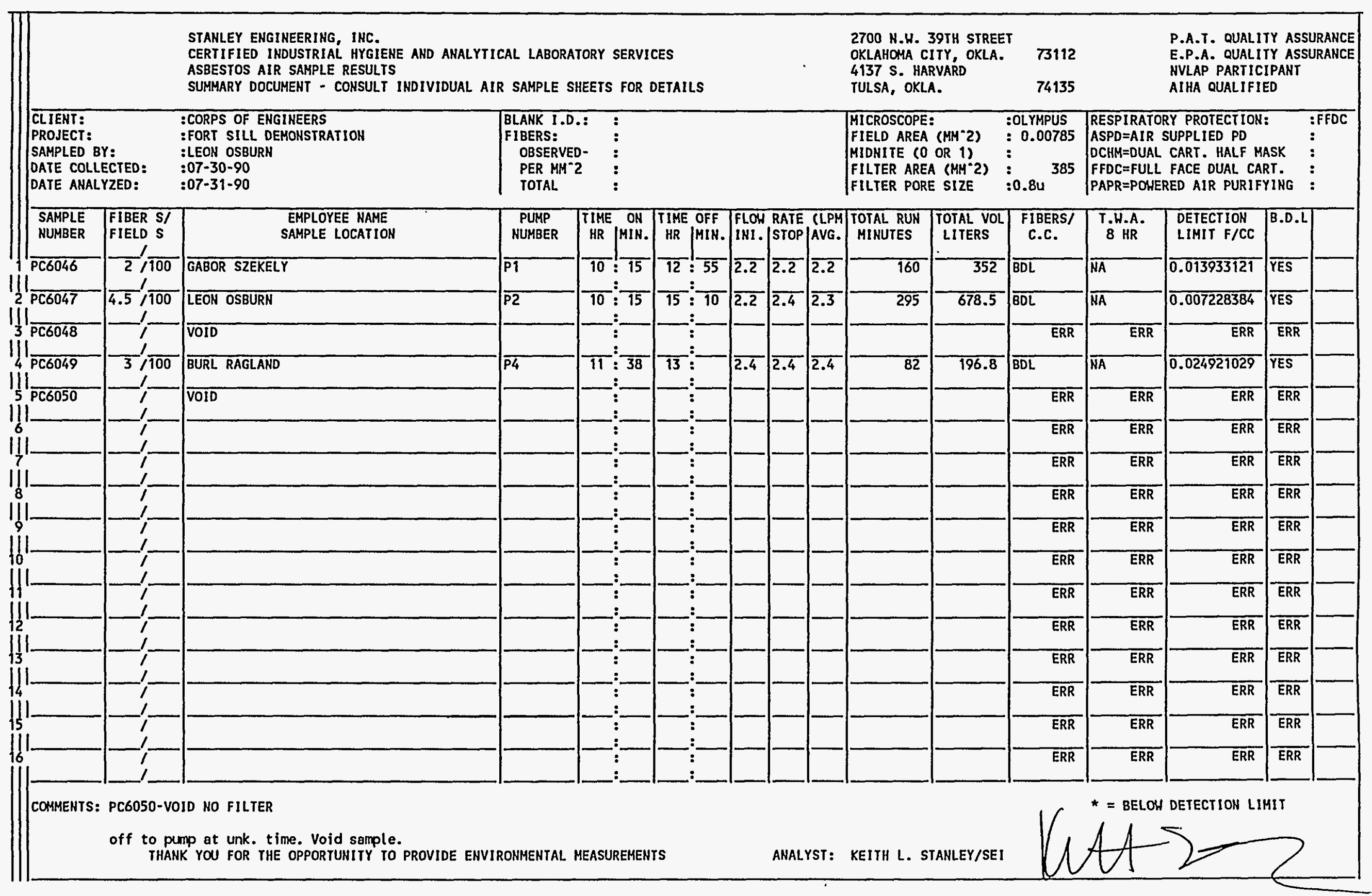




\begin{tabular}{|c|c|c|c|c|c|c|c|c|c|c|c|c|c|c|c|}
\hline & & \multicolumn{7}{|c|}{$\begin{array}{l}\text { STANLEY ENGINEERING, INC. } \\
\text { CERTIFIED INDUSTRIAL HYGIENE AND ANALYTICAL LABORATORY SERVICES } \\
\text { ASBESTOS AIR SAMPLE RESULTS } \\
\text { SUMMARY DOCUMENT - CONSULT INDIVIDUAL AIR SAMPLE SHEETS FOR DETAILS }\end{array}$} & \multicolumn{4}{|c|}{$\begin{array}{l}2700 \text { N.H. 39TH STREET } \\
\text { OKLAHOMA CITY, OKLA. } \\
4137 \text { S. HARVARD } \\
\text { TULSA, OKLA. }\end{array}$} & \multicolumn{3}{|c|}{$\begin{array}{l}\text { P.A.T. QUALITY ASSURANCE } \\
\text { E.P.A. QUALITY ASSURANCE } \\
\text { NVLAP PARTICIPANT } \\
\text { AIHA QUALIFIED }\end{array}$} \\
\hline \multicolumn{2}{|c|}{$\begin{array}{l}\text { CLIENT: } \\
\text { PROJECT: } \\
\text { SAMPLED BY: } \\
\text { DATE COLLECTED: } \\
\text { DATE ANALYZED: }\end{array}$} & $\begin{array}{l}\text { :CORP OF ENGINEERS } \\
\text { :FORT SILL }--B 1436 \\
\text { :LEON OSBURN/SEI } \\
: 7-30-90 \\
: 8-3-90\end{array}$ & \multicolumn{3}{|c|}{\begin{tabular}{|ll} 
BLANK 1.D.: & $:$ C1161 \\
FIBERS: & $:$ \\
OBSERVEO- & $: 0$ \\
PER MM-2 & $: 00$ \\
TOTAL & $: 0$
\end{tabular}} & & & & \multicolumn{2}{|c|}{$\begin{array}{l}\text { MICROSCOPE: } \\
\text { FIELD AREA }\left(M M^{-2} 2\right) \\
\text { MIDNITE (O OR } 1) \\
\text { FILTER AREA }\left(M M^{\wedge} 2\right) \\
\text { FILTER PORE SIZE }\end{array}$} & $\begin{array}{lr}: O L Y M P U S \\
: 0.00785 \\
: & 0 \\
: 0.8 u^{385} & \end{array}$ & \multicolumn{3}{|c|}{$\begin{array}{l}\text { RESPIRATORY PROTECTION: } \\
\text { ASPD=AIR SUPPLIED PD } \\
\text { DCHM=DUAL CART. HALF MASK } \\
\text { FFOC=FULL FACE DUAL CART. } \\
\text { PAPR=POWERED AIR PURIFYING }\end{array}$} & $\begin{array}{l}: \text { NA } \\
\vdots \\
\vdots \\
\vdots\end{array}$ \\
\hline $\begin{array}{l}\text { SAMPLE } \\
\text { NUMBER }\end{array}$ & $\begin{array}{l}\text { FIBER SI } \\
\text { FIELD S }\end{array}$ & $\begin{array}{l}\text { EMPLOYEE NAME } \\
\text { SAMPLE LOCATION }\end{array}$ & $\begin{array}{l}\text { PUMP } \\
\text { NUMBER }\end{array}$ & \begin{tabular}{|l|l|} 
TIME ON \\
HR MIN.
\end{tabular} & $\begin{array}{l}\text { TIME OFF } \\
\text { HR |MIN. }\end{array}$ & $\begin{array}{l}\text { FLOH } \\
\text { INI. }\end{array}$ & $\begin{array}{l}\text { RATE } \\
\text { STOP }\end{array}$ & $\begin{array}{l}\text { (LPM } \\
\text { AVG. }\end{array}$ & \begin{tabular}{|l|} 
TOTAL RUN \\
MINUTES
\end{tabular} & $\begin{array}{l}\text { TOTAL VOL } \\
\text { LITERS }\end{array}$ & $\begin{array}{l}\text { FIBERS/ } \\
\text { C.C. }\end{array}$ & $\begin{array}{l}\text { T.W.A. } \\
8 \text { HR }\end{array}$ & $\begin{array}{l}\text { DETECTION } \\
\text { LIMIT F/CC }\end{array}$ & B.D.L & \\
\hline$\overline{c 1155}$ & $11 / \overline{100}$ & HALLWAY INSIDE CONTAINMENT & & $12: 16$ & $\overline{16}: \overline{20}$ & $\overline{6}$ & $\overline{5.9}$ & $\overline{5.9}$ & 244 & $\overline{1451.8}$ & $\overline{0.003716}$ & $\overline{\mathrm{NA}}$ & $\overline{0.003378191}$ & No & \\
\hline$\overline{c 1156}$ & $4 / \overline{100}$ & CELL II & & $12: \overline{17}$ & $\overline{16}: \overline{21}$ & 6 & 6 & 6 & 24 & 1464 & $\overline{\mathrm{BDL}}$ & $\overline{\mathrm{NA}}$ & 0.00335004 & $\overline{Y E S}$ & \\
\hline C1157 & $21 / 100$ & CLEAN ROOM & $\overline{\mathrm{CR} 1}$ & $12: \overline{8}$ & $\overline{16}: \overline{1}$ & $\overline{6.3}$ & $\overline{7.4}$ & $\overline{6.8}$ & $\overline{233}$ & 1596.05 & $\overline{0.006453}$ & $\overline{0.003132}$ & 0.003072872 & $\overline{\text { NO }}$ & \\
\hline$\overline{c 1158}$ & $6 / \overline{100}$ & OFFICE & $T$ & $12: \overline{4}$ & $\overline{15}: \overline{55}$ & $\overline{6}$ & 6 & $\overline{6}$ & $\overline{231}$ & 1386 & $\overline{B D L}$ & $\overline{\mathrm{NA}}$ & $\overline{0.003538570}$ & $\overline{\text { YES }}$ & \\
\hline$\overline{C 1159}$ & $\overline{1.5} / \overline{100}$ & AREA SOUTH OF CONTAIMMENT & $\overline{93}$ & $12:-2$ & $\overline{15}: \overline{58}$ & $\overline{5.8}$ & $\overline{6}$ & 5.9 & 236 & 1392.4 & $\overline{B D L}$ & $\overline{\mathrm{NA}}$ & $\overline{0.003522305}$ & $\overline{Y E S}$ & \\
\hline$\overline{c 1160}$ & $3 / \overline{100}$ & AREA EAST OF CONTAINMENT & $\overline{\mathrm{ER} 1}$ & $12:-6$ & $\overline{15}: \overline{59}$ & $\overline{5.8}$ & $\overline{6}$ & $\overline{5.9}$ & $\overline{233}$ & 1374.7 & $\overline{\mathrm{BDL}}$ & $\overline{\mathrm{NA}}$ & $\overline{0.003567657}$ & $\overline{\text { YES }}$ & \\
\hline c1161 & $0 / \overline{100}$ & $\overline{\text { BLANK }}$ & & 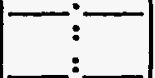 & & & & $\overline{0}$ & $\overline{0}$ & 0 & $\overline{\text { ERR }}$ & ERR & $\overline{\text { ERR }}$ & $\overline{\text { ERR }}$ & \\
\hline & & & & 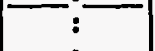 & & & & $\overline{0}$ & & $\overline{0}$ & $\overline{\text { ERR }}$ & ERR & $\overline{E R R}$ & $\overline{\text { ERR }}$ & \\
\hline & & & & $:$ & & & & $\overline{0}$ & $\overline{0}$ & $\overline{0}$ & $\overline{\text { ERR }}$ & $\overline{E R R}$ & $\overline{\text { ERR }}$ & $\overline{\text { ERR }}$ & \\
\hline & & & & $\because-$ & & & & $\overline{0}$ & $\overline{0}$ & $\overline{0}$ & $\overline{\text { ERR }}$ & $\overline{\text { ERR }}$ & $\overline{\text { ERR }}$ & $\overline{\text { ERR }}$ & \\
\hline & & & & $:$ & & & & $\overline{0}$ & $\overline{0}$ & $\overline{0}$ & $\overline{\text { ERR }}$ & $\overline{E R R}$ & $\overline{\text { ERR }}$ & $\overline{\text { ERR }}$ & \\
\hline & 1 & & & $\because$ & & & & 0 & 0 & $\overline{0}$ & $\overline{\text { ERR }}$ & $\overline{\text { ERR }}$ & $\overline{\text { ERR }}$ & $\overline{\text { ERR }}$ & \\
\hline & 1 & & & 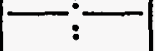 & & & & 0 & & & $\overline{\text { ERR }}$ & ERR & $\overline{\text { ERR }}$ & $\overline{\text { ERR }}$ & \\
\hline & & & & $\because-$ & & & & $\overline{0}$ & $\overline{0}$ & $\overline{0}$ & $\overline{E R \bar{R}}$ & $\overline{E R R}$ & $\overline{\text { ERR }}$ & $\overline{\text { ERR }}$ & \\
\hline & & & & & & & & $\overline{0}$ & $\overline{0}$ & 0 & $\overline{\text { ERR }}$ & $\overline{\text { ERR }}$ & $\overline{\text { ERR }}$ & $\overline{\text { ERR }}$ & \\
\hline & & & & : & & & & $\overline{0}$ & 0 & $\overline{0}$ & $\overline{\text { ERR }}$ & ERR & $\overline{\text { ERR }}$ & $\overline{\text { ERR }}$ & \\
\hline COMMENTS: & $\begin{array}{l}\text { PCM } 7-30- \\
\text { FLOOR TIL } \\
\text { HASHABLE } \\
\text { THAN }\end{array}$ & $\begin{array}{l}90 \text { C1155-C1161 ABATEMENT } \\
\text { E REMOVED HITH STRAIGHT HOE. ALL WORKERS } \\
\text { AB SUITS, GLOVES, AND RUBBER BOOTS. } \\
\text { K YOU FOR THE OPPORTUNITY TO PROVIDE ENVIR }\end{array}$ & $\begin{array}{l}\text { HORE FFDC } \\
\text { RONMENTAL }\end{array}$ & $\begin{array}{l}\text { RESPIRATOR } \\
\text { MEASUREMENT }\end{array}$ & & & ANALY & YST : & KEITH STANL & EY/SEI & & $\star=$ & TION LIS & & \\
\hline
\end{tabular}




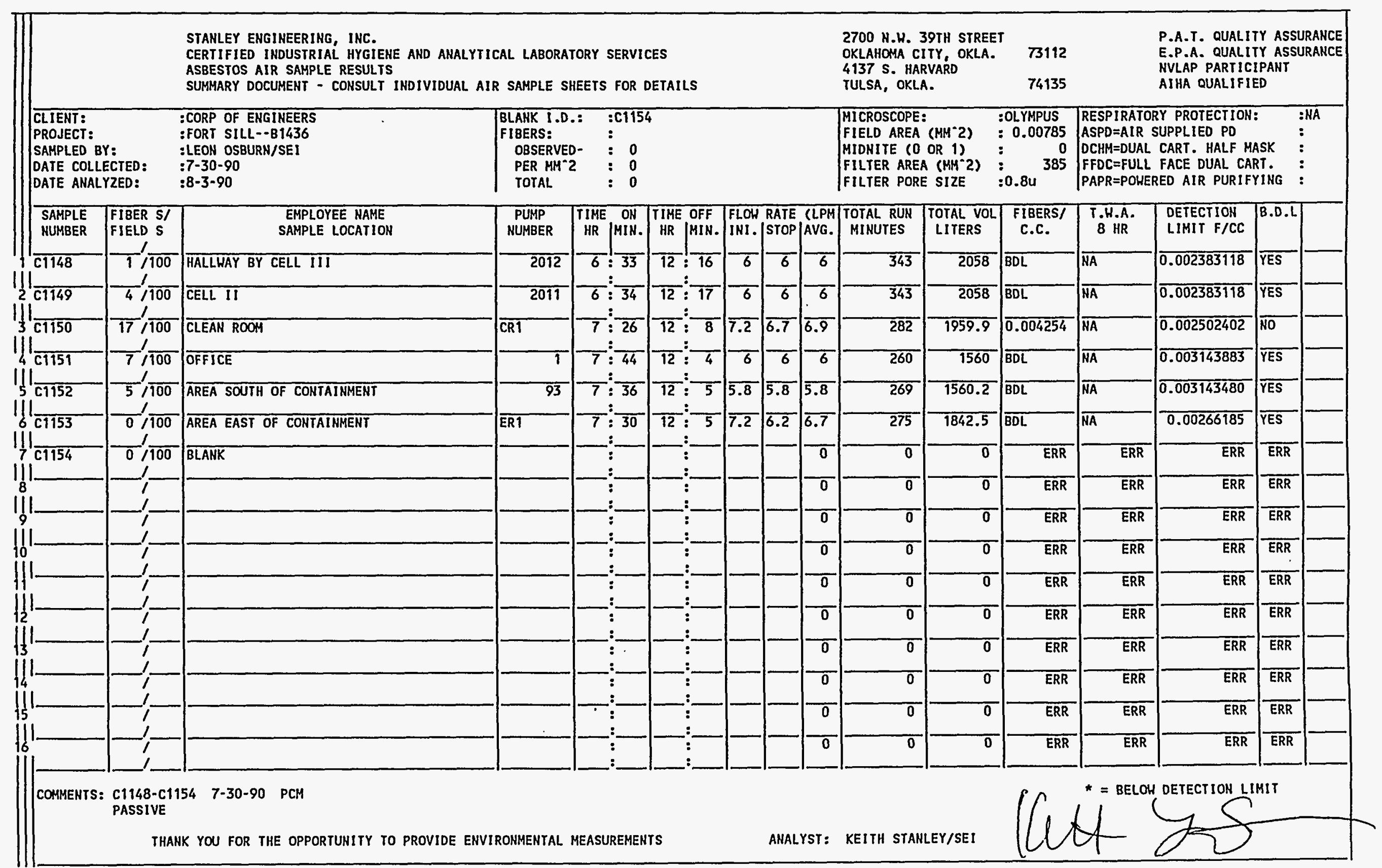




\begin{tabular}{|c|c|c|c|c|c|c|c|c|c|c|c|c|c|c|c|}
\hline & & \multicolumn{7}{|c|}{$\begin{array}{l}\text { STANLEY ENGINEERING, INC. } \\
\text { CERIIFIED INDUSTRIAL HYGIENE AND ANALYTICAL LABORATORY SERVICES } \\
\text { ASBESOS AIR SAMPLE RESULTS } \\
\text { SUMMARY DOCUMENT - CONSULT INDIVIDUAL AIR SAMPLE SHEETS FOR DETAILS }\end{array}$} & \multicolumn{2}{|c|}{$\begin{array}{l}2700 \text { N.H. 39TH STREET } \\
\text { OKLAHOMA CITY, OKLA. } \\
4137 \text { S. HARVARD } \\
\text { TULSA, OKLA. }\end{array}$} & $\begin{array}{l}73112 \\
74135\end{array}$ & & \multicolumn{3}{|c|}{$\begin{array}{l}\text { P.A.T. QUALITY ASSURANCE } \\
\text { E.P.A. QUALITY ASSURANCE } \\
\text { NVLAP PARTICIPANT } \\
\text { AIHA QUALIFIED }\end{array}$} \\
\hline \multicolumn{2}{|c|}{$\begin{array}{l}\text { CLIENT: } \\
\text { PROJECT: } \\
\text { SAMPLED BY: } \\
\text { DATE COLLECTED: } \\
\text { DATE ANALYZED: }\end{array}$} & \multirow{2}{*}{ 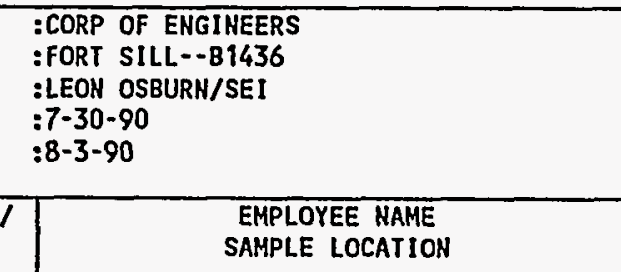 } & \multicolumn{3}{|c|}{\begin{tabular}{|ll} 
BLANK I.D.: & $:$ C1172 \\
F1BERS: & $\vdots$ \\
OBSERVED- & $\vdots 1$ \\
PER MM-2 & $\vdots 1.2$ \\
TOTAL & $: 490$
\end{tabular}} & & & & \multicolumn{2}{|c|}{$\begin{array}{l}\text { MICROSCOPE: } \\
\text { FIELD AREA (MM-2) } \\
\text { MIDNITE }(0 \text { OR } 1) \\
\text { FILTER AREA }\left(M M^{-2} 2\right) \\
\text { FILTER PORE SIZE }\end{array}$} & $\begin{array}{l}: \text { OLYMPUS } \\
: 0.00785 \\
: \quad 1 \\
: \quad 385 \\
: 0.8 u^{r}\end{array}$ & \multicolumn{3}{|c|}{$\begin{array}{l}\text { RESPIRATORY PROTECTION: } \\
\text { ASPD=AIR SUPPLIED PD } \\
\text { DCHH=DUAL CART. HALF MASK } \\
\text { FFDC=FULL FACE DUAL CART. } \\
\text { PAPR=POWERED AIR PURIFYING }\end{array}$} & $\begin{array}{l}: N A \\
\vdots \\
\vdots \\
\vdots\end{array}$ \\
\hline $\begin{array}{l}\text { SAMPLE } \\
\text { NUMBER }\end{array}$ & $\begin{array}{l}\text { FIBER S/ } \\
\text { FIELD S }\end{array}$ & & $\begin{array}{l}\text { PUMP } \\
\text { NUMBER }\end{array}$ & $\begin{array}{c}\text { TIME } \\
\text { ON } \\
\text { OHIN. }\end{array}$ & $\begin{array}{l}\text { TIME OFF } \\
\text { HR | } \text { MIN. }\end{array}$ & $\begin{array}{l}\text { FLOW } \\
\text { INI. }\end{array}$ & $\begin{array}{l}\text { RATE } \\
\text { STOP }\end{array}$ & $\begin{array}{l}\text { (LPM } \\
\text { AVG. }\end{array}$ & $\begin{array}{l}\text { TOTAL RUN } \\
\text { MINUTES }\end{array}$ & $\begin{array}{l}\text { TOTAL VOL } \\
\text { LITERS }\end{array}$ & $\begin{array}{l}\text { FIBERSI } \\
\text { C.C. }\end{array}$ & $\begin{array}{l}\text { T.H.A. } \\
8 \mathrm{HR}\end{array}$ & $\begin{array}{l}\text { DETECTION } \\
\text { LIMIT F/CC }\end{array}$ & B.D.L & \\
\hline${ }^{\mathrm{c1162}}$ & $6 / 100$ & HALLHAY BY CELLL I & 2011 & $16: 20$ & 4 & 6 & $\overline{5.8}$ & $\overline{5.9}$ & 735 & 4336.5 & $\overline{B D L}$ & $\overline{N A}$ & $\overline{0.001130971}$ & $\overline{\text { YES }}$ & \\
\hline $2^{21163}$ & $4 / 100$ & CELL II & 2012 & $16: 20$ & $\overline{35}$ & $\overline{5.9}$ & 5 & $\overline{5.9}$ & 735 & 4373.25 & BDL & $\overline{N A}$ & 0.001121467 & Y̌ES & \\
\hline 311 & $3 / \overline{100}$ & AREA NORTHEAST OF CELL I & 2019 & $16: \overline{28}$ & $4: \overline{37}$ & $\overline{6}$ & $\overline{5.6}$ & $\overline{5.8,}$ & 729 & 4228.2 & $\overline{B D L}$ & $\overline{\text { MA }}$ & 0.001159940 & $\overline{\text { YES }}$ & \\
\hline c1165 & $7 / \overline{100}$ & AREA HORTH CENTER OF CELL I & $\overline{2013}$ & $\overline{16}: \overline{28}$ & $\overline{4}: \overline{37}$ & $\overline{6}$ & $\overline{5.8}$ & $\overline{5.9}$ & 729 & 4301.1 & BOL & $\overline{M A}$ & $\overline{0.001140280}$ & $\overline{\text { YES }}$ & \\
\hline 61166 & $75, \overline{100}$ & AREA NORTH HEST OF CELL I & 2021 & $16: \overline{28}$ & $4: \overline{37}$ & $\overline{6}$ & 6 & 6 & 729 & $\overline{4374}$ & 0.001569 & 0.002384 & $\overline{0.001121275}$ & $\overline{\text { NO }}$ & \\
\hline$6 \longdiv { 6 1 1 6 7 }$ & $\overline{8} / \overline{100}$ & AREA SOUYH HEST OF CELL I & 2017 & $16: \overline{28}$ & $4: \overline{37}$ & 6 & $\overline{5.8}$ & $\overline{5.9}$ & 729 & 4301.1 & $\overline{B D L}$ & $\overline{\mathrm{NA}}$ & $\overline{0.001140280}$ & YES & \\
\hline $7 \overline{c 1168}$ & $3 / \overline{100}$ & EAST ROOM & $\overline{\text { ER1 }}$ & $\overline{15}: \overline{59}$ & $\overline{4}: \overline{37}$ & $\overline{5.8}$ & 6 & $\overline{5.9}$ & 758 & 4472.2 & $\overline{B D L}$ & $\overline{\text { HA }}$ & $\overline{0.001096654}$ & $\overline{\text { YES }}$ & \\
\hline $8 \overline{c 1169}$ & $4 / \overline{100}$ & AREA SOUTH OF CONTAIMMENT & $\overline{93}$ & $15: \overline{58}$ & $6:-15$ & $\overline{6.2}$ & 6 & $\overline{6.1}$ & $\overline{857}$ & 5227.7 & $\overline{B D L}$ & $\overline{N A}$ & $\overline{0.000938167}$ & $\overline{\mathrm{YES}}$ & \\
\hline c1170 & $5 / 100$ & OFFICE & $T$ & $15: 55$ & $6: \overline{12}$ & 6 & $\overline{6.6}$ & $\overline{6.3}$ & 857 & 5399.1 & BDL & $\overline{\mathrm{NA}}$ & $\overline{0.000908384}$ & $\overline{\text { YES }}$ & \\
\hline $10 \overline{\mathrm{c1171}}$ & $17 / \overline{100}$ & CLEAN ROOM & $\overline{\mathrm{CR} 1}$ & $\overline{16}: \overline{2}$ & & $\overline{5.9}$ & & $\overline{2.9}$ & $\overline{478}$ & 1410.1 & $\overline{0.005564}$ & $\overline{0.005541}$ & $\overline{0.003478092}$ & $\overline{N O}$ & \\
\hline fil & $1 / 100$ & BLANK & & $\because$ & & & & $\overline{0}$ & 1440 & $\overline{0}$ & $\overline{\text { ERR }}$ & $\overline{\text { ERR }}$ & $\overline{\text { ERR }}$ & $\overline{\text { ERR }}$ & \\
\hline 12 & & & & $:$ & & & & $\overline{0}$ & 1440 & $\overline{0}$ & ERR & $\overline{\text { ERR }}$ & $\overline{E R R}$ & $\overline{\text { ERR }}$ & \\
\hline 13 & i & & & : & & & & $\overline{0}$ & 1440 & $\overline{0}$ & $\overline{\text { ERR }}$ & $\overline{\text { ERR }}$ & $\overline{\text { ERR }}$ & $\overline{\text { ERR }}$ & \\
\hline & $f$ & & & : & & & & 0 & $\overline{1440}$ & $\overline{0}$ & ERR & $\overline{\text { ERR }}$ & ERR & $\overline{E R R}$ & \\
\hline & - & & & & & & & $\overline{0}$ & 1440 & $\overline{0}$ & $\overline{\text { ERR }}$ & ERR & ERR & $\overline{\text { ERR }}$ & \\
\hline & & & & & & & & $\overline{0}$ & $\overline{1440}$ & 0 & $\overline{\text { ERR }}$ & $\overline{E R R}$ & $\overline{\text { ERR }}$ & $\overline{E R R}$ & \\
\hline COMHENTS & $\begin{array}{l}\text { PCH } 7.30 \\
\text { AFTER ABA } \\
\text { NO ACTIVI } \\
\text { THAN }\end{array}$ & $\begin{array}{l}-90 \text { C1162-C1172 } \\
\text { TEMENT } \\
\text { TY IN CELL DURING CLEARANCE } \\
\text { IK YOU FOR THE OPPORTUNITY T }\end{array}$ & 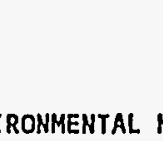 & ASUREMEI & & & Al & YST: & si & LEY/SEI & & $*=B E$ & & & \\
\hline
\end{tabular}




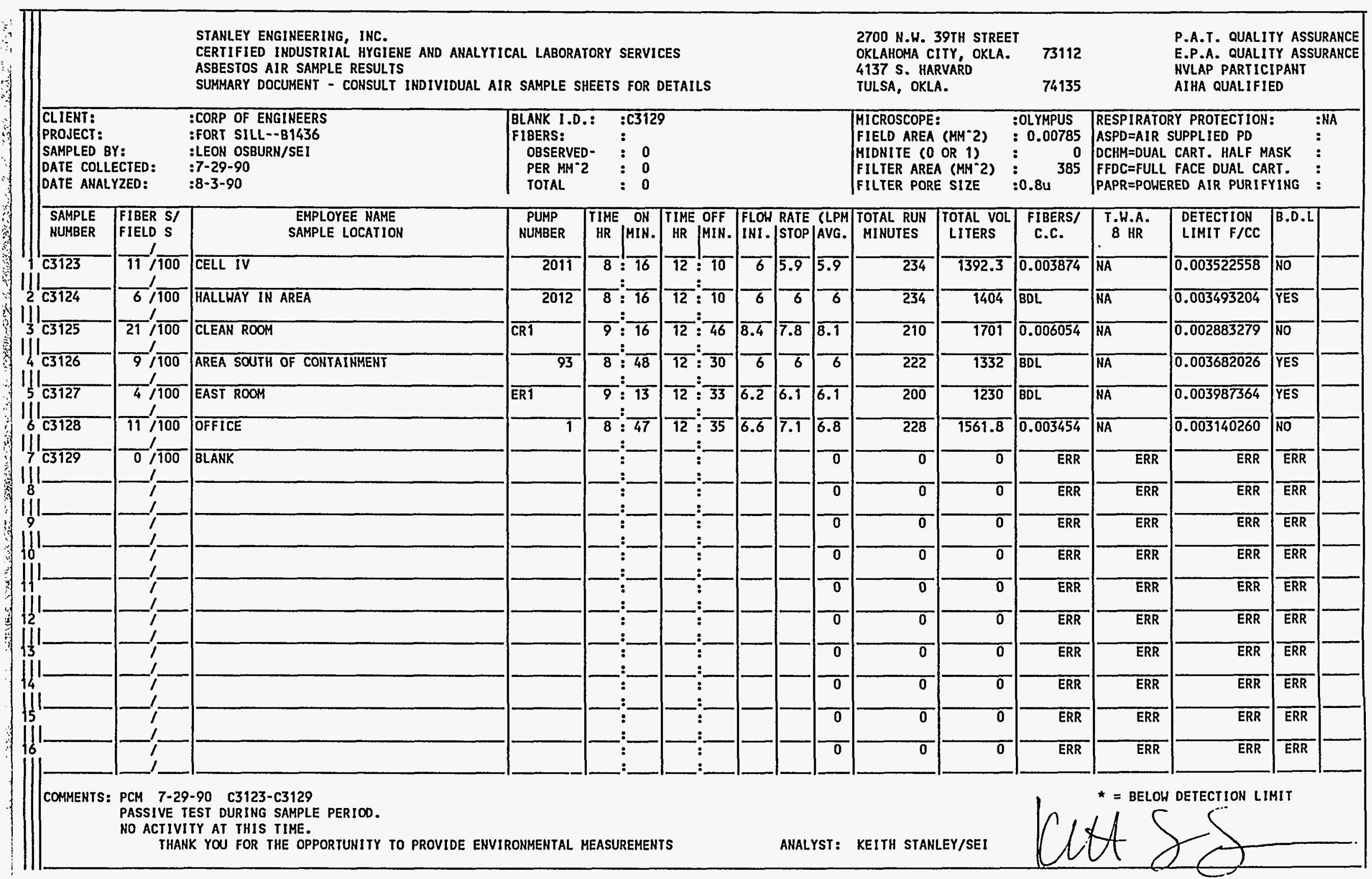




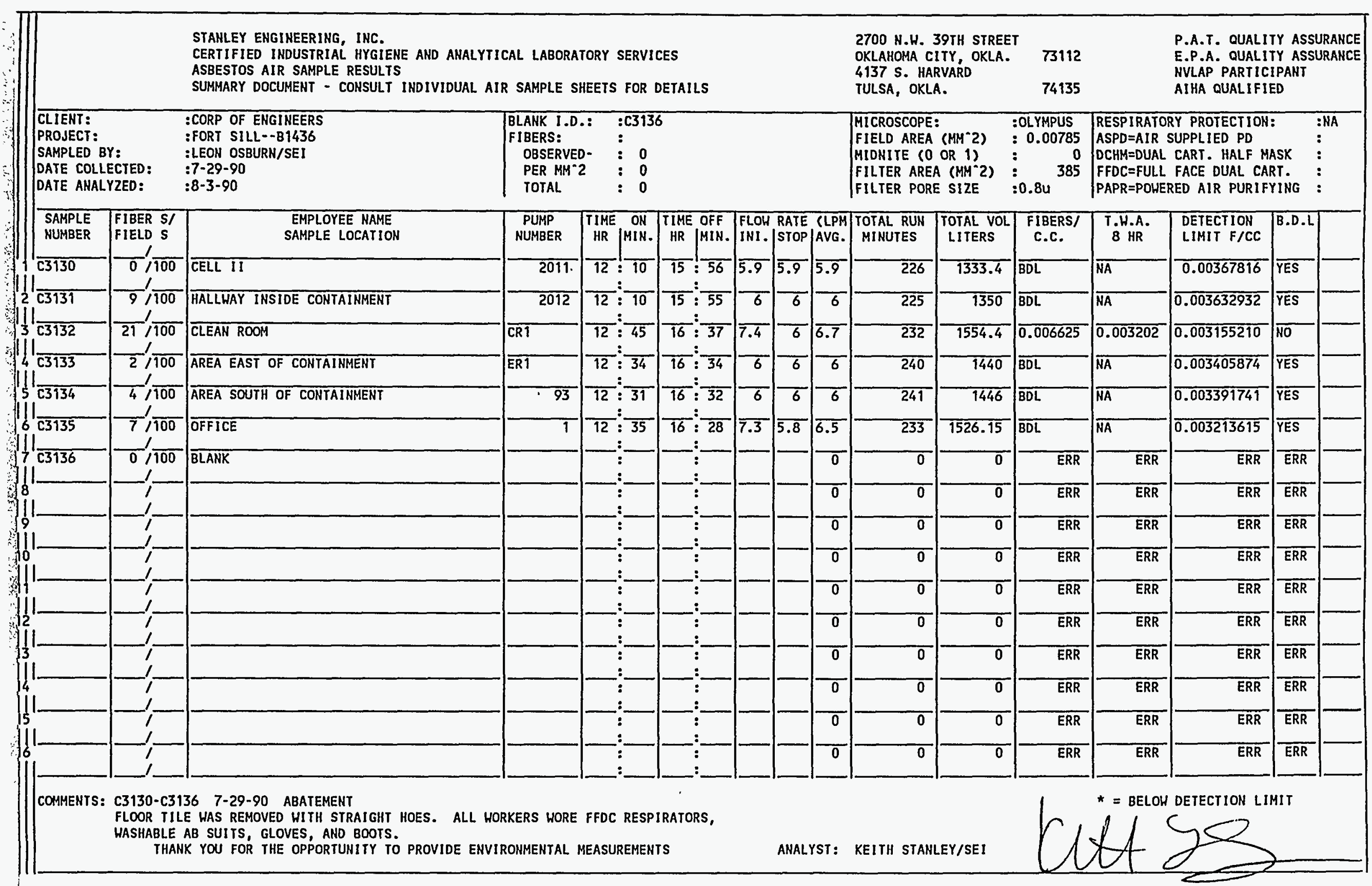




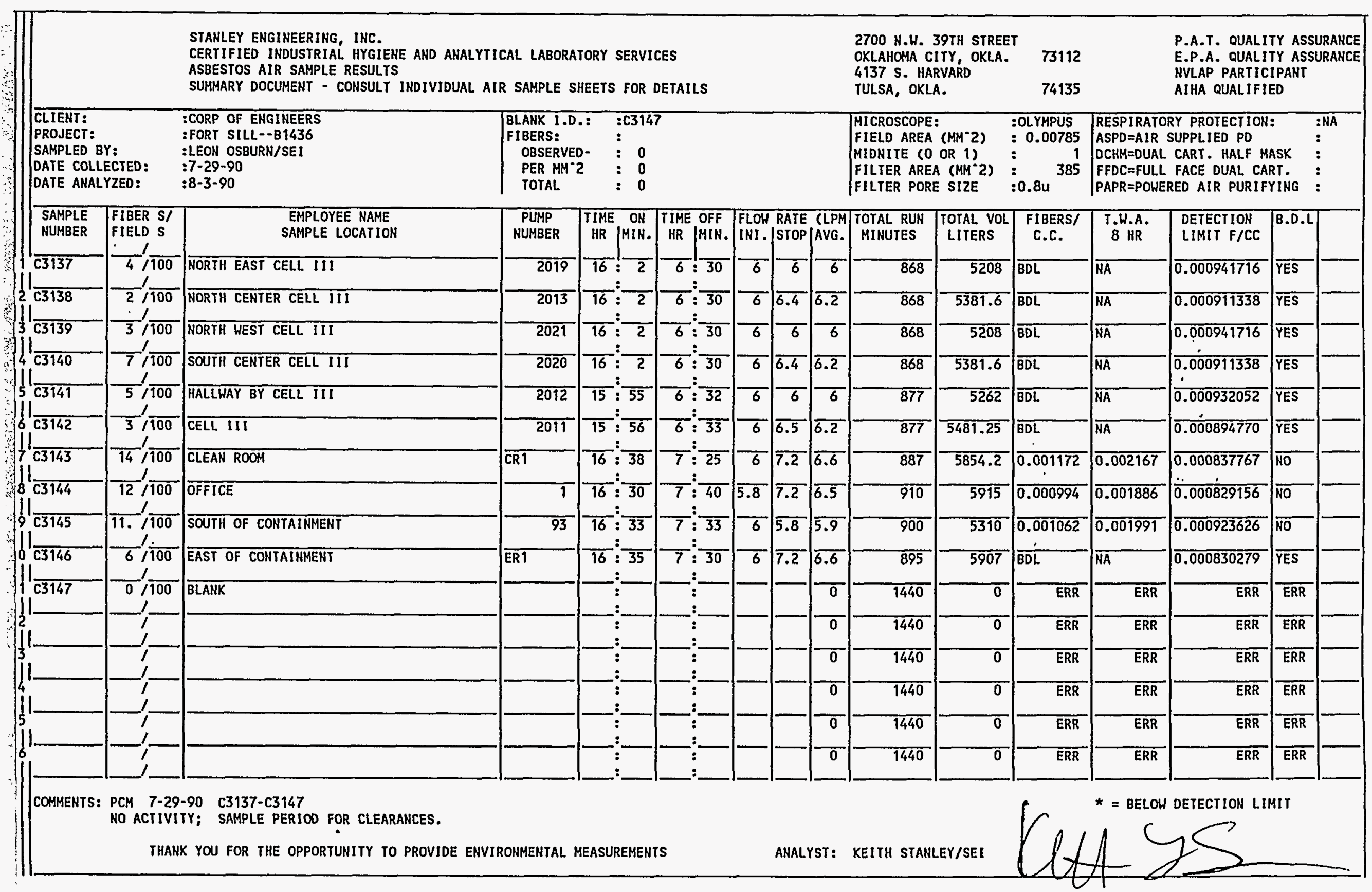




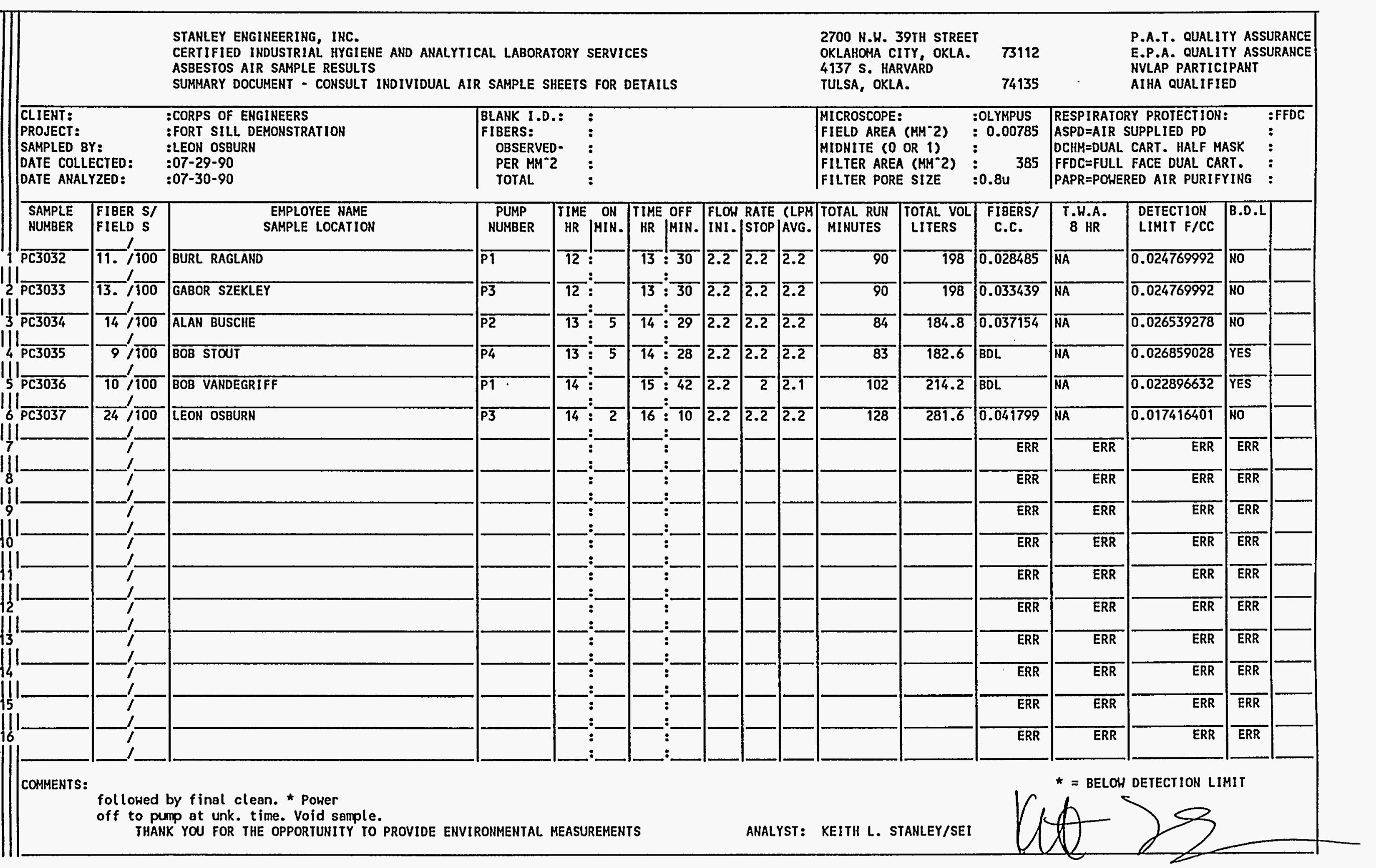




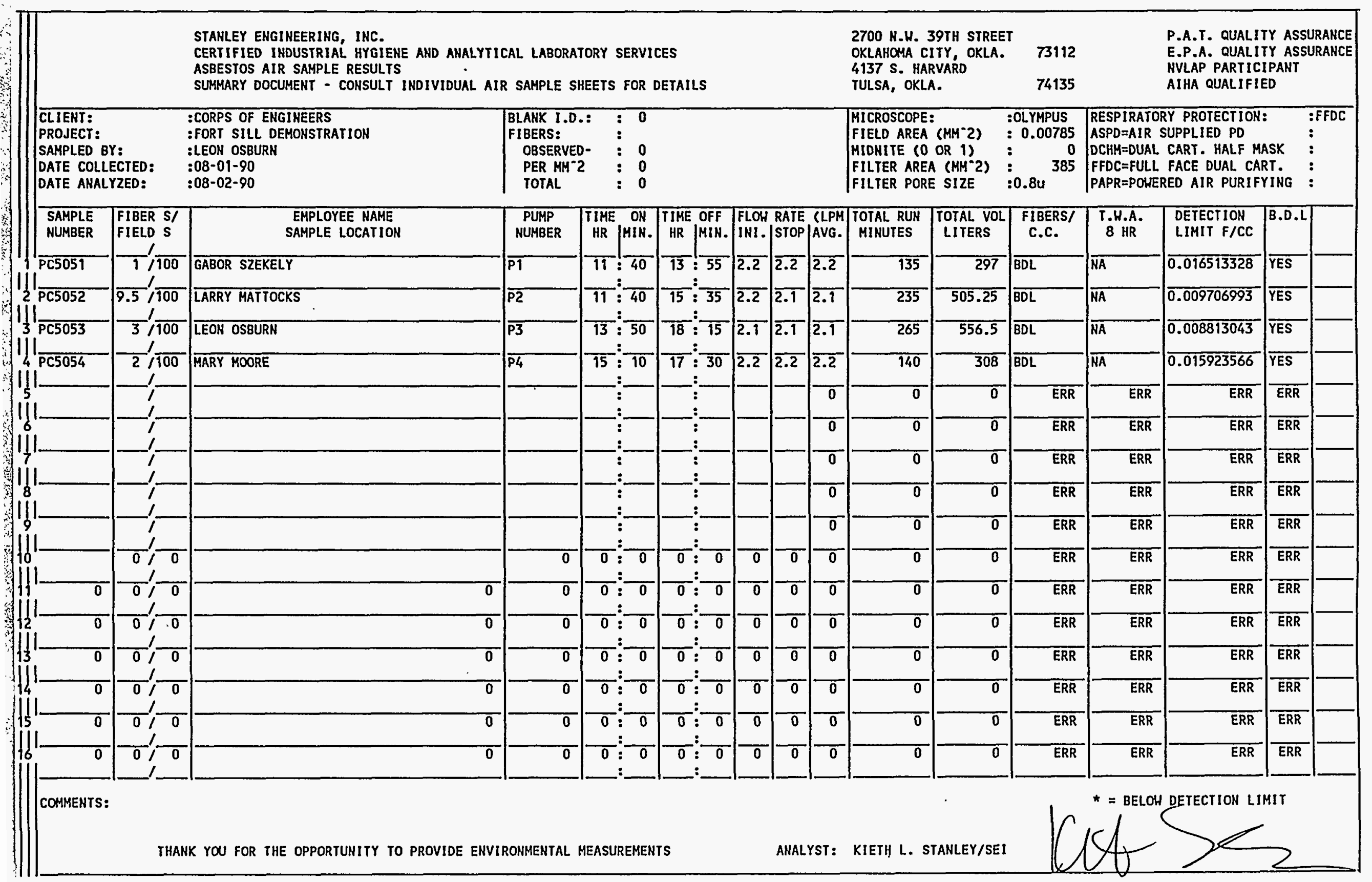




\begin{tabular}{|c|c|c|c|c|c|c|c|c|c|c|c|c|c|c|c|}
\hline & & \multicolumn{7}{|c|}{$\begin{array}{l}\text { STANLEY ENGINEERING, INC. } \\
\text { CERTIFIED INDUSIRIAL HYGIENE AND ANALYTICAL LABORATORY SERVICES } \\
\text { ASBESTOS AIR SAMPLE RESULIS } \\
\text { SUHHARY DOCUHENT - CONSULT INDIVIDUAL AIR SAMPLE SHEETS FOR DETAILS }\end{array}$} & \multicolumn{4}{|c|}{$\begin{array}{l}2700 \text { N.H. 39TH STREET } \\
\text { OKLAHOHA CITY, OKLA. } \\
4137 \text { S. HARVARD } \\
\text { TULSA, OKLA. }\end{array}$} & \multicolumn{3}{|c|}{$\begin{array}{l}\text { P.A.T. QUALIIY ASSURANCE } \\
\text { E.P.A. QUALITY ASSURANCE } \\
\text { HVLAP PARTICIPANT } \\
\text { AIHA QUALIFIED }\end{array}$} \\
\hline \multicolumn{2}{|c|}{$\begin{array}{l}\text { CLIENT: } \\
\text { PROJECI: } \\
\text { SAMPLED BY: } \\
\text { SATE COLLECTED: } \\
\text { OATE AHALYZED: }\end{array}$} & $\begin{array}{l}\text { :CORP OF ENGINEERS } \\
: \text { FORT SILL - }-1436 \\
: \text { LEOH OSBURH/SEI } \\
: 8-3-90 \\
: 8-8-90\end{array}$ & \multicolumn{3}{|c|}{\begin{tabular}{|ll} 
BLAMK I.D.: & $:$ C4266 \\
FIBERS: & $:$ \\
OBSERVED- & $\vdots 2$ \\
PER MM-2 & $: 2.5$ \\
TOTAL & $: 980$
\end{tabular}} & & & & \multicolumn{2}{|c|}{$\begin{array}{l}\text { HICROSCOPE: } \\
\text { FIELD AREA }(M M-2) \\
\text { MIDHITE }(0 \text { OR } 1) \\
\text { FILTER AREA (MM-2) } \\
\text { FILITER PORE SIZE }\end{array}$} & $\begin{array}{l}\text { :OLYMPUS } \\
: 0.00785 \\
: \quad 0 \\
: \quad 385 \\
: 0.8 u^{2}\end{array}$ & \multicolumn{3}{|c|}{$\begin{array}{l}\text { RESPIRATORY PROTECIIOH: } \\
\text { ASPD =AIR SUPPLIED PD } \\
\text { OCHM=DUAL CART. HALF MASK } \\
\text { FFDC=ULL FAC DUAL CART. } \\
\text { PAPR =POWERED AIR PURIFYING }\end{array}$} & $\begin{array}{l}: \text { NA } \\
\vdots \\
\vdots \\
\vdots\end{array}$ \\
\hline $\begin{array}{l}\text { SAMPLE } \\
\text { NUHBER }\end{array}$ & $\begin{array}{l}\text { FIBER S/ } \\
\text { FIELD S }\end{array}$ & $\begin{array}{l}\text { EMPLOYEE NAME } \\
\text { SAMPLE LOCATIOH }\end{array}$ & $\begin{array}{l}\text { PUMP } \\
\text { NUMBER }\end{array}$ & \begin{tabular}{|c|c|} 
TIME & ON \\
HR & MIN. \\
\end{tabular} & \begin{tabular}{|c|} 
TIME OFF \\
HR \\
HIN.
\end{tabular} & $\begin{array}{l}\text { FLOH } \\
\text { IHI. }\end{array}$ & $\begin{array}{l}\text { RATE } \\
\text { STOP }\end{array}$ & $\begin{array}{l}\text { (LPM } \\
\text { AVG. }\end{array}$ & $\begin{array}{l}\text { TOTAL RUN } \\
\text { MINUTES }\end{array}$ & \begin{tabular}{|l} 
TOTAL VOL \\
LITERS
\end{tabular} & $\begin{array}{c}\text { FlBERS/ } \\
\text { C.C. }\end{array}$ & $\begin{array}{l}\text { T.H.A. } \\
8 \mathrm{HR}\end{array}$ & $\begin{array}{l}\text { DETECTION } \\
\text { LIMIT F/CC }\end{array}$ & B.D.L & \\
\hline PC4261 & $2 / \overline{100}$ & HALLWAY NORTH & 2011 & $6: \overline{20}$ & $10: 14$ & 6 & $\overline{5.8}$ & $\overline{5.9}$ & 234 & 1380.6 & $\overline{B D L}$ & $\overline{\mathrm{NA}}$ & $\overline{0.003552411}$ & $\overline{\text { YES }}$ & \\
\hline PC4262 & $12 / \overline{100}$ & HALLHAY SOUTH & 2012 & $6: \overline{20}$ & $10: \overline{15}$ & 6 & $\overline{5.8}$ & $\overline{5.9}$ & 235 & 1386.5 & $\overline{\mathrm{BDL}}$ & $\overline{\mathrm{NA}}$ & $\overline{0.003537294}$ & $\overline{Y E S}$ & \\
\hline PC4263 & $18 / 100$ & CLEAH ROOH & $\overline{C R 1}$ & $6: \overline{30}$ & $10: 38$ & $\overline{6.2}$ & $\overline{6.2}$ & $\overline{6.2}$ & 248 & 1537.6 & 0.005103 & $\overline{\mathrm{NA}}$ & 0.003189684 & NO & \\
\hline$\overline{P C 4264}$ & $1.5 / 100$ & वITSIDE MORTH & $\overline{93}$ & $\overline{6}: \overline{31}$ & $\overline{10}: \overline{37}$ & $\overline{5.8}$ & $\overline{5.8}$ & $\overline{5.8}$ & 246 & 1426.8 & $\overline{B D L}$ & $\overline{\mathrm{NA}}$ & $\overline{0.003437383}$ & $\overline{Y E S}$ & \\
\hline PC4265 & $3 / \overline{100}$ & OFFICE & $\overline{1}$ & $6: \overline{32}$ & $\overline{10}: \overline{35}$ & 6 & $\overline{6}$ & $\overline{6}$ & 243 & 1458 & $\overline{B D L}$ & $\overline{N A}$ & $\overline{0.003363826}$ & $\overline{\text { YES }}$ & \\
\hline PC4266 & $2 / \overline{100}$ & BLANK & & $:$ & $\because$ & & & $\overline{0}$ & 0 & - & ERR & $\overline{\text { ERR }}$ & $\overline{\text { ERR }}$ & ERR & \\
\hline & & & & : & & & & $\overline{0}$ & $\overline{0}$ & $\overline{0}$ & ERR & $\overline{\text { ERR }}$ & $\overline{\text { ERR }}$ & $\overline{\text { ERR }}$ & \\
\hline & ? & & & : & $\because$ & & & 0 & 0 & 0 & $\overline{E R R}$ & $\overline{\text { ERR }}$ & $\overline{\text { ERR }}$ & $\overline{E R R}$ & \\
\hline & & & & $:$ & $\because$ & & & 0 & $\overline{0}$ & 0 & $\overline{\text { ERR }}$ & $\overline{\text { ERR }}$ & $\overline{\text { ERR }}$ & ERR & \\
\hline & 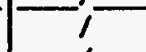 & & & $\because$ & 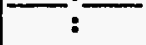 & & & $\overline{0}$ & $\overline{0}$ & 0 & $\overline{\text { ERR }}$ & $\overline{\text { ERR }}$ & ERR & $\overline{\text { ERR }}$ & \\
\hline & & & & $\because$ & $\because$ & & & $\overline{0}$ & 0 & 0 & ERR & $\overline{\text { ERR }}$ & $\overline{\text { ERR }}$ & $\overline{\text { ERR }}$ & \\
\hline & 1 & & & $\because$ & : & & & $\overline{0}$ & $\overline{0}$ & $\overline{0}$ & $\overline{\text { ERR }}$ & $\overline{\text { ERR }}$ & $\overline{\text { ERR }}$ & $\overline{E R R}$ & \\
\hline & 1 & & & $:$ & $\because$ & & & $\overline{0}$ & $\overline{0}$ & $\pi$ & $\overline{\text { ERR }}$ & $\overline{E R R}$ & $\overline{\text { ERR }}$ & $\overline{\text { ERR }}$ & \\
\hline & & & & $:$ & & & & $\overline{0}$ & $\overline{0}$ & $\overline{0}$ & $\overline{\text { ERR }}$ & $\overline{\text { ERR }}$ & $\overline{\text { ERR }}$ & $\overline{\text { ERR }}$ & \\
\hline & 1 & & & $=$ & $\because$ & & & 0 & $\overline{0}$ & $\overline{0}$ & ERR & $\overline{\text { ERR }}$ & $\overline{\text { ERR }}$ & ERR & \\
\hline & & & & & & & & $\overline{0}$ & $\overline{0}$ & $\overline{0}$ & ERR & $\overline{\text { ERR }}$ & $\overline{\text { ERR }}$ & $\overline{\text { ERR }}$ & \\
\hline COMMENTS & $\begin{array}{l}\text { PCM 8-3 } \\
\text { HO ACTIV }\end{array}$ & $\begin{array}{l}-90 \text { C4261-C4266 PASSIVE (CELLS } 4 \text { \& } 7 \text { ) } \\
\text { ITY IN CELL DURING PASSIVE TEST. }\end{array}$ & & & & & & & & & & $=$ BEL & ON LIR & & \\
\hline
\end{tabular}




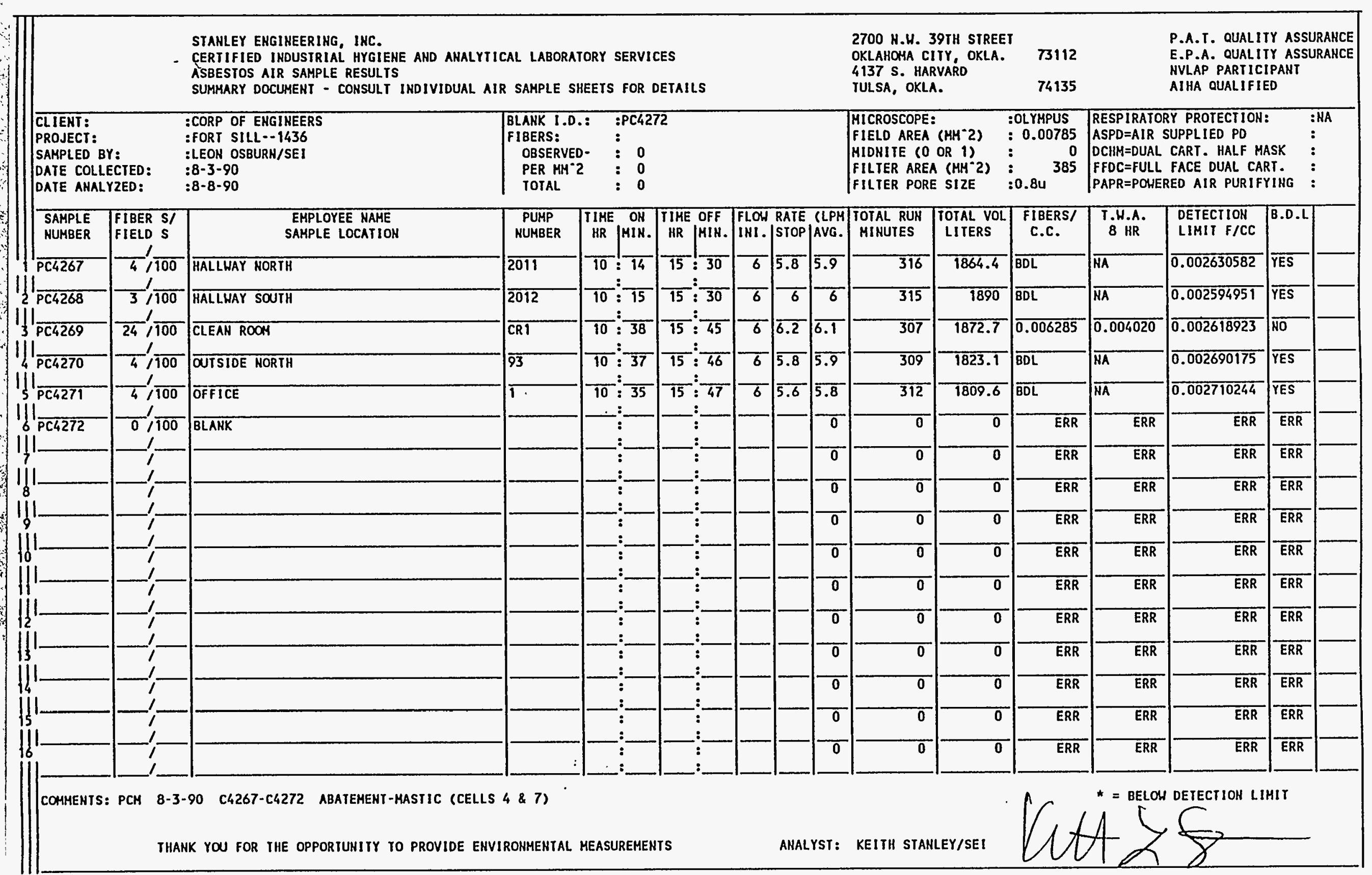




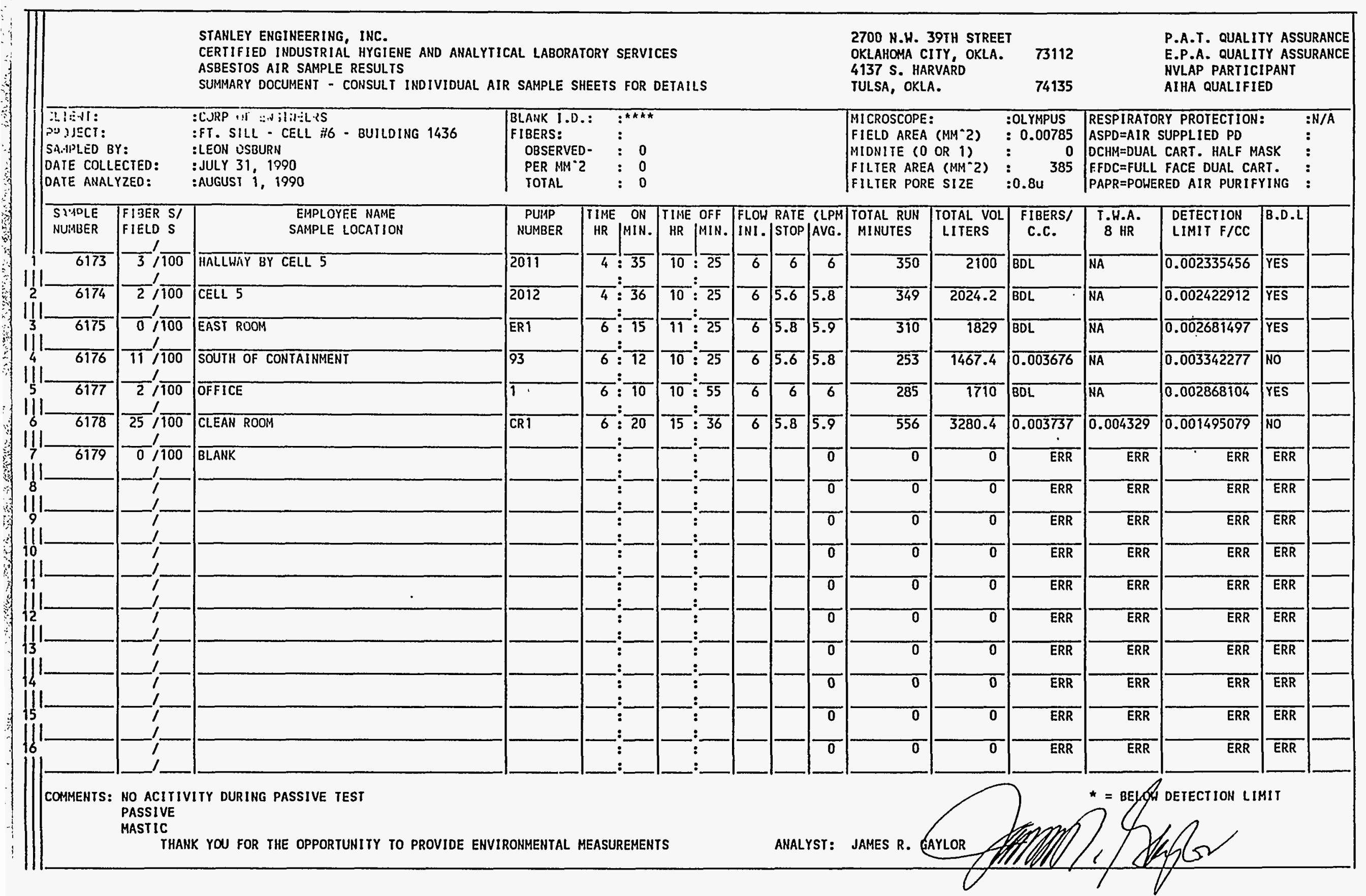




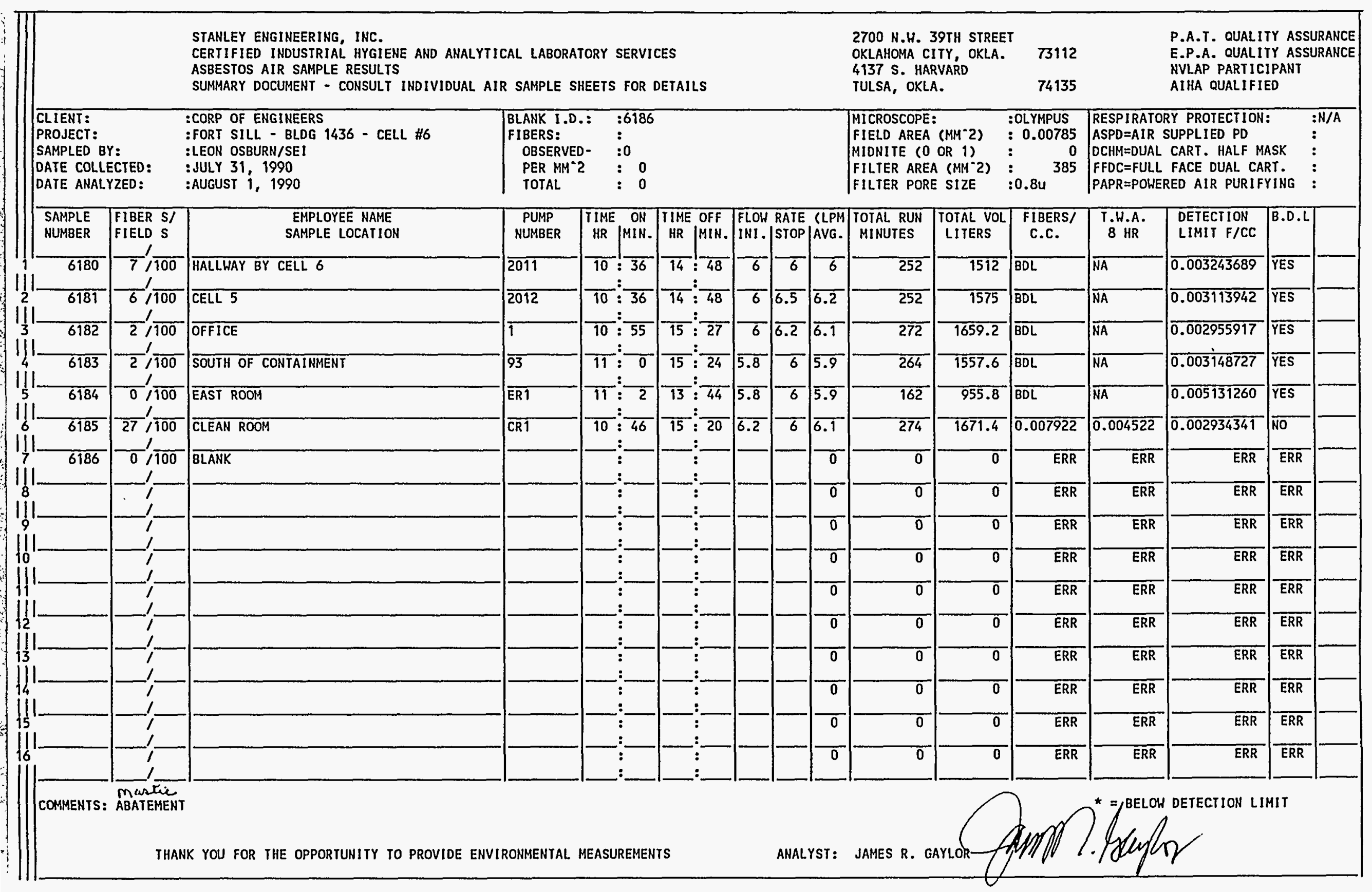




\begin{tabular}{|c|c|c|c|c|c|c|c|c|c|c|c|c|c|c|c|}
\hline & & \multicolumn{7}{|c|}{$\begin{array}{l}\text { STANLEY ENGINEERING, INC. } \\
\text { CERTIFIED INDUSRIAL HYGIENE AND ANALYTICAL LABORATORY SERVICES } \\
\text { ASBESTOS AIR SAMPLE RESULTS } \\
\text { SUMMARY DOCUMENT - CONSULT INDIVIDUAL AIR SAMPLE SHEETS FOR DETAILS }\end{array}$} & \multicolumn{2}{|c|}{$\begin{array}{l}2700 \text { N.H. 39TH STREET } \\
\text { OKLAHOMA CITY, OKLA. } \\
4137 \text { S. HARVARD } \\
\text { TULSA, OKLA. }\end{array}$} & $\begin{array}{ll}\text { ET } & \\
73112 \\
74135\end{array}$ & & \multicolumn{3}{|c|}{$\begin{array}{l}\text { P.A.T. QUALITY ASSURANCE } \\
\text { E.P.A. QUALITY ASSURANCE } \\
\text { NVLAP PARTICIPANT } \\
\text { AIHA QUALIFIED }\end{array}$} \\
\hline \multicolumn{2}{|c|}{$\begin{array}{l}\text { CLIENT: } \\
\text { PROJECT: } \\
\text { SAMPLED BY: } \\
\text { OATE COLLECTED: } \\
\text { DATE ANALYZED: }\end{array}$} & $\begin{array}{l}\text { :CORP OF ENGINEERS } \\
\text { :FORT SILL - BLDG } 1436-\text { CELL \#6 } \\
\text { :LEON OSBURN/SEI } \\
\text { :UULY } 31,1990 \\
\text { :AUGUST } 1,1990\end{array}$ & \multicolumn{3}{|c|}{\begin{tabular}{|ll} 
BLANK I.D.: & $: \star \star \star \star$ \\
FIBERS: & $\vdots$ \\
OBSERVED- & $\vdots 1$ \\
PER MM-2 & $: 1.2$ \\
TOTAL & $: 490$
\end{tabular}} & & & & \multicolumn{2}{|c|}{$\begin{array}{l}\text { MICROSCOPE: } \\
\text { FIELD AREA (MH-2) } \\
\text { MIDNITE (0 OR } 1) \\
\text { FILTER AREA (MM-2) } \\
\text { FILTER PORE SIZE }\end{array}$} & $\begin{array}{lr} & : \text { OLYMPUS } \\
: & 0.00785 \\
: & 0 \\
: & 385 \\
: 0.8 u^{2}\end{array}$ & \multicolumn{3}{|c|}{$\begin{array}{l}\text { RESPIRATORY PROTECTION: } \\
\text { ASPD=AIR SUPPLIED PD } \\
\text { DCHM=DUAL CART. HALF MASK } \\
\text { FFDCFULL FACE DUAL CART. } \\
\text { PAPR=POHERED AIR PURIFYING }\end{array}$} & $\begin{array}{l}\text { :N/A } \\
\vdots \\
\vdots \\
\vdots \\
\vdots\end{array}$ \\
\hline $\begin{array}{l}\text { SAMPLE } \\
\text { NUMBER }\end{array}$ & \begin{tabular}{|c} 
FIBER SI \\
FIELD S \\
\end{tabular} & $\begin{array}{l}\text { EMPLOYEE NAME } \\
\text { SAMPLE LOCATION }\end{array}$ & $\begin{array}{l}\text { PUMP } \\
\text { NUMBER }\end{array}$ & $\begin{array}{ccc}\text { TIME } & \text { ON } \\
\text { HR } & \text { MIN. }\end{array}$ & $\begin{array}{l}\text { TIME OFF } \\
\text { HR PIN. }\end{array}$ & $\begin{array}{l}\text { FLOH } \\
\text { INI. }\end{array}$ & $\begin{array}{l}\text { RATE } \\
\text { STOP }\end{array}$ & $\begin{array}{l}\text { SLPM } \\
\text { |AVG. }\end{array}$ & $\begin{array}{l}\text { TOTAL RUN } \\
\text { MINUTES }\end{array}$ & $\begin{array}{l}\text { TOIAL VOL } \\
\text { LITERS }\end{array}$ & $\begin{array}{l}\text { FIBERSI } \\
\text { c.C. }\end{array}$ & $\begin{array}{l}\text { T.H.A. } \\
8 \mathrm{HR}\end{array}$ & $\begin{array}{l}\text { DETECTION } \\
\text { LIMIT F/CC }\end{array}$ & B.D.L & \\
\hline 6187 & $2 / \overline{100}$ & NORTH EAST CELL 6 & & $74: \overline{44}$ & $78: \overline{13}$ & 6 & 6 & 6 & 209 & 1254 & $\overline{\mathrm{BOL}}$ & $\overline{N A}$ & 0.003911051 & YES & \\
\hline 6188 & $2 / \overline{100}$ & NORTH CENTER CELL 6 & & $14: \overline{44}$ & $18: \sqrt{13}$ & 6 & $\overline{5.8}$ & 5.9 & 209 & 1233.1 & $\overline{B D L}$ & $\overline{N A}$ & 0.003977340 & $\overline{Y E S}$ & \\
\hline 6189 & $0 / \overline{100}$ & SOUTH EAST CELLL 6 & & $\overline{14}: \overline{44}$ & $18: \overline{13}$ & 6 & 7 & 6.5 & 209 & 1358.5 & $\overline{\mathrm{BDL}}$ & $\overline{N A}$ & $\overline{0.003610201}$ & $\overline{\text { YES }}$ & \\
\hline 6190 & $1 / \overline{100}$ & SOUTH CENTER CELLL 6 & & $\sqrt{14}: \overline{44}$ & $18: \overline{13}$ & 6 & 6 & 6 & 209 & 1254 & $\overline{B D L}$ & $\overline{N A}$ & 0.003911051 & YES & \\
\hline 6191 & $5 / \longdiv { 1 0 0 }$ & HALLWAY BY CELLL 6 & 2011 & $14:-$ & $17: \overline{59}$ & 6 & $\overline{5.8}$ & 5.9 & 191 & 1126.9 & $\overline{B D L}$ & $\overline{N A}$ & $\overline{0.004352168}$ & $\overline{Y E S}$ & \\
\hline 6192 & $3 / \overline{100}$ & CELL 5 & 2012 & $14: \overline{48}$ & $17:-$ & $\overline{6}$ & 6 & 6 & 191 & 1146 & $\overline{\mathrm{BDL}}$ & $\overline{N A}$ & $\overline{0.004279632}$ & YES & \\
\hline 6193 & $0 / 100$ & OFFICE & $r$ & $15: \overline{27}$ & $\overline{18}: \overline{32}$ & $\overline{6.2}$ & $\overline{6.4}$ & $\overline{6.3}$ & $\overline{185}$ & 1165.5 & $\overline{B D L}$ & $\overline{N A}$ & 0.004208029 & $\overline{Y E S}$ & \\
\hline 6194 & $0 / 100$ & SOUTK OF CONTAINMENT & 93 & $15: 24$ & $18: \overline{32}$ & 6 & 6 & 6 & 188 & 1128 & $\overline{\mathrm{BDL}}$ & $\overline{N A}$ & 0.004347924 & $\overline{Y E S}$ & \\
\hline 6195 & $18 / \overline{100}$ & CLEAN ROOM & CRI & $15: \overline{21}$ & $18: 35$ & $\overline{6.2}$ & $\overline{5.8}$ & 6 & 194 & 1164 & $\overline{0.007162}$ & $\overline{\mathrm{NA}}$ & 0.004213452 & NO & \\
\hline 6196 & $1 / \overline{100}$ & BLANK & & $\because$ & & & & 0 & 0 & $\overline{0}$ & ERR & ERR & $\overline{E R R}$ & $\overline{\text { ERR }}$ & \\
\hline & 1 & & & :: & :: & & & 0 & $\overline{0}$ & $\overline{0}$ & ERR & ERR & ERR & $\overline{E R R}$ & \\
\hline & 1 & & & $:$ & $\because$ & & & 0 & $\overline{0}$ & 0 & ERR & ERR & ERR & ERR & \\
\hline & & & & $:$ & & & & $\overline{0}$ & $\overline{0}$ & $\overline{0}$ & ERR & ERR & ERR & $\overline{\text { ERR }}$ & \\
\hline & 1 & & & $:$ & & & & 0 & $\overline{0}$ & $\overline{0}$ & ERR & ERR & ERR & $\overline{E R R}$ & \\
\hline & & & & $:$ & & & & $\overline{0}$ & $\overline{0}$ & $\overline{0}$ & ERR & ERR & ERR & $\overline{\text { ERR }}$ & \\
\hline & $i$ & & & : & & & & $\overline{0}$ & 0 & $\overline{0}$ & ERR & ERR & ERR & $\overline{E R R}$ & \\
\hline$m$ & ER A & MENT & & & & & & & & & & & & & \\
\hline
\end{tabular}




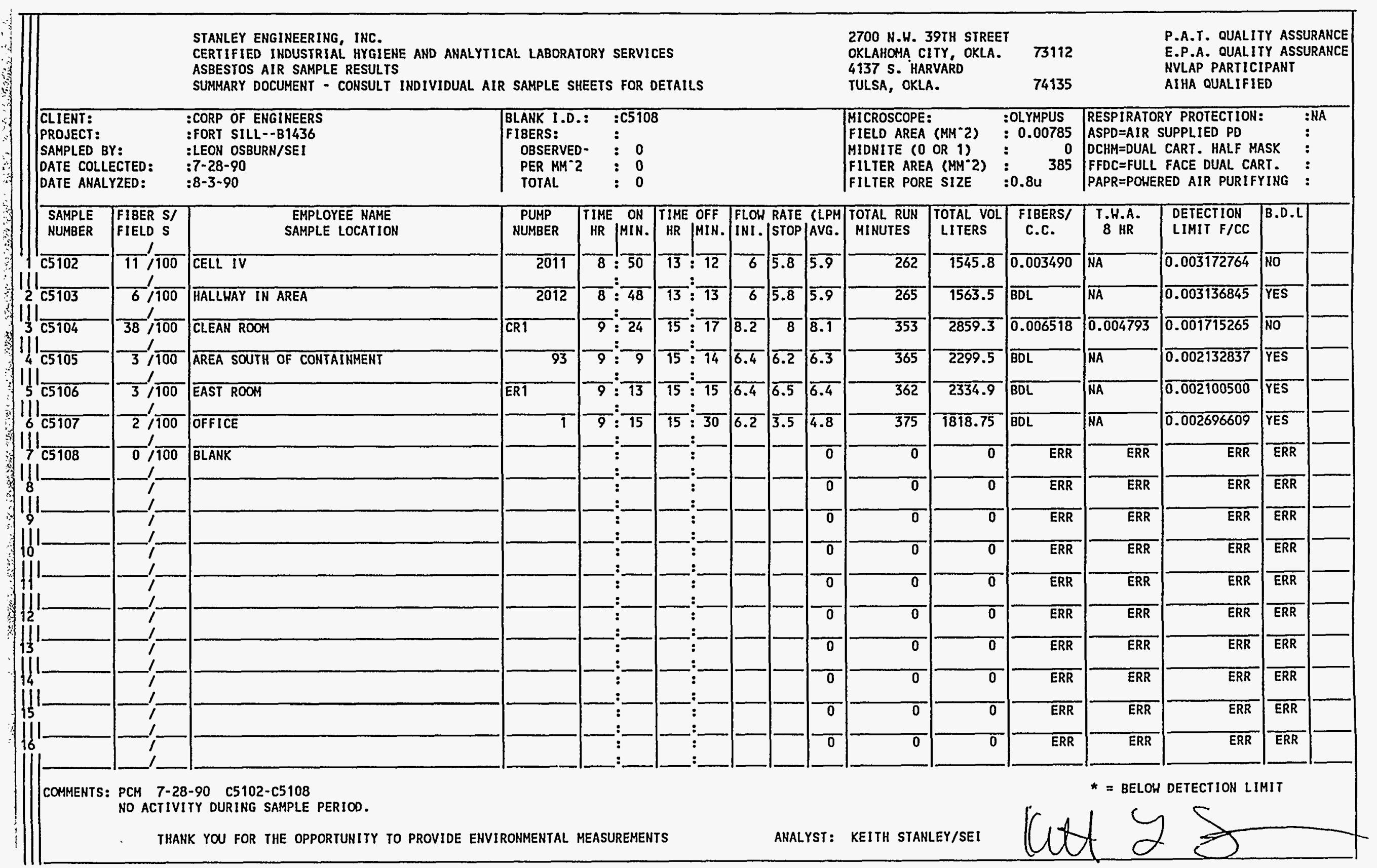




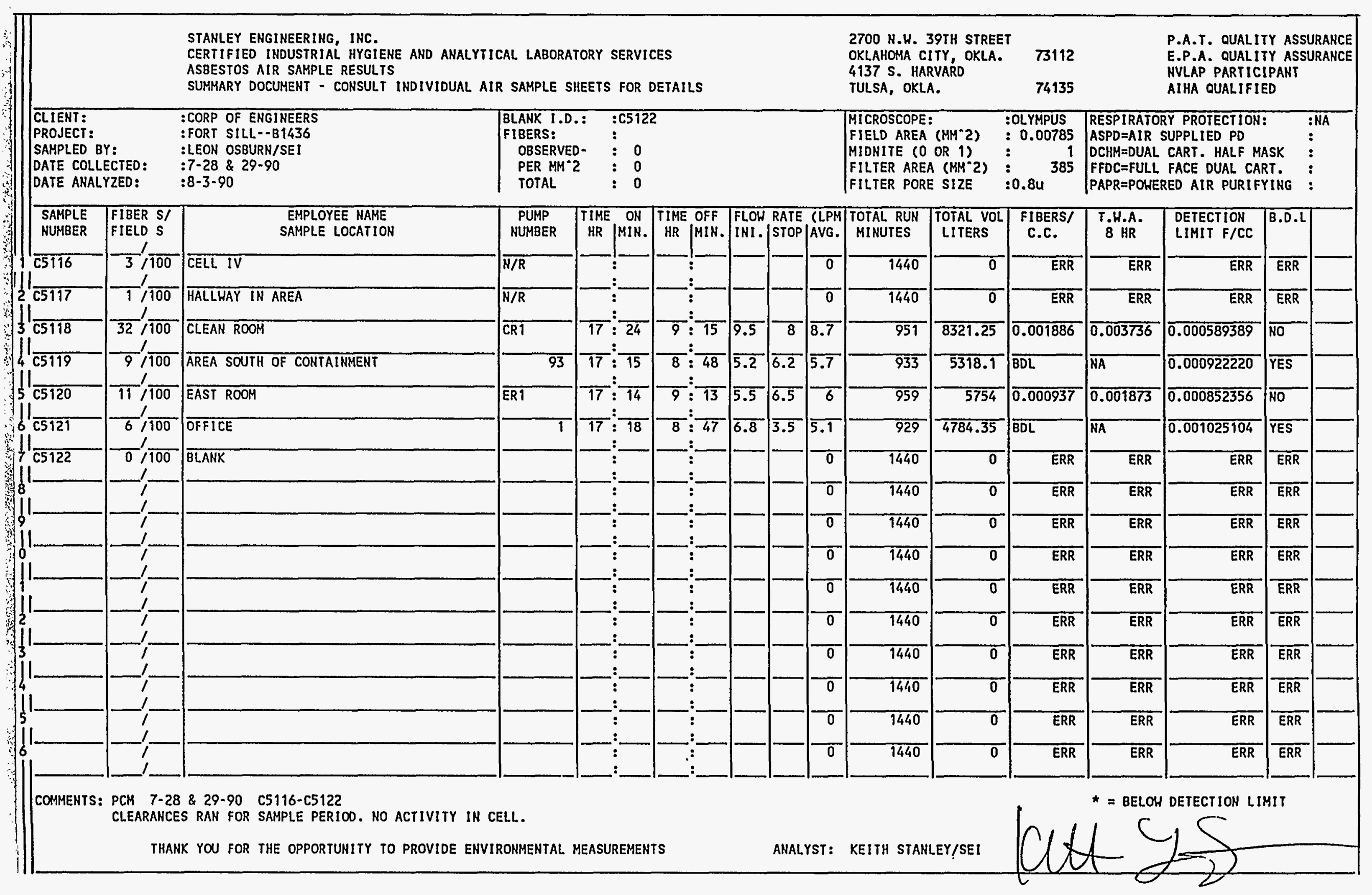


THE EVALUATION OF ASBESTOS FIBER RELEASE

FROM THE REMOVAL OF ASBESTOS CONTAINING FLOOR TILE AND THE RESULTING EXPOSURE POTENTIAL UTUIZING MULTIPLE

AGGRESSIVE TECHNIQUES OF REMOVAL

CONTRACT NO. DACA56-88-D-0054 DELIVERY ORDER NO. "3" TESTING/SAMPLING PROTOCOL FOR THE REMOVAL OF NONFRIABLE

ASBESTOS (FLOOR TILE)

MAY, 1990

DEVELOPED BY:

STANLEY ENGINEERING INC.

2700 N.W. 39TH STREET

OKLAHOMA CITY, OKLAHOMA 73112

(405) 948-6505 


\section{INTRODUCTION}

The following asbestos testing protocols are established for the purpose of determining the extent of asbestos fiber release while utilizing varying aggressive techniques to remove asbestos containing floor tile. Through this effort, it is the intent that the following test protocol will reflect an extent of sampling/testing sufficient to provide for an adequate defense of findings when presented to the scientific community, regulators, or others who would find value from the conclusions of testing. Only current state-of-the-art testing methodologies will be utilized as a basis from which conclusions of this study shall be drawn.

In order to assure that this project has as a basis a known and quantifiable base from which to begin, prior to the initiation the removal techniques, floor tile shall be tested in order to determine both the percent and type of asbestos within each visually distinguishable type of floor tile. Beyond this, the mastic shall also be tested and quantified with regard to percent and type of asbestos. These determinations shall be accomplished by utilizing Polarized Light/Dispersion Staining Microscopy with follow up analysis by Scanning Electron Microscopy (SEM) and/or Transmission Electron Microscopy (TEM) as may be necessary in order to achieve reliability in the quantification of asbestos. Beyond this, requirements of the Asbestos Hazard Emergency Response Act (AHERA) shall be implemented in sample collection within homogeneous areas and use constraints as well as sampling strategies and personnel qualifications. The precise methodologies utilized for preparation of samples will be detailed as apart of the final report, but shall be those methodologies considered to be current in the state-of-the-art.

Background air sampling shall be accomplished within each structure to include as a minimum, the area of the proposed floor tile removal, adjacent to the removal area and external to the removal area. In all instances where monitoring is accomplished, adequate volumes of air will be collected to assure that the minimum detection limit will be achieved.

It is not anticipated that this project will utilize a decontamination facility, negative air pressure, negative air pressure recording, room air changes, or complete preparation on walls and ceiling. It is anticipated that personnel protective equipment will be adequate as demonstrated by sampling results. Should results reflect the need to establish a decontamination facility, negative air pressure, etc., then this will be accomplished in order for the project to proceed.

\section{ANALYTICAL METHODS}

This testing program has been designed in order to assure that test results are complete, accurate and meaningful. This program of testing includes both Phase Contrast Microscopy (PCM), a total fiber counting technique, as well as Transmission Electron Microscopy (TEM), an asbestos fiber differentiating technique and may include, if necessary, Scanning Electron Microscopy (SEM) should a recognized need be established. The purpose of employing these techniques, is in order to assure that fibers counted as a result of asbestos containing floor tile ("tile") removal, are asbestos. 
Bulk materials shall be analyzed by the utilization of Polarized Light/Dispersion Staining Microscopy as specified by EPA Interim Method for the Determination of Asbestos in Bulk Insulation Samples (latest revision).

Only recognized methodologies shall be utilized in the development of analytical results inclusive of NIOSH 7400, Revised 5-15-89, further analysts shall be participants in the Proficiency Analytical Testing (PAT) Program for the past six successive rounds. Additionally, laboratory shall be a participant in the National Institute of Science and Technology (NIST), National Voluntary Laboratory Accredation Program (NVLAP). Analysts shall have attended McCrones Institute as a minimum.

Beyond this, all personnel involved in the collection of samples with specific regard to this project, shall have, as a minimum, attended a forty hour NIOSH 582 Course or its equivalency in "Sampling and Analysis for Asbestos Dust" Each individual shall further, have been involved, in a minimum of five asbestos abatement projects, with the responsibility for the collection and analysis of samples by the NIOSH 582 Procedure and Phase Contrast Microscopy. All work shall be accomplished at the specific direction of a Certified Industrial Hygienist, in the Comprehensive Practice of Industrial Hygiene.

Finally, a minimum of five percent of all air samples collected shall be analyzed by an equivalently qualified (NVLAP, PAT, etc.) outside laboratory. Duplicate sampling shall also constitute a portion of this project, with the intent and interest being reproducibility of samples collected.

\section{ALTERNATTVE FLOOR TILE REMOVAL PROCEDURES}

Seven alternative scenarios for removal of floor tile, have been identified to be included within the Scope of this project. Each alternative must have, as a basis, an adequate in-place floor tile, which will accommodate the required removal time to accomplish an adequate air sample duration. This is necessary in order to assure that a sufficient detection limit can be achieved while at the same time allowing for representative quantification of potential fiber releases due to the applied scenario.

This project will proceed by starting with the least aggressive removal technique and after a review of quantified air sample results, the project will then proceed to the next scenario, which will employ more aggressive removal techniques capable of demonstrating greater intensity of fiber release.

Facility selection considerations have included the following:

$\begin{array}{ll}* & \text { Building location } \\ * & \text { Availability } \\ * & \text { Building prior use } \\ * & \text { Facility age } \\ * & \text { Floor tile type } \\ & \text { vinyl asbestos } \\ & \text { asphaltic asbestos } \\ * \quad & \text { Adhesive type } \\ \text { asbestos containing }\end{array}$


non-asbestos containing

* Building Friable Asbestos Content

background concentrations

physical presence (extent)

* Square Footage of Floor Tile Present asbestos content

To date, considerable effort has gone into locating building candidate for this project. At present the following building candidate have been identified.

* $\quad$ Building No.: 4379, Ft. Sill, Oklahoma

Total Floors: 2; Gross Square Footage: 4,525

* $\quad$ Building No.: 4380, Ft. Sill, Oklahoma

Total Floors: 2: Gross Square Footage: 4,525

* $\quad$ Building No.: 4375, Ft. Sill, Oklahoma (being considered)

Total Floors: 1; Gross Square Footage: 1,311; this building has a layer of sheet flooring below the floor tile.

* Building No: 4363, at present will be the only structure with a wood floor.

Alternatively, our most available source for candidates to be utilized within this project, appears to be houses with $9 \times 9$ floor tile.

Additional building candidate continue to be reviewed and it is anticipated that at the project start date, the additional necessary facilities to carry out the demonstration project, will have been identified. 


\section{REMOVAL SPECIFICATIONS}

\section{DEFINITION OF TERMS}

Terms presented below may be applicable to the project as developed. A more indepth presentation of terms may be found in the Oklahoma State Department of Labor Rules and Regulations (current edition).

A. Abatement: Procedures to control fiber release from asbestos-containing materials. Includes removal, encapsulation, enclosures, repair, demolition and renovation activities.

B. Accredited or Accreditation: when referring to a person or a laboratory means that such a person or laboratory has met the training, experience, and/or quality control requirements to perform work in accordance with AHERA.

C. AHERA: means the Asbestos Hazard Emergency Response Act of 1986 (PL99-519) and rules and regulations enacted by EPA for its implementation.

D. AHERA Abatement Project Designer: means a person who develops plans and specifications for the abatement of asbestos. For the Purposes of these rules, Abatement Project Designers will be considered to be a category of contractors.

E. Airlock: A system for permitting ingress and egress with minimum air movement between a contaminated area and an uncontaminated area, typically consisting of two curtained doorways separated by a distance of at least three feet ( $\left.3^{\prime}\right)$ such that one passes through one doorway into the airlock, allowing the doorway sheeting to overlap and close off the opening before proceeding through the second doorway, thereby preventing flowthrough contamination.

F. Air Monitoring: The process of measuring the fiber content of a known volume of air collected during a specific period of time.

G. Amended Water: Water to which a surfactant has been added.

H. Asbestos: The asbestiform varieties of serpentine (chrysotile), rie beckite (crocidolite), cummingtonite-grunerite (amosite), anthrophylite, actinolite, and tremolite.

I. Asbestos Containing Material (ACM): Material composed of asbestos of any type and in any amount, either alone or mixed with other fibrous or non-fibrous materials.

J. Asbestos Containing Waste Material: Asbestos containing material or asbestos contaminated objects requiring disposal.

K. Authorized Visitor: The Building Owner, inspecting architect, and any representative of a regulatory or other agency having jurisdiction over the project.

L. Building Owner: The Owner or his authorized representative. 
M. Clean Room: An uncontaminated area or room which is a part of the worker decontamination enclosure system with provisions for storage of worker's street clothes and clean protective equipment.

N. Commissioner: means the Oklahoma Commissioner of Labor.

O. Contractor: The individual and/or business with which the Building Owner contracts to perform the asbestos abatement.

P. Curtained Doorway: A device to allow ingress or egress from one room to another while permitting minimal air movement between the rooms, typically constructed by placing three overlapping sheets of plastic over an existing or temporarily framed doorway, securing each along the top of the doorway, securing the vertical edge of the first and third sheets along one vertical side of the doorway and securing the vertical edge of the second sheet along the opposite vertical side of the doorway.

Q. Decontamination Enclosure System: A series of connected rooms, separated from the work area and from each other by air locks, for the decontamination of workers and equipment.

R. DOL: Oklahoma State Department of Labor.

S. DOT: Oklahoma State Department of Transportation.

T. Encapsulant: A liquid material which can be applied to asbestos containing material, controlling the possible release of asbestos fibers from the material, either by creating a membrane over the surface (bridging encapsulant) or by penetrating into the material and binding its components together (penetrating encapsulant).

U. Encapsulation: The application of an encapsulant.

V. Enclosure: The construction of an air-tight, impermeable; permanent barrier around asbestos-containing material to control the release of asbestos fibers into the air.

W. EPA:

U.S. Environmental Protection Agency

401 M. Street S.W.

Washington, D.C. 20460

X. Equipment Decontamination Enclosure System: That portion of a decontamination enclosure system designed for controlled transfer of materials and equipment into or out of the work ares, typically consisting of a washroom and holding area.

Y. Equipment Room: A contaminated area or room which is part of the worker decontamination enclosure system with provisions for storage of contaminated clothing and equipment.

Z. Friable Asbestos Material: means any material that contains asbestos of one percent $(1 \%)$ or more that can be crumbled, pulverized or reduced to powder by hand pressure. 
AA. Glovebag Technique: A method with limited applications for removing small amounts of friable asbestos-containing material from HVAC ducts, short piping runs, valves, joints, elbows, and other non-planar surfaces in a non-contained (plasticized) work area. The glovebag assembly is a manufactured or fabricated device consisting of a glovebag (typically constructed of $6 \mathrm{mil}$ transparent polyethylene or polyvinylchloride plastic), two inward projecting longsleeves, an internal tool pouch, and an attached, labeled receptacle for asbestos waste. The glovebag is constructed and installed in such a manner that it surrounds the object or material to be removed and contains all the asbestos fibers released during the process. All workers who are permitted to use the glovebag technique must be highly trained, experienced and skilled in this method.

BB. HEPA Filter: HEPA means High Efficiency Particulate Absolute. A HEPA air filter is capable of removing particles $>0.3$ microns in diameter with $99.97 \%$ efficiency.

CC. HEPA Vacuum: A vacuum system equipped with HEPA filtration.

DD. Holding Area: A chamber in the equipment decontamination enclosure located between the washroom and an uncontaminated area. The holding area comprises an airlock.

EE. Lockdown: Lockdown is the procedure of applying a protective coating or sealant to a surface from which asbestos-containing material has been removed. Its primary function is to control and minimize the amount of airborne asbestos fiber generation that might result from any residual asbestos-containing debris on the substrate. Though the substrate may appear to be clean, miniscule fibers may have become lodged in cracks or crevices that were inaccessible.

FF. Movable Object: A piece of equipment or furniture in the work area which can be removed from the work area.

GG. Negative Pressure Ventilation System: A portable system equipped with HEPA filtration and capable of maintaining a constant low velocity air flow into contaminated areas from adjacent uncontaminated areas.

HH. NIOSH:

The National Institute for Occupational Safety and Health Building J N.E. - Room 3007

Atlanta, Georgia 30333

II. On-site Day: means any day in which work is being done on an asbestos abatement project that would require the services of a licensed asbestos worker, including but not limited to site preparation, establishment of decontamination facilities, removal and encapsulation, lock-down and removal of preparation.

JJ. OSHA:

The Occupational Safety and Health Administration

200 Constitution Avenue

Washington, D.C. 20210

KK. Plasticize: To cover floors and walls with plastic sheeting in an approved manner. 
LL. Removal: The stripping of any asbestos-containing materials from surfaces or components of a facility.

MM. Shower Room: A room between the clean room and the equipment room in the worker decontamination enclosure with hot and cold or warm running water controllable at the tap and suitably arranged for complete showering during decontamination.

NN. Sprayback: Sprayback is the process of replacing the asbestos-containing material that was originally removed with an effective substitute. This material should have architectural properties and capabilities adequate to meet specifications and requirements originally set for the space (i.e., acoustical insulation, fireproofing). Caution should be used when choosing a sprayback material to ensure that other adverse problems will not result (i.e., potentially harmful vapors generated during application), and that the lockdown material is fully compatible with the sprayback material.

OO. Staging Area: Either the holding area or some area near the waste transfer airlock where containerized asbestos waste has been placed prior to removal from the work area.

PP. Surfactant: A chemical wetting agent added to water to improve penetration.

QQ. Waste Transfer Airlock: A decontamination system utilized for transferring containerized waste from inside to outside the work area.

RR. Wet Cleaning: The process of eliminating asbestos contamination from building surfaces and objects by using cloths, mops, or other cleaning utensils which have been dampened with water and afterwards thoroughly decontaminated or disposed of as asbestoscontaminated waste.

SS. Worker: Any employee of a contractor physically engaged in the abatement of asbestos, or performing a task for the contractor in which direct contact with asbestos is likely.

TT. Work Area: Designated rooms; spaces, or areas of the project in which asbestos abatement actions are to be undertaken or which may become contaminated as a result of such abatement actions. A contained work area is a work area which has been sealed, plasticized, and equipped with a decontamination enclosure system. A non-contained work area is an isolated or controlled access work area which has not been plasticized nor equipped with a decontamination enclosure system.

UU. Worker Decontamination Enclosure: A decontamination system consisting of a clean room, a shower room, and an equipment room separated from each other and from the work area by airlocks and curtained doorways.

\section{STANDARDS AND GUIDELINES}

A. All work shall be done in strict accordance with all applicable Federal, State and Local regulations, standards and codes governing asbestos abatement and any other trade work done in conjunction with the abatement. 
B. The most recent edition of any relevant regulation, standard, document or code shall be in effect. Where conflict among the requirements exists, the most stringent requirements apply.

C. Copies of all standards, regulations, codes and other applicable documents, shall be available at the worksite.

D. Specific requirements shall include:

1. Occupational Safety and Health Administration (OSHA):

a) Title 29 Code of Federal Regulations Section 1910.1001 - General Industry Standard for Asbestos.

b) Title 29 Code of Federal Regulations Section 1910.134 - General Industry Standard for Respiratory Protection.

c) Title 29 Code of Federal Regulations Section 1926 Construction Industry.

d) Title 29 Code of Federal Regulations Section 1910.2 - Access to Employee Exposure and Medical Records.

e) Title 29 Code of Federal Regulations Section 1910.1200 - Hazard Communication.

2. Environmental Protection Agency (EPA):

a) Title 40 Code of Federal Regulations part 61 Subparts $A$ and $M$ (Revised Subpart B) - National Emission Standard for Asbestos.

3. Individuals will abide by all other applicable laws, rules and regulations, including but not limited to those relating to:

a) Worker's Compensation

b) Prevailing wager rates

c) Liability Insurance

d) EPA, OSHA, DOT, and DOL rules and regulations

\section{SITE SECURITY}

During the entirety of this project, i.e., during those times when elevated fiber concentrations are in evidence at each facility, site security shall be required. Security shall consist of a complete listing of all persons who enter and exit the controlled area. The time of entrance, the time of exit, as well as the nature of the business conducted, and with whom they are employed, address and phone number, shall also be recorded. At the present time it is not known who will provide site security, however, this will be in place for the duration of the project. 
The controlled area will be established by the utilization of barrier tape and shall extent a minimum of twenty-five feet in all directions from the periphery of the immediate project.

The area shall be further defined by the posting of signs which carry the wording "CAUTION ASBESTOS HAZARDS - DO NOT ENTER".

\section{BUILDING PREPARATIONS}

Each facility from which floor tile is to be removed, shall be prepared in such a manner as to assure that workers, are not exposed to elevated levels of fiber concentrations which may adversely impact their health or life quality. Likewise, near and far, adjacent occupants, segments of the public, bystanders, and others shall be protected by interception of airborme fibers at the source and through the utilization of various control techniques. Beyond this, no individual who is involved within the project, shall at any time, leave the area of removal, without first having demonstrated that all traces of contamination have been removed from his person.

Prior to the start of the project, and as a first action, each building shall have the electricity disconnected in the area where the removal is to be accomplished. All power to the area shall be re-established through the utilization of ground fault circuit breakers.

\section{Critical Barriers}

Critical barriers shall be established at the boundary of each area of floor tile to be removed. These barriers shall consist of a minimum of one layer of six mil polyethylene sheeting which shall be held firmly in place by stapling and taping. Additional layers may be applied as necessary in order to assure the integrity of the critical barriers. Entrances to the area shall be addressed with a minimum of a double flap. During the course of removal activity, all entrances and exits from the area shall be securely closed. Beyond this, all air supply and return ducts shall be securely sealed in order to assure that contamination of the same does not occur.

Provided that windows and doors are complete, and as an alternative to polyethylene sheeting, spray "poly" or "vinyl" may be utilized in any facility scheduled for demolition.

\section{Occupied vs Non-Occupied Facility}

Measures of control shall not differ on the basis of whether a facility is occupied or not occupied. Control measures shall be complete and in effect throughout the entirety of the project and sampling results shall be utilized as the basis of reflecting the adequacy of the control strategy.

\section{PROTECTIVE EQUIPMENT}

Protective equipment shall be in evidence and properly worn at all times when individuals (workers, supervisory personnel, visitors, etc) are within the confines of the controlled area. 
This action is required with regard to abatement worksites and in order to maintain compliance with the Occupational Safety and Health Act (OSHA) regulations (Title 29 Code of Federal Regulations Part $1910 \mathrm{Sec}$ 1910.1001) Alternatively, individuals associated with the project, shall not be outside the controlled area while wearing protective equipment. Each worker will be supplied with the necessary disposable uniforms and respirator filter changes per each work day, for the complete duration of the project. Work clothes will consist of disposable fully body coveralls, disposable head cover, disposable gloves, disposable footwear, and eye protection. Hard hats and rubber boots (steel toed) shall also be worn by workers. Additional uniforms will be available for individuals performing inspection tasks at the work site.

\section{Respirators}

The respirator protection program, Attachment I, shall be in full force and effect with regard to the floor tile removal/demonstration project.

\section{Decontamination Protocol}

Decontamination involves the progressive removal of oneself from a contaminated area to a clean area without the transportation of the material of contamination from one area to the other. Basically, four steps are involved.

1. Gross contamination removal.

Prior to an individual leaving the area of floor tile removal, gross contamination should be removed from disposable clothing including head and foot covers. This is normally accomplished by one individual assisting another individual and utilizing a HEPA vacuum.

2. Wash and remove footware/respirator cartridges.

Footwear shall be washed and removed immediately and at the exit of the floor tile removal area. The respirator shall then be washed, cleaned and disposable filters or cartridges then discarded as contaminated wasted. Masks as a minimum should be wiped out with a cloth soaked in alcohol and disinfectant, and hung to dry. Finally, the mask shall be placed in a clean plastic bag for storage.

3. Removal of contaminating clothing.

All individuals involved within the project shall double suit. Following gross contamination removal, and immediately outside the entrance to the removal area, the exterior suit of clothing shall be removed and bagged.

4. Leave controlled area.

Each individual should then proceed to the clean area to remove the final disposable coverall and dress in street clothing. 
Two important rules will be strictly enforced i.e., 1) there shall be no eating, drinking or smoking within any contaminated area (controlled area); 2) no individual shall remove their respirator for any reason while in an activated work area.

An alternative to the double suiting procedure, shall be the utilization of a portable shower and clean room. 


\section{PRE-PROJECT BRIEFING}

All asbestos removal projects have basic similarities, however, each will have differing problems requiring different solutions. Each individual having responsibility in this demonstration project, will receiving a briefing prior to each phase which will detail special considerations and/or conditions which may be encountered. These considerations will include:

1. Type of material to be removed, i.e., percent of asbestos, positive mastic, negative mastic, etc.

2. The control procedures to be employed, placement of critical barriers, doorways, loadouts, decontamination, etc.

3. Protective clothing to be worn, any hazards other than asbestos i.e., heat, cold, chemical, the need for hard hats, etc.

4. Specifics with regard to respiratory protection, needed/required.

5. Decontamination procedures, passage ways, emergency routes, and escape procedures.

6. Location of specific hazards, i.e., hot pipes, sharp edges, dangerous steps, etc.

7. Specific job assignments, prior job progress, future tasks, etc.

8. Awareness of unusual hazards i.e., limited vision, hearing and movement restrictions due to protective clothing, and respirators, uncertain footing, due to wet conditions on plastic and/or floor tile. 


\section{AIR MONITORING/TESTING}

The primary purpose of testing and air monitoring, is the documentation of environmental contamination is not occurring as a result of floor tile removal (abatement) and to insure and document that the respiratory protection selected, is sufficient for the airborne concentrations present and finally to ensure that elevated levels of airborne asbestos fibers, which may have resulted from removal procedures, have been sufficiently reduced to a level equal to or less than that existing before project initiation.

Two different types of analysis may be performed in order to accomplish data reflective of airborne fiber concentrations, Phase Contrast Microscopy (NIOSH 7400) and the more sophisticated Transmission Electron Microscopy. Optical microscopy is limited in that it cannot discern the thinner kinds of asbestos fibers. This represents a significant deficiency since it is generally accepted that the largest number of fibers which constitutes typical airborne asbestos contamination in buildings, tends to be of the thinner variety.

Stanley Engineering will provide air monitoring and industrial hygiene oversite during the length of the project. All air monitoring will be conducted in compliance with sampling requirements made by the Oklahoma State Department of Labor at the time of "Prep Inspection", (if requested). Allowable minimum sampling rates will be determined at that point in time. Sampling will be as follows and may be changed, only at the specific direction of Dr. Keith L. Stanley, P.E.,C.I.H.

\section{Background Monitoring}

1. Collect/analyze 3 samples within each area.

2. Collect/analyze 1 samples in each adjacent area (minimum of 3 samples)

3. Collect/analyze 3 ambient air sample

Samples collected in these areas are not expected to exceed 0.005 fibers per $c c$ of air. All other samples are not expected to exceed 0.005 fibers per $c c$ of air. Any result which exceeds the anticipated level will be explained or resampling will be required.

\section{Monitoring During Preparation for the Removal of Floor Tile}

1. Collect/analyze a minimum of 1 personnel air samples per individual worker per shift

2. Collect/analyze 3 area samples per shift.

3. Collect/analyze 3 air samples in adjacent areas per shift.

Any result greater than 0.01 fibers per $c c$, within the abatement area, will be considered excessive and will require the utilization of Personnel Protective Equipment. A minimum of dual cartridge half mask and tyvek will be necessary. 
Any result greater than background ( 0.005 fibers per $c c)$ in adjacent areas will be considered excessive and a complete wipe down of the area will be required.

\section{Required Monitoring During Abatement Activity}

1. Monitor $100 \%$ of all personnel on a daily (shift) basis.

2. Collect/analyze 3 area samples within the work area during each shift.

3. Collect/analyze 1 area samples in adjacent areas (outside each critical barrier) each shift (minimum of 3 samples).

4. Collect/analyze 1 area sample outside the loadout facility, per shift, while it is in use.

5. Collect/analyze 3 area sample per shift within the controlled area.

6. Collect/analyze 3 area samples per shift outside the controlled area. (one upwind and two downwind)

7. A minimum of 3 off shift area samples within each work area, shall be collected.

8. A minimum of 3 off shift area samples outside critical barriers shall be collected.

Results within the removal area will be consistent with the personal protection worn.

Any result outside the removal area shall not exceed 0.005 fibers per cc or background, whichever is greater. Results greater than 0.005 fibers per cc or background will require a complete wipe down of the area and retesting to demonstrate that control of the area has been re-established. All removal work will stop until such a time as control of the adjacent area has been established as demonstrated by air monitoring.

Following the conclusion of the removal project, clean air samples will be collected to demonstrate the return of the area to a level of fiber concentrations equal to or less than background concentrations or detection limits as established during the background monitoring of the facility. Samples shall be collected as follows:

1. A minimum of five samples shall be collected from within the removal area, of sufficient duration and volume to demonstrate a minimum detection. No less than six hours of collection per sample, will be required, and a minimum of 3000 liters of air.

2. Similarly and simultaneously, a minimum of five samples will be collected within the controlled area, external to critical barriers.

3. Similarly and simultaneously, as in one and two above, samples shall be collected for analysis by Transmission Electron Microscopy. Additionally, however, two field blanks and one laboratory blank shall also be analyzed.

The following specification will be utilized for clean air monitoring. 
1. Sampling will not begin until all surfaces are dry and the area will be stirred with a fan or leaf blower.

2. A total of approximately 3,000 liters of air shall be collected at 7 LPM. Under no circumstances will less than six hours of sample duration be achieved.

\section{REMOVAL SCENARIOS/TECHNIQUES}

Each technique utilized to remove asbestos containing floor tile shall be documented in order to fully describe every detail utilized within that specific scenario of removal. As a minimum, each scenario shall be described as follows:

* A statement of the room condition prior to the start of removal.

** Square feet of floor tile

** Floor tile types

** Square footage of windows

** Number of Doors

** Prior use

* Present a full description of the preparation accomplished with regard to the removal area.

$\begin{array}{ll}* * & \text { Initial cleaning } \\ * * & \text { Window coverings } \\ * * & \text { Door seals } \\ * * & \text { Pressures }\end{array}$

* Tools of removal

** Shovels

** Hoes

** Water

** Air cleaning devises

** Others

* Time required for removal

* Manpower utilized during removal

* Work rate
** Maximum
** Breaks
** Other 
* Identification of removal stages

$\begin{array}{ll}* * & \text { Wetting } \\ * * & \text { Removal } \\ * * & \text { Pick up and Bag } \\ * * & \text { Wipedown and Loadout }\end{array}$

+s

$\begin{array}{ll}\text { Disposal } \\ * * & \text { Method } \\ * * & \text { Location } \\ * * & \text { Control measures in route } \\ * * & \text { Offloading }\end{array}$

\section{PROPOSED SCENARIOS OF REMOVAL}

The following brief description is provided which describes the proposed removal method.

1. Wet Removal of Floor Tile by Hand Exercising Ordinary Care

1a Remove Mastic Utilizing Current State-of-the-Art Techniques, differentiated by floor substrate (wood, concrete)

2. Dry Removal of Floor Tile by Hand Exercising Ordinary Care

3. Strip with Power Stripped utilizing commercial strip liquid and recommended stripper pad.

3.a Aggressive dry removal from wood floor, by hand utilizing maximum workers to accomplish removal task in minimal time.

4. Aggressive dry removal from concrete floor, by hand, utilizing maximum workers to accomplish removal task in minimal time.

4.a Grind to remove mastic (wet sand and power grinder)

5. Wet vacuum to clean the floor prior to wetting floor with amended water, cover with visqueen, on concrete, allow to stand (time treatment) removal by hand.

6. Water soak with amended water, cut through wood floor, and completely remove in, tact, four by eight sheet of wood flooring bearing tile. Alternative sizes may be appropriate.

7. Aggressive dry removal from concrete floor, by hand, utilizing maximum workers to accomplish removal task in minimal time. To be accomplished without the utilization of critical barriers. Wind speeds not to exceed $15 \mathrm{mph}$, measurements of airborne fiber concentrations shall be intensified.

8. Power removal floor tile by commercial removal methods utilizing prototype machine of Cooper and Associates. 
Each scenario will be concluded with a final cleanup of the area, which will, as a minimum, include HEPA vacuuming of each floor from which the tile has been removed, as well as wet mopping and wet wiping. It is anticipated that samples will be collected to reflect the content of dust remaining on the floor, prior to the utilization of the HEPA vacuum, and following the wet wiping.

A typical building floor tile demonstration project as anticipated at this time, will be accomplished on wooden structures with either wood or concrete floors. It is not anticipated that the structures will be occupied at the time of floor tile removal, however, the intensity of air monitoring will be of an extent to evaluate adjacent areas with regard to airborne fiber concentrations. A typical building floor plan, Attachment II, reflects both floors within a single structure and will present some variations to the monitoring schedule and removal scheme in order to assure proper documentation of all efforts and activities. 


\section{Attachment I}

\section{RESPIRATOR PROGRAM AND POLICY}

\section{GENERAL}

Stanley Engineering is committed to employee safety. Our employees are the most important aspect of asbestos abatement and their protection from harm is a first priority. This "Respirator Program and Policy" will be fully utilized by all employees and management personnel.

Federal and State laws required that respirators be worn if asbestos concentrations exceed 1 fibers/cc (those fibers that are 5 micrometers and larger). The present day method of measuring fiber concentrations requires air monitoring followed by laboratory analysis that involves a 12 to 24 hour time lag. We therefore require that respirator be worn during all removal and cleanup operations, and during encapsulation to be certain of protection.

Applicable rules to this policy are as follows:

EPA $\quad 40$ CFR $763.120 \& 121$

Regulations Governing Asbestos Abatement Projects

OSHA 29 CFR 1910.134

Respiratory Protection

29 CFR 1926.58

Asbestos Rules for the

Practices for Respiratory

ANSI $\quad \mathrm{Z} 88.2$

Protection

Construction Industry

STATE Title 40, Sections

451 through 457 of

Oklahoma Statutes

Rules for Abatement of Friable Asbestos Materials 


\section{ADMINISTRATION}

This policy shall be administered by a designated person that will be identified as the Program Coordinator.

\section{EQUTPMENT}

All respirators furnished for the employee will have an approved MSHA and NIOSH rating. Type " $C$ " supplied-air respirators shall be used during the beginning shift of an abatement job when a containment is used. Personnel air sample results will be used to determine what level of protection will be necessary for the shifts that follow.

Upgrading or downgrading in respiratory equipment will be based on maintaining a level of 0.01 fibers/cc inside the mask. The following table will decide what will be required.

\section{AIRBORNE CONCENTRATION OF ASBESTOS}

NOT IN EXCESS OF 0.1 F/CC

NOT IN EXCESS OF $0.5 \mathrm{~F} / \mathrm{CC}$

NOT IN EXCESS OF 1.0 F/CC

NOT IN EXCESS OF $10 \mathrm{~F} / \mathrm{CC}$

NOT IN EXCESS OF 10 F/CC

\section{REOUIRED \\ RESPIRATOR}

HALF-FACE AIR-PURIFYING RESPIRATORS EQUIPPED WITH HEPA FILTERS

FULL-FACE AIR $\quad$ PURIFYING
RESPIRATOR EQUIPPED WITH HEPA
FILTERS

ANY POWERED AIR PURIFYING RESPIRATORY EQUIPPED WITH HEPA FILTER(S)
FULL-FACE SUPPLIED-AIR RESPIRATOR OPERATED IN A CONTINUOUS FLOW MODE

$\begin{array}{llr}\text { FULL FACE } & \text { SUPPLIED } & \text { AIR } \\ \text { RESPIRATOR } & \text { OPERATED } \\ \text { PRESSURE DEMAND MODE } & & \text { A }\end{array}$

Special situations may arise where the necessary or preferred equipment is a self contained breathing apparatus. When this is the case, it will be the Program Coordinator's responsibility to advise the regulating agency (i.e., ODOL, Corps of Engineers, etc.) of the intent and the justification.

The Contractor will provide respirators. If the employee finds the unit he is furnished with to be uncomfortable, he will be offered other types until he is satisfied with the fit. The employee may request a power air purifying respirator (PAPR). Provided that a fit cannot be achieved, the employee will not be allowed within abatement areas. 


\section{STANDARD OPERATING PROCEDURES}
A. Each employee will be issued a respirator, properly selected and fit tested.
B. Each employee will label his or her respirator with name and issue date, and will be held responsible for it.
C. No employee will borrow or share another employee's respirator, unless they receive a supervisor's approval and the unit has been thoroughly checked and disinfected.
D. After each day's use, or more often if necessary, the employee must clean, inspect, and store his/her respirator in a convenient and sanitary location, preferably a sealed plastic bag in the clean change room.

E. Employees will be fit tested (qualitative) at least every six months.

Employees will read the literature furnished with the respirator before attempting to wear the unit.

Replacement filters and parts will be stored at a central location designated by job conditions. All employees must know this location. Only filters and replacement parts specified by the respirator manufacturer for job hazard conditions (asbestos) may be used. If in doubt, contract the Program Coordinator. Do not guess; it could be harmful to your health. Health effects from breathing hazardous substances often take many years to result in disease.

Respirators will be donned in the clean room/area and doffed in the shower after thorough wetting of filters. Filters shall be changed after each shower. Filters must be changed when breathing becomes difficult or any changes in the filter function are noted. Examine your filters when you clean and inspect your respirator each day. Replace them if necessary, especially if breathing becomes difficult. Replaced filters must be discarded in plastic bags and disposed of properly. Filters must be replaced outside the work area in clean air.

\section{MEDICAL}

All employees must be physically fit to work while wearing a respirator. Air purifying respirators with cartridges make breathing more difficult because the filters can reduce the flow of air. If you have heart or lung problems, wearing a respirator could present an unacceptable risk.

All employees are required to take a company-furnished medical examination within 30 days of starting employment. This examination includes chest $\mathrm{x}$-rays and lung capacity tests as well as a general medical history, examination of the respirator system and audiometric examination.

Accurate medical records of all employees will be kept by the Contractor and will be provided upon required to all employees.

Additional medical requirements for employees engaged in asbestos removal work include: 
1. Employees should not smoke. Studies have indicated that smoking increases the risk of contracting lung cancer by 92 times.

2. Employees must be clean shaven with short sideburns and hair if they wear disposable or cartridge type respirators. Beards and sideburns interfere with the respirator face seal so the air, instead of going through the filter, actually goes around the respirator. Long hair provides a place for all asbestos fall-out to collect, even though protected by clothing and head covers.

3. Employees must not chew gum or tobacco if they wear disposable or cartridge type respirators because it causes excess facial movement which can break the face seal.

4. Employees who wear prescription glasses must wear a respirator that accommodates glasses without interfering with the protection to the wearer.

\section{FITTING}

A respirator should fit snugly and comfortabie. It should not leave red marks, deep indentations on face, or make it difficult to turn your head.

The Program Coordinator will demonstrate two types of fitting tests as described below.

\section{POSITIVE PRESSURE TEST}

Close the exhalation valve by placing the palm of your hand over the opening. The exhalation valve cover will have to be removed for this test. Exhale gently into the facepiece. This test is satisfactory if a slight positive pressure can be built up inside the facepiece without feeling leakage of air at the face seal. Replace the valve cover and proceed to work.

\section{NEGATIVE PRESSURE TEST}

Close off the inlet openings of the cartridges using the fit check filter cover, if available, with the palm of your hands or with plastic. Inhale gently so that the facepiece collapses slightly, and hold your breath for ten seconds. If the facepiece remains collapsed and no inward leakage of air is detected around the face seal, the respirator fits satisfactorily.

Positive and negative fit testing shall be conducted by the wearer each time the respirator is put on or re-positioned.

\section{IRRITANT FUME TEST}

You may be asked, by your supervisor, to be fit tested under simulated work conditions, using irritant fume. This test on your respirator will be performed by a Certified Industrial Hygienist or his technician who will challenge the seal to a series of head and face movements and breathing both normal and then deep. 


\section{CLEANING}

Respirator will be washed and cleaned in a designated area and either taken home by employee or stored in a clean location on job site.

Cleaning will be accomplished utilizing the following method.

a. Wash with a detergent and disinfectant (Hypochlorite solution) in warm water and with a brush.

b. Rinse in clean water or rinse over with a disinfectant and once with warm water. Rinsing with water is required to remove traces of detergent and disinfectant left on the mask which can cause dermatitis (skin rash).

\section{DRYING}

Respirators can be dried on a rack or clothes line so that the facepiece rubber will not "set" crooked as it dries. Also, care should be taken not to damage the respirator unit.

\section{STORAGE}

The units will be stored in plastic bags so they are protected from dust, sunlight, heat, extreme cold, excessive moisture and damaging or contaminating chemicals.

\section{INSPECTION}

All respirator protective equipment must be inspected:

Before and after each use, and;

During cleaning

Air purifying respirators (Quarter-Mask, Half-Mask, Full Facepiece)

A facepiece-excessive dirt (clean all dirt from facepiece)

Facepiece cracks, tears or holes (obtain a new respirator from your supervisor).

Facepiece distortion (allow facepiece to "sit" free from any constraints and see if distortion disappears: if not, obtain a new respirator.

\section{POWERED AIR-PURIFYING RESPIRATORS (PAPR)}

A. Face-seal - clean daily and check for wear - replace as needed.

B. Face-shield - clean daily or more often as necessary. 
C. Head-piece - clean daily or more often as necessary.

D. Battery - charge after each day's use and replace when needed.

E. Filters - inspect pre-filter and replace if dirty. Check with supervisor for replacement of HEPA filter.

\section{HEADSTRAPS - CHECK FOR:}

A. Breaks or tears - replace.

B. Loss of elasticity - replace.

C. Broken or malfunctioning buckles or attachments - replace.

D. Excessively worn serrations on the head harness which might allow the facepiece to slip - replace.

\section{INHALATION VALVE, EXHALATION VALVE - CHECK FOR:}

A. Detergent residue, dust particles, dirt on valve or valve seat.

B. Cracks, tears or distortion in the valve material or valve seat.

C. Missing or defective valve cover.

\section{FUTER ELEMENTS - CHECK FOR:}

A. Proper filter for the hazard.

B. Approval designation.

C. - Missing or worn gaskets.

D. Worn threads - both filter and facepiece threads.

E. Cracks or dents in filter housing.

\section{CORRUGATED BREATHING TUBE (POWERED AIR) CHECK FOR:}
A. Cracks or holes.
B. Missing or loose hose clamps.
C. Broken or missing end connectors. 


\section{REPAIRS}

Only manufacturer's approved parts shall be used to repair respirators. Aithough some parts may look the same, the manufacturer will only guarantee their respirators if their parts are used. If in doubt, ask your supervisor.

All repairs must be performed at approved locations that are clean and sanitary inside work area.

\section{SURVEILLANCE OF WORK AREA AND RESPIRATOR PROGRAM}

The Contractor's representative will review the work practices and respirator maintenance procedures by the employees to insure the above practices are followed. This will be performed to insure that workers understand the importance and intent of the respirator program.

Air monitoring will be conducted for all asbestos abatement projects.

\section{STANDARD SAFETY PRACTICES}

The following are basic safety practices this company recommends. Please provide any added practices you feel we should incorporate in this standard.

\section{GENERAL}

1. All persons shall follow safe work practices.

2. All unsafe conditions or practices shall be reported to the proper authority.

3. Safety meetings will be held whenever improper work habits are noted.

4. Anyone known to be under the influence of drugs or intoxicating liquor shall not be allowed on the job.

5. Horseplay and scuffling are prohibited.

6. All work shall be well planned to prevent accidents.

7. All injuries or accidents shall be reported promptly to job supervisor.

8. When lifting $\mathrm{h}$ heavy objects, use legs, not back muscles.

9. Shoes shall have proper soles and shall not be badly worn out.

10. No material or tools shall be dropped from structures or scaffolding without taking proper precautions. 
11. Notify supervisor of any equipment or tools required for proper performance of work.

\section{INJURIES AND FIRST AID}

1. There will be one person qualified in first aid on the job at all times.

2. All employees must be informed as to the nearest source of first aid. If unsure employee must ask this location.

3. A properly equipped industrial first aid kit must be available and on the job at all times. Employees should know its location and, if not, ask supervisor.

4. Report all accidents or injuries as soon as they occur.

5. When working in an asbestos contaminated atmosphere, do not waste time in decontamination procedure to remove injured worker.

\section{HOUSEKEEPING}

1. Keep all traffic aisles clean and clear of obstructions.

2. All supplies and tools are to be kept in proper place.

3. All electric cords, air and water hoses shall be kept coiled when not in use.

\section{WORK PRACTICE}

It is important to minimize the risk of exposure to asbestos. This means following the above procedures and instructions, but...more importantly, to keep air concentrations of asbestos as low as reasonably possible. This means following good work practices that include:

1. Keeping wet, all asbestos that is being removed or has been removed.

2. Disturb the asbestos as little as possible through removal and clean-up techniques.

3. Clean-up removed asbestos promptly and keep it wet.

4. Removing contaminated clothing, leaving the work site.

\section{SPECIAL OPERATING CONDITIONS}

\section{EMPLOYEE'S RESPONSIBILITIES}

The employee has the following responsibilities as part of this program. 
A. Use respirators as instructed.

B. Guard against damaging the respirator.

C. Go immediately to an area of "clean" air if your respirator malfunctions.

D. Reports any malfunctions of respirators to your supervisor. These include: Discomfort Resistance to breathing Fatigue due to respirator usage Interface with vision or communication Restriction to movement

Use of respirators in cold temperature causes fogging of facepiece and freezing of the exhalation valve to seal, due to moisture in the exalted air.

Use of respirator in hot environment can put additional stress on the user. Employees should be aware of heat stress and reduce respirator work time. 


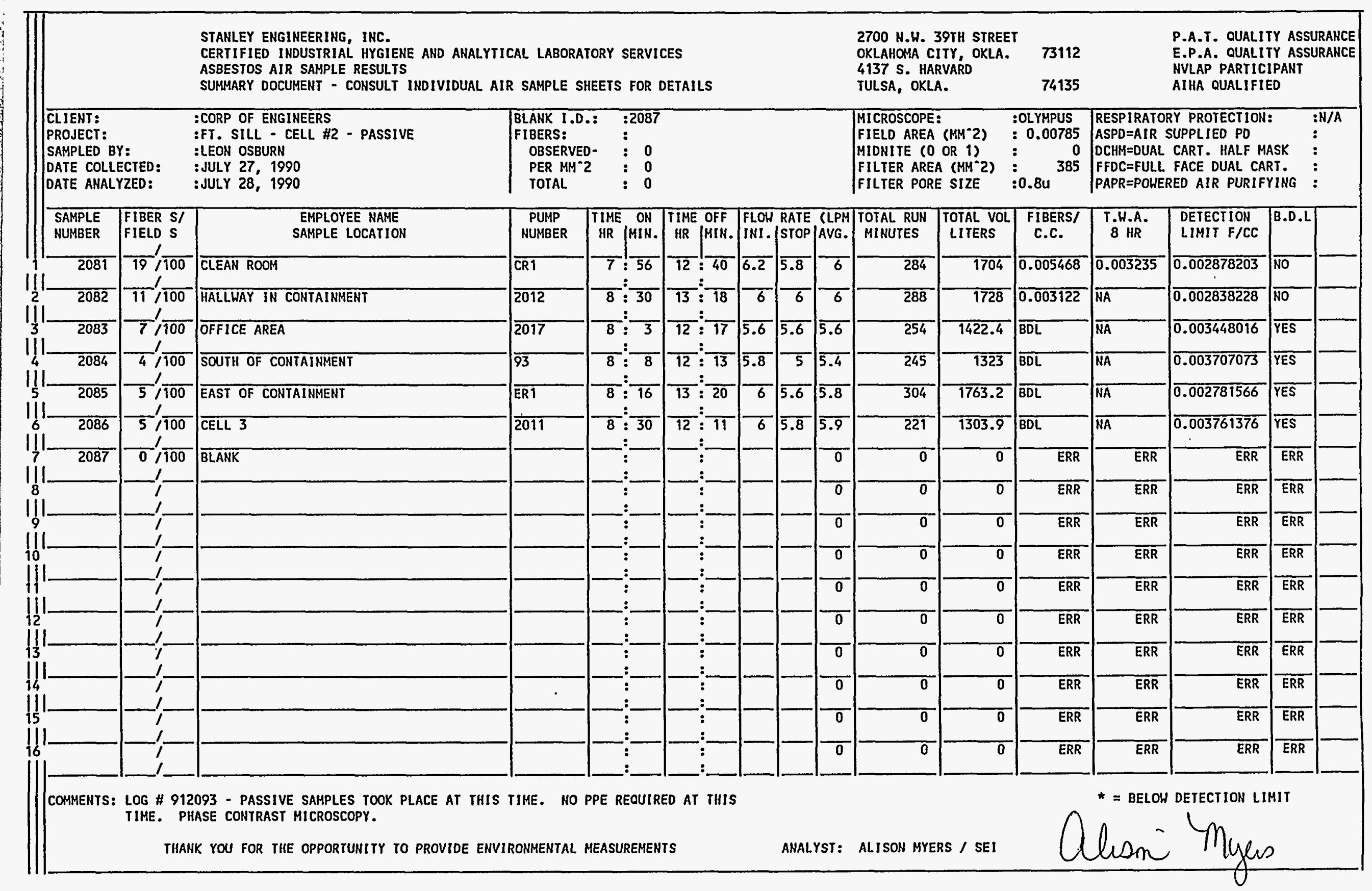




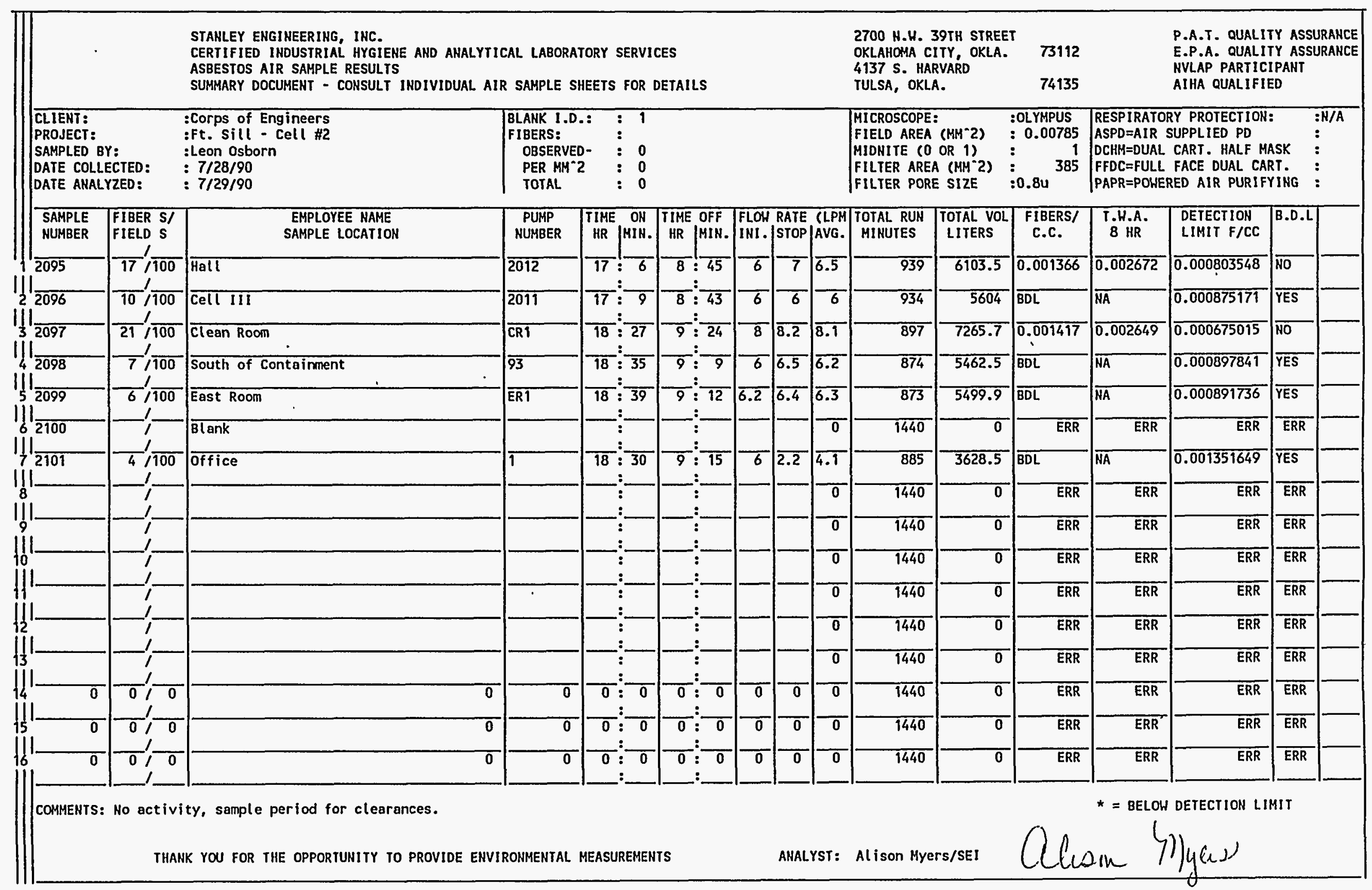




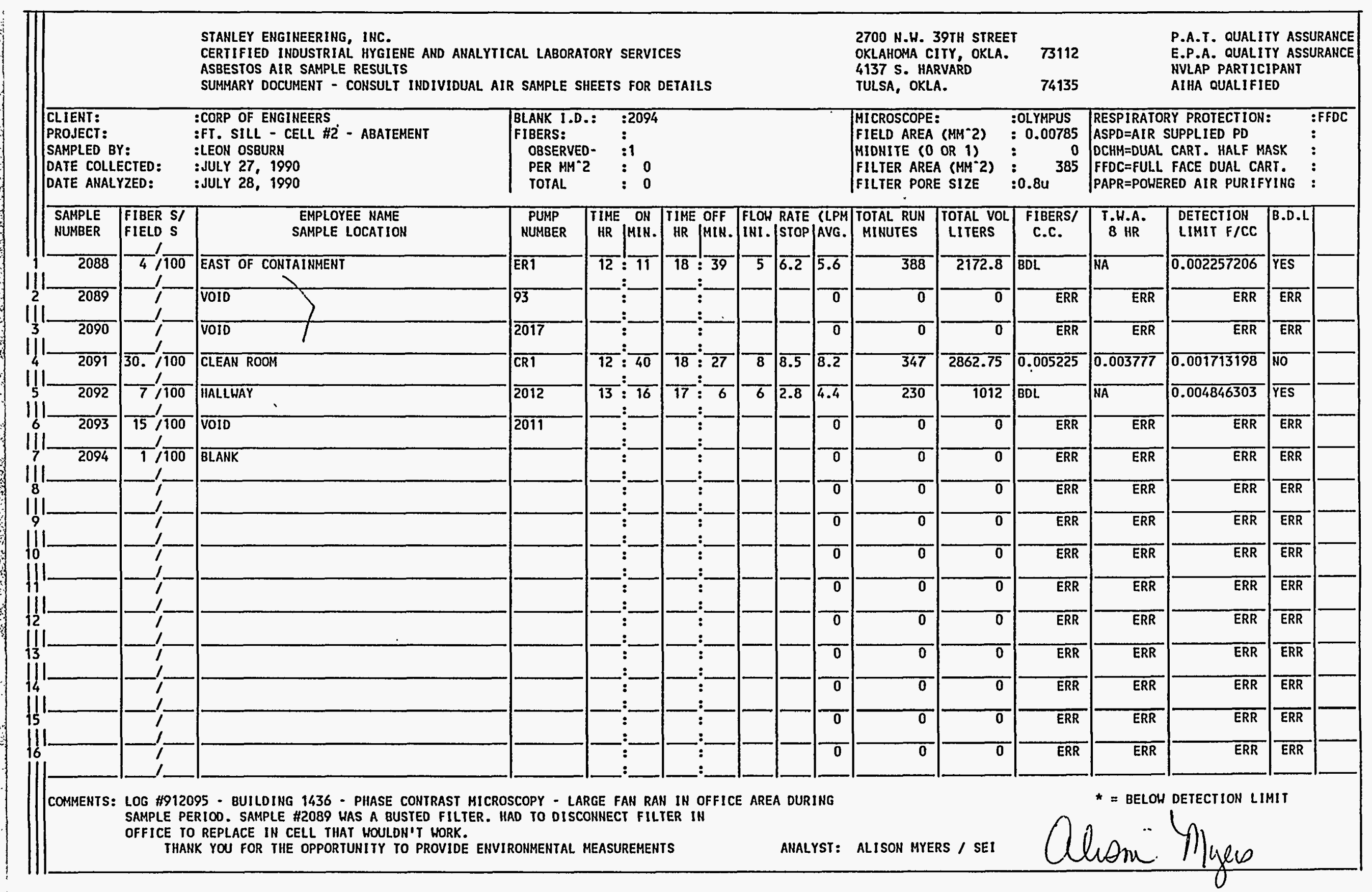




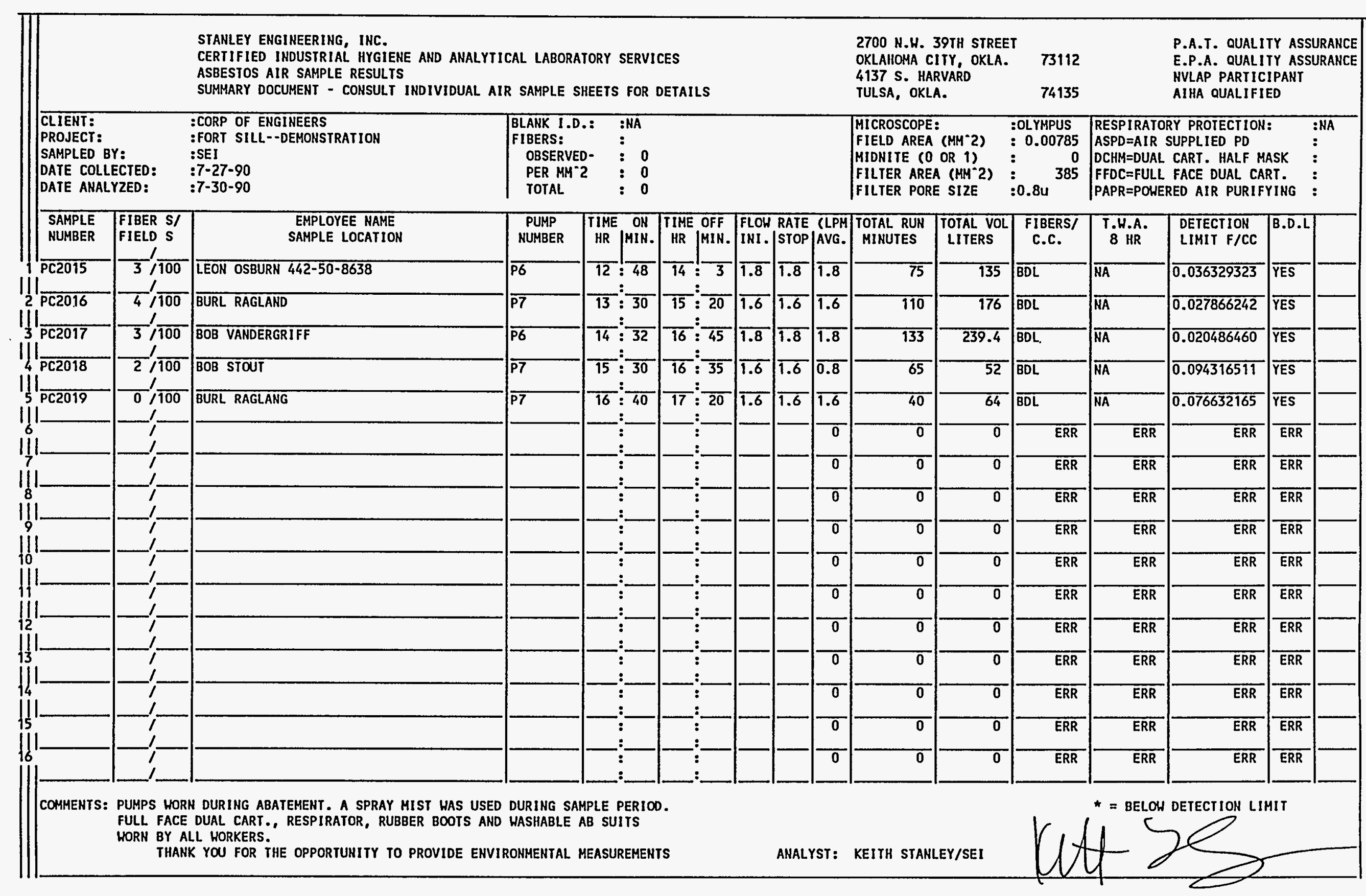




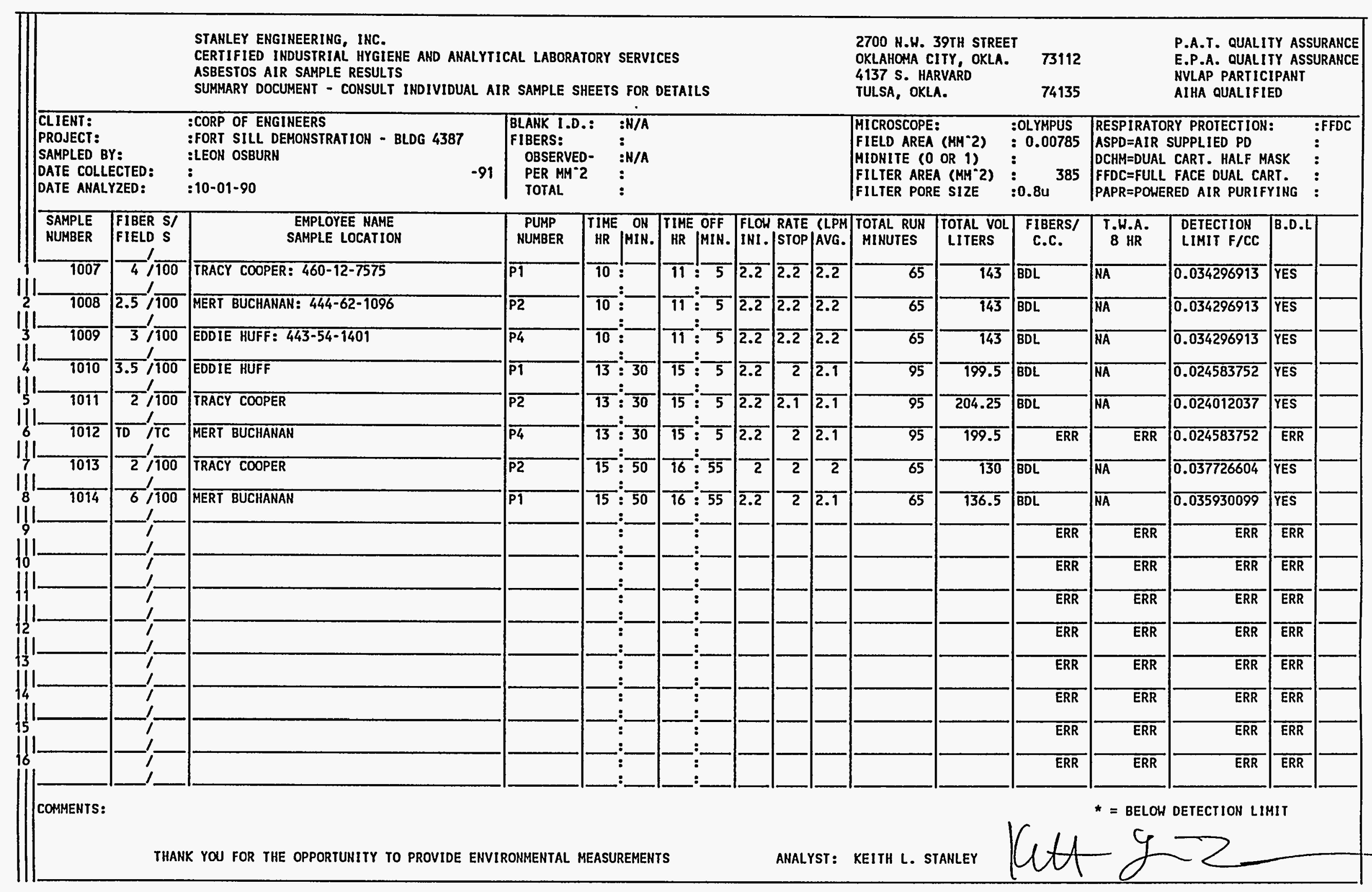




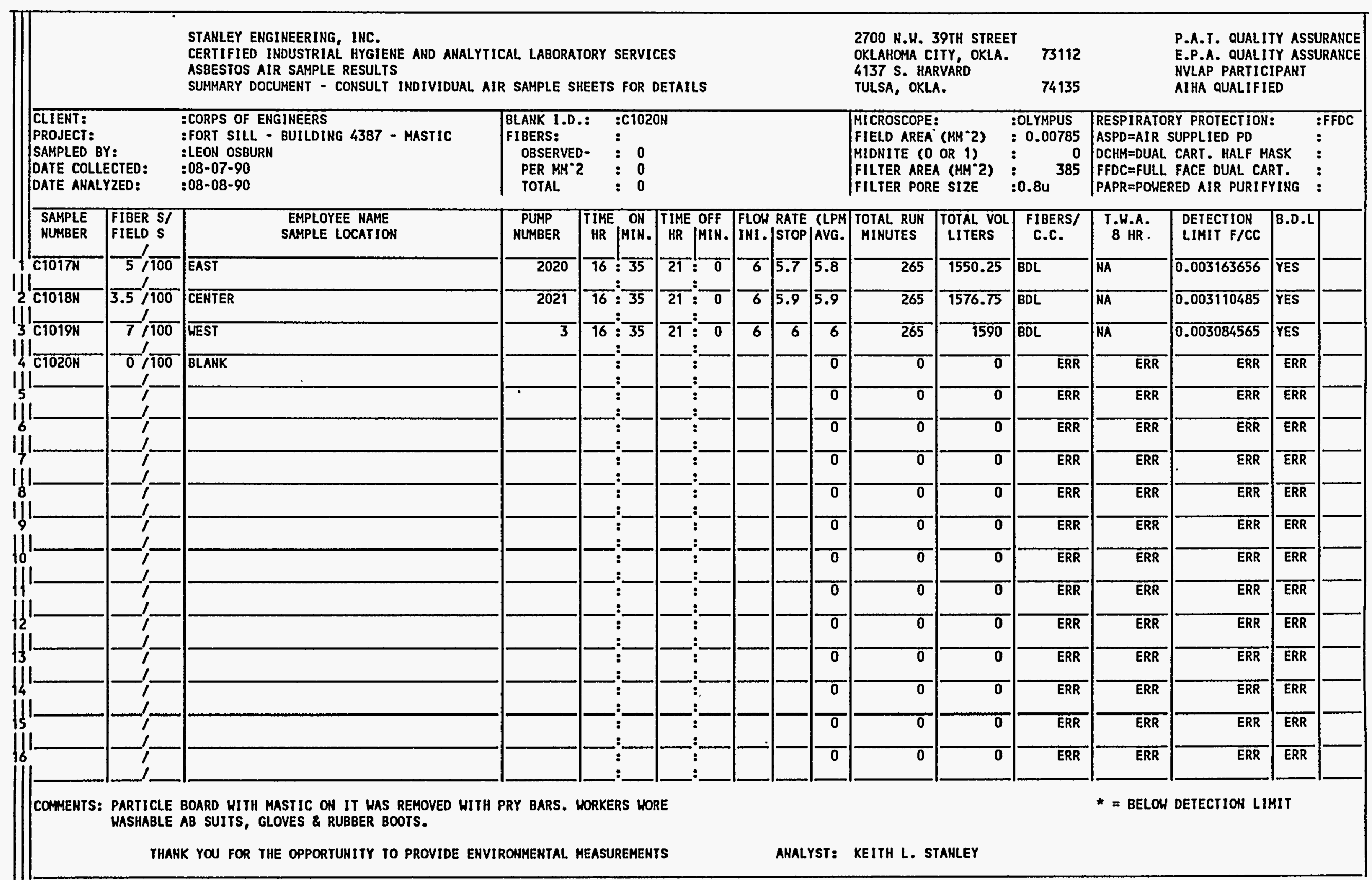




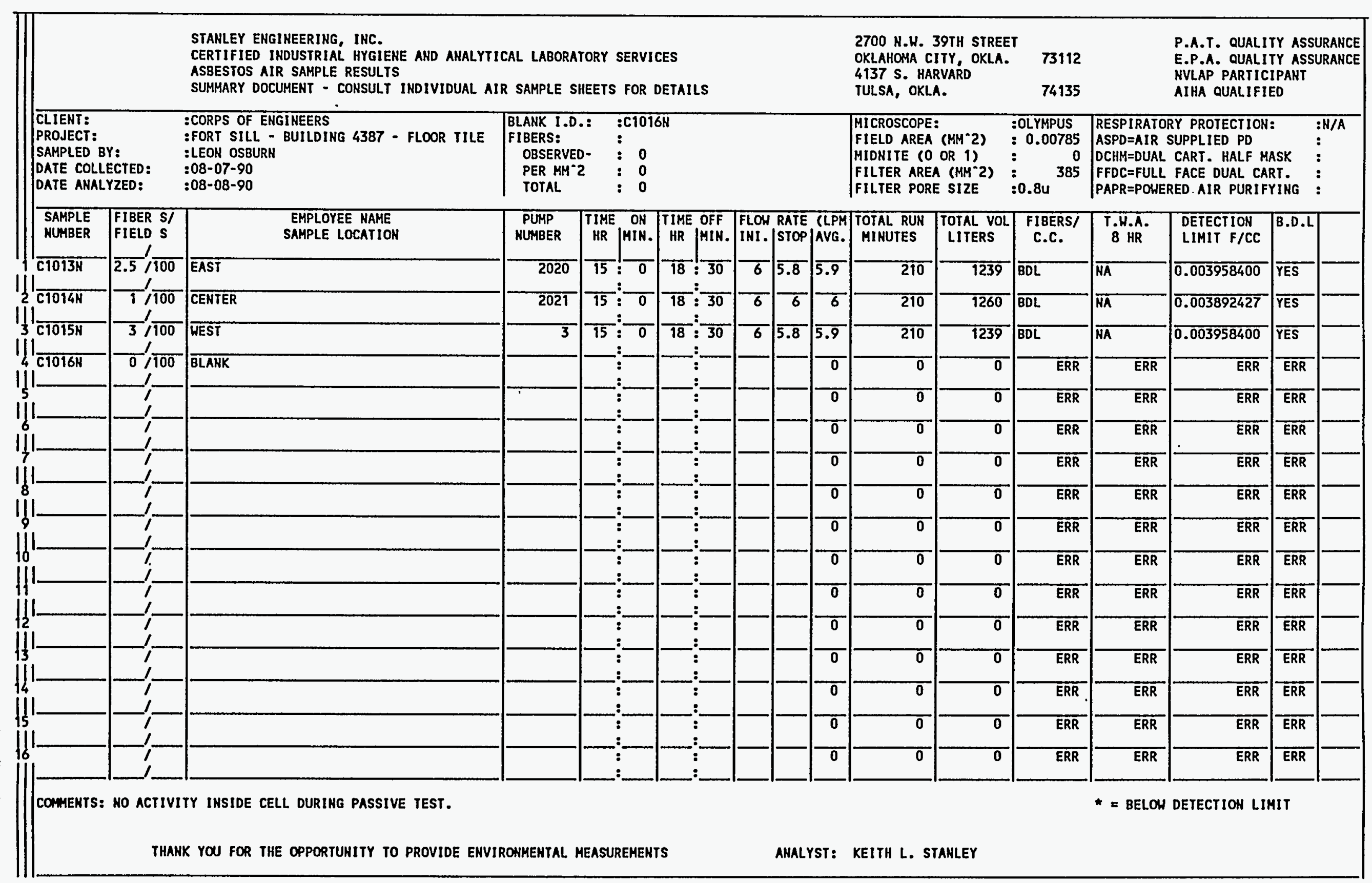




\begin{tabular}{|c|c|c|c|c|c|c|c|c|c|c|c|c|c|c|c|}
\hline & & \multicolumn{7}{|c|}{$\begin{array}{l}\text { STANLEY ENGINEERING, INC. } \\
\text { CERTIFIED INDUSTRIAL HYGIENE AND ANALYTICAL LABORATORY SERVICES } \\
\text { ASBESTOS AIR SAMPLE RESULTS } \\
\text { SUMMARY DOCUMENT - CONSULT INDIVIDUAL AIR SAMPLE SHEETS FOR DETAILS }\end{array}$} & \multicolumn{2}{|c|}{$\begin{array}{l}2700 \text { N.H. 39TH STREET } \\
\text { OKLAHOKA CITY, OKLA. } \\
4137 \text { S. HARVARD } \\
\text { TULSA, OKLA. }\end{array}$} & 73112 & & \multicolumn{3}{|c|}{$\begin{array}{l}\text { P.A.T. QUALITY ASSURANCE } \\
\text { E.P.A. QUALITY ASSURANCE } \\
\text { MVLAP PARTICIPANT } \\
\text { AIHA QUALIFIED }\end{array}$} \\
\hline \multicolumn{2}{|c|}{$\begin{array}{l}\text { CLIENT: } \\
\text { PROJECT: } \\
\text { SAMPLED BY: } \\
\text { DATE COLLECTED: } \\
\text { DATE ANALYZED: }\end{array}$} & \multirow{2}{*}{$\begin{array}{l}\text { :CORP OF ENGIMEERS } \\
\text { :FORT SILL - BUILDING } 4387 \\
\text { :LEON OSBURH / S.E.1. } \\
\text { :08-06-90 } \\
: 08-07-90 \\
7 \mid \begin{array}{c}\text { EMPLOYEE NAME } \\
\text { SAMPLE LOCATION }\end{array} \\
\end{array}$} & \multicolumn{3}{|c|}{\begin{tabular}{|ll} 
BLANK I.D.: & $:$ C1004H \\
FIBERS: & $\vdots$ \\
OBSERVED- & $\vdots 0$ \\
PER MH^2 & $\vdots 0$ \\
TOTAL & $: 0$
\end{tabular}} & & & & \multicolumn{2}{|c|}{$\begin{array}{l}\text { MICROSCOPE: } \\
\text { FIELD AREA }(M M-2) \\
\text { MIDNITE CO OR } 1) \\
\text { FILTER AREA (MM-2) } \\
\text { FILTER PORE SIZE }\end{array}$} & $\begin{array}{l}: \text { OLYMPUS } \\
: 0.00785 \\
\vdots \\
: 0.8 u^{385}\end{array}$ & \multicolumn{3}{|c|}{$\begin{array}{l}\text { RESPIRATORY PROTECTION: } \\
\text { ASPD =AIR SUPPLIED PD } \\
\text { DCHM=DUAL CART. HALF MASK } \\
\text { FFDC=FULL FACE DUAL. CART. } \\
\text { PAPR=POHERED AIR PURIFYING }\end{array}$} & $\begin{array}{l}1+N / A \\
\vdots \\
\vdots \\
\vdots\end{array}$ \\
\hline $\begin{array}{l}\text { SAMPLE } \\
\text { NUMBER }\end{array}$ & $\begin{array}{l}\text { FIBER S/ } \\
\text { FIELD S }\end{array}$ & & $\begin{array}{l}\text { PUMP } \\
\text { NUMBER }\end{array}$ & \begin{tabular}{|l|l|} 
TIME & ON \\
HR & MIN. \\
\end{tabular} & \begin{tabular}{|l|l|} 
TIME OFF \\
HR
\end{tabular} & $\begin{array}{l}\text { FLOH } \\
\text { INI. }\end{array}$ & $\begin{array}{l}\text { RATE } \\
\text { STOP }\end{array}$ & $\begin{array}{l}\text { (LPPM } \\
\text { AVG. }\end{array}$ & $\begin{array}{c}\text { TOTAL RUN } \\
\text { MINUTES }\end{array}$ & $\begin{array}{l}\text { TOTAL VOL } \\
\text { LITERS }\end{array}$ & $\begin{array}{l}\text { FIBERS/ } \\
\text { C.C. }\end{array}$ & $\begin{array}{l}\text { T.H.A. } \\
8 \mathrm{HR}\end{array}$ & $\begin{array}{l}\text { DETECTION } \\
\text { LIMIT } \mathrm{F} / \mathrm{CC}\end{array}$ & B.D.L & \\
\hline C1001N & $7 / \overline{100}$ & NORTHEAST INSIDE CELL 4 & 2020 & $16 ! 48$ & $\overline{20}: \overline{30}$ & $\overline{6}$ & $\overline{6.4}$ & $\overline{6.2}$ & $\overline{222}$ & 1376.4 & $\overline{\mathrm{BDL}}$ & $\overline{N A}$ & $\overline{0.003563250}$ & $\overline{\text { YES }}$ & \\
\hline C1002N & $\overline{3} / \overline{100}$ & MORHT CENTER INSIDE CELL 4 & 2021 & $16: \overline{48}$ & $\overline{20}: \overline{30}$ & 6 & 5.9 & $\overline{5.9}$ & $\overline{222}$ & $\overline{1320.9}$ & $\overline{\mathrm{BDL}}$ & $\overline{N A}$ & $\overline{0.003712967}$ & $\overline{Y E S}$ & \\
\hline $\mathrm{C1003N}$ & $4 / \overline{100}$ & NORTHWEST INSIDE CELLL & 3 & $16: \overline{48}$ & $\overline{20}: \overline{30}$ & 6 & 6 & 6 & $\overline{222}$ & 1332 & $\overline{B D L}$ & $\overline{N A}$ & $\overline{0.003682026}$ & $\overline{\text { YES }}$ & \\
\hline $\mathrm{C1004 \textrm {N }}$ & $0 / \overline{100}$ & BLANK & & $\because-$ & $\because-$ & & & $\overline{0}$ & $\overline{0}$ & $\overline{0}$ & $\overline{E R R}$ & $\overline{\text { ERR }}$ & $\overline{\text { ERR }}$ & $\overline{\text { ERR }}$ & \\
\hline & & & & - & $\because$ & & & $\overline{0}$ & $\overline{0}$ & $\overline{0}$ & $\overline{\text { ERR }}$ & $\overline{\text { ERR }}$ & $\overline{\text { ERR }}$ & $\overline{E R R}$ & \\
\hline & & & & $\because$ & $\because$ & & & $\overline{0}$ & 0 & $\overline{0}$ & $\overline{\text { ERR }}$ & $\overline{\text { ERR }}$ & $\overline{\text { ERR }}$ & $\overline{\text { ERR }}$ & \\
\hline & & & & 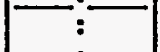 & $\because$ & & & $\overline{0}$ & $\overline{0}$ & $\overline{0}$ & $\overline{\text { ERR }}$ & $\overline{\text { ERR }}$ & $\overline{\text { ERR }}$ & $\overline{\text { ERR }}$ & \\
\hline & & & & & : & & & o & $\overline{0}$ & $\overline{0}$ & $\overline{\text { ERR }}$ & $\overline{\text { ERR }}$ & $\overline{\text { ERR }}$ & ERR & \\
\hline & & & & $\because$ & $\because$ & & & $\overline{0}$ & $\overline{0}$ & $\overline{0}$ & $\overline{\text { ERR }}$ & $\overline{\text { ERR }}$ & $\overline{\text { ERR }}$ & $\overline{\text { ERR }}$ & \\
\hline & & & & $:-$ & $\because$ & & & $\overline{0}$ & $\overline{0}$ & $\overline{0}$ & $\overline{\text { ERR }}$ & $\overline{\text { ERR }}$ & $\overline{\text { ERR }}$ & $\overline{E R R}$ & \\
\hline & & & & $\because$ & $\because$ & & & $\overline{0}$ & 0 & 0 & ERR & $\overline{\text { ERR }}$ & $\overline{\text { ERR }}$ & $\overline{\text { ERR }}$ & \\
\hline & & & & $:$ & 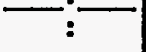 & & & $\overline{0}$ & $\overline{0}$ & $\overline{0}$ & $\overline{\text { ERR }}$ & $\overline{\text { ERR }}$ & $\overline{\text { ERR }}$ & $\overline{\text { ERR }}$ & \\
\hline & & & & $\because$ & $\because$ & & & $\overline{0}$ & $\overline{0}$ & $\overline{0}$ & $\overline{\text { ERR }}$ & $\overline{\text { ERR }}$ & $\overline{\text { ERR }}$ & $\overline{\text { ERR }}$ & \\
\hline & & & & 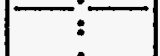 & . & & & $\overline{0}$ & $\overline{0}$ & $\overline{0}$ & $\overline{\text { ERR }}$ & $\overline{\text { ERR }}$ & $\overline{\text { ERR }}$ & $\overline{\text { ERR }}$ & \\
\hline & & & & $:$ & : & & & $\overline{0}$ & $\overline{0}$ & $\overline{0}$ & $\overline{\text { ERR }}$ & $\overline{\text { ERR }}$ & ERR & $\overline{\text { ERR }}$ & \\
\hline & & & & $:-$ & :- & & & 0 & 0 & $\overline{0}$ & $\overline{E R R}$ & $\overline{\text { ERR }}$ & $\overline{\text { ERR }}$ & $\overline{E R R}$ & \\
\hline COMMENTS: & $\begin{array}{l}\text { CELL } 1 \text { HA } \\
\text { DURING TE } \\
\text { PASSIVE-M } \\
\text { THAN }\end{array}$ & $\begin{array}{l}\text { AS HEPA VACED BEFORE PASSIVE TEST BEGAN. } \\
\text { EST. } \\
\text { METHOD } 7402 \\
\text { NK YOU FOR THE OPPORTUNITY TO PROVIDE ENVI }\end{array}$ & $\begin{array}{l}\text { NO OTHER A } \\
\text { RONMENTAL }\end{array}$ & $\begin{array}{l}\text { CTIVITY TOO } \\
\text { MEASUREMENT }\end{array}$ & K PLACE & & ANALY & & KEITH L. ST & TANLEY & & $*=$ BELOW & DETECTION LII & IHIT & \\
\hline
\end{tabular}




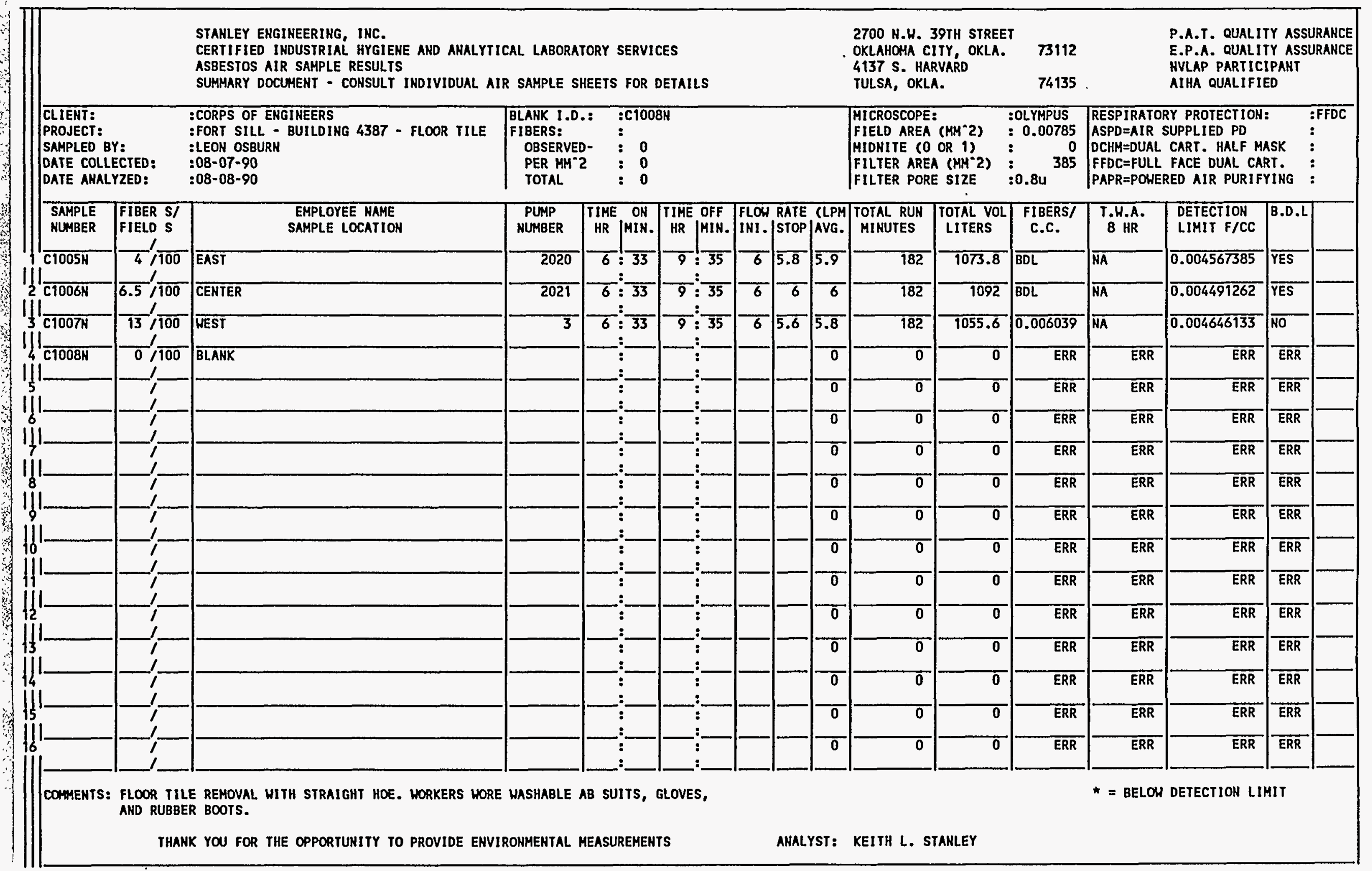




\begin{tabular}{|c|c|c|c|c|c|c|c|c|c|c|c|c|c|c|c|}
\hline & & \multicolumn{7}{|c|}{$\begin{array}{l}\text { STANLEY ENGINEERING, INC. } \\
\text { CERTIFIED INDUSTRIAL HYGIENE AND ANALYTICAL LABORATORY SERVICES } \\
\text { ASBESTOS AIR SAMPLE RESULTS } \\
\text { SUMHARY DOCUMENT - CONSULT INDIVIDUAL AIR SAMPLE SHEETS FOR DETAILS }\end{array}$} & \multicolumn{2}{|c|}{$\begin{array}{l}2700 \text { N.H. 39TH STREET } \\
\text { OKLAHOHA CITY, OKLA. } \\
\text { 4137 S. HARVARD } \\
\text { TULSA, OKLA. }\end{array}$} & $\begin{array}{ll} & \\
& \\
& 74135\end{array}$ & & \multicolumn{3}{|c|}{$\begin{array}{l}\text { P.A.T. QUALITY ASSURAHCE } \\
\text { E.P.A. QUALITY ASSURANCE } \\
\text { HVLAP PARTICIPANT } \\
\text { AIHA QUALIFIED }\end{array}$} \\
\hline \multicolumn{2}{|c|}{$\begin{array}{l}\text { CLIENT: } \\
\text { PROJECT: } \\
\text { SAMPLED BY: } \\
\text { DATE COLLECTED: } \\
\text { DATE ANALYZED: }\end{array}$} & \multirow{2}{*}{$\begin{array}{l}\text { :CORP OF ENGINEERS - CLAN UP } 43755 \\
\text { :FT. SILL - CEELE- - CLEAN } \\
\text { :LEON OSBURN / SEI } \\
\text { :AUGUST 23, 1990 } \\
\text { :OCTOBER 1\%, } 1990 \\
\text { EMMLOYEE NAME } \\
\text { SAMPLE LOCATION }\end{array}$} & \multicolumn{3}{|c|}{\begin{tabular}{|ll} 
BLANK I.D.: & $:$ N/A \\
FIBERS: & $:$ \\
OBSERVED- & $:$ N/A \\
PER MM-2 & $: 0$ \\
TOTAL & $: 0$
\end{tabular}} & & & & \multicolumn{2}{|c|}{$\begin{array}{l}\text { MICROSCOPE: } \\
\text { FIELD AREA }\left(M M^{-} 2\right) \\
\text { MIDNITE (O OR } 1) \\
\text { FILTER AREA (MM`2) } \\
\text { FILTER PORE SIZE }\end{array}$} & $\begin{array}{lr}\text { :OLYMPUS } \\
: 0.00785 \\
: & 0 \\
: & 385 \\
: 0.8 u^{3}\end{array}$ & \multicolumn{3}{|c|}{$\begin{array}{l}\text { RESPIRATORY PROIECTION: } \\
\text { ASPD=ATR SUPPLIED PD } \\
\text { DCHM=DUAL CART. HALF MASK } \\
\text { FFCC=FULL FACE DUAL CART. } \\
\text { PAPR =POWERED ALR PURIFYING }\end{array}$} & $\begin{array}{l}\text { DCHM } \\
\vdots \\
\vdots\end{array}$ \\
\hline $\begin{array}{l}\text { SAMPLE } \\
\text { NUMBER }\end{array}$ & $\begin{array}{l}\text { FIBER S/ } \\
\text { FIELD S }\end{array}$ & & $\begin{array}{l}\text { PUMP } \\
\text { NUMBER }\end{array}$ & \begin{tabular}{|c|c|} 
TIME & ON \\
HR & MIN. \\
\end{tabular} & \begin{tabular}{|c|} 
TIME OFF \\
HR \\
HIN.
\end{tabular} & $\begin{array}{l}\text { FLOW } \\
\text { INI. }\end{array}$ & $\begin{array}{l}\text { RATE } \\
\text { STOP }\end{array}$ & $\begin{array}{l}\text { ILPM } \\
\text { AVG. }\end{array}$ & $\begin{array}{l}\text { TOTAL RUN } \\
\text { MINUTES }\end{array}$ & $\begin{array}{l}\text { TOTAL VOL } \\
\text { LITERS }\end{array}$ & $\begin{array}{l}\text { FIBERS/ } \\
\text { C.C. }\end{array}$ & $\begin{array}{c}\text { T.H.A. } \\
8 \mathrm{HR}\end{array}$ & $\begin{array}{l}\text { DETECTION } \\
\text { LIMIT F/CC }\end{array}$ & $\overline{B . D . L}$ & \\
\hline 5008 & 1,100 & NORTH OF BUILDING & 2019 & $9 !-$ & $12: \overline{55}$ & $\overline{6}$ & $\overline{5.8}$ & $\overline{5.9}$ & $\overline{230}$ & 1357 & $\overline{B D L}$ & $\overline{\mathrm{NA}}$ & $\overline{0.003614192}$ & $\overline{Y E S}$ & \\
\hline 5009 & $8, \overline{100}$ & SOUTHWEST ROOH & 2017 & $9: \overline{55}$ & $\overline{12}: \overline{50}$ & $\overline{6}$ & 5.9 & $\overline{5.9}$ & $\overline{175}$ & 1041.25 & $\overline{\mathrm{BDL}}$ & $\overline{N A}$ & $\overline{0.004710164}$ & $\overline{\text { YES }}$ & \\
\hline (3010 & $5 / 100$ & SOUTHWEST OF BUILDING & 2021 & & $\overline{12}: \overline{56}$ & $\overline{6}$ & 6 & 6 & $\overline{186}$ & 1116 & $\overline{\mathrm{BDL}}$ & $\overline{N A}$ & 0.004394676 & $\overline{\text { YES }}$ & \\
\hline$4 \longdiv { 5 0 1 1 }$ & $\overline{7.5} / \overline{100}$ & EAST ROOM & 3 & $9: \overline{55}$ & $12: \overline{50}$ & 6 & $\overline{5.7}$ & $\overline{5.8}$ & $\overline{175}$ & 1023.75 & $\overline{B D L}$ & $\overline{\mathrm{NA}}$ & $\overline{0.004790679}$ & $\overline{Y E S}$ & \\
\hline$5 \longdiv { 5 0 1 2 p }$ & 4,100 & GABOR SZEKELYY & $\overline{p 1}$ & $10: \overline{0}$ & $\overline{12}: \overline{50}$ & $\overline{2}$ & 2 & $\overline{2}$ & $\overline{170}$ & 340 & $\overline{B D L}$ & $\overline{N A}$ & $\overline{0.014424878}$ & $\overline{\text { YES }}$ & \\
\hline$5 \longdiv { 5 0 1 3 \mathrm { P } }$ & 3,100 & $\overline{\text { BURL RAGLAND }}$ & $\overline{\mathrm{P2}}$ & $10:-0$ & $12: \overline{50}$ & 2 & 2 & $\overline{2}$ & $\overline{170}$ & 340 & $\overline{B D L}$ & $\overline{\mathrm{NA}}$ & $\overline{0.014424878}$ & $\overline{Y E S}$ & \\
\hline $5014 \mathrm{P}$ & $\overline{10} / \overline{100}$ & LEON OSBURN & $\overline{\mathrm{P3}}$ & $\begin{array}{c}10: 0 \\
\end{array}$ & $\begin{array}{l}12: 50 \\
\end{array}$ & $\overline{2}$ & 2 & 2 & 170 & 340 & $\overline{8 D L}$ & $\overline{N A}$ & 0.014424878 & YES & \\
\hline & & & & : & I: & & & 0 & 0 & 0 & ERR & ERR & $\overline{\text { ERR }}$ & ERR & \\
\hline & & & & & $\because:$ & & & $\overline{0}$ & $\overline{0}$ & 0 & ERR & ERR & $\overline{\text { ERR }}$ & $\overline{\text { ERR }}$ & \\
\hline & & & & & & & & $\overline{0}$ & $\overline{0}$ & $\overline{0}$ & ERR & $\overline{E R R}$ & $\overline{E R R}$ & $\overline{\text { ERR }}$ & \\
\hline & & & & & & & & $\overline{0}$ & $\overline{0}$ & 0 & ERR & $\overline{\text { ERR }}$ & $\overline{\text { ERR }}$ & $\overline{E R R}$ & \\
\hline & & & & & & & & 0 & 0 & 0 & ERR & $\overline{\text { ERR }}$ & $\overline{\text { ERR }}$ & $\overline{\text { ERR }}$ & \\
\hline & & & & & & & & $\overline{0}$ & $\overline{0}$ & 0 & $\overline{\text { ERR }}$ & $\overline{\text { ERR }}$ & $\overline{\text { ERR }}$ & $\overline{\text { ERR }}$ & \\
\hline & & & & & & & & $\overline{0}$ & 0 & $\overline{0}$ & ERR & ERR & $\overline{E R R}$ & $\overline{\text { ERR }}$ & \\
\hline & & & & & & & & 0 & $\overline{0}$ & $\overline{0}$ & ERR & $\overline{E R R}$ & $\overline{\text { ERR }}$ & $\overline{\text { ERR }}$ & \\
\hline & & & & $:$ & & & & $\overline{0}$ & $\overline{0}$ & 0 & $\overline{\text { ERR }}$ & ERR & $\overline{E R R}$ & $\overline{\text { ERR }}$ & \\
\hline CO & $\begin{array}{l}\text { LOG \#912 } \\
\text { DOUBLE WI } \\
\text { GLOVES AI } \\
\text { THA }\end{array}$ & $\begin{array}{l}\text { O01 - PHASE CONTRAST MICROSCOPY - BUILD } \\
\text { RAPPED HIT SECOND SHEET OF } 6 \text { MIL POLY. } \\
\text { ND RUBBER BOOS. } \\
\text { NK YOU FOR THE OPPORTUNITY TO PROVIDE E }\end{array}$ & ERS I & $\begin{array}{l}\text { TILE } \\
\text { CHM RI } \\
\text { ASUREI }\end{array}$ & $\begin{array}{l}\text { PLACED I } \\
\text { DISPOSA }\end{array}$ & $\begin{array}{l}\text { N } \\
\text { gL }\end{array}$ & $\begin{array}{l}\text { THEN } \\
\text { B SUIY } \\
\text { ANALY }\end{array}$ & HRAPI & PED IN 6 MII & $L$ POLY. BO & OX WA & $\begin{array}{r}=\text { BEL } \\
=\end{array}$ & & & \\
\hline
\end{tabular}




\begin{tabular}{|c|c|c|c|c|c|c|c|c|c|c|c|c|c|c|c|}
\hline & & \multicolumn{7}{|c|}{$\begin{array}{l}\text { STANLEY ENGINEERING, IHC. } \\
\text { CERIIFIED INDUSTRIAL HYGIENE AND ANALYTICAL LABORATORY SERVICES } \\
\text { ASBESTOS AIR SAMPLE RESULTS } \\
\text { SUMMARY DOCUMENT - CONSULT INDIVIDUAL AIR SAMPLE SHEETS FOR DETAILS }\end{array}$} & \multicolumn{2}{|c|}{$\begin{array}{l}2700 \text { N.H. 39TH STREET } \\
\text { OKLAHOHA CITY, OKLA. } \\
\text { 4137 S. HARVARD } \\
\text { TULSA, OKLA. }\end{array}$} & $\begin{array}{ll} & \\
& \\
& 74112\end{array}$ & & \multicolumn{3}{|c|}{$\begin{array}{l}\text { P.A.T. QUALITY ASSURANCE } \\
\text { E.P.A. QUALITY ASSURANCE } \\
\text { NVLAP PARIICIPANT } \\
\text { AIHA QUALIFIED }\end{array}$} \\
\hline \multicolumn{2}{|c|}{$\begin{array}{l}\text { CLIENT: } \\
\text { PROJECT: } \\
\text { SAMPLED BY: } \\
\text { DATE COLLECTED: } \\
\text { DATE ANALYZED: }\end{array}$} & \multirow{2}{*}{ 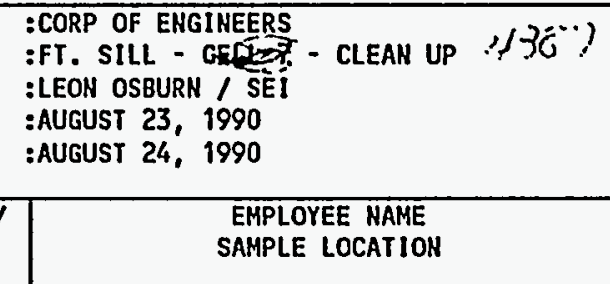 } & \multicolumn{3}{|c|}{\begin{tabular}{|ll} 
BLANK I.D.: & :H/A \\
FIBERS: & $\vdots$ \\
OBSERVED- & $:$ N/A \\
PER MH 22 & $\vdots 0$ \\
TOTAL & $: 0$
\end{tabular}} & & & & \multicolumn{2}{|c|}{$\begin{array}{l}\text { MICROSCOPE: } \\
\text { FIELD AREA }\left(M M^{-2} 2\right) \\
\text { MIDNITE (O OR } 1) \\
\text { FILTER AREA (MM-2) } \\
\text { FILTER PORE SIZE }\end{array}$} & 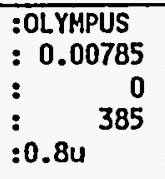 & \multicolumn{3}{|c|}{$\begin{array}{l}\text { RESPIRATORY PROTECTION: } \\
\text { ASPD=AIR SUPPLIED PD } \\
\text { DCHM=DUAL CART. HALF MASK } \\
\text { FFDCFULL FACE DUAL CART. } \\
\text { PAPR =POHERED AIR PURIFYING }\end{array}$} & $\begin{array}{l}: \text { DCHM } \\
\vdots \\
\vdots \\
\vdots\end{array}$ \\
\hline $\begin{array}{l}\text { SAMPLE } \\
\text { NUMBER }\end{array}$ & $\begin{array}{l}\text { FIBER S/ } \\
\text { FIELD S }\end{array}$ & & $\begin{array}{c}\text { PUMP } \\
\text { NUMBER }\end{array}$ & \begin{tabular}{|l|l|} 
TIME & ON \\
HR & MIN.
\end{tabular} & \begin{tabular}{|c|} 
TIME OFF \\
HR PIN.
\end{tabular} & \begin{tabular}{|l} 
FLOH \\
INI.
\end{tabular} & $\begin{array}{l}\text { RATE } \\
\text { STOP }\end{array}$ & $\begin{array}{l}\text { (LPH } \\
\text { AVG. }\end{array}$ & \begin{tabular}{|} 
TOTAL RUM \\
MINUTES
\end{tabular} & \begin{tabular}{|l|} 
TOTAL VOL \\
LITERS
\end{tabular} & $\begin{array}{l}\text { FIBERS/ } \\
\text { C.C. }\end{array}$ & $\begin{array}{c}\text { T.H.A. } \\
8 \mathrm{HR}\end{array}$ & $\begin{array}{l}\text { DETECTIOH } \\
\text { LIMIT F/CC }\end{array}$ & B.D.L & \\
\hline 7001 & TD & HEST & $\overline{2017}$ & $5: \overline{0}$ & $9: 20$ & 7 & $\overline{5.9}$ & 5.9 & 260 & $\overline{1547}$ & $\overline{\text { ERR }}$ & $\overline{\text { ERR }}$ & $\overline{0.003170302}$ & $\overline{E R R}$ & \\
\hline$\overline{7002}$ & $\overline{\mathrm{TD}} / \overline{\mathrm{TC}}$ & $\overline{\text { EAST }}$ & $\overline{3}$ & $5:-$ & $9: \overline{20}$ & $\overline{6}$ & (6) & 6 & $\overline{260}$ & $\overline{1560}$ & $\overline{\text { ERR }}$ & $\overline{\text { ERR }}$ & $\overline{0.003143883}$ & $\overline{\text { ERR }}$ & \\
\hline 7003 & $2 / 100$ & NORTH (OUTSIDE) & $\overline{2021}$ & $4: \overline{45}$ & $\overline{9}: \overline{30}$ & 6 & $\overline{5.7}$ & $\overline{5.8}$ & 285 & 1667.25 & $\overline{B D L}$ & $\overline{N A}$ & $\overline{0.002941645}$ & $\overline{Y E S}$ & \\
\hline 7004 & $2.5 / \overline{100}$ & SOUTH (OUTSIDE) & 2019 & $4: \overline{45}$ & $9: \overline{30}$ & 6 & $\overline{5.7}$ & $\overline{5.8}$ & 285 & 1667.25 & $B \overline{B D L}$ & $\overline{M A}$ & $\overline{0.002941645}$ & $\overline{\mathrm{YES}}$ & \\
\hline$\overline{7005 P}$ & $6 / \overline{100}$ & GABOR SZEKELY: $446-84-8118$ & $\overline{p-1}$ & $5: \overline{0}$ & $9: \overline{20}$ & $\overline{2}$ & $\overline{2}$ & 2 & $\overline{260}$ & $\overline{520}$ & $\overline{B D L}$ & $\overline{\mathrm{NA}}$ & $\overline{0.009431651}$ & $\overline{Y E S}$ & \\
\hline 70068 & $8 / \overline{100}$ & BURL RAGL.AND: 447-38-1989 & $\overline{p-2}$ & $5: \overline{0}$ & $9: \overline{20}$ & 2 & $\overline{2}$ & 2 & 260 & 520 & $\overline{\mathrm{BDL}}$ & $\overline{N A}$ & 0.009431651 & $\overline{Y E S}$ & \\
\hline $7007 \mathrm{P}$ & $9, \overline{100}$ & LEON OSBURN: $442-50-8638$ & $\overline{\mathrm{p}-3}$ & $5: 5$ & $\overline{9}: \overline{20}$ & $\overline{2}$ & $\overline{2}$ & 2 & 260 & 520 & $\overline{B D L}$ & $\overline{\mathrm{NA}}$ & $\overline{0.009431651}$ & $\overline{\text { YES }}$ & \\
\hline & & & & : & & & & $\overline{0}$ & 0 & $\overline{0}$ & $\overline{\text { ERR }}$ & $\overline{\text { ERR }}$ & $\overline{E R R}$ & ERR & \\
\hline & & & & $:$ & : & & & 0 & $\overline{0}$ & 0 & $\overline{\text { ERR }}$ & $\overline{\text { ERR }}$ & ERR & ERR & \\
\hline & & & & $\because:$ & : & & & 0 & $\overline{0}$ & $\overline{0}$ & $\overline{E R R}$ & $\overline{E R R}$ & ERR & $\overline{\text { ERR }}$ & \\
\hline & & & & $\because$ & 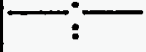 & & & 0 & $\overline{0}$ & $\overline{0}$ & $\overline{\text { ERR }}$ & $\overline{E R R}$ & $\overline{E R R}$ & $\overline{E R R}$ & \\
\hline & & & & $\because$ & $:$ & & & 0 & $\overline{0}$ & $\overline{0}$ & $\overline{\text { ERR }}$ & $\overline{\text { ERR }}$ & $\overline{\text { ERR }}$ & $\overline{\text { ERR }}$ & \\
\hline & & & & : & & & & 0 & $\overline{0}$ & $\overline{0}$ & $\overline{\text { ERR }}$ & $\overline{\text { ERR }}$ & ERR & $\overline{\text { ERR }}$ & \\
\hline & & & & : & $\because-$ & & & 0 & $\overline{0}$ & $\overline{0}$ & $\overline{\text { ERR }}$ & $\overline{\text { ERR }}$ & $\overline{\text { ERR }}$ & ERR & \\
\hline & & & & & & & & 0 & $\overline{0}$ & $\overline{0}$ & $\overline{\text { ERR }}$ & $\overline{\text { ERR }}$ & $\overline{\text { ERR }}$ & $\overline{\text { ERR }}$ & \\
\hline & & & & & & & & $\overline{0}$ & $\overline{0}$ & $\overline{0}$ & $\overline{\text { ERR }}$ & $\overline{\text { ERR }}$ & $\overline{E R R}$ & $\overline{\text { ERR }}$ & \\
\hline COMHENTS & $\begin{array}{l}\text { LOG } \\
\text { POLY THEI } \\
\text { HERE THE }\end{array}$ & $\begin{array}{l}\text { DOO - PHASE CONTRAST MICROSCOPY - BUILDI } \\
\text { DOUBLE WRAPPED HITH SECOND SHEET OF } 6 \\
\text { PLACED INSIDE AB TRAILER. WORKERS HORE } \\
\text { UK YOU FOR THE OPPORTUNITY TO PROVIDE EN }\end{array}$ & $\begin{array}{l}\text { 4387: } \\
\text { IL POLY. } \\
\text { IHM RES } \\
\text { IRONMENT }\end{array}$ & $\begin{array}{l}\text { OR TILE HA } \\
\text { JBLE HRAPP } \\
\text { DISPOSABL } \\
\text { UEASUREMEN }\end{array}$ & $\begin{array}{l}\text { S PLACED I } \\
\text { ED BOXES } \\
\text { E AB SUITS } \\
\text { TS }\end{array}$ & IN BOX & $\begin{array}{l}\text { HRAPI } \\
\text { VES AI } \\
\text { ANAL }\end{array}$ & $\begin{array}{l}\text { PED IN } \\
\text { IND RUE } \\
\text { YST: }\end{array}$ & $\begin{array}{l}\text { N } 6 \text { MIL. } \\
\text { JBBER BOOTS. } \\
\text { ALI SON MYE }\end{array}$ & & & BELOW & ON LI & & \\
\hline
\end{tabular}




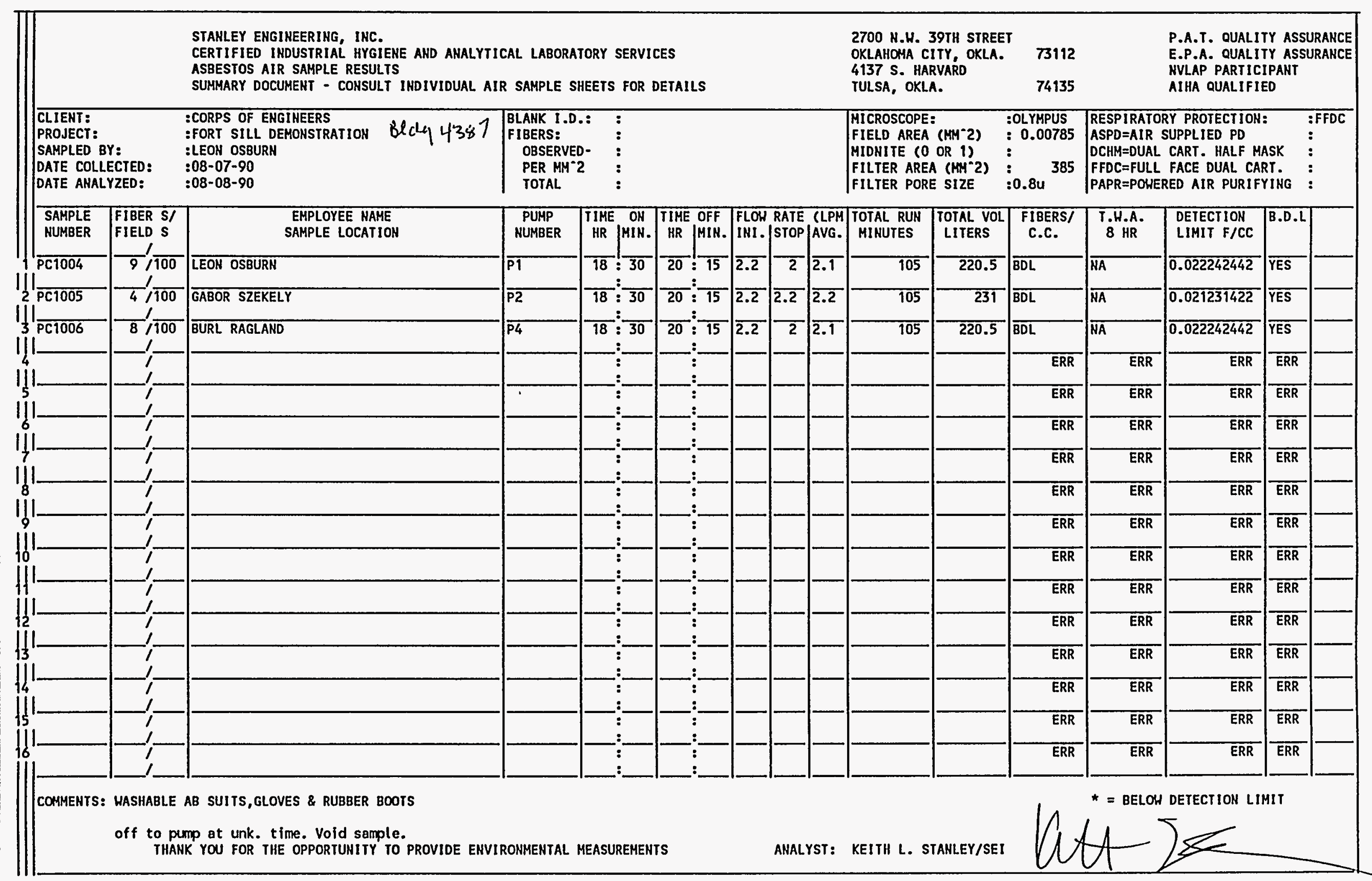




\begin{tabular}{|c|c|c|c|c|c|c|c|c|c|c|c|c|c|c|c|}
\hline & & \multicolumn{7}{|c|}{$\begin{array}{l}\text { STANLEY ENGINEERING, INC. } \\
\text { CERTIFIED INDUSTRIAL HYGIENE AND ANALYTICAL LABORATORY SERVICES } \\
\text { ASBETOS AIR SAMPLE RESULS } \\
\text { SUMMARY DOCUMENT - CONSULT INDIVIDUAL AIR SAMPLE SHEETS FOR DETAILS }\end{array}$} & \multicolumn{2}{|c|}{$\begin{array}{l}2700 \text { N.H. 39TH STREET } \\
\text { OKLAHOMA CITY, OKLA. } \\
4137 \text { S. HARVARD } \\
\text { TULSA, OKLA. }\end{array}$} & 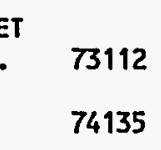 & & \multicolumn{3}{|c|}{$\begin{array}{l}\text { P.A.T. QUALITY ASSURANCE } \\
\text { E.P.A. QUALITY ASSURANCE } \\
\text { NVLAP PARTICIPANT } \\
\text { AIHA QUALIFIED }\end{array}$} \\
\hline \multicolumn{2}{|c|}{$\begin{array}{l}\text { CLIENT: } \\
\text { PROJECT: } \\
\text { SAMPLED BY: } \\
\text { DATE COLLECTED: } \\
\text { DATE ANALYZED: }\end{array}$} & $\begin{array}{l}\text { :CORP OF ENGINEERS } \\
\text { :FORT SILL - BLDG } 4387-\text { CELL \#1 } \\
\text { :LEON OSBURN/SE1 } \\
\text { :AUGUST 7, } 1990 \\
\text { :AUGUST } 7,1990\end{array}$ & \multicolumn{3}{|c|}{\begin{tabular}{|ll} 
BLANK 1.D.: & $: 1020$ \\
FIBERS: & $:$ \\
OBSERVED- & $: 0$ \\
PER MM 22 & $: 0$ \\
TOTAL & $: 0$
\end{tabular}} & & & & \multicolumn{2}{|c|}{$\begin{array}{l}\text { MICROSCOPE: } \\
\text { FIELD AREA }(M M-2) \\
\text { MIDNITE }(0 \text { OR } 1) \\
\text { FILTER AREA (MM²) } \\
\text { FILTER PORE SIZE }\end{array}$} & $\begin{array}{l}: \text { OLYMPUS } \\
: 0.00785 \\
\vdots \\
: 0.8 u^{\quad 385}\end{array}$ & \multicolumn{3}{|c|}{$\begin{array}{l}\text { RESPIRATORY PROTECTION: } \\
\text { ASPD=AIR SUPPLIED PD } \\
\text { DCHM=DUAL CART. HALF MASK } \\
\text { FFOC=FULL FACE DUAL CART. } \\
\text { PAPR=POHERED AIR PURIFYING }\end{array}$} & $\begin{array}{l}\text { :N/A } \\
\vdots \\
\vdots \\
\vdots \\
\vdots\end{array}$ \\
\hline $\begin{array}{l}\text { SAMPLE } \\
\text { NUMBER }\end{array}$ & $\begin{array}{l}\text { FIBER SI } \\
\text { FIELD S } \\
\end{array}$ & $\begin{array}{l}\text { EMPLOYEE NAME } \\
\text { SAMPLE LOCATION }\end{array}$ & $\begin{array}{l}\text { PUMP } \\
\text { NUMBER }\end{array}$ & $\begin{array}{|cc|}\text { TIME } & \text { ON } \\
\text { HR } & \text { MIN. } \\
\end{array}$ & $\begin{array}{l}\text { TIME OFF } \\
\text { HR |MIN. }\end{array}$ & $\begin{array}{l}\text { FLOH } \\
\text { INI. }\end{array}$ & $\begin{array}{l}\text { RATE } \\
\text { STOP }\end{array}$ & $\begin{array}{l}\text { (LPM } \\
\text { AVG. }\end{array}$ & \begin{tabular}{|c|} 
TOTAL RUN \\
MINUTES
\end{tabular} & $\begin{array}{l}\text { TOTAL VOL } \\
\text { LITERS }\end{array}$ & $\begin{array}{l}\text { FIBERS/ } \\
\text { C.C. }\end{array}$ & $\begin{array}{l}\text { T.H.A. } \\
8 \mathrm{HR}\end{array}$ & $\begin{array}{l}\text { DETECTION } \\
\text { LIMIT F/CC }\end{array}$ & B.D.L & \\
\hline 1017 & $\overline{15} / \overline{100}$ & CLEAN ROOM & 7 & $17: 55$ & $79: 40$ & 6 & $\overline{6.2}$ & $\overline{6.1}$ & 105 & 640.5 & $\overline{0.011485}$ & $\overline{N A}$ & 0.007657234 & NO & \\
\hline 1018 & $5 / \overline{100}$ & NORTH EAST & 2017 & $\overline{17}: \overline{56}$ & $\overline{19}: \overline{41}$ & $\overline{6}$ & $\overline{5.9}$ & $\overline{5.9}$ & 105 & 624.75 & $\overline{B D L}$ & NA & $\overline{0.007850273}$ & $\overline{\mathrm{YES}}$ & \\
\hline 1019 & $12 / \overline{100}$ & SOUTH WEST & $\overline{2013}$ & $17: \overline{57}$ & $\overline{19}: \overline{42}$ & 6 & 6 & 6 & 105 & 630 & 0.009341 & $\overline{\mathrm{NA}}$ & $\overline{0.007784854}$ & No & \\
\hline 1020 & $0 / \overline{100}$ & $\overline{\text { BLANK }}$ & & $:$ & $:$ & & & 0 & D & $\overline{0}$ & $\overline{\text { ERR }}$ & $\overline{\text { ERR }}$ & $\overline{\text { ERR }}$ & $\overline{\text { ERR }}$ & \\
\hline & & & & & $\because$ & & & $\overline{0}$ & $\overline{0}$ & $\overline{0}$ & $\overline{E R R}$ & $\overline{E R R}$ & $\overline{\text { ERR }}$ & $\overline{\text { ERR }}$ & \\
\hline & & & & : & $:$ & & & 0 & 0 & 0 & ERR & $\overline{\text { ERR }}$ & ERR & ERR & \\
\hline & & & & $:$ & $\because$ & & & $\overline{0}$ & 0 & 0 & $\overline{E R R}$ & $\overline{\text { ERR }}$ & $\overline{E R R}$ & $\overline{\text { ERR }}$ & \\
\hline & & & & & $\therefore$ & & & 0 & 0 & $\overline{0}$ & $\overline{E R R}$ & $\overline{\text { ERR }}$ & $\overline{E R R}$ & $\overline{\text { ERR }}$ & \\
\hline & & & & & & & & $\overline{0}$ & $\overline{0}$ & $\overline{0}$ & ERR & $\overline{\text { ERR }}$ & $\overline{\text { ERR }}$ & $\overline{\text { ERR }}$ & \\
\hline & 1 & & & & $\because$ & & & $\overline{0}$ & $\overline{0}$ & $\overline{0}$ & $\overline{\text { ERR }}$ & $\overline{\text { ERR }}$ & $\overline{\text { ERR }}$ & $\overline{\text { ERR }}$ & \\
\hline & & & & $:$ & : & & & 0 & $\overline{0}$ & 0 & ERR & $\overline{\text { ERR }}$ & $\overline{\text { ERR }}$ & $\overline{\text { ERR }}$ & \\
\hline & , & & & $\because-$ & $\because-$ & & & $\overline{0}$ & 0 & $\overline{0}$ & ERR & $\overline{\text { ERR }}$ & $\overline{\text { ERR }}$ & $\overline{\text { ERR }}$ & \\
\hline & & & & : & & & & $\overline{0}$ & $\overline{0}$ & $\overline{0}$ & ERR & ERR & ERR & ERR & \\
\hline & - & & & $\because-$ & $\because$ & & & $\overline{0}$ & $\overline{0}$ & $\overline{0}$ & ERR & ERR & ERR & $\overline{\text { ERR }}$ & \\
\hline & & & & $:$ & & & & $\overline{0}$ & 0 & $\overline{0}$ & ERR & ERR & ERR & $\overline{E R R}$ & \\
\hline & & & & : & & & & $\overline{0}$ & 0 & $\overline{0}$ & ERR & $\overline{E R R}$ & ERR & $\overline{E R R}$ & \\
\hline COMMENTS: & $\begin{array}{l}\text { ABATEME } \\
\text { MASTIC } \\
\text { MASTIC } \\
\text { TH }\end{array}$ & $\begin{array}{l}\text { VVAL INSIDE CELL } 1 \\
\text { YOU FOR THE OPPORTUNITY TO PROVIDE El }\end{array}$ & & ISUREM & & & & & & & & & & & \\
\hline
\end{tabular}




\begin{tabular}{|c|c|c|c|c|c|c|c|c|c|c|c|c|c|c|c|c|}
\hline \multirow{2}{*}{\multicolumn{2}{|c|}{$\begin{array}{l}\text { CLIENT: } \\
\text { PROJECT: } \\
\text { SAMPLED BY: } \\
\text { DATE COLLECTED: } \\
\text { DATE ANALYZED: }\end{array}$}} & \multicolumn{8}{|c|}{$\begin{array}{l}\text { STANLEY ENGINEERING, INC. } \\
\text { CERTIFIED INDUSTRIAL HYGIENE AND ANALYTICAL LABORATORY SERVICES } \\
\text { ASBESTOS AIR SAMPLE RESULTS } \\
\text { SUMMARY DOCUMENT - CONSULT INDIVIDUAL AIR SAMPLE SHEETS FOR DETAILS }\end{array}$} & \multicolumn{2}{|c|}{$\begin{array}{l}2700 \text { N.H. 39TH STREET } \\
\text { OKLAHOMA CITY, OKLA. } \\
4137 \text { S. HARVARD } \\
\text { TULSA, OKLA. }\end{array}$} & \multicolumn{2}{|l|}{$\begin{array}{l}73112 \\
74135\end{array}$} & \multicolumn{3}{|c|}{$\begin{array}{l}\text { P.A.T. QUALITY ASSURANCE } \\
\text { E.P.A. QUALIIY ASSURANCE } \\
\text { MVLAP PARTICIPANT } \\
\text { AIHA QUALIFIED }\end{array}$} \\
\hline & & $\begin{array}{l}\text { :Corps of Engineers } \\
\text { :Ft. Sill - Cell } \# 5 \text {-Abatement } \\
\text { :Leon Osborn } \\
: 8 / 8 / 90 \\
: 9 / 27 / 90\end{array}$ & $\frac{4375}{15}$ & $\begin{array}{l}\text { BLANK I.D } \\
\text { FIBERS: } \\
\text { OBSERVE } \\
\text { PER MM- } \\
\text { TOTAL }\end{array}$ & $\begin{array}{lll}\therefore & : & 1 \\
& \vdots & 1 \\
-1 & : 1.2 \\
& : 490\end{array}$ & & & & & $\begin{array}{l}\text { MICROSCOPE: } \\
\text { FIELD AREA } \\
\text { MIDNITE }(0 \\
\text { FILTER AREA } \\
\text { FILTER PORE }\end{array}$ & $\begin{array}{l}:\left(M M^{-2}\right): \\
\text { OR } 1) \\
\text { A }\left(M M^{-2} 2\right): \\
\text { E SIZE }:\end{array}$ & $\begin{array}{lr}\text { :OLYMPUS } \\
: 0.00785 \\
: & 0 \\
: & 385 \\
: 0.8 u^{2}\end{array}$ & $\begin{array}{l}\text { RESPIRATO } \\
\text { ASPD=AIR } \\
\text { DCHM=DUAL } \\
\text { FFDC=FULL } \\
\text { PAPR=POHES }\end{array}$ & $\begin{array}{l}\text { RY PROTECIION } \\
\text { SUPPLIED PD } \\
\text { CART. HALF } \\
\text { FACE DUAL CA } \\
\text { RED AIR PURIF }\end{array}$ & $\begin{array}{l}: \\
\text { IASK } \\
\text { IAT. } \\
\text { YING }\end{array}$ & $\begin{array}{l}: \text { N/A } \\
\vdots \\
\vdots \\
\vdots\end{array}$ \\
\hline $\begin{array}{l}\text { SAMPLE } \\
\text { NUMBER }\end{array}$ & $\begin{array}{l}\text { FIBER SI } \\
\text { FIELD S }\end{array}$ & $\begin{array}{l}\text { EMPLOYEE NAME } \\
\text { SAMPLE LOCATION }\end{array}$ & & $\begin{array}{l}\text { PUMP } \\
\text { NUMBER }\end{array}$ & \begin{tabular}{|l|l|} 
TIME ON \\
HR & MIN. \\
\end{tabular} & \begin{tabular}{|l} 
TIME OFF \\
HR \\
MIN.
\end{tabular} & $\begin{array}{l}\text { FLOH } \\
\text { INI. }\end{array}$ & $\begin{array}{l}\text { RATE } \\
\text { STOP }\end{array}$ & $\begin{array}{l}\text { CLPM } \\
\text { AVG. }\end{array}$ & $\begin{array}{l}\text { TOTAL RUN } \\
\text { MINUTES }\end{array}$ & \begin{tabular}{|c|} 
TOTAL VOL \\
LITERS
\end{tabular} & $\begin{array}{l}\text { FIBERS/ } \\
\text { C.C. }\end{array}$ & $\begin{array}{l}\text { T.W.A. } \\
8 \text { HR }\end{array}$ & $\begin{array}{l}\text { DEIECTION } \\
\text { LIMIT F/CC }\end{array}$ & B.D.L & \\
\hline$\overline{8021}$ & $2 / \overline{100}$ & West Room & & $\overline{2013}$ & $75: 30$ & $22: 15$ & $\overline{6}$ & $\overline{5.9}$ & 5.9 & 405 & 2409.75 & $\overline{B D L}$ & $\overline{N A}$ & 0.002035256 & $\overline{Y E S}$ & \\
\hline B022 & $4 / \overline{100}$ & South East Room & & $\overline{7}$ & $\overline{15}: \overline{30}$ & $\overline{22}: \overline{15}$ & 6 & $\overline{5.8}$ & 5.9 & 405 & 2389.5 & $\overline{B D L}$ & $\overline{N A}$ & $\overline{0.002052504}$ & $\overline{Y E S}$ & \\
\hline$3 \longdiv { 8 0 2 3 }$ & $7 / \overline{100}$ & North East Room & & $\overline{2017}$ & $\overline{15}: \overline{30}$ & $\overline{22}: \overline{15}$ & 6 & $\overline{5.9}$ & $\overline{5.9}$ & 405 & $\overline{2409.75}$ & $\overline{\mathrm{BDL}}$ & $\overline{\mathrm{NA}}$ & 0.002035256 & $\overline{Y E S}$ & \\
\hline$\overline{8024}$ & $1 / 100$ & $\overline{B \operatorname{lank}}$ & & & $:$ & $:$ & & & 0 & $\overline{0}$ & $\overline{0}$ & $\overline{E R R}$ & $\overline{\text { ERR }}$ & ERR & ERR & \\
\hline & 1 & & & & $:$ & $:$ & & & 0 & $\overline{0}$ & $\overline{0}$ & ERR & ERR & ERR & $\overline{\text { ERR }}$ & \\
\hline & & & & & $:$ & & & & 0 & $\overline{0}$ & $\overline{0}$ & $\overline{\text { ERR }}$ & $\overline{\text { ERR }}$ & ERR & $\overline{\text { ERR }}$ & \\
\hline & 1 & & & & $:$ & & & & 0 & $\overline{0}$ & $\overline{0}$ & $\overline{\text { ERR }}$ & $\overline{\text { ERR }}$ & ERR & $\overline{\text { ERR }}$ & \\
\hline & & & & & $\because$ & & & & 0 & 0 & $\overline{0}$ & ERR & ERR & ERR & $\overline{\text { ERR }}$ & \\
\hline & & & & & & $\because$ & & & 0 & $\overline{0}$ & $\overline{0}$ & $\overline{\text { ERR }}$ & $\overline{\text { ERR }}$ & $\overline{\text { ERR }}$ & $\overline{\text { ERR }}$ & \\
\hline & & & & & : & : & & & 0 & $\overline{0}$ & $\overline{0}$ & $\overline{\text { ERR }}$ & $\overline{\text { ERR }}$ & $\overline{E R R}$ & $\overline{\text { ERR }}$ & \\
\hline & & & & & $\because$ & $\because$ & & & 0 & 0 & 0 & $\overline{\text { ERR }}$ & ERR & ERR & ERR & \\
\hline & & & & & $\therefore$ & $\therefore$ & & & 0 & $\overline{0}$ & $\overline{0}$ & ERR & ERR & ERR & $\overline{\text { ERR }}$ & \\
\hline & & & & & 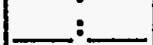 & & & & & & & & & & & \\
\hline & $i$ & & & & $\because$ & & & & $\overline{0}$ & 0 & $\overline{0}$ & ERR & ERR & ERR & ERR & \\
\hline & 0,0 & & $\overline{0}$ & $\overline{0}$ & $0: \overline{0}$ & $0: \overline{0}$ & 0 & 0 & $\overline{0}$ & $\overline{0}$ & $\overline{0}$ & $\overline{\text { ERR }}$ & $\overline{\text { ERR }}$ & ERR & $\overline{\text { ERR }}$ & \\
\hline & 0,0 & & 0 & 0 & $0: \frac{0}{0}$ & $0: \overline{0}$ & 0 & 0 & $\overline{0}$ & $\overline{0}$ & $\overline{0}$ & ERR & ERR & $\overline{\text { ERR }}$ & $\overline{\text { ERR }}$ & \\
\hline 0 & 0,0 & & 0 & 0 & $0: \overline{0}$ & $0: \overline{0}$ & 0 & $\overline{0}$ & $\overline{0}$ & $\overline{0}$ & 0 & $\overline{\text { ERR }}$ & $\overline{E R R}$ & ERR & $\overline{\text { ERR }}$ & \\
\hline COMMENTS: & $\begin{array}{l}\text { No activ } \\
\qquad 1+i\end{array}$ & 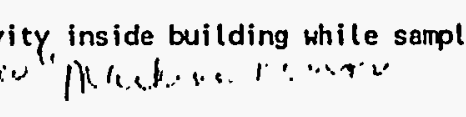 & es were tak & en. Map & back of & location & $m$ & & & & & & $*=B E L O$ & TION LI & & \\
\hline
\end{tabular}




\begin{tabular}{|c|c|c|c|c|c|c|c|c|c|c|c|c|c|c|c|}
\hline \multirow{2}{*}{\multicolumn{2}{|c|}{$\begin{array}{l}\text { CLIENT: } \\
\text { PROJECT: } \\
\text { SAMPLED BY: } \\
\text { DATE COLLECTED: } \\
\text { DATE ANALYZED: }\end{array}$}} & \multicolumn{7}{|c|}{$\begin{array}{l}\text { STANLEY ENGINEERING, INC. } \\
\text { CERTIFIED INDUSTRIAL HYGIENE AND ANALYIICAL LABORATORY SERVICES } \\
\text { ASBESTOS AIR SAMPLE RESULTS } \\
\text { SUMMARY DOCUMENT - CONSULT INDIVIDUAL AIR SAMPLE SHEETS FOR DETAILS }\end{array}$} & \multicolumn{4}{|c|}{$\begin{array}{l}\text { 2700 H.H. 39TH STREET } \\
\text { OKLAHOMA CITY, OKLA. } \\
4137 \text { S. HARVARD } \\
\text { TULSA, OKLA. }\end{array}$} & \multicolumn{3}{|c|}{$\begin{array}{l}\text { P.A.T. QUALITY ASSURANCE } \\
\text { E.P.A. QUALITY ASSURANCE } \\
\text { HVLAP PARTICIPANT } \\
\text { AIHA QUALIFIED }\end{array}$} \\
\hline & & $\begin{array}{l}\text { :CORP OF ENGINEERS } \\
\text { :FT. SILL - CELLL \#1 - BUILDING } 4387 \\
\text { :LEON OSBURN } \\
\text { :AUGST } 7,1990 \\
\text { :AUGUST } 8,1990\end{array}$ & \multicolumn{3}{|c|}{\begin{tabular}{|ll} 
BLANK I.D.: & $: 1012$ \\
FIBERS: & $:$ \\
OBSERVED- & $: 0$ \\
PER MM-2 & $: 0$ \\
TOTAL & $: 0$
\end{tabular}} & & & & \multicolumn{2}{|c|}{$\begin{array}{l}\text { MICROSCOPE: } \\
\text { FIELO AREA (MM־2) } \\
\text { MIDNITE }(0 \text { OR } 1) \\
\text { FILTER AREA }\left(M M^{-2} 2\right) \\
\text { FILTER PORE SIZE }\end{array}$} & $\begin{array}{l}\text { :OLYMPUS } \\
: 0.00785 \\
\vdots \\
\vdots \\
: 0.8 u^{285}\end{array}$ & \multicolumn{3}{|c|}{$\begin{array}{l}\text { RESPIRATORY PROTECTION: } \\
\text { ASPD=AIR SUPPLIED PD } \\
\text { DCHM=DUAL. CART. HALF MASK } \\
\text { FFDC=FULL. FACE DUAL CART. } \\
\text { PAPR=POWERED AIR PURIFYING }\end{array}$} & $\begin{array}{l}: \text { FFDC } \\
\vdots \\
\vdots \\
\vdots\end{array}$ \\
\hline $\begin{array}{l}\text { SAMPLE } \\
\text { NUMBER }\end{array}$ & $\begin{array}{l}\text { FIBER Sf } \\
\text { FIELD S }\end{array}$ & $\begin{array}{l}\text { EMPLOYEE NAME } \\
\text { SAMPLE LOCATION }\end{array}$ & $\begin{array}{l}\text { PUMP } \\
\text { NUMBER }\end{array}$ & $\begin{array}{l}\text { TIME ON } \\
\text { HR J MIN. }\end{array}$ & \begin{tabular}{|l|l} 
TIME OFF \\
HR \\
MIN.
\end{tabular} & $\begin{array}{l}\text { FLOW } \\
\text { IN?. }\end{array}$ & $\begin{array}{l}\text { RATE } \\
\text { STOP }\end{array}$ & $\begin{array}{l}\text { (LPM } \\
\text { AVG. }\end{array}$ & $\begin{array}{l}\text { TOTAL RUN } \\
\text { MINUTES }\end{array}$ & $\begin{array}{l}\text { TOTAL VOL } \\
\text { LITERS }\end{array}$ & $\begin{array}{l}\text { FIBERS/ } \\
\text { C.C. }\end{array}$ & $\begin{array}{c}\text { T.H.A. } \\
8 \text { HR }\end{array}$ & $\begin{array}{l}\text { DETECTION } \\
\text { LIMIT F/CC }\end{array}$ & $\overline{B . D . L}$ & \\
\hline 1009 & $12 / \overline{100}$ & CLEAN ROOM & 2013 & $6: \overline{28}$ & $9: \overline{45}$ & 6 & $\overline{6.2}$ & 6.1 & $\overline{197}$ & $\overline{1201.7}$ & 0.004897 & $\overline{N A}$ & $\overline{0.004081267}$ & NO & \\
\hline 1010 & $0 / \overline{100}$ & NORTHHEST OF BUILDING & 7 & $6: \overline{29}$ & $9: \overline{46}$ & $\overline{6}$ & $\overline{5.8}$ & 5.9 & $\overline{197}$ & 1162.3 & $\overline{B D L}$ & $\overline{\mathrm{NA}}$ & $\overline{0.004219615}$ & $\overline{Y E S}$ & \\
\hline 1011 & $4 / \overline{100}$ & SOUTHWEST OF BUILDING & 2017 & $6: \overline{30}$ & $9: \overline{47}$ & $\overline{6}$ & $\overline{5.8}$ & 5.9 & $\overline{197}$ & 1162.3 & $\overline{\mathrm{BDL}}$ & $\overline{N A}$ & $\overline{0.004219615}$ & $\overline{\text { YES }}$ & \\
\hline 1012 & $0 / \overline{100}$ & BLANK & & $\because$ & $\because$ & & & 0 & $\overline{0}$ & $\overline{0}$ & $\overline{E R R}$ & ERR & $\overline{\text { ERR }}$ & $\overline{\text { ERR }}$ & \\
\hline & & & . & $\because$ & $\because$ & & & 0 & $\overline{0}$ & 0 & ERR & ERR & $\overline{\text { ERR }}$ & $\overline{\text { ERR }}$ & \\
\hline & & & & $\because$ & : & & & 0 & 0 & 0 & ERR & ERR & $\overline{\text { ERR }}$ & $\overline{\text { ERR }}$ & \\
\hline & &. & & $:$ & $\because$ & & & 0 & $\overline{0}$ & 0 & ERR & ERR & $\overline{\text { ERR }}$ & $\overline{\text { ERR }}$ & \\
\hline & & & & 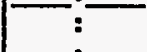 & $\because$ & & & 0 & $\overline{0}$ & $\overline{0}$ & ERR & $\overline{\text { ERR }}$ & $\overline{\text { ERR }}$ & $\overline{\text { ERR }}$ & \\
\hline & & & & : & :: & & & 0 & $\overline{0}$ & 0 & ERR & ERR & $\overline{\text { ERR }}$ & $\overline{\text { ERR }}$ & \\
\hline & & & & $:$ & $\because:$ & & & 0 & $\overline{0}$ & 0 & ERR & ERR & $\overline{E R R}$ & ERR & \\
\hline & & & & $\because$ & $\because$ & & & 0 & 0 & $\overline{0}$ & $\overline{E R R}$ & $\overline{\text { ERR }}$ & $\overline{E R R}$ & $\overline{\text { ERR }}$ & \\
\hline & 1 & & & $:$ & $:$ & & & 0 & $\overline{0}$ & 0 & $\overline{E R R}$ & $\overline{E R R}$ & $\overline{\text { ERR }}$ & $\overline{E R R}$ & \\
\hline & 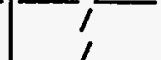 & & & $\because$ & 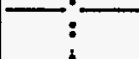 & & & 0 & $\overline{0}$ & 0 & $\overline{\text { ERR }}$ & $\overline{\text { ERR }}$ & ERR & $\overline{\text { ERR }}$ & \\
\hline & 1 & & & $:$ & $:$ & & & 0 & $\overline{0}$ & 0 & ERR & ERR & ERR & ERR & \\
\hline & 1 & & & $:$ & $:$ & & & 0 & 0 & $\overline{0}$ & ERR & ERR & ERR & $\overline{\text { ERR }}$ & \\
\hline & , & & & : & $:$ & & & 0 & 0 & $\overline{0}$ & ERR & $\bigcap^{\text {ERR }}$ & $\overline{E R R}$ & ERR & \\
\hline COMMENTS: & $\begin{array}{l}\text { FLOOR TILI } \\
\text { ASBESTOS } \\
\text { ABATEMENT } \\
\text { THAN! }\end{array}$ & $\begin{array}{l}\text { E REMOVED HITH STRAIGHT HOE. WORKER } \\
\text { SUITS, GLOVES \& RUBBER BOOTS. } \\
\text { IK YOU FOR THE OPPORTUNITY TO PROVIDE }\end{array}$ & FFOC RI & PIRATORS, & & & & & & & & & & & \\
\hline
\end{tabular}




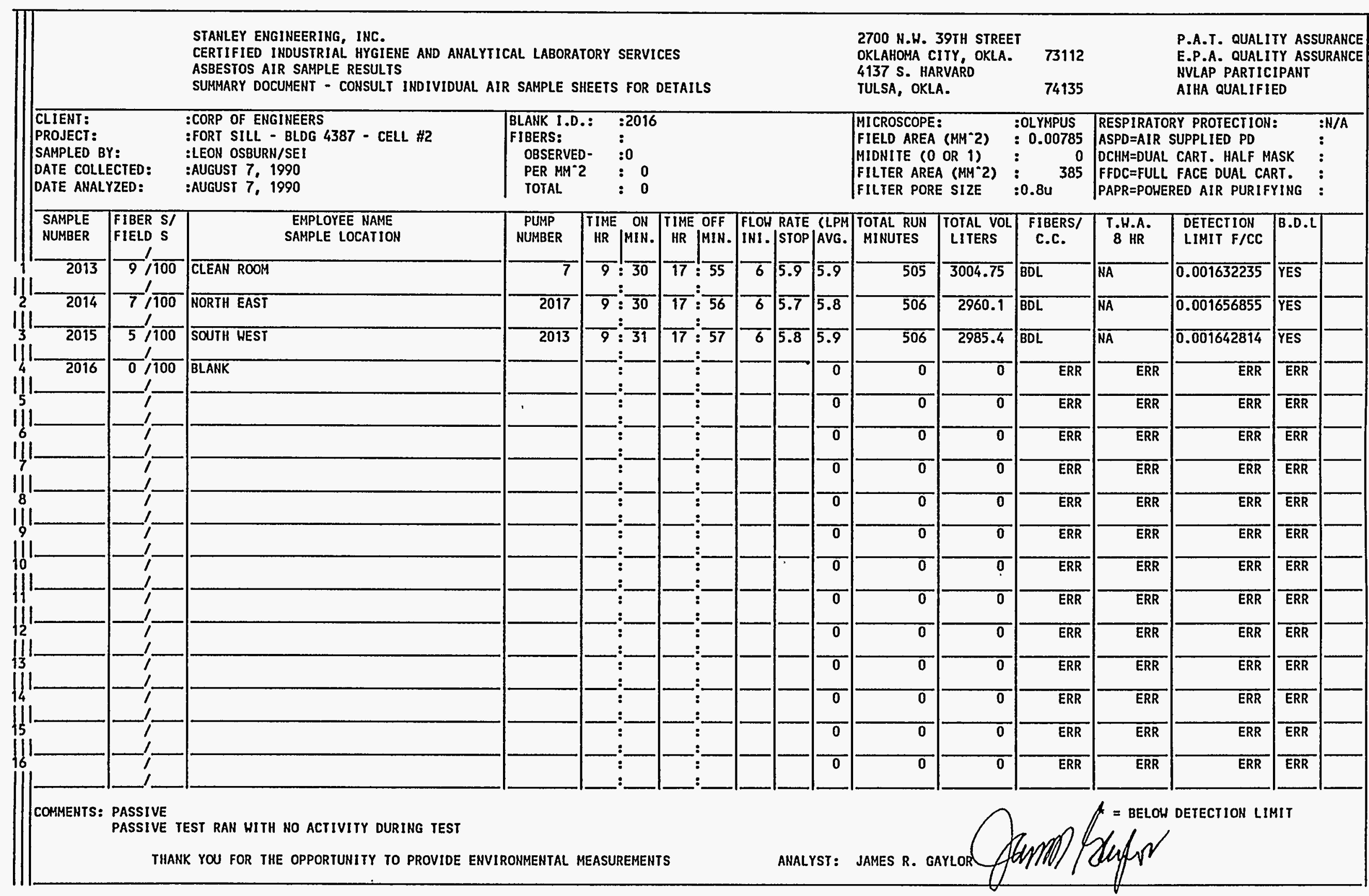




\begin{tabular}{|c|c|c|c|c|c|c|c|c|c|c|c|c|c|c|c|}
\hline & & \multicolumn{7}{|c|}{$\begin{array}{l}\text { STANLEY ENGINEERING, INC. } \\
\text { CERTIFIED INDUSTRIAL HYGIENE AND ANALYTICAL LABORATORY SERVICES } \\
\text { ASBESTSS AIR SAMPLE RESULTS } \\
\text { SUMMARY DOCUMENT - CONSULT INDIVIDUAL AIR SAMPLE SHEETS FOR DETAILS }\end{array}$} & \multicolumn{2}{|c|}{$\begin{array}{l}2700 \text { N.H. 39TH STREET } \\
\text { OKLAHOHA CITY, OKLA. } \\
4137 \text { S. HARVARD } \\
\text { TULSA, OKLA. }\end{array}$} & $\begin{array}{l}73112 \\
74135\end{array}$ & & \multicolumn{3}{|c|}{$\begin{array}{l}\text { P.A.T. QUALITY ASSURANCE } \\
\text { E.P.A. QUALITY ASSURANCE } \\
\text { NVLAP PARTICIPANT } \\
\text { AIHA QUALIFIED }\end{array}$} \\
\hline \multicolumn{2}{|c|}{$\begin{array}{l}\text { CLIENT: } \\
\text { PROJECT: } \\
\text { SAMPLED BY: } \\
\text { DATE COLLECTED: } \\
\text { DATE ANALYZED: }\end{array}$} & $\begin{array}{l}\text { :CORP OF ENGINEERS } \\
\text { :FORT SILL - BLDG } 4387 \text { - CELL \#1 } \\
\text { :LEON OSBURN/SEI } \\
\text { :AUGUST 7, } 1990 \\
\text { :AUGUST 7, } 1990\end{array}$ & \multicolumn{3}{|c|}{\begin{tabular}{|ll} 
BLANK 1.D.: & $: 1008$ \\
FIBERS: & $\vdots$ \\
OBSERVED- & $: 0$ \\
PER HM-2 & $\vdots 0$ \\
TOTAL & $: 0$
\end{tabular}} & & & & \multicolumn{2}{|c|}{$\begin{array}{l}\text { MICROSCOPE: } \\
\text { FIELD AREA (MM-2) } \\
\text { MIDNITE }(0 \text { OR } 1) \\
\text { FILTER AREA (MM-2) } \\
\text { FILTER PORE SIZE }\end{array}$} & 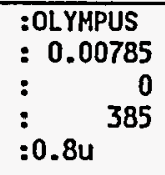 & \multicolumn{3}{|c|}{$\begin{array}{l}\text { RESPIRATORY PROTECTION: } \\
\text { ASPD=AIR SUPPLIED PD } \\
\text { DCHM=DUAL CART. HALF MASK } \\
\text { FFDC=FULL FACE DUAL CART. } \\
\text { PAPR=POHERED AIR PURIFYING }\end{array}$} & $\begin{array}{l}: N / A \\
\vdots \\
\vdots\end{array}$ \\
\hline $\begin{array}{l}\text { SAMPLE } \\
\text { NUMBER }\end{array}$ & $\begin{array}{l}\text { FIBER SI } \\
\text { FIELD S }\end{array}$ & $\begin{array}{l}\text { EMPLOYEE NAME } \\
\text { SAMPLE LOCATION }\end{array}$ & $\begin{array}{l}\text { PUMP } \\
\text { NUMBER }\end{array}$ & \begin{tabular}{|c|c|c|} 
TIME & ON \\
HR & MIN.
\end{tabular} & $\begin{array}{l}\text { TIME OFF } \\
\text { HR } \\
\text { HIM. }\end{array}$ & $\begin{array}{l}\text { FLOH } \\
\text { INI. }\end{array}$ & $\begin{array}{l}\text { RATE } \\
\text { |STOP | }\end{array}$ & $\begin{array}{l}\text { (LPM } \\
\text { JAVG. }\end{array}$ & $\begin{array}{l}\text { TOTAL RUN } \\
\text { MINUTES }\end{array}$ & $\begin{array}{l}\text { TOTAL VOL } \\
\text { LITERS }\end{array}$ & $\begin{array}{l}\text { FIBERS/ } \\
\text { C.C. }\end{array}$ & $\begin{array}{c}\text { T.H.A. } \\
8 \text { HR }\end{array}$ & $\begin{array}{l}\text { DETECTION } \\
\text { LIMIT F/CC }\end{array}$ & B.D.L & \\
\hline 1005 & $12 / 100$ & CLEAN ROOM & 7 & $16: 55$ & $.20 ! 35$ & $\overline{6}$ & $\overline{6.3}$ & 6.1 & $\overline{220}$ & 1353 & 0.004349 & $\overline{N A}$ & 0.003624877 & NO & \\
\hline 9906 & $4 / 100$ & SOUTH WEST OF ,BUILDING & 2017 & $16: \overline{58}$ & $20: \overline{36}$ & 6 & $\overline{6.5}$ & $\overline{6.2}$ & $\overline{218}$ & 1362.5 & $\overline{B D L}$ & $\overline{W A}$ & $\overline{0.003599602}$ & $\overline{Y E S}$ & \\
\hline$\overline{1007}$ & $2 / 100$ & NORTH EAST OF BUILDING & $\overline{2013}$ & $16: \overline{59}$ & $20: \overline{37}$ & 6 & 7 & 6.5 & $\overline{218}$ & $\overline{1417}$ & $\overline{B D L}$ & $\overline{\text { WA }}$ & $\overline{0.003461156}$ & $\overline{\mathrm{YES}}$ & \\
\hline 1008 & $\overline{0} / \overline{100}$ & BLANK & & $\because$ & & & & 0 & $\overline{0}$ & $\overline{0}$ & ERR & $\overline{\text { ERR }}$ & $\overline{E R R}$ & $\overline{\text { ERR }}$ & \\
\hline & & & & $:$ & $:$ & & & 0 & $\overline{0}$ & $\overline{0}$ & ERR & $\overline{\text { ERR }}$ & $\overline{E R R}$ & $\overline{\text { ERR }}$ & \\
\hline & & & & $:$ & & & & 0 & 0 & $\overline{0}$ & ERR & $\overline{\text { ERR }}$ & $\overline{\text { ERR }}$ & $\overline{\text { ERR }}$ & \\
\hline & & & & $\vdots$ & : & & & $\overline{0}$ & 0 & $\overline{0}$ & ERR & $\overline{\text { ERR }}$ & $\overline{\text { ERR }}$ & $\overline{\text { ERR }}$ & \\
\hline & ! & & & $:$ & 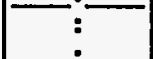 & & & 0 & 0 & $\overline{0}$ & ERR & $\overline{\text { ERR }}$ & $\overline{\text { ERR }}$ & $\overline{\text { ERR }}$ & \\
\hline & i & & & : & & & & 0 & $\overline{0}$ & $\overline{0}$ & ERR & $\overline{\text { ERR }}$ & ERR & $\overline{\text { ERR }}$ & \\
\hline & & & & : & $:$ & & & $\overline{0}$ & 0 & $\overline{0}$ & ERR & $\overline{\text { ERR }}$ & $\overline{\text { ERR }}$ & $\overline{E R R}$ & \\
\hline & 1 & & & : & $:$ & & & 0 & 0 & $\overline{0}$ & ERR & $\overline{\text { ERR }}$ & $\overline{E R R}$ & $\overline{\text { ERR }}$ & \\
\hline & 1 & & & : & : & & & 0 & 0 & $\overline{0}$ & ERR & $\overline{\text { ERR }}$ & $\overline{\text { ERR }}$ & $\overline{E R R}$ & \\
\hline & 1 & & & $\because$ & $\because$ & & & $\overline{0}$ & 0 & 0 & ERR & $\overline{\text { ERR }}$ & $\overline{\text { ERR }}$ & $\overline{\text { ERR }}$ & \\
\hline & 1 & & & : & & & & 0 & $\overline{0}$ & 0 & $\overline{E R R}$ & ERR & $\overline{E R R}$ & ERR & \\
\hline & & & & : & : & & & $\overline{0}$ & 0 & $\overline{0}$ & ERR & $\overline{\text { ERR }}$ & $\overline{E R R}$ & $\overline{\text { ERR }}$ & \\
\hline & 1 & & & $:$ & $:$ & & & 0 & 0 & $\overline{0}$ & ERR & $\overline{\text { ERR }}$ & $\overline{\text { ERR }}$ & $\overline{E R R}$ & \\
\hline COMMENT & $\begin{array}{l}\text { CELL } 11 \\
\text { PASSIVE }\end{array}$ & BEGAN. & DTHEE & IVITY & OK PLACE & IG & TEST. & & & & & & & & \\
\hline
\end{tabular}




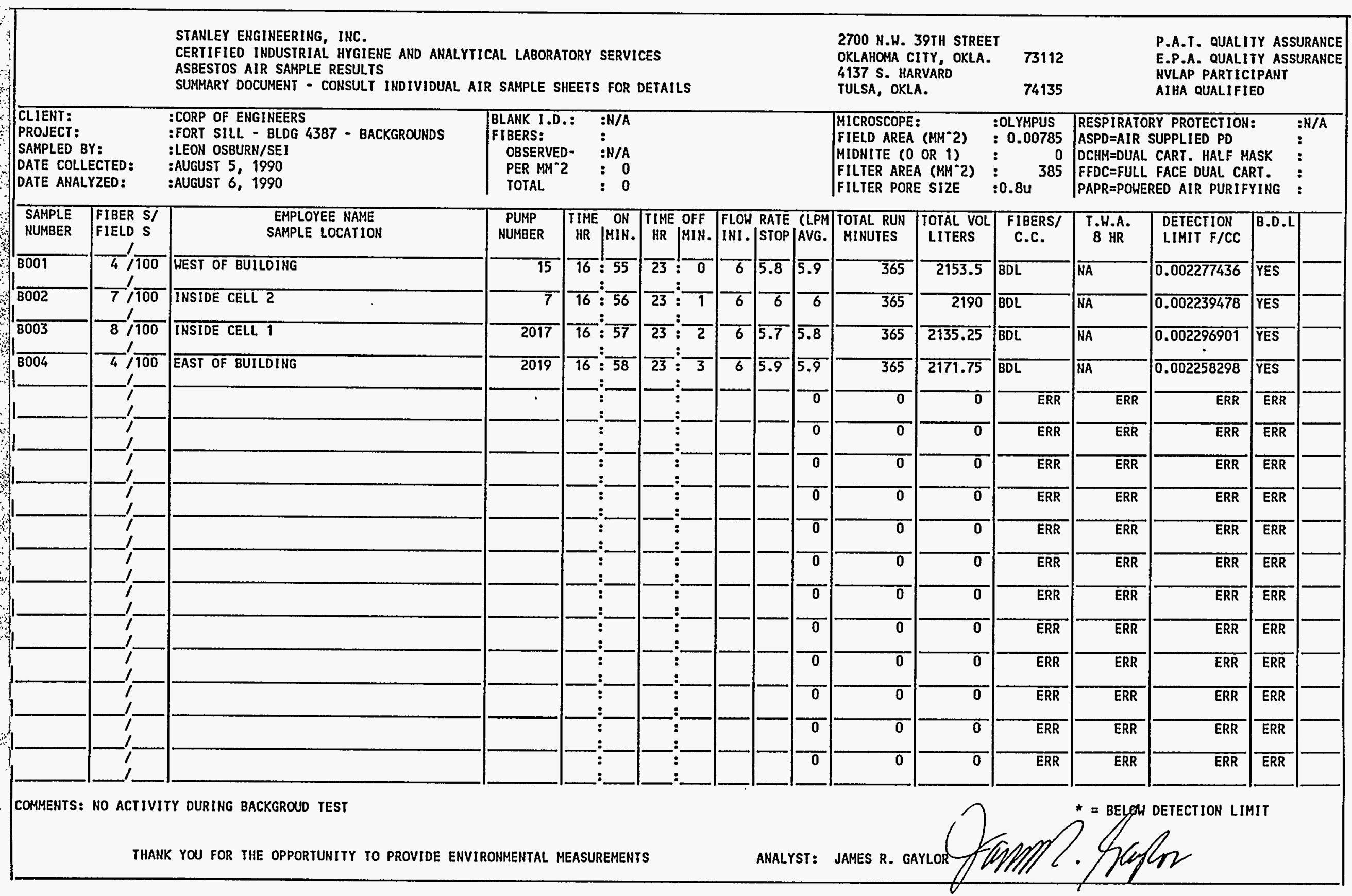




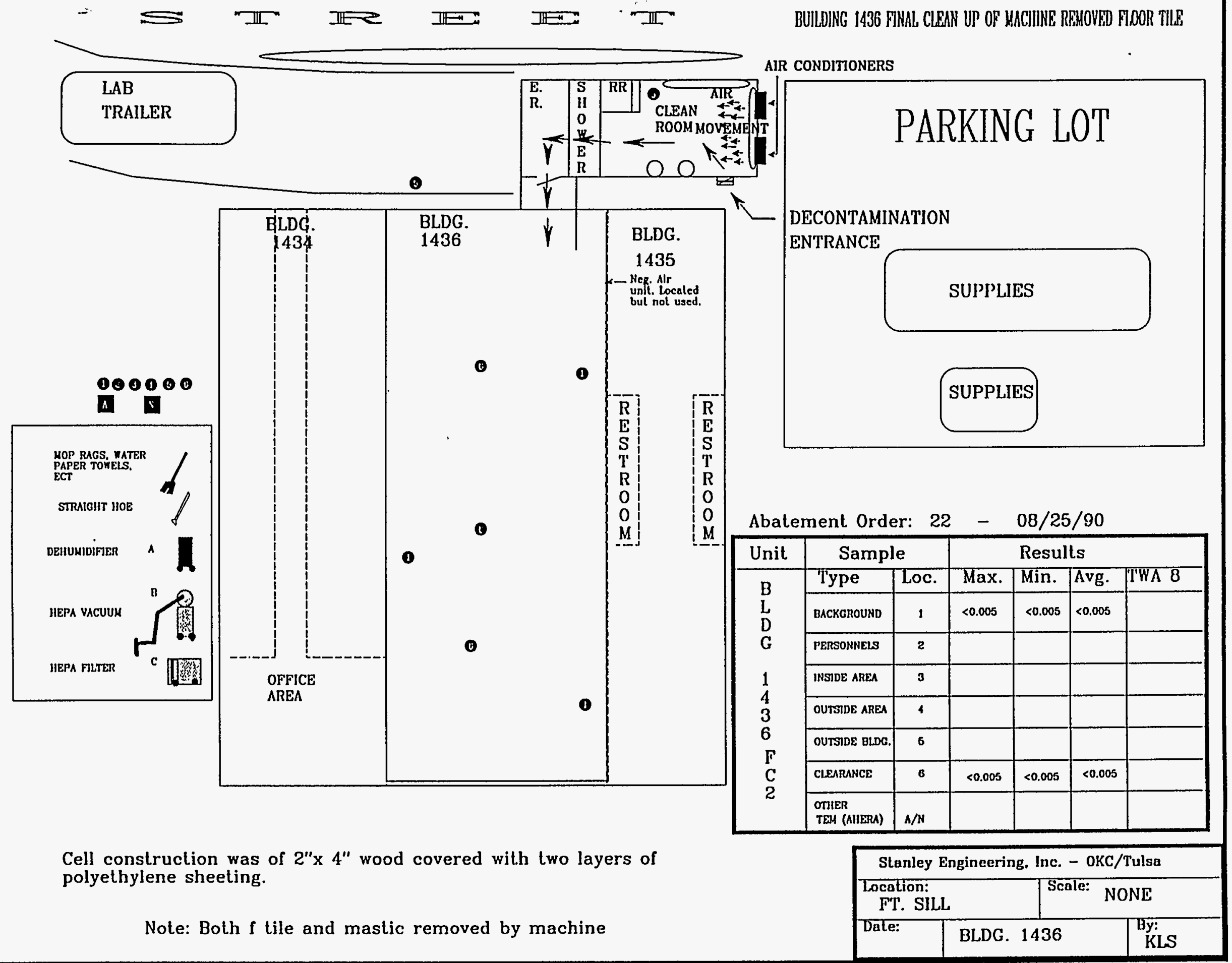




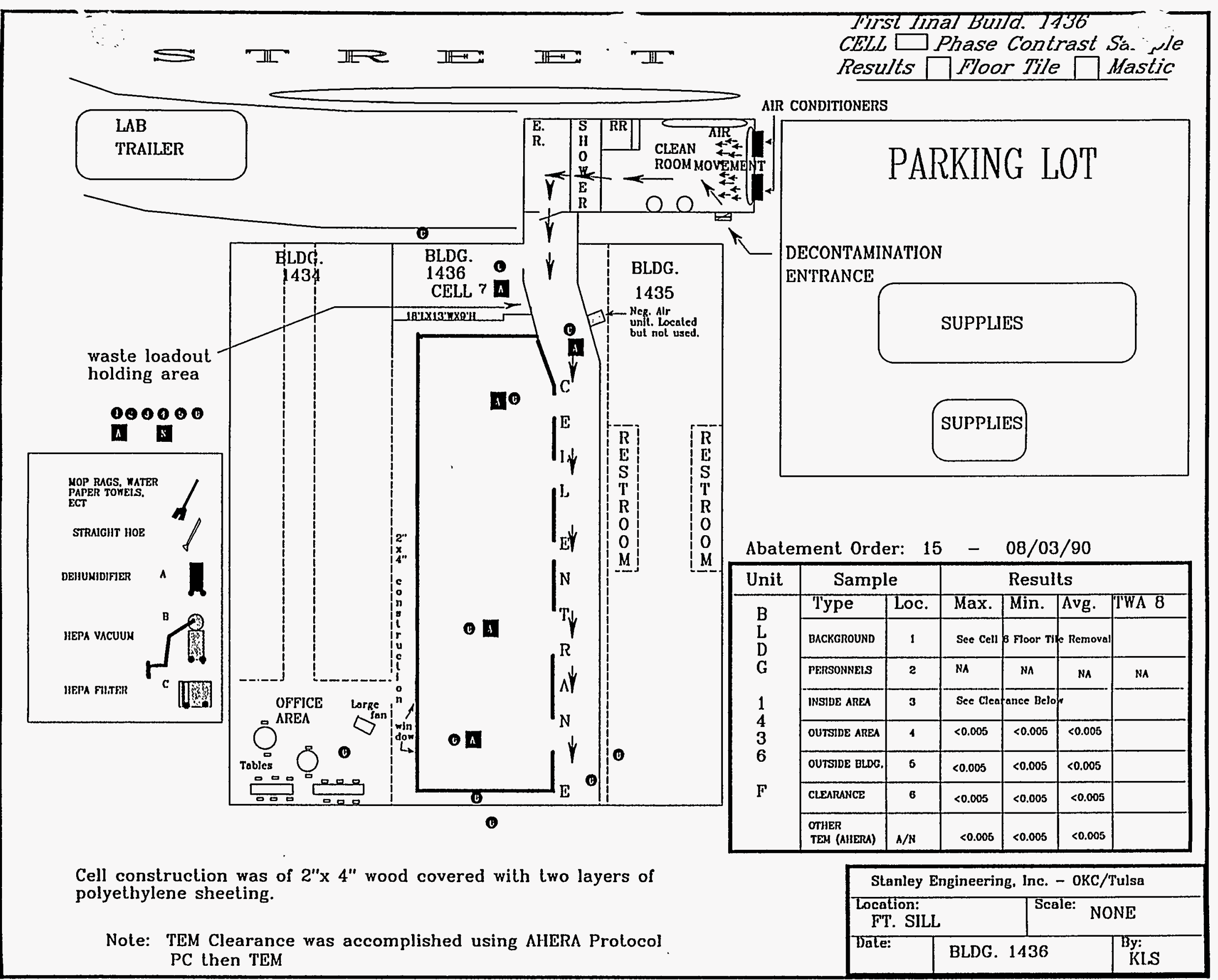




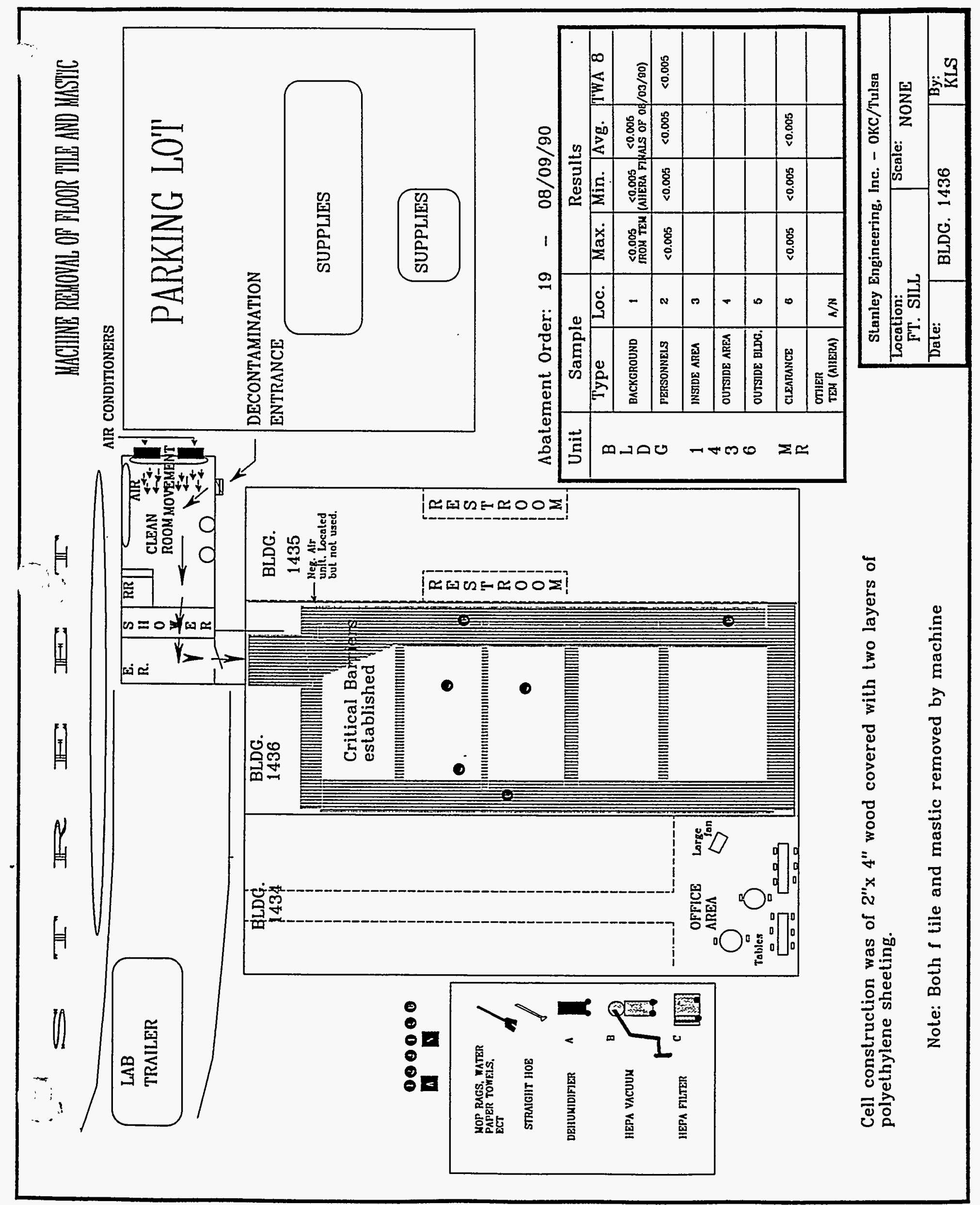




\section{REFERENCE}

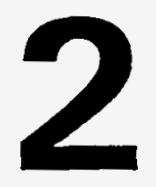




$$
\text { AUG } 22 \text { 1891 }
$$

\title{
EVALUATION OF TILE AND MASTIC REMOVAL AT FORT SILL \\ - Draft Report
}

\author{
Prepared by: \\ DAVID C. COX \& ASSOCIATES \\ 1620 22nd street, N.W. \\ Washington, D.C. 20008 \\ Under subcontract to: \\ PEI ASSOCIATES, INC. \\ 11499 Chester Road \\ Cincinnati, OH 45246 \\ EPA Contract No. 68-C0-0016 \\ PEI Subcontract No. 1045-91 \\ Work Assignment No. 0-10
}

\section{For the:}

Risk Reduction Engineering Iaboratory office of Research and Development

U.S. Environmental Protection Agency 26 West Martin Luther King Drive cincinnati, OH 45268 


\section{Study Objectives}

Vinyl asbestos floor tile and the underlying mastic adhesive were removed by a variety of methods in 7 distinct areas ("cells") in a single building at the Fort sill U.S. Army base in Oklahoma. Tile was removed by either a dry method or a wet method (there were two different wet methods, one involving soaking the tile prior to removal, and the other involving misting of the cell during removal, but both are considered "wet" removal for the purposes of this analysis). Mastic was removed either using citric acid and towels, or using a sand machine (two varieties of sand were used, but we will not distinguish between them here).

Airborne asbestos levels were monitored before and during the removals using transmission electron microscopy (TEM), with a direct preparation technique. Samples included in this evaluation were collected under passive conditions, i.e., without disturbance of the air beyond that caused by the activity being monitored.

Numerous comparisons are of interest in this analysis. First, we want to compare airborne asbestos levels during removal by each of the 4 methods to those measured before removal. Second, we want to develop confidence intervals for the average airborne asbestos levels during removal by each of the 4 methods. Third, we want to compare levels during removal by each of the 4 methods. Finally, it is of interest to determine whether airborne asbestos levels during mastic removal, irrespective of method, are different from those during tile removal, irrespective of method.

\section{Study Data}

The assignment of removal methods to cells in the study is shown in 
TABLE I

ABSIGNMENT OF REMOVAL METHODS TO CELLS

\begin{tabular}{|c|c|c|}
\hline CELI NUMBER & TILE REMOVAL & MASTIC REMOVAL \\
\hline 1 & Dry & Citric Acid/Towels \\
\hline 2 & Wet (Mist) & Sand (blasting) \\
\hline 3 & Dry & Citric Acid/Towels \\
\hline 4 & Dry & Sand (blasting) \\
\hline 5 & Dry & Sand (blasting) \\
\hline 6 & Dry & Sand (masonry) \\
\hline 7 & Wet & Citric Acid/Towels \\
\hline
\end{tabular}

Three airborne asbestos samples were taken in each cell before and after both tile and mastic removal (the cells were thoroughly cleaned after tile removal), for a total of 84 . The 3 filters from Cell 5 during tile removal were too heavily loaded to be analyzed, so that a total of 81 TEM measurements are actually available. A listing of the study database in given in the appendix.

\section{Descriptive statistics}

Figures 1, 2, and 3 give a graphical portrayal of the data. Figure 1 shows airborne asbestos levels by cell before and during tile removal; Figure 2 is the corresponding graph for mastic removal. on both figures, a "1" denotes a measurement before removal, while 
a "2" denotes a measurement during removal. Some of the "1's" represent multiple measurements close together. Figure 3 shows asbestos levels during removal for both tile and mastic removals. The measurements are coded numerically by removal method: $1=$ dry tile removal; 2 = wet tile removal; 4 = sand machine mastic removal; 5 = citric acid/towels mastic removal.

Table II is a companion to Figures 1 and 2 combined. It shows arithmetic average airborne asbestos levels by cell before and during both tile and mastic removal.

TABLE II

ARITHMETIC AVERAGE AIRBORNE ASBESTOS LEVELS (S/CC), BY CELI, BEFORE AND DURING TILE AND MASTIC REMOVAL

\begin{tabular}{|c|c|c|c|c|c|c||}
\hline \multirow{2}{*}{ CELL } & \multicolumn{3}{|c|}{ TILE } & \multicolumn{3}{c|}{ MASTIC } \\
\cline { 2 - 7 } & METHOD & BEFORE & DURING & BEFORE & DURING & METHOD \\
\hline 1 & DrY & 0 & 0.130 & 0.008 & 0.290 & Citric \\
\hline 2 & Wet & 0.001 & 0.067 & 0.022 & 0.103 & Sand \\
\hline 3 & DrY & 0 & 0.169 & 0.001 & 0.261 & Citric \\
\hline 4 & DrY & 0 & 0.125 & 0.003 & 0.925 & Sand \\
\hline 5 & DrY & 0.005 & N/A & 0.003 & 0.223 & Sand \\
\hline 6 & DrY & 0 & 0.116 & 0.001 & 0.056 & Sand \\
\hline 7 & Wet & 0 & 0.066 & 0 & 0.190 & Citric \\
\hline ALI & & 0.001 & 0.112 & 0.005 & 0.292 & \\
\hline
\end{tabular}


Several observations can be made based on Figures 1 - 3 and Table II:

* asbestos levels before removal are very low, especially those before tile removal (19 of 21 measurements before tile removal were 0 ).

* levels during removal (of tile or mastic) are typically 2 orders of magnitude higher than those before removal, with the exception of mastic removal in Cell 2, where the difference is a factor of 5 .

* with the exception of sand removal of mastic, asbestos levels in different cells with the same removal method are quite consistent. The sand mastic removal levels are very variable from cell to cell.

* Dry tile removal appears to result in higher asbestos levels than wet tile removal.

* Mastic removal appears to generally result in higher levels than tile removal, irrespective of removal methods.

\section{Statistical Tests}

\subsection{Statistical Significance of Elevation of Levels During Removal}

Table II suggests that airborne asbestos levels during both tile and mastic removal are elevated compared to levels before removal, and that there is a large relative difference between the "during" and "before" levels. In testing the statistical significance of these elevated levels, it is therefore natural to focus on the ratio of levels during removal to levels before removal. An 
immediate difficulty arises in that many of the "before" levels are zero (19 of 21 for tile removal; 10 of 21 for mastic removal). We therefore replaced zero levels by one-half the corresponding analytical sensitivity. The analytical sensitivity is the airborne asbestos concentration corresponding to a single asbestos structure found on 10 grid openings.

This procedure is conservative in the sense that plausible arguments exist for replacing the zeros with a lower value. For example, since 19 "before" levels for tile removal are zero, it might be reasonable to replace these zeros by one-half of onenineteenth of the average analytical sensitivity. The replacement procedure adopted in this analysis is therefore, by comparison with other plausible replacement procedures, less likely to lead to a conclusion that levels during removal are statistically significantly elevated compared to levels before removal.

Having replaced zeros with one-half the analytical sensitivity, the data was transformed by a natural logarithm. Average values were then calculated for the logarithms in each cell before and during tile and mastic removal. Paired t-tests were then applied to the "before" and "after" cell means for each removal method. This procedure is equivalent to applying an analysis of variance (ANOVA) model, separately for each removal method, with TIME (before and after) as a fixed effect, and CELL as a random effect. Thus, we are testing the difference between before and after removal levels assuming that the cells actually tested represent a random sample from the universe of cells to which the method could be applied.

Table III shows the t-statistic, the number of degrees of freedom (one less than the number of cells available for the comparison), and the one-sided p-value for testing the hypothesis that the geometric mean level during removal exceeds the geometric mean level before removal. 
TABLE III

PAIRED T-TESTS FOR: GEOMETRIC MEAN AIRBORNE ABBESTOB LEVEL DURING REMOVAL GREATER THAN GEOMETRIC MEAN IEVEL BEFORE REMOVAL

\begin{tabular}{|l|c|c|c|}
\hline REMOVAL METHOD & T-STATISTIC & DEGREES OF FREEDOM & P-VALUE \\
\hline Dry Tile & 30.02 & 3 & $<0.0001$ \\
\hline Wet Tile & 33.83 & 1 & 0.0094 \\
\hline Citric Mastic & 17.63 & 2 & 0.0016 \\
\hline Sand Mastic & 4.82 & 3 & 0.0085 \\
\hline
\end{tabular}

When 4 simultaneous comparisons are made, an overall significance level of 0.05 is achieved when significance for individual comparisons is recognized for $p$-values less than $0.05 / 4=0.0125$. By this criterion, all comparisons in the table are significant. We conclude that airborne asbestos levels during removal of tile or mastic are elevated as compared to levels before removal irrespective of removal method.

\subsection{Confidence Intervals for Average Levels During Removal}

These confidence intervals can be calculated for both the arithmetic and the geometric mean levels during removal. The calculation for geometric means is formally the same as the calculation for arithmetic means, except that it is carried out on the log scale and the results exponentiated back to the measurement scale. The confidence intervals in both cases are based on the $t-$ statistic with the appropriate number of degrees of freedom, which is one less than the number of cells in which the method was applied. Thus, the confidence interval calculation accounts for 
the variability between cells, and assumes that the cells are a random sample of all cells to which the methods could be applied. Table IV shows the point estimates (arithmetic or geometric means) and associated $95 \%$ confidence intervals.

TABLE IV

ESTIMATED ARITHMETIC AND GEOMETRIC MEAN AIRBORNE ABBESTOS LEVELبS (8/CC) DURING TILE AND MASTIC REMOVAL, BY REMOVAL METHOD, FITH ASSOCIATED 95\% CONFIDENCE INTERVALS

\begin{tabular}{||c|c|c|c|c||}
\hline $\begin{array}{c}\text { REMOVAL } \\
\text { METHOD }\end{array}$ & $\begin{array}{c}\text { ARITHMETIC } \\
\text { MEAN }\end{array}$ & $\begin{array}{c}\text { 95\% } \\
\text { CONFIDENCE } \\
\text { INTERVAL }\end{array}$ & $\begin{array}{c}\text { GEOMETRIC } \\
\text { MEAN }\end{array}$ & $\begin{array}{c}\text { 95\% } \\
\text { CONFIDENCE } \\
\text { INTERVAI }\end{array}$ \\
\hline $\begin{array}{c}\text { DIY } \\
\text { Tile }\end{array}$ & 0.135 & $0.098-0.173$ & 0.132 & $0.101-0.173$ \\
\hline $\begin{array}{c}\text { Wet } \\
\text { Tile }\end{array}$ & 0.066 & $0.058-0.075$ & 0.061 & $0.048-0.076$ \\
\hline $\begin{array}{c}\text { Citric } \\
\text { Acid }\end{array}$ & 0.247 & $0.119-0.374$ & 0.241 & $0.141-0.410$ \\
\hline $\begin{array}{c}\text { Sand } \\
\text { Machine }\end{array}$ & 0.326 & $-0.317-0.970$ & 0.183 & $0.027-1.248$ \\
\hline
\end{tabular}

The arithmetic and geometric means, and associated confidence intervals, are in close agreement for dry and wet tile removal, and for mastic removal by citric acid/towels. For sand machine removal of mastic, however, the situation is different. The arithmetic mean is almost twice the geometric mean, indicating a positively 
skewed distribution. The confidence interval for the arithmetic mean includes the value 0 , again indicating a skewed distribution with high variability. The confidence interval for the geometric mean covers the range of confidence intervals for the other three methods combined. The major contributor to this variability in levels during sand machine removal can be found in cell 4, where the levels are far higher than in other cells.

\subsection{Comparison of Levels During Removal by Different Methods}

With 4 removal methods, there are 6 pairwise comparisons of airborne asbestos levels during removal. As the study design turned out, only 2 of the 6 comparisons can be based on more than a single matched cell: Cells 1 and 3 for the Dry Tile - Citric Mastic comparison; Cells 4 and 6 for the Dry Tile - Sand Mastic comparison. Clearly, it is better to compare Dry Tile in 4 cells to citric Mastic in 3 cells, without pairing the methods by cell, than to restrict the comparison to the 2 paired cells. The same is true for Dry Tile - Sand Mastic (4 cells each for the unpaired comparison versus 2 for the paired comparison). Accordingly, twosample t-tests were carried out on the log-transformed data. For each comparison, an F-test of equality of variance between the two groups was first performed. If the $F$ statistic was significant at the $5 \%$ level, the unequal variance version of the two-sample t-test was used. Otherwise, the equal variance version was used.

Table $\mathrm{V}$ shows the results of the two-sample t-tests. In assessing the significance of these results, the appropriate p-value for an overall significance level of 0.05 is, by the Bonferroni method, $0.05 / 6=0.008$, since 6 simultaneous comparisons are being made. By this criterion, the Dry Tile/Citric Mastic comparison is barely non-significant. Since the Bonferroni method is conservative, and an argument can be made for basing these comparisons on a one-sided rather than a two-sided test (which would cut the p-values in half), we will count this comparison as significant. Hence, we 
conclude that airborne asbestos levels during Citric Acid/Towels removal of mastic are statistically significantly higher than those during tile removal by either a dry or a wet method. Also, Dry Tile removal results in significantly higher levels than wet Tile removal. There are no statistically significant differences between Sand Removal of mastic and Dry or Wet tile removal, or Citric Acid removal of mastic.

TABLE V

TWO-SAMPLE T-TESTS FOR EQOALITY OF GEOMETRIC MEAN AIRBORNE ASBESTOS LEVELS DURING REMOVAI OF TILE OR MASTIC BY VARIOUS METHODS

\begin{tabular}{|c|c|l|}
\hline COMPARISON & P-VALUE (2-SIDED) & T-TEST VERSION \\
\hline Dry Tile/Wet Tile & 0.004 & Equal Variance \\
\hline Dry Tile/Citric Mastic & 0.009 & Equal Variance \\
\hline Dry Tile/Sand Mastic & 0.63 & Unequal Variance \\
\hline Wet Tile/Citric Mastic & 0.003 & Equal Variance \\
\hline Wet Tile/Sand Mastic & 0.17 & Unequal Variance \\
\hline Citric Mastic/Sand Mastic & 0.69 & Unequal Variance \\
\hline
\end{tabular}

\subsection{Overall Comparison of Tile and Mastic Removal}

An ANOVA model with REMOVAL TYPE (tile or mastic) as a fixed effect and CELL $(1,2,3,4,6,7)$ as a random effect was fit to the "during" data on both the measurement and the log scale. In neither case was REMOVAL TYPE a statistically significant effect. Thus, the apparent elevation of airborne asbestos levels during mastic removal as compared to tile removal, evident in Figure 3 , is not 
statistically significant. This negative conclusion is due to the variability in asbestos levels between cells during sand machine removal of mastic.

\section{Conclusions}

The principal conclusions of this analysis are as follows:

(i) Airborne asbestos levels during removal of tile or mastic were statistically significantly elevated compared to levels before removal, irrespective of removal method.

(ii) Airborne asbestos levels before removal were very low, averaging $0.001 \mathrm{~s} / \mathrm{cc}$ before tile removal, and $0.005 \mathrm{~s} / \mathrm{cc}$ before mastic removal.

(iii) Airborne asbestos levels averaged $0.135 \mathrm{~s} / \mathrm{cc}$ during dry tile removal, $0.066 \mathrm{~s} / \mathrm{cc}$ during wet tile removal, 0.247 $s / c c$ during removal of mastic using citric acid and towels, and $0.326 \mathrm{~s} / \mathrm{cc}$ during sand machine mastic removal.

(iv) Airborne asbestos levels during removal of mastic by sand machine were much more variable than levels during removal of tile or mastic by the other methods.

(v) Airborne asbestos levels were statistically significantly higher during citric acid removal of mastic as compared to tile removal by either method. Levels during dry tile removal were significantly higher than during wet tile removal. However, the differences between sand machine removal of mastic and all other removal methods were not significant, because of the much larger variability of the sand machine data. 
ASBESTOS LEVELS FOR TILE REMOVAL ( 1 = BEFORE; 2 = DURING)

A
I
R
B
O
R
N
E

I

$A$
$S$
$B$
$E$
$S$
$T$
$O$
$S$

c

$\mathrm{N}$

C

$\dot{\mathrm{R}}$

A

T

(1)

N

S

I

$\mathrm{s}$

I

c

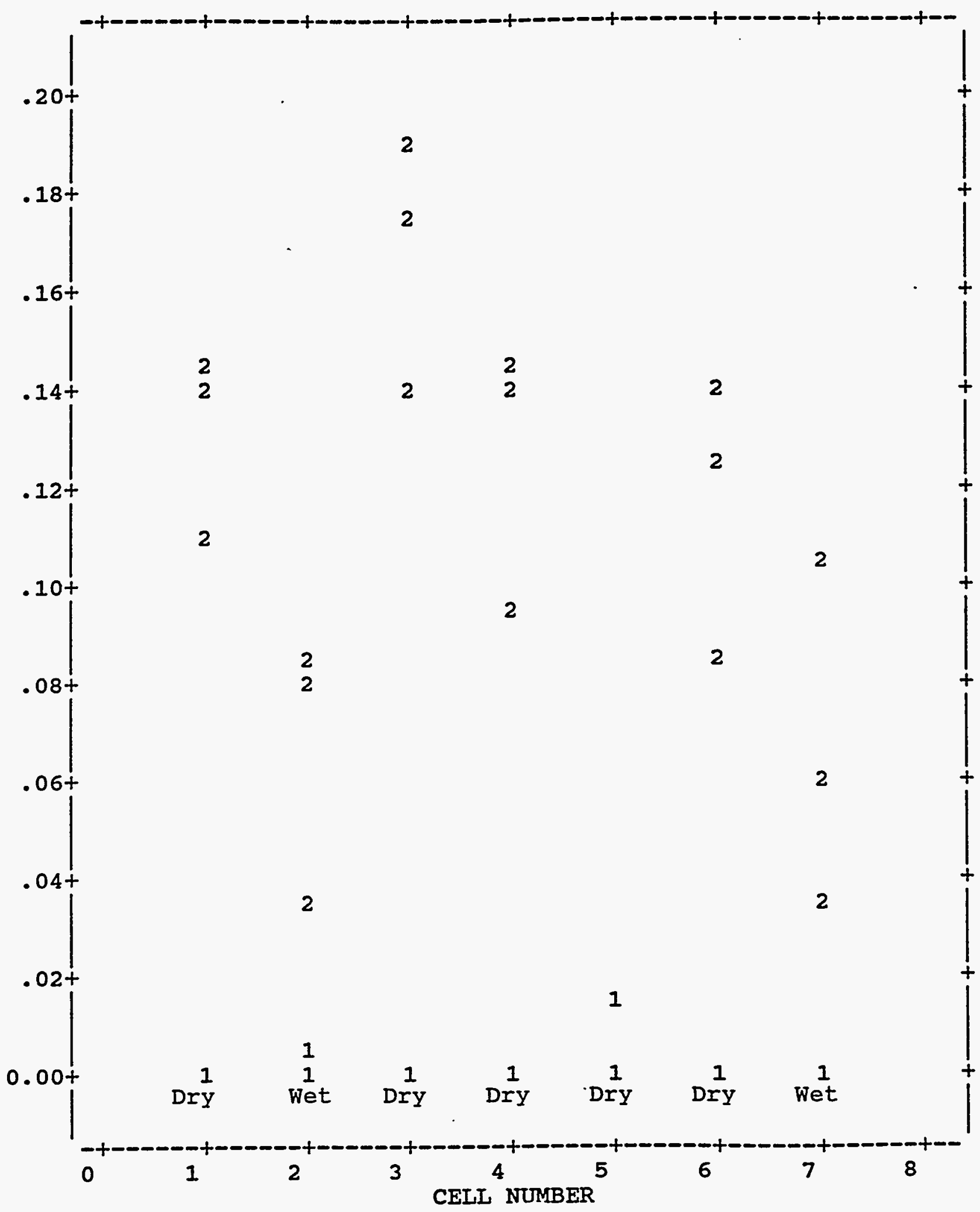

Figure 1 
ASBESTOS LEVELS FOR MASTIC REMOVAI ( 1 = BEFORE; 2 = DURING)

A
I
R
B
O
R
N
E

$1.20 t$

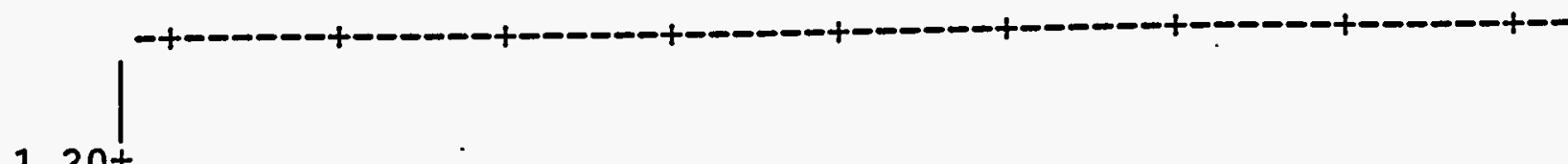

2

$1.08+$

R

A

$S$

B

E

S

T

S

c

0

N
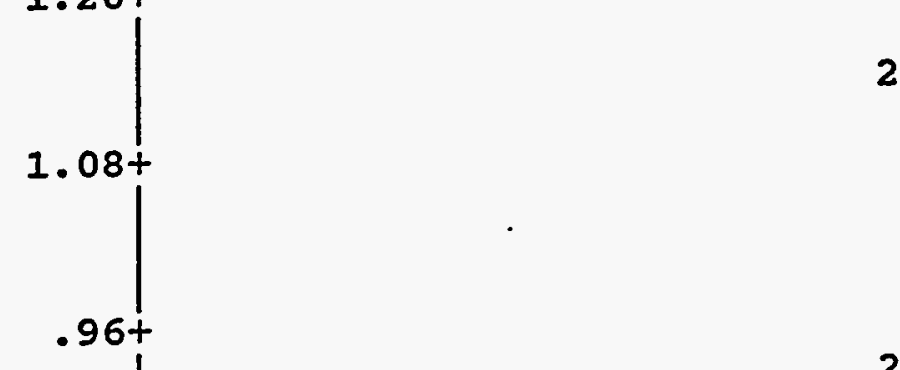

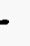
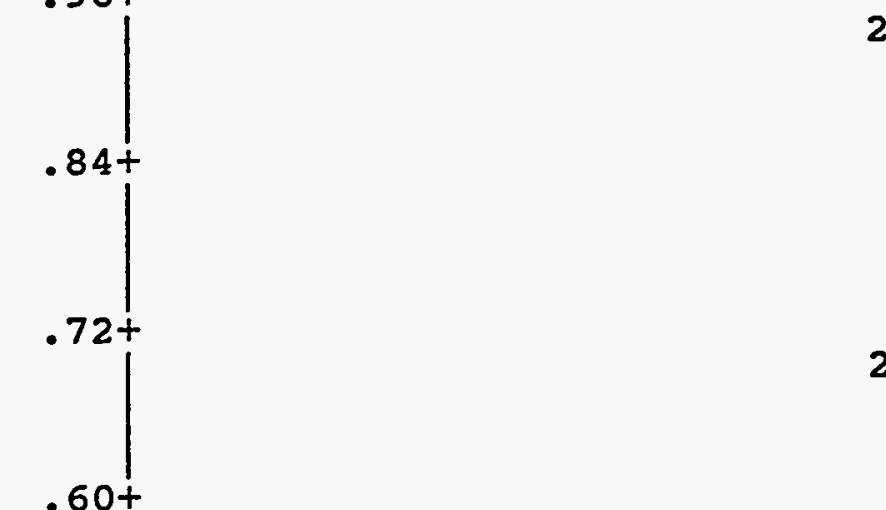

2

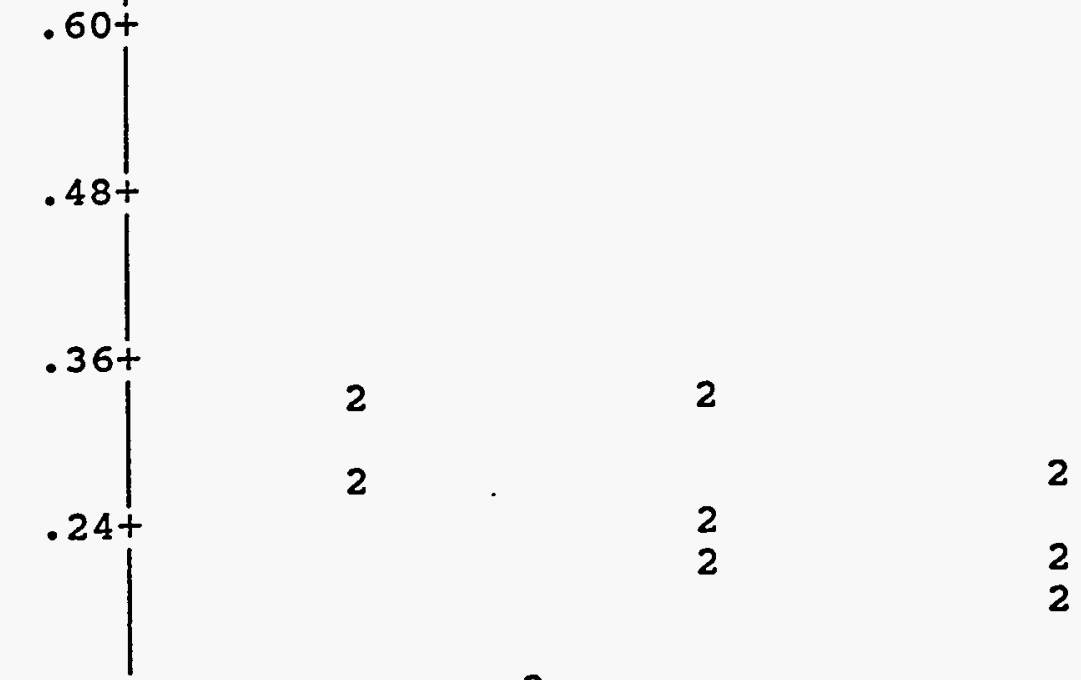

2

I

s

l

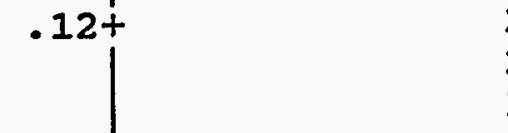

2
2
1

2

2

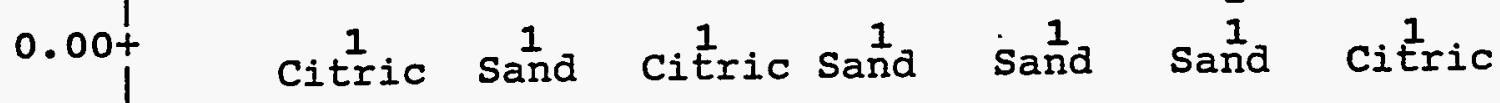

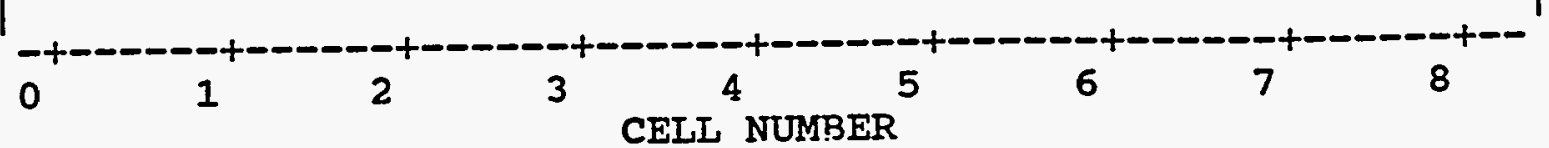

Figure 2 
IEVELS DURING REMOVAL (1=DRY; 2=WET; 4=SAND; 5=CITRIC)

A
I
R
B
O
R
N
E

A

$S$

B

E

S

T

S

c

0

N

$T$

R

A

T

I

N

$\mathrm{S}$

I

$\stackrel{I}{N}$

s

l

C

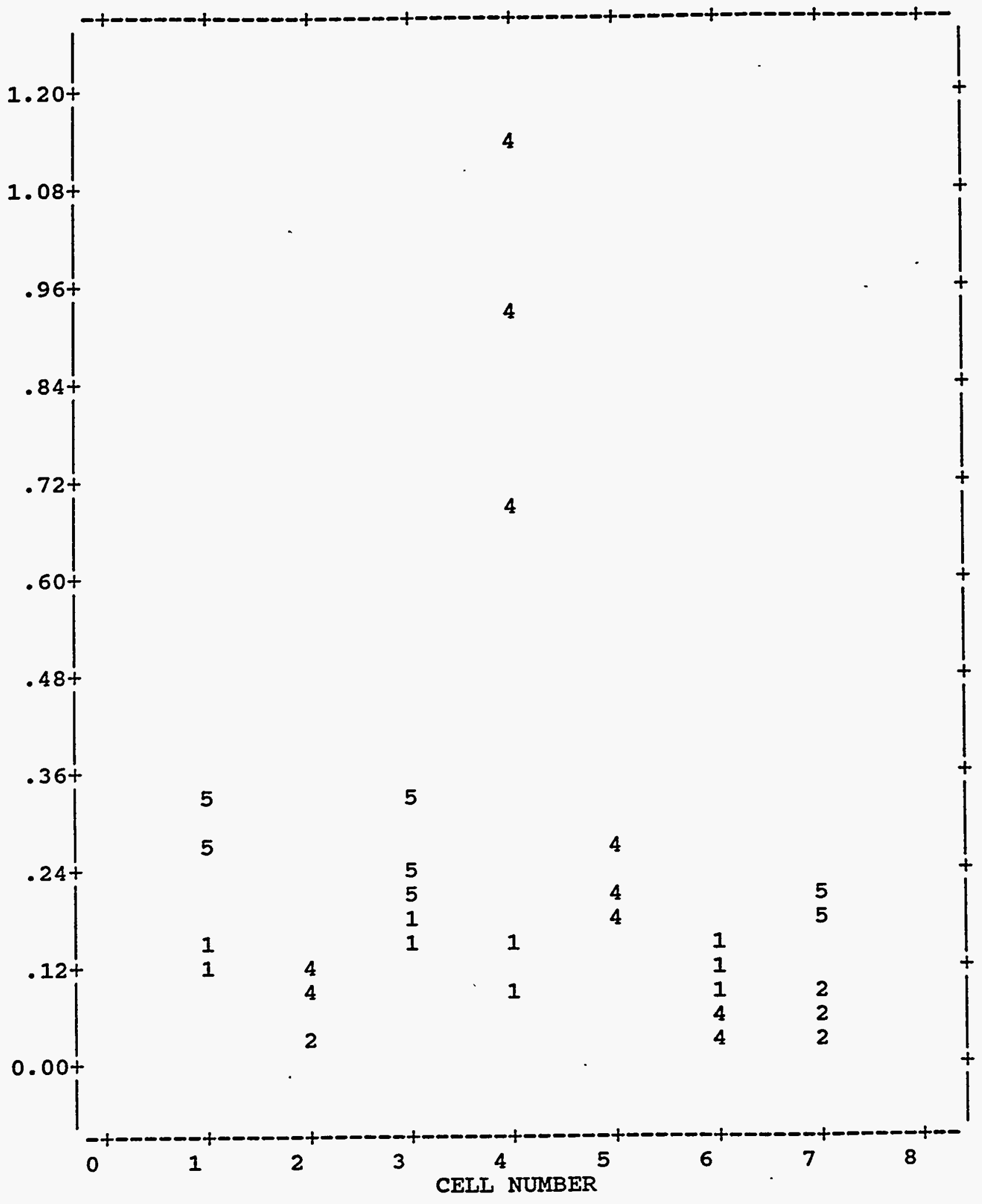

Figure 3 
APPENDIX

FORT SIIL ANALYSIS DATASET

\begin{tabular}{|c|c|c|c|c|c|c|}
\hline SAMPIEID & LOCATION & TIME & AIRCONC $(S / C C)$ & CELL & REMTYPE & REM METH \\
\hline 1057 & EAST & PRE & 0 & 1 & Tile & Dry \\
\hline 1058 & CENTER & PRE & 0 & 1 & Tile & Dry \\
\hline 1059 & WEST & PRE & 0 & 1 & Tile & Dry \\
\hline 1061 & EAST & DUR & 0.143 & 1 & Tile & Dry \\
\hline 1062 & CENTER & DUR & 0.138 & 1 & Tile & Dry \\
\hline 1063 & WEST & DUR & 0.11 & 1 & Tile & Dry \\
\hline 1105 & EAST & PRE & 0.014 & 1 & Mastic & Citric Acid \\
\hline 1106 & CENTER & PRE & 0 & 1 & Mastic & Citric Acid \\
\hline 1107 & WEST & PRE & 0.009 & 1 & Mastic & citric Acid \\
\hline 1113 & EAST & DUR & 0.345 & 1 & Mastic & Citric Acid \\
\hline 1114 & CENTER & DUR & 0.267 & 1 & Mastic & Citric Acid \\
\hline 1115 & WEST & DUR & 0.257 & 1 & Mastic & Citric Acid \\
\hline 2025 & EAST & PRE & 0 & 2 & Tile & Mist \\
\hline 2026 & CENTER & PRE & 0.004 & 2 & Tile & Mist \\
\hline 2027 & WEST & PRE & 0 & 2 & Tile & Mist \\
\hline 2029 & EAST & DUR & 0.082 & 2 & Tile & Mist \\
\hline 2030 & CENTER & DUR & 0.034 & 2 & Tile & Mist \\
\hline 2031 & WEST & DUR & 0.085 & 2 & Tile & Mist \\
\hline 2093 & EAST & PRE & 0.009 & 2 & Mastic & Sand - blasting \\
\hline 2094 & CENTER & PRE & 0.049 & 2 & Mastic & Sand - blasting \\
\hline 2095 & WEST & PRE & 0.009 & 2 & Mastic & Sand - blasting \\
\hline 2097 & EAST & DUR & 0.085 & 2 & Mastic & Sand - blasting \\
\hline 2098 & CENTER & DUR & 0.111 & 2 & Mastic & Sand - blasting \\
\hline 2099 & WEST & DUR & 0.114 & 2 & Mastic & Sand - blasting \\
\hline 3041 & EAST & PRE & 0 & 3 & Tile & Dry \\
\hline 3042 & CENTER & PRE & 0 & 3 & Tile & Dry \\
\hline 3043 & WEST & PRE & 0 & 3 & Tile & Dry \\
\hline 3053 & EAST & DUR & 0.191 & 3 & Tile & Dry \\
\hline 3054 & CENTER & DUR & 0.176 & 3 & Tile & Dry \\
\hline 3055 & WEST & DUR & 0.141 & 3 & Tile & Dry \\
\hline 3073 & EAST & PRE & 0 & 3 & Mastic & Citric Acid \\
\hline 3074 & CENTER & PRE & 0.004 & 3 & Mastic & Citric Acid \\
\hline 3075 & WEST & PRE & 0 & 3 & Mastic & Citric Acid \\
\hline 3077 & EAST & DUR & 0.2225 & 3 & Mastic & Citric Acid \\
\hline 3078 & CENTER & DUR & 0.331 & 3 & Mastic & Citric Acid \\
\hline 3079 & WEST & DUR & 0.228 & 3 & Mastic & Citric Acid \\
\hline 4017 & EAST & PRE & 0 & 4 & Tile & Dry \\
\hline 4018 & CENTER & PRE & 0 & 4 & Tile & Dry \\
\hline 4019 & WEST & PRE & 0 & 4 & Tile & Dry \\
\hline 4021 & EAST & DUR & 0.093 & 4 & Tile & Dry \\
\hline 4022 & CENTER & DUR & 0.145 & 4 & Tile & Dry \\
\hline 4023 & WEST & DUR & 0.138 & 4 & Tile & Dry \\
\hline 4125 & EAST & PRE & 0.005 & 4 & Mastic & Sand - blas \\
\hline 4126 & CENTER & PRE & 0 & 4 & Mastic & Sand - bla \\
\hline
\end{tabular}




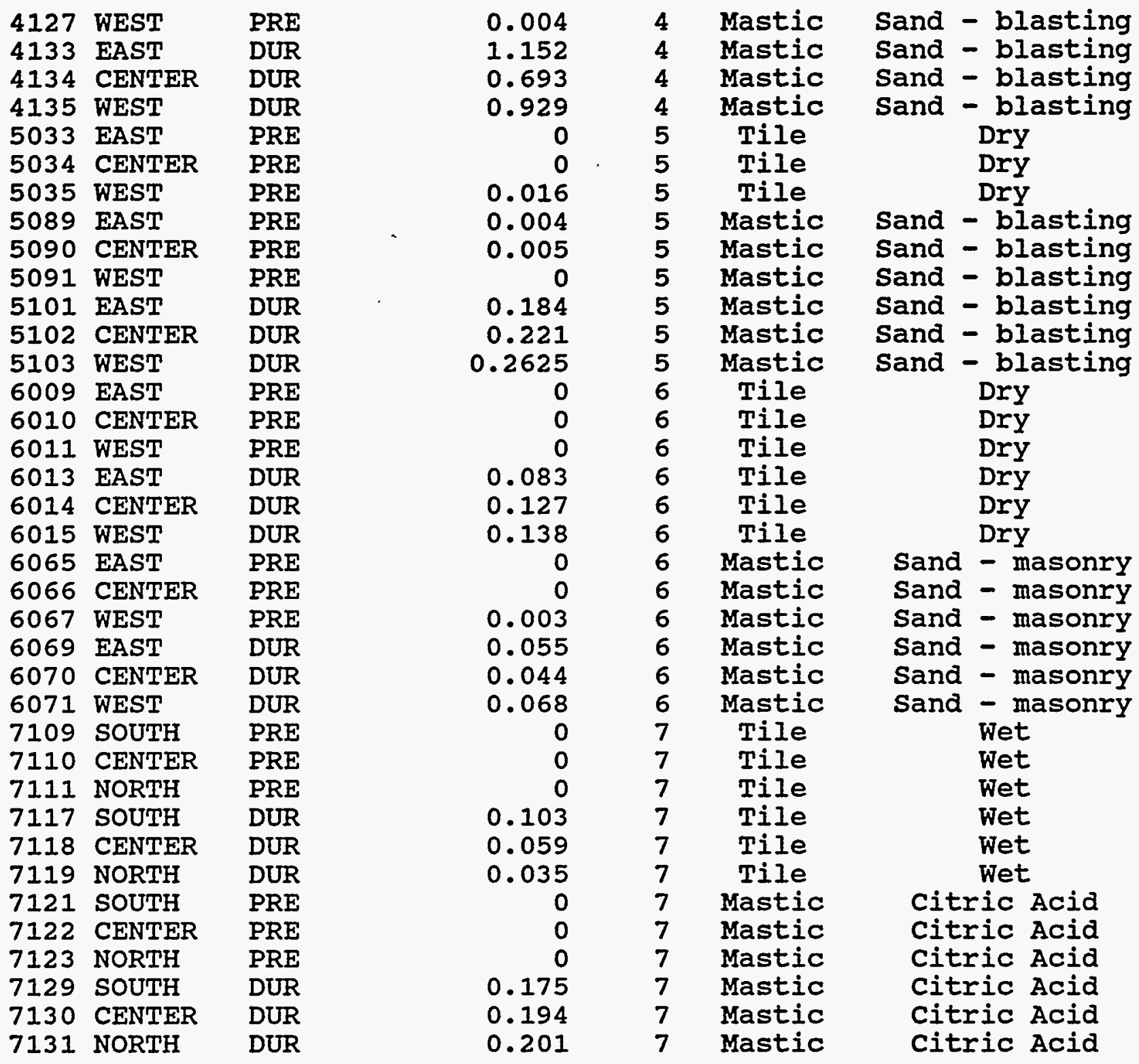

